\title{
Allergic bronchopulmonary aspergillosis (ABPA)
}

Crameri, R

DOI: https://doi.org/10.1111/pai.12563

Posted at the Zurich Open Repository and Archive, University of Zurich

ZORA URL: https://doi.org/10.5167/uzh-140904

Book Section

Published Version

Originally published at:

Crameri, R (2016). Allergic bronchopulmonary aspergillosis (ABPA). In: Matricardi, P M; Kleine-Tebbe, J; Hoffmann, H J; Valenta, R; Ollert, M. EAACI Molecular Allergology User's Guide. Zurich: European Academy of Allergy and Clinical Immunology, 131-136.

DOI: https://doi.org/10.1111/pai.12563 

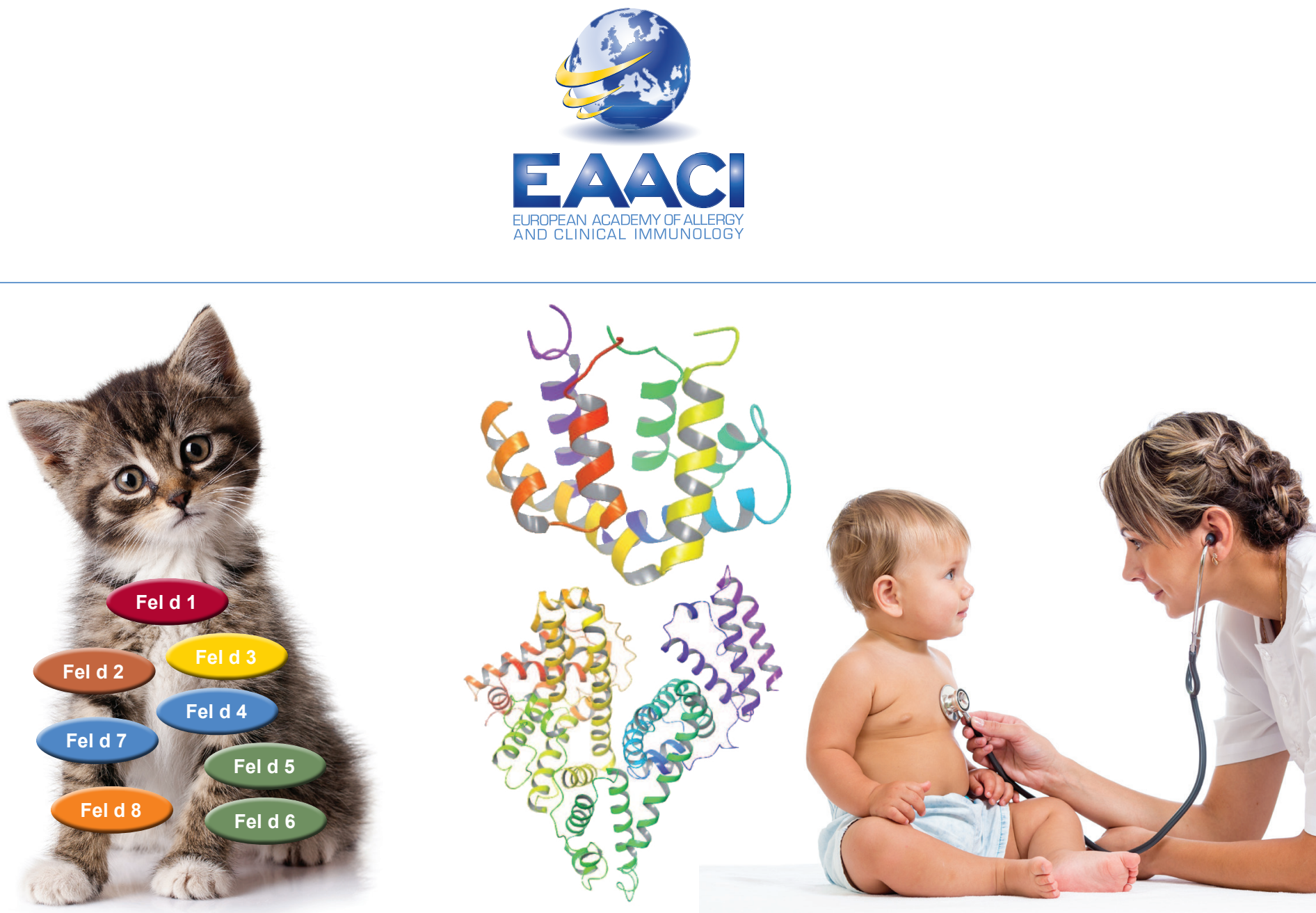

\section{EAACI}

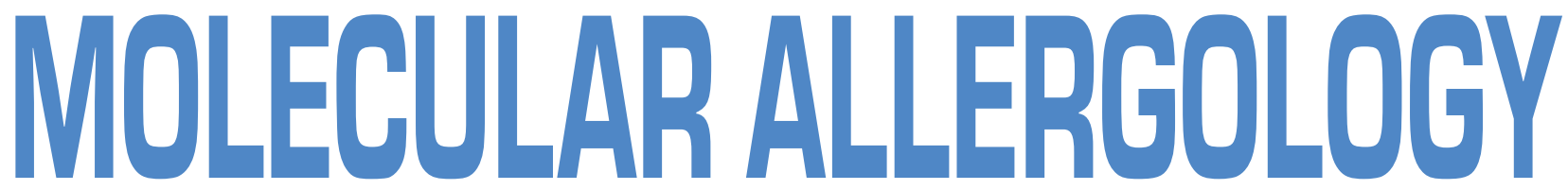

\section{USER'S GUIDE}

Published by the European Academy of Allergy and Clinical Immunology 2016 


\section{PART A}

MOLECULAR

ALLERGOLOGY: GENERAL CONCEPTS

PART B

USING MOLECULAR ALLERGOLOGY IN THE CLINICAL PRACTICE
Allergology from Extracts to Molecules: Integrating Tradition with Innovation

Allergens and the Allergenic Composition of Source Materials

Molecular Allergy Diagnostics in Clinical

Practice

Methods for IgE Antibody Testing: Singleplex and Multiplex Assays

Skin test, Basophil Activation Test (BAT) and Provocation Tests Allergen Families and Databases

Tree Pollen Allergy

Grass Pollen Allergy

Weed Pollen Allergy

Dust Mite Allergy

Cockroach Allergy

Allergy to Cat, Dog and Horse

Allergic Bronchopulmonary Aspergillosis (ABPA)

Role of Microbial Allergens in Atopic Dermatitis

Molecular Diagnostics in the Management of Food Allergy

Cow's Milk Allergy

Allergy to Egg

Allergy to Fish

Allergy to Crustacean and Mollusks (Shellfish)

Allergy to Mammalian Meat

Allergy to Fruits and Vegetables

Wheat Allergy

Soy Allergy

Peanut Allergy

Tree Nut and Seed Allergy

Bee Venom Allergy

Vespid Allergy

Latex Allergy

Allergy to Laboratory Animals

Profilins

PR-10-like Allergens

Non-specific Lipid Transfer Proteins (nsLTPs)

Serum Albumins

Tropomyosins

Polcalcins

Lipocalins

Parvalbumins
Paolo Maria Matricardi, Jörg Kleine-Tebbe, Hans Jürgen Hoffmann, Rudolf Valenta, Markus Ollert

Ronald van Ree, Rob C. Aalberse

Jörg Kleine-Tebbe, Thilo Jakob

Robert G. Hamilton, Jörg Kleine-Tebbe

Nikos Douladiris, Peter Korosec, Hans Jürgen Hoffmann

Heimo Breiteneder

Enrico Scala, Riccardo Asero, Verena Niederberger

Janet M. Davies, Paolo M. Matricardi, Johannes Schmid

Gabriele Gadermaier, Janet M. Davies, Thomas Hawranek, Richard Weber

Thomas Platts-Mills, Luis Caraballo

Luis Caraballo, Thomas Platts-Mills

Marianne van Hage, Christiane Hilger

Reto Crameri

Martin Glatz, Philipp P. Bosshard, Reto Crameri, Peter Schmid-Grendelmeier

Helen A. Brough, Gideon Lack, Jörg Kleine-

Tebbe, Antonella Muraro

Anna Nowak-Wegrzyn, Antonella Muraro

Philippe A. Eigenmann, Jean-Christoph Caubet, Antonella Muraro

Lars K. Poulsen, Martine Morisset, Annette Kuehn

Andreas L. Lopata, Sandip D. Kamath

Marianne van Hage, Tilo Biedermann,

Thomas A.E. Platts-Mills

Barbara Ballmer-Weber, Karin Hoffmann-

Sommergruber

Mika Mäkelä

Jörg Kleine-Tebbe, Kirsten Beyer, Motohiro Ebisawa

Jörg Kleine-Tebbe, Kirsten Beyer, Motohiro Ebisawa

Edward Knol, Magnus Wickman

Markus Ollert, Simon Blank

M. Beatrice Bilò, Markus Ollert

Monika Raulf

Monika Raulf

\section{Riccardo Asero, Domingo Barber}

Heimo Breiteneder, Jörg Kleine-Tebbe

Elide Pastorello, Domingo Barber

Christiane Hilger

Luis Caraballo

Joaquín Sastre

Christiane Hilger

Annette Kuehn, Lars K. Poulsen, Martine Morisset

\section{PART D}

100 Important Allergenic Molecules and their Characteristics

Glossary of Immunological Assays in Molecular Allergology
Stephanie Hofmaier, Karin HoffmannSommergruber, Heimo Breiteneder 

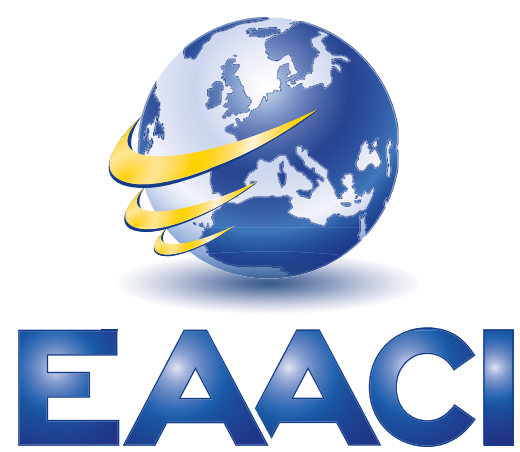

EUROPEAN ACADEMY OF ALLERGY AND CLINICAL IMMUNOLOGY
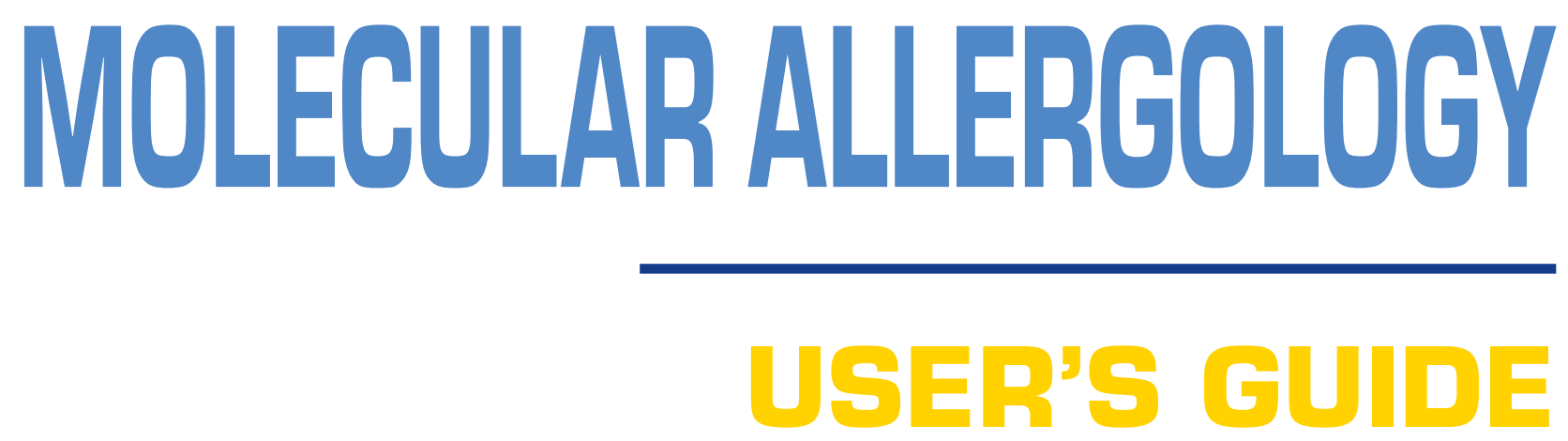

Editors

Paolo Maria Matricardi Jörg Kleine-Tebbe Hans Jürgen Hoffmann Rudolf Valenta Markus Ollert

Published by the European Academy of Allergy and Clinical Immunology 2016 
(c) John Wiley \& Sons A/S. Published by John Wiley \& Sons Ltd

The content of this book was first published as a supplement of the journal

„Pediatric Allergy and Immunology“ (Pediatr Allergy Immunol 2016;27(suppl23):1-250.)

Published by:

The European Academy of Allergy and Clinical Immunology (EAACI)

Hagenholzsltrasse 111

3rd Floor

8050 Zurich

Switzerland

The European Academy of Allergy and Clinical Immunology, EAACl, is a non-profit organisation active in the field of allergic and immunologic diseases such as asthma, rhinitis, eczema, occupational allergy, food and drug allergy and anaphylaxis. EAACl was founded in 1956 in Florence and has become the largest medical association in Europe in the field of allergy and clinical immunology. It includes over 9300 members from 121 countries, as well as 54 National Allergy Societies.

This book contains information obtained from authentic and highly regarded sources. Reasonable efforts have been made to publish realiable data and information, but the authors and the publisher make no representations or warranties with respect to, nor assume responsibility for, the validity or accuracy of all materials or the consequences of their use and shall not be liable for any damages arising from reliance thereon. If professional advice or other expert assistance is required, the services of a competent professional should be sought. The authors and publishers have attempted to trace the copyright holders of all material reproduced in this publication and appologize to copyright holders if permission to publish in this form has not been obtained. If any copyright material has not been acknowledged, please contact us so we may specify in any future reprint.

Printed in Vienna, Austria, 2016

ISBN 978-3-033-05653-4 


\section{EAACl \\ MOLECULAR \\ ALLERGOLOGY \\ USER'S GUIDE}

\section{AUTHORS}

\section{Rob C. Aalberse}

Sanquin Research, Dept Immunopathology, Amsterdam, The Netherlands, and Landsteiner Laboratory, Academic Medical Centre, University of Amsterdam, Amsterdam, The Netherlands

\section{Ioana Agache}

Department of Allergy and Clinical Immunology, Faculty of Medicine, Transylvania University of Brasov, Brasov, Romania

\section{Riccardo Asero}

Ambulatorio di Allergologia, Clinica San Carlo, Paderno Dugnano (MI), Italy

\section{Barbara Ballmer-Weber}

Allergy Unit, Department of Dermatology, University Hospital of Zürich, Zürich, Switzerland

\section{Domingo Barber}

IMMA-School of Medicine, University CEU San Pablo, Madrid, Spain

\section{Kirsten Beyer}

Department of Paediatric Pneumology and Immunology, Charité University Medical Centre, Berlin, Germany

\section{Tilo Biedermann}

Department of Dermatology and Allergology, Technical University Munich, Munich, Germany

\section{Beatrice Bilò}

Allergy Unit, Department of Internal Medicine, University Hospital Ospedali Riuniti di Ancona, Ancona, Italy

\section{Simon Blank}

Center of Allergy and Environment (ZAUM), Technical University of Munich and Helmholtz Center Munich, Munich, Germany

\section{Barbara Bohle}

Division of Experimental Allergology, Department of Pathophysiology and Allergy Research Center of Pathophysiology, Infectiology \& Immunology Medical University of Vienna

\section{Philipp P. Bosshard}

Allergy Unit, Department of Dermatology, University Hospital of Zürich, Zürich, Switzerland

\section{Heimo Breiteneder}

Department of Comparative Medicine, Messerli Research Institute of the University of Veterinary Medicine Vienna, the Medical University of Vienna and the University of Vienna, Vienna, Austria

\section{Helen Brough}

Paediatric Allergy, Department of Asthma, Allergy and Respiratory Science, King's College London, Guys' Hospital, London, United Kingdom

Luis Caraballo

Institute for Immunological Research, The University of Cartagena, Cartagena de Indias, Colombia

\section{Jean-Cristoph Caubet}

Pediatric Allergy Unit, Department of Child and Adolescent, University Hospitals of Geneva, Geneva, Switzerland 


\section{Reto Crameri}

Swiss Institute of Allergy and Asthma research, University of Zürich, Davos, Switzerland

Janet M. Davies

School of Biomedical Sciences, Institute of Biomedical Innovation, Queensland University of Technology, Australia

Nikos Douladiris

Allergy Unit, 2nd Paediatric Clinic, National \& Kapodistrian University, Athens, Greece

\section{Motohiro Ebisawa}

Department of Allergy, Clinical Research Center for Allergology and Rheumatology, Sagamihara National Hospital, Kanagawa, Japan

Philippe A. Eigenmann

Pediatric Allergy Unit, Department of Child and Adolescent, University Hospitals of Geneva, Geneva, Switzerland

Montserrat Fernández Rivas

Allergy Department, Hospital Clinico San Carlos IdISSC, Madrid, Spain

\section{Fatima Ferreira}

Department of Molecular Biology, University of Salzburg, Salzburg, Austria

Gabriele Gadermaier

Department of Molecular Biology, University of Salzburg, Salzburg, Austria

\section{Martin Glatz}

1. Allergy Unit, Department of Dermatology, University Hospital of Zürich, Zürich, Switzerland

2. Christine Kühne Center for Allergy Research and Education CK-CARE, Davos, Switzerland

Robert G. Hamilton

Department of Medicine, Johns Hopkins University School of Medicine, Baltimore, MD USA

\section{Thomas Hawranek}

Department of Dermatology, Paracelsus Private Medical University Salzburg, Austria

Peter Hellings

1. Department of Otorhinolaryngology, University Hospitals Leuven, Leuven, Belgium

2. Department of Otorhinolaryngology, Academic Medical Center (AMC), Amsterdam, The Netherlands

Christiane Hilger

Department of Infection \& Immunity, Luxembourg Institute of Health, Esch-sur-Alzette, Luxembourg

\section{Stephanie Hofmaier}

Department of Paediatric Pneumology and Immunology, Charité University Medical Centre, Berlin, Germany

\section{Hans Jürgen Hoffmann}

Department of Respiratory Diseases and Allergy, Institute of Clinical Medicine, Aarhus University and Aarhus University Hospital, Denmark

Karin Hoffmann-Sommergruber

Dept. of Pathophysiolology and Allergy Research, Medical University of Vienna, Austria

Thilo Jakob

Department of Dermatology and Allergology, University Medical Center Giessen and Marburg, Justus Liebig University Giessen, Germany

Uta Jappe

1. Div. of Clinical and Molecular Allergology, Research Center Borstel; Airway Research Center North (ARCN), Member of the German Center for Lung Research (DZL), Borstel, Germany

2. Interdisciplinary Allergy Division, Dept. of Pneumology, University of Lübeck, Germany

Marek Jutel

Department of Clinical Immunology, Wrocław Medical University, and "ALL-MED" Medical Research Institute, Wrocław, Poland

Sandip Kamath

Molecular Allergy Research Laboratory, Centre for Biodiscovery and Molecular Development of Therapeutics, Australian Institute of Tropical Health and Medicine, James Cook University, Queensland, Australia

Jörg Kleine-Tebbe

Allergy \& Asthma Center Westend, Outpatient Clinic Ackermann, Hanf, \& Kleine-Tebbe, Berlin, Germany

\section{Edward Knol}

Departments of Immunology and Dermatology/ Allergology, University Medical Center Utrecht, Utrecht, The Netherlands

Peter Korosec

University Clinic of Respiratory and Allergic Diseases, Golnik, Slovenia

Annette Kühn

Department of Infection and Immunity, Luxembourg Institute of Health (LIH), Luxembourg

\section{Gideon Lack}

King's College London, MRC \& Asthma UK centre in Allergic Mechanisms of Asthma, Division of Asthma, Allergy and Lung Biology, Guy's and St Thomas' NHS Foundation Trust, London, UK

Andreas L. Lopata Molecular Allergy Research Laboratory, Centre for Biodiscovery and Molecular Development of Therapeutics, Australian Institute of Tropical Health and Medicine, James Cook University, Queensland, Australia 
Paolo Maria Matricardi

Department of Paediatric Pneumology and Immunology, Charité University Medical Centre, Berlin, Germany

\section{Mika Mäkelä}

Skin and Allergy Hospital, Helsinki University Central Hospital and University of Helsinki, Finland

Martine Morisset

National Service of Immuno-Allergology, Centre Hospitalier Luxembourg (CHL), Luxembourg

Antonella Muraro

The Referral Centre for Food Allergy Diagnosis and Treatment Veneto Region. Department of Mother and Child Health, University of Padua, Padua, Italy

Verena Niederberger

Department of Otorhinolaryngology, Medical University of Vienna, Vienna, Austria

\section{Anna Nowak-Węgrzyn}

Pediatric Allergy and Immunology, Jaffe Food Allergy Institute, Icahn School of Medicine at Mount Sinai, New York, NY, USA

\section{Markus Ollert}

1. Department of Infection and Immunity, Luxembourg Institute of Health (LIH), Esch-sur-Alzette, Luxembourg

2. Department of Dermatology and Allergy Center, Odense Research Center for Anaphylaxis, University of Southern Denmark, Odense, Denmark

Nikolaos G. Papadopoulos

1. Centre for Paediatrics and Child Health, Institute of Human Development, University of Manchester, Manchester, UK

2. Allergy Department, 2nd Paediatric Clinic, University of Athens, Athens, Greece

Elide Pastorello

Unit of Allergology and Immunology, Niguarda Ca' Granda Hospital, Milan, Italy

\section{Gabrielle Pauli}

Service de Pneumologie, Hôpitaux Universitaires de Strasbourg, Strasbourg, France

Thomas Platts-Mills

1. Department of Microbiology \& Immunology, University of Virginia School of Medicine, Charlottesville, VA, USA

2. Carter Immunology Center, University of Virginia Health System, Charlottesville, VA, USA; Department of Medicine, University of Virginia Health System, Charlottesville, VA, USA

\section{Daniela Posa}

Department of Paediatric Pneumology and Immunology, Charité University Medical Centre, Berlin, Germany

Lars K. Poulsen

Allergy Clinic, Copenhagen University Hospital, Denmark

\section{Monika Raulf}

Institute for Prevention and Occupational Medicine of the German Social Accident Insurance, Ruhr-University Bochum (IPA), Bochum, Germany

Joaquín Sastre Allergy Division, Fundación Jimenez Díaz, Madrid, Spain.

Enrico Scala

Experimental Allergy Unit, IDI-IRCCS, Rome, Italy

Johannes Schmid

Department for Allergy and Respiratory Medicine, Aarhus University Hospital, Denmark

Peter Schmid-Grendelmeier

1. Allergy Unit, Department of Dermatology, University Hospital of Zürich, Zürich, Switzerland

2. Christine Kühne Center for Allergy Research and Education CK-CARE, Davos, Switzerland

\section{Rudolf Valenta}

Division of Immunopathology, Department of Pathophysiology and Allergy Research, Center of Pathophysiology, Infectiology and Immunology, Medical University of Vienna, Vienna, Austria

\section{Marianne van Hage}

Department of Medicine Solna, Clinical Immunology and Allergy Unit, Karolinska Institutet and Karolinska University Hospital, Stockholm, Sweden

\section{Ronald van Ree}

Departments of Experimental Immunology and of Otorhinolaryngology, Academic Medical Center, University of Amsterdam, Amsterdam, The Netherlands

Stefan Vieths

Department of Allergology, Paul-Ehrlich-Institut, Langen, Germany

\section{Richard Weber}

School of Medicine, University of Colorado, Denver, and Department of Medicine, National Jewish Health Service, Denver, United States of America

Magnus Wickman Sachs' Children's Hospital, Karolinska Institutet, Stockholm, Sweden 



\section{EAACl MOLECULAR ALLERGOLOGY USER'S GUIDE}

\section{TABLE OF CONTENTS}

PART A

MOLECULAR

ALLERGOLOGY: GENERAL CONCEPTS
A01 03 Allergology from Extracts to Molecules: Integrating Tradition with Innovation Paolo Maria Matricardi, Jörg Kleine-Tebbe, Hans Jürgen Hoffmann, Rudolf Valenta, Markus Ollert

A02 $11 \mid$ Allergens and the Allergenic Composition of Source Materials

Ronald van Ree, Rob C. Aalberse

A03 $21 \mid$ Molecular Allergy Diagnostics in Clinical Practice Jörg Kleine-Tebbe, Thilo Jakob

A04 35 Methods for IgE Antibody Testing: Singleplex and Multiplex Assays

Robert G. Hamilton, Jörg Kleine-Tebbe

A05 51 Skin test, Basophil Activation Test (BAT) and Provocation Tests

Nikos Douladiris, Peter Korosec, Hans Jürgen Hoffmann

A06 $57 \mid$ Allergen Families and Databases

Heimo Breiteneder 
PART B

USING MOLECULAR ALLERGOLOGY IN THE CLINICAL PRACTICE
B01 71 | Tree Pollen Allergy

Enrico Scala, Riccardo Asero, Verena Niederberger

B02 $85 \mid$ Grass Pollen Allergy

Janet M. Davies, Paolo M. Matricardi, Johannes Schmid

B03 $95 \mid$ Weed Pollen Allergy

Gabriele Gadermaier, Janet M. Davies, Thomas Hawranek, Richard Weber

B04 105 | Dust Mite Allergy

Thomas Platts-Mills, Luis Caraballo

B05 115 | Cockroach Allergy

Luis Caraballo, Thomas Platts-Mills

B06 123 | Allergy to Cat, Dog and Horse

Marianne van Hage, Christiane Hilger

B07 131 | Allergic Bronchopulmonary Aspergillosis (ABPA)

Reto Crameri

B08 137 | Role of Microbial Allergens in Atopic Dermatitis Martin Glatz, Philipp P. Bosshard, Reto Crameri, Peter Schmid-Grendelmeier

B09 147 Molecular Diagnostics in the Management of Food Allergy

Helen A. Brough, Gideon Lack, Jörg Kleine-Tebbe, Antonella Muraro

B10 157 | Cow's Milk Allergy

Anna Nowak-Wegrzyn, Antonella Muraro

B11 167 | Allergy to Egg

Philippe A. Eigenmann, Jean-Christoph Caubet, Antonella Muraro

B12 173 | Allergy to Fish Lars K. Poulsen, Martine Morisset, Annette Kuehn

B13 181 | Allergy to Crustacean and Mollusks (Shellfish) Andreas L. Lopata, Sandip D. Kamath

B14 193 Allergy to Mammalian Meat

Marianne van Hage, Tilo Biedermann, Thomas A.E. Platts-Mills

B15 199 Allergy to Fruits and Vegetables Barbara Ballmer-Weber, Karin Hoffmann-Sommergruber

B16 $213 \mid$ Wheat Allergy

Mika Mäkelä

B17 225 | Soy Allergy

Jörg Kleine-Tebbe, Kirsten Beyer, Motohiro Ebisawa

B18 $235 \mid$ Peanut Allergy

Jörg Kleine-Tebbe, Kirsten Beyer, Motohiro Ebisawa 
B19 245 | Tree Nut and Seed Allergy Edward Knol, Magnus Wickman

B20 255 | Bee Venom Allergy Markus Ollert, Simon Blank

B21 265 | Vespid Allergy

M. Beatrice Bilò, Markus Ollert

B22 277 | Latex Allergy

Monika Raulf

B23 285 | Allergy to Laboratory Animals Monika Raulf

PART C

CROSS-REACTIVE MOLECULES \& THEIR CLINICAL RELEVANCE

\author{
C01 $293 \mid$ Profilins \\ Riccardo Asero, Domingo Barber \\ C02 299 PR-10-like Allergens \\ Heimo Breiteneder, Jörg Kleine-Tebbe \\ C03 311 Non-specific Lipid Transfer Proteins (nsLTPs) \\ Elide Pastorello, Domingo Barber

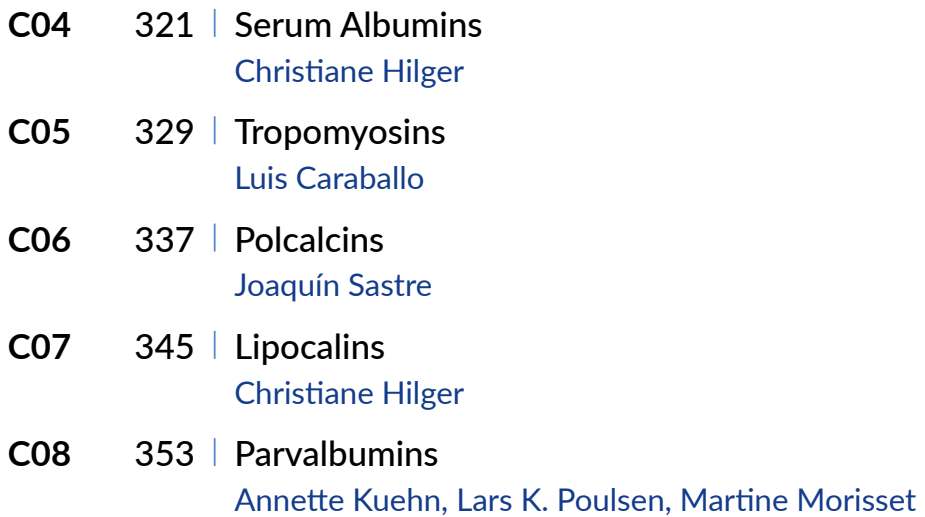

PART D

\section{APPENDICES}

D01 365100 Important Allergenic Molecules and their Characteristics

Stephanie Hofmaier, Karin Hoffmann-Sommergruber, Heimo Breiteneder D02 $375 \mid \begin{aligned} & \text { Glossary of Immunological Assays } \\ & \text { in Molecular Allergology }\end{aligned}$

D03 379 | Abbreviations and Acronyms

D04 383 | Acknowledgements 



\section{PREFACE FROM THE EAACI PRESIDENT}

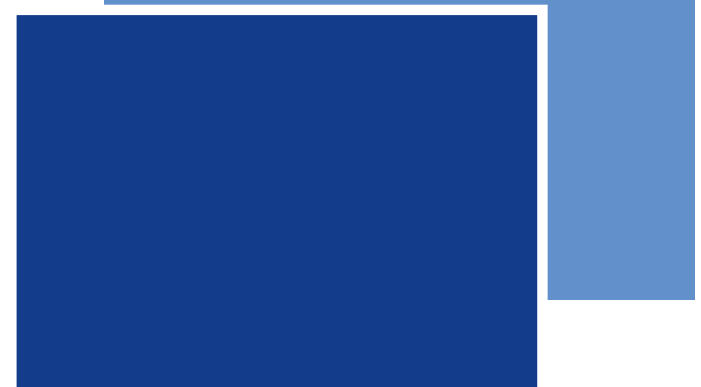

Allergic diseases are still steadily rising and affecting a large number of individuals who are also facing an overall worsening of their clinical manifestations at any point during their life-time.

Many children suffer from severe food allergy and anaphylaxis, many adolescents and young adults are underperforming due to asthma and allergic rhinitis and many adults are missing work due to co-morbidities of allergic diseases. Allergy is currently a public health issue although hugely underestimated and under -recognized. There is a need to improve the care of allergic patients through better education of health professionals and the public. As part of its Mission the European Academy of Allergy and Clinical Immunology -EAACI has started a project aimed to translate best knowledge into best practice. Atlases and Guidelines represent a comprehensive set of documents that are distributed for improving appraisal and proper management of allergy.

This User's Guide for Molecular Allergology will be another breakthrough in this regard.

Recombinant technology in the field of allergology has brought tremendous advances in allergen characterization as well as in the knowledge of immune mechanisms involved in allergic diseases. Molecular allergology takes diagnostics one-step further. It can enable you to get better perspectives on the risk connected with sensitization. Sensitization to the components is measured individually helping to pinpoint on an exact molecular level which component the patient is sensitized to. In light of this, allergen extracts are being step-by-step replaced by molecule-based products. The new developments will allow clinicians to obtain detailed information on sensitization patterns and more accurate interpretation of allergic symptoms. This information provides the basis for a refined, earlier, diagnosis of the allergic reactions and ultimately for a tailored individualized management of the patient, including opportunities for prevention.

In this regard, molecular allergology is a further, excellent example of how allergy is linked to precision medicine and drives precision health forward.

Moreover, this book is also the culmination of the EAACl spirit of team working. It comes from an outstanding effort from many: the EAACI Board of Officers, the Executive Committee and the 100 contributors who have volunteered in this activity to get it finalized. Without their enthusiasm and continuous support this achievement would not have been accomplished. 
However, a special thank you must go to the leadership of the Interest Group of Allergy Diagnosis, Paolo Matricardi, Hans-Jurgen Hoffman, Markus Ollert, Jorg Kleine - Tebbe, Rudolph Valenta. They have been the key drivers of the initiative and their vision and commitment has helped in tackling many issues.

This User's guide is supposed to be a straightforward guide on molecular allergology describing what are components, the clinical benefits of testing for components as well as how to interpret results including understanding cross-reactions. Our hope is that it will be an essential daily pocket guide for allergists and for all those health professionals who are dealing everyday with allergic patients

I am confident that the dissemination of this User's Guide will positively impact the perception of allergy at the global level changing significantly the approach to patients world-wide.

Antonella Muraro

President of the European Academy of Allergy and Clinical Immunology-EAACI 


\section{PREFACE FROM THE EDITORS}

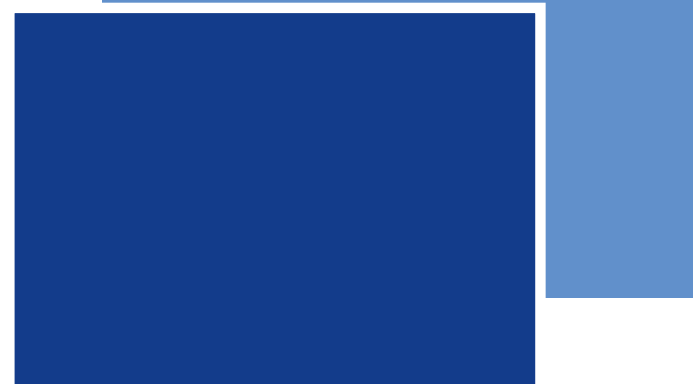

Allergology is experiencing a technological revolution, which is transforming into a rapid clinical practice "evolution". Our knowledge as "traditional" allergists, inherited since the first years of 1900, is progressively challenged by new concepts. Our tools, based on sometimes poorly standardized and highly variable allergenic preparations, become clearly defined and allow more analyses in depth. Similar to other areas of Medicine, these changes are facilitated by a new era of biosiences and technologies, including advanced molecular and structural biology. This process is part of a broader concept, coined "Precision Medicine" promoting personalized treatments based on a detailed patient's disease phenotype. Thanks to this technological revolution, a growing number of allergenic molecules and their isoforms have been purified, sequenced, cloned, and produced in large quantities. They allowed to study the immunological reactivity of these molecules, their degree of cross-reactivity and to define complex IgE repertoires. New diagnostic methods have been made available for clinicians and improved therapeutic strategies will be further developed in the future.

The results of these studies are regularly published in medical journals with high impact factors. Up to now more than one thousand molecules from diverse allergen sources have been characterized; many others will be identified, purified and cloned in the future. Over 100 of these molecules have already been developed as diagnostic reagents for routine use in clinical practice. Many more will be introduced not far from today. Altogether, this knowledge has created a new branch establishing novel "allergomics" or "allergologic proteomics". The main information on physico-chemical and biological features of allergenic molecules, their sources, abundance, regional distribution and nomenclature are accessible in several web-based databanks. Symposia and Congresses are nowadays providing and promoting exiting topics regarding Molecular Allergology.

However, this "scientific revolution" has not yet fully translated into an improved management of the allergic patient. The main reason for this "gap" between science and routine clinical practice is in part the complexity of the new subject, hampering instant utilization and interpretation of these new diagnostic tools by an average physician. Based on this scientific revolution in allergy practice, the medical education programs as well as the training programs for allergy specialists should be re-written and adapted accordingly. 
Nevertheless, molecular tests are already available and clinicians need guidance for proper use. For this purpose, the EAACI has established a Task Force to compose a handbook on molecular allergology for clinicians. This document will introduce the current position of the EAACI on in vitro methods of Molecular Allergology, their advantages and limitations and how they can be used in an up-to-date diagnostic work-up of the allergic patients. With this perspective, most immediate hypersensitivity reactions and allergic diseases are taken into account, including allergic rhinoconjunctivitis and asthma to pollens, mites and other airborne allergens, food allergies, atopic eczema, insect venom hypersensitivity and occupational allergies. For each of these diseases the most important allergenic molecules and their potential role in our diagnostic work-up will be discussed, providing a unique EAACI position on their current use. Molecular Allergology is rapidly evolving. Therefore this text will regularly be updated, approximately every three years, to monitor technological and scientific progress, as well as new evidence coming from clinical studies.

The idea of a Task Force on Molecular Allergology was originally proposed in 2011 by Dr Adriano Mari, a visionary initiator in this field, and then re-vitalized by Markus Ollert and Paolo Matricardi. The goal of a handbook has been established after an constructive and articulate debate within the EAACl. The workplan has been further shaped during a meeting in London in November 2014 among the five editors. More than 50 authors from over 15 countries and five continents have drafted the 40 chapters composing this first edition of the Handbook. These drafts have been discussed in a three day workshop in Berlin (15-17 April 2015) and further elaborated by their contributors. A long reviewing process has involved all the authors and, at a second stage, six additional reviewers that had not participated in the Berlin meeting. The final draft of the text has subsequently been presented to the EAACI Executive Committee, approved and released for publication.

We are all grateful to the former EAACI President Nikos Papadopoulos (2013-2015), who has moderated and guided our initiative during its infancy including delicate debates on the proper orientation and structure of the Handbook. We also thank our current EAACI President Antonella Muraro, who has not only contributed as an author, but has strongly supported our Task Force activities. The Handbook would have never been produced without the constant assistance of Dr. Stephanie Hofmaier, who has not only contributed to the scientific content of the Handbook (PART-D, appendices), but also coordinated the Berlin team in the organization of meetings, telephone conferences and the long and difficult peer-reviewing and publication process. We are also particularly grateful to Dr. Christiane Hilger who has not only contributed various chapters of the User's Guide, but has also provided clear examples of the editorial style with her first contribution, being followed by many other authors. Last but not least we are of course grateful to all the authors, opinion leaders in their own area, who have all enthusiastically accepted to join us in this ambitious project and have dedicated so much of their time to share, discuss and elaborate their knowledge and expertise in such a broad area. We do really hope that this group of experts will continue to work together in the next years to develop updates of the Handbook, to transform it into a novel informatics platform, to foster translation in several languages and to develop didactic tools for the dissemination of this new tool for doctors and their patients.

Paolo Maria Matricardi

Jörg Kleine-Tebbe

Hans Jürgen Hoffmann

Rudolf Valenta

Markus Ollert 


\section{PART A}

MOLECULAR ALLERGOLOGY: GENERAL CONCEPTS 

ALLERGOLOGY FROM EXTRACTS TO MOLECULES: INTEGRATING TRADITION WITH INNOVATION

Paolo Matricardi, Jörg Kleine-Tebbe, Hans Jürgen Hoffmann, Rudolf Valenta, Markus Ollert

Immunoglobulin E (IgE) antibodies are both a marker and major contributor in immediate hypersensitivity reactions and atopic diseases (1). Since the late sixties serological tests for allergen-specific IgE antibodies were used to identify triggers of IgE-mediated allergic symptoms (2). Notably, the composition and amount of an allergenic extract strongly affects the result of traditional extract-based IgE assays (3). Therefore, IgE assays produced by different companies generate often various quantitative levels and sometimes nonconcordant qualitative results (positive versus negative).

The following rationales support the targeted use of allergen molecules and improve test properties: (I) increased test sensitivity ("analytical sensitivity"), particularly when important allergens are underrepresented or lacking in the extract; (II) improved test selectivity (analytical specificity), particularly when the selected IgE repertoire against an allergen yields additional information on: (a) potential risks, (b) possible cross-reactivity, or (c) primary (species-specific) sensitization. Allergen-specific lgE tests utilizing individual allergenic molecules are subsequently considered a more precise and informative option, particularly in polysensitized

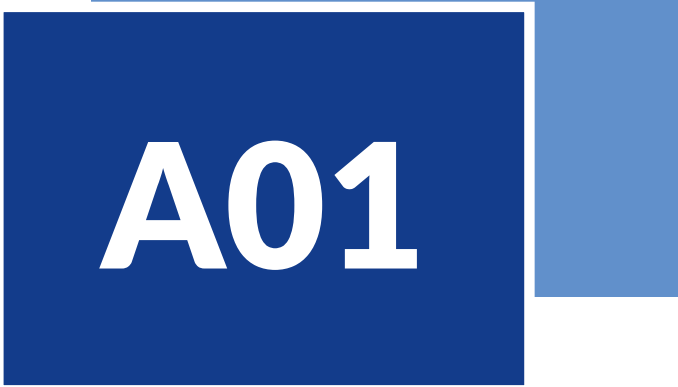

The transition from allergen extracts to molecules for the diagnostic work-up of IgE mediated reactions/diseases has great potential, but requires detailed knowledge and intelligent interpretation

Diagnostic algorithms traditionally start with the history ("top-down"), followed by skin tests, then IgE assays adding allergen molecules for "component-resolved diagnosis" (CRD)

Novel diagnostics invert the test order ("bottom-up") and start with a multiplex (i.e. microarray-based) IgE assay and many components. Then the history is reviewed for concordance to establish a broad CRD

Combining both strategies, "top-down" and "bottom-up", creates a "U-shaped" sequence. Traditional diagnostic work-up can thus be expanded by a broad screening for lgE-binding molecules unfolding missing details and feeding back to a molecular allergy diagnosis 
patients compared to those tests based on allergenic extracts (4). A growing spectrum of molecules, representing single allergens of clinical relevance have been identified, characterized and produced for commercial in vitro assays. This "component resolved diagnosis" (CRD) thus allows a detailed molecular profiling of the polyclonal IgE repertoire of the allergic patient (5).

CRD is pushing Allergology in the coming Era of "Precision Medicine", an approach integrating novel individual genetic or molecular data for improved geno-/phenotyping and proper selection of personal treatments (6). Accordingly, the need of learning to handle and interpret "allergomics" data is a true challenge for the practicing allergist (7). Several databases of allergenic molecules and advanced webplatforms are already available for this purpose (8).

The list of important allergenic molecules, cloned or purified and introduced for diagnostic purposes, is still incomplete. Moreover, the costs of IgE assays using individual allergens are about 10\% higher compared to extract-based tests; microarrayed multiple allergenic molecules will produce even higher costs. It is therefore conceivable that the use of molecular assays in allergology will be balanced by a general need of careful stewarding the scarce resources of public health systems (9). This may be particular relevant for IgE microarrays testing of more than 100 individual allergenic molecules in a single analysis (10). In other words, "Precision Allergology" will progressively grow, but confined in a "Choosing Wisely" scenario. Given these premises, both, the in vitro IgE assays based on allergenic extracts and those based on allergenic molecules, will probably co-exist in clinical practice for many years to come.

\section{DISCREPANCIES BETWEEN ALLERGEN EXTRACT AND ALLERGEN MOLECULE- BASED IGE ASSAY RESULTS (11)*}

The coexistence of two laboratory strategies to test serum IgE antibodies to allergens (extracts vs molecules) will inevitably lead to discrepancies between their outcomes. In an ideal world, a "positive" IgE-extract assay result should always be paralleled by

(*this paragraph is reprinted with permission from the publisher from: Matricardi PM, Kleine-Tebbe J. Molecular Allergology between Precision Medicine and the Choosing Wisely initiative. Clin Exp Allergy 2016; in press.) a "positive" outcome in one or more of the lgE assays utilizing the extract's individual molecules. Similarly, a "negative" IgE-extract assay should always correspond to "negative" outcomes in molecular based IgE assays covering all the extract's components.

Nonconcordant outcomes among extract- and molecular-based IgE assays can have several characteristics and explanations (Table 1):

A. Allergenextractpositive butitsmoleculesnegative - The serum IgE antibodies might recognize one/ several particular molecules in the extract, which is/are simply not available in corresponding molecular assay/s. Another explanation would be a lower Limit of Quantitation (LoQ) of the extract assay compared to the molecular one in case of rather low specific IgE levels. In other words, the serum IgE antibodies levels to the implicated molecules could be below the LoQ of the molecular-assay but above the LoQ of the extract-assay.

B. Allergen extract negative but its molecules positive - The serum might carry IgE to components recognized in a molecular assay, but missing or underrepresented in the corresponding allergen extract. The same discrepancy can also be explained if the extract assay is analytically less sensitive (higher LoQ) than the molecular one and specific IgE levels are low. In other words, the level of serum IgE antibodies to the implicated molecules could be above the LoQ of the molecular-assay but below the LoQ of the extract-assay.

C. Allergen extract positive but its genuine components are negative - In this case the serum's IgE might recognize only minor, highly crossreactive components of an allergenic extract, but not its genuine, species-specific (major) components. Patients sensitized to panallergens frequently show positive IgE results to many different allergenic extracts containing a member of that highly cross-reactive family and falsely appear to be highly "polysensitised". Similarly, patients sensitized to cross-reactiv carbohydrate determinants (CCD) only, show positive result to extracts but not to corresponding molecules produced as recombinant proteins lacking posttranslational modifications like CCDs.

D. Allergen extract less positive than the added molecular assay levels - The level of serum lgE to 
Table 1

Discrepancies between allergen extract and allergen molecular lgE assay results

\begin{tabular}{|c|c|c|c|}
\hline & Extracts & Molecular & Explanations \\
\hline \multicolumn{4}{|c|}{ Absolute Disagreement (qualitative differences) } \\
\hline A & positive & negative & $\begin{array}{l}\text { a) serum IgE binds only to extract's molecules that are not (yet) } \\
\text { available in molecular assays; } \\
\text { b) molecular assays less analytically sensitive than the extract based } \\
\text { assay }\end{array}$ \\
\hline B & negative & positive & $\begin{array}{l}\text { c) serum IgE binds to molecules tested as components which } \\
\text { missing or of low abundance in the extract; } \\
\text { d) extract assay less analytically sensitive than the molecular assay }\end{array}$ \\
\hline \multicolumn{4}{|c|}{ Relative Disagreement (quantitative differences) } \\
\hline C & positive & $\begin{array}{l}\text { negative to major } \\
\text { components }\end{array}$ & $\begin{array}{l}\text { serum IgE binds only to highly cross reactive, minor allergenic } \\
\text { molecules or CCD determinants }\end{array}$ \\
\hline D & lower levels & higher levels & $\begin{array}{l}\text { serum IgE binds to molecules tested as components being of low } \\
\text { abundance in the extract }\end{array}$ \\
\hline
\end{tabular}

modified from (11) with permission

an allergenic extract could be much lower than the sum of the lgE levels to all its components. Extractsbased IgE assays tend to underestimate the IgE levels of highly sensitized patients, as they might miss or contain only limited amounts of particular molecules recognized by the patient's IgE. As a consequence, the sum of IgE levels obtained by testing individual molecules is often higher than the level measured by testing the corresponding allergenic extract. An additional explanation is potential (partial) cross-reactivity between structurally non-related allergens (i.e. Ara h 1, 2 and 3) from different protein families, leading to arbitrarily high sum values after adding molecular assay results in comparison to the extract result.

In summary, studying discrepancies between outcomes of allergen extract-based vs molecular IgE assays is of crucial relevance for improving our capability to screen for IgE sensitizations in allergic patients with high precision in a cost-effective way, as recently reviewed elsewhere (6). In other words, the use of "customized", properly balanced selection of allergenic molecules in IgE assays will be a valuable compromise between "tradition" and "modernity" in serological in vitro allergy testing and the best strategy to harmonize "Precision Medicine" with a "Choosing Wisely" approach in the field of allergology.

\section{INTEGRATING TRADITION AND INNOVATION: EXTRACTS AND MOLECULES}

How should a molecular approach be integrated in the diagnostic work-up of the allergic patient? There is no conclusive answer to this question. New technologies require years before being fully integrated in the routine clinical practice. Moreover, only daily clinical practice can ultimately disclose their advantages and limitations in different settings. Currently, doctors practicing a molecular approach as part of their allergy diagnosis can be roughly divided into those pursuing an integration of allergy molecular tests in a traditional diagnostic workup which includes the use of allergen extracts, and those that prefer to generally omit the use of allergenic extracts and conceive a systematic molecular diagnosis. The first ones privilege the use of singleplex tests, i.e. an inductive method based on the doctor's choice which allergen should be used. This approach has been termed "TOP-DOWN" diagnostic approach, including first clinical history with SPT or lgE assays to extracts and then the molecular step. The others prefer to use microarrays providing a broad and analytical vision of the sensitization profile of the patient. This can be defined as "BOTTOM-UP" approach, moving from a microarray test to the clinical history (Fig. 1). In the following section seven arguments for the first and the second approach are listed: 


\section{Arguments raised pro a TOP-DOWN approach or against a BOTTOM-UP approach.}

1. Extracts are essential screening tools that most doctors are accustomed to; they cannot be removed as routine diagnostics since doctors would loose basic tools for proper allergy work-up.

2. Microarrays are too complex and detailed; doctors are not yet prepared to interpret them properly.

3. Microarrays offer useful information - if linked to individual clinical symptoms - but also information on sensitizations not being linked to any symptoms. This extra information can generate conceptual, ethical and legal problems and it is difficult to be communicated to the patient.

4. The use of individually selected reagents, covering extracts and molecules for testing, facilitates the use of the singleplex approach to molecular diagnosis, which is inductive and less expensive than the use of microarrays.

5. Extract-based reagents contain more molecules than those included in a microarray or in catalogues for singleplex tests. Some of these missing molecules are essential for a diagnosis.

6. SPT based on extracts offer information on biological function of IgE antibodies, not only their presence/absence.

7. Degranulating cells in polysensitized patients (the vast majority) react to complex protein mixtures in the allergen extract and not only to individual molecules.

\section{Arguments raised pro a BOTTOM-UP approach or against a TOP-DOWN approach}

1. Patients should be examined with a multiplexed approach (microarray) to get a global evaluation of their individual IgE repertoire

2. Any step or approach using extracts is outdated and unreliable, because extracts are not always properly characterized and standardized; different extracts tend to give different results leading to different decisions in the same patients.

3. Patients should be examined with a multiplexed approach (microarray) for a global evaluation of the entire sensitization profile, then the physician should properly address the clinical history, and make a comprehensive evaluation of the atopic conditions.
4. Positive results in a microarray, even if not clinically relevant at present, may predict future allergic symptoms.

5. The bottom-up approach, performed with a microarray, would reduce the number of contacts patient-doctor-lab while a singleplex "reflex" approach would require several consultations (and therefore higher costs) before all the molecules relevant for a final diagnosis are tested.

6. Broader use of microarrays will induce companies to reduce the prices in view of a broader distribution and increase the likelihood of reimbursement from insurance companies and public health systems.

7. The resistance of allergists to the progress of molecular allergology, which may reduce their earnings based on SPT, should be overcome.

\section{THE EAACI PROPOSAL: A "U-SHAPED" APPROACH}

The debate between the two diagnostic approaches to molecular allergology is still open. Nevertheless, the Task Force of EAACl suggests to integrate both of them in a so called "U-SHAPED" approach (Fig. 1). The patient undergoes, as in usual care, clinical history, physical examination, skin test or IgE assay with extracts and selected molecules, chosen according to the clinical history and results of the tests with extracts. Then IgE responses to allergenic molecules of those allergen sources to which clinical relevant sensitizations have been detected by the previous two diagnostic steps ["top-down" approach (part II)]. In some patients, IgE sensitization to highly crossreacting molecules belonging to the allergenic source may have been demonstrated. Here comes the advice of further investigating the patient for potential clinical consequences of cross-reactive sensitization: (1) by testing IgE sensitization to other molecules of the same family, but limited cross-reactivity (i.e. seed storage proteins); (2) by expanding the clinical history with questions related to symptoms triggered by other allergen sources containing the other molecules of the same family ["bottom-up" approach (part III)]. Arguments in favour of this "U-SHAPED" approach are listed below:

1. An "exclusive" bottom-up approach would not be cost-effective. In addition, the microarray test is not reimbursed in most countries. The bottom- 


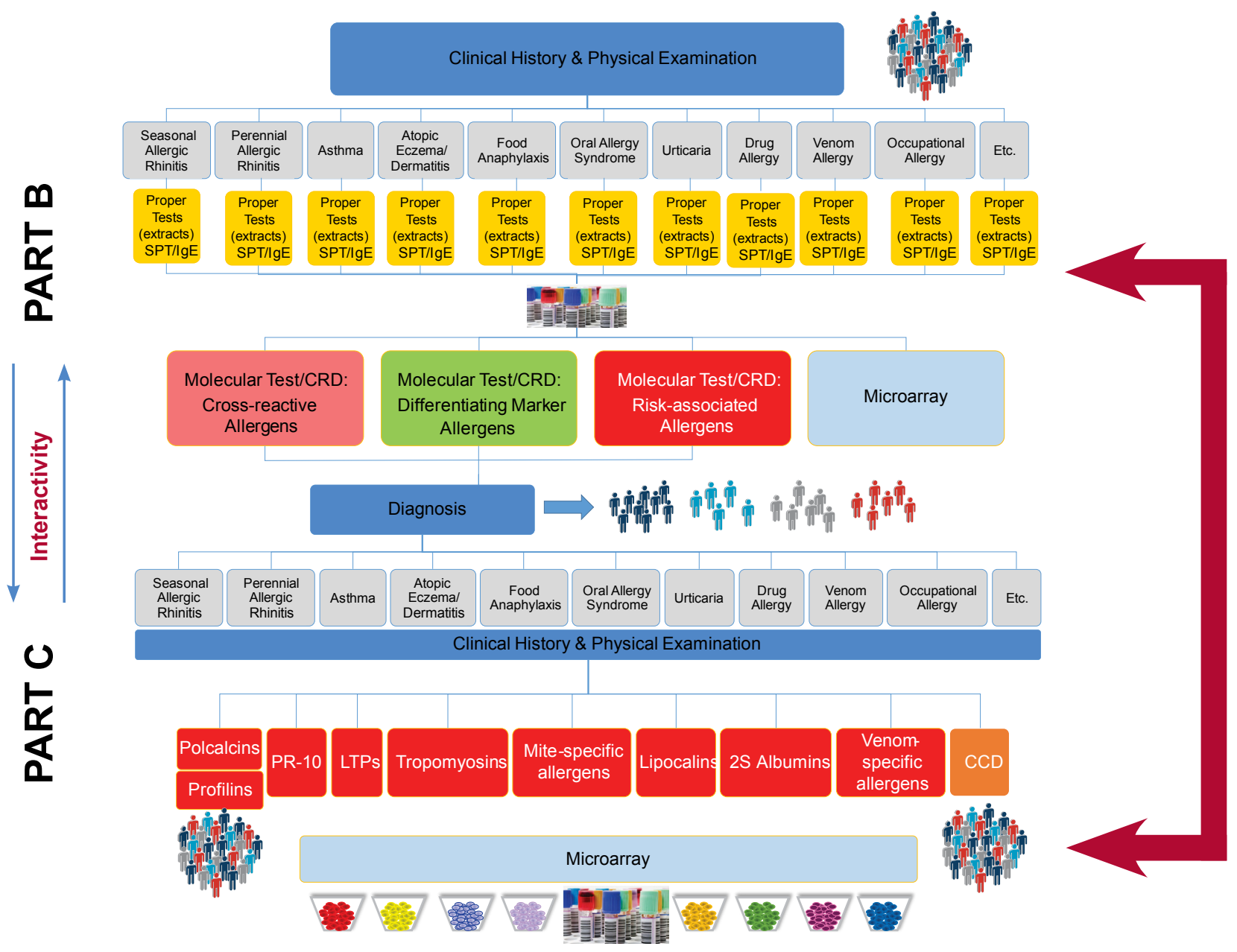

Figure 1

The combined "U-shaped" approach.

up approach, based on a microarray, is a visionary one, but only the traditional diagnostic algorithm using extracts and singleplex approach is the one presently reimbursed.

2. Most doctors will continue using extracts and working with a top-down approach. A subsequent "U-shaped" approach should make molecular allergolgy accessible to more allergists and not only to the very few "enthusiasts" of molecular allergology and microarrays.

3. The new way of thinking should be introduced step-by-step. The use of microarrays will become easier after doctors have learnt to use individual molecules (singleplex).

4. We should prevent the legal/QoL problems generated by knowing sensitization to molecules (e.g. LTP) with potentially no clinical relevance (e.g. in south Europe) but always tested in a microarray, independently from the patient's diagnostic question. The handling of occasional or bystander results in medicine (e.g. those obtained in total body $\mathrm{CT}$ or NMR) is always difficult.

\section{THE HANDBOOK OF MOLECULAR \\ ALLERGOLOGY: GENERAL CONCEPTS, ALLERGEN SOURCES, AND ALLERGEN FAMILIES}

In keeping with the concepts expressed above, the Handbook is divided in three parts: 


\section{A INTRODUCTION TO MOLECULAR} ALLERGOLOGY - this part is dedicated to introduce the reader to basic scientific and clinical concepts of molecular allergology. Five sections describe (A02) the major properties of an allergenic molecule, including structural characteristics; biological functions; immunological and allergenic features are described; (A03) the use of a molecular approach in the clinical practice; (A04) the essentials of in vitro IgE assays and the most important performance characteristics and parameters of reliability; specific features, advantages and limitations, of singleplex assays and multiplex assay; (A05) how information obtained with skin prick tests, challenge tests and basophil activation tests can be interpreted in the framework of a molecular approach to allergy diagnosis and (A06) the concept of cross-reactivity and how the complex diversity of shapes are shared at different degrees by molecules belonging to similar or unrelated species (allergen families).

\section{B MOLECULAR DIAGNOSIS BY ALLERGENIC} SOURCE - This part describes systematically how to use and integrate a molecular assay in a classical allergy diagnostic work-up, which starts with clinical history, physical examination and extract-based assays. 23 Sections (B01-B23) are dedicated to all major allergenic sources, including pollens, mites and cockroaches, animals, molds, occupational allergens, major food allergens, insect venoms. In each section, general information on the characteristics of the allergen source: nature, classification, allergenic tissues, cross-reacting species, exposure, are given as in a "traditional" textbook. Then some characteristics of major and relevant minor allergenic molecules are described (e.g. MW, functions, biochemical properties, localization, isoforms, etc).

The clinical relevance of a sensitization to individual molecules is discussed providing information on

- the frequency and intensity of IgE sensitizations,

- the potential role in triggering and shaping the patient's symptoms, and

- the relevance for prognosis or response to therapy.
When appropriate, a diagnostic algorithm is proposed by the authors, i.e. when a patient is consistently positive to the allergenic source. These diagnostic algorithms are also based on expert opinion and not necessarily backed up by various studies or meta-analyses. They can be useful to orient the doctor in the use of molecular diagnostic tests but they are not intended to provide a fixed, rigid approach to diagnosis. One or more index clinical cases are then presented to provide examples of how molecular diagnosis may help understanding the patient's allergy and assist clinical decisions.

C FAMILIES OF HIGHLY CROSS-REACTIVE MOLECULES - This part is dedicated to present major allergen families, their clinical relevance and how to use this information to improve the management of the allergic patient in the daily clinical practice. The families taken into consideration are: Profilins, PR-10-like molecules, nsLTP, Serum Albumins, Tropomysins, Polcalcins, Lipocalins, Parvalbumins (section C01-C08). Each section describes features of members from that protein family: MW, functions, biochemical characteristics, distribution, tertiary structure; a list of major members of the family is given, with the range of homogeneity, and a phylogenetic tree. When appropriate, the routes of exposure, the symptoms triggered, aggravating and protecting features, cofactors, frequency, epidemiology of sensitization are also discussed. Then the impact of sensitization to members of the allergen family is discussed with the respect to clinical decision and the management of the patient. Finally, one or more index clinical cases are then presented to provide examples of how molecular diagnosis may help understanding the patient's allergy and assist clinical decisions.

D APPENDICES - Four appendixes are given at the end oft he text, including a list summarizing the characteristics of 100 relevant allergenic molecules ("the "100VIM", [D01]) and a list of a few definitions, which are relevant especially for molecular in vitro diagnostic tests ("Glossary" [D02]).

\section{References}

1. Ishizaka K, Ishizaka T. Identification of gammaE-antibodies as a carrier of reaginic activity. J Immunol 1967;99:1187-1198.

2. Wide L, Bennich H, Johansson SGO. Diagnosis 
of allergy by an in vitro test for allergen antibodies. Lancet 1967;2:1105-1107.

3. Hamilton RG. Clinical laboratories worldwide need to report IgE antibody results on clinical specimens as analytical results and not use differential positive thresholds. JAllergy Clin Immunol 2015;136:811-812.

4. Kleine-Tebbe J, Jakob T. Molecular allergy diagnostics using IgE singleplex determinations: methodological and practical consideration for use in clinical routine - Part 18 of the Series Molecular Allergology. Allergo J Int 2015;24:185-197.

5. Valenta R, Lidholm J, Niederberger V, Hayek B, Kraft D, Grönlund H. The recombinant allergenbased concept of component-resolved diagnostics and immunotherapy (CRD and CRIT). Clin Exp Allergy 1999;29:896-904.

6. Mirnezami R, Nicholson J, Darzi A. Preparing for precision medicine. N Engl J Med 2012;366:489-491.
7. Mari A. Importance of databases in experimental and clinical allergology. Int Arch Allergy Immunol 2005;138:88-96.

8. Mari A, Scala E. Allergome: a unifying platform. Arb Paul Ehrlich Inst Bundesamt Sera Impfstoffe Frankf A M 2006;95:29-39.

9. Morden NE, Colla CH, Sequist TD, Rosenthal MB. Choosing wisely-the politics and economics of labeling low-value services. N Engl J Med 2014;370:589-592.

10. De Knop KJ, Bridts $\mathrm{CH}$, Verweij MM, Hagendorens MM, De Clerck LS, Stevens WJ, et al. Componentresolved allergy diagnosis by microarray: potential, pitfalls, and prospects. Adv Clin Chem 2010;50:87101.

11. Matricardi PM, Kleine-Tebbe J. Molecular Allergology between Precision Medicine and the Choosing Wisely initiative. Clin Exp Allergy 2016;46:664-667. 



\section{ALLERGENS AND THE ALLERGENIC COMPOSITION OF SOURCE MATERIALS}

\author{
Ronald van Ree, Rob C. Aalberse
}

Allergenic sources can vary from biological sources with very complex composition such as pollen, house dust mites (inhalant allergy) or foods (food allergy), to single molecules such as chemicals (occupational allergy) or drugs (drug allergy). In this chapter we will focus on the molecular composition of more complex biological allergen sources that are implicated in causing hay fever, allergic asthma and food allergy. Around the late sixties and early seventies of last century the first reports were published in which individual molecules were identified that were responsible for binding IgE within different allergen sources such as grass pollen (1), ragweed pollen (2), cod fish (3) and house dust mite (4). By now probably the most important allergens of the most relevant allergen sources have been identified (www.allergen. org; www.allergenonline.org; www.allergome.org).

Before we can discuss the allergens, we have to introduce the nomenclature of molecularly defined allergens (Textbox 1).

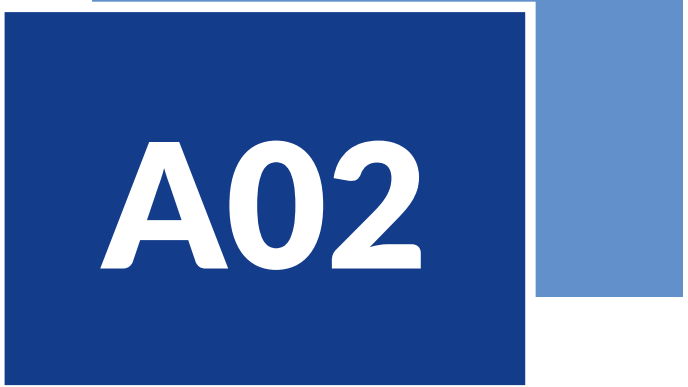

Many different types of proteins are allergenic.

The context of a protein may be a major determinant for its allergenicity.

Some lgE inducers are not really allergens at all because they don't induce symptoms. This negatively impacts specificity of diagnostic tests, certainly of extract-based tests but also still of molecular tests.

Allergen extracts are imperfect but not yet obsolete.

Molecular sensitization profiles: potential biomarkers for disease phenotypes and progression

The initial response to an allergen source is possibly characterized by IgE antibodies to one or two "initiator" allergens. 


Allergen nomenclature
Allergen names are based on the scientific (Latin)
name of the plant or animal species from which the
allergen originates (5, 6). For example, the major
allergen from birch pollen Bet $v 1$ is named after
the scientific name of the tree Betula verrucosa, in
which Betula is the genus and verrucosa the spe-
cies. The first three letters of the genus (Bet) and
the first letter of the species (v) together form the
basis of the allergen name, followed by a number. In
principle the number is given in order of discovery,
so Bet $v 1$ was the first allergen from birch pollen
that was discovered. Related (often cross-reactive)
allergens from different species, genus, family or
even order, get the same number, if still available.
So, the homologue of Bet $v 1$ in hazel is Cor a 1 and
in apple is Mal d 1 , but in peanut is Ara $h 8$ because
numbers 1 to 7 were already occupied by peanut
allergens described earlier.
Many allergens have molecular variants (isoforms).
One example is Cor a 1. One isoform is mainly
found in hazel pollen (Cor a 1.01 ), the other main-
ly in hazelnut (Cor a 1.04 ). Some isoforms are so
closely related (>90\% sequence identity) that they
can usually be considered identical. If they need to
be distinguished, two more digits are added to the
name, e.g. Cor a 1.0101 and Cor a 1.0102.

\section{WHAT IS AN ALLERGEN?}

The broadest definition of an allergen is that it is any molecule that binds IgE antibodies (7). Allergens can differ in several ways (Textboxes 2 and 3 ). In this introduction, the practical consequences of four of these differences will be discussed.

\section{SENSITIZING VERSUS NON-SENSITIZING ALLERGENS}

Most, but not all, allergens are sensitizing which is defined as the ability to induce allergen-specific IgE antibodies. Non-sensitizing allergens can only cause allergic symptoms if previous contact with a related (cross-reactive) allergen has caused sensitization. A prototypic example of a sensitizer is birch allergen Bet $v 1$ and a cross-reactive non-sensitizer is the homologous apple allergen Mal d 1 (8).

\section{Textbox 2}

The long road from allergen-coding DNA to a molecule interacting with IgE on the mast cell surface

Much of our information on allergen structure comes from proteins that are produced with recombinant DNA technologies (conveniently, but scientifically incorrectly, referred to as "recombinant allergens"). Most currently available recombinant allergens are prepared based on a direct translation of their genomic information. In the real world, the allergen that is knocking at the mast cell's door is often modified. Some of the modifications are well-characterized intra-cellular biochemical processes known as co-translational or post-translational modifications, such as homo-and hetero-oligomerization, glycosylation, cleavage of a leader peptide, propeptide and other proteolytic events, binding of metallic cofactors or organic ligands and oxidation of proline to hydroxyproline. Others are due to more random extracellular processes often influenced by environmental conditions (humidity, UV, ozone). Examples are nitration, methionine oxidation, deamidation and cross-linking by transglutaminases and glycation (a non-enzymatic process also known as the Maillard reaction). Upon water loss, excreted proteins attach to various substrates, both on a nano-scale (homo- and hetero-aggregation) and on a micro-scale (attachment to fibers and dusty particles). The effect of these modifications of the structure of the allergen on allergenicity has only just started to be investigated (9).

\section{AIRBORNE VERSUS FOOD ALLERGENS: CROSSING DIFFERENT BARRIERS}

The two most common sites of entry into the body are the mucosal surfaces of the airways in which the allergen is delivered as part of an airborne particle or aerosol droplet and the digestive tract which includes the oral cavity where it is introduced as part of a food or drink (Textbox 4). Also the skin has been proposed as a route for sensitization, a hypothesis that gained significance since the discovery of filaggrin SNPs associated the development of allergy. Prototypic examples of true food allergens include the shrimp muscle protein tropomyosin that varies in nomenclature depending on the type of shrimp (Pen a 1, Cra c 1, Met e 1, Lit v 1 etc). All these are highly cross-reactive allergens, see the official IUIS website [www.allergen.org] or the Allergome website [www. 
Textbox 3

\section{The exception: some allergens are NOT proteins}

Examples of non-protein allergens are drugs such as penicillin, chlorhexidine and other pharmacological compounds such as rocuronium. It is generally assumed that these compounds depend for their allergenicity on a strong (covalent) interaction with a carrier protein, but this has not always been demonstrated convincingly. One explanation might be that a metabolite is the allergologically active substance.

The above-mentioned allergic drug-protein complexes are often referred to as hapten-carrier complexes. Substances other than pharmacological compounds can also act as hapten. An important category of hapten-like structures are naturally occurring chains of simple sugars, referred to as glycans. The role of glycans as IgE-reactive structure is a source of some confusion. It is generally assumed that pure glycans are unable to induce IgE antibodies. This fits with the general scheme of IgE antibody production as a process that depends on signals provided by $\mathrm{TH} 2$ cells, as described above. Classical MHC-II molecules are very efficient at interacting with peptides, but are unable to combine with pure glycans. However, for glycans coupled to a protein carrier, the situation is different. The cell-anchored antibody on some B cells can interact with the glycan. These B cells bind the glycoprotein via the antibody-glycan interaction. Next, the B cell ingests and digests the glycoprotein and presents the peptides in its $\mathrm{MHCll}$ to the T cell. The T cell receptor interacts with the peptide-MHC-II complex on the B cell, which results in activation of the T cell. The T cell activates the B cell, which results in differentiation of the $B$ cell to an antibody-secreting plasma cell. The important point is that the conventional $\mathrm{TH} 2$ cell does not recognize the glycan and yet it can induce the B cell to produce anti-glycan antibodies. It is possible that glycans can be allergenic not only as glycoprotein, but also as glycolipid, potentially via sources of IL-4 other than TH2 cells. This is presumably mostly relevant for immune responses to invertebrate parasites such as helminths and ticks.

Two prototypic glycans with well-established IgE-binding activity are known as CCD (10) and the alpha-Gal epitope (11). CCD (cross-reactive carbohydrate determinant) refers to a group of related glycans that are characterized by a fucose and/or a xylose that are linked in a specific way to the core of the glycan. Such glycans are produced by invertebrates and plants, but not by vertebrates. Because of the widespread presence of such structures in plant foods, patients with such IgE antibodies demonstrate cross-reactivity to virtually all plant foods (12). In contrast, alpha-Gal (in full: the Gal- $\alpha 1-3 \mathrm{Gal}-\beta 1-3 \mathrm{GlcNAc}$ epitope) is produced by non-primate mammals, but not by humans. This structure, now commonly referred to as alpha-Gal, has been associated with allergy to red meat. For yet unknown reasons, the onset of systemic (skin) symptoms observed upon consumption of meat is not immediate but delayed. Where induction of IgE antibodies against CCD is thought to be driven by pollen exposure and/or insect stings, IgE antibodies against aGAL most likely occur in response to tick bites.

A convenient way to distinguish peptide-based epitopes from glycan-based epitopes is the use of proteolytic enzymes or glycan-destroying chemicals (periodate). Some IgE antibodies show dual recognition towards a glycoprotein allergen: their epitope consists of a combination of part of the glycan and part of the protein (13).

allergome.org]); other invertebrate allergens, such as hemocyanin and hemoglobin, are more likely to sensitize via the airways or via skin contact in an occupational setting (seafood preparation, fish food production) (14).

INITIATOR ALLERGENS VERSUS SECONDARY RESPONDING ALLERGENS

The initial response to an allergen source is possibly characterized by IgE antibodies to one or two "initiator" allergens, which tend to dominate the subsequent more complex lgE response to the allergen source in question. It is therefore an attractive hypothesis that within an allergen source some allergens are more important than others. It might be tempting to call these "major" allergens, but traditionally, an allergen is referred to as "major" if it is recognized by $>50 \%$ of the patients that are sensitized to the source (Textbox 5). Not all these "major" allergens seem to act as "initiator" allergens. 


\section{INNOCENT VERSUS DANGEROUS ALLERGENS}

As expected, a close association is often found between allergen exposure and allergic symptoms. However, for some sensitizers this association is very weak. Several factors may explain the position of an allergen in the allergenicity risk spectrum. In addition to all 3 above-mentioned features, it is presumably

Allergens have to get into our body to sensitize and
to do harm
Allergenic proteins have to be in solution in order
to get into our tissues, both for the sensitization
phase (the interaction reaction with a profession-
al antigen-presenting cell, T cell and B cell) and for
elicitation phase (the interaction with the lgE-anti-
body on the surface of the mast cell or basophilic
leukocyte). For inhaled allergens the allergen will be
attached to a particle (pollen grain, mold spore, mite
fecal particle, a skin flake, hair, a textile fiber or a
fluid droplet) The size of the allergen-carrying par-
ticle (typically $5-20$ micron) is important, because
this determines the most likely site of deposition
(upper or lower airways). The allergen has to be re-
leased into the mucosal fluid to pass through the
airway epithelial barrier. For this passage also a size
limit exists. The diameter of a typical globular aller-
gen molecule is some 1000 times smaller than the
allergen-carrying particle (2-10 nm, in molecular
mass units: $5-50$ kDa).
For food allergens the biophysical requirements for
allergenicity are different in at least 2 ways. First-
ly food processing can substantially change the
solubility of some proteins and in some cases also
change allergenicity. In addition to the destructive
effects of cooking on many allergens, another well-
known example is the loss of allergenicity following
the mincing of apples, due to oxidative browning
of apple polyphenols. This results in denaturation
of apple proteins by the tannin-like structures.
Secondly, the digestive system could increase al-
lergenicity by releasing small soluble allergenic
fragments from poorly soluble conglomerates, or
decrease allergenicity by more extensive fragmen-
tation. In addition to proteases, also the low pH in
the stomach and the detergent action of bile salts
are important in modifying the allergenicity of in-
gested proteins.

relevant that some relatively innocuous sensitizers are associated with relatively high $\operatorname{lgG} / \mathrm{lgE}$ ratios. A prototypic example is tetanus toxoid (15). The allergenic risk spectrum is further discussed below.

\section{WHAT MAKES AN ANTIGEN AN ALLERGEN?}

Some, but not all, antigens that pass through our epithelial barrier trigger an IgE response (Textbox 6). There is an ongoing debate on the features of allergenic proteins (if any) that distinguish these from the more mundane, only lgG-inducing, antigens. It has been proposed that there are few restrictions on the properties of antigens that can induce IgE antibodies $(6,12)$. On the other hand, it has been argued that only a very restricted set of antigens has been found to induce IgE antibodies. This view has been promoted among others by Breiteneder et al. (16).

It is clear that many different types of proteins are allergenic. Some features are not an intrinsic to the protein itself, but rather a consequence of an extrinsic feature: the context of the protein. One such extrinsic feature is its introduction of the protein in the presence of bacterial cell wall components. If a protein enters our body as part of a bacterium, the cytokine response induced by this bacterial infection usually evokes the production of TH1-type cytokines (such as gamma interferon) that are needed to efficiently fight the infection. Gamma interferon prevents the

What are major and minor allergens?
Officially a major allergen is an allergen that is
recognized by lgE antibodies of $>50 \%$ of patients
allergic to the allergen source. A minor allergen is
recognized by by < $50 \%$ of the allergic population.
Although the terminology "major" and "minor" may
suggest major and minor clinical relevance, this is
not necessarily automatically true. Some allergens
may be recognized by $>50 \%$ of patients, but bind
only a small fraction of the overall IgE response
against the allergen source. However, in most cas-
es, major allergens bind a large fraction of the al-
lergen-source specific IgE and are (most likely) of
dominant clinical importance. Most major allergens
also occupy the lower numbers in the nomenclature
system, simply because researchers tended to iden-
tify the most dominant allergens first.


production of TH2-type cytokines (particularly IL-4) that are needed for the switch to IgE.

Regarding intrinsic features, the situation is not so clear-cut. While there is good evidence to support the claim that proteolytic activity may enhance the allergenicity of a protein (example: the mite allergen Der $p$ 1), most allergens are not proteases. Some allergens have enzymatic activities that are unlikely to have an effect on human pathophysiology (example: pectinase activity of pollen allergens). Similarly, many allergens can bind small ligands, but the type of ligand varies considerably. It is not uncommon to find more than 10 different allergenic proteins in a single allergen source material (mite, pollen, peanut, shrimp etc).

It has been suggested that proteins that have close homologues to human proteins are intrinsically more likely to be allergenic. Proteins of the lipocalin family are taken as an example (17). It is clear that some proteins are more allergenic than others. Many factors are known that contribute to these differences, but prediction of the allergenicity (i.e. sensitization risk of a protein not cross-reactive with a known allergen) of a novel protein is as uncertain as next week's weather forecast. The allergenicity debate will undoubtedly go on. From a practical point of view it is relevant that some source materials are more allergenic than others. Illustrative examples include allergens from cooked legumes that are less allergenic than those from roasted peanuts and the lipocalins from dogs (Can $\mathrm{f} 1$, Can $\mathrm{f} 2$ and Can $\mathrm{f} 4$ as major allergens) are less allergenic than the major allergen from cats (Fel d 1), which is a NOT a lipocalin.

\section{CLINICAL RELEVANCE OF INDIVIDUAL ALLERGENIC PROTEINS}

Some inhalant allergen sources contain a single dominant major allergen. The clearest example is Bet $v 1$ in birch pollen, which is responsible for most of the IgE binding to the allergen source. For tree pollen allergic patients in Northwestern and Central Europe, Bet $v 1$ is of decisive clinical importance because there is no "competing" major allergen. In contrast, multiple major allergens have been described for grass pollen [i.e. group 1 (e.g. $\mathrm{Phl} \mathrm{p} \mathrm{1)} \mathrm{and} \mathrm{group} 5$ (e.g. Phl p 5)] and house dust mite [group 1 (e.g. Der $p 1$ ) and group 2 (e.g. Der p 2)]. Currently, it is assumed that in these cases both allergens clinically play an important role. Whether individually they play a role in determining

\section{Textbox 6
Short introduction on the production of IgE anti-
bodies to conventional protein allergens}

A cardinal feature of an allergen is the ability to induce the production of IgE antibodies. The first step to initial IgE antibody production is the activation and expansion of naïve allergen-reactive IgM-producing $B$ cells. This process depends on the interaction between various cells (in particular dendritic antigen presenting cells and $T$ helper cells) and the production of many cytokines.

IgE antibody production is a process that depends on signals provided by $\mathrm{TH} 2$ cells. Major Histocompatibility Complex-II or MHC-II molecules present peptides derived from the protein to $\mathrm{TH} 2$ cells. The $\mathrm{T}$ cell receptor interacts with the peptide-MHC-II complex, which results in activation of the $T$ cell. This activated $T$ cell can activate $B$ cells, but only if these B cells have the same peptide in their surface-anchored MHC-II. In contrast to the antigen-presenting cell, the $B$ cell can only ingest antigens if the antigen binds to the surface-anchored unique antibody of that $B$ cell. These $B$ cells ingest and digest the protein and present the peptides in its MHC-II to the T cell. The B cell further activates the $\mathrm{T}$ cell, which results in the production of cytokines by the T cell. The T cell activates the $B$ cell, which results in differentiation of the $B$ cell to an antibody-secreting plasma cell. The activated IgM-producing B cells can differentiate to change their isotype by a process called class-switch recombination (which results in a change in isotype production from $\lg M$ to $\lg G_{1}, \lg G_{4}, \lg A_{1}$ etc, and sometimes to $\operatorname{lgE}$ ) and to increase their affinity by a process called somatic hypermutation. Both processes result in irreversible changes in the DNA of the $B$ cell. The discovery of the crucial role of IL-4 produced by $\mathrm{TH} 2$-cells (and other cells) in the generation of IgE responses is a milestone in the history of allergy $(18,19)$.

clinical phenotypes is still unknown. Future studies will have to elucidate whether specific molecules are associated with different clinical presentations such as allergic rhinitis or asthma, or with severity phenotypes.

For food allergy, individual allergen molecules have clearly been associated with both defined clinical phenotypes and the severity of allergic symptoms (20). There are three ways one can become allergic to a specific food, (a) by direct exposure to that food 


\section{Textbox 7}

\section{Allergen cross-reactivity and its assessment}

Two allergens are cross-reactive if antibodies exist that recognize both allergens. The antibody will usually have a preference for one allergen over the other. This preferential recognition provides a clue as to identify of the more relevant of the two allergens. A single allergen molecule has several lgE-binding regions (called epitopes). Among IgE antibodies to the birch allergen, Bet $v 1$, that are induced by inhaling birch pollen, two types of antibody populations can be distinguished based on their reactivity with Mal $\mathrm{d} 1$, the homologous protein of apple. Some IgE anti-Bet $v 1$ antibodies will not react with Mal d 1, because they are directed to a non-conserved part of Bet $v 1$. Other IgE antibodies will react not only with Bet $v 1$, but also with Mal $d 1$. The latter reaction will usually be of lower affinity. In this example, Bet $v 1$ can be shown to be a more complete allergen than Mal $d$ 1. Grass pollen extract does not at all inhibit the binding between IgE anti Bet $v 1$ to Mal $d 1$ (because grass pollen extract does not contain a cross-reactive Bet $v 1$ homologue). In this way it is possible to rank allergen source materials (such as birch, apple, celery and peanut) in a cross-reactivity hierarchy. This is most reliably done by using a quantitative bi-directional cross-inhibition protocol (21), but less demanding protocols may also be informative. Some cross-reactions are relatively restricted (example: cross-reactivity among grasses). Others are broader (example: Bet $v 1 /$ Mal d 1, with much lower cross-reactivity to the homologous protein in peanut, celery and potato and no cross-reactivity with grasses). Others cross wider phylogenetic barriers (examples: cross-reactivity between pollen from birch and grass due to profilin $(22,23)$ and cross-reactivity between shrimp and mites due to tropomyosin (24)). Among the glycan epitopes, CCDs tend to be even more cross-reactive (example: cross-reactivity between bee venom and potato (10). Glycan-based cross-reactivity is different from protein-based cross-reactivity, because the degree and fine structure of glycosylation is variable among glycoproteins, even at the single-cell level (25). It is not unusual to find that 2 allergen source materials share several distinct cross-reactive molecules. An example is the cross-reactivity among birch pollen, vegetables and fruits, which was found to be due to at least 3 cross-reactive structures (26).

most likely via the oral route, but the skin has also been proposed, (b) by cross-reactivity between foods, and (c) by cross-reactivity between respiratory allergen sources and foods. The best-known example of the latter is fruit and tree nut allergy as comorbidity with birch pollen allergy (27). The mechanism behind this association is cross-reactivity of Bet v 1-specific lgE with structurally homologous allergens in foods like apple, peach, hazelnut and peanut (Textbox 7). The clinical phenotype observed in such patients is characterized by mild to moderate symptoms restricted to the oral cavity. The explanation for the lack of (severe) systemic symptoms is thought to reside in the labile nature of the Bet $v$ 1-related food allergens that are readily digested in the gastro-intestinal tract (28). This characteristic also explains why sensitization to these Bet $v$ 1-related food allergens is never seen in patients without birch pollen allergy. Apple Mal d 1 or peach Pru p 1, the Bet $\checkmark 1$ homologues of these fruits, are completely digested before they can directly sensitize.

This does not mean that a fruit like peach cannot directly sensitize atopic subjects. For yet unknown reasons this is mainly observed in patients living around the Mediterranean Sea (29). The implicated allergen for peach allergy in countries like, Spain, Italy and Greece is the non-specific lipid transfer protein (LTP), i.e. Pru p 3. IgE antibodies against Pru p 3 can cross-react quite broadly to other fruits, as well as to tree nuts, legumes and some vegetables (30), and they are associated with an increased risk for severe systemic reactions (31). This more "dangerous" profile of LTPs has been attributed to their high degree of protease (and food-processing) resistance (32).

In addition to Bet $v$ 1-related allergens and LTPs, tree nuts, legumes and seeds contain far more abundant seed storage proteins, such as $2 \mathrm{~S}$ albumins, and $7 \mathrm{~S}$ and $11 \mathrm{~S}$ globulins. These proteins are involved in direct sensitization, which often occurs at younger ages. As reported for LTPs, IgE antibodies against these seed storage proteins are remarkably stable and thus better markers for predicting a positive double-blind placebo-controlled food challenge, i.e. for clinical allergy. They are also associated with more severe symptoms. Hazelnut $2 \mathrm{~S}$ (Cor a 14) and $11 \mathrm{~S}$ (Cor a 9) play such a role (33), however for hazelnut allergy as a whole they would not qualify as major allergens because birch pollen-associated hazelnut allergy is the dominant phenotype (34). This illustrates that minor allergens can be of major clinical relevance. 


\section{THE ALLERGENIC RISK SPECTRUM}

Some allergens are considered more dangerous than others in that they elicit more severe allergic symptoms. In contrast, some lgE inducers are not really allergens at all because they don't induce symptoms. The prevalence of such cases has often been underestimated, because they are rarely detected in the doctor's office. They used to be identified mostly in population surveys and birth cohort studies. This situation is changing, particularly since the introduction of the large allergen microarrays, as discussed in Section A04. Peanut is a prototypic example of an allergenic source material to which many people have IgE antibodies, but they can freely consume peanuts. Finding IgE to peanuts in peanuttolerant subjects is particularly common among pollen-sensitized patients (46). This association is due to IgE cross-reactivity between allergens from pollen and (glyco-) proteins in peanut and many other vegetable sources. Bet $v$ 1-related crossreactivity has been discussed above. Peanut contains a cross-reactive homologue, Ara h 8 . However, crossreactivity has also been evident between peanut and grasses, which don't have a cross-reactive Bet $v 1$ homologue. Profilin and CCD have been found to be the most likely additional cross-reactive substances (35). It has been convincingly demonstrated that CCD-specific IgE antibodies are of limited if any clinical relevance (36).

It is tempting to assume that all non-sensitizing cross-reactive allergens are relatively safe. While this is true in many cases, severe reactions caused by exposure to such presumed non-sensitizing crossreactive allergens have been reported.

The often relatively low biological activity of crossreacting allergens may reflect lower epitope density and lower affinity of the IgE-allergen interaction, but it has been disappointingly difficult to predict biological activity on the basis of immunochemical characteristics in individual cases.

The use of a bioassay such as the basophil-activation test is a promising alternative. Allergen sources are complex, heterogeneous mixtures of proteins. They contain harmless IgE-binding structures such as CCD, molecules that induce mild symptoms only and molecules that are associated with severe symptoms including food or insect venom-induced anaphylaxis. Moreover, molecules can inform us about the origin and route of sensitization, sometimes reflected in clear geographic differences. Dissection of these molecular characteristics of allergen sources is of the utmost importance to improve allergy diagnosis, prevention and therapy.

\section{MOLECULAR SENSITIZATION PROFILES: BIOMARKERS FOR DISEASE PROGRESSION?}

A relatively unexplored area is whether IgE recognition profiles have predictive value for disease progression. This field will most likely develop rapidly in the near future. Some evidence from the field of food allergy suggests that recognition profiles of specific epitopes on major food allergens, using short synthetic peptides, can predict outgrowth or persistence (37). Another study reported that persistence of peanut allergy is associated with the number of peanut allergens recognized (38). These studies are just the beginning and illustrate the importance of carefully dissecting molecular composition of allergen sources.

\section{ALLERGEN EXTRACTS: IMPERFECT BUT NOT YET OBSOLETE}

Both allergy diagnostics and allergen immunotherapy (AIT) still heavily depend on extracts of the allergen sources. In particular in diagnostics, molecular approaches are gaining ground rapidly (39), but extracts can certainly not be dismissed (40). Usually, allergen extracts are simple aqueous extracts of the crude allergen source. In most cases, extraction is carried out at neutral or close to neutral $\mathrm{pH}$, followed by a defatting step, and dialysis. In the case of food extracts the source material may sometimes be partially processed before extraction, e.g. peanut meal of mildly roasted peanuts.

What are the potential shortcomings of allergen extracts? Allergen sources are biological products with inherent variability of composition. Extraction with aqueous buffers at neutral $\mathrm{pH}$ may not optimally extract all possible allergens, especially those that are lipid soluble. This is particularly relevant for food extracts because the natural route of exposure through the stomach includes exposure to low $\mathrm{pH}$. A good example of a food allergen that is not optimally extracted at neutral $\mathrm{pH}$ is LTP from legumes such as peanut and lentils (41). This phenomenon may also be the explanation for the huge variability in 
LTP content reported for a series of commercially available skin test reagents for hazelnut allergy (42). Extraction at low $\mathrm{pH}$ has proven to be the solution. Another problem encountered when preparing diagnostic food extracts, in particular of fruits and vegetables, is that enzymatic oxidative processes are initiated when the food tissue is disrupted. In particular Bet $v$ 1-related food allergens such as Mal d 1 in apple, Pru p 1 in peach or Cor a 1 in hazelnut are sensitive to these processes and they lose their IgE-binding capacity. Finally, the defatting step has been implicated in loss of lipophilic allergens such as oleosins in legumes, nuts and seeds (43). Together, these shortcomings are probably the main reason that skin testing for many foods is done using the poorly standardized but more sensitive prick-toprick method with fresh foods. To overcome the loss of sensitivity for detecting IgE antibodies against hazelnut Cor a 1, the extract can effectively be improved by spiking with recombinant Cor a 1 . Although GMP-requirements do not facilitate broad in vivo application of recombinant allergens, e.g. recombinant Mal $d 1$ has been successfully used in skin testing and oral challenges $(44,45)$.

\section{CONCLUSION}

Overall, one can conclude that the multitude of factors influencing extract composition results in batchto-batch and company-to-company differences, which may lead to differences in the diagnostic and therapeutic management of patients. For several reasons, this is particularly true for skin test reagents. Traditionally, these products are provided for free by allergen manufacturers to support the selection and subsequent sales of immunotherapy products. Regulatory pressure now requires skin test reagents to be registered. This development that has resulted in many "less important" allergen specificities being removed from the market, because they would require too big an investment in documentation of their clinical performance. Potential solutions such as spiking with recombinant allergens are not really an option either, because recombinant allergens used in vivo need to be produced under GMP conditions and tested in toxicity studies. Again, this is too large an investment. In the future the number of skin test reagents available will therefore be rather limited, and extracts for in vitro diagnosis will continue to be improved by the use of different extraction methods and/or spiking. Increasingly, molecular diagnostics will supplement and partly replace extract-based tests, to overcome the imperfections of extracts and this should also lead to allow improved risk assessment and subsequent advice to patients.

\section{References}

1. Johnson P, Marsh DG. 'Isoallergens' from rye grass pollen. Nature 1965;206:935-937.

2. King TP, Norman PS, Connell JT. Isolation and characterization of allergens from ragweed pollen. Biochemistry 1964;3:458-468.

3. Aas K, Jebsen JW. Studies of hypersensitivity to fish. Partial purification and crystallization of a major allergenic component of cod. Int Arch Allergy Appl Immunol 1967;32:1-20.

4. Chapman MD, Platts-Mills TA. Purification and characterization of the major allergen from Dermatophagoides pteronyssinus-antigen P1. J Immunol 1980;125:587-592.

5. King TP, Hoffman D, Løwenstein H, Marsh DG, Platts-Mills TA, Thomas W. Allergen nomenclature. Allergy 1995;50:765-774.

6. Radauer C, Nandy A, Ferreira F Goodman RE, Larsen $\mathrm{JN}$, Lidholm J et al. Update of the WHO/IUIS Allergen Nomenclature Database based on analysis of allergen sequences. Allergy 2014;69:413-419.

7. Aalberse RC. Structural biology of allergens. J Allergy Clin Immunol 2000;106:228-238.

8. Aalberse RC, Akkerdaas J, van Ree R. Cross-reactivity of IgE antibodies to allergens. Allergy 2001;56:478490.

9. Ackaert C, Kofler S, Horejs-Hoeck J, Zulehner N, Asam $\mathrm{C}$, von Grafenstein S et al. The impact of nitration on the structure and immunogenicity of the major birch pollen allergen Bet v 1.0101. PLoS One 2014;9:e104520.

10. Aalberse RC, Koshte V, Clemens JG. Immunoglobulin $\mathrm{E}$ antibodies that crossreact with vegetable foods, pollen, and Hymenoptera venom. J Allergy Clin Immunol 1981;68:356-364.

11. Platts-Mills TA, Schuyler AJ, Tripathi A, Commins SP. Anaphylaxis to the Carbohydrate Side Chain Alphagal. Immunol Allergy Clin North Am 2015;35:247-260.

12. Van Ree R. Carbohydrate epitopes and their relevance for the diagnosis and treatment of allergic diseases. Int Arch Allergy Immunol 2002;129:189-197.

13. Aalberse RC, Crameri R. IgE-binding epitopes: a reappraisal. Allergy 2011;66:1261-1274.

14. Pascal M, Grishina G, Yang AC et al. Molecular Diagnosis of Shrimp Allergy: Efficiency of Several Allergens to Predict Clinical Reactivity. J Allergy Clin Immunol Pract 2015;3:521-529. 
15. Grüber C, Lau S, Dannemann A, Sommerfeld C, Wahn $\mathrm{U}$, Aalberse RC. Down-regulation of IgE and IgG4 antibodies to tetanus toxoid and diphtheria toxoid by covaccination with cellular Bordetella pertussis vaccine. J Immunol 2001;167:2411-2417.

16. Breiteneder H, Mills EN. Molecular properties of food allergens. J Allergy Clin Immunol 2005;115:14-23.

17. Virtanen $T$, Kinnunen $T$, Rytkönen-Nissinen $M$. Mammalian lipocalin allergens--insights into their enigmatic allergenicity. Clin Exp Allergy 2012;42:494504.

18. Coffman RL, Ohara J, Bond MW, Carty J, Zlotnik A, Paul WE. B cell stimulatory factor-1 enhances the IgE response of lipopolysaccharide-activated B cells. J Immunol 1986;136:4538-45341.

19. Paul WE. History of interleukin-4. Cytokine 2015;75:37.

20. Nicolaou N, Poorafshar M, Murray C et al. Allergy or tolerance in children sensitized to peanut: prevalence and differentiation using component-resolved diagnostics. J Allergy Clin Immunol 2010;125:191197.

21. Aalberse RC. Assessment of allergen crossreactivity. Clin Mol Allergy 2007;5:2.

22. ValentaR,DuchêneM,PettenburgerKetal.Identification of profilin as a novel pollen allergen; IgE autoreactivity in sensitized individuals. Science 1991;253:557-560.

23. van Ree R, Voitenko V, van Leeuwen WA, Aalberse RC. Profilin is a cross-reactive allergen in pollen and vegetable foods. Int Arch Allergy Immunol 1992;98:97104.

24. Witteman AM, Akkerdaas JH, van Leeuwen J, van der Zee JS, Aalberse RC. Identification of a cross-reactive allergen (presumably tropomyosin) in shrimp, mite and insects. Int Arch Allergy Immunol 1994;105:56-61.

25. Altmann F. The role of protein glycosylation in allergy. Int Arch Allergy Immunol 2007;142:99-115.

26. Vieths S, Scheurer S, Ballmer-Weber B. Current understanding of cross-reactivity of food allergens and pollen. Ann N Y Acad Sci 2002;964:47-68.

27. Calkhoven PG, Aalbers M, Koshte VL, Pos O, Oei HD, Aalberse RC. Cross-reactivity among birch pollen, vegetables and fruits as detected by IgE antibodies is due to at least three distinct cross-reactive structures. Allergy 1987;42:382-390.

28. Sancho Al, Wangorsch A, Jensen BM et al. Responsiveness of the major birch allergen Bet $v$ 1 scaffold to the gastric environment: impact on structure and allergenic activity. Mol Nutr Food Res 2011;55:1690-1699.

29. Reuter A, Lidholm J, Andersson $\mathrm{K}$ et al. A critical assessment of allergen component-based in vitro diagnosis in cherry allergy across Europe. Clin Exp Allergy 2006;36:815-823.
30. Asero R. In patients with LTP syndrome food-specific IgE show a predictable hierarchical order. Eur Ann Allergy Clin Immunol 2014;46:142-146.

31. Fernández-Rivas M, Bolhaar S, González-Mancebo E et al. Apple allergy across Europe: how allergen sensitization profiles determine the clinical expression of allergies to plant foods. J Allergy Clin Immunol 2006;118:481-488.

32. Asero R, Mistrello G, Roncarolo D et al. Lipid transfer protein: a pan-allergen in plant-derived foods that is highly resistant to pepsin digestion. Int Arch Allergy Immunol 2000;122:20-32.

33. Masthoff LJ, Mattsson L, Zuidmeer-Jongejan L et al. Sensitization to Cor a 9 and Cor a 14 is highly specific for a hazelnut allergy with objective symptoms in Dutch children and adults. J Allergy Clin Immunol 2013;132:393-399.

34. Datema MR, Zuidmeer-Jongejan L, Asero R et al. Hazelnut allergy across Europe dissected molecularly: A EuroPrevall outpatient clinic survey. J Allergy Clin Immunol 2015;136:382-391.

35. van der Veen MJ, van Ree R, Aalberse RC et al. Poor biologic activity of cross-reactive IgE directed to carbohydrate determinants of glycoproteins. J Allergy Clin Immunol 1997;100:327-334.

36. Mari A, Ooievaar-de Heer $P$ et al. Evaluation by double-blind placebo-controlled oral challenge of the clinical relevance of IgE antibodies against plant glycans. Allergy 2008;63:891-896.

37. Järvinen KM, Beyer K, Vila L, Bardina L, Mishoe M, Sampson HA. Specificity of IgE antibodies to sequential epitopes of hen's egg ovomucoid as a marker for persistence of egg allergy. Allergy 2007;62:758-765.

38. Agabriel C, Ghazouani O, Birnbaum J et al. Ara h 2 and Ara h 6 sensitization predicts peanut allergy in Mediterranean pediatric patients. Pediatr Allergy Immunol 2014;25:662-667.

39. Skamstrup Hansen K, Poulsen LK. Component resolved testing for allergic sensitization. Curr Allergy Asthma Rep 2010;10:340-348.

40. Aalberse JA, Aalberse RC. A lesson from componentresolved testing: we need better extracts. J Allergy Clin Immunol Pract 2014;2:635-636.

41. Akkerdaas J, Finkina El, Balandin SV et al. Lentil (Lens culinaris) lipid transfer protein Len c 3: a novel legume allergen. Int Arch Allergy Immunol 2012;157:51-57.

42. Akkerdaas $\mathrm{JH}$, Wensing $M$, Knulst $A C$ et al. How accurate and safe is the diagnosis of hazelnut allergy by means of commercial skin prick test reagents? Int Arch Allergy Immunol 2003;132:132-140.

43. Zuidmeer-Jongejan L, Fernández-Rivas $M$, Winter MG et al. Oil body-associated hazelnut allergens including oleosins are underrepresented in diagnostic extracts but associated with severe symptoms. Clin Transl 
Allergy 2014;4:4.

44. Bolhaar ST, Zuidmeer L, Ma $Y$ et al. A mutant of the major apple allergen, Mal d 1, demonstrating hypo-allergenicity in the target organ by doubleblind placebo-controlled food challenge. Clin Exp
Allergy 2005;35:1638-1644.

45. Kollmann D, Geroldinger-Simic M, Kinaciyan T et al. Recombinant Mal d 1 is a reliable diagnostic tool for birch pollen allergen-associated apple allergy. J Allergy Clin Immunol 2013;132:1008-1010. 


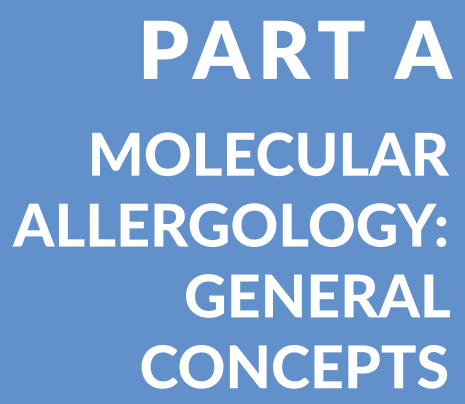
CONCEPTS

\section{MOLECULAR ALLERGY DIAGNOSTICS IN CLINICAL PRACTICE}
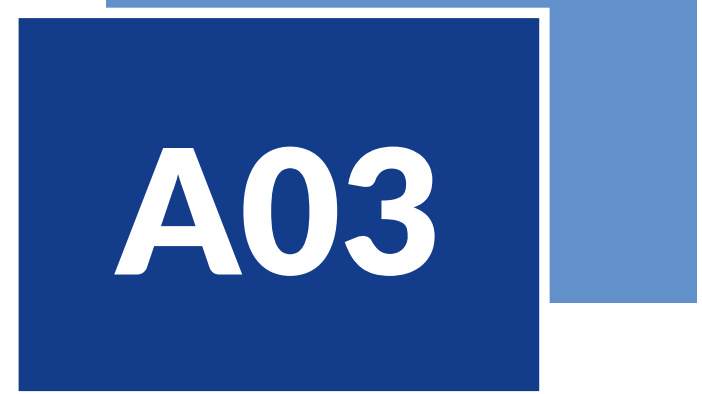

Jörg Kleine-Tebbe, Thilo Jakob

Conventional allergy diagnostics are based on detecting specific IgE antibodies in the blood or skin with reactivity for allergen extracts obtained from various allergen sources such as pollen grains, house dust mite, or cat dander. These extracts contain many components (glycosylated and unglycosylated proteins lipids etc.) the majority of which are irrelevant for the allergic reaction and allergy diagnostics.

Progress in molecular biology over the last 3 decades has allowed us to identify and characterize single allergens in detail at a molecular level. Large allergen data banks have been established (e.g. www.allergen. org, www.allergome.org) in which information on identified allergens is accessible for the scientific and medical community. Currently as of June 2015, more than 3000 different allergens (plus approx. 1400 isoforms) have been described (www.allergome. org), almost 1500 of which have been expressed as recombinant proteins. Some of these allergens have already and will become available for in vitro allergy diagnostics, either as highly purified native or recombinant proteins.

The use of single allergenic molecules (instead of extracts) has introduced a new area of high-resolution molecular allergy diagnostics (also designated
Diagnostic work-up for IgE mediated allergic reactions/diseases starts with the history, followed by sensitization tests (skin, lgE and basophil tests) and optional challenge tests (Top-down approach)

Molecular allergens for IgE testing provide additional information, particularly in polysensitized patients and with allergens of low abundance, low stability or associated risks

IgE reactivity to members of the same allergen family reflect the degree of protein homology and IgE cross-reactivity. If it is high, the relevance needs to be sorted out clinically. In case it is low, selected IgE testing of other family members can provide additional information.

Proper interpretation should complete diagnostic testing: Positive sensitizations to allergen extracts or molecules are only clinically relevant in case of corresponding symptoms 
"component-resolved diagnostics", CRD (1)) and changed our understanding of sensitization profiles and cross-reactivity (2). Daily routine molecular allergy diagnostics offers a number of benefits that give us a higher diagnostic precision and allow for better management of the patient. To utilize the full potential in clinical practice, an in-depth general knowledge of molecular allergology as well as a clear rationale for its use are needed as it relates to when and how allergenic molecules are to be used for diagnostic purposes ("always think molecular - use molecules, when needed").

This section

- summarizes general considerations for the diagnostic work-up of allergic patients in the age of molecular allergology,

- provides a number of universal reasons to utilize molecular diagnostics, and

- describes the rationale behind different approaches ("from symptoms to molecules"; "from molecules to symptoms")

that allow us to make the optimal use of molecular allergy diagnostics in clinical practice.

\section{GENERAL CONSIDERATIONS FOR THE DIAGNOSTIC WORK-UP OF ALLERGY PATIENTS}

In patients with suspected IgE-mediated reactions and/or diseases, the diagnostic algorithm should include the following sequential steps (Fig. 1 and 2):

A. Clinical evaluation and examination

a. allergy-related history including information on co-morbidities, differential diagnoses

b. clinical examination

B1. Sensitization test(s) with allergen extracts, like skin prick tests (SPT) (3), IgE tests (4), and basophil activation tests (BAT) (5) if needed, providing information on the allergic sensitization, that is "the risk for allergy"

B1. indirect or direct evidence of allergenspecific IgE

B3. interpretation of sensitization test result(s) (clinical relevance or not?)

B2. Sensitization test with allergenic molecules (applying allergen-specific lgE tests)

B2. direct evidence of present or absent allergen-specific IgE to defined allergens?

B3. interpretation of sensitization test result(s) (clinical relevance or not?)

C. Challenge test (optional, depending on the allergen source in question)

a. demonstration of clinical symptoms upon allergen exposure

b. interpretation (qualitative conclusion: positive or negative?)

For each of these steps certain general considerations may be helpful to make the best out of our expanding knowledge of the molecular nature of allergens. These considerations are listed below and combined with examples from clinical practice (italic font).

A. Some history-related information might immediately suggest certain underlying allergenic molecules ("think molecular"), due to

- temporal relationship of symptoms with particular exposures (i.e. pollen, furry animals, house dust, certain food items)

- patient's observation of certain triggers representing particular pattern (related triggers, i.e. indicative selection of food items),

- degree and variety of symptoms indicating involvement of certain molecules (either mild oropharyngeal or severe systemic symptoms to i.e. legumes, tree nuts or seeds)

\section{Examples:}

Oropharyngeal symptoms after eating raw apples, hazelnuts, carrots and/or soy AND symptoms of allergic rhinoconjunctivitis during the birch pollen season: Suggestive for the presence of IgE to major birch pollen allergen Bet $v$ 1-specific with subsequent serological (and clinical) cross reactions (primary inhalant sensitization, but due to structural similarity of Bet $v 1$ and its homologs secondary symptoms in the oral cavity upon exposure).

Oropharyngeal symptoms after eating various (nonrelated) fruits and vegetables, including melon, citrus fruits, banana, avocado, peanuts AND symptoms of allergic rhinoconjunctivitis during the grass pollen season: Suggestive for IgE to minor (grass) pollen allergen profilin (i.e. timothy profilin $\mathrm{Phl} p$ 12) with subsequent serological (and clinical?) cross reactions.

Anaphylaxis in the context of exercise after consumption of wheat containing food stuff, which is suggestive for IgE to omega-5 gliadin Tri a 19. 


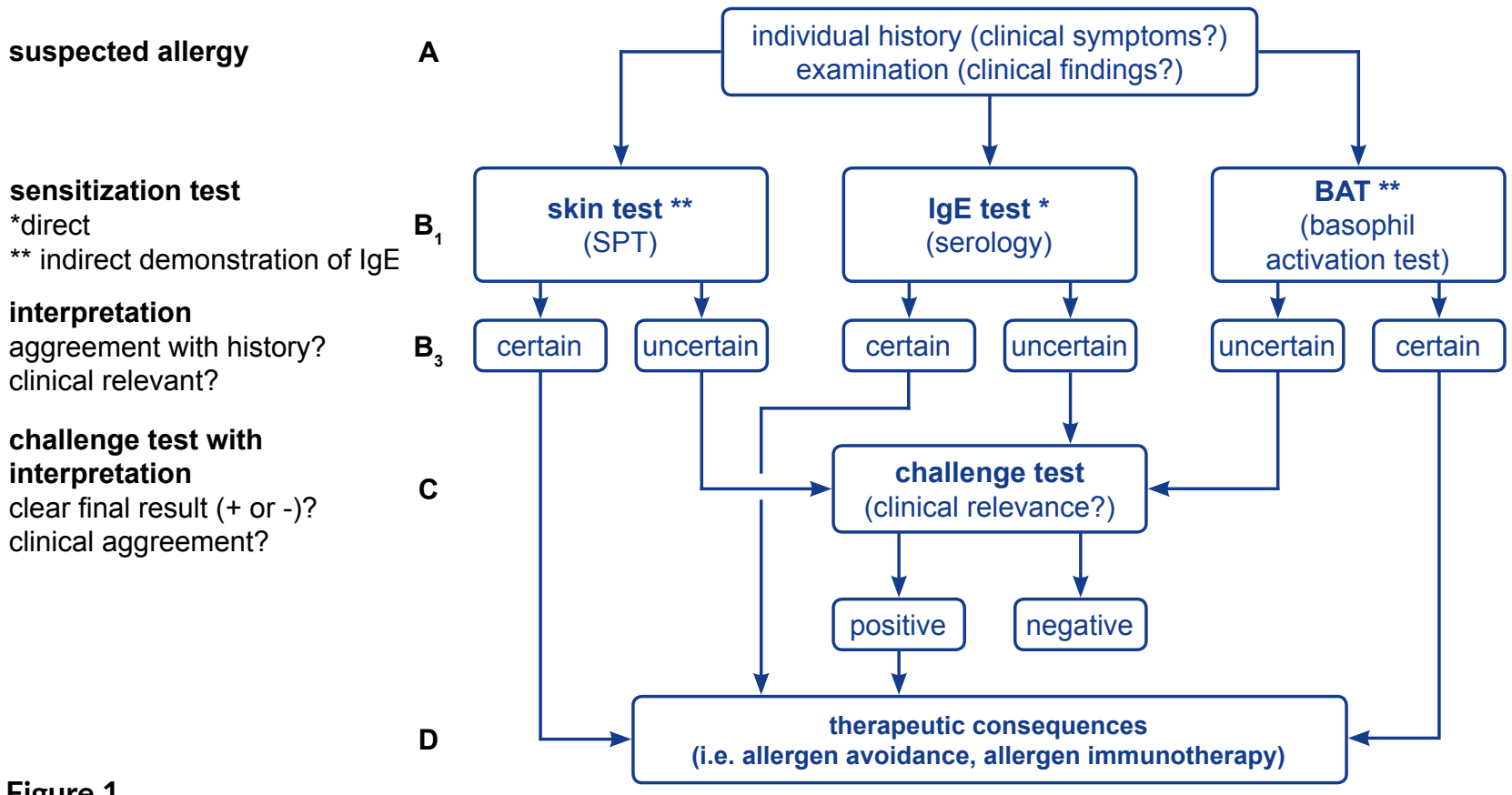

Figure 1

General diagnostic work-up of IgE-mediated allergic reactions and diseases. After collecting the allergy history and performing a physical examination (A) appropriate sensitization tests are applied (B1). IgE-sensitizations are directly demonstrated by serological allergen-specific IgE determination (4) and/or indirectly by skin prick tests (SPT) (3) or basophil activation tests (BAT) (5), if indicated. Sensitization tests should be completed by careful interpretation (B3), validating the agreement with the history to ultimately evaluate the clinical relevance of the obtained results. In case of uncertainty (B3), i.e. due to a non-conclusive history, challenge test are applied (C) to induce allergic symptoms under controlled conditions ( 7 , 8). A clear outcome will support the decision on the clinical relevance of suspected allergen triggers and provide the basis for potential therapeutic consequences (D).

B1. Some sensitization test results with extracts, either by SPT or serology, might immediately suggest certain underlying allergenic molecules, i.e.:

- particular pattern of sensitization, pointing to cross-reactive molecules

- unusual pattern or magnitude of sensitization test results (i.e. to non-related allergen sources)

Examples:

Positive reactions to fagales tree (hazel, alder, birch, beech, oak) pollen, potentially with symptoms during the tree pollen season:

Suggestive for the presence of IgE to major birch pollen allergen Bet $v 1$ with subsequent serological (and potential clinical) cross reactions to related fagales trees.

Positive reactions to non-related pollen plants, sometimes all pollen sources, with various, not necessarily corresponding symptoms:
Suggestive for the presence of IgE to pan-pollen allergens (profilins, i.e. Bet v 2 or Phl p 12 and/or polcalcins, i.e. Bet $v 4$ or Phl $p$ 7) with subsequent serological (and clinical?) cross reactions to profilin-containing pollen and plant foods as well as polcalcin-containing pollen. In case of double sensitization to profilin AND polcalcin: commonly positive reactions to ALL pollen species can be expected and it is not possible to define the precise sensitization specificity with allergen extracts.

Multiple sensitization to different furry animals which is suggestive of IgE reactivity to the animal pan allergen serum albumin or certain lipocalins

B2. Tests for allergen-specific IgE to molecules (Fig. 2) can be applied with one reagent as single test (singleplex) (4) or with many reagents (multiplex) (6) for i.e. screening purposes (see section A04 for more technical information). Reasons for molecular 


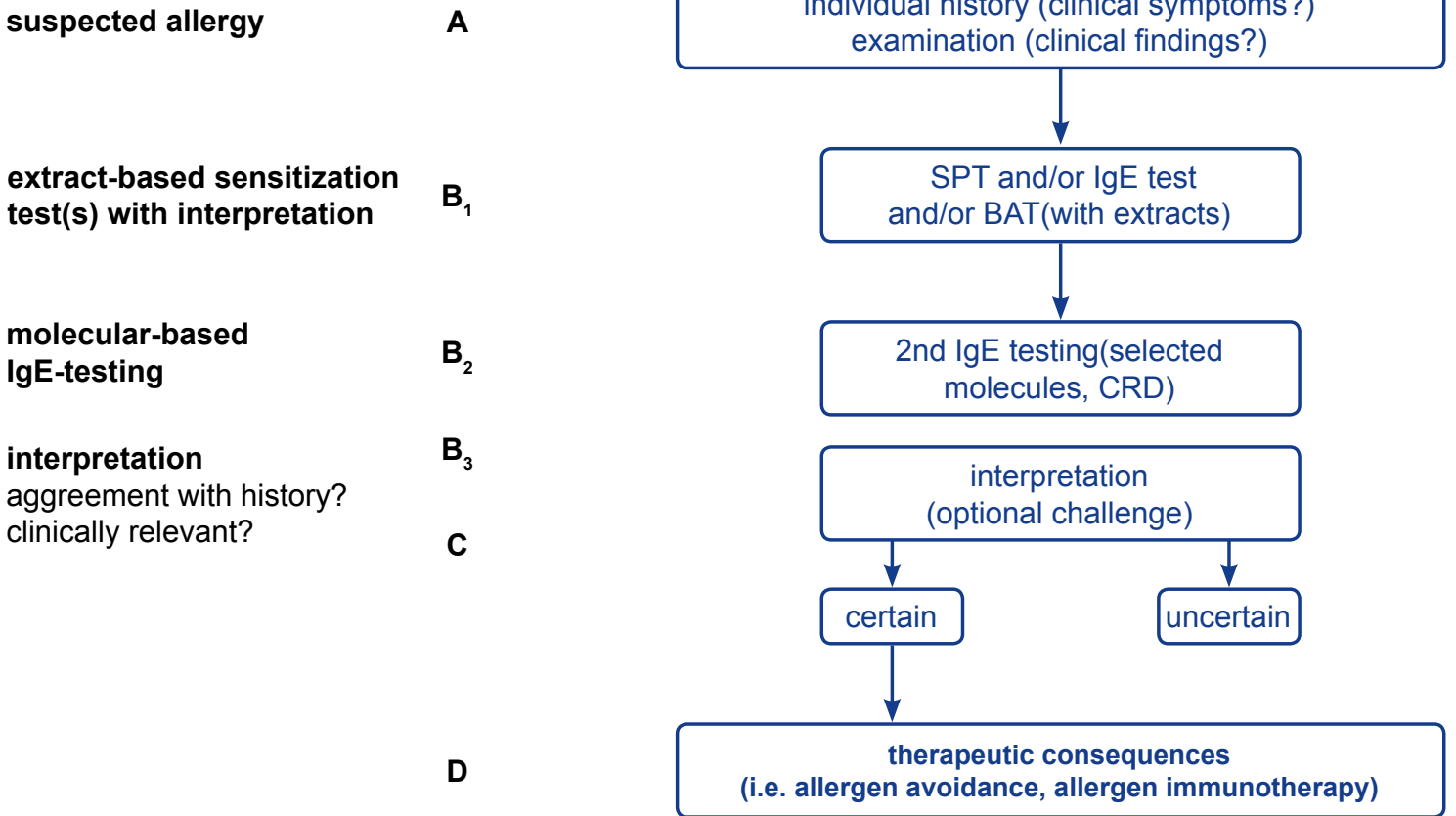

\section{Figure 2}

Diagnostic work-up with targeted (singleplex) molecular-based lgE-testing. After sensitization testing (B1) with allergen extracts, more detailed information regarding the IgE-repertoire is obtained with molecular-based testing (B2). This diagnostic approach, coined "top-down" is followed by a thorough interpretation (B3) including challenge tests (C), if needed.

IgE testing, either singleplex or multiplex, will be summarized below (see also section 5 ).

Following options will be extensively discussed in the following sections:

- "Classical" diagnostic work-up (Fig. 1) with extractbased sensitization test(s), before employing allergen-specific IgE-testing with molecules (Fig. 2) ("top-down approach", section 3)

- Novel diagnostic work-up with primarily allergen molecule-related information, i.e. allergenspecific IgE to a panel of related molecules to explain diverse clinical reactions or diseases ("bottom-up approach", section 4)

- Integrated use of both approaches, first working from the history, applying extract-based sensitization tests (SPT, IgE) before exploring the entire individual IgE repertoire with an extended panel of allergen molecules ("U-shape approach", section 5).

C. Finally, if the information provided by the patient's history and/or their sensitization test results is inconclusive and does not allow for a clear decision on the clinical relevance of the suspected allergen source, additional challenge tests should be applied. They should ultimately demonstrate or rule out clinical symptoms following allergen exposure.

In case of inhalants (pollen, mites, molds, furry animals) standardized extracts of the suspected allergen source are applied on the mucosal surface (i.e. conjunctiva, nose) (7). In case of food allergy (plant foods, i.e. fruits, vegetables, legumes) increasing doses of the suspected allergen are given orally, ideally in a double-blind, placebo-controlled fashion (8, 9).

Subsequent immediate or delayed (i.e. exacerbation of an atopic eczema) type allergic symptoms would prove current clinical relevance; in contrast, a lack of any objective clinical reaction would rule out a previously suspected allergy (provided that no additional co-factors are required to elicit the allergic reaction, as exemplified by all forms of fooddependent, exercise induced anaphylaxis (10)). 
Subsequently, interpretation needs to be integral part of any suspected sensitization (i.e. demonstrated by SPT, IgE, BAT) as well of a challenge test outcome (Fig. 1).

- A positive test result is only clinically relevant in the case of corresponding allergic symptoms that are temporally associated with a defined allergen exposure.

- A negative test (i.e. allergen-specific IgE) result against one recombinant allergen molecule or a mixture of natural isoforms of one single allergen can indicate exclusion of allergic sensitization or risk of allergy to the allergen specificity in question (see section 5 for details), provided that

a) the total IgE is high enough (i.e. > $20 \mathrm{kUA} / \mathrm{l}$ )

b) the allergen reagent is of sufficient abundance, fully intact, and presenting all its epitopes

c) the analytical performance of the $\lg E$ antibody assay has been optimized for a low limit of quantitation (i.e. $0.1 \mathrm{kUA} / \mathrm{L}$ ).

In conclusion, the clinical relevance of an allergic sensitization (i.e. presence of allergen-specific $\mathrm{IgE}$, independent of the use of allergen extracts or molecules for diagnostic purposes) can ultimately only be determined by the physician and not by the test. Therefore, the complete diagnostic results of sensitizations as well as challenge test results will always have to be interpreted within the clinical context and on the basis of the individual's case history.

\section{COMMON REASONS TO UTILIZE MOLECULAR DIAGNOSTICS AND THEIR LIMITATIONS}

There are a number of general reasons that speak in favor of using single allergens as compared to allergen extracts (Fig. 3). They are principally related to an improved assay performance (i.e. assay sensitivity and analytical specificity) and/or to additional levels of interpretation such as risk assessment or differentiation between genuine ("primary") sensitization and cross reactivity, particularly in presumed polysensitizations.

While these arguments clearly support the use of single allergens in clinical routine, we need to be careful not to over interpret results of molecular allergy diagnostics, which have clear limitations when it comes to predicting clinical outcomes. slgE test results - regardless of using extracts or single molecules - only reflect the status of sensitization and always have to be interpreted in the context of the clinical data. The benefits and limitations of molecular allergy diagnostics are outlined below.

Factors improving assay performance are able to warrant the use of allergenic molecules instead of extracts:

\section{Molecules of low abundance and/or weak stability}

If allergen molecules, being of low abundance or missing in the extract, are able to improve the assay's analytical sensitivity (LoQ) of an IgE test, their use is meaningful and important (i.e. Gly $m$ 4 vs soy extract, omega-5-gliadin vs wheat extract).

\section{Risk- or severity-associated molecules}

If allergen molecules provide improved analytical specificity ("selectivity") and allow additional clinical assumption(s) (i.e. increased risk association, clinical severity or other associated clinical features of an IgE-sensitization), their use is again meaningful and recommended (i.e. storage proteins Ara h 1, 2, 3, 6 vs whole peanut extract).

\section{Indicator of cross reactivity}

Certain allergen molecules can serve as indicators for serological cross sensitizations through the binding of cross-reactive IgE. In case of a positive result they can demonstrate the lack of analytical specificity of an IgE test with allergen extracts (in affected subjects with potential cross reactions) (e.g. profilin or polcalcin, members of plant panallergen families),

\section{Marker of genuine (species-specific) sensitization}

Particular allergen molecules (often major allergens) can serve as markers for a primary, "genuine", family- or species-specific sensitization. They provide improved analytical specificity compared to allergen extracts (particularly in affected subjects with potential cross reactions) (e.g. marker allergens Ves $v 1$ and Ves $v 5$ from yellow jacket venom and marker allergens Api m 1, Api m 3, Api $m 10$ from honey bee venom vs hymenoptera whole venom preparations from the corresponding species). 


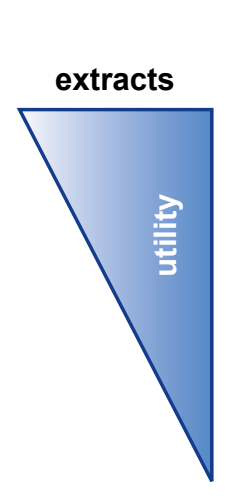

A. Sensitization profile

$$
=\lg E \text { repertoire }
$$

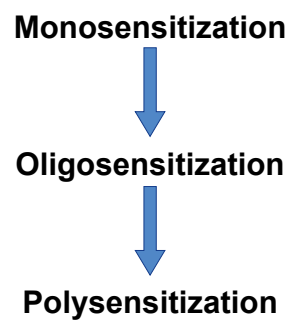

C. Abundance

in whole extract

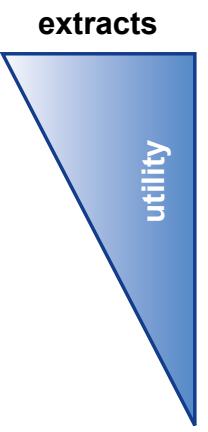

in whole extract

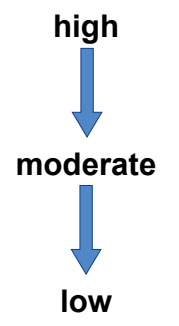

B. Associated risks for clinical reactions
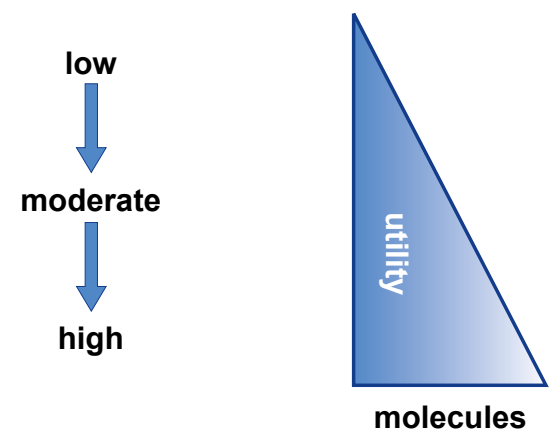

D. Stability

of single allergen

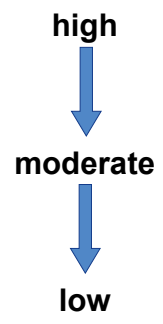

Figure 3

Utility of allergen extracts and allergenic molecules for diagnostic work-up Mono-/limited oligo-sensitizations (A) and/or minor clinical risks (B) as well as high abundance allergen molecules in the allergen source suspected (C) and/or allergens of high stability (D) indicate suitability of allergen extracts for proper diagnostic work-up. In case of polysensitizations and/or allergen triggers associated with high clinical risks as well as low abundant and/or labile allergenic molecules in the extract, the diagnostic work-up should consider the use of molecular components for lgE detection.

The above rationale is primarily based on the status of sensitization (presence or absence of IgE antibody) and not on the clinical manifestations of the subjects. Examples are given in more detail below in Section 2.

\section{Limitations in improving predictions on clinical outcome from (isolated) molecular based sensitization test results}

In contrast to parameters that describe the analytical assay performance (analytical sensitivity, analytical specificity, see above), clinical diagnostic criteria are required when it comes to making predictions on the clinical outcome. General clinical diagnostic criteria in the field of (molecular) allergology include:
I. diagnostic sensitivity (proportion of positive IgE antibody tests in patients with allergic symptoms/ disease)

II. diagnostic specificity (proportion of negative lgE antibody tests in asymptomatic/healthy individuals)

III. indicator of clinical cross reactivity (allergic symptoms to allergenic sources that did not elicit the primary sensitization)

IV. prediction of clinical reactions (positive predictive value, PPV, negative predictive value, NPV, thresholds, likelihood ratio etc.)

All of the above criteria require a thorough individual interpretation of each test result based on the previous history and if needed additional proof of 
interpretation match with history? clinically relevant?

\section{molecular-based IgE-testing broad panels of components, if needed \\ complex allergy or previous testing with complex outcome}

\section{Figure 4}

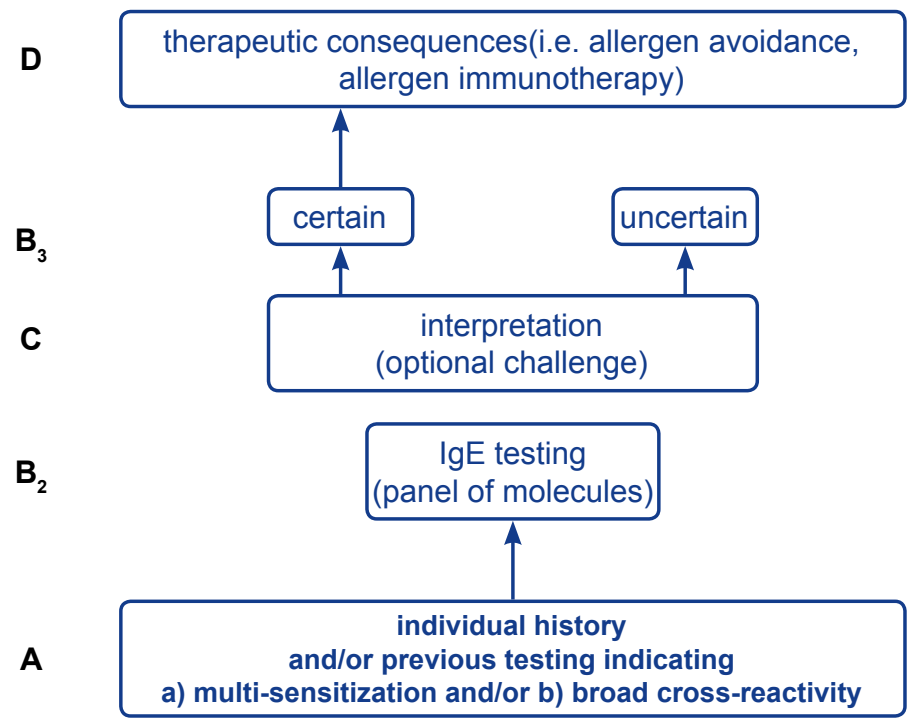

Diagnostic work-up with broad (multiplex) molecular-based IgE-testing: In complex cases and/or inconclusive diagnostic outcomes after previous testing $(A)$ a panel of molecular allergens might be applied for subsequent (multiplex) lgE testing (B2). After final interpretation (B3) with an optional challenge (C) this approach, coined "bottom-up", might facilitate superior decisions on therapeutic consequences.

reproducible and objective symptoms in the affected allergic subject upon exposure (i.e. challenge test). Subsequently, these clinical criteria will go beyond the essential ("raw") assay result of an allergen-specific lgE test (IgE-sensitization in question: yes or no).

In general, clinical diagnostic criteria are

- less suitable and sometimes misleading for a proper assay evaluation of sensitization tests

- largely not needed to demonstrate the advantage of single allergenic molecules in IgE assays and

- often burdened with unsatisfactory study results due to the per se limited and imperfect prediction of clinical outcomes (clinical reactivity) by sensitization tests.

In conclusion, the above listed advantages of molecular allergy diagnostics mostly refer to an improved detection and discrimination of allergic sensitization. Molecular allergy diagnostics, however, have clear limitations for improving predictions of the clinical outcome. After all, the detection of slgE is primarily an indicator of "sensitization" and - despite various attempts to integrate clinical data and results of challenge tests - not a decisive predictor of clinical reactivity.

\section{FROM SYMPTOMS TO MOLECULES: THE "TOP-DOWN APPROACH"}

Based on the experience that detection of allergen specific IgE does not equal clinical relevance, current guidelines on allergy diagnostics (4) recommend that the diagnostic workup should be primarily guided by the clinical symptoms. Random screening for IgE sensitization is discouraged since the number of positive lgE results to a certain allergen source usually exceeds by far the number of clinical relevant allergies (11). This "top-down" approach - from the symptoms to the allergen source also applies to molecular allergy diagnostics and can be defined as follows:

Definition: Diagnostic work-up from symptoms to molecules ("top-down approach", (Fig. 2) aims for more detailed characterization of the lgE-repertoire unfolding important molecular IgE-sensitizations that provide information beyond the extract based test results.

In practice, taking the case history and performing a symptom guided diagnostic work-up with extractbased SPT and/or IgE-testing usually allows the identification (or exclusion) of IgE-sensitizations to potentially involved allergen sources (Fig. 1). Here two main scenarios are usually encountered: 
A) Limited numbers of positive extract based sensitization test results

In case of rather restricted IgE antibody responses with only few positive results to inhalants like tree or grass or weed pollen, certain molds, one or two furry animals, only a single insect venom (bee or wasp venom) or only selected food items, the analytical specificity of an extractbased sensitization test might be sufficient to identify the underlying allergen source. No further testing would be required, if the extractbased sensitization test permits a proper and specific diagnostic work-up. The exceptions are potentially false negative sensitization tests in case of underrepresented or unstable single allergens.

B) Broad panel of positive extract based sensitization test results

More frequently we encounter the scenario in which rather broad IgE antibody responses occur with many positive results to extracts from inhalants or reported symptoms to many (plant) foods. This indicates possible cross-reactivities and a lack of analytical specificity of the extract based test approach.

In this setting, further work-up ("top-down approach", (Fig. 2)) with allergen molecules may allow a more detailed and meaningful characterization of the lgE-repertoire, identifying important molecular IgE-sensitizations. Examples of situations in which molecular allergy diagnostics provide additional information beyond the extract-based tests are listed below:

\section{Examples of situations for further molecular diagnostic work-up}

A) Allergen source with potentially competing clinically relevant allergen sources

- Multiple sensitizations to (non-related) pollen species (i.e. from trees, grasses, weeds) with overlapping seasons

Examples: pollinating plants (trees, grasses, weeds) with overlapping seasons. Here the use of marker allergens and pan allergens allows discrimination between genuine sensitization and cross reactivity.

- Symptoms to multiple (non-related) plant foods due to potential cross-reactivity

\section{Examples:}

a. apples, hazelnuts, cherries, plums, peaches, carrots, soy (suggesting Bet $v$ 1-cluster, predominantly in the northern hemisphere with birch trees)

b. melon, banana, apples, nuts, peanut, citrus and others (suggesting profilin-cluster, often due to high regional grass pollen exposure)

c. peaches, other fruits of the Rosacea family, grapes, berries (black, blue), citrus and others (suggesting LTP-cluster, mainly in the mediterranean region)

- Multiple sensitizations to furry animals (with potential clinical consequences)

Examples: i.e. cats, dogs, horses, furry animals (suggesting serum albumin or certain lipocalins as cross reacting allergens)

B) Allergen source with a variety of different single allergens, either resembling cross reactive or genuine molecules.

- Anaphylactic Hymenoptera sting reaction and sensitization to both honey bee and yellow jacket venom.

Both allergen sources contain potentially crossreactive allergens such as Api $m$ 2, Ves $\vee 2$ (hyaluronidases), Api $m 5$, Ves $\vee 3$ (Dipetidylpepitdases), Api $m$ 12, Ves $\vee 6$ (vitellogenins), and marker allergens that are specific for honey bee venom (Api m 1, Api m 3, Api $m$ 4, Api $m$ 10) or yellow jacket venom (Ves v 1, Ves $\checkmark 5)$. Use of marker allergens allows discrimination between genuine sensitization and cross reactivity.

- Variable symptoms to certain plant foods

Examples: fruits, vegetables

- Severe reactions to plant foods

Examples: peanut, soy, tree nuts, seeds

\section{Criteria for selecting appropriate molecules} (from an allergen source) (Fig. 5)

The general reasons given in section 2.a provide criteria to select certain molecules for further diagnostic work-up:

1. molecules of low abundance and/or weak stability

Examples: use major birch pollen allergen Bet $v 1$ as a representative to demonstrate potential crossreactivities to low abundant, labile Bet $v$ 1-homologs i.e. Cor a 1.04 (hazelnut), Act $d 8$ (kiwi), Pru $p 1$ (peach), Gly $m 4$ (soy) and others 

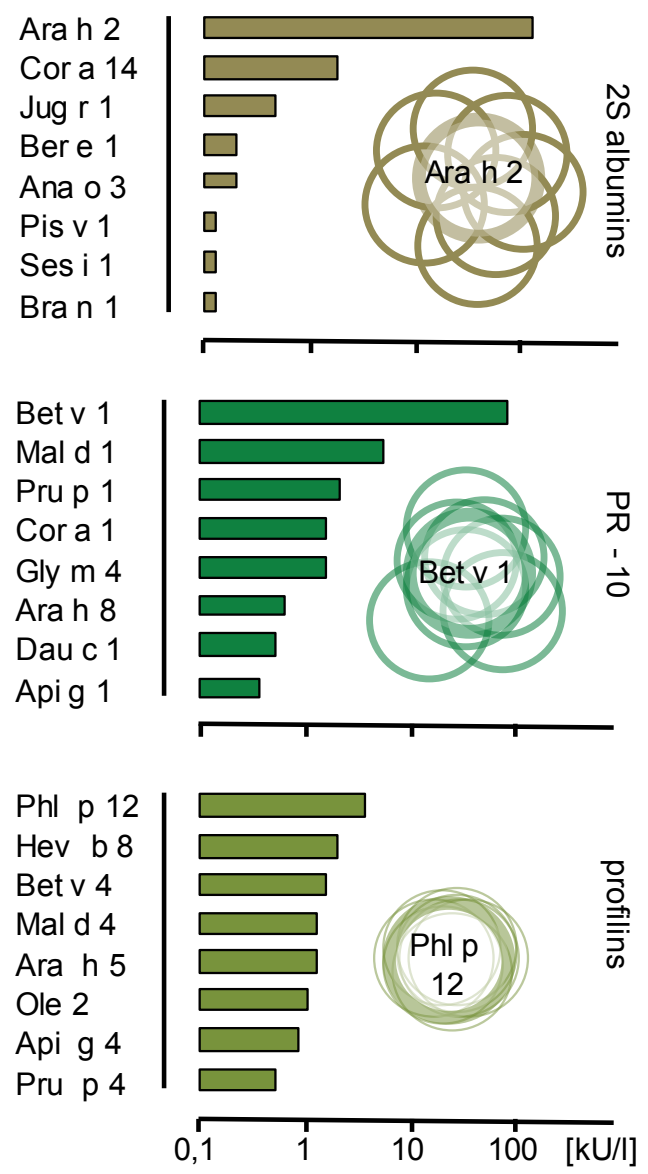

Other examples of not well represented allergens are the peanut allergens Ara h 10, Ara h 11, Ara h 14, Ara $h 15$ (oleosins), the wheat allergen Tri a 19 (omega-5-gliadin), and natural rubber latex allergen (Hev b 5 (acidic structural protein).

2. risk- or severity-associated molecules

Examples: 25 albumins, i.e. Ara $h$ 2, Ara $h$ 6/7 (peanut), Cor a 14 (hazelnut), Gly $m 8$ (soy),

other seed storage proteins Ara h 1, Ara h 3, Cor a 9 , Cor a 11, Gly m 5, Gly m 6,

nsLTP, i.e. Pru $p 3$ (LTP marker in peach, mediterranean), Cor a 8 (hazelnut, mediterranean), Ara $h 9$ (peanut, mediterranean),

other examples: alpha-GAL (delayed type red meat allergy)

3. indicators of cross reactivity

Examples: Fel d 2, Can f 3, Equ c 3 (serum albumins); Bet v 1, Act d 8, Ara h 8, Prup 1, (Bet v 1-homologs); Amb a 8, Ara h 5, Art v 4, Bet v 2, Ole e 2*, Phl $p$ 12, Pru $p 4$ (profilins, pan-allergen in pollen and

\section{Figure 5}

Allergen-specific IgE concentrations to various
allergen molecules depending on structural sim-
ilarity within one allergen family.
A. Variable, limited cross reactions (illustrated by
circles with limited overlap) between different
2S-albumins (stabile seed storage proteins in
nuts, legumes and seeds) and corresponding IgE
values (bars of various sizes)
B. Cross reactions of moderately limited variabil-
ity (illustrated by circles with moderately limited
overlap) between different Bet v 1-homologous
food allergens
C. High degree of cross reactivity (illustrated by
largely overlapping circles) between highly con-
served, similar stuctures and epitopes of profi-
lins (in pollen, foods and natural rubber latex)
with corresponding IgE concentrations (bars of
almost identical size)
(Adapted from (13) with kind permission of
Springer Science and Business Media).

plant foods); Amb a 10, Art v 5, Bet v 4, Ole e 3, $\mathrm{Phl} p 7$ (polcalcins, pan-allergen in pollen); CCD (crossreactive carbohydrate determinants)

4. markers of genuine (species-specific) sensitization

Examples: Fel d 1 (cat), Api m 1, Api m 3, Api m 4, Api $m 10$ (honey bee venom), Ves $v 1$, Ves $v 5$ (Vespula species), Bet $v 1$ (fagales), Ole e 1 (olive tree, plantane), Phl p 1, Phl p 5 (grass) Art v 1 (mugwort), Amb a 1(ragweed)

bold letters indicate availability as reagents mainly non-USA, (eg. Europe, Japan); regular letters: not (yet) available as reagents,

See also section 2.a for explanations.

In summary, after taking the history and performing extract-based sensitization tests a diagnostic workup including specific IgE to allergenic molecules is useful to increase assay sensitivity for single allergens of low abundance (in extracts) or weak stability. An increased analytical specificity will help to identify risk- or severity-associated allergens, indicators for 
cross-reactivity and marker allergens of genuine (primary) sensitization.

Interpretation is an integral part of each sensitization test: Positive results are only clinically relevant in case of corresponding symptoms; negative results can ideally rule out an allergic sensitization and subsequent clinical reaction to the tested allergen specificity.

\section{FROM MOLECULES TO SYMPTOMS: THE "BOTTOM-UP APPROACH"}

Instead of performing symptom-oriented focused molecular allergy diagnostics ("top-down approach", see above), one can simply turn this approach around and start from the bottom i.e. with the molecules (12). In an ideal scenario, diagnostic tools would allow us to characterize the entire IgE repertoire to all potential allergens that a patient has been exposed to. It would then be conceivable that we first analyze the entire IgE repertoire and then start talking to the patient to find out which of the detected IgE sensitizations are clinically relevant. This would be a "broad bottom up approach" i.e. turning the diagnostic pyramid upside down. However, a number of reasons suggest that in real life this is not appropriate:

a) We are far from being able to characterize the entire $\lg \mathrm{E}$ repertoire, i.e. the individual $\lg \mathrm{E}$ response to the entire allergome - currently only approx. 200 of the 3000 known allergens are available for diagnostic purposes.

b) An entire IgE repertoire characterization would be exceedingly expensive and yield enormous amounts of information that require processing and interpretation.

c) At present molecular allergy research attempts and multiplex technologies still depend on the availability of allergens for diagnosis. Many research projects have so far focused on certain molecules, i.e. Bet $v$ 1-homologous proteins in various sources, leading to a broad spectrum of available proteins. However, this does not mean that this group is more relevant than other allergens to which less attention was paid in the past or which are more difficult to be produced as recombinant allergens.

d) Finally, the number of positive IgE results to a certain allergen source usually exceeds by far the number of clinical relevant allergies. Screening the IgE response to the entire allergome thus would most likely result in generation of large proportions of positive test results that have no clinical relevance.

In conclusion, screening of IgE sensitization profiles to large panels of allergens irrespective of the clinical history ("broad bottom up approach") is of limited value for the management of the allergy patient. However, there are a number of situations, in which a "targeted bottom up approach", i.e. using molecular information and asking for corresponding symptoms can be helpful for patient management and consultation in clinical practice as outlined below:

Definition: Diagnostic work-up from (cross-reactive=) molecules to clinical implications ("targeted bottomup approach ", (Fig. 4)) aims for more detailed characterization of the IgE-repertoire or clinical reaction pattern unfolding important hints from a panel of potential molecular IgE-mediated crossreactions.

This diagnostic algorithm "targeted bottom-up approach" (Fig. 4) is designated for molecular workup in case of positive IgE to a certain, potentially clinically relevant cross-reactive molecule (protein family). The finding might explain broad crossreactivities to other allergen sources, if the candidate belongs to a protein family with many members of similar structure and a high degree of cross-reactive lgE-recognition.

In clinical practice, the diagnostic work up in such cases (IgE to potentially clinically relevant crossreactive molecules) can be based purely on clinical assessment to determine the relevance of potential symptom driving cross reactivities, extending the clinical history or applying optional challenge tests with the allergen source in question.

Alternatively or in addition, molecular lgE serology using the "targeted bottom up approach" i.e. screening for slgE to corresponding cross-reactive allergens, may provide useful information on the presence or absence of cross-sensitizations.

The decision on which allergenic molecules have to be tested in this context should be based on the known degree of cross reactivity. Here, two different pattern emerge:

1. Protein families with highly cross-reactive allergens do not require further IgE-testing, but thorough clinical work-up to identify relevant clinical cross-reactions. 
- A single IgE test is sufficient to demonstrate cross-reactivity to a prominent (most IgEbinding) member of an allergen family with broad cross-reactivity.

- Additional tests would only demonstrate more cross reactions (Fig. $5 \mathrm{~B}, \mathrm{C}$ ) without addressing the clinical consequences (i.e. symptoms, clinical reactions)

- Subsequently, detailed clinical work-up is required to clarify potential clinically relevant cross-reactivities.

Reasoning: Further IgE tests would potentially create many (more) positive results with questionable clinical relevance. Therefore, the physician should sort out potential clinically relevant cross-reactions to related allergen sources in questions containing a cross-reactive member of the same allergen family. In conclusion, it is commonly sufficient to test only one member of a highly cross-reactive allergen family.

\section{Examples:}

Bet $v 1$ and its homologs, nsLTPs, profilins, polcalcins (Ca++-binding proteins), serum albumins, grass pollen major group 1 and 5 allergens, parvalbumins, tropomyosins

2. Protein families with allergens of limited cross reactivity are an option for further IgE-testing, if an IgE test to a member of the same allergen family has been positive.

- In general, the highest IgE concentration to a member of the same family might indicate the primary sensitizer (Fig. $4.5 \mathrm{~A}$ ). A negative result would generally exclude an IgEsensitization and make subsequent clinical reactions highly unlikely.

- However, in the case of a positive IgE result, only a thorough clinical work-up would be able to clarify potential clinically relevant cross-reactivities and subsequent reactions.

- If the case history is not informative, a challenge test with the allergen source in question has to be applied to ultimately address the question of potential clinical relevance.

Reasoning: In case of allergens of limited cross reactivity (Fig. $5 \mathrm{~A}$ ) an appropriate panel of related allergens (from the same protein family) could be used to demonstrate or exclude subsequent (serological) cross-reactivities. Therefore, additional IgE testing with related allergen molecules of the same family might establish a hierarchy of allergen-specific IgE values (Fig. 5): Ideally the one with the highest IgE antibody level will represent the primary sensitizer. A negative result could exclude serological (and subsequently clinical) cross-reactivity. A positive result, however, would indicate serological cross-reactivity which should be addressed with the patient according to his individual symptoms. Only in the case of corresponding symptoms, sometimes backed by an oral challenge, these cross reactivities have to be considered in terms of present clinical relevance.

\section{Examples:}

Seed storage proteins like 2S-albumins, 7S-globulins (vicilins), 11S-globulins (legumins);

\section{lipocalin subfamilies}

In conclusion, dissecting the relevance of a panel of related, cross-reactive allergens can be obtained by a) a purely clinical work-up and/or b) a further introduction of related, cross-reactive molecules. In case of negative IgE-tests, serological as well as clinical cross-reactions can be ruled out with certainty. Positive IgE results would confirm serological cross-reactivity, the clinical relevance needs to be addressed with the patient according to his individual symptoms. This approach is only recommended for protein families with a low or limited degree of crossreactivity (i.e. seed storage proteins, nsLTP), where the individual's IgE repertoire is highly variable and its binding to related molecules cannot be predicted.

\section{"U-SHAPED" MOLECULAR DIAGNOSTICS IN IGE-MEDIATED DISEASES}

Definition: A previous diagnostic work-up from symptoms to molecules ("top-down approach", (Fig. 2) is combined with a subsequent diagnostic sequence from molecules to clinical implications ("targeted bottom-up approach", (Fig. 4)), coined "U-shaped molecular diagnosis" (Fig. 6), dissecting the relevance of potential molecular lgE-mediated cross-reactions.

Satisfactory diagnostic conclusions after applying selected molecules ("top-down approach) for lgEtesting would end with appropriate advice to the patient without the need of further work-up (i.e. "bottom-up approach). 


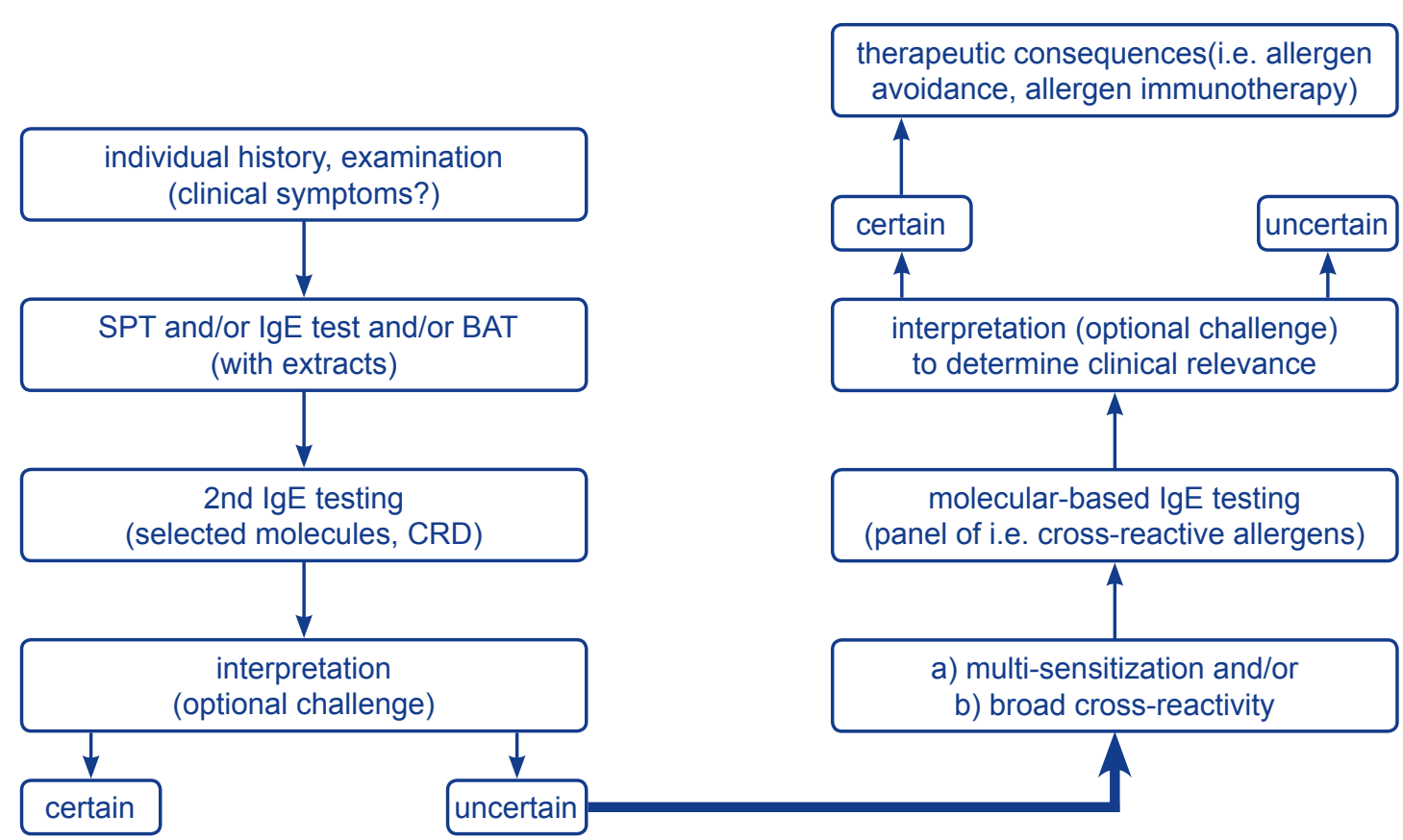

\section{Figure 6}

Combined diagnostic work-up with "top-down" and "bottom-up" aproach. The diagnostic flow chart starts with the history, extract diagnostics, molecular diagnostics and subsequent application of extended molecular panels for further differentiation of the allergen-specific IgE repertoire. The approach, coined "U-shape", has been proposed for complex cases.

However, if open questions remain, regarding the implications of potentially cross-reactive allergens after identification of one key allergen, the U-shaped molecular diagnosis (Fig. 7) might help to solve the diagnostic problem. Subsequently, criteria for further molecular work-up after a previous "top-down approach" with the "bottom-up approach" have to be applied on an individual basis depending on the diagnostic outcome after the initial diagnostic steps.

The question remains if singleplex or multiplex testing should be applied:

- if possibly a large number of allergens from one family are involved, multiplex testing might carry some advantages;

- singleplex testing, however, offers an enhanced assay sensitivity, allowing the ultimate exclusion of IgE-mediated sensitization to the allergen in question if the allergen-specific IgE does not exceed the cut-off of $0.1 \mathrm{kUA} / \mathrm{l}$;

- therefore, the decision of singleplex or multiplex testing should take into account the number of allergens to be tested and the preferred test sensitivity (very low or not as low).

Examples: seed storage proteins like 2S-albumins, 7S-globulins (vicilins), 11S-globulins (legumins). Note of caution: The number of storage proteins of different nuts, legumes and seeds that are available for diagnostics is still limited. This does not allow one to check for the presence of allergen-specific lgE to a full panel of these stable and risk-associated allergens. Therefore, allergen extracts are still needed, to indirectly get information on the potential relevance of the risk-associated storage proteins.

In conclusion, certain scenarios require a complete molecular diagnostic work-up after taking the history and performing focused extract- and molecule-based sensitization tests. This approach explores the degree and potential clinical relevance of further crossreactivities to related molecules of a protein family. Singleplex assays would guarantee maximum assay sensitivity; multiplex assays would rather provide a broad panel of related, cross-reactive molecules for 
further definition of the IgE-repertoire. The clinical relevance has to be determined by the physician and not by the test, based on patient's history and outcome of challenge tests if needed.

\section{References}

1. Valenta R, Lidholm J, Niederberger V, Hayek B, Kraft D, Gronlund H. The recombinant allergenbased concept of component-resolved diagnostics and immunotherapy (CRD and CRIT). Clin Exp Allergy 1999;29:896-904.

2. Werfel T, Asero R, Ballmer-Weber BK, Beyer K, Enrique $E$, Knulst AC et al. Position paper of the EAACl: food allergy due to immunological cross-reactions with common inhalant allergens. Allergy 2015;70:10791090.

3. Heinzerling L, Mari A, Bergmann KC, Bresciani $M$, Burbach G, Darsow $U$ et al. The skin prick test European standards. Clin Transl Allergy 2013;3:3.

4. Hamilton RG, Matsson PNJ, Chan S, van Cleve M, Hovanec-Burns D, Magnusson C, et al. Analytical performance characteristics, quality assurance and clinical utility of immunological assays for human immunoglobulin E (IgE) antibodies of defined allergen specificities. Third Edition, I/LA20-A3, International CLSI-Guideline 2016 (in press).

5. Hoffmann HJ, Santos AF, Mayorga C, Nopp A, Eberlein B, Ferrer $M$, et al. Clinical utility of the basophil activation test in diagnosis and monitoring of allergy. Allergy 2015;70:1393-1405.

6. Chapman MD, Wuenschmann S, King E, Pomés A. Technological Innovations for High-Throughput
Approaches to In Vitro Allergy Diagnosis. Curr Allergy Asthma Rep 2015;15:36.

7. Agache I, Bilo M, Braunstahl GJ, Delgado L, Demoly P, Eigenmann $P$, et al. In vivo diagnosis of allergic diseases-allergen provocation tests. Allergy 2015;70:355365.

8. Muraro A, Werfel $\mathrm{T}$, Hoffmann-Sommergruber $\mathrm{K}$, Roberts G, Beyer K, Bindslev-Jensen C, et al. EAACl food allergy and anaphylaxis guidelines: diagnosis and management of food allergy. Allergy 2014;69:10081025.

9. Sampson HA, Aceves S, Bock SA, James J, Jones $\mathrm{S}$, Lang $\mathrm{D}$, et al. Food allergy: a practice parameter update-2014. J Allergy Clin Immunol 2014;134:10161025.

10. Ansley L, Bonini M, Delgado L, Del Giacco S, Du Toit $G$, Khaitov M, et al. Pathophysiological mechanisms of exercise-induced anaphylaxis: an EAACl position statement. Allergy 2015;70:1212-1221.

11. Incorvaia $C$, Mauro $M$, Ridolo $E$, Makrì $E$, Montagni M, Ciprandi G. A Pitfall to Avoid When Using an Allergen Microarray: The Incidental Detection of IgE to Unexpected Allergens. J Allergy Clin Immunol Pract 2015;3:879-882.

12. Giangrieco I, Rafaiani C, Liso M, Palazzo P, Pomponi D, Tuppo L, et al. Allergens in allergy diagnosis: a glimpse at emerging new concepts and methodologies. Transl Med UniSa 2012;4:27-33.

13. Kleine-Tebbe J, Jakob T. Molecular allergy diagnostics using IgE singleplex determinations: methodological and practical consideration for use in clinical routine - Part 18 of the Series Molecular Allergology. Allergo J Int 2015;24:185-197. 

METHODS FOR IgE ANTIBODY TESTING: SINGLEPLEX AND MULTIPLEX ASSAYS

\author{
Robert G. Hamilton, Jörg Kleine-Tebbe
}

\section{INTRODUCTION}

The serological measurement of IgE antibodies provides the clinician with a measure of a patient's allergic sensitization profile. Two fundamental types of IgE antibody assays are performed in the clinical immunology laboratory. "Singleplex" or "monoplex" assays refer to laboratory methods in which one analyte is measured per analysis. "Multiplex" assays permit more than one analyte to be detected and quantified in a single assay analysis (1). This report examines the technology, performance and application of singleplex and multiplex IgE antibody assays that utilize allergen extracts and allergenic molecules (components) in the diagnosis and subsequent management of human allergic disease.

\section{IMMUNOCHEMISTRY DESIGN CONSIDERATIONS}

Two fundamental assay chemistries that have been referred to as "classical' or "reverse" assay formats have been used to detect IgE antibody (Table 1).
IgE antibody tests are run as singleplex (one), multi-allergen $(<10)$ and multiplex (>100 allergen specificities) assays, all with particular design and performance features.

Allergen extracts remain the principal reagents for IgE assays; allergenic molecules supplement labile or missing allergens in extracts or are analyzed individually.

Allergenic molecules enhance the IgE assay's analytical sensitivity, and improve its analytical specificity by separating serological cross-reactivity from primary (genuine) sensitization to an allergen source or by identifying risk-associated allergens.

The relevance of positive allergen-specific IgE antibody responses, either to extracts or molecules, can only be determined by the physician based on the clinical context (history, challenge) and not by the test itself. 
Table 1

Different formats and features of allergen-specific IgE-assays

\begin{tabular}{|c|c|c|}
\hline & "classical" IgE assay format & "reverse" IgE assay format \\
\hline 1st assay step & $\begin{array}{l}\text { allergen-specific lgE and other antibody } \\
\text { isotypes (e.g., lgG) bind allergen reagent }\end{array}$ & $\begin{array}{l}\text { Immobilized Anti-lgE ideally binds entire (total) IgE in } \\
\text { the reaction vessel }\end{array}$ \\
\hline 2nd assay step & $\begin{array}{l}\text { Labeled anti-lgE binds only allergen- } \\
\text { specific IgE }\end{array}$ & $\begin{array}{l}\text { Labeled allergen reagent binds only allergen-specific } \\
\text { lgE }\end{array}$ \\
\hline $\begin{array}{l}\text { Advantages } \\
\text { (pro) }\end{array}$ & $\begin{array}{l}\text { Detection of the entire allergen-specific } \\
\text { lgE-repertoire (low and high affinity) }\end{array}$ & $\begin{array}{l}\text { detection of allergen-specific IgE principally of higher } \\
\text { affinity } \\
\text { no competitive inhibition through allergen-specific } \\
\text { immunoglobulins other than IgE, (i.e. IgG) }\end{array}$ \\
\hline $\begin{array}{l}\text { Limitations } \\
\text { (con) }\end{array}$ & $\begin{array}{l}\text { competitive inhibition by allergen- } \\
\text { specificimmunoglobulins other than lgE, } \\
\text { (i.e. IgG antibody after subcutaneous } \\
\text { immunotherapy or natural exposure) }\end{array}$ & $\begin{array}{l}\text { large amounts of anti-lgE needed for sera with high } \\
\text { total IgE levels } \\
\text { biased results (lower values) in samples with a low } \\
\text { specific to total IgE-ratio } 6\end{array}$ \\
\hline $\begin{array}{l}\text { Potential use } \\
\text { in multiplex } \\
\text { assay formats }\end{array}$ & $\begin{array}{l}\text { micronization of binding chemistry and } \\
\text { limited amounts of allergen reagents } \\
\text { required }\end{array}$ & $\begin{array}{l}\text { less useful due to the need of multiple labeled } \\
\text { allergens (to be put into one reaction vessel) }\end{array}$ \\
\hline References & Wide et al. (2); Ekins (3) & Ricci et al. (4); Petersen et al. (5) \\
\hline
\end{tabular}

\section{"Classical" IgE assay format: Basis of most current singleplex and multiplex assays}

The noncompetitive, heterogeneous (separation of free and bound), immunometric (labeled antibody) assay that employs allergen immobilized on a solid phase "allergosorbent" to bind specific antibodies of all isotypes from serum is the design that has endured in both singleplex and multiplex assays that are used in clinical laboratories (Fig. 1). Following a buffer wash to separate free and bound human antibody, radionuclide-, enzyme- or fluorescence-labeled antihuman IgE is added to detect IgE antibodies that have bound to immobilized allergen. The magnitude of the response (counts per minute-radioactivity, optical density, chemiluminescence, or fluorescence) after the final buffer wash is proportional to the quantity of allergen-specific lgE antibody in the original test serum.

\section{Reverse IgE assay format: Basis of certain singleplex assays}

A reverse or capture anti-lgE assay design uses a second step liquid-phase allergen to detect allergenspecific IgE antibody. In this assay (Fig. 1-bottom panel], all IgE (in theory) is initially captured from serum by a paramagnetic particle solid-phase anti-lgE in molar excess to the amount of IgE in most test sera. Following the capture of human IgE, allergen-specific IgE antibody is detected with limited quantities of labeled allergen. The reverse phase assay format has been used for IgE antibody quantitation in the ADVIA Centaur $(4,5)$.

The principal advantage of the reverse phase assay over the classical allergosorbent-based singleplex assay is its tendency to measure principally high affinity IgE antibody that is assumed to be "more" clinically relevant (Table 1 ). In contrast, assays that use molar excess amounts of allergen that have been immobilized on an allergosorbent tend to more broadly detect both low as well as high affinity IgE antibody. The reverse assay format also addresses the concern of competitive inhibition caused by allergen-specific antibodies of non-IgE isotypes origin such as IgG anti-allergen that can achieve microgram per $\mathrm{ml}$ levels in sera from individuals receiving immunotherapy. This reverse assay format is, however, less amenable to use in multiplex assays where multiple labeled allergens would have to be added to the same reaction vessel. It suffers from a requirement for large amounts of anti-lgE capture 
A

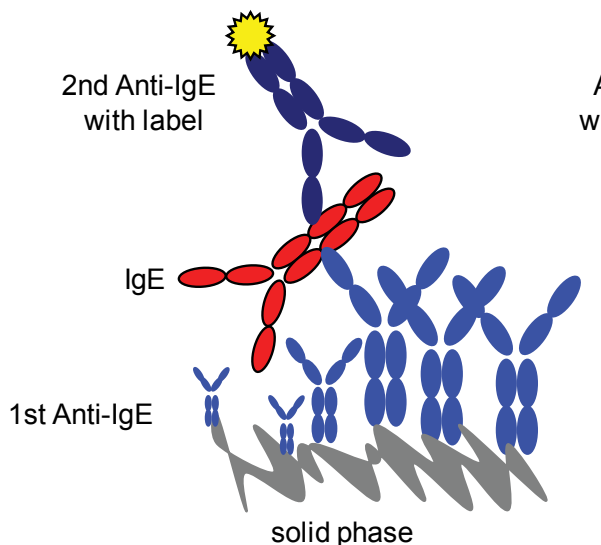

Allergen-specific IgE „classical“ assay format

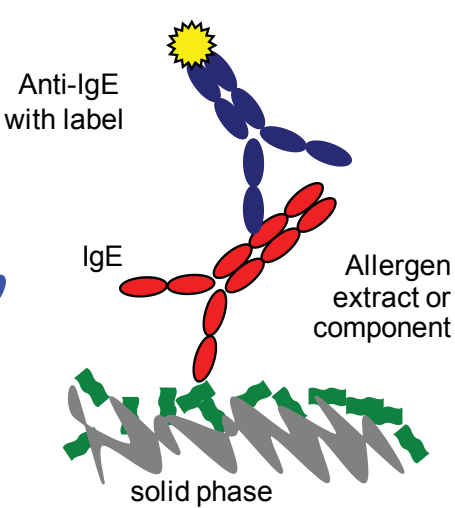

(well, polymer, chip, bead)

B

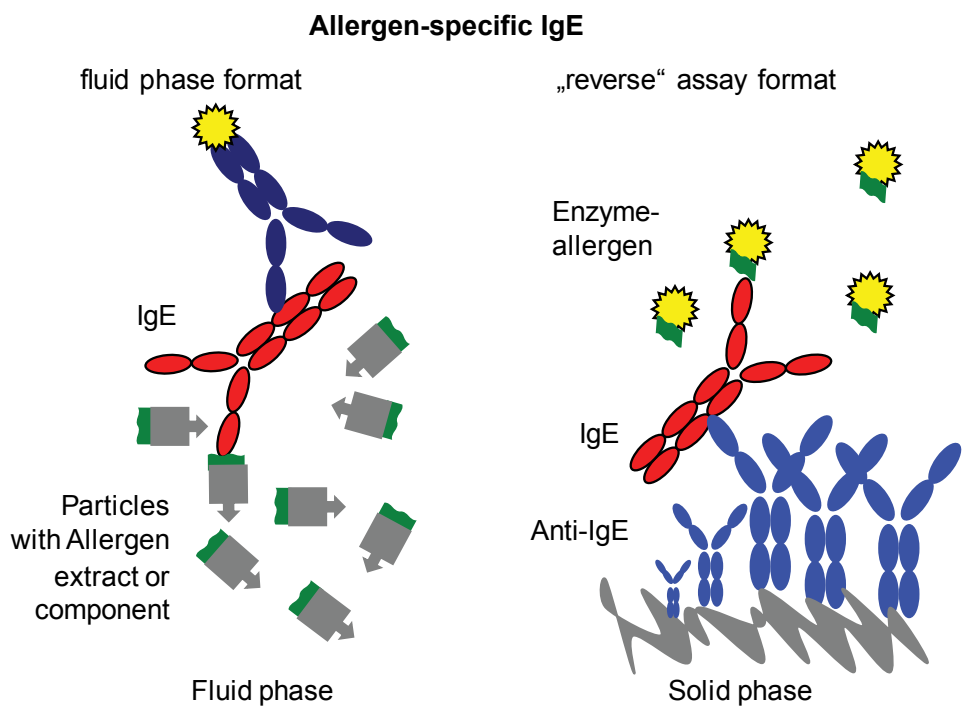

Figure 1

A. General principle of "classical" IgE assay formats for total and allergen-specific lgE quantification. B.

General principle of the "reverse" IgE assay format for allergen-specific IgE quantification.

antibody to insure the binding of all IgE molecules from the test serum. The reverse assay design can also show a major bias because its performance depends on the fraction of the total IgE that is specific for the allergen of interest. These assay design constraints have resulted in the disappearance of the reverse assay format from use in clinical laboratories in the USA and elsewhere.

\section{Heterologous calibration based on total IgE for singleplex allergen-specific IgE systems}

Consensus has been established that a single generic total serum IgE calibration system is the only workable calibration strategy for use in clinical IgE antibody assays (Figure 1, top panel) (1). It allows interpolation of IgE antibody results from any of the hundreds of allergen specificities as long as the total serum IgE and allergen-specific IgE portions of the assay dilute out in parallel with each other. The total serum IgE "heterologous" calibration system that is used in all regulatory cleared singleplex assays is traceable to the World Health Organization's recently depleted $75 / 502$ and currently used third 11/234 human IgE Reference Preparations (6). This calibration system allows interpolation of $\mathrm{IgE}$ antibody results from a limit of quantitation of $0.1 \mathrm{kUA} / \mathrm{L}$ to $100 \mathrm{kUA} / \mathrm{L}$ 
levels of IgE antibody. While rarely performed in clinical testing, serum levels of IgE antibody greater than $100 \mathrm{kUA} / \mathrm{L}$ can be accurately determined by reanalysis of the serum at a dilution and subsequent mathematical correction for the dilution factor. The alternative to the total serum IgE-based heterologous interpolation scheme is the use of individual allergenspecific calibrations, one for each allergen specificity. Early attempts as the use of this approach exposed its major limitation which involved a demand for liter quantities of IgE positive sera for each specificity (7). This made the multiple specific IgE antibody calibration strategy impractical, especially since there are no internationally recognized polyclonal human IgE antibody reference preparations.

\section{Multi-allergen versus Multiplex Assays}

A true multiplex antibody assay allows many specificities of a single antibody isotype (e.g. IgE) to be individually detected and semi-quantified in a single analysis (1). This assay design can be distinguished from a "multi-allergen" screening assay in which many allergen specificities from a common group (aeroallergens or food allergens) are mixed and immobilized as extracts or components on a single solid phase. This multi-allergen reagent is typically used in a singleplex assay format to simultaneously detect specific IgE to multiple antibody specificities in a single reaction. A single qualitative (positive or negative) result is generated for each specimen based on a positive/negative cutpoint. However, the actual allergen specificities that produce a positive IgE antibody response in the multi-allergen screen cannot be definitively identified by the requesting physician without further analysis using additional singleplex assay analyses, one for each of the individual allergen specificities on the multi-allergosorbent. One widely used multi-aeroallergen screening assay measures IgE antibody to 10 or more aeroallergens (8). The allergen specificities immobilized on a single solid phase are carefully selected because they are known to be unique or cross-reactive with the major specificities that induce the majority of aeroallergen-related allergic symptoms. Due to its high negative predictive value, this particular multiaeroallergen assay serves as a cost-effective screen to rule out allergic sensitization in an individual with a questionable respiratory allergy history and to define the atopic status of individuals enrolling in asthma studies (9).

\section{Heterogeneity in IgE Antibody Measurements from Different Assay Types and Manufacturers}

In most multiplex assays, the small quantities of the individual allergens bound to a solid phase contrast with the higher IgE antibody binding capacity present on individual hydrophilic polymer and beadbased allergosorbents that are used clinically. Law of Mass Action constraints cause these assays to detect different distributions of allergen-specific lgE antibody in any given serum. The amount of antibody detected in the assay is dependent on multiple factors including the IgE antibody's concentration, affinity, epitope specificity, IgE specific activity (specific to total IgE ratio) (10) and level of non-IgE antibody specific for the allergen (1). The more antigenlimiting multiplex allergosorbents tend to bind more allergen-specific IgE antibody when it is higher in concentration, has a higher affinity, the serum has a higher specific IgE antibody to total IgE ratio and a lower concentration of competing allergen-specific non-lgE (typically lgG) antibody. These mass action considerations have important assay performance consequences, especially when analyzing sera with nanogram quantities of IgE antibody that are present with high microgram/ml levels of allergen-specific IgG antibody. Such high IgG anti-allergen levels can result from inadvertent natural exposure to high levels of allergen or hyper-immunization through allergen immunotherapy. IgG antibody competes with the lower nanogram $/ \mathrm{ml}$ levels of IgE antibody for limited allergen binding sites on the multiplex chip allergosorbent (11). This constraint has been cited as an advantage of the multiplex assay format in that its lower level of detected allergen-specific IgE antibody in the presence of high allergen specific IgG may more closely reflect the true biological consequence of IgG interference with allergen binding to IgE attached to effector cells.

\section{Advantages and Limitations of Multiplex Assays}

Table 2 summarizes commonly cited performance and assay design advantages and limitations of a multiplex assay in comparison with the singleplex assay. Multiplex assays are attractive because they tend to have a shorter turn-around time for result generation. They tend to use less specimen volume by simultaneously testing multiple IgE antibody specificities in a small surface area on the solid phase. Their assay design tends to be simpler, with fewer reagents and less technician time that reduces overall costs. Multiplex assays, especially in a hand- 


\section{Table 2}

Advantages and Limitations of Singleplex and Multiplex Assay Technology for Allergen-specific IgE Testing that Utilizes Allergenic Molecules (Components)

\section{Performance Related Advantages (pro)}

* Increased assay analytical sensitivity (lower Limit of Quantitation, LoQ)

* Potentially more precise quantification and precision, facilitating comparisons between different allergen reagents (extracts versus molecules)

* More established internal and external quality control measures (proficiency testing)

\section{Assay Design and Cost Related Advantages (pro)}

* Traceable of allergen-specific IgE values to a total human IgE International Reference Preparation

* Similar units for total IgE and allergen-specific IgE due to heterologous calibration (permits calculation of allergen-specific lgE/total IgE-ratio)

* Global availability in many countries

* In case of limited number of samples more cost efficient

* Minimizes unneeded testing

\section{Performance Limitations (con)}

More costly due to increased need for reagents More technical intervention

Limited answers in case of few samples per subject

Expensive in case of large scale screening (i.e. multisensitized subjects)
* Potentially lower analytical sensitivity for each analyte specificity measured (higher limit of detection, LoD)

* Reduced ability to accurately quantify each IgE antibody

* Encouragement of abusive testing which involves the measurement of unwanted or unneeded IgE antibody specificities

\section{Assay Design and Cost Related Limitations (con)}

\begin{tabular}{ll}
\hline & $*$ Less global availability \\
& $*$ Cost of the new instrumentation and reagents \\
* Momples & $*$ Greater challenge in managing different levels of non- \\
* Potentially slower analysis & specific binding \\
$*$ Likely more sophisticated assay format & Enhanced challenges in optimizing, balancing and \\
& standardizing assay reagents and assay quality control
\end{tabular}

held cassette format, are attractive for use as point of care tests (12). These advantages are offset by the multiplex assays' potentially lower limit of quantitation, reduced ability to provide quantitative levels of antibody for each respective IgE specificity, and an increased challenge in optimizing the assay which involves simultaneous quality control of many immobilized allergens. There is the potential for greater inter-lot variability as a result of the need to balance multiple reagents in different spots on a single allergosorbent. The fixed allergen menus of the multiplex assay encourages the testing of IgE antibody for unwanted or unnecessary specificities. Finally, there can be additional expense associated with the need to purchase new equipment to perform a multiplex assay. 


\section{CURRENT ASSAY TECHNOLOGY}

\section{Common IgE assay systems based on singleplex technology}

Many versions of the "classical" IgE assay format have been cleared by governmental regulators over the years. Worldwide, three singleplex autoanalyzers that use the "classical" allergosorbent design dominate the current clinical laboratory market. These are the ImmunoCAP (Thermofisher Scientific/Phadia); Immulite (Siemens Healthcare Diagnostics) and the HyTEC88 (Hycor Biomedical). The latter Hycor assay is being replaced with a new autoanalyzer called the Falcon. In Europe, there are additional assays with the EU mark that use a similar assay design but that are not available worldwide for use in clinical laboratories. The performance characteristics of the three predominant singleplex autoanalyzers have been assessed using masked patient specimens and inter-laboratory proficiency data (13). All three singleplex autoanalyzers use an analogous total IgE calibration curve. They display good precision, reproducibility and they report down to the same $0.1 \mathrm{kUA} / \mathrm{L}$ limit of quantitation. Multiple studies have confirmed, however, that they report different levels of IgE antibody for any given specificity, which indicates that they detect different distributions of allergen-specific IgE antibody (13-15). This is most probably due to the use of different allergencontaining reagents and possibly a result of slightly different procedures for assay calibration and data computation.

\section{Introduction of single molecules (components) into singleplex and multiplex assays}

The single most important scientific advance to impact on the use of multiplex assays in the diagnostic allergy laboratory has been the identification since 2000 and purification of allergenic components from principal aero-, food and venom allergens as discussed extensively throughout this book. Molecular biology techniques have been employed to generate recombinant forms of many of the allergens and others are isolated from extracted native sources using various purification procedures. Allergen libraries have been created as illustrated by the food allergen library from the EuroPrevall project that has established rigorous verification and purity requirements for allergenic molecules. Well-characterized allergenic components from cow's and goat's milk, chicken egg, fish, shrimp, hazelnut, peanut, celery and fruits from the Rosaceae family (apple and peach) have been produced. Documentation of these allergenic components has involved extensive analytical, immunochemical and 3-dimensional structural analyses.

The availability of unlimited quantities of the molecular allergens has allowed multiplex chip microarray based assay methods to be used for rapid simultaneous evaluation of human sera for IgE antibodies to multiple allergen specificities. The most important illustration of technology transition from singleplex to multiplex assays has involved the chipbased multiplex IgE antibody assay initially reported by Hiller et al. (16). The original chip-based microarray utilized 94 purified allergen molecules, which were covalently immobilized in fixed microdot arrays on a pre-activated glass slide. IgE antibody profiles of allergic individuals were evaluated to diseasecausing allergens in a single multiplex analysis using 40 microliters of undiluted serum. With this report, serious clinical application of both allergenic components and multiplex assay methods because available to evaluate individuals for allergic disease. From this initial proof of concept, the repertoire of allergens has increased and the assays' lower limit of quantitation and reproducibility have continued to improve. The commercially available version of this assay is the immune solid phase allergen chip or ISAC (Thermofisher Scientific/Phadia) which requires 40 microliters of serum to detect IgE antibody to 112 individual allergenic molecules that are in a static or planar array on a glass slide (17-19). The ISAC reports IgE antibody levels in ISU units, which are considered semi-quantitative (18). A good correlation exists between the summed IgE anti-cow's milk components levels (Bos d 4, 5, 6, 8 and lactoferrin, r2=0.66) as measured in 44 sera from clinically milk allergic individuals by the singleplex ImmunoCAP ( $x$-axis) and multiplex ISAC (y axis) (Fig. 2 upper panel). The correlation remains impressive when one compares the individual IgE anti-cow's milk components (Bos d 4, 5, 6, 8 and lactoferrin, $r 2=0.77$ ) as measured in the same sera by ImmunoCAP and ISAC (Fig. 2 lower panel). The lower analytical sensitivity of the ISAC, however, depends on the allergen in question (18, 19) and is evident with many strongly positive IgE antibody levels as detected in the ImmunoCAP that are undetectable in the same sera when analyzed in the ISAC. 


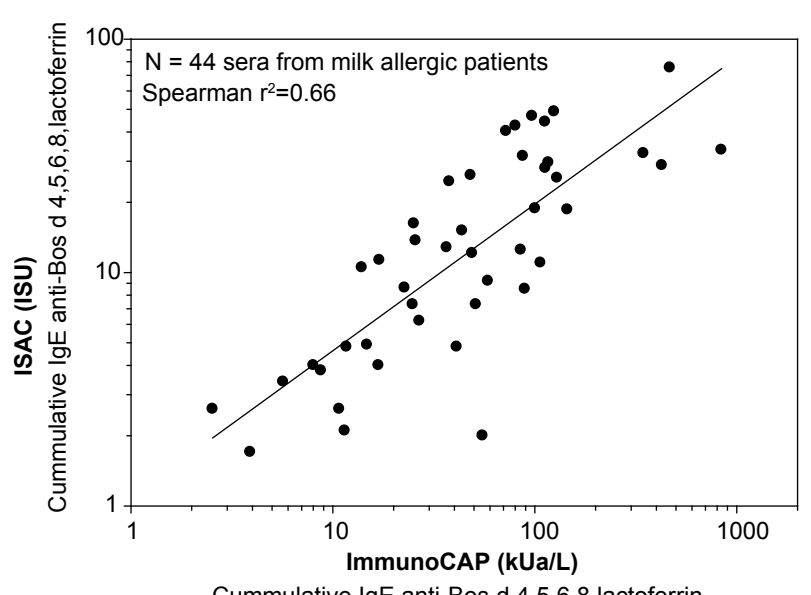

Cummulative IgE anti-Bos d 4,5,6,8, lactoferrin

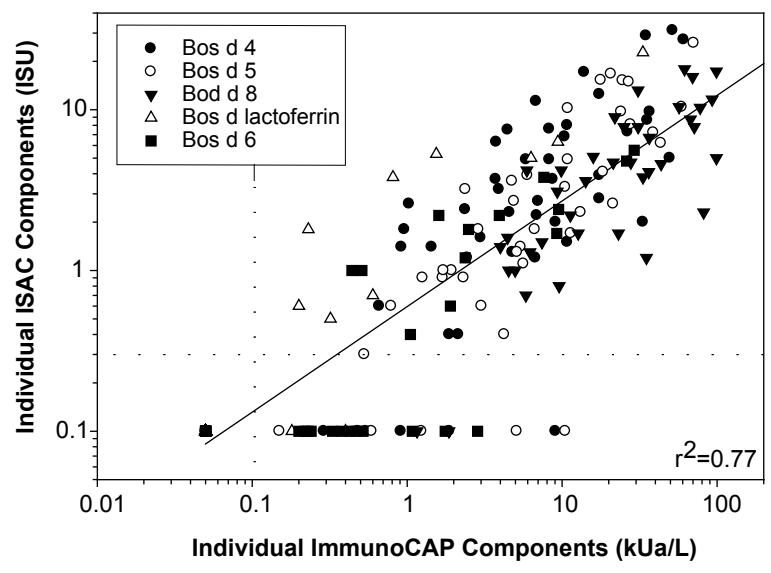

Figure 2

Correlation between the summed IgE anti-cow's milk (Bos domesticus [Bos d]) components as measured in the ImmunoCAP and ISAC (Bos d 4:alpha lactalbumin + Bos d 5: beta lactoglobulin + Bos d 6: bovine serum albumin; Bos d 8: casein + Bos d lactoferrin). (Lower Panel) Correlation between individual IgE anti-cow's milk (Bos domesticus [Bos d]) molecules as measured in the ImmunoCAP and ISAC (Bos d 4:alpha lactalbumin; Bos d 5: beta lactoglobulin; Bos d 6: bovine serum albumin; Bos d 8: casein; Bos d lactoferrin) The dashed lines indicate the positive negative cutoff for each assay: ImmunoCAP $0.1 \mathrm{kUA} / \mathrm{L}$; ISAC: 0.3 ISU. Reproduced from Reference 20.

\section{Additional multiplex IgE assays used in research or in development}

While the ISAC has been designed and thoroughly evaluated (18) for use with purified recombinant and native allergenic molecules, other assays have employed allergen extracts immobilized in chip microarrays and multiplex assay modifications.

A. A research version of the ISAC called the "Mechanisms for the Development of ALLergy" or MeDALL allergen chip has been produced with 170 allergen molecules to more broadly study $\lg \mathrm{E}$ and $\operatorname{IgG}$ antibody development in children (11) Using defined concentrations of chimeric lgE and IgG antibodies specific for Bet $v 1$, the study demonstrated that the simultaneous presence of IgG blocking antibodies can effectively inhibit IgE antibody binding to Bet $v 1$ allergen that has been immobilized on the multiplex chip. In contrast, the same levels of IgG anti-Bet v1 produce minimal competitive interference in the more antigen laden singleplex ImmunoCAP. The authors suggest that the inhibition of IgE binding by IgG antibodies of the same specificity to limited allergen immobilized on the chip may more closely reflect biological responses under conditions of natural allergen exposure. However, the clinical relevance of this inhibition needs further investigation. The smaller amount of allergen on the chip also reduces the working range of the IgE antibody assay in comparison to the singleplex ImmunoCAP, whose allergosorbent has 10,000,000 times more allergen coupled (11).

B. In 2015, Williams et al. (21) reported comparative testing with ISAC, ImmunoCAP and puncture skin testing of a chip-based multiplex autoanalyzer called the MicrotestDx In contrast to the ISAC, it uses 100 microliters of serum and employs 19 allergen extracts and 16 allergenic molecules covering a total of 26 aero- and food-allergen specificities that are covalently immobilized onto a precoated chip in triplicate. This is a scaled down version of a proof of concept assay that used 95 allergen extracts and 8 recombinant proteins which were immobilized on aldehyde-activated glass microscope slides (22). These initial IgE antibody comparative data are encouraging as qualitative positive/negative agreement between the methods ranged from $81 \%$ to $87 \%$ and semi-quantitative (negative, low, moderate and high) agreement among the 4 methods ranged from $60 \%$ to $77 \%$ (86). 
C. Wiltshire et al. spotted a small number of allergen extracts on activated microarray slides and used an interesting rolling DNA circle amplification strategy to detect IgE antibody bound to immobilized allergen (23). Feyzkhanova et al. (24) photo-induced copolymerization of 21 allergens [15 extracts and 6 molecular allergens] into a hydrogel covered chip and used 60 microliters of serum to perform IgE antibody microarray analyses. Renault et al. (25) reported a microarray assay in which 350 defatted and extracted foods were imprinted on slides (4800 dots per slide) and human $\operatorname{lgG}$, IgA, IgM and IgE antibodies were simultaneously detected in serum using a 4 laser scanner. Joshi et al. (26) reported on an ultrasensitive carbohydrate-peptide surface plasmon resonance imaging microarray in which they immobilized peptide and xylosyl glycoside of Ara $\mathrm{h} 2$ onto carboxylated gold slides and amplified the response with 1 micron diameter magnetic beads coated with $\sim 60,000$ polyclonal anti-lgE molecules. These proof of concept microarrays that have used novel imaging systems and allergen extracts bound to chips raise theoretical concerns about the analytical sensitivity of the assays and whether the limited binding capacity of microdot surface on an activated glass chip can immobilize sufficient molar concentrations of allergen (especially from allergen extracts) to quantitatively bind IgE antibody in the presence of other antibody isotypes.

D. Alternative multiplex technologies are capable of detecting IgE antibody in human serum. The Luminex bead based suspension array assay uses fluorescent microspheres are coupled with allergen, one specificity per bead type. Each bead type emits a different internal fluorescence that allows them to be distinguished from each other in a flow cytometer when they are mixed together. Each well of a microtiter plate is loaded with a mixture of bead types (50 microliters; 2000 beads) and serum (50 microliters at 1:4). Following an incubation and wash, bound IgE antibody is detected with biotinylated anti-IgE and avidinphycoerythrin. The fluorescence intensity on the surface of the individual bead types is quantified and interpolated from a (fluorescent intensity vs total serum IgE) calibration curve. In a proof of concept study, this assay was able to measure lgE antibody specific for 6 aeroallergen components from dust mites (Der p 1, Der p 2), cat dander (Fel d 1), dog dander (Can $f$ 1), birch tree pollen (Bet v 1) and Timothy grass pollen (Phl p 5) (27).

E. Adifferent multi-array approach has been employed by Meso-Scale Discovery (28). In an unpublished proof of concept analysis, a-lactalbumin, $\beta$-lactoglobulin A/B, $\alpha-\beta$ - $k$-casein, lactoferrin and BSA proteins were individually biodotted onto separate spots in NPT 9-spot plates. Each spot within the same reaction well permitted a separate antibody specificity to bind. Following reaction with milk allergic sera, bound IgE antibody was detected with Sulfo-Tag-labeled anti-human IgE antibody. Bound labeled antibody when exposed to an electrical pulse generated chemiluminescence through an oxidation-reduction reaction that was measured in an automated reader. Response levels were interpolated from a calibration curve into IgE antibody units. In unpublished pilot studies, significant correlations have been shown between the NPT9-spot plate assay and the singleplex ImmunoCAP generated estimates of IgE antibody levels.

F. A proof of concept study investigated a vertical flow allergen microarray assay with 10 purified allergenic molecules at 3 concentrations that were immobilized on 0.1 um pore size nitrocellulose membranes (29). Bound IgE antibodies from human sera were detected with gold nanoparticle bound anti-lgE using a colorimetric readout. Its precision and relative concordance with the singleplex ImmunoCAP were encouraging. However, to apply the vertical flow strategy, additional verification analyses are needed to further validate the technique using direct comparison studies with clinical specimens that have been analyzed in parallel with established single and multiplex IgE assays.

G. A novel nanotechnology biosensor point of care test reported by Abionic has been developed in which serum is mixed with fluorescently labeled anti-lgE and the mixture added to a capsule containing 10 allergenic molecules coupled to a biosensor surface. Capillary action drives allergen-specific $\lg \mathrm{E}$ to bind to immobilized allergen and fluorescent molecular complexes are then optically measured by the abioSCOPE reading unit. The fluorescent response is finally translated to an IgE antibody dose. This is graphically overviewed by Chapman et al. (30). 


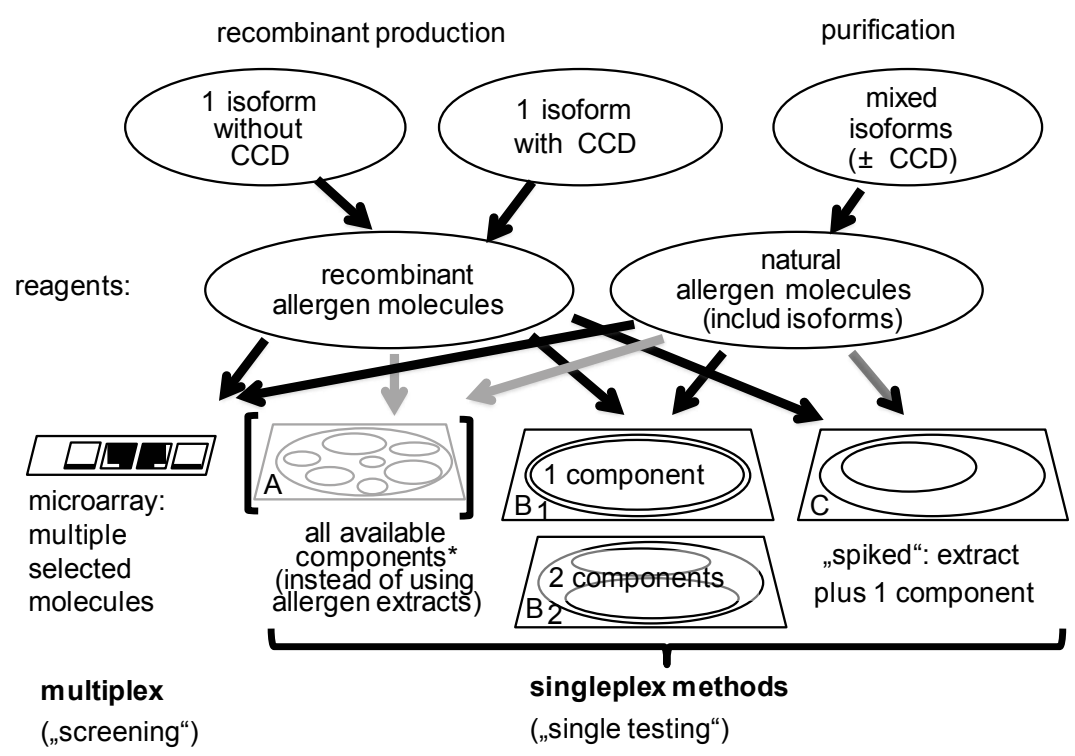

Figure 3

Use of allergenic molecules in singleplex and multiplex IgE antibody assays. Modified from (37) with kind permission of Dustri-Verlag, Deisenhofen-Munich, Germany.

\section{RATIONALE FOR THE INTRODUCTION OF ALLERGENIC MOLECULES INTO CLINICAL IGE ANTIBODY ASSAYS}

The use of single allergens (molecules/components) that have been prepared by purification from native sources or molecular recombinant methods can enhance the clinical performance of serological lgEassays in different ways (Fig. 3 and 4). Recombinant allergens can be generated with or without crossreactive carbohydrate determinants while allergenic molecules purified from native sources will have a mixture of isoforms and carbohydrate determinants. All available allergenic molecules of one allergen source can be used as a mixture in place of a complex natural allergen extract mixture (Fig. 3A). While this approach is theoretically feasible, so far it has not been considered as a serious option since it is considered cumbersome, possibly not all inclusive of relevant allergens, expensive and thus of questionable benefit. A second approach is to use allergenic molecules individually as single reagents in a singleplex or as individual replicate spots in multiplex microarray assays for targeted allergen-specific IgE detection (Fig. 3B-1 component). This is at present the most common use of molecular allergens. Third, selected single molecular allergens of a given allergen specificity can be combined and used as single molecular mixtures for allergen-specific IgE detection (Fig. 3B-2 component). To illustrate this approach, an equal molar mixture of the unique marker allergens $\mathrm{Phl} \mathrm{p} 1$ and $\mathrm{Phl}$ p 5 for Timothy grass sensitization are also representative of sweet vernal grasses. Alternatively, a combination of highly cross reactive allergenic molecules like $\mathrm{Phl}$ p 7 and Phl p 12 which are the polcalcin and profilin representatives of Timothy grass pollen can be used to identify a patient's sensitization to other cross-reactive polcalcin and/or profilin pan-allergen specificities. Fourth, single components can be added to allergenic extracts ("spiked") to increase assay sensitivity. This has been particularly useful for Hevea brasiliensis latex where Hev $b 5$ is underrepresented as a result of being in low abundance or missing from certain extracts (Figures $3 \mathrm{C}$ and $4 \mathrm{~A}$ ). While, spiking physiological allergen extracts can enhance the assay's limit of quantitation and increase its analytical sensitivity, it can lead to problems. For instance, supplementation of hazelnut ImmunoCAP (F17) with recombinant Cor a 1 caused Bet v 1-specific IgE to be increasingly detected. This led unsuspecting clinicians to puzzle over the elevated values of IgE anti-hazelnut in the serum of their patients who subsequently were confirmed to have birch pollen allergy (32). The use of all available components (Figure $3 \mathrm{~A}$ ) in a microarray format can allow targeted and more precise differentiation of the individual's sensitization profile from their allergen- 


\begin{abstract}
Allergen source/extract
\end{abstract} (A, B, C)

Reasoning for using allergen molecules

\section{Allergen} molecules

Effects on assay results

Variants for improvement:
A

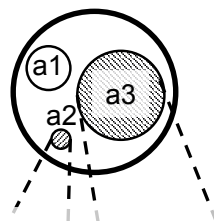

missing or low abundance

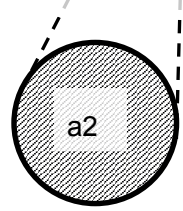

$\downarrow$ limit of quantitation

( $\uparrow$ analytical specificity) defined clinical risk/role

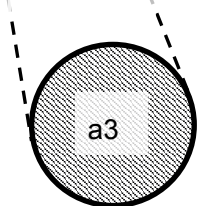

( $\downarrow$ limit of quantitation)
$\uparrow$ analytical
specificity

2
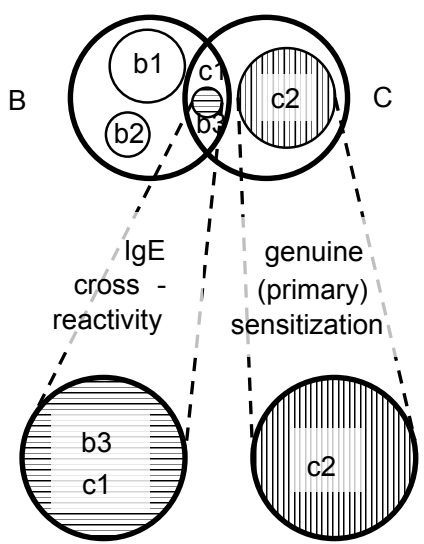

indicators of

cross-reactivity

marker

allergen

\section{Figure 4}

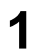

3

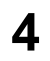

Reagent patterns based on allergen sources/extracts (upper row), with typical reasons why it is valuable to use allergen molecules as reagents in allergen-specific singleplex IgE assays (middle row) and how IgE anti-allergenic molecule results can enhance the final analytical sensitivity and specificity of the generated assay results. Table 3 provides specific examples that correspond with the conditions depicted in depicted in this figure (adapted from (31) with kind permission of Springer Science and Business Media).

\section{Table 3}

Utility of allergen molecules as reagents from various allergen sources/extracts (left column), reasons and improved assay performance (upper row) will vary due to the individual diagnostic question and the specific allergen applied.

\begin{tabular}{|c|c|c|c|c|}
\hline Variants & 1 & 2 & 3 & 4 \\
\hline $\begin{array}{l}\text { Examples } \\
\text { (allergen source, } \\
\text { allergen carrier) }\end{array}$ & $\begin{array}{l}\text { Increased analytic } \\
\text { sensitivity }\end{array}$ & $\begin{array}{l}\text { Increased analytical } \\
\text { specificity/selectivity }\end{array}$ & $\begin{array}{l}\text { Cross-reactive } \\
\text { allergens }\end{array}$ & $\begin{array}{l}\text { Species/family- } \\
\text { specific major } \\
\text { allergens }\end{array}$ \\
\hline cat & & Fel d 2 & Fel d 2 & Fel d 1 \\
\hline hazelnut & $\begin{array}{c}\text { Cor a } 1 \\
\text { (Bet v 1-homologue) }\end{array}$ & $\begin{array}{c}\text { Cor a } 14 \text { (2S albumin) } \\
\text { Cor a } 9 \text { (11S globulin) } \\
\text { Cor a } 8 \text { (LTP, mediterranean) }\end{array}$ & & \\
\hline kiwi & $\begin{array}{c}\text { Act d } 8 \\
\text { (Bet v 1-homologue) }\end{array}$ & & $\begin{array}{l}\text { Act d } 8 \text { (Bet v } \\
\text { 1-homolog) }\end{array}$ & \\
\hline peach & $\begin{array}{c}\text { Pru p } 1 \\
\text { (Bet v 1-homologue) }\end{array}$ & $\begin{array}{l}\text { Pru p } 3 \text { (LTP, marker, } \\
\text { mediterranean) }\end{array}$ & $\begin{array}{l}\text { Pru p } 1 \text { (Bet v } \\
\text { 1-homolog), } \\
\text { Pru p } 4 \text { (Profilin) }\end{array}$ & \\
\hline peanut & $\begin{array}{l}\text { Ara h 10, Ara h } 11 \\
\text { (oleosins) }\end{array}$ & $\begin{array}{c}\text { Ara h } 1 \text { (7S globulin) } \\
\text { Ara h } 2 \text { (2S albumin) } \\
\text { Ara h } 3 \text { (11S globulin) } \\
\text { Ara h 6/7 ( } 2 \text { S albumin) } \\
\text { Ara h } 9 \text { (LTP, mediterranean) }\end{array}$ & $\begin{array}{l}\text { Ara h } 8 \text { (Bet v } \\
\text { 1-homolog) } \\
\text { Ara h 5* }\end{array}$ & \\
\hline
\end{tabular}


Table 3 (continued)

\begin{tabular}{|c|c|c|c|c|}
\hline Variants & 1 & 2 & 3 & 4 \\
\hline $\begin{array}{l}\text { Examples } \\
\text { (allergen source, } \\
\text { allergen carrier) }\end{array}$ & $\begin{array}{l}\text { Increased analytic } \\
\text { sensitivity }\end{array}$ & $\begin{array}{l}\text { Increased analytical } \\
\text { specificity/selectivity }\end{array}$ & $\begin{array}{l}\text { Cross-reactive } \\
\text { allergens }\end{array}$ & $\begin{array}{l}\text { Species/family- } \\
\text { specific major } \\
\text { allergens }\end{array}$ \\
\hline soy & $\begin{array}{c}\text { Gly m } 4 \\
\text { (Bet v 1- homolog) }\end{array}$ & Glym 5, Gly m 6 & & \\
\hline wheat & $\begin{array}{c}\text { Tri a } 19 \\
\text { (omega-5-gliadin) }\end{array}$ & & & \\
\hline meat & alpha-GAL & alpha-GAL & & \\
\hline honeybee venom & $\begin{array}{l}\text { Api } m 3 \text {, Api } m 4 \text {, } \\
\text { Api } \mathrm{m} 10\end{array}$ & $\begin{array}{c}\text { Api } m \text { 1, Api m 3, Api } m \text { 4, } \\
\text { Api } m 10\end{array}$ & & $\begin{array}{l}\text { Api } m \text { 1, Api } m 3 \text {, } \\
\text { Api } m \text { 4, Api } m 10\end{array}$ \\
\hline yellow jacket venom & Ves v 5 & Ves $v 1$, Ves $v 5$ & & Ves $v 1$, Ves $v 5$ \\
\hline $\begin{array}{l}\text { birch (hazel, alder, birch } \\
\text { pollen) and beech trees } \\
\text { (beech, oak pollen) }\end{array}$ & & Bet v 1 & $\begin{array}{l}\text { Bet v 2*, } \\
\text { Bet v } 4^{* *}\end{array}$ & Bet v 1 \\
\hline $\begin{array}{l}\text { oleaceae (ash, olive } \\
\text { pollen) }\end{array}$ & & Ole e 1 & $\begin{array}{l}\text { Ole e } 2^{*} \\
\text { Ole e } 3^{* *}\end{array}$ & Ole e 1 \\
\hline $\begin{array}{l}\text { poaceae (pollen } \\
\text { from moderate climate } \\
\text { grasses) }\end{array}$ & & $\begin{array}{l}\text { Phl p } 1 \\
\text { Phl p } 5\end{array}$ & $\begin{array}{l}\text { Phl p } 12^{*} \\
\text { Phl p } 7^{* *}\end{array}$ & $\begin{array}{l}\text { Phl p 1, } \\
\text { Phl p } 5\end{array}$ \\
\hline mugwort pollen & & Art v 1 & $\begin{array}{l}\text { Art } \vee 4^{*} \\
\text { Art } \vee 5^{* *}\end{array}$ & Art v 1 \\
\hline ragweed pollen & & Amb a 1 & $\begin{array}{c}\text { Amb a } 8^{*} \\
\text { Amb a } 10^{* *}\end{array}$ & Amb a 1 \\
\hline
\end{tabular}

bolded letters indicate availability as reagents mainly non-USA, (eg Europe, Japan); regular letters: not (yet) available as reagents, * profilin (panallergen in pollen and plant foods), ${ }^{* *}$ polcalcin (pan-allergen in pollen); (adapted from (31) with kind permission of Springer Science and Business Media)

specific IgE response. This approach has been coined "Component-Resolved-Diagnostic" (CRD) (31) and it represents the most important option in molecular allergy diagnosis.

The utility of single allergenic molecules can be justified by four conditions (Fig. 4 and Table 3)(4). First, the assay's sensitivity can be improved by lowering its limit of quantification (LoQ, Textbox 1) while increasing its analytical specificity. Second, if allergen molecules are in low abundance or missing in the extract such as Cor a 1 in hazelnut or Gly $\mathrm{m} 4$ in soy, supplementation improves the assay's sensitivity (LoQ). Third, if allergen molecules are unique to a specificity such as Fel $d 1$ for cat or Bet $v 1$ for birch, their use can improve the analytical specificity ("selectivity") of the assay. This allows additional clinical assumption(s) such as assessing increased risk for severe symptoms. Finally, certain allergenic molecules such as Ara h 8 (Bet $v 1$ homologue) for peanut and $\mathrm{Phl} p 7$ and 12, the polcalcin and profilin representatives in Timothy grass, can serve as indicators for serological cross sensitizations through the binding of cross reactive IgE. In case of a positive result, they can demonstrate the lack of analytical specificity of an IgE test with allergen extracts in affected subjects with potential cross-reactions.

\section{ASSAY PERFORMANCE EVALUATION: ASSAY SENSITIVITY AND ANALYTICAL SPECIFICITY (SELECTIVITY)}

The analytical performance characteristics of laboratory tests and their predictive value in defining 


\begin{abstract}
Textbox 1
Common definitions to describe performance characteristics of a laboratory test (i.e. allergen-specific lgE assay)

Analytical sensitivity is equivalent to the slope of the calibration curve of an (immuno)assay. In contrast assay sensitivity in real terms (=lowest test "cut-off") is currently calculated and provided with following, internationally harmonized variables:

- limit of blank, LoB (i.e. signal of a serum sample without allergen-specific lgE)

- limit of detection, LoD (i.e. signal of a serum sample with the lowest detectable allergen-specific lgE)

- limit of quantitation, LoQ (i.e. signal of a serum sample with the lowest allergen-specific lgE at a predefined assay precision)

Analytical specificity of an allergen-specific IgE assay can, first, be related to the specificity of the detected Immunoglobulin class, meaning the test will indeed measure IgE and not immunoglobulins of other classes (IgA, $\lg \mathrm{D}, \lg \mathrm{g}$ or $\lg \mathrm{M}) 6$.

A second definition relates analytical specificity to a targeted, more selective IgE-detection against single allergenic molecules. While an allergen extract, consisting of complex protein mixtures, ideally binds the entire IgE-repertoire to a specific allergen source, the use of single allergen molecules will only detect a part of the IgE-repertoire. Thus, the analytical specificity (selectivity) will be increased.
\end{abstract}

the presence and severity clinical disease have been internationally defined by variables such as sensitivity and specificity (Textbox 1) (1). Two pairs of definitions separate the IgE antibody test's analytical sensitivity and specificity (Table 4, left column, 1-4) from its diagnostic sensitivity and specificity (Table 5 , right column, I-IV) that discriminate among the various clinical allergy phenotypes. These definitions have been adopted as part of international guidelines for IgE antibody assays through the Clinical Laboratory Standards Institute (CLSI) (1). They are particularly important to consider when allergenic molecules are substituted into an IgE antibody assay (31). Their importance stems from the observation that introducing single allergenic molecules into an IgE antibody assay frequently improves the analytical variables in the left column of Table 4 and this has a direct effect on changing the diagnostic clinical discrimination of disease as defined by the parameters in the right column of Table 4.

The extent to which an assay performance improvement translates into improved diagnostic clinical discrimination of disease depends on (a) the cohort of individuals being evaluated in terms of their age, disease spectrum and severity, (b) the availability and selection of the specific allergenic molecules used in the IgE assay, and (c) the preselected study endpoints defined by the clinician (33). This means that the diagnostic-clinical criteria [right column, I-IV, Table
4] need a thorough individual interpretation based on each IgE antibody test result using the patient's previous history and if needed, additional proof of reproducible and objective symptoms in the affected allergic subject upon allergen exposure (i.e., challenge test). As a consequence, these clinical criteria extend beyond the essential "raw" allergen-specific IgE antibody assay result (e.g., IgE sensitization in question: yes or no). Together these facts support the conclusion that it can be misleading to use sensitization test results to define the diagnostic clinical criteria of an IgE antibody test (31, 34).

One example is enhancement of the analytical sensitivity of an IgE antibody assay by supplementing an extract with a labile allergen molecule (Hev b 5 into the Hevea brasiliensis extract) prior to use in preparing the allergosorbent. The additional Hev b 5 improves the analytical sensitivity by lowering the assay's limit of quantitation (LoQ), and thus increasing the test's diagnostic sensitivity without compromising the analytical specificity of the test. Alternatively, in patients with wheat-dependent, exercise-induced anaphylaxis (WDEIA), only $20-30 \%$ of the subjects have IgE-mediated sensitization to wheat flour extract. However, $80-90 \%$ of these cases demonstrate allergen-specific IgE to Tri a 19 (Omega5-gliadin). This gliadin is often responsible for WDEIA, however, it suffers from a poor aqueous solubility and is therefore not well represented in wheat extracts. 


\section{Table 4}

Potential criteria for assessing assay improvement of sensitization tests following the replacement of allergen extracts with allergenic molecules

\begin{tabular}{|c|c|c|c|}
\hline & $\begin{array}{l}\text { analytical criteria } \\
\text { (potential assay improvement) }\end{array}$ & & $\begin{array}{l}\text { diagnostic-clinical criteria } \\
\text { (potential clinical advantages) }\end{array}$ \\
\hline 1. & $\begin{array}{l}\text { >"analytical" sensitivity } \\
\text { <limit of quantitation (LoQ) }\end{array}$ & I. & $\begin{array}{l}\text { > diagnostic sensitivity (proportion of positive IgE antibody } \\
\text { tests in patients with allergic disease) }\end{array}$ \\
\hline 2. & >analytical specificity & II. & $\begin{array}{l}\text { > diagnostic specificity (proportion negative IgE antibody } \\
\text { tests in healthy individuals ) }\end{array}$ \\
\hline 3. & $\begin{array}{l}\text { indicator of serological } \\
\text { cross reactivity }\end{array}$ & III. & $\begin{array}{l}\text { indicator of clinical crossreactivity (allergic symptoms to } \\
\text { allergenic sources that did not elicit the primary sensitization) }\end{array}$ \\
\hline 4. & $\begin{array}{l}\text { marker of primary/genuine } \\
\text { sensitization }\end{array}$ & IV. & $\begin{array}{l}\text { prediction of clinical reactions (PPV, NPV, thresholds, } \\
\text { likelihood ratio etc.) }\end{array}$ \\
\hline
\end{tabular}

(adapted from (31) with kind permission of Springer Science and Business Media)

By using recombinant Tri a 19 as a reagent in the lgE antibody assay, the assay's sensitivity (lowered LoQ) is immediately improved.

Another asset of the use of defined allergenic molecules is the restriction that it provides to the assay. This is especially important when IgE immune responses need to be detected to allergenic specificities that are highly stable or in relatively high abundance (i.e. Ara h 2 or Cor a 14). Their use makes the measurement of IgE antibody more targeted or analytically specific. Identification of IgE immune response patterns to the $2 \mathrm{~S}$ albumins, Ara h 2 and Cor a 14 (Table 3), have been repeatedly associated with an increased risk for severe reactions to foods and they can facilitate decisions about the possible elimination of an oral food challenge. Alternatively, in a non-selected population study, more than $10 \%$ of German children and adolescents demonstrate allergen-specific IgE to peanut extract that is predominantly linked to serological pollen-associated cross reactions (35). Moreover, diagnostic tests with the stable and risk-associated peanut storage allergen (Ara h 2) show elevated allergen-specific IgE in only a small proportion of the general children/adolescent population (approximately $0.2 \%$ to $0.4 \%$ ) (Kirsten Beyer, personal communication). Thus IgE anti-Ara h 2 provides a much higher analytical specificity ("selectivity") than the use of a peanut extract based allergosorbent.

\section{CLINICAL EVALUATION: DIAGNOSTIC SENSITIVITY AND SPECIFICITY}

Diagnostic sensitivity and specificity are related to a clinical history and physical examination based assessment of affected and non-affected subjects. Requirements for proper calculation and interpretation of the diagnostic sensitivity and specificity of IgE antibody tests of sensitization require sound clinical data from the subject's case history and in some cases additional challenge tests to back up the clinical diagnosis (Table 4, right column). However, the presence of allergen-specific IgE is strictly a marker for allergic sensitization (risk for allergy) and it alone cannot predict the probability of a clinical reaction per se $(31,34)$. Thus, concordant results (case history and allergen-specific IgE with a positive clinical or challenge outcome) are effectively considered as clinically relevant (rather than being labeled as true positive). The same applies for concordant negative results, which are used to exclude a clinical state of allergy and an underlying state of allergic sensitization. In case of positive allergen-specific IgE results and a negative case history or provocation test, however, consideration should be given to labeling these discordant results as clinically irrelevant (rather than false positive). Labeling clinically irrelevant as false positive results actually misses the key point of the analysis, since the presence of allergen-specific IgE itself should not be disputed, but rather considered valid in its own right as a marker for atopy and IgE-sensitization. 
A number of clinical studies have explored diagnostic sensitivity and specificity of IgE tests with single allergens from a particular allergen source. The use of previously missing or less represented allergens in IgE antibody assays was able to increase its diagnostic sensitivity through the improvement of assay sensitivity, usually by lowering the assay's limit of assay quantitation (Tables 3 and 4). As a consequence, higher rates of sensitization were found, in general, even among subjects without clinically relevant reactions or disease.

The reciprocity of diagnostic sensitivity and specificity is a general feature of diagnostic tests. It is usually depicted as Receiver Operating Characteristics (ROC) curves (general example see Figure 5B). Some single allergens like Ara h 2 or other risk-associated allergens belonging to the seed storage protein family of $2 \mathrm{~S}$-albumins have been found to increase diagnostic sensitivity and specificity of a patient's evaluation by assessing the risk of severe clinical reactions. Using risk-associated 2S-albumins, predictive allergenspecific IgE-thresholds ("decision points") have been defined to forecast a positive or negative oral challenge in children with peanut or hazelnut allergy (35).

Despite the performance of elaborate and elegant clinical studies, they have produced rather variable outcomes with group effects, displaying considerable overlap between i.e. clinically affected and nonaffected (tolerant) individuals (Fig. 5C). Presenting data with smoothed probability plots (Fig. 5D) suggests a close relationship, suitable for individual prediction with the collected data. However, results of sensitization tests like the allergen-specific IgE assays cannot yet (and presumably never will) reliably predict clinical reactions (or their nonappearance) (36). Therefore, future studies on the diagnostic value of allergenic molecules should establish as their primarily goal, the improvement of well-defined methodological variables (Table 3, left column) that are linked to the analytical performance characteristics of the assay. This should be done even without a complete clinical evaluation of the assay which includes the assessment of the assay's diagnostic sensitivity and specificity, predictive values and likelihood ratio (Table 3 , right column), the analytical performance of IgE assays can be substantially improved in many cases by the use of allergenic molecules that are used in parallel with or in place of allergen extracts. This conceptual view has already been adopted by international guidelines on allergen-specific IgE assays (1). It should further
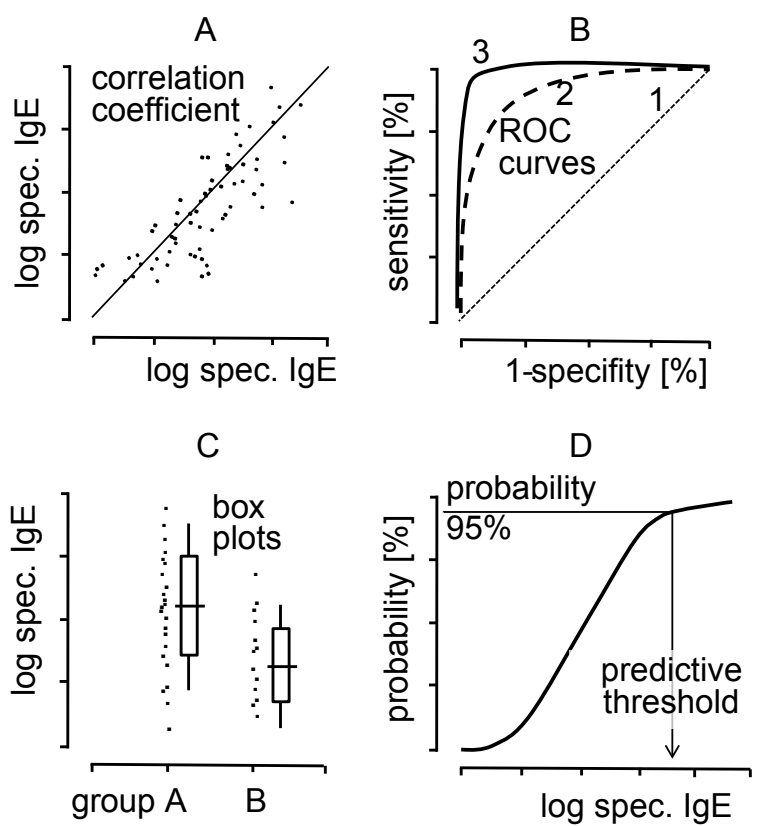

Figure 5

Typical data analysis of IgE antibody laboratory data with single allergenic molecules. A: Association of log distributed allergen-specific IgE concentrations to a natural ( $x$ axis) versus recombinant (y-axis) allergen molecules. B: Diagnostic efficacy (Receiver Operating Characteristics, ROC) displaying performance of IgE antibody assays with allergen molecules as compared to extracts; C. Single values of allergen molecule-specific IgE antibody with the median, $25 \%$ and $75 \%$ percentiles that are presented to compare groups. D: Threshold levels of IgE antibody that are used to predict the probability of a clinical reaction (i.e. 95\% probability of a positive oral food challenge). (Reproduced from (37) with kind permission of Dustri-Verlag, Deisenhofen-Munich, Germany).

facilitate and possibly accelerate the evaluation and clinical acceptance of allergenic molecules into the diagnostic algorithm for human allergic disease.

\section{Determination of the Clinical Relevance of an IgE Antibody Assay}

The ultimate and essential question with diagnostic allergy testing is "what is the clinical relevance of an allergen-specific IgE measurement"? Even in this era with the availability of molecular allergens, the basic rule still applies. Namely, a positive allergen-specific 
IgE result represents a state of allergic sensitization (risk for allergic disease), but not proof of allergic disease $(31,36)$. A positive IgE antibody response is only clinically relevant in the case that there are objectively defined corresponding allergic symptoms that are temporally associated with a known allergen exposure. A negative allergen-specific IgE result against one recombinant allergen molecule or a mixture of natural isoforms of one single allergen can generally exclude an allergic sensitization or risk of allergy to that allergen specificity in question. This is, however, only possible if the total $\lg E$ is high enough (i.e. $>20 \mathrm{kUA} / \mathrm{L}$ ), the allergen reagent is in sufficient abundance, fully intact, and presenting all its epitopes and the analytical performance of the lgE antibody assay has been optimized for a low limit of quantitation (i.e. $0.1 \mathrm{kUA} / \mathrm{L}$ ).

In conclusion, the clinical relevance of an allergic sensitization (i.e. presence of allergen-specific $\operatorname{lgE}$ ) independent of the use of allergen extracts or molecules for diagnostic purposes can ultimately only be determined by the physician and not by the test $(1,31,34,36)$. Therefore, the complete diagnostic results of sensitizations tests, including allergenspecific IgE assays that have been improved by the use of allergenic molecules, will always have to be interpreted in the clinical context and on the basis of the individual's case history.

\section{SUMMARY}

For the foreseeable future, several clinically validated singleplex assays that use allergen extract-based reagents will remain the principal assays employed worldwide by clinical immunology laboratories to serologically document sensitization (IgE antibody) in patients with a positive history of allergic disease. Novel molecular allergen-based multiplex assays will remain invaluable research assays. Allergen extract-based reagents used in the singleplex assays will currently be judiciously supplemented with an increasing number of allergenic molecule-based reagents. Principal among these are molecular allergens from peanut and hazelnut that (a) improve analytical sensitivity by providing molar excess of missing or low abundant allergens (e.g. Ara $\mathrm{h} 8$ in the peanut extract; Cor a 1 in the hazelnut extract), (b) enhance the assay's analytical specificity by defining a clinical risk for systemic reactions (Ara h 1,2,3 [severe] versus Ara $h 8$ [more mild]) and (c) distinguish cross-reactivity versus (d) genuine (primary) sensitization (Cor a 9,14 [genuine] versus Cor a 1 [Bet $\vee 1$ cross-reactive] sensitization to hazelnut). The clinical relevance of allergen-specific IgE detection in a patient's serum is strictly as a marker for allergic sensitization (risk for allergy) and it alone cannot predict the probability of an allergic reaction. The determination of the diagnostic sensitivity and specificity of IgE antibody assays will thus remain difficult to definitively determine because of the lack of an absolute method of defining the presence of allergic disease. This means that the clinical relevance of an allergic sensitization (i.e. presence of allergenspecific $\lg \mathrm{E}$ ) independent of the use of allergen extracts or molecules for diagnostic purposes will ultimately be determined only by the physician and not by the test.

\section{Refences}

1. Hamilton RG, Matsson PNJ, Chan S, van Cleve M, Hovanec-Burns D, Magnusson C, et al. Analytical performance characteristics, quality assurance and clinical utility of immunological assays for human immunoglobulin $\mathrm{E}$ (IgE) antibodies of defined allergen specificities. Third Edition, I/LA20-A3, International CLSI-Guideline 2016 (in press).

2. Wide L, Bennich $\mathrm{H}$, Johansson SGO. Diagnosis of allergy by an in vitro test for allergen antibodies. Lancet 1967; 2: 1105-1107.

3. Ekins RP. Ligand assays: from electrophoresis to miniaturized microarrays. Clin Chem 1998;44:2015-2030.

4. Ricci G, Capelli M, Miniero R, Menna G, Zannarini L, Dillon $P$ et al. A comparison of different allergometric tests, skin prick test, Pharmacia UniCAP and ADVIA Centaur, for diagnosis of allergic diseases in children. Allergy 2003;58:38-45.

5. Petersen $A B$, Gudmann $P$, Milvang-Gronager $P$, Mørkeberg R, Bøgestrand S, Linneberg $A$ et al. Performance evaluation of a specific IgE assay developed for the ADVIA centaur immunoassay system. Clin Biochem 2004;37:882-892.

6. Thorpe S, Heath A, Fox B, Patel D, Egner W. The 3rd international standard for serum IgE: International collaborative study to evaluate a candidate preparation. Clin Chem Lab Med 2014;52:1283-1289.

7. Ownby DR, Adkinson NF, Hamilton RG, Homburger HA, Zeitz HJ, Kowalski D et al. Multi-center comparison of ABBOTT MATRIX Aero to Pharmacia Standard RAST, Modified RAST and skin puncture tests. Eur J Clin Chem Clin Biochem 1994;32:631-637.

8. Merrett J, Merrett TG. Phadiatop - a novel antibody screening test. Clinical Allergy 1987;17:409-416.

9. Szefler SJ, Wenzel S, Brown R, Erzurum SC, Fahy JV, Hamilton RG et al. Asthma outcomes: biomarkers. J Allergy Clin Immunol 2012;129:S9-23. 
10. Hamilton RG, MacGlashan DW Jr, Saini SS. IgEantibodyspecific activity in human allergic disease. Immunol Res 2010;47:273-284.

11. Lupinek C, Wollmann E, Baar A, Banerjee S, Breiteneder $\mathrm{H}$, Broecker BM et al. Advances in allergen-microarray technology for diagnosis and monitoring of allergy: the MeDALL allergen-chip. Methods 2014;66:106-119.

12. Hedlin G, Moreno C, Petersson CJ, Lilja G, Toledano FL, García AN et al. Allergy Diagnosis in Children and Adults: Performance of a New Point-of-Care Device, ImmunoCAP Rapid. World Allergy Organ J 2009;2:138-143.

13. Hamilton RG. Proficiency Survey Based Evaluation of Clinical Total and Allergen-Specific IgE assay performance. Arch Path Lab Med 2010;134:975-982.

14. Wood RA, Segall N, Ahlstedt S, Williams PB. Accuracy of IgE antibody laboratory results. Ann Allergy Asthma Immunol 2007;99:34-41.

15. Wang J, Godbold JH, Sampson HA. Correlation of serum allergy (IgE) tests performed by different assay systems. J Allergy Clin Immunol 2008;121:1219-1224.

16. Hiller R, Laffer S, Harwanegg C, Huber M, Schmidt WM, Twardosz A, et al. Microarrayed allergen molecules: diagnostic gatekeepers for allergy treatment. FASEB J 2002;16:414-416.

17. Martínez-Aranguren R, Lizaso MT, Goikoetxea MJ, García BE, Cabrera-Freitag P, Trellez $\mathrm{O}$ et al. Is the determination of specific IgE against components using ISAC 112 a reproducible technique? PLoS One 2014;9:e88394.

18. Jakob T, Forstenlechner $\mathrm{P}$, Matricardi $\mathrm{P}$, Kleine-Tebbe J. Molecular allergy diagnostics using multiplex assays: methodological and practical considerations for use in research and clinical routine. Allergo J Int 2015;24:320-332.

19. Huss-Marp J, Gutermuth J, Schäffner I, Darsow U, Pfab F, Brockow K et al. Comparison of molecular and extract-based allergy diagnostics with multiplex and singleplex analysis. Allergo J Int 2015;24:46-53.

20. Hamilton RG. Multiplex IgE antibody testing as a tool for diagnosis and defining the allergic (atopic) status of study populations. Arb Paul Ehrlich Inst Bundesinstitut Impfstoffe Biomed Arzneim Langen Hess 2013;97:82-90.

21. Williams P, Önell A, Baldracchini F, Hui V, Jolles S, ElShanawany T. Evaluation of a novel automated allergy microarray platform compared with three other allergy test methods. Clin Exp Immunol 2016;184:1-10.

22. Dottorini T, Sole G, Nunziangeli L, Baldracchini F, Senin $\mathrm{N}$, Mazzoleni $\mathrm{G}$ et al. Serum IgE reactivity profiling in an asthma affected cohort. PLoS One 2011;6:e22319.

23. Wiltshire S, O'Malley S, Lambert J, Kukanskis K, Edgar D, Kingsmore SF et al. Detection of multiple allergenspecific IgEs on microarrays by immunoassay with rolling circle amplification. Clin Chem 2000;46:1990-1993.

24. Feyzkhanova GU, Filippova MA, Talibov VO,
Dementieva El, Maslennikov VV, Reznikov YP et al. Development of hydrogel biochip for in vitro allergy diagnostics. J Immunol Methods 2014;406:51-57.

25. Renault NK, Gaddipati SR, Wulfert F, Falcone FH, Mirotti $\mathrm{L}$, Tighe $\mathrm{PJ}$ et al. Multiple protein extract microarray for profiling human food-specific immunoglobulins $A, M$, $\mathrm{G}$ and E. J Immunol Methods 2011;364:21-32.

26. Joshi AA, Peczuh MW, Kumar C, Rusling JF. Ultrasensitive carbohydrate-peptide SPR imaging microarray for diagnosing IgE mediated peanut allergy. Analyst 2014;139:5728-5733.

27. King EM, Vailes LD, Tsay A, Satinover SM, Chapman MD. Simultaneous detection of total and allergen-specific IgE by using purified allergens in a fluorescent multiplex array. J Allergy Clin Immunol 2007;120:1126-1131.

28. Bastarache JA, Koyama T, Wickersham NE, Ware LB. Validation of a multiplex electrochemiluminescent immunoassay platform in human and mouse samples. $J$ Immunol Methods 2014;408:13-23.

29. Chinnasamy T, Segerink LI, Nystrand M, Gantelius J, Andersson Svahn H. Point-of-care vertical flow allergen microarray assay: proof of concept. Clin Chem 2014;60:1209-1216.

30. Chapman MD, Wuenschmann S, King E, Pomes A. Technological innovations for high hroughtput approaches to in vitro allergy diagnosis. Curr Allergy Asthma Rep 2015;15:35-42.

31. Kleine-Tebbe J, Jakob T. Molecular allergy diagnostics using IgE singleplex determinations: methodological and practical considerations for use in clinical routine. Allergo J Int 2015;24:185-197.

32. Sicherer SH, Dhillon G, Laughery KA, Hamilton RG, Wood RA. Caution: the Phadia hazelnut ImmunoCAP (F17) has been supplemented with recombinant Cor a 1 and now detects Bet v 1-specific IgE, which leads to elevated values for persons with birch pollen allergy. $J$ Allergy Clin Immunol 2008;122:413-414.

33. Heiss S, Mahler V, Steiner R, Spitzauer S, Schweiger C, Kraft D et al. Component-resolved diagnosis (CRD) of type I allergy with recombinant grass and tree pollen allergens by skin testing. J Invest Dermatol 1999;113:830-837.

34. Hamilton RG. Allergic sensitization is a key risk factor for but not synonymous with allergic disease. J Allergy Clin Immunol 2014;134:360-361.

35. Beyer K, Grabenhenrich L, Härtl M Beder A, Kalb B, Ziegert $M$ et al. Predictive values of componentspecific IgE for the outcome of peanut and hazelnut food challenges in children. Allergy 2015;70:90-98.

36. Hamilton RG, Kleine-Tebbe J. Molecular allergy diagnostics: analytical features that support clinical decisions. Curr Allergy Asthma Rep 2015;15:57.

37. Kleine-Tebbe J, Jappe U. Molecular allergy diagnostic tests: development and relevance in clinical practice. Allergologie 2013;36:327-49. 
SKIN TEST, BASOPHIL ACTIVATION TEST (BAT) AND PROVOCATION TESTS

\author{
Nikos Douladiris, Peter Korosec, Hans \\ Jürgen Hoffmann
}

\section{INTRODUCTION}

The issues covered in this section (in vivo testing with recombinant allergens (1) and basophil activation (2) have recently been reviewed.

\section{Think molecular when taking clinical history}

The first line approach to the diagnosis of allergic disease is with a thorough clinical history and physical examination of the patient. When allergic symptoms appear on exposure to a relevant allergen, specific IgE sensitization is confirmed with second line investigation, i.e. with allergen- extract-based in vivo skin prick tests and /or serology. If there is a mismatch between the history and these primary diagnostic tests, basophil activation testing could assess the allergenic activity ex vivo. When required, a third line provocation test (conjunctival, nasal or bronchial allergen challenge, placebo controlled food challenge) may confirm the diagnosis.

However, an increasing proportion of patients have inconclusive clinical history, symptoms profile and allergen extract testing, which may be solved by including component resolved diagnosis. These are
Skin and other provocation testing with molecular allergens is safe and may provide important information beyond extract testing.

Basophil activation testing of molecular allergens is a good alternative prior to skin or other provocation testing 
patients with complex symptomatology that mainly originates from:

a) Poly-sensitization to three or more inhalant allergens with overlapping exposure periods to natural and work environment, with graded symptoms. Molecular allergens can contribute to efficient identification of genuine sensitization to eliciting allergens and reveal co-sensitization and/or cross-sensitization of closely related or widely different allergen sources.

b) Sensitization to one or more food allergens with graded severity of symptoms that appears on ingestion of graded quantity and/or degree of processing of food (raw, cooked, baked). Molecular allergens can be used to characterize the severity and assess the future risk of the reaction in relation to stability and any process of the offending (food) allergen.

c) Co-sensitization to inhalant and food allergens present with symptoms of known or obscure aetiology. Molecular allergens can be used to optimize the decision process of provocation tests, avoiding costly, time consuming, and potentially life-threatening reactions and improve allergen avoidance recommendations.

d) Sensitization to more than one insect venom allergen in patients with anaphylactic sting reaction, but confused culprit history of insect stings. Molecular allergens can contribute to efficient identification of genuine sensitization to culprit venom and reveal co-sensitization and/ or cross sensitization of different insect venoms and can facilitate accurate prescription of venom immunotherapy.

e) In case of suspecting idiopathic anaphylaxis allergen microarrays allow the simultaneous detection of patients' antibody reactivity profiles towards a large variety of each of the molecular allergens, and this information is very helpful in establishing reactivity profiles and identifying of possible trigger.

Integrating component resolved diagnosis in first and second line investigations solves a number of difficult diagnoses.

\section{SKIN TESTS}

The usefulness of commercial whole-allergen extracts for skin testing is limited by the variation in the content of major and minor allergens, as well as by non-allergenic compounds they contain. Recombinant allergens or natural purified allergen molecules (referred to as: molecular allergens or allergen components or components) have been produced from all major allergen sources (e.g. pollens, mites, fungi, Hymenoptera venom, and food) and may be used for diagnosis and provocation testing; they were reviewed recently in detail (1).

Current evidence suggests skin allergy testing as a safe procedure, although in extremely rare occasions, fatalities have been observed with both skin provocation tests and - with a slightly higher rate with intradermal tests (3). No such reaction has been reported so far with molecular allergens. Allergen components are well defined and highly purified molecules with a narrow concentration range for optimal sensitivity and specificity. A comparison of possible advantages vs. disadvantages of available biological tests with allergen components is listed in Table 1.

Molecular allergens can promote diagnosis in skin testing significantly, contributing to both a more precise determination of sensitization pattern and a more targeted therapeutic approach. To that end, components could be used according to algorithms either complementary to, or instead of whole extracts, as they are proposed for in vitro use, in other sections of this handbook. The inclusion of recombinant or natural purified allergen molecules in current in vivo diagnostic methodology of allergic disease is a very promising task. Recent application of Molecular allergens in skin testing are listed in Table 2. Current high demands set by EU-imposed GMP and GCP may make this impossible, or at best an option for the distant future (4).

\section{BASOPHIL ACTIVATION TESTING}

Serum IgE reactivity to allergens informs about the concentration of specific IgE but not the individual allergenic activity of the measured specific IgE antibodies. As basophil activity depends on both reactivity and sensitivity, a basophil activation test (BAT) with molecular allergens not only identifies the allergen the patient is IgE sensitized to, but also the allergenic activity of these specific IgE antibodies.

Basophil activation is based on the fact that mast cells and basophil granulocytes share the pathway 


\section{Table 1}

Advantages and disadvantages of allergen extract vs. molecular allergens in allergy diagnostics

\begin{tabular}{|lll|}
\hline \multicolumn{1}{|c|}{ Allergen extracts } & Molecular allergens \\
\hline \multirow{2}{*}{ Advantages } & Easy preparation & Detection of genuine sensitization. \\
& Inexpensive & Contain many allergenic proteins \\
& Easy market authorisation & Identification of cross reactivity as cause of a \\
& & s-lgE measurement \\
& & Highly defined proteins \\
Disadvantages & Highly purified proteins \\
& Difficult standardization & Structure - amount of protein precisely known \\
& Unknown allergen composition & More safe \\
& Contains undefined components, i.e. endotoxins & Uncompleted yet panel of allergens for some \\
& Endogenous degradation - low sensitivity & allergen sources \\
& Proteins mixture complexity - low specificity & Difficult market authorization \\
\hline
\end{tabular}

\section{Table 2}

An update of provocation and BAT assessment of recombinant allergens since 2013

\begin{tabular}{|c|c|c|c|c|c|c|c|}
\hline Allergen Source & $\begin{array}{l}\text { Molecular } \\
\text { allergen }\end{array}$ & Modification & Test & $\begin{array}{c}\mathrm{Nr} \\
\text { Patients }\end{array}$ & $\begin{array}{c}\mathrm{Nr} \\
\text { controls }\end{array}$ & Conc & $\begin{array}{c}\text { Ref } \\
\text { (PMID) }\end{array}$ \\
\hline Peanut & Ara h 1, 2, 3 & $\begin{array}{l}\text { Hypo- } \\
\text { allergenic }\end{array}$ & $\begin{array}{c}\text { Rectral } \\
\text { provocation }\end{array}$ & 10 & 5 & $10-3063 \mu \mathrm{g}$ & (15) \\
\hline $\begin{array}{l}\text { Dermatophagoides } \\
\text { farinae }\end{array}$ & Der f 27 & - & SPT & 19 & . & $10 \mu \mathrm{g} / \mathrm{ml}$ & (16) \\
\hline $\begin{array}{l}\text { Dermatophagoides } \\
\text { farinae }\end{array}$ & Der f 29 & - & SPT & 14 & - & Not listed & (17) \\
\hline $\begin{array}{l}\text { Chenopodium } \\
\text { album }\end{array}$ & $\begin{array}{c}\text { Che a } 1, \\
\text { Che a } 2 \text { and } \\
\text { Che a } 3\end{array}$ & $\begin{array}{l}\text { Hypo- } \\
\text { allergenic, } \\
\text { natural }\end{array}$ & SPT & 17 & 4 & $1 \mathrm{mg} / \mathrm{ml}$ & (18) \\
\hline $\begin{array}{l}\text { Dog } \\
\text { Canis familiaris }\end{array}$ & $\begin{array}{l}\text { Can f } 1 \text {, Can } \\
\text { f 2, Can f } 4 \\
\text { and Can f } 6\end{array}$ & Fusion protein, & BAT & & & & (19) \\
\hline $\begin{array}{l}\text { Grass } \\
\text { Phleum partense }\end{array}$ & $\mathrm{Phl} \mathrm{p} 4$ & $\begin{array}{l}\text { Natural, non- } \\
\text { glycosylated }\end{array}$ & BAT & & & & (20) \\
\hline Cockroach & Per a 10 & - & $\begin{array}{c}\text { Basophil } \\
\text { histamine release }\end{array}$ & & & & (21) \\
\hline Siberian hamster & Pho s 1, 2, 3 & - & BAT & 2 & 2 & $\begin{array}{c}0-.1-10 \\
\mu \mathrm{g} / \mathrm{ml}\end{array}$ & $(22)$ \\
\hline Felix domesticus & Fel d 1, 2, 3 & $\begin{array}{l}\text { Fusion protein, } \\
\text { mosaic protein }\end{array}$ & BAT & 21 & 1 & $\begin{array}{l}0.1-100 \\
\mathrm{ng} / \mathrm{ml}\end{array}$ & (23) \\
\hline $\begin{array}{l}\text { Dermatophagoides } \\
\text { pterinussinus }\end{array}$ & Der $\mathrm{p} 2$ & - & BAT & 10 & 12 & $\begin{array}{c}100 \mathrm{fg} / \mathrm{ml}- \\
100 \mathrm{ng} / \mathrm{ml}\end{array}$ & (24) \\
\hline
\end{tabular}


for anaphylactic degranulation after crosslinking of the high affinity receptor for the IgE (FceRI) by IgE and allergen on the cell surface. Individual basophils with anaphylactic degranulation can be identified and distinguished from marginally activated basophils by upregulation of CD63 on activated basophils (2).

An allergen induces response in basophils through FceRI (this is basophil reactivity), in concentrations where non-allergic patients would not react (this is basophil sensitivity). Basophil reactivity has been shown to correlate with symptoms. Once reactivity is documented, it could be useful to know the sensitivity of blood basophils to allergen - this requires testing at a broad range of allergen concentrations (typically in 3- or 10-fold steps of dilution). On the basis of this series of tests, basophil sensitivity can be determined from a non-linear line of regression. The sensitivity has been associated with patients' threshold in allergen provocation (2). For example, diagnosis of peanut allergy usually requires a provocation as follow up on a positive skin prick test. Basophil sensitivity of sensitised patients was assessed before provocation testing. Basophil sensitivity with Ara h 2 gave the same clinical outcome as testing with peanut allergen (2). All patients tested negative in the basophil sensitivity test also passed a provocation test.

Insect venom allergy can be life threatening and is treated with a long course of AIT. The diagnostic process starts with measurement of specific IgE that often is positive for both bee and wasp whole allergen extracts. In contrast, in certain cases (e.g. mastocytosis) both tests may be negative. Assessment of reactivity to allergen components such as Api $\mathrm{m} 1$ (honey bee) and/or Ves v5/Ves v1 (wasp) has better diagnostic performance than measurement of serum IgE to whole extracts (5). Upgrading of those tests with component BAT demonstrates a further diagnostic improvement (6).

Basophil reactivity with olive pollen extracts and nOle e 1 was useful in the diagnosis of local allergic rhinitis (7).

Basophil testing is useful in the evaluation of the biological relevance of newly described molecular allergens. Reactivity to the recently discovered panallergen Der $\mathrm{p} 23$ was demonstrated, and the lack of response of a modified hypoallergenic form of Der $p$ 23 was confirmed by basophil testing (7). In the last few years all novel allergen components developed for diagnostic use or constructed as hypoallergenic vaccine (Table 2 ) were characterized by BAT for their allergenic activity $(1,2,9,10)$.

There are three major advantages of basophils testing compared with provocation testing:

a. The patient is not exposed to allergen. This saves both the patient and the health care system for resources, and requires only good manufacturing and laboratory procedures.

b. A number of single allergen molecules can be tested simultaneously as $75-100 \mu \mathrm{l}$ blood is required for a single test, and allergen components can be combined to mirror a real life exposure.

c. CD63 upregulation is a precise marker of anaphylactic degranulation, and thus has a potential to reflect the severity of allergic reaction (2).

\section{PROVOCATION TESTS}

Allergen provocation testing has been among the finest tools in the allergologist's quiver, setting the golden standard in certain conditions or being neglected due to standardization or safety issues in many others (11). Used mostly when observing discrepancies between clinical history and test results, provocation tests can provide critical information in clinical practice, along with serving for research (12).

Nasal allergen provocation testing is important when there are inconsistencies between clinical history and sensitization patterns. An important condition diagnosed by BAT or nasal provocation is the subgroup of patients suffering from local allergic rhinitis, with negative serum specific IgE and skin test results. Provocation with olive pollen extracts detected more sensitised patients with LAR than provocation with nOle e 1 , but provocation with nOle e1 may be more specific (7). Nasal provocation should comply with $E A A C l$ guidelines. It is the provocation modality first and most frequently used for in vivo testing of molecular allergens (1) because the nose is accessible, and symptoms are easily standardised. Nasal provocation with component Bet $\checkmark 1$ and Art $v 1$ results in similar effects as provocation with natural pollen extracts (1) and skin testing with molecular allergens (Bet v1, Bet $\vee 2$ and Phl p 1, $\mathrm{Phl} \mathrm{p} \mathrm{2,} \mathrm{Phl} \mathrm{p} \mathrm{5)} \mathrm{reflects} \mathrm{the} \mathrm{nasal} \mathrm{response} \mathrm{better}$ than concentrations of serum IgE (13). The only trial with bronchial provocation of asthmatic patients 
found comparable reactivity of component Bet $v$ 1 and natural allergen in skin prick tests, nasal and bronchial allergen provocation testing (14). Similarly, conjunctival provocation with component Bet $\mathrm{v} 1$ and birch extract found comparable allergenic reactivity of recombinant and natural products (1).

\section{CONCLUSION}

Molecular allergens may develop into a useful supplement in the clinic. Testing with allergen components is a part of the development of novel allergy vaccines and identification of nonallergenic isoforms. Development of recombinant allergen products has been delayed by the large difference in requirements for marketing natural extracts and recombinant products. Due to those requirements it seems unlikely to have recombinant allergen molecules for in vivo test in near future. However, we expect dissemination of recombinant allergen molecules in BAT testing. CRD is useful in identification of patients genuinely sensitised to allergen sources and identification of patients crossreacting to pan allergens. These groups need to be treated differently. For now however, CRD need to be accompanied by clear explanation of the relevance and implication of positive and negative tests.

\section{References}

1. Niederberger V, Eckl-Dorna J, Pauli G. Recombinant allergen-basedprovocationtesting. Methods 2014;66: 96-105.

2. Hoffmann HJ, Santos AF, Mayorga C, Nopp A, Eberlein $B$, Ferrer $M$, et al. The Clinical Utility of Basophil Activation Testing in Diagnosis and Monitoring of Allergic Disease. Allergy 2015;70:1393-1405.

3. Galindo-Pacheco LV, O'Farrill-Romanillos PM, Amaya-Mejía AS, Almeraya-García P, López-Rocha E. Anaphylaxis secondary to prick-to-prick tests to foods and its risk factors. Rev Alerg Mex 2014;61:24-31.

4. Klimek L, Hoffmann HJ, Renz H, Demoly P, Werfel T, Matricardi PM, et al. Diagnostic test allergens used for in vivo diagnosis of allergic diseases are at risk: a European Perspective. Allergy 2015;70:1329-1331.

5. Mittermann I, Zidarn M, Silar M, Markovic-Housley Z, Aberer W, Korosec P, et al. Recombinant allergenbased IgE testing to distinguish bee and wasp allergy. J Allergy Clin Immunol 2010;125:1300-1307.

6. Balzer L, Pennino D, Blank S, Seismann H, Darsow U, Schnedler M, et al. Basophil Activation Test Using Recombinant Allergens: Highly Specific Diagnostic
Method Complementing Routine Tests in Wasp Venom Allergy. PLoS ONE 2014;9:e108619.

7. Campo P, Villalba M, Barrionuevo E, Rondón C, Salas M, Galindo L, et al. Immunologic responses to the major allergen of Olea Europaea in local and systemic allergic rhinitis subjects. Clin Exp Allergy 2015;45:1703-1712.

8. Banerjee S, Weber M, Blatt K, Swoboda I, Focke-TejkI $M$, Valent $P$, et al. Conversion of Der p 23, a new major house dust mite allergen, into a hypoallergenic vaccine. J Immunol 2014;192:4867-4875.

9. Campana R, Vrtala S, Maderegger B, Jertschin P, Stegfellner G, Swoboda I, et al. Hypoallergenic derivatives of the major birch pollen allergen Bet $\vee 1$ obtained by rational sequence reassembly. J Allergy Clin Immunol 2010;126:1024-1031.

10. Focke-Tejkl M, Weber M, Niespodziana K, Neubauer A, Huber $\mathrm{H}$, Henning $\mathrm{R}$, et al. Development and characterization of a recombinant, hypoallergenic, peptide-based vaccine for grass pollen allergy. J Allergy Clin Immunol 2015;135:1207-1217.

11. Glaumann S, Nopp A, Johansson SGO, Borres MP, Nilsson C. Oral Peanut Challenge Identifies an Allergy but the Peanut Allergen Threshold Sensitivity Is Not Reproducible. PLOS ONE 2013;8:e53465.

12. Santos AF, Douiri A, Bécares N, Wu S-Y, Stephens A, Radulovic $S$, et al. Basophil activation test discriminates between allergy and tolerance in peanut-sensitized children. J Allergy Clin Immunol 2014;134:645-652.

13. Niederberger V, Stübner P, Spitzauer S, Kraft D, Valenta $\mathrm{R}$, Ehrenberger K, et al. Skin test results but not serology reflect immediate type respiratory sensitivity: a study performed with recombinant allergen molecules. J Investig Dermatol 2001;117:848-851.

14. Godnic-Cvar J, Susani M, Breiteneder H, Berger A, Havelec L, Waldhör T, et al. Recombinant Bet v 1, the major birch pollen allergen, induces hypersensitivity reactions equal to those induced by natural Bet $v 1$ in the airways of patients allergic to tree pollen. J Allergy Clin Immunol 1997;99:354-359.

15. Wood RA, Sicherer SH, Burks AW, Grishin A, Henning $A K$, Lindblad $R$, et al. A phase 1 study of heat/ phenol-killed, E. coli-encapsulated, recombinant modified peanut proteins Ara $\mathrm{h} 1$, Ara $\mathrm{h} \mathrm{2}$, and Ara $h 3$ (EMP-123) for the treatment of peanut allergy. Allergy 2013;68:803-808.

16. Lin J, Li M, Liu Y, Jiang C, Wu Y, Wang Y, et al. Expression, purification and characterization of Der $f$ 27, a new allergen from dermatophagoides farinae. Am J Transl Res 2015;7:1260-1270.

17. Jiang C, Fan X, Li M, Xing P, Liu X, Wu Y, et al. Characterization of Der $f$ 29, a new allergen from dermatophagoides farinae. Am J Transl Res 2015;7:1303-1313. 
18. Nouri HR, Varasteh A, Vahedi F, Chamani J, Afsharzadeh D, Sankian M. Constructing a hybrid molecule with low capacity of IgE binding from Chenopodium album pollen allergens. Immunol Lett 2012;144:67-77.

19. Nilsson $O B$, Neimert-Andersson $T$, Bronge $M$, Grundström J, Sarma R, Uchtenhagen H, et al. Designing a multimer allergen for diagnosis and immunotherapy of dog allergic patients. PLOS ONE 2014;9:e111041.

20. Zafred D, Nandy A, Pump L, Kahlert H, Keller W. Crystal structure and immunologic characterization of the major grass pollen allergen $\mathrm{Phl} p$ 4. J Allergy Clin Immunol 2013;132:696-703.

21. Govindaraj D, Gaur SN, Arora N. Characterization of recombinant per a 10 from Periplaneta americana. Clin Vaccine Immunol 2013;20:262-268.
22. Torres JA, de Las Heras M, Maroto AS, Vivanco F, Sastre J, Pastor-Vargas C. Molecular and immunological characterization of the first allergenic lipocalin in hamster: the major allergen from Siberian hamster (Phodopus sungorus). J Biol Chem 2014;289:2338223388.

23. Curin $M$, Weber $M$, Thalhamer $T$ et al. Hypoallergenic derivatives of Fel $\mathrm{d} 1$ obtained by rational reassembly for allergy vaccination and tolerance induction. Clin Exp Allergy 2014;44:882-894.

24. Krohn IK, Sverrild A, Lund G, Dahl R, Erjefält JS, Backer V, et al. Cultured mast cells from patients with asthma and controls respond with similar sensitivity to recombinant Der p2-induced, IgE-mediated activation. Scand J Immunol 2013;78:352-356. 


\title{
ALLERGEN FAMILIES AND DATABASES
}

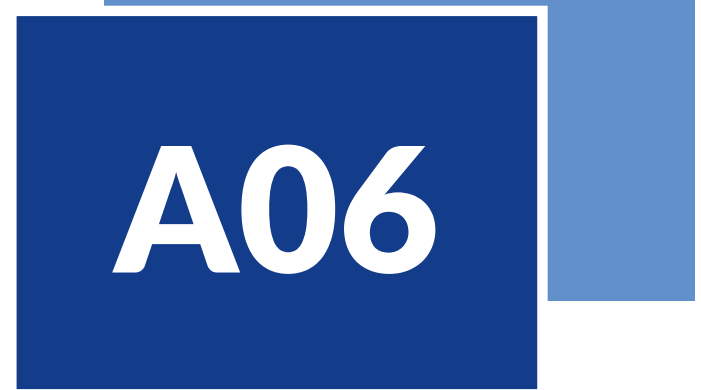

\author{
Heimo Breiteneder
}

\section{INTRODUCTION}

The current version 29.0 of the Pfam (protein family) database (http://pfam.xfam.org/) describes 16,295 protein families (1). Families are grouped together into superfamilies - called clans in Pfam (e.g. the prolamin superfamily), and some families are further divided into subfamilies (e.g. the PR-10like proteins are a subfamily of the Bet $v 1$ family). A protein family is a group of proteins that share a common evolutionary origin, which is reflected first and foremost by their similar overall structure and topology (Fig. 1). Members of a protein family may also possess related biological functions and immunological characteristics as well as similar amino acid sequences.

The allergenicity of proteins is determined by a small number of protein architectures and thus are found in a rather limited number of protein families. The SDAP (https://fermi.utmb.edu/; accessed on March $7,2015)$ database of structures of allergenic proteins assigns all presently known allergens to 130 of the 16,230 Pfam families. Proteins that are described worldwide as the most important allergens can be classified into roughly 30 to 40 protein families.
Proteins that share a common evolutionary origin - which is reflected first and foremost by their similar overall structure and topology - are grouped into families. Families with a common origin are grouped into superfamilies.

Very few of the almost 16,300 protein families as described by the protein family database Pfam families harbour allergens.

Proteins that are described worldwide as allergens can be classified roughly into 30 to 40 protein families.

Based on their molecular, biologic and biochemical properties, allergenic proteins are able to initiate both innate and adaptive immune responses during the sensitization process that ultimately result in the production of allergen-specific lgE.

Allergen databases make curated information available on the allergen nomenclature, allergen structures, allergen protein families, and the risk of allergenic cross-reactivity, or offer an uncurated extensive collection of information on allergens. 


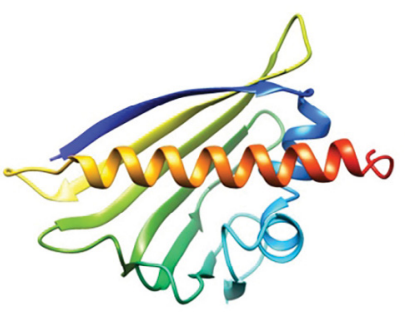

Bet $v 1$

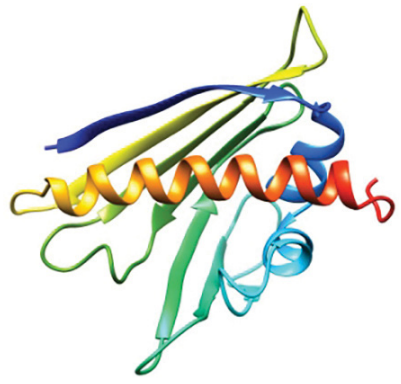

Pru av 1

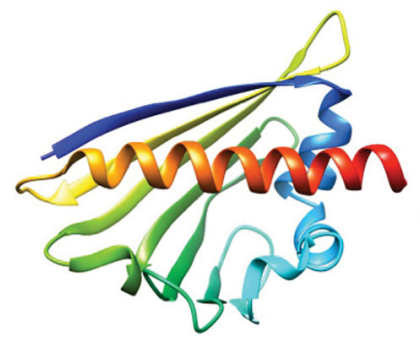

Api g 1

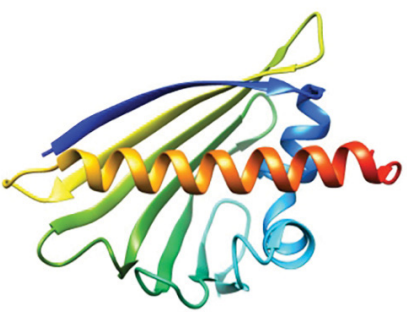

Ara h 8

Figure 1

Four representative members of the Bet $v 1$ family of proteins. Bet $v 1$ is from birch pollen, Pru av 1 from cherry, Api g 1 from celery and Ara h 8 from peanut.

The most important families and selected allergenic member proteins are discussed here (Table 1).

Our understanding why exactly these types of proteins are able to trigger Th2-dominated immune responses is incomplete. Allergenic proteins that are able to sensitize predisposed individuals initiate both innate and adaptive immune responses. Such proteins are recognized by epithelial cells and dendritic cells that produce signals to polarize the immune response towards a Th2 phenotype. It has been clearly shown that the decision whether a protein will be treated as an allergen by the organism is made by the innate immune system (2-4). It is highly likely that allergenic proteins possess molecular features that enable them to activate the pattern recognition receptors of innate immune cells to induce a Th2 polarization of the ensuing immune response. These features include (i) the ability to bind lipids and to activate Tolllike receptors - shown for Der $\mathrm{p} 2$ from house dust mite (5) and for Fel d 1 from cat (6), (ii) the presence of glycosylation and thus the ability to bind to C-type lectin receptors - shown for allergens from house dust mite, pollen and peanut (7-9), or (iii) the presence of protease activity which allows the activation of the protease-activated receptor PAR-2 - shown for Der $p$ 3 and Der $\mathrm{p} 9$ from house dust mite (10).

\section{PROLAMIN SUPERFAMILY}

The prolamin superfamily derives its name from the alcohol-soluble proline- and glutamine-rich storage proteins of cereal grains. Members of this superfamily are characterized by the presence of an alpha-helical globular domain. This domain contains a conserved pattern of cysteine residues that form three to five intra-molecular disulfide bonds. Apart from the conserved cysteine pattern, only little sequence similarities exist between the members of different families. Members of the prolamin superfamily include the cereal prolamin seed storage proteins (gliadins and glutenins) and several families of disulfide-rich small proteins belonging to the bifunctional inhibitors, the $2 \mathrm{~S}$ albumin seed storage proteins, and the non-specific lipid transfer proteins.

\section{Cereal prolamins}

Cereal prolamins are seed storage proteins that are exclusively found in the grains of cereal grasses. In contrast to the low molecular weight members of the superfamily, the $\alpha$-helical domain of the cereal prolamins has been disrupted by an insertion of repetitive sequences (11). Gliadins and glutenins represent the members of the cereal prolamin family. Gliadins are soluble in alcohol and they are classified into $\alpha / \beta$-, $\gamma$ - and $\omega$-gliadins. Glutenins are polymeric proteins that are held together by interchain disulfide bonds. They are divided into high and low molecular weight groups (Fig. 2) (12).

Wheat (Triticum aestivum) contains several allergenic cereal prolamins. Tri a 19 is an $\omega$-5-gliadine, Tri a 21 an $\alpha / \beta$-gliadine, and Tri a 26 a high molecular weight glutenine from wheat.

\section{Bifunctional inhibitors}

Like the cereal prolamins, the bifunctional inhibitors are only present in cereal grains. These allergens sensitize either via the respiratory tract by inhalation of the flour or via the gastro-intestinal tract by eating foods that contain wheat, barley or rice. The 
Table 1

Overview of the most important allergen superfamilies and families with their corresponding sources

\begin{tabular}{|c|c|c|}
\hline Superfamily & Family & Allergen sources \\
\hline \multirow{4}{*}{ Prolamin } & $\begin{array}{l}\text { Cereal } \\
\text { prolamins }\end{array}$ & $\begin{array}{l}\text { Grains of cereal } \\
\text { grasses }\end{array}$ \\
\hline & $\begin{array}{l}\text { Bifunctional } \\
\text { inhibitors }\end{array}$ & $\begin{array}{l}\text { Grains of cereal } \\
\text { grasses }\end{array}$ \\
\hline & $2 \mathrm{~S}$ albumins & $\begin{array}{l}\text { Tree nuts, peanuts, } \\
\text { legumes, seeds }\end{array}$ \\
\hline & $\begin{array}{l}\text { Non-specific } \\
\text { lipid-transfer } \\
\text { proteins }\end{array}$ & $\begin{array}{l}\text { Fruits, tree nuts, } \\
\text { peanuts, vegetables, } \\
\text { tree and weed } \\
\text { pollen, latex }\end{array}$ \\
\hline \multirow[t]{2}{*}{ EF-hand } & Polcalcins & $\begin{array}{l}\text { Tree, grass, and } \\
\text { weed pollen }\end{array}$ \\
\hline & Parvalbumins & Fish \\
\hline Profilin-like & Profilin & $\begin{array}{l}\text { Tree, grass, and } \\
\text { weed pollen, fruits, } \\
\text { vegetables, latex }\end{array}$ \\
\hline $\begin{array}{l}\text { Tropomyosin- } \\
\text { like }\end{array}$ & Tropomyosin & $\begin{array}{l}\text { Crustaceans, } \\
\text { mollusks, fish } \\
\text { parasite Anisaki } \\
\text { simplex, mites, } \\
\text { cockroaches }\end{array}$ \\
\hline \multirow{2}{*}{ Cupin } & Vicilins & $\begin{array}{l}\text { Tree nuts, peanuts, } \\
\text { legumes, seeds }\end{array}$ \\
\hline & Legumins & $\begin{array}{l}\text { Tree nuts, peanuts, } \\
\text { legumes, seeds }\end{array}$ \\
\hline Bet v 1-like & Bet $\vee 1$ & $\begin{array}{l}\text { Fagales tree pollen, } \\
\text { fruits, vegetables, } \\
\text { legumes, tree nuts }\end{array}$ \\
\hline Calycin & Lipocalins & $\begin{array}{l}\text { Mammals, mites, } \\
\text { cockroaches }\end{array}$ \\
\hline \multirow{2}{*}{$\begin{array}{l}\text { Double-psi } \\
\text { beta-barrel }\end{array}$} & DPBB_1 & Grass pollen \\
\hline & $\begin{array}{l}\text { Pollen_- } \\
\text { allerg_1 }\end{array}$ & Grass pollen \\
\hline
\end{tabular}

bifunctional inhibitors are $12-$ to $16 \mathrm{kDa}$ proteins that are held together by 4 to 6 disulfide bonds (13). Monomeric, heterodimeric and heterotetrameric forms are distinguished according to the degree of aggregation of their subunits. They are the major cause of baker's asthma but also play a role as plant food allergens (14).
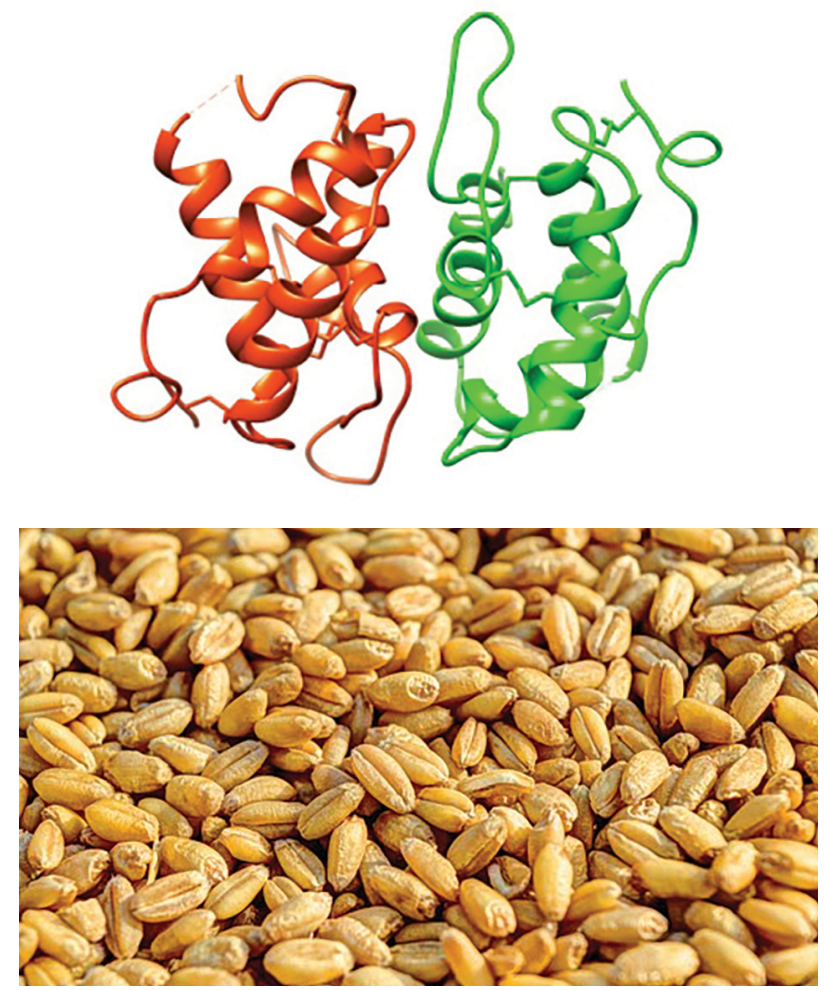

Figure 2

Dimeric Tri a 28 from wheat.

Hor $v 15$ is a monomeric $\alpha$-amylase inhibitor from barley. Tri a 28 is a dimeric and Tri a 29 a tetrameric $\alpha$-amylase inhibitor from wheat.

\section{$2 \mathrm{~S}$ albumins}

The $2 \mathrm{~S}$ albumins are a major group of plant seed storage proteins. Most $2 \mathrm{~S}$ albumins are synthesized as single-chain proteins that are subsequently cleaved into a small and a large subunit. Both subunits are held together as compact $\alpha$-helical molecules by 4 to 5 disulfide bonds (15). Many of the important seed and tree nut allergens are $2 \mathrm{~S}$ albumins (Fig. 3).

- Allergenic $2 S$ albumins include Ara h 2 and Ara h 6 from peanut, Ber e 1 from Brazil nut, Cor a 14 from hazelnut, Jug $r 1$ from English walnut, Ses i 1 from sesame seeds, and Sin a 1 from yellow mustard.

\section{Non-specific lipid transfer proteins (nsLTPs)}

nsLTPs have been suggested to mediate the transfer of phospholipids between vesicles and membranes. 

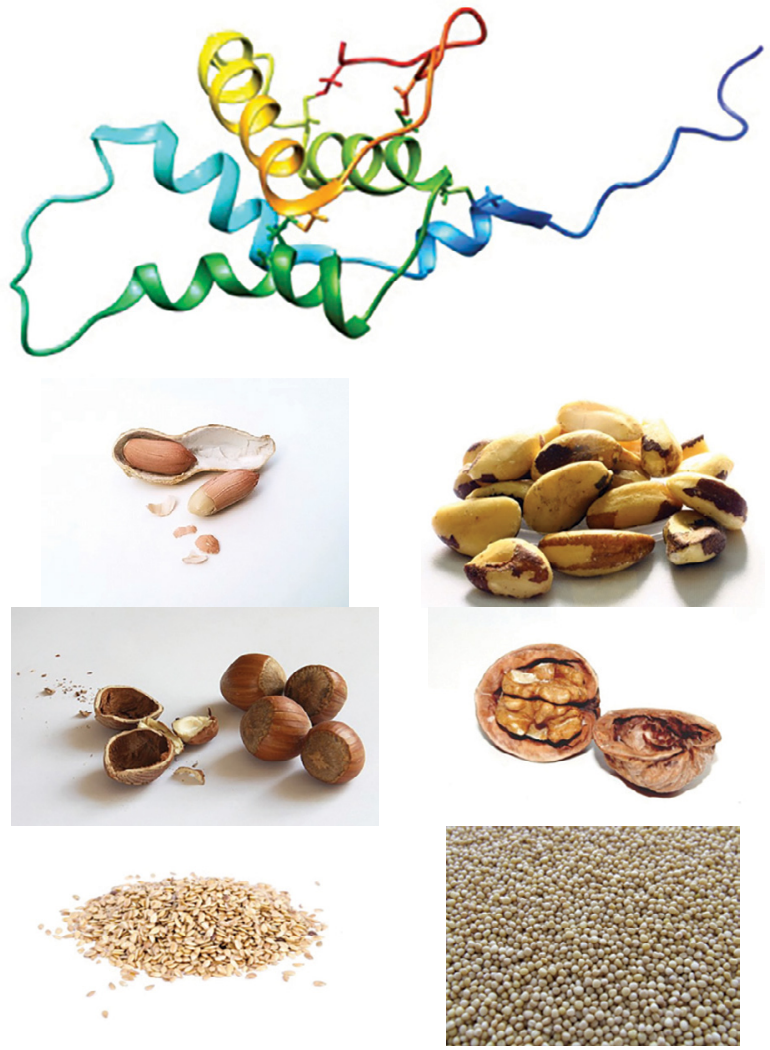

Figure 3

Ara h 6 from peanut and other sources of allergenic $2 S$ albumins: Brazil nut, hazelnut, walnut, sesame seeds, and yellow mustard.

However, plants have use the three-dimensional scaffold of the nsLTPs in various ways and many nsLTPs play a role in plant defense against fungi and bacteria. Allergenic nsLTPs are a large group of proteins that are resistant to heat and digestion (16). nsLTPs are found in high concentrations in epidermal tissues of fruits. They are major allergens of fruits from the Rosaceae family. In addition, allergenic nsLTPs are present in nuts, seeds, vegetables, and Hevea brasiliensis latex. Besides their presence in plant foods, nsLTPs are also expressed in pollen of weeds, olive, and plane (Fig. 4).

- Roseacea fruit nsLTP include Mal d 3 from apple and Pru $\mathrm{p} 3$ from peach. Representative allergenic nsLTPs from tree nuts are Cor a 8 from hazelnut and Jug $r 3$ from walnut. Pollen nsLTPs include Pla a 3 from plane tree and Art $v 3$ from mugwort. Zea $\mathrm{m} 14$ is the nsLTP from maize.
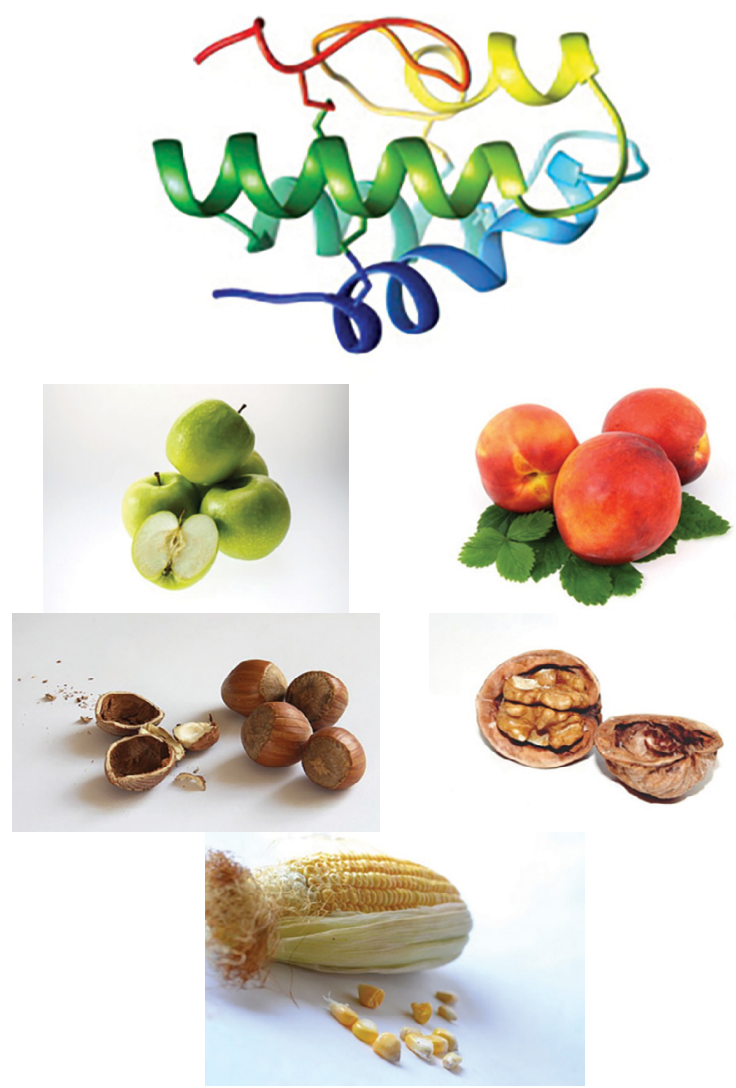

Figure 4

Zea m 14 from maize and other sources of allergenic nsLTPs: apple, peach, hazelnut, maize, and walnut.

\section{EF-HAND SUPERFAMILY}

A wide range of calcium-binding proteins share a conserved domain consisting of a 12 residue calciumbinding loop flanked on both sides by a-helices of 12 residues in length (17). The biological functions of these proteins include signaling and calcium buffering or transport.

\section{Polcalcins}

Polcalcins are $9 \mathrm{kDa}$ calcium-binding pollen proteins of unknown biological function. While regular polcalcins contain two EF hand domains, several polcalcinrelated allergens with three or four EF hand domains were described (18). Polcalcins were shown to be minor albeit highly cross-reactive allergens identified in pollen from diverse plant families (Fig. 5). 


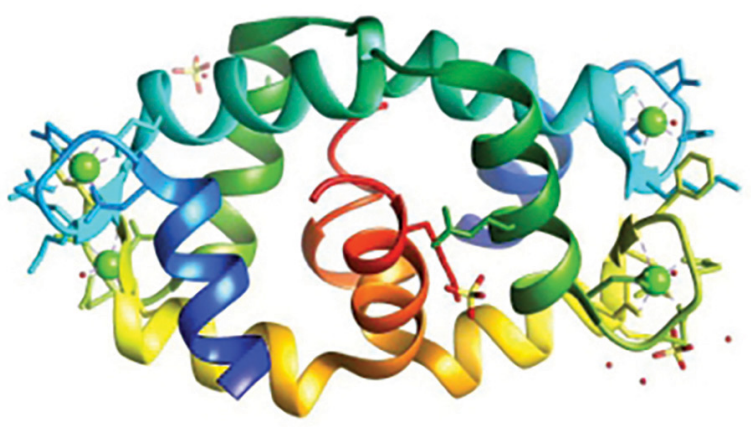

Figure 5

Phl p 7 dimer with bound calcium ions.
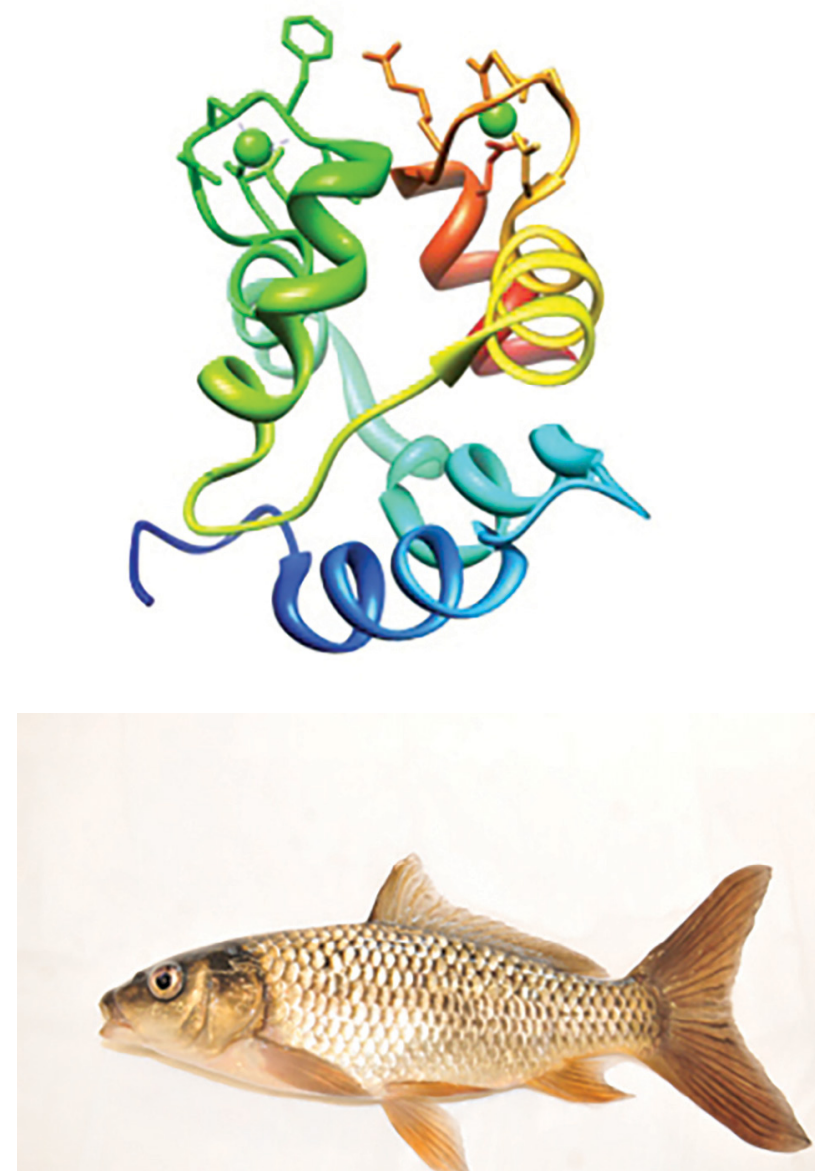

Figure 6

Gad m 1 from cod with bound calcium ions (green spheres) and a young carp (picture kindly provided by Clinical Unit of Fish Medicine, University of Veterinary Medicine, Vienna, Austria), a source of Cyp c 1.
- Allergenic polcalcins include the monomeric Bet $v 4$ from birch, the dimeric Phl $\mathrm{p} 7$ from timothy grass, and the tetrameric Che a 3 from Chenopodium album.

\section{Parvalbumins}

Parvalbumins are $12 \mathrm{kDa}$ proteins that contain two EF hand domains. They are found in fast-twitch muscle fibers of vertebrates and bind calcium ions during muscle relaxation. Parvalbumins from fishes and amphibians are major food allergens eliciting IgE responses in most fish-allergic individuals (Fig. 6) (19).

- Allergenic parvalbumins include Gad m 1 from Atlantic cod, Sal s 1 from Atlantic salmon, and Cyp c 1 from carp

\section{PROFILIN-LIKE SUPERFAMILY}

Profilins are small cytosolic proteins that are found in all eukaryotic cells. Profilins bind to monomeric actin and various other muscle proteins, thus regulating the dynamics of actin polymerization during processes such as cell movement, cytokinesis, and signaling. Profilins from higher plants constitute a family of highly conserved proteins that show sequence identities of at least $75 \%$ even between members from distantly related organisms (20). Since profilinspecific IgE usually cross-reacts with homologues from virtually every plant source, sensitization to these allergens has been considered a risk factor for pollen-associated food allergy (21). However, no substantial cross-reactivity between plant and human profilins has been shown so far (Fig. 7).

- Allergenic profilins include Art v 4 from mugwort pollen, Bet $v 2$ from birch pollen, Ole e 2 from olive pollen, Cit s 2 from oranges, Cuc $\mathrm{m} 2$ from melons, and Mus a 1 from bananas.

\section{TROPOMYOSIN-LIKE SUPERFAMILY}

Tropomyosins are present in muscle and nonmuscle cells. In striated muscle, they mediate the interactions between the troponin complex and actin to regulate muscle contraction. Tropomyosin is an a-helical protein that forms a coiled-coil structure of two parallel helices containing two sets of seven alternating actin binding sites. Tropomyosins were identified as animal food allergens in crustaceans, 

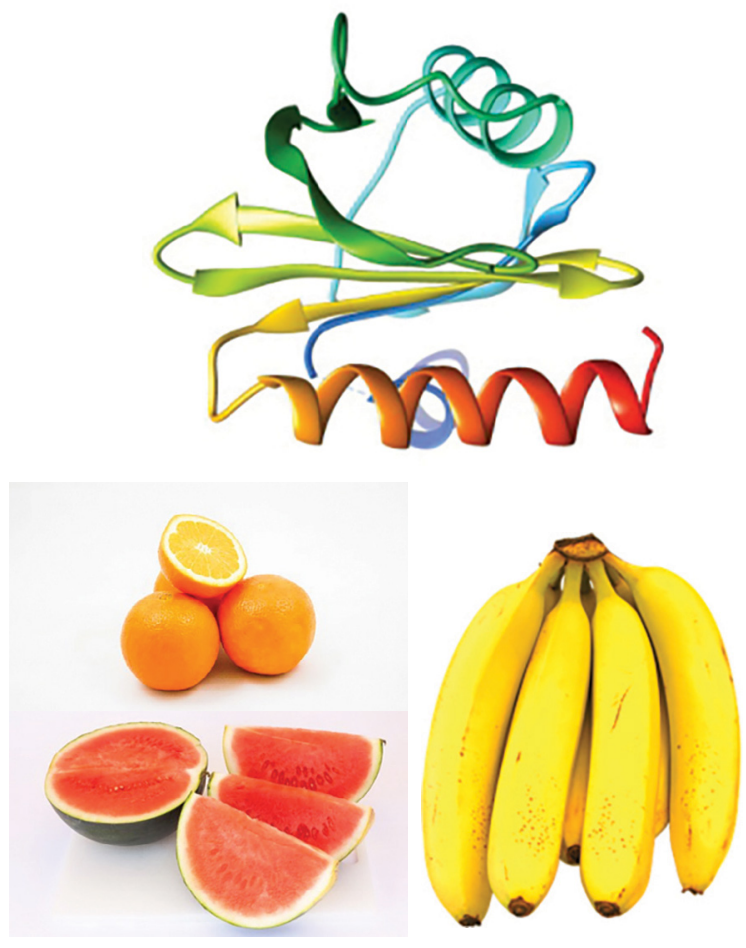

Figure 7

Bet $\vee 2$ from birch pollen and other sources of allergenic profilins.

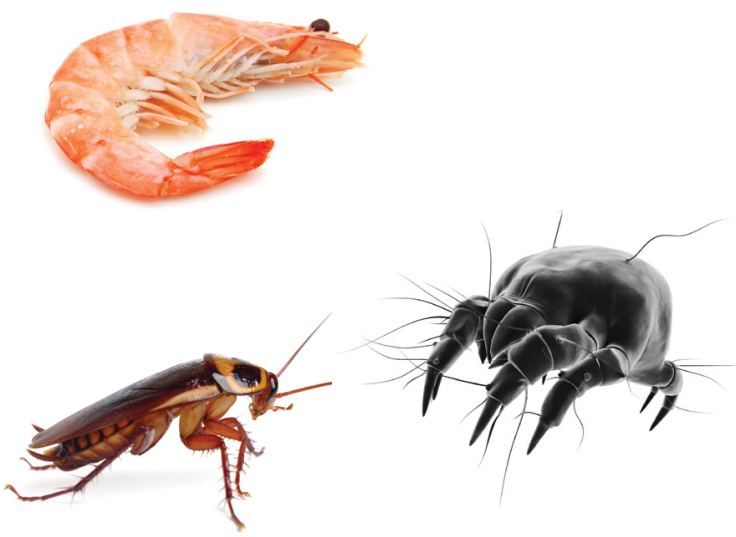

Figure 8

Examples for sources of allergenic tropomyosins: Indian prawn, German cockroach, and house dust mite. mollusks, and the fish parasite Anisakis simplex (22). Tropomyosins were also identified as inhalant allergens in arthropods (mites, cockroaches). Tropomyosin sequences are highly conserved, which explains the frequent cross-sensitization among tropomyosin-containing allergen sources (Fig. 8) (23).

- Pen i 1 from the Indian prawn, Bla g 1 from the German cockroach, and Der $p 10$ from house dust mite are well-known allergenic tropomyosins.

\section{CUPIN SUPERFAMILY}

The cupins are a large and functionally immensely diverse superfamily of proteins that have a common origin and whose evolution can be followed from bacteria to eukaryotes including animals and higher plants. Cupin proteins are currently classified into 57 protein families of this superfamily. The largest families of bicupins are the 7/8S and $11 \mathrm{~S}$ seed storage globulins that are the major components of plant seeds and are not only important sources of proteins for the human diet and but also of allergens $(24,25)$.

\section{Vicilins}

These are homotrimeric proteins of about 150 to $190 \mathrm{kDa}$. Their detailed subunit compositions vary considerably due to differences in proteolytic processing and glycosylation of the monomers (Fig. 9).

- Allergenic vicilins include Ara h 1 from peanut, Gly $m 5$ from soybean, Jug $r 2$ from walnut, and Ses i 3 from sesame.

\section{Legumins}

In mature legumins, two trimers associate to form hexameric proteins (Fig. 10).

- Allergenic legumins include the peanut allergen Ara h 3, Gly $m 6$ from soybean, Ber e 2 from Brazil nut, and Fag e 1 from buckwheat.

\section{BET V 1-LIKE SUPERFAMILY}

The Bet v 1-like superfamily currently harbors 103,375 proteins from 1,452 species with sequences related to the major birch (Betula verrucosa) pollen allergen Bet $v$ 1. These proteins are distributed between 14 families. The members of all 14 families (one of which is the Bet v 1 family) in this superfamily share the same structure 

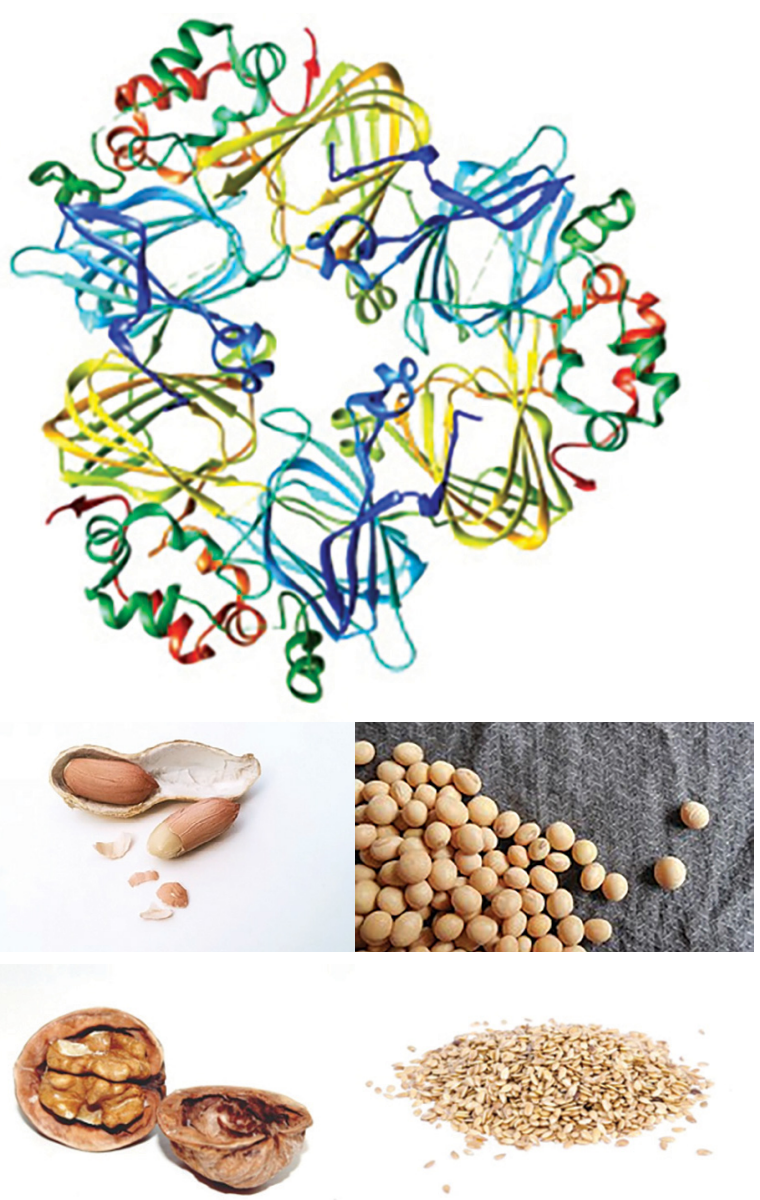

Figure 9

Ara h 1 homotrimer from peanut and other sources of allergenic vicilins: soybean, walnut, sesame seeds.

which is composed of 7 antiparallel $\beta$-sheets and 3 $\alpha$-helices (26). There is a cavity between the $\beta$-sheet and a long $C$ terminal $\alpha$-helix. The cavity appears to be able to bind a variety of lipid and bioflavonoid molecules. So far, allergens were identified in 3 of the 11 subfamilies of the Bet $v 1$ family.

\section{Pathogenesis-related protein subfamily 10 (PR- 10)}

The PR-10 proteins are actually one of the 11 subfamilies of the Bet $v 1$ family. The expression of these proteins is either induced by pathogen attack or abiotic stress, or it is developmentally regulated. PR-10 proteins are expressed in high concentrations in reproductive tissues such as pollen, seeds and
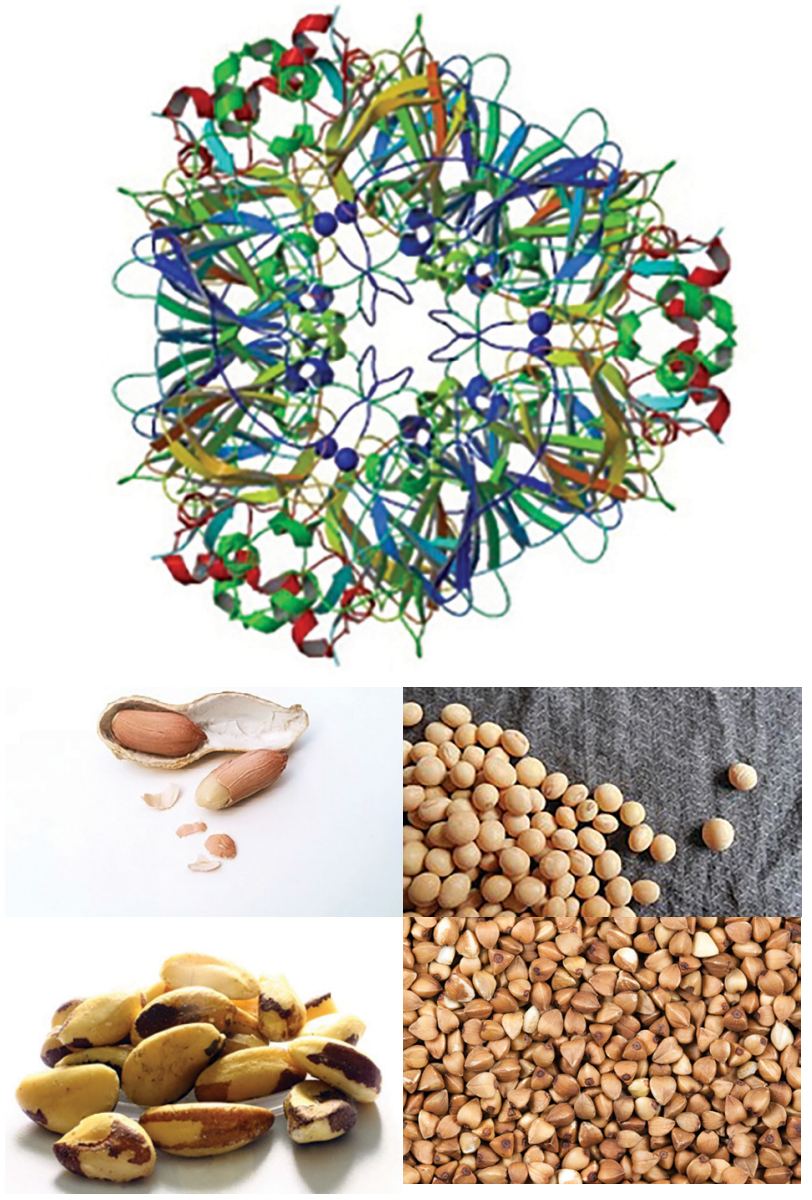

Figure 10

Ara h 3 from peanut and other sources of allergenic legumins: soybean, Brazil nut, and buckwheat.

fruits. Many birch pollen-allergic patients show allergic reactions to various fruits and vegetables, which are caused by IgE cross-reactivity between Bet $v 1$ and homologous allergens from plant foods. Most Bet $v$ 1-related food allergens have been found in members of certain plant families: Rosaceae (apple, pear, stone fruits), Apiaceae (celery, carrot), and Fabaceae (soybean, peanut; Fig. 11).

\section{RRP (ripening-related proteins)/MLP (major latex proteins) family}

The RRP/MLP group of proteins is another subfamily of the Bet $v 1$ family. Act $d 11$ is the first described Bet $v 1$-related allergen from kiwi from the RRP/MLP subfamily (Fig. 12) (27). 

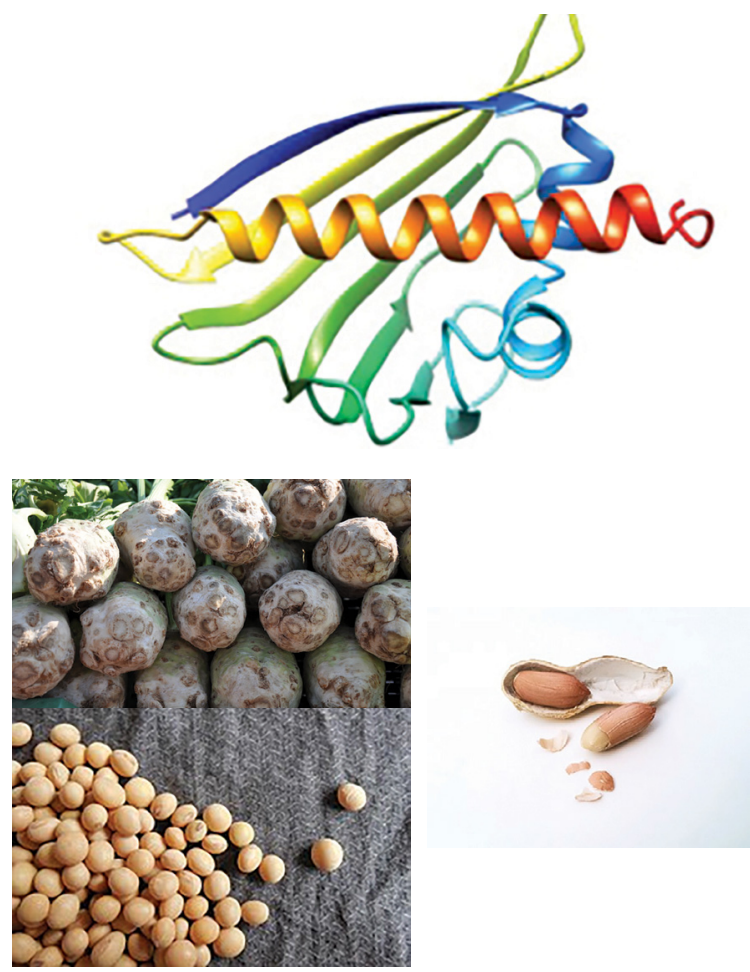

\section{Figure 11}

Bet $\vee 1$ from birch pollen and other sources of allergens belonging to the pathogenesis-related protein subfamily 10: celery, soybean, and peanut.
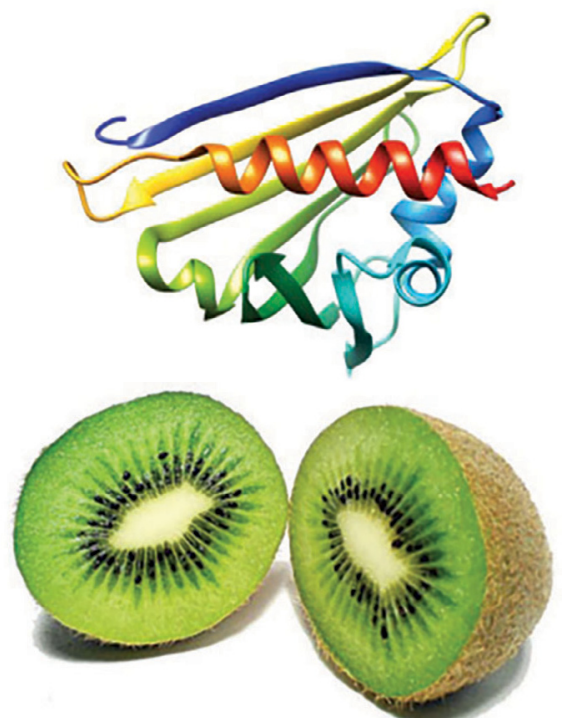

Figure 12

Act d 11 from kiwi.
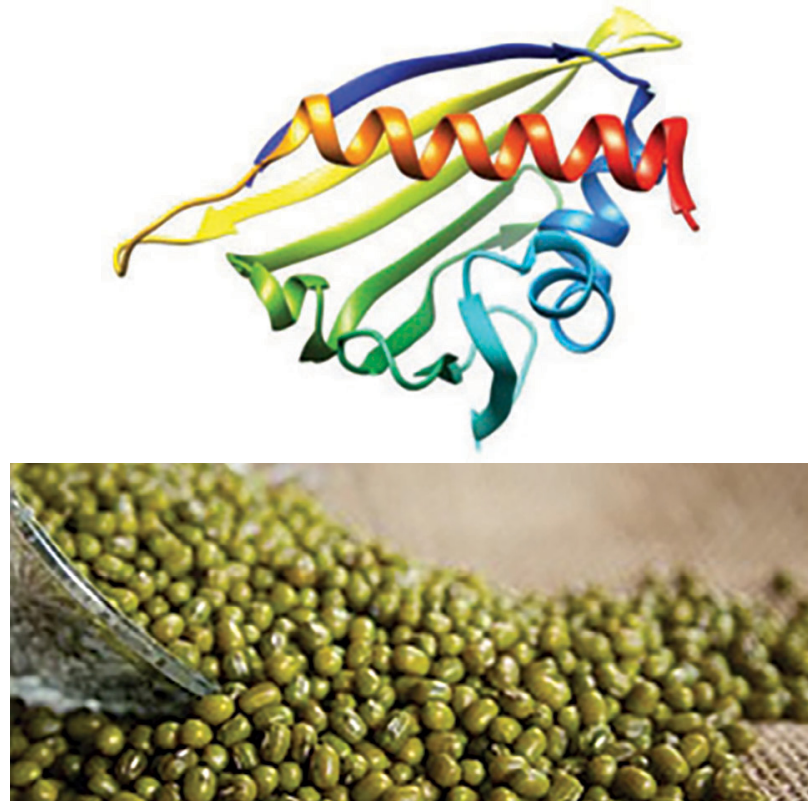

Figure 13

Vig $r 6$ from mungbean.

\section{CSBP (cytokinin-specific binding proteins) subfamily}

Vig $r 6$ from the mung bean is the first described Bet $\checkmark$ 1-related allergen from the CSBP subfamily of the Bet $v 1$ family Bet $v 1$ family (Fig. 13) (28).

\section{CALYCIN SUPERFAMILY}

The calycin superfamily comprises 16 families. Although structurally similar, calycins have rather low sequence similarities. The calycin architecture is based on an eight-stranded $\beta$-barrel which can bind a variety of different ligands (29).

\section{Lipocalins}

Lipocalins are transporters for small hydrophobic molecules, such as lipids, steroid hormones, bilins, and retinoids. Lipocalins fold into an eight-stranded antiparallel $\beta$-barrel. Allergens from this protein family include $\beta$-lactoglobulin, mammalian dander allergens, and cytoplasmic fatty acid binding proteins $(30,31)$. $\beta$-lactoglobulin is the major whey protein of ruminant species. Bos d 5 is a major cow's milk allergen. Cross- 


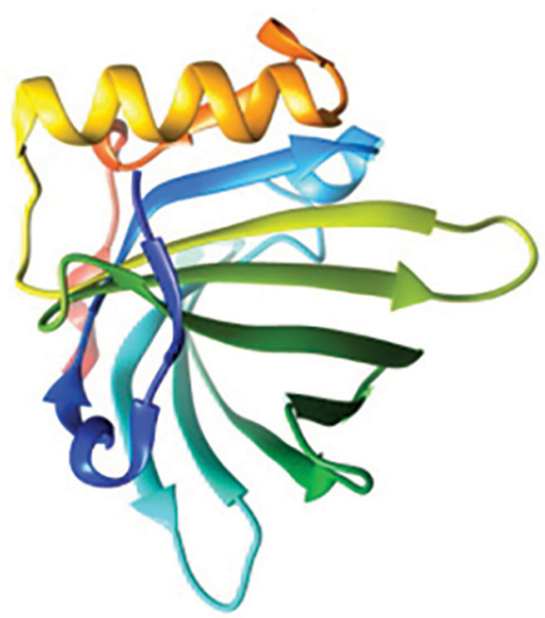

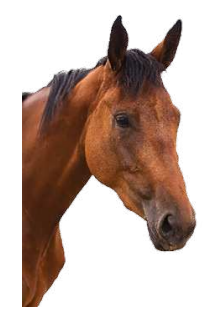

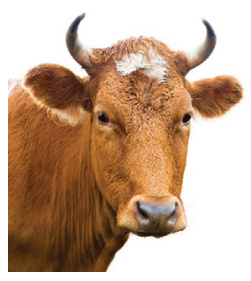

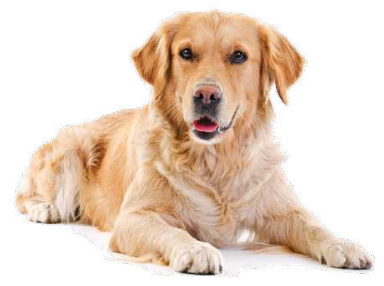

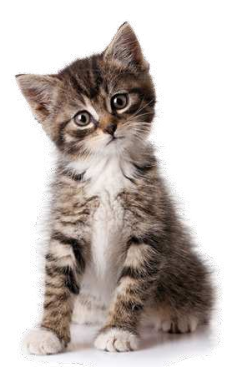

Figure 14

Equ c 1 from horse and other sources of allergenic lipocalins: dog, cow, and cat.

reactions to milk proteins from other species have also been described. Lipocalins constitute the vast majority of mammalian dander allergens (Fig. 14). Cytoplasmic fatty acid binding proteins are distantly related to extracellular lipocalins and $\beta$-lactoglubulins. They were identified as minor allergens in mites (group 13) and cockraoches.

- Examples for mammalian allergenic lipocalins are Equ c 1 from horse, Bos d 2 from cattle, Can $f 1$ and Can $\mathrm{f} 2$ from dog, and Fel $d 4$ from cat.

\section{DPBB (DOUBLE-PSI BETA BARREL) SUPERFAMILY}

$\beta$-barrels are frequently found structural elements of proteins. Barrels of a given number of strands can have different strand connections, or topologies. One such topology consisting of a certain arrangement of six $\beta$-strands is called a double-psi $\beta$-barrel (32). Group 1 grass pollen allergens are found in the DPBB_1 family of the DPBB superfamily. They are most closely related to the $\beta$-expansins and - like them contain two domains. Domain 1, which assumes the six-stranded double-psi $\beta$-barrel topology, is distantly related to glycoside hydrolase family 45 proteins, and domain 2 , which consists of two stacked $\beta$-sheets with an immunoglobulin-like fold, is also called group 2 grass pollen allergen domain (33). Expansin activity is often associated with cell wall loosening activity in growing cells including penetration of pollen tubes through the stigma and style (34). Group 2 and 3 grass pollen allergens belong to the Pollen_allerg_1 family and are related to the domain 2 of $\beta$-expansins. As they lack the domain 1, it is assumed that these allergenic proteins evolved from a truncated copy of a $\beta$-expansin gene (Fig. 15) (33).

- Examples for group 1, grass pollen allergens are Lol $\mathrm{p} 1$ from rye grass, Phl $\mathrm{p} 1$ from timothy grass, and Poa $p 1$ from Kentucky bluegrass. Group 2/3 grass pollen allergens include Dac $g$ and Dac $g$ 3 from orchard grass, Lol p 2 and Lol $p 3$ from rye grass, and Phl p 2 and Phl p 3 from timothy grass.

\section{DATABASES}

1. WHO/IUIS allergen nomenclature database (http://www.allergen.org/)

Theallergen nomenclature database is a repository of allergens that underwent a submission and evaluation process and were accepted by a panel of experts in allergen characterization, structure and function. This is the only body officially able to assign allergen designations. The database contains links for each allergen to the nucleotide and protein databases of the NCBI (National Center for Biotechnology Information; http:// www.ncbi.nlm.nih.gov/), the UniProt database (http://www.uniprot.org/), and the Protein Databank (PDB) of macromolecular structures (http://www.rcsb.org/pdb/). 


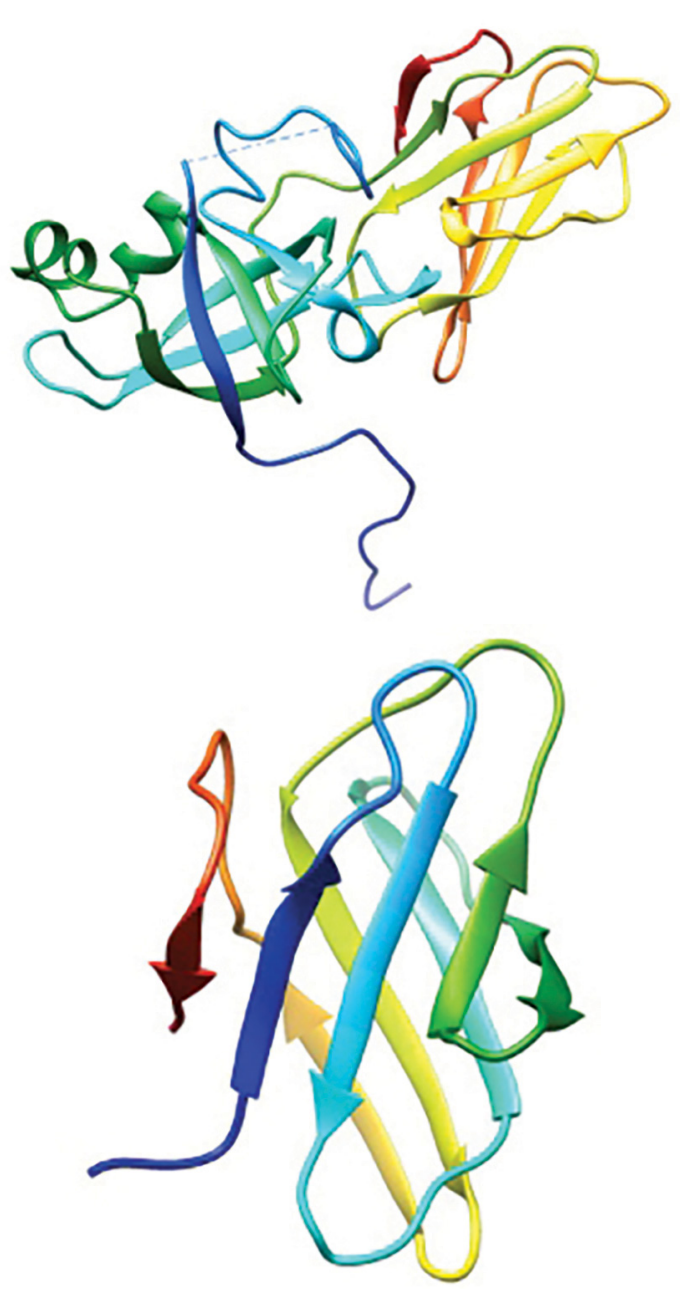

Figure 15

Phl p 1 and Phl 2 from timothy grass.

2. SDAP (structural database of allergenic proteins, https://fermi.utmb.edu/)

The SDAP is a repository of allergen sequences, IgE epitopes, structures and models. It offers various computational tools onsite that can assist structural biology studies related to allergens. The SDAP is curated by host scientists with oversight by an international review panel. The SDAP was developed based on the allergen list from the WHO/IUIS allergen nomenclature database.

3. AllFam (allergen protein families, http://www. meduniwien.ac.at/allergens/allfam/)

The AllFam database is a resource for classifying allergens into protein families. AllFam groups allergens according to the protein family classification from the Pfam database. AllFam is curated by the host scientists and provides a good overview on allergen families and their most important member proteins. A new version of the Allfam database will become available in June 2016.

4. AllergenOnline (http://www.allergenonline.org/) AllergenOnline provides access to a peerreviewed allergen list and sequence searchable database intended for the identification of proteins that may present a potential risk of allergenic cross-reactivity. The website was designed to help in assessing the safety of proteins that may be introduced into foods through genetic engineering or through food processing methods.

5. Allergome (http://www.allergome.org/)

Allergome a non peer-reviewed database has the most extensive collection of information on allergens. It is based on the literature published since the early sixties and is continuously updated. Allergome lists all allergens that have been identified and characterized in the literature including those that are not present on the WHO/IUIS allergen nomenclature website.

6. Pfam (http://pfam.xfam.org/)

Pfam is the most extensive database of manually curated protein families. The current release of Pfam, version 29.0, contains 16,295 families. The Pfam database was used to classify allergens into protein families.

\section{References}

1. Finn RD, Bateman A, Clements J, Coggill P, Eberhardt RY, Eddy SR et al. Pfam: the protein families database. Nucleic Acids Res 2014;42:D222-230.

2. Ruiter B, Shreffler WG. Innate immunostimulatory properties of allergens and their relevance to food allergy. Semin Immunopathol 2012;34:617-632.

3. Platts-Mills TA, Woodfolk JA. Allergens and their role in the allergic immune response. Immunol Rev 2011;242:51-68.

4. Wills-Karp M. Allergen-specific pattern recognition receptor pathways. Curr Opin Immunol 2010;22:777782.

5. Trompette A, Divanovic S, Visintin A, Blanchard C, Hegde RS, Madan R et al. Allergenicity resulting from functional mimicry of a Toll-like receptor complex protein. Nature 2009;457:585-588. 
6. Herre J, Grönlund H, Brooks H, Hopkins L, Waggoner L, Murton $\mathrm{B}$ et al. Allergens as immunomodulatory proteins: the cat dander protein Fel $\mathrm{d} 1$ enhances TLR activation by lipid ligands. J Immunol 2013;191:1529-1535.

7. Royer PJ, Emara M, Yang C, Al-Ghouleh A, Tighe $\mathrm{P}$, Jones $\mathrm{N}$ et al. The mannose receptor mediates the uptake of diverse native allergens by dendritic cells and determines allergen-induced $T$ cell polarization through modulation of IDO activity. $J$ Immunol 2010;185:1522-1531.

8. Hsu SC, Chen $\mathrm{CH}$, Tsai SH, Kawasaki $\mathrm{H}$, Hung $\mathrm{CH}$, Chu $\mathrm{YT}$ et al. Functional interaction of common allergens and a C-type lectin receptor, dendritic cell-specific ICAM3-grabbing non-integrin (DC-SIGN), on human dendritic cells. J Biol Chem 2010;285:7903-7910.

9. Shreffler WG, Castro RR, Kucuk ZY, Charlop-Powers Z, Grishina G, Yoo $S$ et al. The major glycoprotein allergen from Arachis hypogaea, Ara $\mathrm{h} 1$, is a ligand of dendritic cell-specific ICAM-grabbing nonintegrin and acts as a Th2 adjuvant in vitro. J Immunol 2006;177:3677-3685.

10. Sun G, Stacey MA, Schmidt M, Mori L, Mattoli S. Interaction of mite allergens Der p3 and Der p9 with protease-activated receptor-2 expressed by lung epithelial cells. J Immunol 2001;167:1014-1021.

11. Shewry PR, Halford NG, Belton PS, Tatham AS. The structure and properties of gluten: an elastic protein from wheat grain. Philos Trans $R$ Soc Lond B Biol Sci 2002;357:133-142.

12. Tatham AS, Shewry PR. Allergens to wheat and related cereals. Clin Exp Allergy 2008;38:1712-1726.

13. Oda Y, Matsunaga T, Fukuyama K, Miyazaki T, Morimoto T. Tertiary and quaternary structures of 0.19 alpha-amylase inhibitor from wheat kernel determined by X-ray analysis at $2.06 \mathrm{~A}$ resolution. Biochemistry 1997;36:13503-13511.

14. Salcedo G, Quirce S, Diaz-Perales A. Wheat allergens associated with Baker's asthma. J Investig Allergol Clin Immunol 2011;21:81-92.

15. Moreno FJ, Clemente A. 2S Albumin Storage Proteins: What Makes them Food Allergens? Open Biochem J 2008;2:16-28.

16. Egger M, Hauser M, Mari A, Ferreira F, Gadermaier G. The role of lipid transfer proteins in allergic diseases. Curr Allergy Asthma Rep 2010;10:326-335.

17. Lewit-Bentley A, Réty S. EF-hand calcium-binding proteins. Curr Opin Struct Biol 2000;10:637-643.

18. Magler I, Nuss D, Hauser M, Ferreira F, Brandstetter H. Molecular metamorphosis in polcalcin allergens by EF-hand rearrangements and domain swapping. FEBS J 2010;277:2598-2610.

19. Kuehn A, Swoboda I, Arumugam K, Hilger C, Hentges F. Fish allergens at a glance: variable allergenicity of parvalbumins, the major fish allergens. Front Immunol 2014;5:179.
20. Radauer C, Willerroider M, Fuchs H, HoffmannSommergruber K, Thalhamer J, Ferreira F et al. Crossreactiveandspecies-specificimmunoglobulinEepitopes of plant profilins: an experimental and structure-based analysis. Clin Exp Allergy 2006;36:920-929.

21. Hauser M, Roulias A, Ferreira F, Egger M. Panallergens and their impact on the allergic patient. Allergy Asthma Clin Immunol 2010;6:1.

22. Reese G, Ayuso R, Lehrer SB. Tropomyosin: an invertebrate pan-allergen. Int Arch Allergy Immunol 1999;119:247-258.

23. Lopata AL, O'Hehir RE, Lehrer SB. Shellfish allergy. Clin Exp Allergy 2010;40:850-858.

24. Radauer $\mathrm{C}$, Breiteneder H. Evolutionary biology of plant food allergens. J Allergy Clin Immunol 2007;120:518525.

25. Willison LN, Sathe SK, Roux KH. Production and analysis of recombinant tree nut allergens. Methods 2014;66:34-43.

26. Radauer $\mathrm{C}$, Lackner $\mathrm{P}$, Breiteneder $\mathrm{H}$. The Bet $\vee 1$ fold: an ancient, versatile scaffold for binding of large, hydrophobic ligands. BMC Evol Biol 2008;8:286.

27. D'Avino R, Bernardi ML, Wallner M, Palazzo $P$, Camardella L, Tuppo L et al. Kiwifruit Act d 11 is the first member of the ripening-related protein family identified as an allergen. Allergy 2011;66:870-877.

28. Guhsl EE, Hofstetter G, Hemmer W, Ebner C, Vieths $S$, Vogel $L$ et al. Vig $r$, the cytokinin-specific binding protein from mung bean (Vigna radiata) sprouts, crossreacts with Bet $\vee$ 1-related allergens and binds IgE from birch pollen allergic patients' sera. Mol Nutr Food Res 2014;58:625-634.

29. Chiu HJ, Bakolitsa C, Skerra A, Lomize A, Carlton D, Miller $\mathrm{MD}$ et al. Structure of the first representative of Pfam family PF09410 (DUF2006) reveals a structural signature of the calycin superfamily that suggests a role in lipid metabolism. Acta Crystallogr Sect F Struct Biol Cryst Commun 2010;66:1153-1159.

30. Virtanen T, Kinnunen T, Rytkonen-Nissinen $M$. Mammalian lipocalin allergens--insights into their enigmatic allergenicity. Clin Exp Allergy 2012;42:494504.

31. Hilger C, Kuehn A, Hentges F. Animal lipocalin allergens. Curr Allergy Asthma Rep 2012;12:438-447.

32. Castillo RM, Mizuguchi K, Dhanaraj V, Albert A, Blundell TL, Murzin AG. A six-stranded doublepsi beta barrel is shared by several protein superfamilies. Structure 1999;7:227-236.

33. Sampedro J, Cosgrove DJ. The expansin superfamily. Genome Biol 2005;6:242.

34. Cosgrove DJ, Bedinger P, Durachko DM. Group I allergens of grass pollen as cell wall-loosening agents. Proc Natl Acad Sci U S A 1997;94:6559-6564. 



\section{PART B}

USING MOLECULAR ALLERGOLOGY IN THE CLINICAL PRACTICE 



\section{TREE POLLEN ALLERGY}

\author{
Enrico Scala, Riccardo Asero, Verena \\ Niederberger
}

\section{THE ALLERGEN SOURCES}

Among over 400,000 plant species (1), about 100 flowering (Angiospermae) and non-flowering (Gymnospermae) trees can induce specific sensitization in predisposed individuals. Besides grass pollen and house dust mites, tree pollens belong to the most important respiratory allergen sources. The knowledge of the taxonomical relationship between different tree species allows the prediction of cross reactivity between closely related plants, which share homologous molecules not found in unrelated plants. The trees most commonly causing allergy belong to the orders Fagales (alder, beech, birch, hazelnut, oak), Lamiales (ash, privet, olive, lilac), Pinales (cypress, Japanese cedar, juniper), and Proteales (plane tree, sycamore) (2).

The geographical distribution of allergenic plants drives patients' sensitization profiles, as a consequence of different local pollen exposure. For instance in the Mediterranean area, as well as in regions with a Mediterranean climate such as North and South Africa, North and South America and Australia, trees belonging to the order Lamiales (i.e. olive tree) or Pinales (i.e. cypress tree) are mainly
Cup a 1 reactivity is the specific marker allergen for a sensitization to the Cupressaceae family.

PR-10 molecules (i.e. Bet $v$ 1) are the major allergens in Fagales pollen, often associated with an oral allergy syndrome.

Ole $e 1$ is the most common sensitizing molecule in olive pollen.

Pla a 1 and Pla a 2 may serve as a marker of primary sensitization to plane tree pollen. 
found, whilst Fagales trees play a role as allergen sources mostly in temperate climate regions such as Northern and Central Europe, North America, East Asia and Northwest Africa (3).

The order Fagales encompasses two main families most frequently implicated in allergies: (i) Betulaceae including the genera Alnus (alder), Betula (birch), Carpinus (hornbeam), Corylus (hazel), and Ostrya (hop hornbeam), and (ii) Fagaceae, comprising the genera Castanea (chestnut), Fagus (beech), and Quercus (oak) (Fig. 1). A high degree of allergenic cross-reactivity among allergens from these plants distributed all over the world has been demonstrated. Birch, followed by alder and hazel, represents the most potent cause of tree pollen allergy within this order. The flowering period of birch begins at the end of March in Western Europe, from the beginning to mid April in central and Eastern Europe and from late April to late May in northern Europe. From 1 to 3 weeks after the beginning of the season higher amounts of pollen in the atmosphere are recorded, and the extent of the pollen season is extremely dependent on weather conditions and thus ranges from 2 to 8 weeks. An alternation of low and high pollen production years has been detected. Hazel and alder florescence starts early from December to April, is followed by birch, hornbeam and hop hornbeam and then by oak and beech in spring. Chestnuts shed pollens in June and July in western and central Europe.

Trees from the family Oleaceae, order Lamilaes, grow on all 5 continents and are among the most important causes of respiratory allergy in the Mediterranean area as well as in some areas of North America, Australia, Japan and North and South Africa, where these trees are intensively cultivated (4). The Oleaceae family comprises 4 main genera: olive (Olea europea), European ash (Fraxinus excelsior), lilac (Syringa vulgaris), and common privet (Ligustrum vulgare), all able to cause IgE sensitization (5) (Fig. 1). The pollination period ranges from April to June in warmer regions, and the occurrence of olive tree allergy is about 30$40 \%$ in Italy and over $80 \%$ in southern Spain. Ash pollen season is during wintertime, rather similar to the birch pollen season, and is very relevant in central Europe (Austria, North and East of France, Switzerland up to $30 \%$ prevalence of pollen allergic patients).

In Mediterranean regions trees of the Cupressaceae family (Fig. 1) from the order Pinales are widely spread. Wind pollination of cypress trees occurs during the winter season, when no other allergenic plants flower, and accounts for up to $40 \%$ of the total pollen count in Mediterranean countries (160). Cypress tree inflorescence covers about 30-40 days, from January to April, showing a high variability from year to year, depending on weather conditions, causing difficulty in identifying the beginning and length of pollen season. The high degree of crossreactivity found among Cupressaceae trees (cypress, juniper and cedar), which have somewhat different but overlapping pollination periods, could extend the cypress pollen season from December to March.

Trees of the Plane-tree familty, from the order Proteales are common species widely spread in southern Europe, with a short but intense pollen season from March to April, characterized by high pollen counts, reaching one hundred billion pollen grains per tree only few days after the florescence time. Clinical surveys have acknowledged plane trees as a major cause of pollen with sensitization rates ranging from 8 to $17 \%$ in exposed populations. Annual airborne pollen counts differ on the basis of weather conditions but also as a function of human activity, mainly pruning, since plane trees or sycamores are widely used for ornamental purposes (7). Temperature, but not rainfall, is the weather parameter mainly affecting the Platanus pollen season, affecting both start-date and daily pollen counts.

\section{MAJOR AND RELEVANT MINOR ALLERGENIC MOLECULES}

Pollen from Fagales trees are one of the most frequent causes of winter/spring respiratory allergy in the temperate areas of the Northern hemisphere. This order includes two main families (Betulaceae and Fagaceae) comprising different trees characterized by a rather limited number of homologous, crossreacting allergens (8) (Table 1 ).

Pathogenesis-related-protein group 10 (PR-10) molecules (i.e., Bet v 1 and homologous allergens) (9) are the major allergens in Fagales pollen and are recognized by virtually all allergic patients, thus representing the major cause of clinical allergy (see also section C02). Several PR-10 family members have been described to date within tree pollen belonging to Fagales order ([i] Betulaceae: Aln $g 1$ from alder, Bet $v 1$ from birch, Car b 1 from hornbeam, Cas $\mathrm{s} 1$ from chestnut and Cor a 1 from hazel, [ii] Fagaceae: Fag s 1 from European beech, Ost c 1 from hop hornbeam, and Que a 1 from oak). 


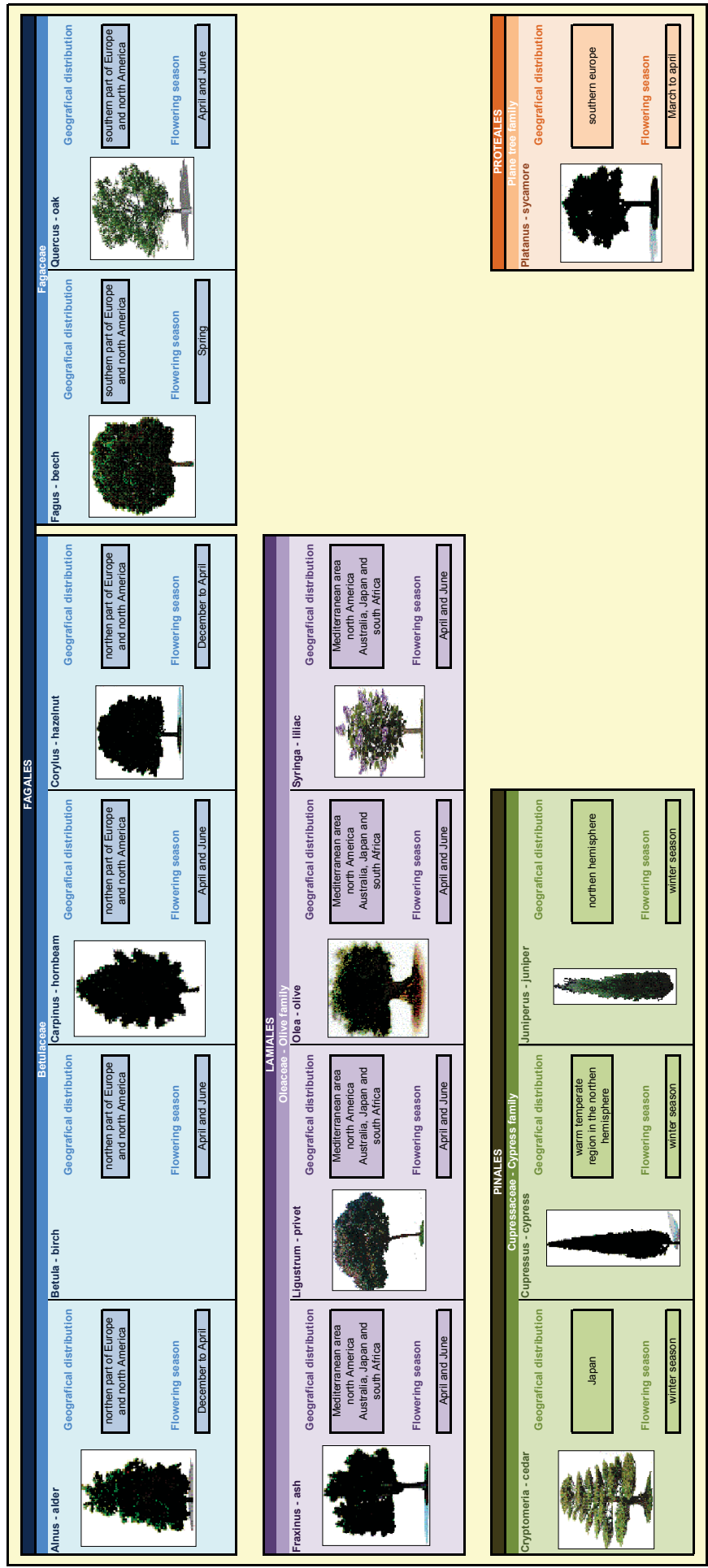

Figure 1

Tree pollen related allergen families. 
Table 1

Allergenic molecules of the two main families (Betulaceae and Fagaceae) of the order Fagales

\begin{tabular}{|c|c|c|c|c|c|}
\hline $\begin{array}{l}\text { Species } \\
\text { (English name) }\end{array}$ & $\begin{array}{l}\text { Allergenic } \\
\text { molecule }\end{array}$ & Biochemical name & $\begin{array}{l}\text { IUIS } \\
\text { code }\end{array}$ & $\begin{array}{c}\text { Prevalence } \\
\text { among patients }\end{array}$ & MW \\
\hline \multicolumn{6}{|l|}{ Betulaceae } \\
\hline \multirow{2}{*}{$\begin{array}{l}\text { Alnus glutinosa } \\
\text { (Alder) }\end{array}$} & Aln g 1 & PR-10, Bet v 1 family member & 19 & $100 \%$ & 18 \\
\hline & Alng 4 & Polcalcin & 20 & $18 \%$ & 6 \\
\hline \multirow{6}{*}{$\begin{array}{l}\text { Betula verrucosa } \\
\text { (Betula pendula) } \\
\text { (European white birch) }\end{array}$} & Bet v 1 & PR-10, Bet $v 1$ family member & 129 & $95 \%$ & 17 \\
\hline & Bet v 2 & Profilin & 130 & $22 \%$ & 15 \\
\hline & Bet v 3 & $\begin{array}{l}\text { "Polcalcin-like protein } \\
\text { (4 EF-hand)" }\end{array}$ & 131 & $10 \%$ & 24 \\
\hline & Bet v 4 & Polcalcin & 132 & $5 \%$ & 7 \\
\hline & Bet v 6 & $\begin{array}{l}\text { "Isoflavone reductase-like and } \\
\text { phenylcoumaran benzylic ether reductase" }\end{array}$ & 133 & $32 \%$ & 35 \\
\hline & Bet v 7 & Cyclophilin & 134 & $21 \%$ & 18 \\
\hline $\begin{array}{l}\text { Carpinus betulus } \\
\text { (Hornbeam) }\end{array}$ & Car b 1 & PR-10, Bet v 1 family member & 182 & & 17 \\
\hline \multirow{10}{*}{$\begin{array}{l}\text { Corylus avellana } \\
\text { (Hazel) }\end{array}$} & Cor a 1 & PR-10, Bet v 1 family member & 228 & $100 \%$ & 17 \\
\hline & Cor a 2 & Profilin & 234 & & 14 \\
\hline & Cor a 6 & Isoflavone reductase homologue & 778 & & 35 \\
\hline & Cor a 8 & Non-specific lipid transfer protein type 1 & 235 & & 9 \\
\hline & Cor a 9 & $11 S$ seed storage globulin (legumin-like) & 236 & $86 \%$ & 40 \\
\hline & Cor a 10 & Luminal binding protein & 229 & & 70 \\
\hline & Cor a 11 & 7S seed storage globulin (vicilin-like) & 230 & & 48 \\
\hline & Cor a 12 & 17 kDa oelosin & 231 & $63 \%$ & 17 \\
\hline & Cor a 13 & 14-16 kDa oleosin & 232 & $63 \%$ & 14 \\
\hline & Cor a 14 & $2 \mathrm{~S}$ albumin & 233 & $33 \%$ & 15 \\
\hline $\begin{array}{l}\text { Ostrya carpinifolia } \\
\text { (European hophornbeam) }\end{array}$ & Ost c 1 & PR-10, Bet $v 1$ family member & 697 & & \\
\hline \multicolumn{6}{|l|}{ Fagaceae } \\
\hline \multirow{4}{*}{$\begin{array}{l}\text { Castanea sativa } \\
\text { (Chestnut) }\end{array}$} & Cas $\mathrm{s} 1$ & PR-10, Bet $v 1$ family member & 185 & $100 \%$ & 22 \\
\hline & Cas s 5 & Chitinase & 186 & & \\
\hline & Cas s 8 & Non-specific lipid transfer protein type 1 & 187 & & 12 \\
\hline & Cas s 9 & Cytosolic class I small heat shock protein & 666 & & 17 \\
\hline $\begin{array}{l}\text { Fagus sylvatica } \\
\text { (European beech) }\end{array}$ & Fag s 1 & PR-10, Bet $v 1$ family member & 696 & & \\
\hline $\begin{array}{l}\text { Quercus alba } \\
\text { (White oak) }\end{array}$ & Que a 1 & PR-10, Bet $v 1$ family member & 566 & $64 \%$ & 17 \\
\hline
\end{tabular}




\section{Table 2}

Allergenic molecules of the olive family

\begin{tabular}{|c|c|c|c|c|c|}
\hline $\begin{array}{l}\text { Species } \\
\text { (English name) }\end{array}$ & $\begin{array}{l}\text { Allergenic } \\
\text { molecule }\end{array}$ & Biochemical name & $\begin{array}{l}\text { IUIS } \\
\text { code }\end{array}$ & $\begin{array}{c}\text { Prevalence } \\
\text { among patients }\end{array}$ & MW \\
\hline \multicolumn{6}{|l|}{ Oleaceae } \\
\hline $\begin{array}{l}\text { Fraxinus excelsior } \\
\text { (Ash) }\end{array}$ & Fra e 1 & Ole e 1-like protein family member & 331 & $87 \%$ & 20 \\
\hline $\begin{array}{l}\text { Ligustrum vulgare } \\
\text { (Privet) }\end{array}$ & Lig v 1 & Ole e 1-like protein family member & 409 & $58 \%$ & 20 \\
\hline \multirow{12}{*}{$\begin{array}{l}\text { Olea europaea } \\
\text { (Olive) }\end{array}$} & Ole e 1 & Common olive group 1 & 454 & $90 \%$ & 16 \\
\hline & Ole e 2 & Profilin & 456 & $50 \%$ & 15 \\
\hline & Ole e 3 & Polcalcin-like protein (4 EF-hands) & 457 & & 9 \\
\hline & Ole e 4 & N.A. & 458 & $80 \%$ & 32 \\
\hline & Ole e 5 & Superoxide dismutase [Cu-Zn] & 459 & $35 \%$ & 16 \\
\hline & Ole e 6 & & 460 & $15 \%$ & 10 \\
\hline & Ole e 7 & putative non-specific lipid transfer protein & 461 & $47 \%$ & 9 \\
\hline & Ole e 8 & $\begin{array}{l}\text { "Polcalcin-like protein } \\
\text { (4 EF-hands)" }\end{array}$ & 462 & & 21 \\
\hline & Ole e 9 & 1 3-beta glucanase & 463 & $68 \%$ & 46 \\
\hline & Ole e 10 & X8 domain containing protein & 455 & $90 \%$ & 11 \\
\hline & Ole e 11 & Pectin methylesterase & 685 & & 39.4 \\
\hline & Ole e 12 & Isoflavone reductase & & $4-33 \%$ & 36 \\
\hline $\begin{array}{l}\text { Syringa vulgaris } \\
\text { (Lilac) }\end{array}$ & Syr v 1 & Ole e 1-like protein family member & 612 & $90 \%$ & 20 \\
\hline
\end{tabular}

In addition to PR-10 proteins, several other allergens have been described. (i) Profilins (e.g. Bet $v 2$ from birch pollen or Cor a 2 from hazel pollen) (10) are plantpan allergens (see section C01) present in the whole plant kingdom. Profilins are recognized by $10-20 \%$ of patients primarily sensitized to birch pollen, but this proportion is higher in areas where grass pollen represents the primary sensitizer. Clinical relevance of profilin as a respiratory allergen is variable (11). Profilins may cause secondary plant food allergy to various fruits and vegetables (see section C01).

(ii) Polcalcin-like proteins (calcium binding proteins; e.g. Bet $v 3$ and Bet $v 4$ from birch, and Aln $g 4$ from alder) are pollen pan-allergens, which generally sensitize less than $10 \%$ of pollen-allergic individuals. They cross-react with homologous allergens from pollen from botanically unrelated species. The clinical relevance is variable and often limited (165); (iii) phenyl-coumaran benzylic ether reductased and isoflavone reductases (e.g. Bet v 6 from birch, Cor a 6 from hazel, Ole e 12 from olive) are minor allergens which are involved in plant defence reactions, showing a sensitization rate of about $32 \%$ among birch allergic people (12); (iv) cyclophilin (Bet $v 7$ from birch) is a minor, potentially cross-reactive, allergen; (v) pectin methylesterase (Bet $v 8$ from birch) (13); (vi) glucanase; (vii) thaumatin-like protein; and (viii) Glutathione-S-transferase (GST).

Olive pollen allergy is caused by Ole e 1 in the majority of cases (about 70\%), (Table 2). The Ole e 1-like protein family comprises several other allergenic glycosylated proteins from tree pollen (Fra e 1, Lig $\vee 1$, and Syr $\vee 1$ ), whose glycan moieties are involved in the allergenic properties of these molecules (14).

Besides Ole e 1, several other molecules have been identified, and a biological function can be associated 


\section{Table 3}

Allergenic molecules of the cypress family

\begin{tabular}{|c|c|c|c|c|c|}
\hline $\begin{array}{l}\text { Species } \\
\text { (English name) }\end{array}$ & $\begin{array}{l}\text { Allergenic } \\
\text { molecule }\end{array}$ & Biochemical name & $\begin{array}{l}\text { IUIS } \\
\text { code }\end{array}$ & $\begin{array}{l}\text { Prevalence } \\
\text { among patients }\end{array}$ & MW \\
\hline \multicolumn{6}{|l|}{ Cupressaceae } \\
\hline \multirow{2}{*}{$\begin{array}{l}\text { Chamaecyparis obtusa } \\
\text { (Japanese cypress) }\end{array}$} & Cha o 1 & Pectate lyase & 193 & $97.50 \%$ & 40.2 \\
\hline & Cha o 2 & Polygalacturonase & 194 & $82.50 \%$ & 45 \\
\hline \multirow{2}{*}{$\begin{array}{l}\text { Cryptomeria japonica } \\
\text { (Sugi) }\end{array}$} & Cry j 1 & Pectate lyase & 245 & $>90 \%$ & $41-45$ \\
\hline & Cry j 2 & Polygalacturonase & 246 & $>90 \%$ & 45 \\
\hline $\begin{array}{l}\text { Cupressus arizonica } \\
\text { (Cypress) }\end{array}$ & Cup a 1 & Pectate lyase & 253 & $100 \%$ & 43 \\
\hline \multirow{2}{*}{$\begin{array}{l}\text { Cupressus sempervirens } \\
\text { (Common cypress) }\end{array}$} & Cup s 1 & Pectate lyase & 254 & & 43 \\
\hline & Cup s 3 & Thaumatin-like protein & 255 & & 34 \\
\hline \multirow{3}{*}{$\begin{array}{l}\text { Juniperus ashei } \\
\text { (Mountain cedar) }\end{array}$} & Jun a 1 & Pectate lyase & 392 & $71.40 \%$ & 43 \\
\hline & Jun a 2 & Polygalacturonase & 393 & $100 \%$ & 43 \\
\hline & Jun a 3 & Thaumatin-like protein & 394 & 42.95 & 30 \\
\hline $\begin{array}{l}\text { Juniperus oxycedrus } \\
\text { (Prickly juniper) }\end{array}$ & Jun o 4 & $\begin{array}{l}\text { Polcalcin-like protein ( } 4 \mathrm{EF} \text { hand } \\
\text { domains) }\end{array}$ & 395 & 14.65 & 29 \\
\hline \multirow{2}{*}{$\begin{array}{l}\text { Juniperus virginiana } \\
\text { (Eastern red cedar) }\end{array}$} & Jun v 1 & Pectate lyase & 397 & 46.15 & 43 \\
\hline & Jun v 3 & Thaumatin-like protein & 398 & & 34 \\
\hline
\end{tabular}

with most of these molecules, such as actin-binding protein (the profilin Ole e 2), polcalcin (Ole e 3 and Ole e 8), glucanase (Ole e 9 and its probable degradation product Ole e 4), superoxide dismutase (Ole e 5) and lipid transfer protein (Ole e 7). Olive tree Ole e 7 , shares less than $20 \%$ of amino acid sequence with Pru $\mathrm{p}$ 3. Even though the homology at the primary sequence level is low, the tertiary structure of nsLTP is rather similar. Immunologically they seem to be distinct, which is also true for Par j 2, the nsLTP from pellitory that does not cross-react with e.g. Pru $p$ 3., Ole e 7 is suspected to be the possible cause of adverse reactions in patients undergoing allergen specific immunotherapy.

The glucanase Ole e 9, despite representing less than $0.3 \%$ of crude olive pollen content, induces sensitization in about $50 \%$ of patients in some Mediterranean regions with high olive pollen counts during pollen season. As previously described for Ole e 7, sensitized patients are at higher risk of suffering adverse side reactions during immunotherapy. Ole e 7 and Ole e 9 lgE recognition have been recently associated with local or systemic reactions to food and Atopic dermatitis, respectively
(15). Ole e 10 (X8 domain containing protein) and the pectin methylesterase Ole e 11 are two other major olive pollen allergens.

In the Cypress family two main groups of proteins have been identified: the pectate lyases and the polygalacturonases (16) (Table 3). The highly related, (95.1\% sequence identity) pectate lyases Cup a 1 (57) and Cup s 1 are found In the Mediterranean area, whilst Cry j 1 and Cha o 1 are mainly found in Japan, sharing $78.6 \%$ sequence identity. The polygalacturonases, Cha o 2, Cry j 2, and Jun a 2 are also major allergens of Pinales pollen, showing high levels sequence identities (71\%-82\%).

The most important allergen from London plane tree (Platanus acerifolia) pollen is Pla a 1 (17), which has an invertase inhibitor function as has the homologous Pla or 1 from Platanus orientalis (Table 4). Pla a 2 and Pla or 2 are major allergens displaying a polygalacturonase activity. The plane tree Pla a 3 belongs to the family of non-specific lipid transfer proteins, showing $58.3 \%$ sequence identity with the nsLTP Pru p 3 from peach (18). 


\section{Table 4}

Allergenic molecules of the plane-tree family

\begin{tabular}{|c|c|c|c|c|c|}
\hline $\begin{array}{l}\text { Species } \\
\text { (English name) }\end{array}$ & $\begin{array}{l}\text { Allergenic } \\
\text { molecule }\end{array}$ & Biochemical name & $\begin{array}{l}\text { IUIS } \\
\text { code }\end{array}$ & $\begin{array}{l}\text { Prevalence } \\
\text { among patients }\end{array}$ & MW \\
\hline \multicolumn{6}{|l|}{ Platanaceae } \\
\hline \multirow{3}{*}{$\begin{array}{l}\text { Platanus acerifolia } \\
\text { (London plane } \\
\text { tree) }\end{array}$} & Pla a 1 & Putative invertase inhibitor & 520 & $87.50 \%$ & 18 \\
\hline & Pla a 2 & Polygalacturonase & 521 & $83 \%$ & 43 \\
\hline & Pla a 3 & $\mathrm{~N}$ on-specific lipid transfer protein 1 & 522 & $45 \%$ & 10 \\
\hline \multirow{3}{*}{$\begin{array}{l}\text { Platanus orientalis } \\
\text { (Oriental plane) }\end{array}$} & Pla or 1 & Putative invertase inhibitor & 524 & $15.80 \%$ & 18 \\
\hline & Pla or 2 & Polygalacturonase & 525 & $26.30 \%$ & 42 \\
\hline & Pla or 3 & nsLTP1 & 526 & $26.30 \%$ & 11 \\
\hline
\end{tabular}

\section{SENSITIZATION TO INDIVIDUAL MOLECULES AND THEIR CLINICAL RELEVANCE}

\section{Epidemiology and sensitization/cross reactivity rates}

In Europe, the prevalence of positive skin prick test to birch pollen allergens ranges from $5 \%$ in The Netherlands to $54 \%$ in Switzerland, while Scandinavian countries have the highest number of patients with exclusive sensitization to Bet $v 1$ (19). Bet $v$ 1-specific IgE levels are not predictive for the development of pollen-related bronchial asthma. PR10 proteins defend plants against fungi and other microorganisms. Their homologs are also present in a large number of plant-derived foods, and thus frequentlycause cross-sensitization and consequently plant-food allergy (oral allergy syndrome, in most cases). For this reason, up to $70 \%$ of patients with sensitization to PR-10 proteins complain about oral symptoms following the ingestion of certain plantfoods (e.g., apples, carrots, hazel nuts and stone fruit, see section C02). No more than $38 \%$ identity is found comparing the sequences of PR-10 tree pollen members belonging to either the Betulaceae or the Fagaceae family (31 identical positions and 57 similar positions). When the sequences of PR-10 proteins from tree pollen of the Betulaceae and Fagaceae families were compared separately, the identity doubled up to $63.75 \%$ and $63.125 \%$, respectively. This indicates from a clinical point of view the need to check at least one representative allergen from both the Betulaceae family (i.e. Bet $v 1$ from birch) and the Fagaceae family (i.e. Que a 1 from oak) in all patients.

Also Olea europaea reactivity seems to be clinically characterized by rhino-conjunctivitis more than bronchial asthma. Moreover, in olive pollen patients a polysensitization is more common than monosensitization. Reactivity to other genera belonging to the Oleaceae family, i.e. Fraxinus excelsior or Ligustrum vulgare, is relevant in several regions in central and southern Europe (20). Olive tree Ole e 1 is a 145 amino acid protein sharing both significant sequence identity (82.76\% of identity with 120 Identical positions and 19 similar positions) and IgE cross-reactivity with all the other related trees belonging to the Olive family (Fra e 1 from ash, Lig $\vee 1$ from privet and Syr $v 1$ from lilac) (21). Several Ole e 1-like molecules have been described in goosefoot (Chenopodium album, Che a 1), timothy (Phleum pretense, $\mathrm{Phl} \mathrm{p} \mathrm{11)}$, rye-grass (Lolium perenne, Lol p 11), English plantain (Plantago lanceolata Pla I 1) and prickly saltwort (Salsola kali, Sal k 5), but the real clinical cross-reactivity of these molecules not belonging to the Olive family with Ole e 1 is somewhat questioned (20).

Patients allergic to Cypress pollen show a $30 \%$ sensitization rate in some areas (22) and $42 \%$ in central and southern Italy (23). Cypress pectate lyases allergy generally causes seasonal allergic rhinitis, and a very low occurrence of bronchial asthma in sensitized patients has been reported in the literature (24), but since asthma is a genetically determined condition, it depends more on the degree of sensitization and exposure rather than on the intrinsic characteristics of the allergens. High sequence identity and IgE cross- 

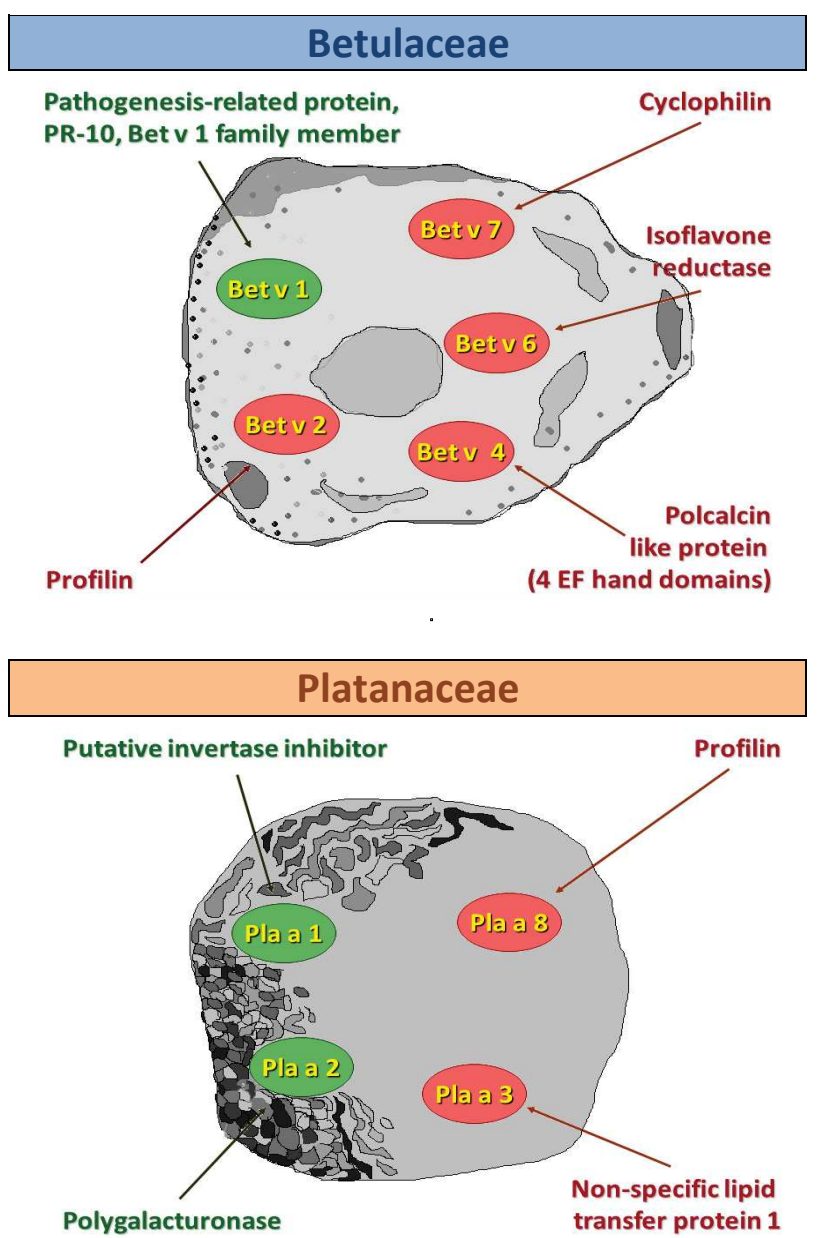
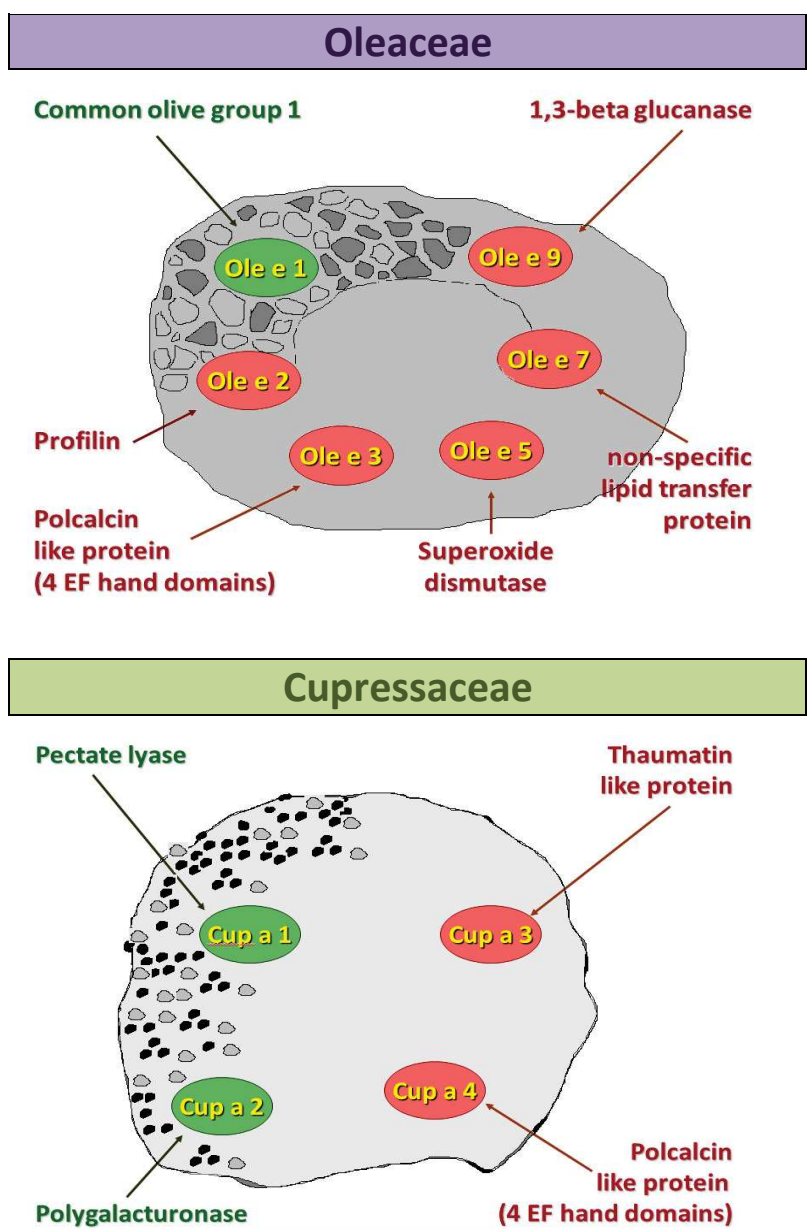

\section{Figure 2}

Genuine markers of sensitization (green) and panallergens (red) in the different pollens.

reactivity among the pectate lyases belonging to the Cupressaceae family (Cha o 1 , Cry j 1, Cup a 1, Cup $\mathrm{s} 1$, Jun a 1 , Jun c 1 , Jun o 1 and Jun $v 1$ ) is observed, with an overall $70 \%$ of similarity ( 262 identical and 74 similar amino acid positions).

A $50 \%$ sequence identity with the ragweed allergenic pectate lyase Amb a 1 without evidence of crossreactivity has been described (25).

In the case of Polygalacturonases an IgE cross reactivity among the homologous allergens belonging to Cupressaceae families (Cri y 2, Cha o 2, Cup a 2, Cup s 2 and Jun a 2) has been observed (26). Polygalacturonase belonging to timothy grass ( $\mathrm{Phl} \mathrm{p} \mathrm{13}$ ) showed also considerable (up to $40 \%$ ) sequence identity with Cri j 2 , without measurable cross-reactivity (27).
Plane tree Pla a 1 has a $98 \%$ sequence identity with Pla or 1. Pla a 2 and Pla or 2 are other major allergens displaying a polygalacturonase activity with only $35 \%$ of sequence identity and no cross reactivity with Cryptomera japonica Cry j 2. The plane tree Pla a 3 exhibits approximately $50 \%$ sequence identity with peach Pru $\mathrm{p} 3$, and thus is possibly implicated in plant food-pollen co-sensitization, although this role has been recently questioned (18).

\section{Clinical relevance and clinical patterns}

Certain proteins are restricted to a given allergenic biological source, and therefore can be considered as a "marker allergens" or the genuine "signature" clinically useful for the identification of patients for whom immunotherapy with a given allergen extract 


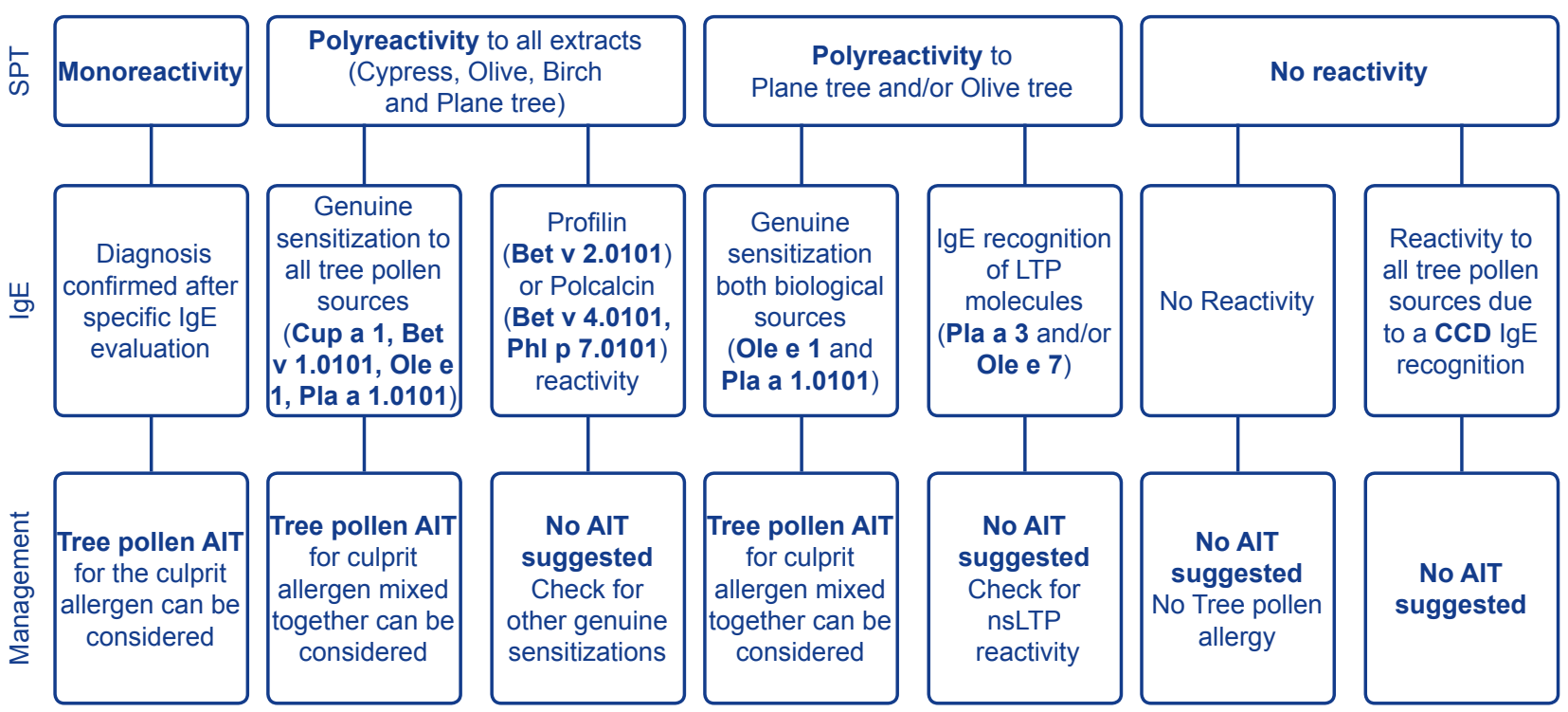

Figure 3

Algorithms to complete the diagnostic work-up of tree pollen allergic patients.

\section{Textbox 1}

Clinical relevance

- Molecular allergens markers of genuine reactivity: Cry j 1 (pectate lyase); Cup a 1 (pectate lyase); Aln g 1.0101 (PR-10 protein); Bet v 1.0101 (PR-10 protein); Cor a 1.0101 (PR-10 protein); Ole e 1 (common olive group 1); Ole e 9.0101 (beta-1,3-glucanase); Pla a 1.0101 (putative invertase inhibitor); Pla a 2 (polygalacturonase)

- Panallergens: Profilin (Bet $v$ 2.0101), Polcalcin (Bet v 4.0101) and nsLTP (Ole e 7 and Pla a 3.0101). Check also bromelain (Ana c 2) or the purified $\mathrm{N}$-glycan from bromelain MUXF3 in the case of multiple tree pollen IgE reactivity, to rule out the possibility of CCD reactivity.

- Tree pollen allergy is associated with a low incidence of respiratory symptoms and asthma.

is appropriate. For instance, in the tree pollen model, the major birch pollen, Bet $\mathbf{v}$, can identify individuals allergic to the Betulaceae family. The olive tree major allergen, Ole e 1, detects sensitization to the Oleaceae family, the cypress pollen major allergen, Cup a 1, reveals sensitization to the Cupressaceae family, and Pla a 1 detects sensitization to the Platanaceae family.
Other allergens exhibit a large cross-reactivity and their distribution is not restricted to a given taxonomical order, but they are rather distributed throughout the entire plant kingdom and are therefore found in all tree pollen families (the socalled panallergens). Polcalcin-like proteins (4 EFhand) and the profilins are typical examples of panallergens. In the case of polcalcin-like proteins, also known as EF-hand calcium-binding allergens (i.e. alder Aln g 4, hornbeam Car b 4, birch Bet v 4, beech Fag s 4, and oak Que a 4), IgE recognition is often associated with a multiple pollen (grass, weed and tree) sensitization (28) and a lower response to immunotherapy. Patients sensitized to profilins (e.g., Aln g 2 from alder, Bet $\vee 2$ from birch, Car b 2 from hornbeam, Cas s 2 from chestnut, Cor a 2 from hazel, Fag s 2 from beech and Que a 2 from oak) are not only reactors to a panallergen found in distinct sources but also true plant food multi-sensitized patients (29). Panallergens reactivity could therefore bring to a misleading interpretation if allergy testing is carried out only using allergenic extracts. A given patient could have positive extract-based tests to several tree pollen extracts, due to IgE recognition of both genuine and/or panallergens, or as a result of an IgE recognition of panallergens in the absence of a genuine reactivity to the marker allergens. Despite the high sequence identity observed among 
constituents of every single group of panallergens, testing of several panallergens could possibly increase assay reliability and the identification of interesting clinical phenotypes (30), albeit in daily clinical practice a less expensive approach may often be necessary (31). IgE reactivity due to cross-reacting carbohydrate determinants (CCDs) should also be ruled out, since all plant extracts can be weakly recognized by patients' IgE specific for CCDs, with no clinical significance (32).

In Fig. 2, genuine markers of sensitization are indicated in green and panallergens found in the different pollens are colored in red.

\section{CLINICAL MANAGEMENT}

Allergen specific Immunotherapy (AIT) should be prescribed only when clinical relevance of a given allergen source has been reliably demonstrated (33). In the presence of a multiple lgE-sensitization the first goal is to distinguish patients genuinely reactive from those misrecognizing a given biological source due to a reactivity to polcalcin, profilin or nsLTPs. Another difficulty in identifying the primary sensitizing source occurs in several countries (i.e. southern Europe) where an overlapping of tree, weed or grass pollination periods takes place. Several molecules have been proposed as markers for the prediction of a better response to AIT: Ole e 1, Cup a 1, Bet v 1, Cor a 1 or Pla a 1 reactivity can be considered as specific signatures for a genuine tree pollen allergy.

Cup a 1 reactivity is the specific marker allergen for a sensitization to pollen of trees of the Cupressaceae family. Also in this case the high sequence identity, associated to a high degree of cross-reactivity among Cupressaceae family members, suggest the use of Cup a 1 as a representative marker of the entire family for both diagnostic testing and therapeutic approaches (Fig. 1 and 2).

Bet $\mathbf{v} 1$ sensitized individuals often experience an oral allergy syndrome due to the intake of food containing PR-10 proteins. It has been suggested that birch pollen AIT can improve not only pollenrelated respiratory symptoms but also-food related adverse reactions (34), but different outcomes without benefit are reported in other studies (35) (see section C02).

Ole e 1 is the most common sensitizing molecule in olive pollen. It is utilized in both diagnostic and therapeutic extracts for standardization purposes and can determine immunological changes after olive pollen AIT. On the other hand, due to the high degree of cross-reactivity among the Ole e 1-like proteins of the Oleaceae family, in olive-free areas, Ole e 1 reactivity could help to identify individuals reacting with ash or privet pollen as suitable for AIT (36). In areas with heavy olive pollen exposure, Ole e 7 and Ole e 9 should be tested to identify patients with a more severe allergic phenotype (15).

Pla a 1 and Pla a 2 may serve as a marker of primary sensitization to plane tree pollen, therefore useful for AIT selection, whilst the nsLTP Pla a 3 has been linked with sensitization to plant-food LTPs (37).

Profilin and polcalcin represent the major causes of cross-reactivity due to their highly conserved structure and ubiquitous distribution (38). Profilin or polcalcin-reactors score positive to all tree pollen after an extract based diagnostic testing (29). Several allergens that are currently available for routine testing (profilins from birch, Bet $\vee 2$, and grass, Phl $\mathrm{p} 12$, and polcalcins from birch, rBet $\vee 4$, and grass, $\mathrm{rPhl} \mathrm{p} \mathrm{7)}$, are marker molecules for the entire group of panallergens, excluding cypress and Parietaria profilins (39). IgE-sensitization to panallergens, despite the ability to induce symptoms in sensitized patients, could affect AIT efficacy in the absence of species-specific molecules reactivity (33) (Fig. 3). Interestingly, panallergens reactors in the vast majority of cases are also co-sensitized to speciesspecific genuine molecules from different pollen (23), thus ideally requiring a multiple pollen AIT to be successful.

Cross-reactive carbohydrate determinants (CCDs) do not behave as allergens in vivo and are therefore clinically insignificant (40), but the presence of IgE to CCDs could lead to a misleading in vitro reactivity also in the case of extract-based testing orwhen using CCD-containing natural purified glycoproteins such as nCyn d 1, nOle e 1, nCup a 1, nSal k 1, nPla a 2 or nArt v 1 (41). Recombinant proteins produced in Escherichia coli bacteria are not affected by CCD recognition, because of the lack of the posttranslational glycosylation of proteins (42). Bromelain (Ana c 2) or the purified $\mathrm{N}$-glycan from bromelain MUXF3 are available to detect CCDs in vitro in the vast majority of pollen sources, with the exception of nArt $\vee 1$ where CCD reactivity is driven by $\mathrm{O}$-glycans and not $\mathrm{N}$-glycans measurable with bromelain or MUXF3. A positive skin prick test 
or in vivo (i.e. nasal, or conjunctival) challenge with the biological source can prove the genuine protein epitopes IgE recognition (40). In Fig. 3 we suggest several algorithms possibly useful to complete the diagnostic work-up in tree pollen allergic patients.

\section{CLINICAL CASES}

\section{Case 1 (original)}

Clinical History: Austria, patient (female, 27y) suffers from allergic rhinitis and conjunctivitis in April, the flowering period of birch and ash. Oral allergy syndrome does not occur.

Test with extracts (A) SPT: A skin prick test is not possible because the patient has dermographism; (B) in-vitro testing: Birch: 8 kUA/L; Ash: 2 kUA/L

Test with molecules: Bet $\vee$ 1: $7 \mathrm{kUA} / \mathrm{L}$; Bet $\vee 2: 3$ kUA/L; Bet v 4: negative; Ole e $1^{*}$ : negative

*Ole e 1 is used here to substitute for Fra e 1, which is highly cross-reactive with Ole e 1 and is not available in the CAP system.

Conclusion: The serology clearly identifies the patient as genuinely sensitized to birch; elevated IgE to ash is caused by cross-reactive panallergens. Immunotherapy with birch pollen extract is possible because the patient is mainly sensitized to the major allergen Bet $v 1$ and should be performed if symptomatic treatment does not sufficiently reduce the symptoms of allergy.

\section{Case 2 (original)}

Clinical History: Male, Italy, born 1994. Patient suffering from a perennial allergic rhinitis. Because of concurrent antihistaminic therapy, patient underwent a routine specific extract IgE evaluation.

Test with extracts: (A) In-vitro testing: birch: 25 kUA/L; olive tree: $18 \mathrm{kUA} / \mathrm{L}$; plane tree; $14 \mathrm{kUA} / \mathrm{L}$; Cypress: $30 \mathrm{kUA} / \mathrm{L}$; D. pteronyssinus: $44 \mathrm{kUA} / \mathrm{L}$. (B) SPT: After discontinuation of antihistaminic therapy, the patient went through cutaneous allergic evaluation that gave negative results for all the four tree pollen tested and mono-reactivity to $D$. pteronyssinus and $D$. farinae.

Test with molecules: ImmunoCAP ISAC: Der p 2: 35 ISU-E; Der f 2: 42 ISU-E; Lep d 2: 2.3 ISU-E; rBet v 1: Negative; nCry j 1: 1.2 ISU-E; nCup a 1: 3.3
ISU-E; nOle e 1: 2.8 ISU-E; rPla a 1: Negative; nPla a 2: 4 ISU-E; rPla a 3: Negative; MUXF3: 18 ISU-E

Conclusion: The serology clearly identifies the patient as genuinely sensitized only to house dust mite. The presence of MUXF3 reactivity indicates a CCD recognition further confirmed by the reactivity to native tree pollen molecules (Ole e 1 , Pla a 2, Cup a 1 and Cry j 1) in the presence of negative result considering recombinant (not CCD bringing) molecules (rBet v 1, rPla a 1, rPla a 3) and in the absence of skin test reactivity to tree pollen extracts (CCD IgE reactivity is never followed by a positive skin test result).

\section{Case 3 (original)}

Clinical History: Female, Italy, born 1973. Patient has been suffering from a seasonal allergic rhinitis and asthma since 2000. After ingestion of 2 walnuts, anaphylactic reaction (abdominal pain, dyspnea, generalized flushing and swelling, low blood pressure), subsequent emergency treatment and hospitalization overnight. No food allergy to peach or other food item known so far.

Test with extracts: (A) SPT: Environmental allergens: Pellitory (Parietaria j): $9 \mathrm{~mm} \times 5 \mathrm{~mm}$; plane tree (Platanus a.): $6 \mathrm{~mm} \times 7 \mathrm{~mm}$; olive tree (Olea e): $7 \mathrm{~mm} \times 10 \mathrm{~mm}$; Mugwort (Artemisia v.): $3 \mathrm{~mm} \times 4 \mathrm{~mm}$. Food allergens: all negative except walnut (Juglans regia nut): $15 \mathrm{~mm} \times 10 \mathrm{~mm}$ (peach negative). (B) Invitro testing: (2014) Total IgE $49.3 \mathrm{kU} / \mathrm{l}$, specific IgE to pellitory (Parietaria j): $2.5 \mathrm{kUA} / \mathrm{l}$; plane tree (Platanus a.): $0.56 \mathrm{kUA} / \mathrm{l}$; Olive tree (olea e): 0.78 kUA/l; Mugwort (Artemisia v.): 0.42 kUA/l; Walnut $3.82 \mathrm{kUA} / \mathrm{l}$; rPru p 3: $0.76 \mathrm{kUA} / \mathrm{l}$

Test with molecules: (ImmunoCAP ISAC): Par j 2: 1.56 ISU-E; Jug r 3: 1.15 ISU-E; Ole e 7: 9.82 ISU-E; Pla a 3: 1.98 ISU-E, (Pru p 3: negative)

Conclusion: Strict avoidance of walnut. AIT prescribed only for Pellitory.

\section{References}

1. Scutt CP, Vandenbussche M. Current trends and future directions in flower development research. Ann Bot 2014;114:1399-1406.

2. Asam $C$, Hofer $H$, Wolf $M$, Aglas L, Wallner $M$. Tree pollen allergens-an update from a molecular perspective. Allergy 2015;70:1201-1211.

3. Moverare R, Westritschnig K, Svensson M, Hayek B, Bende M, Pauli $G$ et al. Different IgE reactivity profiles in birch pollen-sensitive patients from six European 
populations revealed by recombinant allergens: an Imprint of local sensitization. Int Arch Allergy Immunol 2002;128:325-335.

4. Kirmaz C, Yuksel H, Bayrak P, Yilmaz O. Symptoms of the olive pollen allergy: do they really occur only in the pollination season? J Investig Allergol Clin Immunol 2005;15:140-145.

5. Quiralte J, Florido F, Arias De Saavedra JM et al. Olive allergen-specific IgE responses in patients with Olea europaea pollinosis. Allergy 2002;57(Suppl 71):4752.

6. D'Amato G, Cecchi L, Bonini S, Nunes C, AnnesiMaesano I, Behrendt $\mathrm{H}$ et al. Allergenic pollen and pollen allergy in Europe. Allergy 2007;62:976-990.

7. Alcazar P, Carinanos P, De Castro C, Guerra F, Moreno C, Dominguez-Vilches E et al. Airborne plane-tree (Platanus hispanica) pollen distribution in the city of Córdoba, South-western Spain, and possible implications on pollen allergy. J Investig Allergol Clin Immunol 2004;14:238-243.

8. Niederberger V, Pauli G, Gronlund H, Froschl R, Rumpold $\mathrm{H}$, Kraft $\mathrm{D}$ et al. Recombinant birch pollen allergens ( $r$ Bet $\vee 1$ and $r$ Bet $\vee 2$ ) contain most of the IgE epitopes present in birch, alder, hornbeam, hazel, and oak pollen: a quantitative IgE inhibition study with sera from different populations. J Allergy Clin Immunol 1998;102:579-591.

9. Breiteneder $\mathrm{H}$, Hassfeld $\mathrm{W}$, Pettenburger $\mathrm{K}$, Jarolim $\mathrm{E}$, Breitenbach $\mathrm{M}$, Rumpold $\mathrm{H}$ et al. Isolation and characterization of messenger RNA from male inflorescences and pollen of the white birch (Betula verrucosa). Int Arch Allergy Appl Immunol 1988;87:19-24.

10. Valenta R, Duchene M, Vrtala S, Valent P, Sillaber C, Ferreira $\mathrm{F}$ et al. Profilin, a novel plant pan-allergen. Int Arch Allergy Immunol 1992;99:271-273.

11. Asero R, Jimeno L, Barber D. Preliminary results of a skin prick test-based study of the prevalence and clinical impact of hypersensitivity to pollen panallergens (polcalcin and profilin). J Investig Allergol Clin Immunol 2010;20:35-38.

12. Karamloo F, Schmitz N, Scheurer S, Foetisch K, Hoffmann A, Haustein D et al. Molecular cloning and characterization of a birch pollen minor allergen, Bet v 5 , belonging to a family of isoflavone reductase- related proteins. J Allergy Clin Immunol 1999;104:991-999.

13. Mahler V, Fischer S, Heiss S, Duchêne M, Kraft D, Valenta R. cDna cloning and characterization of a crossreactive birch pollen allergen: identification as a pectin esterase. Int Arch Allergy Immunol 2001;124:64-66.

14. Rodríguez R, Villalba M, Monsalve RI, Batanero E. The spectrum of olive pollen allergens. Int Arch Allergy Immunol 2001;125:185-195.

15. Scala E, Abeni D, Pomponi D, Paganelli R, Locanto $M$, Giani $\mathrm{M}$ et al. Ole e 1, Ole e 7 and Ole e 9: identifying distinct clinical subsets of Olive tree-allergic patients. J Allergy Clin Immunol 2016;137:629-631.

16. Di Felice G, Barletta B, Tinghino R, Pini C. Cupressaceae pollinosis: identification, purification and cloning of relevant allergens. Int Arch Allergy Immunol 2001;125:280-289.

17. Asturias JA, Ibarrola I, Bartolomé B, Ojeda I, Malet A, Martínez A. Purification and characterization of Pla a 1, a major allergen from Platanus acerifolia pollen. Allergy 2002;57:221-227.

18. Scala E, Till SJ, Asero R, Abeni D, Guerra EC, Pirrotta $L$ et al. Lipid transfer protein sensitization: reactivity profiles and clinical risk assessment in an Italian cohort. Allergy 2015;70:933-943.

19. D'Amato G, Spieksma FT, Liccardi G, Jager S, Russo $\mathrm{M}$, Kontou-Fili $\mathrm{K}$ et al. Pollen-related allergy in Europe. Allergy 1998;53:567-578.

20. Asero R. Analysis of hypersensitivity to oleaceae pollen in an olive-free and ash-free area by commercial pollen extracts and recombinant allergens. Eur Ann Allergy Clin Immunol 2011;43:77-80.

21. Niederberger $V$, Purohit $A$, Oster JP, Spitzauer $S$, Valenta $\mathrm{R}$, Pauli G. The allergen profile of ash (Fraxinus excelsior) pollen: cross- reactivity with allergens from various plant species. Clin Exp Allergy 2002;32:933-941.

22. Charpin D, Calleja M, Lahoz C, Pichot C, Waisel Y. Allergy to cypress pollen. Allergy 2005;60:293-301.

23. Scala E, Alessandri C, Bernardi ML, Ferrara R, Palazzo $\mathrm{P}$, Pomponi $\mathrm{D}$ et al. Cross-sectional survey on immunoglobulin E reactivity in 23,077 subjects using an allergenic molecule-based microarray detection system. Clin Exp Allergy 2010;40:911-921.

24. Agea E, Bistoni O, Russano A et al. The biology of cypress allergy. Allergy 2002;57:959-960.

25. Ace Aceituno E, del Pozo V, Minguez A, Arrieta I, Cortegano I, Cardaba B et al. Molecular cloning of major allergen from Cupressus arizonica pollen: Cup a 1. Clin Exp Allergy 2000;30:1750-1758.

26. Asam C, Hofer $H$, Wolf M, Aglas L, Wallner M. Tree pollen allergens-an update from a molecular perspective. Allergy 2015;70:1201-1211.

27. Swoboda I, Grote M, Verdino P, Keller W, Singh MB, de Weerd $\mathrm{N}$ et al. Molecular characterization of polygalacturonases as grass pollen-specific marker allergens: expulsion from pollen via submicronic respirable particles. J Immunol 2004;172:6490-6500.

28. Kazemi-Shirazi L, Niederberger V, Linhart B, Lidholm $J$, Kraft D, Valenta R. Recombinant marker allergens: diagnostic gatekeepers for the treatment of allergy. Int Arch Allergy Immunol 2002;127:259-268.

29. Villalta $D$, Asero R. Analysis of the allergenic profile of patients hypersensitive to pollen pan-allergens living in two distinct areas of northern Italy. Eur Ann Allergy Clin Immunol 2011;43:54-57. 
30. Scala E, Alessandri C, Palazzo P, Pomponi D, Liso M, Bernardi ML et al. IgE recognition patterns of profilin, PR-10, and tropomyosin panallergens tested in 3,113 allergic patients by allergen microarray-based technology. Plos One 2011;6:e24912.

31. Villalta D, Asero R. Is the detection of IgE to multiple Bet $v$ 1-homologous food allergens by means of allergen microarray clinically useful? J Allergy Clin Immunol 2010;125:1158-1161.

32. Aalberse RC. Food allergens. Environ Toxicol Pharmacol 1997;4:55-60.

33. Douladiris N, Savvatianos S, Roumpedaki I, Skevaki C, Mitsias D, Papadopoulos NG. A molecular diagnostic algorithm to guide pollen immunotherapy in southern Europe: towards component-resolved management of allergic diseases. Int Arch Allergy Immunol 2013;162:163-172.

34. Asero R. Fennel, cucumber, and melon allergy successfully treated with pollen- specific injection immunotherapy. Ann Allergy Asthma Immunol 2000;84:460-462.

35. Kinaciyan T, Jahn-Schmid B, Radakovics A, Zwolfer B, Schreiber C, Francis JN et al. Successful sublingual immunotherapy with birch pollen has limited effects on concomitant food allergy to apple and the immune response to the Bet v 1 homolog Mal d 1. J Allergy Clin
Immunol 2007;119:937-943.

36. Rodriguez R, Villalba M, Batanero E, Palomares $O$, Quiralte J, Salamanca G et al. Olive pollen recombinant allergens: value in diagnosis and immunotherapy. $J$ Investig Allergol Clin Immunol 2007;17(Suppl 1):4-10.

37. Lauer I, Miguel-Moncin MS, Abel T, Foetisch K, Hartz C, Fortunato $\mathrm{D}$ et al. Identification of a plane pollen lipid transfer protein (Pla a 3) and its immunological relation to the peach lipid-transfer protein, Pru p 3. Clin Exp Allergy 2007;37:261-269.

38. Hauser M, Roulias A, Ferreira F, Egger M. Panallergens and their impact on the allergic patient. Allergy Asthma Clin Immunol 2010;6:1.

39. Asero R, Monsalve R, Barber D. Profilin sensitization detected in the office by skin prick test: a study of prevalence and clinical relevance of profilin as a plant food allergen. Clin Exp Allergy 2008;38:1033-1037.

40. Malandain H, Giroux F, Cano Y. The influence of carbohydrate structures present in common allergen sources on specific IgE results. Eur Ann Allergy Clin Immunol 2007;39:216-220.

41. Altmann F. The Role of Protein Glycosylation in Allergy. Int Arch Allergy Immunol 2007;142:99-115.

42. Demain AL, Vaishnav P. Production of recombinant proteins by microbes and higher organisms. Biotechnol Adv 2009;27:297-306. 



\title{
GRASS POLLEN ALLERGY
}

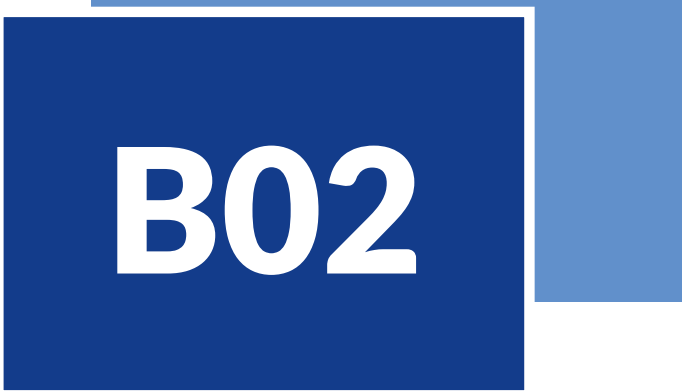

\author{
Janet M. Davies, Paolo M. Matricardi, \\ Johannes Schmid
}

\section{THE ALLERGEN SOURCES}

Grasses are ubiquitous throughout the entire world. In places with a temperate climate, members of the Pooideae subfamily (1) like Timothy grass (Phleum pratense), Orchard grass (Dactylus glomerata), Perennial ryegrass (Lolium perenne) and bluegrass (Poa pratensis) are the most common pollen sources. The pollen of this Pooideae subfamily shows extensive IgE crossreactivity. Grasses have a pollinating season from May to August in Central Europe, peaking in June. In Northern Europe, the grass pollen season starts later, while pollination lasts for a longer period in Mediterranean Europe. The grass pollen season overlaps with weed pollen (mugwort, ragweed) in most parts of Europe and with tree pollen (olive, plane) in Southern Europe. In subtropical and tropical regions of the world, grass pollen seasons can be perennial (2). Subtropical sources of allergenic grass pollens include those of the Panicoideae subfamily; Bahia grass (Paspalum notatum) and the prolific weed Johnson grass (Sorghum halepense), as well as the Chloridoideae subfamily; Bermuda grass (Cynodon dactylon) (3). Timothy grass is originally native to Europe and adjacent regions in Africa and Asia. It is widely cultivated throughout most temperate regions of the world for pasture and hay production. Despite
IgE to group 1 allergens (eg Phl $p$ 1) is a marker of true grass pollen sensitisation.

Whilst IgE to other major grass pollen allergens are rarely observed in the absence of lgE to $\mathrm{Phl} p 1$, lgE to $\mathrm{Phl} p 5$ or Phl $p 2$ can serve as a markers of true grass pollen sensitisation.

A number of allergen families exist in grass pollens, but lgE reactivity to $\mathrm{Phl} p 4, \mathrm{Phl} p 7, \mathrm{Phl} p 11$ or $\mathrm{Phl} 12$ may be due to cross-reactivity (CCD or panallergenicity).

Early onset of IgE sensitisation to grass pollen allergens, particularly $\mathrm{Phl} p 1$, and a high number of sensitisations (Phl p 5, 7 and 12) may be prognostic markers of disease progression but further studies are needed.

In temperate climates, patient serum IgE shows broad cross-reactivity between similar allergen components from different temperate grass pollens.

Group 1 allergens of subtropical grass pollens (Pas $n$ 1 , Sor $h 1$ and Cyn d 1) are more relevant allergens for patients in subtropical regions. 


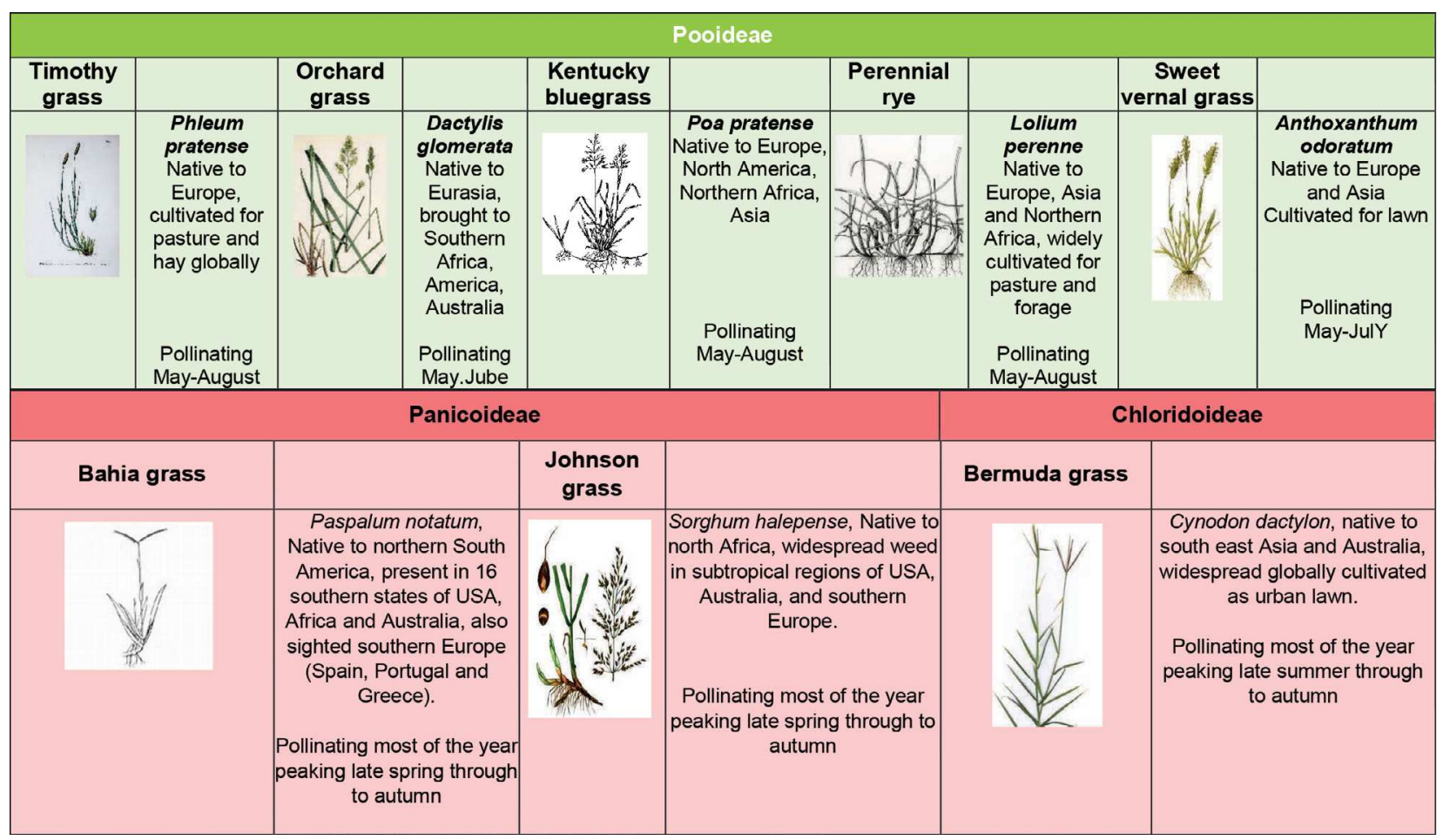

\section{Figure 1}

Clinically important examples of common temperate (Pooideae) and subtropical (Panicoideae and Chloridoideae) grass pollen allergen sources. Species origin, geographical distribution and peak pollinating periods in common regions are included. Timing of pollination of Pooideae species refers to the northern hemisphere.

of substantial geographical variations, grass pollen is the most prevalent sensitizing pollen in Europe with a median prevalence of $16.5 \%$ (4).

\section{MAJOR AND RELEVANT MINOR ALLERGENIC MOLECULES}

At present, nine allergenic molecules from Timothy grass pollen have been officially listed by the IUIS Allergen Nomenclature Sub-Committee. Phl p 2, p 5 and $\mathrm{p} 6$ are specific for grasses from the Pooideae subfamily. Orthologues of the group 1 grass pollen allergens represented by $\mathrm{Phl} \mathrm{p} 1$ of Timothy grass are present in all grass pollens thus being specific for grasses from the Poaceae family. Phl p 3 is another Pooideae specific allergen molecule with similarities to Phl p 2 (5) not yet listed in the IUIS list. Phl p 13, a polygalacturonase protein, is a grass pollen-specific allergen molecule as well.
Sensitization to $\mathrm{Phl} p 1$ usually precedes other grass pollen sensitizations and is the most prevalent component sensitization in grass pollen allergic patients (6). It is a useful marker for primary grass pollen sensitization. Phl p 1 is a beta-expansin, bound to the cell wall and important for pollen tube penetration. $\mathrm{Phl} \mathrm{p} 1$ is a major grass pollen allergen, with more than $80 \%$ homology to group 1 allergens from other members of the Pooideae subfamily (7). $\mathrm{Phl} \mathrm{p} 1$ shares epitopes with group 1 allergens from other grasses and shows IgE cross-reactivity to most other group 1 allergens from grasses, corns and monocots (8).

$\mathrm{Phl}$ p 5 is another major pollen allergen of temperate grasses with a lower sensitization prevalence, but often with high lgE-levels. Phl p 5 is a cytoplasmatic ribonuclease, important in the enzymatic degradation of RNA. It shows broad IgE cross reactivity with other group 5 allergens from the Pooideae subfamily of temperate grasses. 


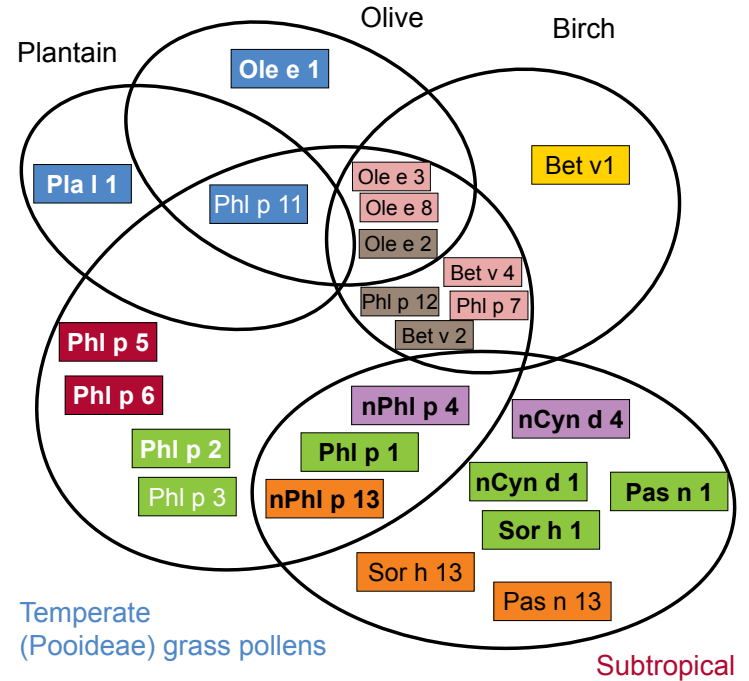

(Chloridoideae \& Panicoideae)

Figure 2

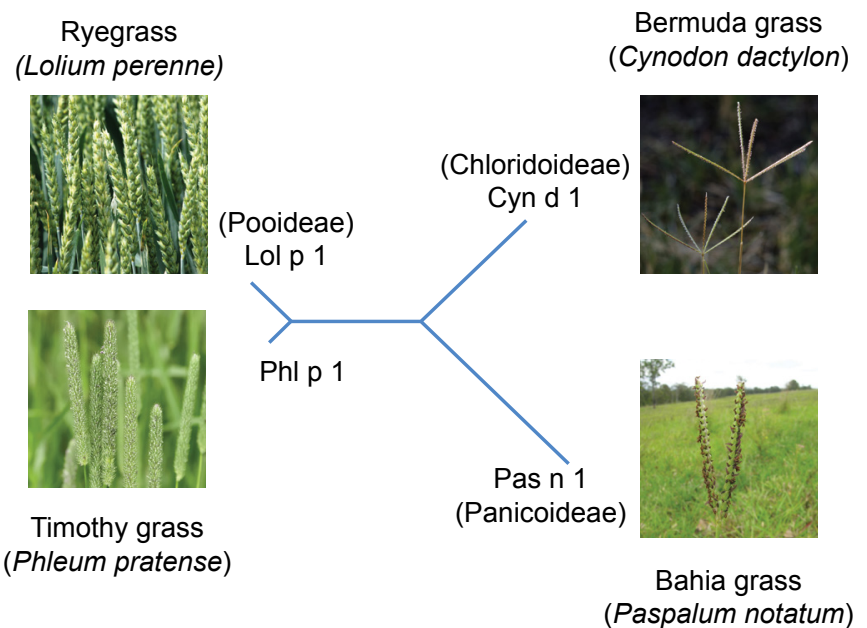

Left panel: A Venn diagram showing allergens of different sources that are similar to temperate and/or subtropical grass pollen allergens. Major allergens are in bold. Allergens of the same biochemical family are shown in boxes of the same color. Panallergens including profilins (brown boxes e.g. Phl p12) and polcalins (pink boxes e.g. Phl p 7) are in small font. Right panel: Relationship between the major group 1 allergen components of temperate (Pooideae) and subtropical grass pollens of Chloridoideae and Panicoideae subfamily (Unrooted phylogentic tree generated by multiple sequence alignment with ClustalW2 using sequences published in Davies et al., 2008 (20).

The $\mathrm{Phl} \mathrm{p} 2$ and $\mathrm{Phl} \mathrm{p} 3$ allergens are proteins with homology to the $C$ terminal domain of the betaexpansin protein family. They show substantial similarities and are specific for the Pooideae subfamily. Their biochemical function is not yet known.

$\mathrm{Phl} \mathrm{p} 6$ is another major grass pollen allergen, specific for the Pooideae subfamily. Its function has not yet been described.

$\mathrm{Phl} \mathrm{p} 13$ is a polygalactorunase which is a hydrolytic enzyme, degrading parts of the pectin network in plant cell walls. It is a major allergen, specific for the Pooideae subfamily.

$\mathrm{Phl}$ p 4 is a tryptase-resistant glycoprotein, berberine bridge enzyme, involved in the synthesis of alkaloids. It can be classified as a major allergen. It shows IgE cross reactivity with other group 4 grass pollen allergens, including with Cyn 4 to some extent. Moreover, cross-reactivity to the major ragweed allergen Amb a 1 and to Oilseed Rape pollen has been demonstrated. Natural Phl p 4 contains CCD, which may lead to IgE cross-reactivity with a wide range of plants and plant products.
$\mathrm{Phl} p 11$ belongs to the Ole e 1 related proteins and hence exhibits a broad range of cross-reactivities to pollen from different plants as olive, ash, privet, saffron crocus, thistle, plantain and corn. It is an acidic polypeptide with homology to the tryptase inhibitor of soybean.

$\mathrm{Phl} \mathrm{p} 7$ and Phl p 12 are minor allergens, representing pan-allergens from the plant world. Phl p 7, polcalcin, is a calcium binding protein present in many different pollens, hence representing a broad cross-reacting allergen: birch, alder, juniper, ragweed, mugwort, olive, goosefoot etc. Phl p 7 sensitization can be used as a marker for a more general pollen sensitization.

$\mathrm{Phl} \mathrm{p} 12$ is a member of the profilin family, an actinbinding protein that is present throughout the whole plant world. As profilins are ubiquitous in plant cells, profilin sensitization gives rise to a long range of cross-reacting plants and plant products as birch, soybean, corn, latex and plant foods.

Subtropical grass pollens of Bahia grass (Paspalum notatum) and Bermuda grass (Cynodon dactylon) show only limited IgE cross-reactivity with the pollens from the Pooideae subfamily (9). Patterns of IgE cross- 
Table 1

Allergenic molecules of timothy grass (205-207)

\begin{tabular}{|c|c|c|c|c|c|c|c|}
\hline Allergen & Allergenicity & Code & Biochemical name & Function & MW & $\begin{array}{l}\text { Iso- } \\
\text { forms }\end{array}$ & $\begin{array}{l}\text { Structure } \\
(14)\end{array}$ \\
\hline \multicolumn{8}{|c|}{ Phleum pratense (temperate grasses) } \\
\hline Phl p 1 & $83-95 \%$ & $\begin{array}{l}549- \\
551\end{array}$ & $\begin{array}{l}\text { CCD-bearing } \\
\text { protein }\end{array}$ & Beta-expansin & 27 & 2 & \\
\hline Phl p 2 & $55-65 \%$ & 555 & Grass group 2 & Unknown & $\begin{array}{l}10- \\
12\end{array}$ & 1 & \\
\hline Phl p 3 & $60 \%$ & 556 & Grass group 3 & unknown & & 2 & \\
\hline Phl p 4 & $70-75 \%$ & 557 & $\begin{array}{l}\text { CCD-bearing } \\
\text { protein }\end{array}$ & $\begin{array}{c}\text { Berberine-bridge } \\
\text { enzyme }\end{array}$ & 55 & 6 & \\
\hline Phl p 5 & $50-955$ & 558 & Grass group 5 & Ribonuclease & 32 & 16 & ans \\
\hline Phl p 6 & $44-75 \%$ & 569 & Grass group 6 & unknown & 11 & 2 & \\
\hline Phl p 7 & $7-10 \%$ & 570 & Polcalcin & $\begin{array}{l}\text { Calcium-binding } \\
\text { protein }\end{array}$ & 6 & 1 & 300 \\
\hline Phl p 11 & $32-43 \%$ & 552 & $\begin{array}{l}\text { Ole e 1-related } \\
\text { protein }\end{array}$ & Trypsin inhibitor & 20 & 1 & \\
\hline Phl p 12 & $15 \%$ & 553 & Profilin & $\begin{array}{l}\text { Actin-binding } \\
\text { protein }\end{array}$ & 14 & 3 & \\
\hline Phl p 13 & $50 \%$ & 554 & Grass group 13 & Polygalacturonase & 55 & 1 & \\
\hline \multicolumn{8}{|c|}{ Subtropical grasses } \\
\hline Cyn d 1 & $76-100 \%$ & Yes & Glycoprotein & Beta-expansin & 32 & 12 & \\
\hline Cyn d 4 & $100 \%$ & Yes & Glycoprotein & $\begin{array}{c}\text { Berberine bridge } \\
\text { enzyme }\end{array}$ & 60 & 1 & \\
\hline Pas n 1 & $85-92 \%$ & Yes & Glycoprotein & Beta-expansin & $\begin{array}{l}29- \\
30\end{array}$ & 2 & \\
\hline Pas n 13 & $48 \%$ & No & Glycoprotein & Polygalacturonase & 55 & 4 & \\
\hline Sor h 1 & $76 \%$ & Yes & Glycoprotein & Beta-expansin & 30 & 2 & \\
\hline
\end{tabular}


reactivity between subtropical and temperate grass pollen appear to depend of the geographical region of the patient population being investigated.

Subtropical grass pollens contain the major beta expansin group 1 allergen family; Pas $n 1$ of Bahia grass, multiple isoforms of Sor $h 1$ of Johnson grass and Cyn d 1 of Bermuda grass. The polygalacturonase components Pas n 13 of Bahia and Sor h 13 of Johnson grasses, are the second most abundant protein and frequently recognized allergens from pollen of Panicoideae family of subtropical subtropical regions. A number of allergens have been described from Bermuda grass pollen including the berberine bridge enzyme orthologue Cyn $d 4$ that is a major allergen. IgE reactivity with a group 2 allergen Sor $\mathrm{h} 2$ of Johnson grass pollen has recently been discovered (19). Notably, however to date no allergen with significant homology with the Pooideae group 5 allergen has been discovered by proteomic or transcriptomic analysis of subtropical grass pollens.

As mentioned above allergen molecules from different members of the Pooideae subfamily are highly IgE cross-reactive (10). As both wild and cultivated grasses in the temperate climate zones belong to the Pooideae subfamily, Phleum pratense allergens can be used for diagnostic and therapeutic purposes in grass pollen allergic patients living in the temperate parts of the world.

\section{SENSITIZATION TO INDIVIDUAL MOLECULES AND ITS CLINICAL RELEVANCE}

The IgE response against grass pollen (e.g. Phleum pratense) usually evolves from a simple, monomolecular stage to an oligomolecular stage and eventually to a polymolecular sensitization stage (6). This phenomenon has been defined as 'molecular spreading', that is, "The sequential development of antibody (IgE) response to distinct non-cross-reacting molecules from the same antigenic (allergenic) source, starting with an "initiator" (allergenic) molecule." (15). Phl $\mathrm{p} 1$ is the probable 'initiator' molecule in most patients, and the response involves then $\mathrm{Phl} \mathrm{p} 4$ or $\mathrm{Phl} \mathrm{p} \mathrm{5}$, thereafter also $\mathrm{Phl} p 2$ and $\mathrm{Phl} p 11$ and at a later stage $\mathrm{Phl} p 12$ or Phl $\mathrm{p}$ 7. This has been confirmed in other birth cohort studies (16). The practical consequence of this phenomenon is that the longer is the duration of disease, the broader is the repertoire of IgE against the different molecules of a single pollen.

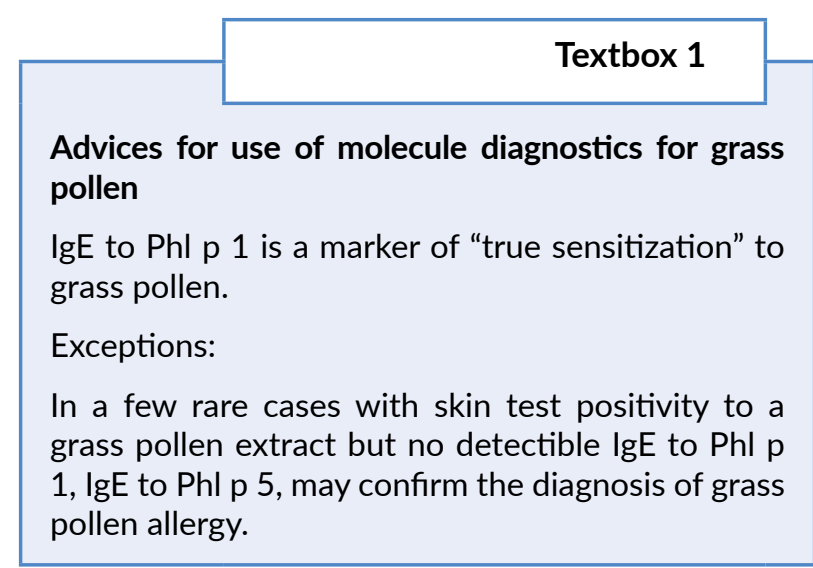

This has also led to the consideration that AIT should be started earlier in a patient's clinical care, possibly even immediately after the first season in which the allergic respiratory symptoms are initiated ("earlyAIT") (15). Interestingly, the molecular spreading process follows different pathways in different children: some patients remain sensitized only to the "initiator" molecule (Fig. 3) while a few patients become sensitized to most or all allergenic molecules. Consequently, a population of grass-pollen allergic patients "apparently" homogeneous if examined with an allergen extract is remarkably heterogeneous when examined with allergenic molecules (Fig. 4) $(16,17)$. The clinical relevance of individual profile of sensitizaion is being tested in large populations both in cross-sectional, observational studies and in longitudinal intervention studies. However, only few data have yet been published; in a recent study a higher risk of asthma at 11 years was observed in children being sensitized at 5 years to almost all grass pollen allergen molecules in comparison with those who had a late onset of sensitization (16).

IgE to Phl p 1 - Clinical relevance - Phl p 1 (or others of the "group 1" antigens of grass pollen, such as Lol $p 1$, from Lolium perenne) is in most patients the "initiator" molecule. Moreover, even in the few grass-pollen allergic patients who start their sensitization process with other molecules, IgE against $\mathrm{Phl} p 1$ are produced quite soon. Therefore, IgE to Phl p 1 is an essential marker in grass pollen allergic patients to establish "true sensitization". The presence of lgE to Phl $p 1$ confirms that the patient with a positive skin test or IgE assay to grass pollen extract is truly sensitized to grass pollen. The absence of lgE to Phl $p 1$ does not exclude "true" sensitization to grass pollen, which might be due (in a few cases) to isolated IgE sensitization to other major allergenic proteins (e.g. Phl p 5) but makes it rather 


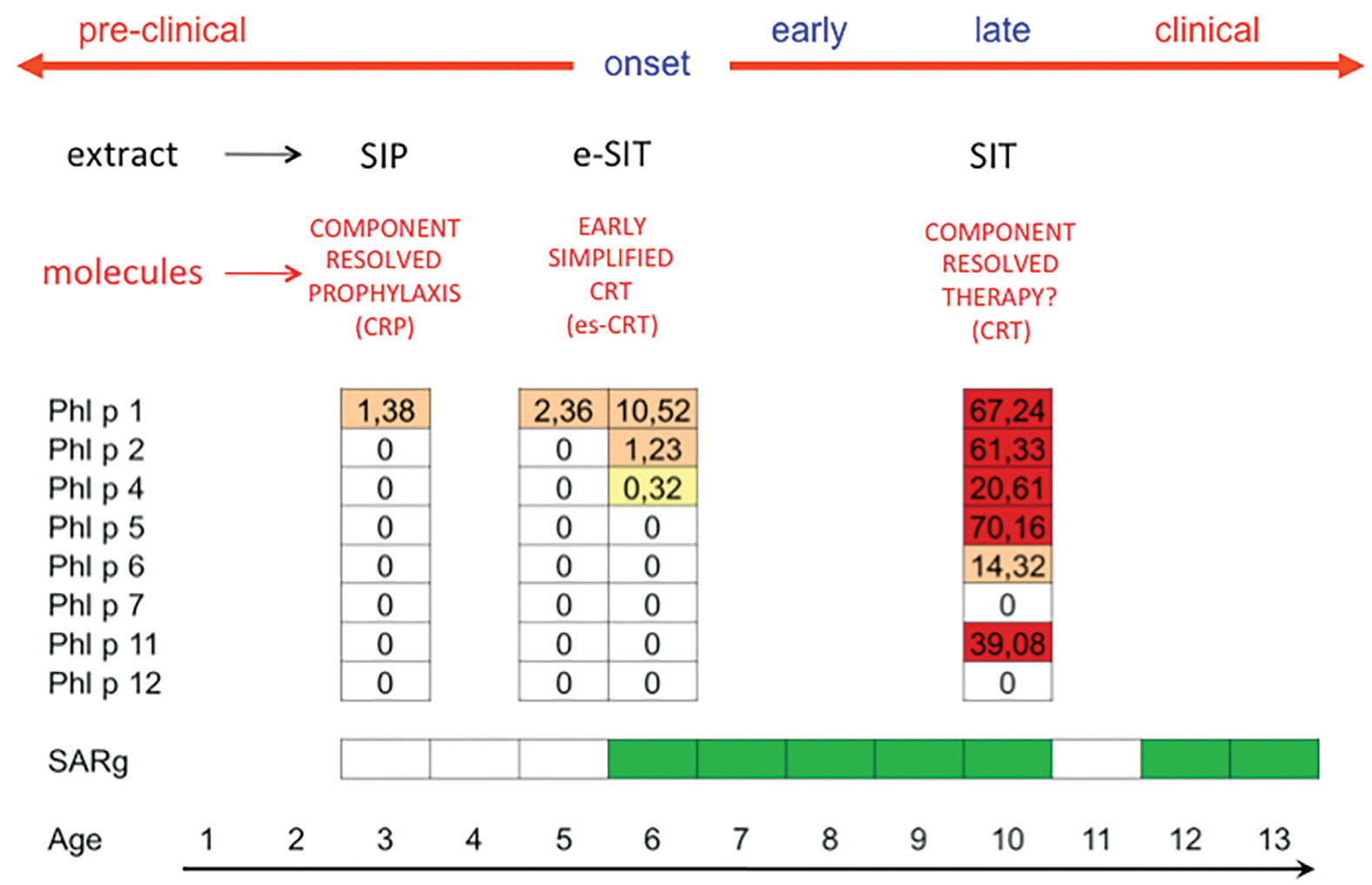

\section{Figure 3}

Molecular spreading of the IgE response to Timothy grass and potential implications for allergen-specific immunological intervention in a child with seasonal allergic rhinitis to grass pollen (SARg). Molecular spreading of the IgE response to Timothy grass and potential implications for allergen-specific immunologic intervention in a child with seasonal allergic rhinitis to grass pollen (SARg). Molecular spreading of the IgE response to Phleum pratense and implications for allergen-specific immunological intervention in one child with hay fever (case from the MAS birth cohort). Numbers refer to serum concentrations of IgE antibodies measured by ISAC (ISU). This child started suffering from hay fever symptoms at the age of 6 years. IgE response against Phleum pratense started 3 years before with a weak, monomolecular sensitization to Phl p 1. This IgE response was stronger and directed also to Phl p 2 and Phl p 4 at disease onset. After disease onset, the IgE response was much stronger and directed also to Phl p 5, Phl p 6, and Phl p 11. In clinical practice, allergen-specific immunotherapy (SIT) would be 'normally' prescribed at this advanced stage, after some years of symptoms (age $10 \mathrm{yrs}$ ). An interesting hypothesis is that SIT would be more efficient if started much earlier, ideally 'at' disease onset (age 6 yrs) (early SIT). Moreover, it could be investigated whether an immune intervention at the earliest, preclinical stages (age $3 \mathrm{yrs}$ ) of disease could even better change the natural history of the sensitization and prevent or delay diseases onset (allergen-specific immunoprohpylaxis, SIP). The use of recombinant allergens would be easier at this stage, as less molecules should be used (component-resolved immune prophylaxis (CRP)). Reprinted with permission from (15).

unlikely. Then patients with skin test-lgE positivity to a grass pollen extract but lacking IgE to $\mathrm{Phl} p 1$ should be tested for IgE to all other Phl p molecules. The group 1 allergens are the major and most clinically important allergen of subtropical Panicoideae grass pollens. Whilst other allergen components are present in subtropical grass pollens, IgE to Pas $\mathrm{n} 1$ of Bahia grass pollens accounts for nearly all of the detectible $\lg \mathrm{E}$ reactivity to the whole extract (18) similarly, IgE reactivity with Sor $\mathrm{h} 1$ of Johnson grass pollen is highly 


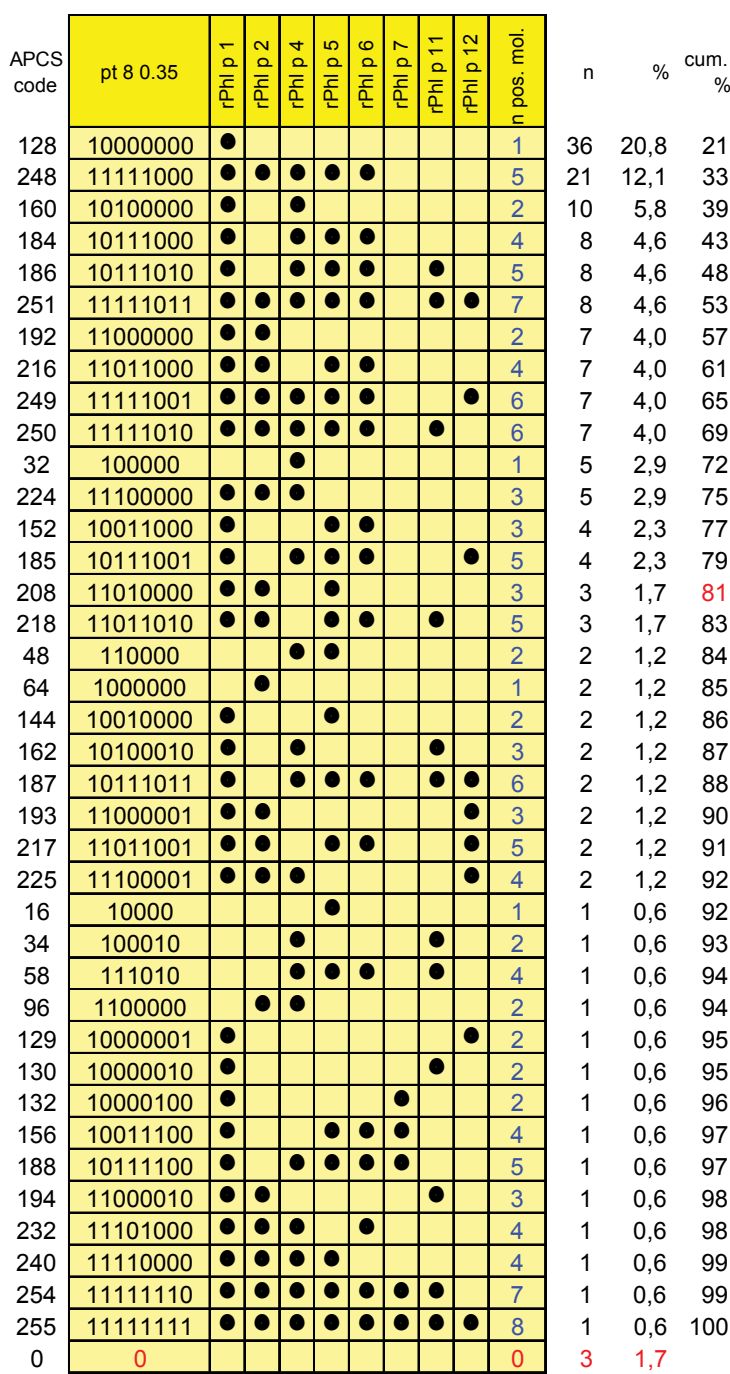

Figure 4

Profiles of IgE sensitization to eight Phleum pratense molecules in 176 sensitized children. Profiles of IgE sensitization to eight Phleum pratense molecules in 176-sensitized children. Profiles of IgE sensitization to eight Phleum pratense moleculesin 176 children with an lgE reaction to Phleum pratense and complete data-set. The Allergen Profile Codification System (APCS) code and the absolute and cumulative frequency are shown. The profiles are ordered by declining frequency and the point at which the arbitrary threshold of $80 \%$ of the patient population has been reached is marked in red. Reprinted with permission from (17).

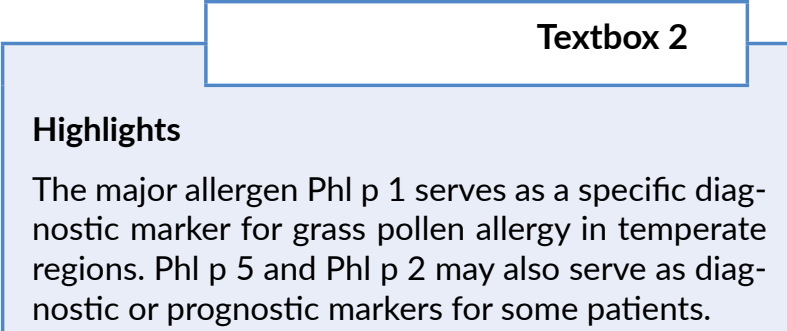

correlated with IgE reactivity with the whole pollen (19). For Bermuda grass pollen, Cyn d 1 is the major allergen but the complexity of described allergen components is broad (3).

IgE to $\mathrm{Phl}$ p 5 - Clinical relevance - Phl p 5 is rarely the only molecule inducing grass pollen sensitization and the presence of IgE antibodies to this $\mathrm{Phl} p 5$ - observed in around $50 \%$ of the European grass pollen allergic patients - confirms that a positive SPT reaction is the expression of true sensitization to grass pollen. However, although IgE to Phl p 5 usually appear later than those to $\mathrm{Phl} \mathrm{p} 1$ in the sensitization process, their concentration grows in many patients rapidly and higher and their contribution to patients' symptoms has been demonstrated. Testing IgE to $\mathrm{Phl}$ p 5 can be useful as a second line test and has been shown to be useful for distinguishing between allergy to grass and olive pollen in Southern Europe. $\mathrm{Phl}$ p 5 specific IgE may have some prognostic value for indicating disease severity or likely progression from allergic rhinitis to asthma, but this needs to be confirmed with well-designed studies. As group 5 allergens have not been found in subtropical grass pollens, Phl p 5 specific lgE may indicate sensitization to temperate grass pollens in particular. This needs to be investigated in relevant patient populations.

IgE to Phl p 12 - Clinical relevance (see also Section C01) - Phl p 12, is the highly cross-reacting profilin of Phleum pratense. As a heat-labile, relatively weak allergenic molecule, IgE sensitization to profilin comes later in the molecular spreading process, reaches only moderate levels of IgE antibodies and only in a minority of patients. Hence, IgE to Phl p 12 mark in general patients with a higher atopic background and/ or longer disease duration. Patients with a positive skin test/lgE to grass pollen extract but no lgE to Phl p 1 and Phl p 5 must be tested for IgE to Phl p 12 as these antibodies - that can be induced by other pollens containing profilin, is the first cause of "false" positivity to assays based on grass pollen extract. In the presence of IgE to $\mathrm{Phl} \mathrm{p} \mathrm{12,} \mathrm{patients} \mathrm{should} \mathrm{be}$ 


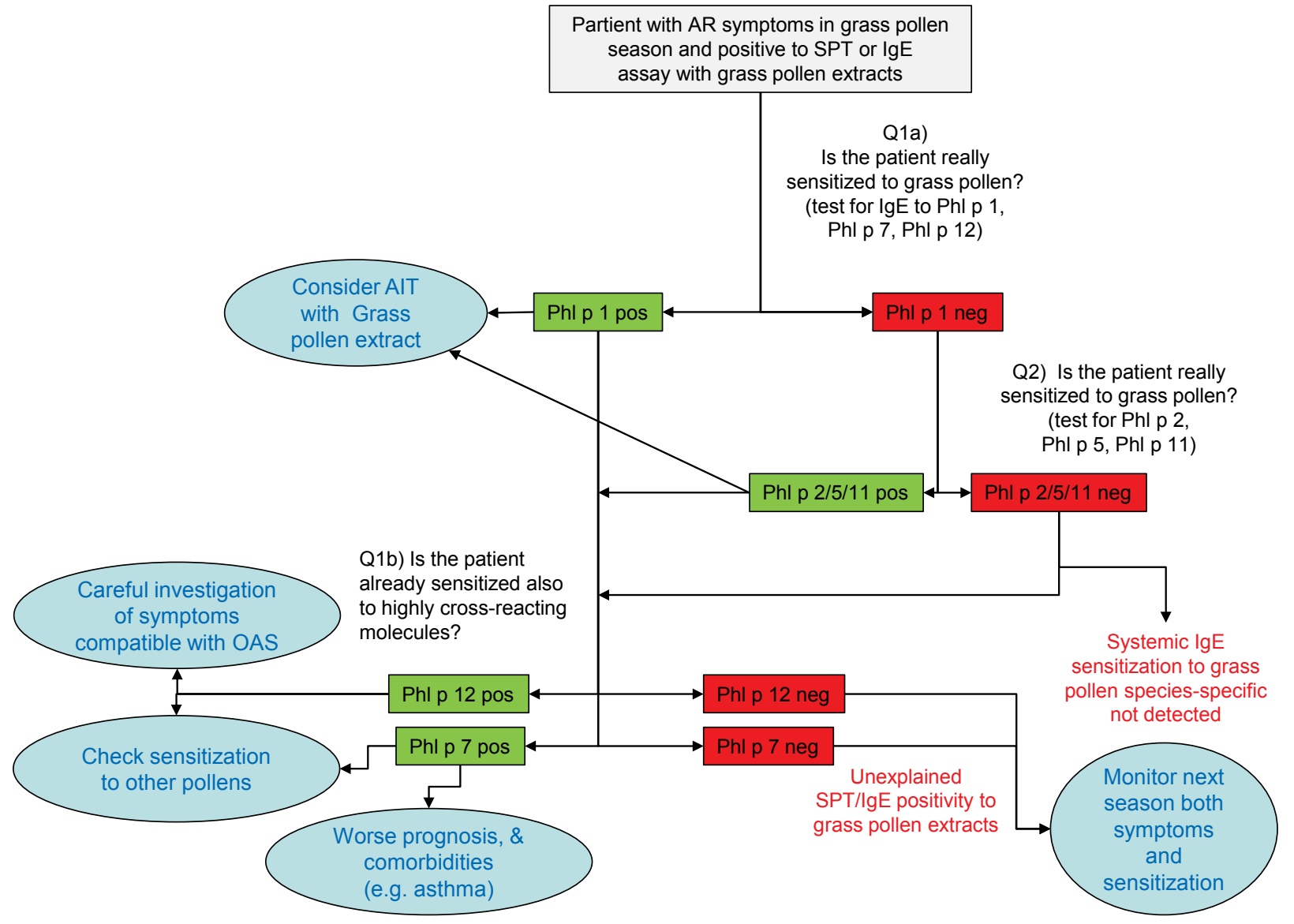

Figure 5

Diagnostic algorithm for AIT prescription in grass pollen allergic patients. Patients with AR symptoms during the grass pollen-season and a positive SPT/IgE assay to grass pollen extracts are further investigated to detect serum IgE antibodies to Phl p 1, Phl p 2, Phl p 5, Phl p 7, Phl p 11, and Phl p 12. The identification of one or more of IgE antibodies to Phl p 1, Phl p 2, Phl p 5 and/or Phl p 11 is followed by the prescription of grass pollen AIT. The identification of IgE to Phl p 12 (profilin) is followed by further investigation of OAS and influences is relevant to better interpret results of SPT/lgE assays with other pollen extracts or vegetables. The identification of IgE to Phl p 7 alerts the doctor of a worse prognosis and greater severity of the disease.

asked about Oral Allergic Syndrome triggered by the ingestion of fruit and vegetables containing profilin, with a focus on apple, celery and birch.

IgE to Phl p 7 - Clinical relevance (see also Section C06) - Phl p 7, is the highly cross-reacting polcalcin of Phleum pratense. This is a heat-stabile, relatively potent allergen that can induce quite high IgE antibody levels. An lgE response to $\mathrm{Phl} p 7$ is observed only infrequently among grass pollen allergic patients and usually many years after the disease onset. IgE to Phl p 7 marks a relatively distinct category of grass pollen allergic patients, with more severe symptoms, a higher prevalence of asthma, and a higher frequency of additional allergic comorbidities. Moreover, many other pollens and allergenic sources contain polcalcin so that the origin sensitization to polcalcin in a grasspollen allergic patient must be carefully searched. These allergenic sources could be indeed responsible of a more severe disease.

IgE to Phl p 4 - Clinical relevance (see also Section A03) - Phl p 4 is a major allergenic protein of grass 
pollen. In its native form, the one still used in most commercialavailableassays, $\mathrm{Phl}$ p 4 contains extremely highly cross-reactive carbohydrate determinants (CCD). This explains why in several epidemiological studies IgE positivity to $\mathrm{Phl}$ p 4 scores over $90 \%$ of the grass pollen allergic patients. However, when the recombinant version of the molecules is used for assays, over $50 \%$ of the positivity is not confirmed anymore. As extracts contain native $\mathrm{Phl} \mathrm{p} \mathrm{4,} \mathrm{a} \mathrm{weak}$ positivity to SPT/IgE test based on grass pollen extracts can in some patients be "false" and simply explained by IgE recognizing CCD determinants. Phl p 4 may also serve as a marker for sensitization to Bermuda grass pollen due to its similarity with Cyn d 4, a major allergen of Bermuda grass pollen, but this needs to be investigated in relevant patient populations.

IgE to Phl p 2, 6, 11 - Phl p 2 and Phl p 11 are both rarely the only molecule inducing grass pollen sensitization and the presence of IgE antibodies to this $\mathrm{Phl} \mathrm{p} 2$ - observed in around $60-80 \% \%$ of the European grass pollen allergic patients - just confirms that a positive SPT reaction is the expression of true sensitization to grass. Phl p 6 is highly cross-reacting with $\mathrm{Phl}$ p 5 and does not add a lot of diagnostic information, once IgE to Phl p 5 has been tested.

\section{CLINICAL MANAGEMENT}

In patients allergic to grass pollens IgE tests should be oriented to answer the following questions:

A) is the patient really sensitized to grass pollen major allergenic proteins? (test $\mathrm{Phl} p 1$, if negative also Phl p 5 and the other molecules);

B) is the patient sensitized also to highly crossreacting molecules? (test $\mathrm{Phl} \mathrm{p} 12$ and $\mathrm{Phl} \mathrm{p} \mathrm{7);}$

C) in the case of negativity to $\mathrm{Phl} p 1$ and other species-specific allergenic molecules and positivity to $\mathrm{Phl} \mathrm{p} 12$ and/or $\mathrm{Phl} \mathrm{p} \mathrm{7),} \mathrm{which} \mathrm{is} \mathrm{the}$ pollen inducing a "false" IgE sensitization to grass pollen extracts?

After having answered these questions the doctor should be able to decide whether the patients' symptoms are consistent or not to IgE sensitization to grass-pollen and consequently can decide whether to prescribe an AIT based on grass-pollen extract. Whilst orthologues such as Cyn d 7 and Sor h 12 have been reported in subtropical grasses, there is currently limited evidence available of IgE reactivity in relevant patients primarily sensitized to subtropical grass pollens. A diagnostic algorithm for a decision making process which summarizes the information provided in the previous section is proposed (Fig. 5).

\section{CLINICAL CASES}

\section{Case 1}

Step 1 - History: A 35 year old male from Central Italy patient presented with allergic rhinitis from April to July but not in September or October. The patient had experienced conjunctival and nasal symptoms that did not respond to antihistamines and were only partly controlled with nasal steroids. He reported the condition was steadily increasing each season and that he, occasionally, experienced a tight chest after spending time outside. Since the last year, he had experienced oral symptoms (pruritus, swelling) after eating either melon or watermelon.

Step 2 - Testing: SPT positive for birch ( $5 \mathrm{~mm})$, timothy grass $(8 \mathrm{~mm})$, pellitory $(4 \mathrm{~mm})$, olive $(3 \mathrm{~mm})$ pollens. Serum IgE antibody levels were $7.1 \mathrm{kU} / \mathrm{L}$ to birch, $17.3 \mathrm{kU} / \mathrm{L}$ to timothy grass, $6.7 \mathrm{kU} / \mathrm{I}$ to pellitory, $3.2 \mathrm{kU} / \mathrm{I}$ to olive extracts.

Step 3 - Treatment: No AIT was started as the doctor was not sure which pollen(s) was/were responsible of the patient's symptoms.

Added CRD value: positive response to $\mathrm{Phl} p 1$ (12.2 $\mathrm{kU} / \mathrm{l}), \mathrm{Phl} p 5(6.5 \mathrm{kU} / \mathrm{l})$, and $\mathrm{Phl} \mathrm{p} 12(4.3 \mathrm{kU} / \mathrm{l})$ but not to Bet $\vee 1$, Ole e 1, and Par j 2. The patient commenced SLIT with grass pollen and responded well to this treatment. OAS was also explained by IgE sensitization to profilin (Phl p 12).

\section{Case 2}

Step 1 - History: A 26 year old Danish woman with a 10 year history of persistent severe seasonal rhinoconjunctivitis during birch and grass pollen season. Symptoms most severe in early summer, with persistent conjunctival (redness, itching, watering, light sensitivity) and nasal (blocked nose, itching and secretion) symptoms. Very poor effect of systemic antihistamines and local antihistamine (eyes and nose) and local corticosteroid (nose). Had some benefit from systemic corticosteroid. Good symptom control during birch pollen season. 
Step 2 - Testing: SPT positive for birch and grass pollen $7 \mathrm{~mm}$ diameter. IgE to grass pollen 10.9 $\mathrm{kU} / \mathrm{l}$, birch pollen $3.9 \mathrm{kU} / \mathrm{I}$

Step 3 - Treatment: Starts standard SCIT with natural grass pollen extract'. Poor clinical effect after 2 years of treatment.

Added $C R D$ value $=$ Missing Step $2 b-$ In vitro testing: We found a sensitizations to $\mathrm{Phl} p 4$ in the grass pollen panel, as well as Bet $\vee 1$. She was not sensitized to typical other $C C D$ reactive natural molecules. The conclusion was that she truly was grass pollen allergic, and she received anti-lgE treatment during grass pollen season with good clinical outcome

\section{References}

1. Laffer $S$, Vrtala $S$, Duchene $M$, van Ree R, Kraft D, Scheiner $O$, et al. IgE-binding capacity of recombinant timothy grass (Phleum pratense) pollen allergens. J Allergy Clin Immunol 1994;94:88-94.

2. Popescu FD. Molecular biomarkers for grass pollen immunotherapy. World J Methodol 2014:26;4:26-45.

3. Davies JM. Grass pollen allergens globally: The contribution of subtropical grasses to burden of allergic respiratory diseases. Clin Exp Allergy 2014;44:790801.

4. Bousquet PJ, Chinn S, Janson C, Kogevinas M, Burney P, Jarvis $D$, et al. Geographical variation in the prevalence of positive skin tests to environmental aeroallergens in the European Community Respiratory Health Survey I. Allergy 2007;62:301-309.

5. Devanaboyina SC, Cornelius C, Lupinek C, Fauland K, Dall'Antonia F, Nandy A, et al. High-resolution crystal structure and IgE recognition of the major grass pollen allergen Phl p 3. Allergy 2014;69:1617-1628.

6. Hatzler L, Panetta V, Lau S, Wagner P, Bergmann RL, Illi $S$, et al. Molecular spreading and predictive value of preclinical IgE response to Phleum pratense in children with hay fever. J Allergy Clin Immunol 2012;130:894901.

7. Suphioglu C, Singh MB. Cloning, sequencing and expression in Escherichia coli of Pha a 1 and four isoforms of Pha a 5, the major allergens of canary grass pollen. Clin Exp Allergy 1995;25:853-865.

8. Focke M, Mahler V, Ball T, Sperr WR, Majlesi Y, Valent P, et al. Nonanaphylactic synthetic peptides derived from $\mathrm{B}$ cell epitopes of the major grass pollen allergen, $\mathrm{Phl} p$ 1, for allergy vaccination. FASEB J 2001;15:2042-2044.
9. Nony E, Timbrell V, Hrabina M, Boutron M, Solley $G$, Moingeon $P$, et al. Specific IgE recognition of pollen allergens from subtropic grasses in patients from the subtropics. Ann Allergy Asthma Immunol 2015;114:214-220.

10. Gangl K, Niederberger V, Valenta R. Multiple grass mixes as opposed to single grasses for allergen immunotherapy in allergic rhinitis. Clin Exp Allergy 2013;43:1202-1216.

11. https://www.allergome.com [homepage on the Internet] 2015.

12. Mari A. Skin test with a timothy grass (Phleum pratense) pollen extract vs. IgE to a timothy extract vs. IgE to $\mathrm{rPhl}$ $\mathrm{p}$ 1, rPhl p 2, nPhl p 4, rPhl p 5, rPhl p 6, rPhl p 7, rPhl $\mathrm{p}$ 11, and $\mathrm{rPhl} \mathrm{p}$ 12: Epidemiological and diagnostic data. Clin Exp Allergy 2003;33:43-51.

13. IUIS: Nomenclature of allergens [homepage on the Internet]. 2015. Available from: http://www.allergen. org/.

14. The protein model portal [homepage on the Internet] 2015. Available from: http://www.proteinmodelportal. org/.

15. Matricardi PM. Allergen-specific immunoprophylaxis: Toward secondary prevention of allergic rhinitis? Pediatr Allergy Immunol 2014;25:15-18.

16. Custovic A, Sonntag HS, Buchan IE, Belgrave D, Simpson A, Mattia $C$ et al.Evolution pathway of IgE responses to grass and mite allergens throughout childhood. J Allergy Clin Immunol 2015;136:16451652.

17. Tripodi S, Frediani T, Lucarelli S, Macri F, Pingitore G, Di Rienzo Businco A, et al. Molecular profiles of IgE to Phleum pratense in children with grass pollen allergy: Implications for specific immunotherapy. J Allergy Clin Immunol 2012;129:834-839.

18. Timbrell VL, Riebelt L, Simmonds C, Solley G, Smith WB, Mclean-Tooke A, et al. An immunodiagnostic assay for quantitation of specific lgE to the major pollen allergen component, Pas $\mathrm{n} 1$, of the subtropical Bahia grass. Int Arch Allergy Immunol 2014;165:219-228.

19. Campbell BC, Gilding EK, Timbrell V, Guru P, Loo D, Zennaro $\mathrm{D}$ et al. Total Total transcriptome, proteome, and allergome of Johnson grass pollen, which is important for allergic rhinitis in subtropical regions. J Allergy Clin Immunol 2015;135:133-142.

20. Davies, JM, Mittag D, Dang TD, Symons K, Voskamp A, Rolland JM et al. Molecular cloning, expression and immunological characterisation of Pas $\mathrm{n}$ 1, the major allergen of Bahia grass Paspalum notatum pollen. Mol. Immunol 2008;46:86-293. 
WEED POLLEN ALLERGY

\author{
Gabriele Gadermaier, Janet M. Davies,
} Thomas Hawranek, Richard Weber

\section{THE ALLERGEN SOURCES}

The term weed does not constitute a botanical family, but rather refers to diverse plants used sometimes as culinary culinary and medicinal herbs, that are ecologically adaptive and invasive segetal plants. Pollen of weeds mediating IgE-related allergic reactions are found in monocot (Poaceae) and dicot (Asteraceae, Urticaceae, Plantaginaceae, Euphorbiaceae, and Amaranthaceae) plant families (1). This section will focus on dicot weeds. Pollen of ragweed, mugwort, sunflower, feverfew, pellitory, English plantain, Annual mercury, goosefoot, Russian thistle and amaranth are considered important allergy eliciting sources (Fig. 1). Poaceae weeds like Johnson grass can be found in section B02.

The impact of climate change on pollen load, allergenicity, distribution, and flowering season is well acknowledged and is of particular interest in regards to weeds since they can dominate groundcover, adapt to various environmental conditions or reside in ecologic niches. Due to globalization, neophytes such as ragweed have been imported to Europe as ballast grain, spreading readily with predictions to reach Northern Europe (2). Furthermore, significant
Common invasive weeds like ragweed, mugwort and plantain, are frequently important allergen sources typically flowering in summer through to autumn.

The biogeographical range and pollination periods of allergenic weeds can overlap confounding accurate allergy diagnosis.

Specific lgE to Amb a 1 can be a useful marker for ragweed sensitization but it shows crossreactivity with Art $v 6$ from mugwort. Likewise specific lgE to Art $v 1$ can be a useful marker for mugwort sensitization, but its shows partial cross-reactivity with Amb a 4 from ragweed.

Art $v 3$ reactivity is frequently associated with LTP sensitization in Mediterranean patients but is rare in patients with mugwort pollenrelated symptoms in non-Mediterranean regions.

Parj 2 is a highly specific marker allergen for pellitory sensitization while Pla I 1 is a useful marker for genuine plantain sensitization. 

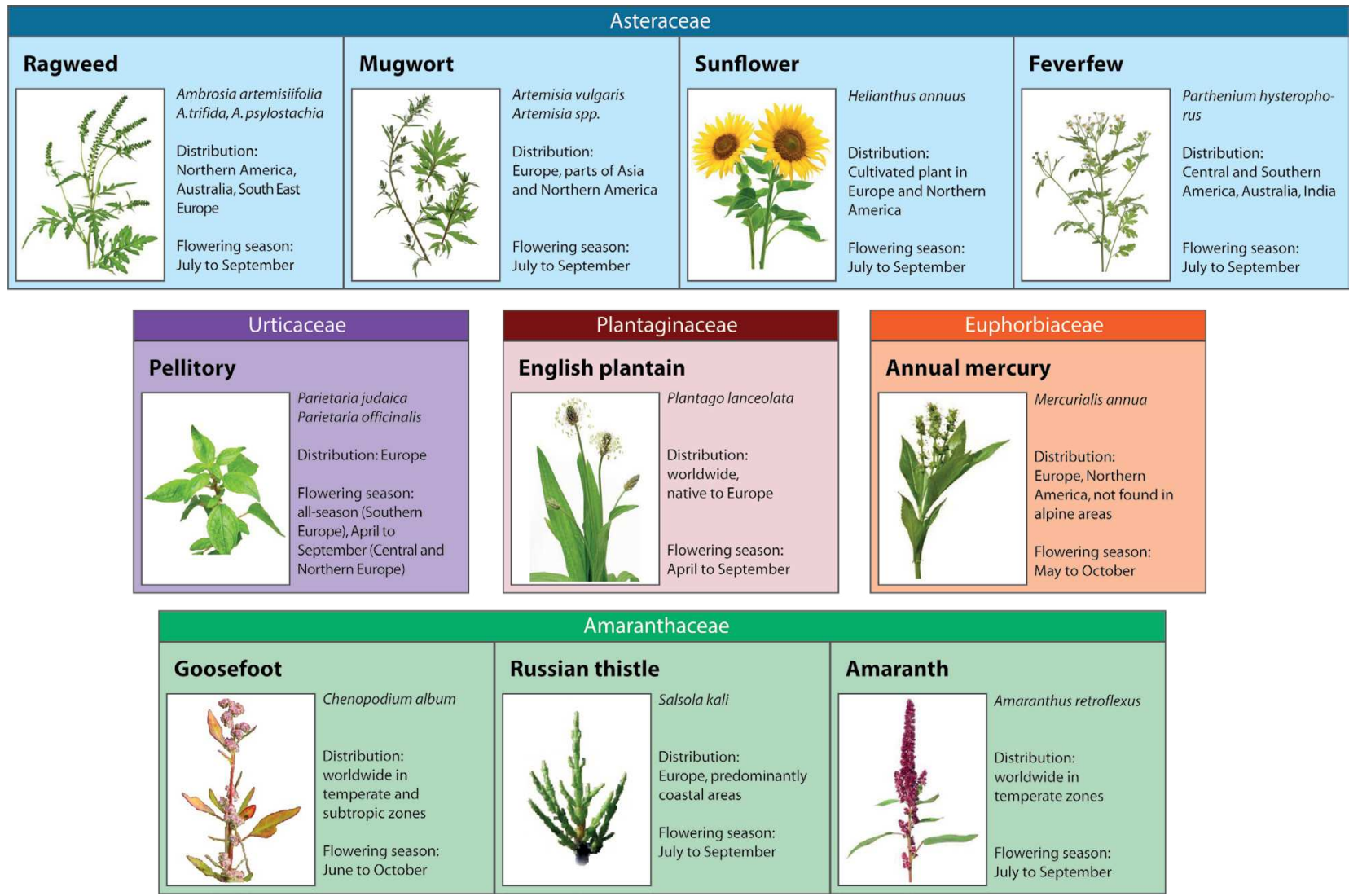

\section{Figure 1}

Important allergenic weeds. Figure adapted from Stemeseder et al. (215). Flowering periods given refer to the Northern Hemisphere.

increases in duration of pollen season of ragweed and pellitory were recorded over the last 20 years. Additional influence on the allergenicity might arise from environmental pollution, as was shown for ragweed pollen collected along high-traffic roads presenting elevated IgE reactivity. Weeds are often considered non-desired invasive species and thus combated using herbicides. However, there are some species actively cultivated for economic purposes, e.g. sunflower for birdseed or Artemisia annua to obtain the anti-malaria drug artemisinin.

Plants of the genus Ambrosia comprise around 50 species native to northern and central America. In the past decades, the neophyte is rapidly spreading in Europe due to the pollen's ability to travel long distances. The genus Artemisia comprises around 350 species and representatives can be found throughout the Northern hemisphere and Australia.
Mugwort is frequently used as herb in traditional Chinese medicine and A. annua is cultivated for harvest of artemisinin. There are parts of Europe and America where the range and pollination of mugwort and ragweed overlap (www.discoverlife.org; Atlas of living Australia and US Department of Agriculture), confounding accurate allergy diagnosis. Common sunflower is primarily grown for commercial use of its oil and birdseed. Parthenium spp are predominately found in Southern US, Central and South America and invasive in India, Australia and parts of Africa. Allergenic members of the Parietaria genus are frequently found in southern and central Europe showing a long pollination season with recurrent flowering periods. The genus Plantago includes around 250 species and was spreading from Europe throughout the world. Mercurialis annua is a highly prevalent weed throughout Europe. Chenopodium album, Salsola kali and Amaranthus retroflexus can be 
found in arid regions of the Northern hemisphere and Australia. Due to use in greening programs or as ornamental flowers, these weeds are highly abundant in Iran, Kuwait or Saudi Arabia.

\section{MAJOR AND RELEVANT MINOR ALLERGENIC MOLECULES}

The most relevant weed pollen allergens belong to the families of pectate lyases, defensin-like proteins, non-specific lipid transfer proteins (nsLTP) and Ole e 1-like proteins (Table 1). Additionally, the panallergens profilin and polcalcin have been identified as crossreactive molecules present in weed pollen (see C01 and $\mathrm{CO} 4$ of this handbook). Currently, 36 weed pollen allergens are listed as allergens by the IUIS Allergen Nomenclature Sub-Committee. In addition, a $60 \mathrm{kDa}$ acidic glycoprotein from mugwort and Par $h$ 1, a defensin-like protein from feverfew pollen were described to be major allergens in the respective sources. Comprehensive information and characteristics on molecules and derivatives as well as functions are reviewed in Gadermaier et al and Villalba et al $(3,4)$.

\section{SENSITIZATION TO INDIVIDUAL MOLECULES AND THEIR CLINICAL RELEVANCE}

\section{Epidemiology and sensitization}

Ambrosia spp are major elicitors of type I pollen allergies in Northern America and 15.3\% adults from Canada were tested positive with ragweed extract. Data from the GA2LEN study showed sensitization rates of $8.7 \%$ (US), while in European countries SPT reactivity was typically lower than $2.6 \%$ in the general population (6). Among allergic patients from Germany, $19.5 \%$ were ragweed sensitized as determined by SPT (7). Sensitization frequencies to mugwort of more than $10 \%$ were found in a German general population cohort. Amongst patients with allergic rhinitis in Korea, the sensitization rate to ragweed was low (2.2\%) but increasing over time (2.8\%). In China, 3.7-6.5\% of asthma and/or rhinitis patients were sensitized to ragweed while reactivity to mugwort was around $11 \%$ (8). There was a markedly higher frequency of specific IgE to Artemisia (58.3\%) correlating to Art $\vee 1$ reactivity (49\%). In contrast, specific IgE to Ambrosia (14.7\%) and Amb a 1 (11.2\%) was lower in frequency and level, not correlated with each other, and uncommon in the absence of specific IgE to Art $v 1$ suggesting primary sensitization with Artemisia species (9). Epidemiological studies on pollen allergic patients performed in Spain identified a frequency of sensitization to Art v 1 of 13\% (10). In Adelaide, South Australia, $37 \%$ and $25 \%$ of over 3000 patients presenting to a respiratory clinic showed SPT to plantain and ragweed respectively (Dr Frank Kett, personal communication). Similar sensitization frequencies for ragweed were found in other regions of Australia ranging from 34-38\% (11). Among sunflower processing workers, a sensitization rate of $23.5 \%$ to sunflower extract was reported (12). Thirty-five percent of autumn pollinosis patients in US were reactive to feverfew extract and among type IV atopic dermatitis patients in India, 35.7\% showed positive type I reactions in skin prick tests (13).

Parietaria is one of the most relevant causes of pollen allergy in people living in the Mediterranean basin. About $30 \%$ of all allergic subjects living in southern Italy are SPT positive to Parietaria judaica pollen extract, with local sensitization rates up to $60 \%$. Although the weed is highly prevalent throughout Europe, sensitization in the non-Mediterranean population is marginal (14). Epidemiological studies on pollen allergic patients performed with Pla I 1 in Spain identified plantain as the second cause of pollinosis in some northern areas, frequently associated to grass sensitization. Recent studies also demonstrated the relevance of Plantago in central/northern European patients $(10,15)$. High levels of reactivity to Mercurialis annua pollen ranging from $28-56 \%$ were observed in several areas of Spain (3).

Due to use of Chenopodium album in greening programs, the weed gained relevance in countries with desert and semi-desert areas accounting for up to $70.7 \%$ sensitization in asthmatic patients. Clinical incidences have been reported in southern Spain and Saudi Arabia, while they even represent the main sensitizer for allergic rhinitis and asthma in Kuweit and Iran (4). The ornamental plant Amaranthus retroflexus is also described as major trigger of allergic reactions in Iran, with a sensitization frequency up to $69 \%$ in SPT (4). Salsola allergy, as determined by specific sensitization to Sal k 1, the major Salsola allergen (homologous molecule absent in Chenopodiaceae), is the overall third cause of pollinosis in Spain. In some of the dryer areas of the south, up to $80 \%$ of the patients suffering from seasonal allergy are sensitized to Sal k 
Table 1

Relevant weed pollen allergens

\begin{tabular}{|c|c|c|c|c|}
\hline $\begin{array}{l}\text { Species } \\
\text { (English name) }\end{array}$ & $\begin{array}{l}\text { Allergenic } \\
\text { molecule }\end{array}$ & Biochemical name & $\begin{array}{l}\text { Prevalence } \\
\text { among patients }\end{array}$ & $\begin{array}{l}\mathrm{MW} \\
(\mathrm{kDa})\end{array}$ \\
\hline \multirow{7}{*}{$\begin{array}{l}\text { Ambrosia artemisiifolia } \\
\text { (ragweed) }\end{array}$} & Amb a 1 & Pectate lyase & $>95 \%$ & 38 \\
\hline & Amb a 4 & Defensin-like protein & $20-40 \%$ & $13-15$ \\
\hline & Amb a 6 & Non-specific lipid transfer protein & $20 \%$ & 10 \\
\hline & Amb a 8 & Profilin & $35-50 \%$ & 14 \\
\hline & Amb a 9 & Polcalcin (2 EF-hand calcium binding protein) & $10-15 \%$ & 9 \\
\hline & Amb a 10 & Polcalcin (3 EF-hand calcium binding protein) & $10-15 \%$ & 17 \\
\hline & Amb a 11 & Cysteine protease & $66 \%$ & 37 \\
\hline \multirow{5}{*}{$\begin{array}{l}\text { Artemisia vulgaris } \\
\text { (mugwort) }\end{array}$} & Art v 1 & Defensin-like protein & $95 \%$ & $13-15$ \\
\hline & Art v 3 & Non-specific lipid transfer protein & $22-70 \%$ & 10 \\
\hline & Art v 4 & Profilin & $35 \%$ & 14 \\
\hline & Art $v 5$ & Polcalcin (2 EF-hand calcium binding protein) & $10-28 \%$ & 10 \\
\hline & Art v 6 & Pectate lyase, Amb a 1-homolog & $26 \%$ & 38 \\
\hline \multirow{2}{*}{$\begin{array}{l}\text { Helianthus annuus } \\
\text { (sunflower) }\end{array}$} & Hel a 1 & $\begin{array}{l}\text { Defensin-like protein (potentially SF18 from } \\
\text { sunflower) }\end{array}$ & $65 \%$ & 34 \\
\hline & Hel a 2 & Profilin & $31 \%$ & 14 \\
\hline \multirow{4}{*}{$\begin{array}{l}\text { Parietaria judaica } \\
\text { (pellitory) }\end{array}$} & Par j 1 & Non-specific lipid transfer protein & $95 \%$ & 15 \\
\hline & Par j 2 & Non-specific lipid transfer protein & $80 \%$ & 11 \\
\hline & Par j 3 & Profilin & nd & 14 \\
\hline & Par j 4 & Polcalcin (2 EF-hand calcium binding protein) & $6 \%$ & 9 \\
\hline $\begin{array}{l}\text { Plantago lanceolata } \\
\text { (English plantain) }\end{array}$ & Pla I 1 & Ole e 1-like protein & $86 \%$ & 15 \\
\hline $\begin{array}{l}\text { Mercurialis annua } \\
\text { (Annual mercury) }\end{array}$ & Mer a 1 & Profilin & $50-60 \%$ & 14 \\
\hline \multirow{3}{*}{$\begin{array}{l}\text { Chenopodium album } \\
\text { (goosefoot) }\end{array}$} & Che a 1 & Ole e 1-like protein & $70 \%$ & 18 \\
\hline & Che a 2 & Profilin & $55 \%$ & 14 \\
\hline & Che a 3 & Polcalcin (2 EF-hand calcium binding protein) & $46 \%$ & 10 \\
\hline \multirow{3}{*}{$\begin{array}{l}\text { Salsola kali (Russian } \\
\text { thistle) }\end{array}$} & Sal k 1 & Pectin methylesterase family & $65 \%$ & 37 \\
\hline & Sal k 4 & Profilin & $46 \%$ & 14 \\
\hline & Sal k 5 & Ole e 1-like protein & $30-60 \%$ & 18 \\
\hline $\begin{array}{l}\text { Amaranthus retroflexus } \\
\text { (Amaranth) }\end{array}$ & Ama r 2 & Profilin & $33 \%$ & 14 \\
\hline
\end{tabular}

Data on sensitization frequency according to Gadermaier et al. (3), except for Amb a 11 (5); the sensitization prevalence to each allergenic molecule among patients sensitized to the natural extract may vary in different geographic regions. 


\section{A) Pectate lyases}

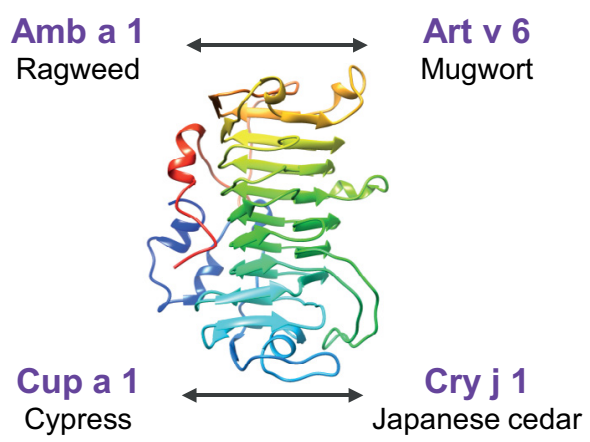

\section{C) Lipid transfer proteins}

\section{B) Defensin-like proteins}

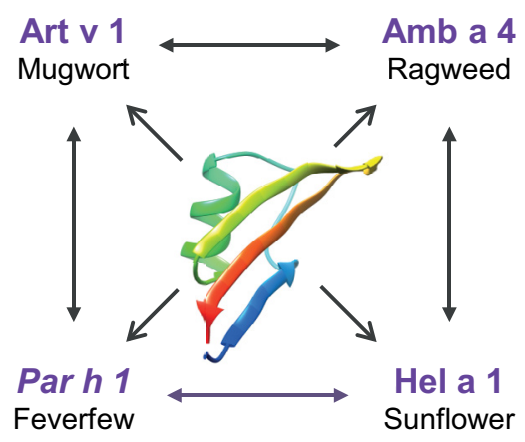

\section{D) Ole e 1-like family}

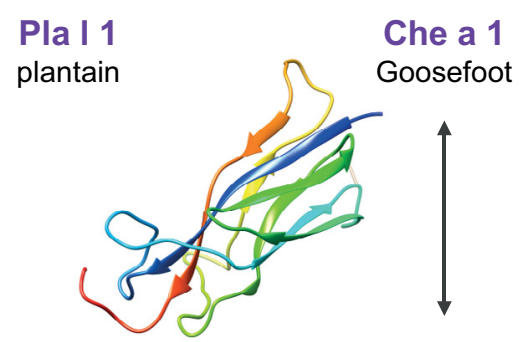

Ole e 1

Olive
Sal k 5

Russian thistle

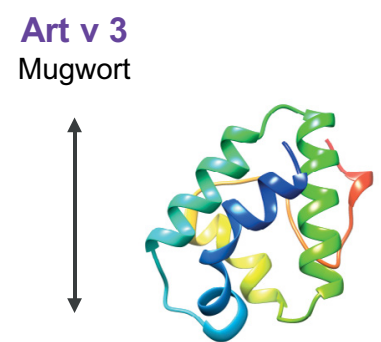

Pru p 3

Peach fruit

Par j 2

Pellitory

\begin{abstract}
Amb a 6
Ragweed
\end{abstract}

\section{Figure 2}

A) Model Amb a 1.0101 (template 1PXZ), B) Structure Art v 1.0101 defensin-domain (2KYP), C) Model Art v 3.0201 (template 2B5S), and D) Structure Pla I 1.0101 (4Z8W). Models were generated using Swiss-Model (www.swissmodel.expasy.org) and ribbon cartons are shown using UCSF Chimera (www.cgl. ucsf.edu/chimera). Lines represent documented IgE cross-reactivity, dotted lines represent potential IgE cross-reactivity based on high sequence identity.

1 , and are frequently monosensitized. In other areas such as the Ebro river valley it is the second cause of pollinosis after grasses $(10,16)$ This allergy is also very prevalent in other dry areas like Iran where up to $72.5 \%$ of pollen allergic patients are sensitized to Salsola $(3,4)$.

\section{Cross-reactivity}

A high degree of IgE cross-reactivity is typically observed among different Artemisia or Ambrosia species and is extendable to allergenic plants of the Asteraceae family $(3,17)$. Frequent levels of moderate IgE cross-reactivity are observed between pectate lyases (Amb a 1 and Art $v$ 6) and defensin-like proteins (Art v 1, Amb a 4, Par h 1, and potentially Hel a 1) (Fig. $2 A$ and $2 B)(3,18)$. Art $v 3$, the nsLTP from mugwort pollen demonstrates frequent cross-reactivity to homologous molecules in plant food, i. e. Pru p 3 (Fig. 2C). Sensitization to Art $\vee 3$ in the absence of pollinosis can indicate primary sensitization to LTP in case of food allergy. In contrast, source constrained sensitizations are observed for the nsLTP Par j 2 and Amb a 6 (19). The Ole e 1-like protein Pla I 1 from plantain presents low sequence identity to other family members and thus presents limited crossreactivity. In contrast, substantial cross-reactivity is 
observed for Che 1 and Sal $k 5$ due to $74 \%$ sequence identity (Fig. 2D) (4, 15). As anticipated, pan-allergens like profilins or polcalcins also present in weed pollen frequently give rise to IgE cross-reactivity beyond close botanical relations.

Pollen-food syndromes mediated by weeds are mainly involving mugwort and ragweed allergic patients (for review see (20). In addition to oral allergy syndromes, more severe clinical pictures as observed e.g. in the celery-mugwort-spice syndrome are reported. Underlying cross-reactive molecules can be found within the family of nsLTP (Art $\vee 3$ ), profilins, and highmolecular weight allergens with CCDs (20).

\section{CLINICAL MANAGEMENT}

\section{Clinical relevance and clinical pattern}

A) Exposure to weed pollen and primary sensitization to major as well as minor allergens predominately leads to rhino-conjunctivitis and/or asthma. Occupational allergies, i. e. to sunflower pollen are observed in workers and citizens in close vicinity.

B) Clinically relevant IgE cross-reactivity to weeds due to primary sensitization to pollen from botanically related sources, i) sunflower or feverfew allergy as a consequence of primary mugwort pollen allergy since the major allergen Art $\vee 1$ demonstrates high sequence similarity, ii) IgE cross-reactivity between Salsola and Chenopodium is observed due to highly homologous Ole e 1-like molecules.

\section{Clinical diagnosis}

Diagnosis of weed pollen allergy can be difficult due to frequent polysensitization and inconclusive anamnesis owing to overlapping flowering seasons with other pollen. Thus molecule-based allergy diagnosis is particularly advantageous and work-ups facilitating diagnosis of some weed pollen allergies are presented in Figure 3.

\section{Case history}

Weed pollen allergic patients typically present seasonal respiratory symptoms (rhinitis and/ or conjunctivitis and/or asthmatic symptoms, sometimes also itching of the throat and/or contact urticaria). Since clinical symptoms coincide with flowering periods of the respective weeds (Fig. 1), principal information can be obtained by narrowing down the eliciting allergen source(s).

\section{Skin prick test (SPT)}

The choice of commercially available weed pollen extracts for SPT is highly depending on pollen exposure and clinical references. Based on current $G^{2} L E N$ recommendations for harmonization of skin prick tests in Europe, mugwort, ragweed and pellitory are included in routine diagnostic panels, while plantain and allergenic pollen of the Amaranthaceae family are not considered. Since the allergological relevance can considerably vary among regions, local modifications are however useful and necessary. Since Parietaria extracts are virtually missing profilin, a positive SPT with this weed is generally indicative of primary sensitization unless the patient is sensitized to the pollen pan-allergen polcalcin (21).

\section{IgE testing}

Apart from in vitro testing using weed pollen extracts, molecule-based approaches offer a valuable tool for refined diagnosis limiting unspecific results due to polysensitization (Fig. 2). All major allergens of weed pollen are commercially available for diagnosis using single components or multiplex assays, except for Che a 1 which is solely present on a multiplex platform. Components are available as recombinant molecules (rPla I 1, rChe a 1, rPar j 2) or CCD (N-glycan)-free, natural molecules like nAmb a 1 (non-glycosylated) and nArtv 1 (O-glycosylated). To discriminate ragweed and mugwort primary sensitization, Art $\mathrm{v} 6$ and Amb a 4 would be additional useful diagnostic markers as they are homologs of the respective major allergens; however these components are not yet available for routine diagnosis. In the case of Sal k 1, falsepositive results might arise due to $\mathrm{N}$-glycosylation. Mer a 1 is so far the only allergen identified from annual mercury and owing to expected broad IgE cross-reactivity with other profilins should not be considered a marker allergen for the source (see C01).

\section{Clinical management}

Besides recommendations to generally limit exposure during pollen season, symptomatic treatment is considered a first line of defense for weed pollen allergic patients. In addition, allergen immunotherapy (AIT) is recommended based on the identification of the primary sensitizer using highly specific marker allergens. Weed allergic patients frequently present multiple sensitizations, and thus typically allergen sources triggering most profound symptoms 


\section{A) Ragweed and mugwort}

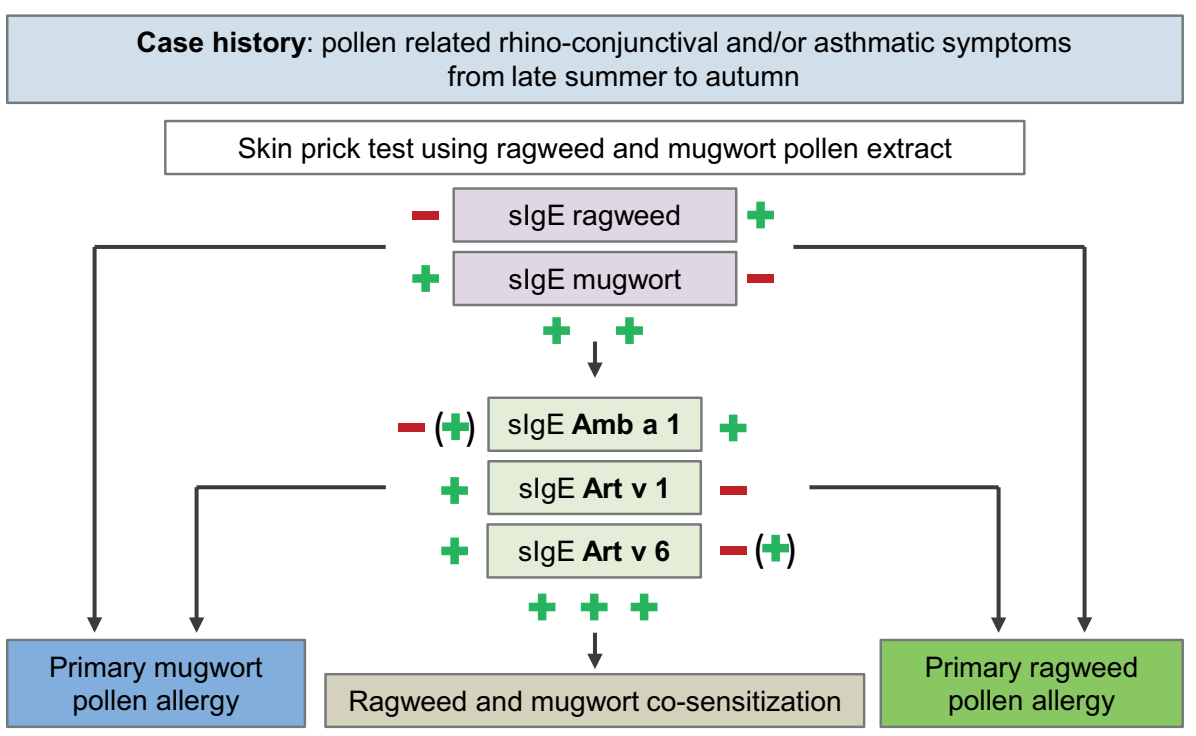

\section{B) Pellitory}
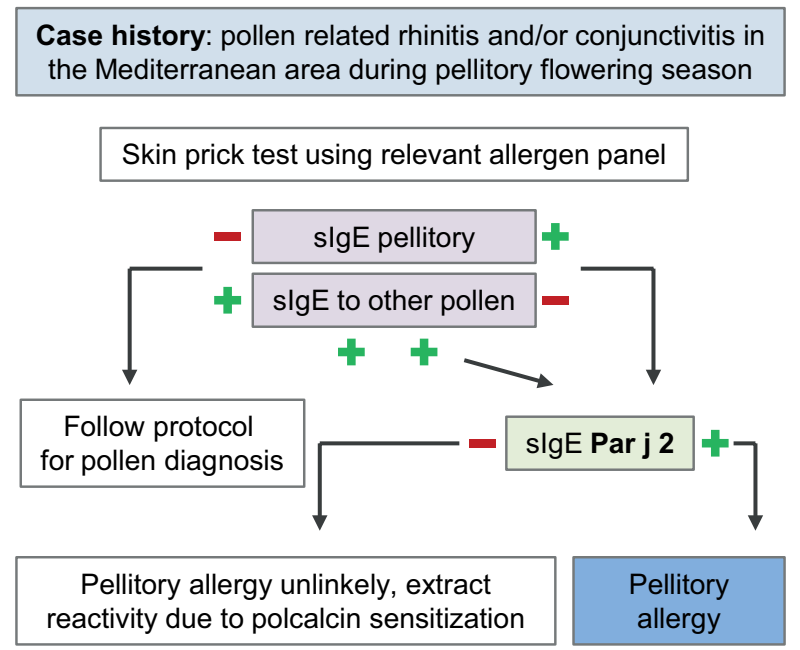

\section{C) English plantain}

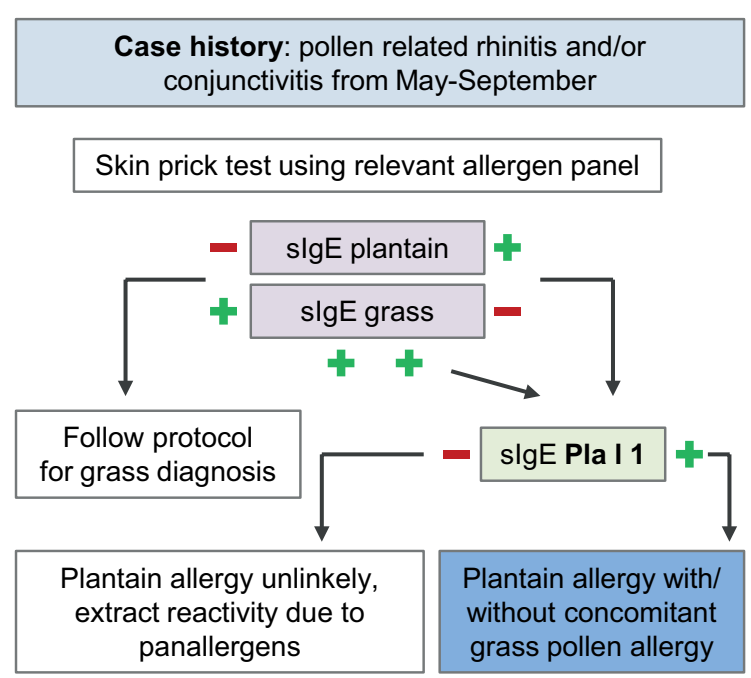

\section{Figure 3}

Diagnostic work-up for A) ragweed and mugwort pollen allergy, B) pellitory allergy and C) English plantain allergy. 


\section{Table 2}

Molecular components for diagnosis of weed pollen allergy

\begin{tabular}{|c|c|c|c|}
\hline Allergen source & $\begin{array}{l}\text { Specific lgE } \\
\text { component }\end{array}$ & Protein family & Diagnostic use \\
\hline Ragweed & Amb a 1 & Pectate lyase & $\begin{array}{l}\text { Marker for ragweed, IgE cross-reactivity with } \\
\text { Art } \vee 6 \text { from mugwort }\end{array}$ \\
\hline Ragweed & Amb a $4^{*}$ & $\begin{array}{l}\text { Defensin-like } \\
\text { protein }\end{array}$ & $\begin{array}{l}\text { Minor ragweed allergen with partial IgE cross- } \\
\text { reactivity to Art } v 1\end{array}$ \\
\hline Mugwort & Art v 1 & $\begin{array}{l}\text { Defensin-like } \\
\text { protein }\end{array}$ & $\begin{array}{l}\text { Marker for mugwort, partial cross-reactivity } \\
\text { with Amb a } 4\end{array}$ \\
\hline Mugwort & Art $v 3$ & nsLTP & $\begin{array}{l}\text { Cross-reactive with nsLTP from food e.g. Pru p } \\
3 \text { from peach or Cor a } 8 \text { from hazelnut }\end{array}$ \\
\hline Mugwort & Art $\vee 6^{*}$ & Pectate lyase & Amb a 1 cross-reactive allergen \\
\hline Pellitory & Par j 2 & nsLTP & $\begin{array}{l}\text { Highly specific marker allergen for pellitory } \\
\text { sensitization }\end{array}$ \\
\hline English plantain & Pla I 1 & Ole e 1-like protein & $\begin{array}{l}\text { Highly specific marker allergen for English } \\
\text { plantain }\end{array}$ \\
\hline Goosefoot & Che a $1^{\S}$ & Ole e 1-like protein & $\begin{array}{l}\text { Marker for goosefoot, partial IgE cross-reactivity } \\
\text { with minor Russian thistle allergen Sal k } 5\end{array}$ \\
\hline Russian thistle & Sal k 1 & $\begin{array}{l}\text { Pectin } \\
\text { methylesterase }\end{array}$ & $\begin{array}{l}\text { Marker allergen for Russian thistle allergy, } \\
\text { contains } N \text {-glycans (result might be false } \\
\text { positive if patient is CCD reactive) }\end{array}$ \\
\hline
\end{tabular}

nsLTP, non-specific lipid transfer protein. Marker allergens shown bold, ${ }^{*}$ not commercially available, Savailable only in multiplex analysis

are selected for therapeutic interventions. For subcutaneous immunization, 5 single extracts and 9 combination products are available and registered for the German market (www.pei.de).

However, economic considerations, regulation and standardisation requirements are prompting some providers to withdraw their weed pollen products. Various weed pollen solutions (mainly combination products) and recently also a tablet for sublingual immunotherapy of ragweed allergy are available in the U.S. (www.fda.gov).

\section{CLINICAL CASES}

\section{Case 1 (educational)}

Clinical history: A 42-year old man from Central Europe with increasing rhino-conjunctival symptoms from February to September for 12 years; additionally asthmatic symptoms for 2 years occurring only between May and September.

Test with extracts: Histamine-equivalent sensitization to tree pollen (hazel, alder, birch, ash) and to mugwort; weak skin prick reactivity to ragweed and goosefoot; strong sensitization to grass pollen. Specific IgE to birch (10.7 kU/L), ash (1.31 kU/L), grass (17.7 kU/L), mugwort (10.4 $\mathrm{kU} / \mathrm{L})$ and ragweed pollen $(1.26 \mathrm{kU} / \mathrm{L})$.

Test with molecules: The patient had positive IgE results to Bet v 1 (13.2 kU/L), Ole e $1(2.56 \mathrm{kU} / \mathrm{L})$, $\mathrm{Phl} \mathrm{p} 1$ (9.26 kU/L), Phl p 5 (3.58 kU/L), Art v 1 (12.8 kU/L), Art v 3 (10.8 kU/L) and Amb a 1 (0.68 $\mathrm{kU} / \mathrm{L})$.

Conclusion: The patient presents a complex pollen sensitization profile involving tree, grass and weed pollen. Symptoms in autumn are mainly caused by 

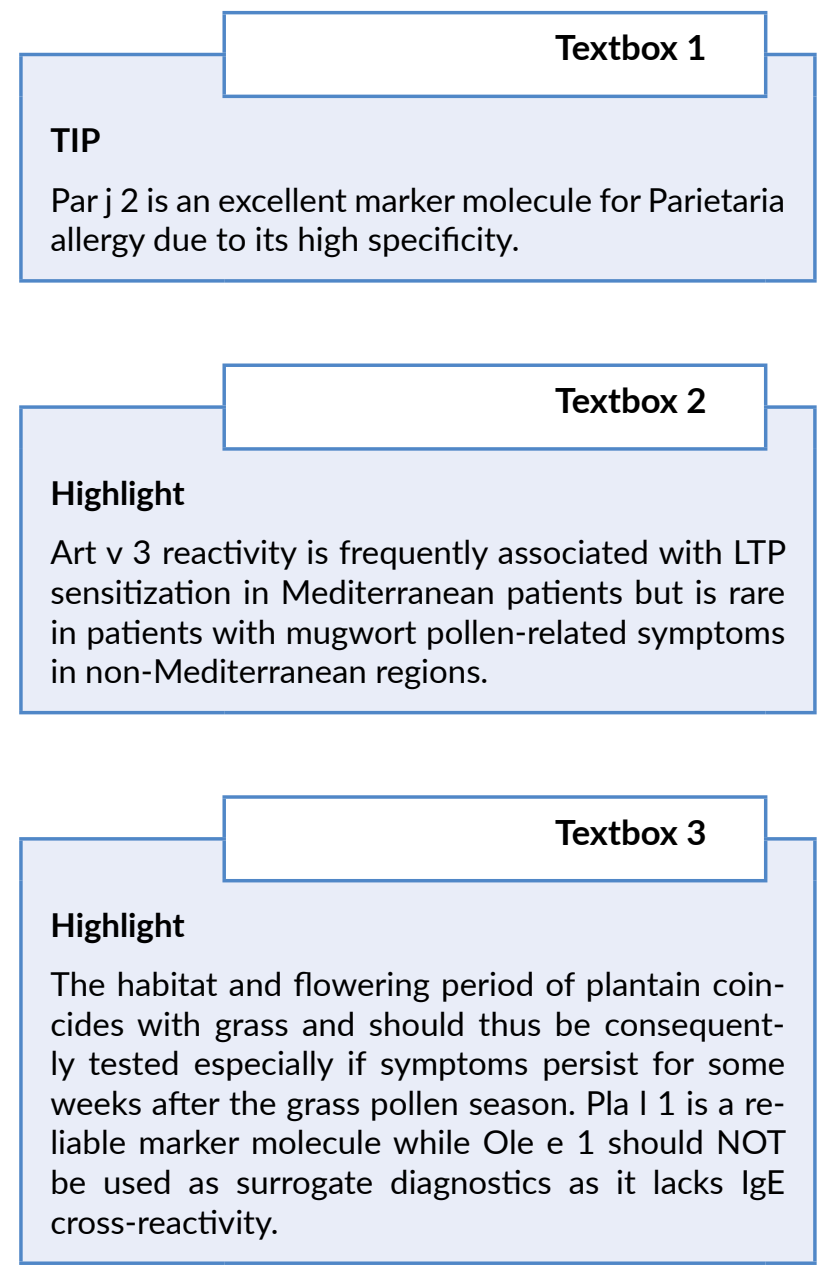

mugwort pollen; primary sensitization to ragweed is not indicated as Amb a 1-levels are very low and might arise due the cross-reactive molecule Art $v$ 6 in mugwort.

\section{Case 2 (original)}

Clinical history: A 20-year old woman from Western Austria with severe rhino-conjunctival symptoms from end of April to the middle of September since early childhood.

Test with extracts: The patient had a weak SPT positivity with hazel pollen, histamine-equivalent reactivity to grass pollen and strong sensitization to English plantain, all other standard inhalant allergens negative. Positive in vitro lgE test to grass $(14.2 \mathrm{kU} / \mathrm{L})$ and plantain pollen $(14.6 \mathrm{kU} / \mathrm{L})$.

Test with molecules: The patient had positive IgE results to $\mathrm{Phl} \mathrm{p} 1(9.72 \mathrm{kU} / \mathrm{L})$ and Pla I 1 (15.5 $\mathrm{kU} / \mathrm{L}$ ), while Phl p 7, Phl p 5, and Phl p 12 were negative. Multiplex results showed sensitization to Cyn d 1 (6.20 ISU), Phl p 1 (29.2 ISU), Phl p 2 (10.2 ISU), Phl p 11 (5.25 ISU) and Pla I 127.2 ISU.

Conclusion: In addition to grass pollen, the patient presents a weed pollen allergy to English plantain which explains symptoms observed in autumn after the grass pollen season.

\section{Case 3 (educational)}

Clinical history: A 36-year-old male living in northern Italy with a long lasting history of mild seasonal rhinitis from the beginning of May to the end of June experiences severe rhino-conjunctivitis associated with asthma at the beginning of March as he moves to Sicily. Gradual worsening symtpoms force the man to ask for assistance at an Emergency Department where systemic corticosteroids were administered.

Test with extracts: Strong skin reactivity to pellitory pollen was observed ( $12 \mathrm{~mm}$ mean wheal diameter) along with a weak sensitivity to grass pollen ( 3 $\mathrm{mm})$.

Test with molecules: The patient had positive IgE results to Par j 2 (37.3 kU/L), Phl p 1 (0.88 kU/L) and Phl p 5 (0.41 kU/L).

Conclusion: Allergy to pellitory pollen is diagnosed which was caused by high pollen exposure in Southern Italy.

\section{References}

1. Stemeseder, T, Hemmer W, Hawranek T, Gadermaier G. Marker allergens of weed pollen - basic considerations and diagnostic benefits in the clinical routine: Part 16 of the Series Molecular Allergology. Allergo J Int 2014;23:274-280.

2. Storkey J, Stratonovitch P, Chapman DS, Vidotto F, Semenov MA. A Process-Based Approach to Predicting the Effect of Climate Change on the Distribution of an Invasive Allergenic Plant in Europe. PLoS One 2014;9: e88156.

3. Gadermaier G, Hauser M, Ferreira F. Allergens of weed pollen: An overview on recombinant and natural molecules. Methods 2014;66:55-66.

4. Villalba M, Barderas R, Mas S, Colás C, Batanero E, Rodríguez R. Amaranthaceae pollens: review of an emerging allergy in the mediterranean area. J Investig Allergol Clin Immunol 2014;24:371-381.

5. Bouley J, Groeme R1, Le Mignon M1, Jain K1, Chabre H1, Bordas-Le Floch V1 et al. Identification of the cysteine protease Amb a 11 as a novel major allergen from short ragweed. J Allergy Clin 
Immunol 2015;136:1055-1064.

6. Bousquet PJ, Chinn S, Janson C, Kogevinas M, Burney P, Jarvis $D$ et al., Geographical variation in the prevalence of positive skin tests to environmental aeroallergens in the European Community Respiratory Health Survey I. Allergy 2007;62:301-309.

7. Ruëff F, Przybilla B, Walker A, Gmeiner J, Kramer $M$, Sabanés-Bové $D$ et al. Sensitization to common ragweed in southern Bavaria: clinical and geographical risk factors in atopic patients. Int Arch Allergy Immunol 2012;159:65-74.

8. Li J, Sun B, Huang Y, Lin X, Zhao D, Tan G et al, A multicentre study assessing the prevalence of sensitizations in patients with asthma and/or rhinitis in China. Allergy 2009;64:1083-1092.

9. Hao GD, Zheng YW, Gjesing B, Kong XA, Wang JY, Song ZJ et al. Prevalence of sensitization to weed pollens of Humulus scandens, Artemisia vulgaris, and Ambrosia artemisiifolia in northern China. J Zhejiang Univ Sci B 2013;14:240-246.

10. Barber D, de la Torre F, Lombardero M, Antépara I, Colas C, Dávila I, et al. Component-resolved diagnosis of pollen allergy based on skin testing with profilin, polcalcin and lipid transfer protein pan-allergens. Clin Exp Allergy 2009;39:1764-1773.

11. Bass D, Delpech V, Beard J, Bass P, Walls RS. Ragweed in Australia. Aerobiologia 2000;16:107-111.

12. Atis $S$, Tutluoglu $B$, Sahin $K$, Yaman $M$, Küçükusta AR, Oktay I. Sensitization to sunflower pollen and lung functions in sunflower processing workers. Allergy 2002;57:35-39.
13. Kumar S, Khandpu S, Rao DN, Wahaab S, Khanna N. Immunological response to Parthenium hysterophorus in Indian Patients with Parthenium sensitive atopic dermatitis. Immunol Invest 2012;41:75-86.

14. D'Amato, G., et al., Allergenic pollen and pollen allergy in Europe. Allergy 2007;62:976-990.

15. Gadermaier G, Eichhorn S, Vejvar E, Weilnböck L, Lang $\mathrm{R}$, Briza $\mathrm{P}$ et al. Plantago lanceolata: An important trigger of summer pollinosis with limited IgE crossreactivity. J Allergy Clin Immunol 2014;134:472-475.

16. Barber D, de la Torre F, Feo F, Florido F, Guardia P, Moreno $\mathrm{C}$ et al. Understanding patient sensitization profiles in complex pollen areas: a molecular epidemiological study. Allergy 2008;63:1550-1558.

17. Gruber P, Gadermaier G, Bauer R, Weiss R, Wagner $S$, Leonard $R$ et al. Role of the polypeptide backbone and post-translational modifications in cross-reactivity of Art $\vee 1$, the major mugwort pollen allergen. Biol Chem 2009;390:445-51.

18. Asero R, Bellotto E, Ghiani A, Aina R, Villalta D, Citterio $\mathrm{S}$. Concomitant sensitization to ragweed and mugwort pollen: who is who in clinical allergy? Ann Allergy Asthma Immunol 2014;113:307-313.

19. Egger M, Mutschlechner S, Wopfner N, Gademeier G, Briza P, Ferreira F. Pollen-food syndromes associated with weed pollinosis: an update from the molecular point of view. Allergy 2006;61:461-476.

20. Asero, R., R. Monsalve, D. Barber. Profilin sensitization detected in the office by skin prick test: a study of prevalence and clinical relevance of profilin as a plant food allergen. Clin Exp Allergy 2008;38:1033-1037. 


\section{DUST MITE ALLERGY}

\author{
Thomas Platts-Mills, Luis Caraballo
}

\section{THE ALLERGEN SOURCES}

Arthropods are divided into five major classes of which three play an important role in allergic disease. Thus both insects and arachnids play important roles, as inhalant allergens while the crustacean are an important source of food allergens. The arthropods separated from other animals approximately 600 million years ago and the major classes were established within 100 million years (Fig. 1). Thus dust mites and cockroaches have been separated for at least 400 million years and it is no surprise that most of these allergens are so different in their primary sequences that they do not cross react. This should be compared to the mammals, which separated approximately 65 million years ago (MYA) and still have extensive similarity between proteins. Of the hundreds of thousands of arthropod species only a few have been recognized as significant sources of indoor allergens. Indeed greater than $90 \%$ of the literature relates to five genera: Dermatophagoides, Blomia, Euroglyphus, Blatella and Periplaneta.

Since this and the next section (Cockroach Allergens) have several points in common a brief introduction is pertinent. The important parallel between mites and
House dust allergy is an important risk factor for asthma and rhinitis

Most recognized house dust mites are Dermatophagoides pteronyssinus, Dermatophagoides farinae and Blomia tropicalis

In the majority of cases skin test with mite extracts is able to detect sensitization and define the specificity of immunotherapy in asthmatic patients

The use of species specific components might be necessary in places like the Tropics where coexposure to non-crossreacting mites genera is common 


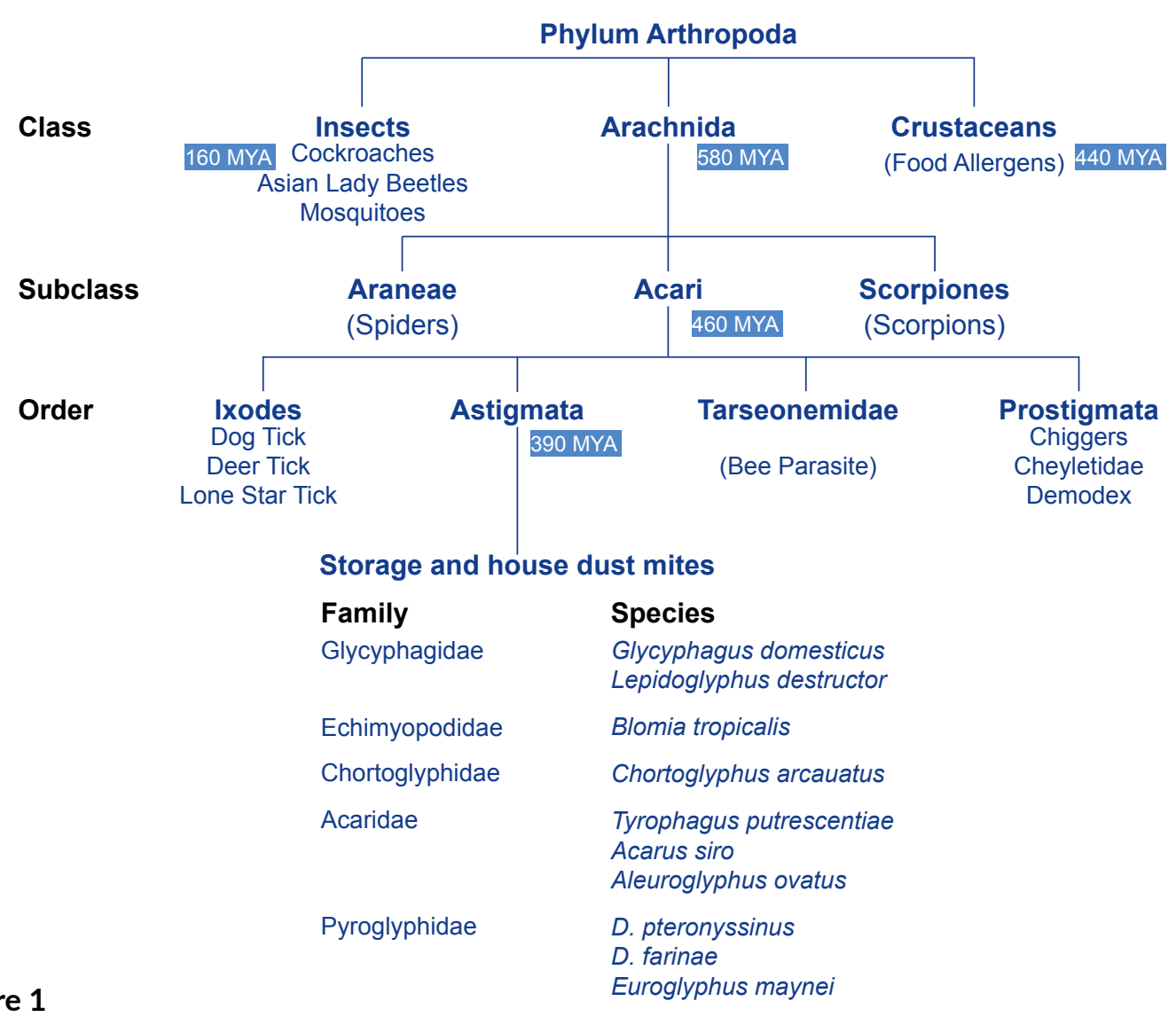

Figure 1

Clinically relevant members of Arthropoda, including storage and house dust mites.

cockroaches is that they each can play a very important role in asthma, without their role being obvious to the patients. While there are many possible explanations for this the most obvious are that exposure is perennial, that the organisms are small and often not noticed and that the particles that carry these allergens are sufficiently large that they do not remain airborne for more than a few minutes after disturbance $(1,2)$. Because the role of exposure to mite or cockroach allergens was not obvious to the patients these species have been central to the arguments about the causal role of allergen exposure in asthma. The relevance of particle size became obvious shortly after the purification of Der $p 1$ and the development of accurate assays for this allergen (3). For $D$. pteronyssinus and $D$. farinae the faecal particles are the cardinal form in which the allergens became airborne $(1,4)$. For cockroach, the dynamics of airborne exposure are similar but the particle is not so well defined (2). The faeces of cockroaches are usually too large being visible like ground pepper i.e. > 100 micron in diameter. Because the particles are not well defined, there is less detailed information about the elements that are inhaled together with cockroach allergens.

The focus on particles carrying allergens is because of the awareness that due to protein molecule size of most allergens, that is, $15,000-50.000 \mathrm{Da}$, they are not volatile, so that significant exposure can only occur on particles. The nature of these particles is relevant to both the induction of the IgE response and the subsequent contribution to inflammation of the nose and lungs. For dust mite particles, it is clear that they not only carry a high concentration of several allergens but also are an important source of other substances both nucleic acids and proteins that act as toll receptor ligands or PAMPS. While this has been well recognized for mites, it may be just as important for cockroach allergens.

A significant feature of the epidemiology of these two sources of allergens is that there are areas of the world where one, both or neither of these two sources are important (Table 1). Both climate and housing conditions play a major role in these differences. However, there 


\section{Table 1}

Environments Where Mites and Cockroaches Play Dramatically Different Roles in Relation to Asthma

I. Dominant Dust Mite: New Zealand, Australia, Europe (West and Central) England, Scotland have temperate climates with damp houses in a climate where because of the climate cockroaches do not flourish outside and most families live in separate homes.

II. Dominant Cockroach Allergens: United States inner city apartments in the North e.g. Chicago or New York, where buildings are heated and very dry so that cockroaches can thrive but mites do not. Many areas of South Asia

III. Both Cockroach and Mite Allergens: United States inner city apartments or houses e.g. New Orleans or Atlanta, but also many areas of South America where homes are both warm and damp so that both species thrive;

IV. either of these arthropods are relevant: Northern areas of Europe e.g. Norbotten where the climate is extremely dry so that mites cannot survive the winter and the outside is too cold for cockroaches.

is also a significant biological difference between the two groups of arthropods. Cockroaches can search for a water source in a building and will find a leaking tap or drip pan behind a refrigerator. By contrast, mites absorb moisture from their environment and are absolutely dependent on the level of humidity in the air or on either carpets or upholstered material, which will retain humidity for long periods of time.

Before 1960, it was well recognized that house dust contained allergens other than those derived from domestic or pet animals as well as pollens or fungi; indeed several groups had attempted to identify a house dust atopen by immunochemical analysis of house dust extracts. However, the breakthrough was made by microscopic identification of dust mites' in house dust. It was fitting that these observations were made in the Netherlands since Van Leeuwenhoek had first described mites in 1693. In addition, to demonstrating that mites of the genus Dermatophagoides were the major source of allergens in house dust Spieksma and Voorhorst also developed the technique for culturing these organisms (5). This in turn made it possible to manufacture dust mite extracts for commercial use and subsequently facilitated the purification of mite allergen (3).
Table 2

The Best Recognized Mites Found in House Dust

\begin{tabular}{|lc|}
\hline Mite Species & $\begin{array}{c}\text { Defined } \\
\text { Allergens }\end{array}$ \\
\hline House Dust Mites & \\
\hline Dermatophagoides pteronyssinus & 17 \\
\hline Dermatophagoides farinae & 27 \\
\hline Euroglyphus maynei & 5 \\
\hline Blomia tropicalis & 13 \\
\hline Storage Mites & \\
\hline Acarus siro & 1 \\
\hline Glycyphagus domesticus & 1 \\
\hline Lepidoglyphus destructor & 5 \\
\hline Tyrophagus putrescentior & 5 \\
\hline
\end{tabular}

Number of specific allergens from each species recognized in www.allergen.org

Dust mites belong to the order Astigmata, which is part of the Arachnids (Fig. 1). This order includes not only the Pyroglyphidae and at least four families of storage mites (Echimyopodidae, Acaridae, Glycyphagidae and Chortoglyphidae) but also Sarcoptes scabiei (the scabies mite). Many different mites have been identified in house dust but only a limited number have been related to allergic disease. The mites generally recognized as house dust mites are: D. pteronyssinus, D. farinae, Euroglyphus maynei, and Blomia tropicalis. However, four species of storage mites, which are best recognized as pests on farms or in food storage have also been recognized in house dust (Table 2). In addition, mites of the family Tarsonemidae may be found in significant numbers in house dust. The best evidence that a given mite species is relevant to allergic disease comes from studies in an area where a given species dominates all other mites in the house dust. This is true for $D$. pteronyssinus in the UK, and New Zealand, for $D$. farinae in some areas of the United States, and for Blomia tropicalis in areas of South America and other tropical areas (6, 7). In high altitude location such as the Alpes, specific species (Euroglyphus manei) are found. Representative allergens from $B$. tropicalis and D. pteronyssinus are shown in Fig. 2. 


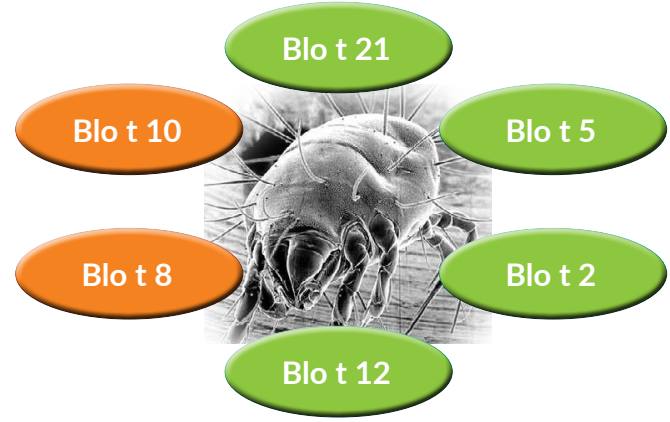

Blomia tropicalis

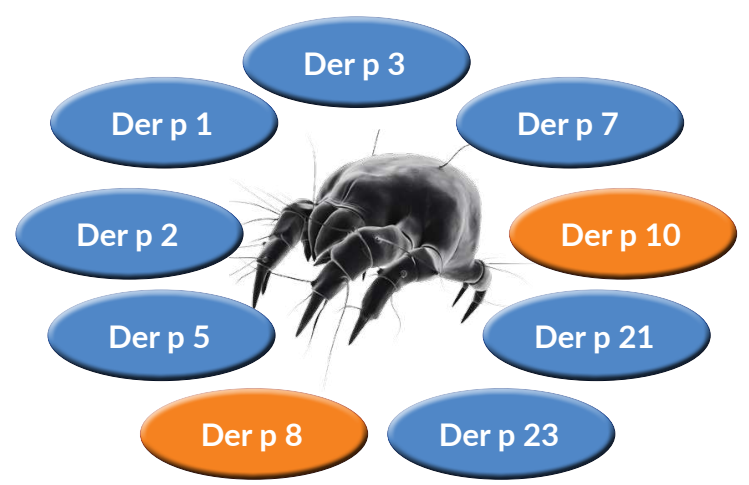

Dermatophagoides pteronyssinus

\section{Figure 2}

Clinically relevant house dust mite allergens. Those with experimentally detected cross-reactivity are shown in orange.

\section{MAJOR AND RELEVANT MINOR ALLERGENIC MOLECULES}

The first dust mite allergens were purified in 1980 and 1982 using classical immunochemical techniques without the benefit of monoclonal antibodies, cloning or modern sequencing techniques $(3,8)$. By contrast the purification of the Group 2 allergens Der $\mathrm{p} 2$ and Der $\mathrm{f} 2$ in 1989 was aided by the development of a monoclonal antibody $(9,10)$. Considerable effort has been made to keep the nomenclature of mite allergens consistent within groups so that Groups 1, 2, 3, 4, 5 and 10 include similar molecules purified from each of these species (Table 3 ). Over the last 20 years, the techniques for cloning and sequencing proteins have become well defined and much simpler. As a result many mite derived proteins have been described with convincing data about their amino acid sequence and in many cases about their tertiary structure. However, the evidence about allergenicity is less robust, and in some cases is limited to non-quantitative immunoblots on a small series of sera. Given the large number of allergens that have been described it is not possible to give a realistic assessment of the clinical significance of the allergens such as Der $\mathrm{f}$ 13-22, and Der $\mathrm{f}$ 24-33 (see www.allergen.org).

For the major allergens in Group 1 and Group 2, we have evidence from random cohorts and birth cohorts as well as measurements of airborne and dust allergens $(11,12)$. Der $p 1$ and Der $p 2$ are present in fecal particles and are strongly associated with asthma. In addition, for Der $\mathrm{p} 1$, there is specific evidence about the effects of reducing exposure from $13 \mu \mathrm{g} / \mathrm{g}$ to $0.2 \mu \mathrm{g} / \mathrm{g}$ on symptoms among mite allergic individuals $(13,14)$. Thus for these allergens there is good evidence that they have an important role in the symptoms of rhinitis and asthma. For other allergens there may be excellent evidence about the prevalence of sensitization but less complete basis for judging their role in disease. Clearly, it is not possible to have an accurate assessment of the relative importance of more than ten different proteins derived from mites. Interestingly, there are two relatively recent identifications, which may have significance. Der $p$ 11 was defined several years ago and is distinctive because of the high molecular weight i.e. 95-100 kDA (15). However in 2014, it was reported that IgE antibodies to Der p 11 are more common in sera from patients with Atopic Dermatitis (AD). Thus sensitization to this allergen may reflect the fact that the eczematous skin allows easy penetration of allergens even with molecular weight as high as 100,000 (15). Blo t 12 is an interesting allergen that has different isoforms in Colombia and Singapore (16). Der p 21 and Blo t 21 have high prevalence of sensitization in allergic children. The other recently described mite allergen is Der $\mathrm{p} 23$, which appears to be a major allergen associated with asthma and has a molecular weight of $8 \mathrm{KD}$. The sequence of Der $p$ 23 identifies it as a peritrophin-like protein and it is found in the peritrophic lining of the gut as well as in fecal particles (17). 


\section{Table 3}

Major and relevant minor mite-allergens for $D$. pteronyssinus, $D$. farinae and B. tropicalis species

\begin{tabular}{|llcc|}
\hline Allergenic molecule & Biochemical name & $\begin{array}{c}\text { Prevalence among } \\
\text { patients (\%) }\end{array}$ & $\begin{array}{c}\text { Molecular } \\
\text { Weight (kDa) }\end{array}$ \\
\hline Der p 1, Der f 1, Blo t 1 & Cysteine protease & $70-100$ & $24-27$ \\
\hline Der p 2, Der f 2, Blo t 2 & $\begin{array}{l}\text { NPC2 protein family (epidermal } \\
\text { secretory proteins) }\end{array}$ & $80-100$ & $15-26$ \\
\hline Der p 3, Der f 3, Blo t 3 & Trypsin-like protein & $16-100$ & $29-31$ \\
\hline Der p 4, Blo t 4 & Alpha-amylase & $25-46$ & 60 \\
\hline Der p 5, Blo t 5 & & $50-70$ & 14 \\
\hline Der p 7, Der f 7 & Lipid binding protein & 50 & $26-31$ \\
\hline Der p 8, Der f 8, Blo t 8 & Glutathione S-transferase & 40 & 27 \\
\hline Der p 10, Der f 10, Blo t 10 & Tropomyosin & $5-18,50-95$ & 36 \\
\hline Der p 11 & Paramyosin & 80 & 103 \\
\hline Blo t 12 & Chitin binding protein & 50 & 14 \\
\hline Der p 21, Blo t 21 & & & 13.2 \\
\hline Der p 23 & Peritrophin-like protein & 74 & 8 \\
\hline
\end{tabular}

\section{SENSITIZATION TO INDIVIDUAL MOLECULES AND ITS CLINICAL SIGNIFICANCE}

\section{Fecal particles as the major airborne form of mite allergens}

Mite fecal particles consist of an amorphous mass of partially digested skin scales and other food materials surrounded by a chitinous peritrophic membrane. These particles contain mite DNA, bacteria, bacterial DNA, bacterial products such as endotoxin as well Group I and Group II allergens and in the case of $D$. pteronyssinus Der $\mathrm{p}$ 23. Airborne measurements of particle size in homes suggest that these particles maintain a size of $>10$ microns and microscopic examination suggest that in general they maintain the structure due to the strength of the peritrophic membrane (4). Thus the diameter of these particles ranges from 15-30 microns, which is in keeping with the fact that most dust mite allergen falls within 20 minutes after a vigorous domestic disturbance (1). In turn, this is in keeping with the fact that very few mite allergic patients report onset of symptoms occurring within minutes of entering a house, which is infested with mites.
The biological and immunological significance of the mite particles includes both the properties of mite allergens and the associated constituents. Not only do the particles contain a very high concentration of mite allergen e.g. Der $p$ 1, Der $p 2$, and Der $p 23$ but they also contain ligands for TLR 4, TLR 9 and Dectin I. Given that Der p 1 is an active cysteine protease and has been shown to cleave CD 25 and CD 23, while Der p 2 has activity comparable to $M D 2$, it is obvious that these particles have different constituents that can contribute to the allergenicity. Furthermore, it is obvious that the constituents of the particle are released rapidly after contact with a liquid milieu such as the mucosal membrane of the nose or the lung lining fluid (1).

\section{CLINICAL MANAGEMENT}

\section{Diagnosis of Sensitization}

As was stated in the introduction the role of dust mite allergy in asthma is generally not obvious to the patients. Indeed histories of specific allergy to mites are not usually clear. Many patients will report sneezing on awakening or sneezing during house cleaning. During vigorous cleaning they may also 
notice eye irritation or wheezing, but conjunctivitis is not a common symptom of dust mite allergy. Skin prick testing is the primary means of diagnosis, and dust mite extracts are included in all inhalant panels. Most authorities would regard a wheal of $3 \mathrm{~mm}$ greater than the negative control as positive. The common practice is to test with both $D$. pteronyssinus and $D$. farinae. In some areas one mite or the other is the dominant cause of sensitization, but in general the results of the two provide convincing positive or negative results. However, D. pteronyssinus extracts might lack important allergens and often show great variability regarding allergen composition (18). Also, testing with B. tropicalis has become a very useful routine in the tropics. In vitro assays for lgE to dust mite are well established and the units are given in $\mathrm{IU} / \mathrm{ml}$ or kUnits /L. In vitro testing for specific IgE can be done using extracts of $D$. pteronyssinus (Dp), $D$. farinae (Df) and B. tropicalis (Bt). Assays for IgE to components (Der p 1, Der p 2, Der p 10, Blo t 5) are also available. There is good evidence that the units used for IgE ab are the same as the units used for IgE i.e. 2.4 ng. Serum assays can provide a wide range of positive results from $0.1 \mathrm{IU} / \mathrm{ml}$ to $>300 \mathrm{IU} / \mathrm{ml}$. There is also good evidence that the size of the wheal or the titer of IgE is a useful predictor of the risk of allergic disease and also of the scale of the added risk with rhinovirus infection (19).

Although the criteria for judging sensitization can be defined convincingly, it is not so easy to define criteria for judging the role of dust mite in individual cases. There are several things that can help. If the patient is only allergic to mite, or the skin tests or IgE assays are much stronger for mite than for those other allergens, which could be relevant for perennial symptoms. Although not widely accepted as a diagnostic procedure nasal provocation test with mite extracts is other important tool for defining the clinical relevance of sensitization and detecting cases of local rhinitis associated to negative skin tests. A measurement of mite allergens in dust from the house can be very helpful. However, the criteria of $>2 \mu \mathrm{g}$ Der $\mathrm{p} 1$ per gram of dust for sensitization and $>10 \mu \mathrm{g}$ Der $\mathrm{p} 1$ per gram of dust for severe symptoms should not be regarded as more than a guide. It is a major advantage to know what the average levels of mite allergen are in homes or apartments in the areas where the patient lives (Table 1).

Cross reactivity between $D$. pteronyssinus and $D$. farinae extracts is high but between Dermatophagoides and Blomia tropicalis is low. The use of species-

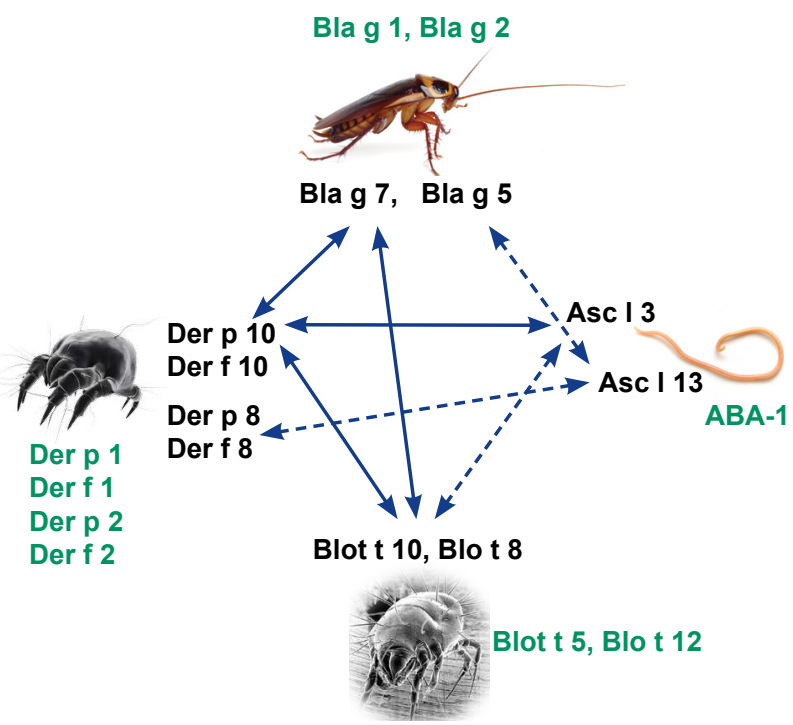

Figure 3

Clinically relecat cross-reactivity of mite allergens. Species-specific components are shown in green.

specific components might be necessary in places like the tropics where co exposure to both genera is common. Tropomyosin is the main cause of cross reactivity among mites, cockroaches, shellfish and helminths (e.g. Ascaris lumbricoides), but Glutathione Transferase may also be involved (Fig. 3).

In the majority of cases skin test with mite extracts is able to detect sensitization and define the specificity of immunotherapy in asthmatic patients. However, the use of component resolved diagnosis could be useful in especial circumstances where genuine sensitization is not clear and has to be defined. Figures 4 and 5 represent algorithms that could be applied for diagnosing mite allergy in temperate and tropical countries. Not all suggested components are commercially available.

\section{Management}

The management of allergic disease in patients who are allergic to dust mites consists of several different phases most of which are similar to those for many other inhalant allergens (Table 4). However education and allergen avoidance require extra care because of the complex biology of dust mites, the fact that their presence in the home is not visible to the patients and the need for significant education in relation to avoidance. 


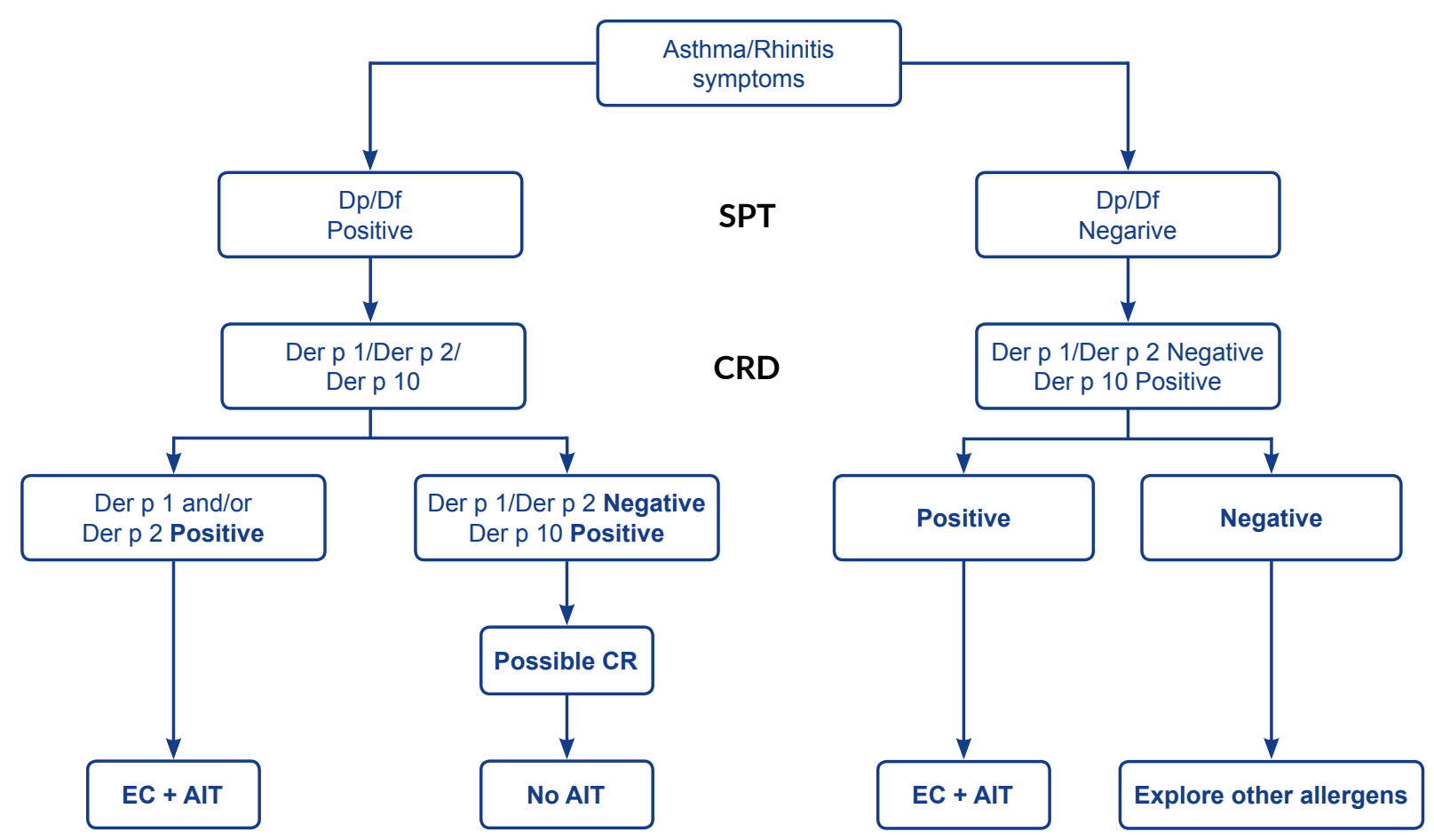

Figure 4

Possible decision algorithm for mite allergy in temperate countries. SPT: Skin Prick Test. CRD: Component Resolved Diagnosis. CR: Cross-reactivity. EC: Environmental Control. AIT: Allergen Immunotherapy.

There is a wide range of evidence that dramatically decreasing exposure to dust mite allergens can help both asthma and rhinitis related to dust mites. This comes both from controlled trials of avoidance and from moving patients to a sanatorium or to a hospital based allergen "free" unit $(13,14,20,21)$. Some of the most dramatic results have come from Sanatoria in the Alps, but these are complicated to interpret because exposure to animal dander and fungi as well as mites will be reduced (21-23). In addition, most of these Sanatoria have regular exercise regimes, which may also contribute to the improved lung function and to the decrease in non-specific bronchial hyper-reactivity (BHR) (21). Following the initial study in Davos further studies were carried out in Briancon in France and Misurina in Italy. The studies in Misurina provided compelling evidence that there was a progressive decrease in inflammatory markers in parallel with decreases in BHR among mite allergic children who spent 3 months in the sanatorium. In order to study the role of mite allergens, mite allergic asthmatics in London spent 3 or more months living in a hospital room which had filtered air and was designed to have no sites where

\section{Table 4}

Management of Allergic Disease Related to Dust Mite
A. Skin tests or serum assays for lgE antibodies using dust mite extract. CRD could be helpful to define allergen immunotherapy
B. Education about the role these allergens can play in both acute and chronic symptoms
C. Advice about avoidance including a written plan and in some cases measurement of mite allergen in houses
D. A plan for pharmaceutical management both for the nose and the lungs
E. Subcutaneous or sublingual Immunotherapy using dust mite allergen
F. In cases of asthma that are poorly controlled treatment with Anti-lgE (omalizumab) may also be recommended




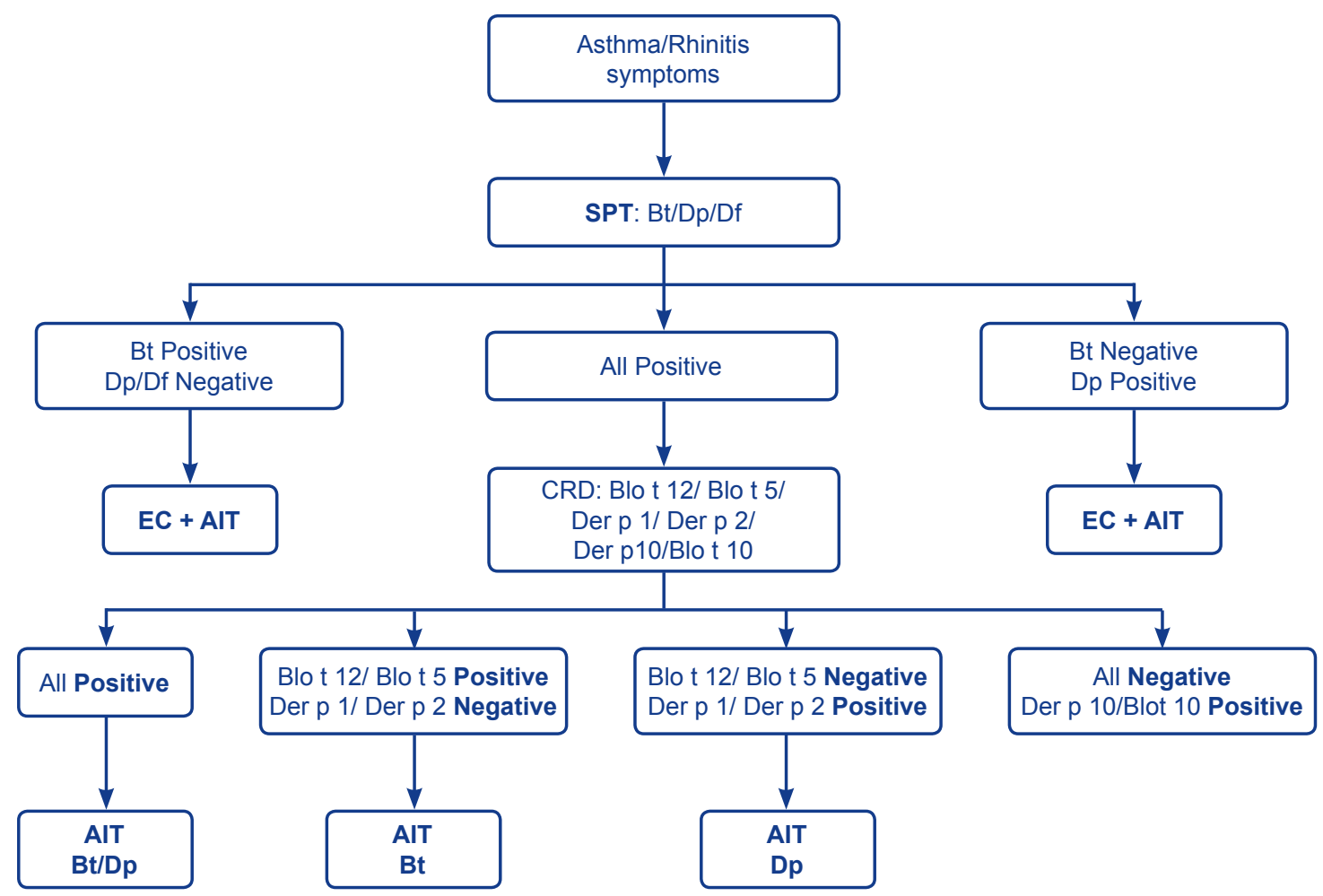

Figure 5

Possible decision algorithm for mite allergy in the tropics. SPT: Skin Prick Test. CRD: Component Resolved Diagnosis. CR: Cross-reactivity. EC: Environmental Control. AIT: Allergen Immunotherapy.

mites could live. The level of mite allergen in dust from their homes was $13.6 \mu \mathrm{g}$ Der $\mathrm{p} 1 / \mathrm{g}$, while dust from the hospital room had less than $0.2 \mu \mathrm{g} / \mathrm{g}$ dust. The patients not only improved their symptoms but also experienced a major decrease in BHR (13).

\section{CLINICAL CASES}

\section{Case 1}

Clinical history: A 32-year old male faculty member in cardiology presented to clinic because of increasing episodes of shortness of breath (SOB) during exercise. He was an enthusiastic runner (up to 10 miles) and had only developed symptoms since moving into a basement apartment one year earlier. His history did not include seasonal nasal symptoms or reactions on exposure to animals. When seen in clinic, his examination and spirometry were normal.
Test with extracts: Prick skin tests were strongly positive for $D$. pteronyssinus and $D$. farinae with $8 \times 8$ and $7 \times 6 \mathrm{~mm}$ wheals. Blood count was unremarkable with AEC of 350. Total IgE 230 $\mathrm{kUA} / \mathrm{L}$; IgE to D. pteronyssinus was $32 \mathrm{UA} / \mathrm{ml}$. He was given a peak flow meter (Mini Wright) and instructed to record values before and after running, in addition, we arranged to collect samples from his apartment.

Test with molecules: Serum assayed for components using ISAC chip showed Der p 1 IgE 28 ISU/ml; Der p 233 ISU/ml ; Der p 10 (tropomyosin) <0.5 $\mathrm{ISU} / \mathrm{ml}$. Peak flow values mean of 3 values, before running was $510+20$. Liters $/ \mathrm{min}$ and fell to 400 $+40 / \mu$ Liters/min and $320+20$, Liters/min 2 minutes and 4 minutes after running for 6 minutes.

Dust samples from his apartment: $8.4 \mu \mathrm{g}$ Der $\mathrm{p} 1 / \mathrm{g}$ carpet dust, $10.6 \mu \mathrm{g}$ Der $\mathrm{p} \mathrm{1/g}$ sofa dust and 4.6 $\mu \mathrm{g}$ Der $\mathrm{p} 1 / \mathrm{g}$ bedding dust 
Treatment Advice and Outcome: Initially he was treated with albuterol inhaler, two puffs $10 \mathrm{~min}$. prior to exercise and inhaled Fluticasone 100 $\mu \mathrm{g}$ bid. In addition, he was advised to move to a second floor apartment without carpeting and with minimal upholstered furniture. He was given routine advice about controlling mites in his bedding. He moved one month later and within 3 months his exercise breathing returned to normal. When seen 1 year later, he was no longer using inhalers and was without significant symptoms.

\section{References}

1. Tovey ER, Chapman MD, Wells CW, Platts-Mills TA. The distribution of dust mite allergen in the houses of patients with asthma. Am Rev Respir Dis 1981;124:630-635.

2. de Blay F, Sanchez J, Hedelin G, Perez-Infante A, Verot A, Chapman MD et al. Dust and airborne exposure to allergens derived from cockroah (Blattella germanica) in low-cost public housing in Strasbourg (France). $J$ Allergy Clin Immunol 1997;99:107-112.

3. Chapman MD, Platts-Mills TA. Purification and characterization of the major allergen from Dermatophagoides pteronyssinus-antigen P1. J Immunol 1980; 125:587-92.

4. Tovey ER, Chapman MD, Platts-Mills TA. Mite faeces are a major source of house dust allergens. Nature 1981;289:592-593.

5. Spieksma F, Voorhorst R. Comparison of skin reactions to extracts of house dust, mites, and human skin scales. Acta Allergol 1969;24:124-146.

6. Caraballo L, Puerta L, Martínez B, Moreno L. Identification of allergens from the mite Blomia tropicalis. Clin Exp Allergy 1994;24:1056-1060.

7. Arruda LK, Fernandez-Caldas E, Naspitz CK, Montealegre F, Vailes LD, Chapman MD. Identification of Blomia tropicalis allergen Blo 5 by cDNAcloning. Int Arch Allergy Appl Immunol 1995;107:456-457.

8. Dandeu J, Le Mao J, Lux M, Rabillon J, David B. Antigens and allergens in Dermatophagoides farinae mite. II. Purification of $A G$ f1, a major allergen in Dermatophagoides Farinae. Immunology 1982;46:679-687.

9. Heymann PW, Chapman MD, Aalberse RC, Fox JW, Platts-Mills TA. Antigenic and structural analysis of group II allergens (Der f II and Der $p$ II) from house dust mites (Dermatophagoides spp). J Allergy Clin Immunol 1989;83:1055-1067.

10. Stewart GA, Ward LD, Simpson RJ, Thompson PJ. The group III allergen from the house dust mite Dermatophagoides pteronyssinus is a trypsin-like enzyme. Immunology 1992;75:29-35.
11. Sporik R, Holgate ST, Platts-Mills TA, Cogswell JJ. Exposure to house-dust mite allergen (Der $\mathrm{pl}$ ) and the development of asthma in childhood. A prospective study. N Engl J Med 1990;323:502-507.

12. Fernández-Caldas E, Puerta L, Caraballo L, Lockey RF. Mite allergens. Clin Allergy Immunol 2008;21:161182.

13. Platts-Mills TA, Tovey ER, Mitchell EB, Moszoro $\mathrm{H}$, Nock P, Wilkins SR. Reduction of bronchial hyperreactivity during prolonged allergen avoidance. Lancet 1982;2:675-678.

14. Platts-Mills TA, Vervloet D, Thomas WR, Aalberse RC, Chapman MD. Indoor allergens and asthma: report of the Third International Workshop. J Allergy Clin Immunol 1997;100:S2-24.

15. Banerjee S, Resch Y, Chen K, Swoboda I, Focke-TejkI $\mathrm{M}$, Batt $\mathrm{K}$ et al. Der $\mathrm{p} 11$ is a major allergen for house dust mite-allergic patients suffering from atopic dermatitis. J Invest Dermatol 2015;135:102-109.

16. Zakzuk J, Jimenez S, Cheong N, Puerta L, Lee BW, Chua KY et al. Immunological characterization of a Blo t 12 isoallergen: identification of immunoglobulin E epitopes. Clin Exp Allergy 2009;39:608-616.

17. Weghofer M, Grote $M$, Resch $Y$, Casset A, Kneidinger $M$, Kopec J et al. Identification of Der p 23, a peritrophin-like protein, as a new major Dermatophagoides pternonyssinus allergen associated with the peritrophic matrix of mite fecal pellets. J Immunol 2013;190:3059-3067.

18. Casset A, Mari A, Purohit A, Resch Y, Weghofer $M$, Ferrara $R$ et al. Varying allergen composition and content affects the in vivo allergenic activity of commercial Dermatophagoides pteronyssinus extracts. Int Arch Allergy Immunol 2012;159:253-262.

19. Soto-Quiros M, Avila L, Platts-Mills TA, Hunt JF, Erdman DD, Carper $\mathrm{H}$ et al. High titers of IgE antibody to dust mite allergen and risk for wheezing among asthmatic children infected with rhinovirus. J Allergy Clin Immunol 2012;129:1499-1505.

20. Platts-Mills TA, Thomas WR, Aalberse RC, Vervloet $\mathrm{D}$, Champman MD. Dust mite allergens and asthma: report of a second international workshop. J Allergy Clin Immunol 1992;89:1046-1060.

21. Kerrebijn K. Endogenous factors in childhood CNSLD: methodological aspect in population studies. Royal Van Gorcum, Assen (The Netherlands) 1970:38-48.

22. Charpin D, KLleisbauer J, Lanteaume A, Razzouk $H$, Vervloet $D$, Toumi $M$ et al. Asthma and allergy to house-dust mites in populations living in high altitudes. Chest 1988;93:758-761.

23. Piacentini G, Del Giudice M, Bodini A, Costella S, Vicentini L, Peroni $D$ et al. Exhaled NO reduced on allergen avoidance. Allergy 2001;56:251-252. 



\title{
COCKROACH ALLERGY
}

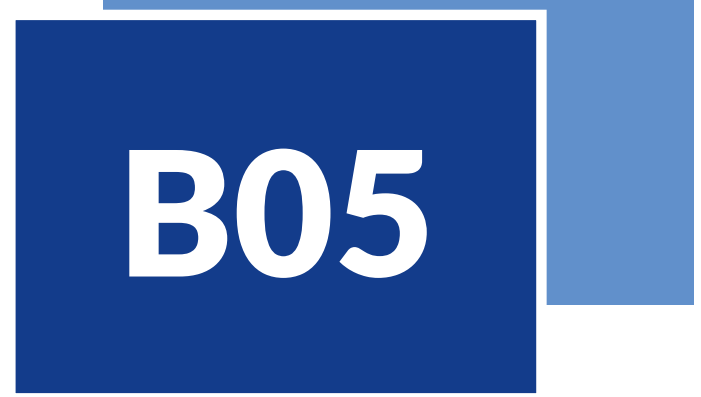

\author{
Luis Caraballo, Thomas Platts-Mills
}

\section{THE ALLERGEN SOURCES}

Cockroaches belong to the phylum Arthropoda; class Insecta, Order Blattaria. Species causing allergy symptoms such as asthma are listed in Table 1. These ubiquitous scavenger organisms have inhabited the planet long ago and domiciliary species are currently a serious problem for humans. Those that live in human dwellings (around 25 species) include American, German, Oriental, and Asian, which, together with the brown-banded and the smoky-brown cockroaches are sources of important allergens, inducers of allergic asthma (1).

Sensitization to cockroach usually occurs by inhalation. Potential sources of relevant allergens in the environment include whole bodies, cast skins, secretions, egg casings, and fecal material. Level of exposure for increased risk of asthma symptoms is $8 \mathrm{U} / \mathrm{g}$ of dust and a US national study found that $10 \%$ of living rooms were above this point. There is inter-species cross reactivity (e.g. American, German, Asian and Oriental) and extra-species cross reactivity ("pan allergy") with a number of other arthropods such as crustaceans (shrimp, crab, and lobster), insects (silverfish, butterflies), arachnids (dust mites)
Cockroach allergens are strong inducers of sensitization and asthma.

Clinically important species include American, German, Oriental, Asian, brownbanded and smoky-brown cockroaches.

Blag 2 and Blag 5 have the higher frequency of IgE positivity among cockroach allergens but there are important differences among individual patients and populations.

Currently, diagnosis is performed by skin testing and/or measurement of specific lgE to cockroach, using crude extracts. 


\section{Table 1}

Clinically relevant cockroach species

\begin{tabular}{|llll|}
\hline Family & Genus/species & Common name & Geographic distribution \\
\hline Blaberidae & Leucophaea maderae & Madeira & Asia, Africa, America, Oceania \\
\hline \multirow{3}{*}{ Blattellidae } & Blatella germanica & German & Mainly temperate dry zones, Europe and USA \\
\cline { 2 - 4 } & Blatella asahinai & Asian & Japan, tropical and subtropical \\
\cline { 2 - 4 } & Supella longipalpa & Brown-banded & Tropical \\
\hline Blattidae & Periplaneta americana & American & Mainly tropical and subtropical \\
\cline { 2 - 4 } & Periplaneta australasiae & Australian & Cosmopolitan \\
\cline { 2 - 4 } & Periplaneta brunnea & Brown & Mainly tropical \\
\cline { 2 - 4 } & Periplaneta fuliginosa & Smoky brown & China, Russia, Korea, Japan, Australia and USA \\
\cline { 2 - 4 } & Blatta orientalis & Oriental & America, United Kindom, Germany \\
\hline
\end{tabular}

and mollusks (oysters, mussels, scallops, clams). Since both exposure and allergy to cockroach are very common, patients with asthma or rhinitis should be routinely evaluated for this type of allergy.

\section{MAJOR AND RELEVANT MINOR ALLERGENIC MOLECULES}

Based in their molecular and biological properties cockroach allergens have been distributed in several groups, most of them are shown in Fig. 1 and Table 2 (2).

Group 1 Bla g 1 is composed by multiple consecutive amino acid repeats originated by gene duplication of an original 100 amino acid domain (3). There is cross reactivity between Bla $\mathrm{g} 1$ and homologous proteins (such as Per a 1, Per $f 1$ and Bla o 1) from other cockroach species, and allergens from other insects. The protein is most prevalent in the midgut, probably because the Bla g 1 gene is exclusively expressed by midgut cells. The presence of Bla g 1 in fecal particles makes this molecule, together with Bla g 2, a good marker of cockroach allergen exposure. Three epitopes of Bla g 1 have been described (www.iedb.org).

Group 2 Blag 2 is an unusual (inactive) aspartic protease with strong allergenic properties. It is the most frequent IgE binding molecule among cockroach allergens (4). The crystal structure (5) and antigenic structure of this allergen was analyzed by $X$ ray crystallography and site directed mutagenesis, providing important information about key residues determining epitopes and antigen antibody interactions $(6,7)$. There is three times more Bla g 2 in cockroach feces compared with the whole extract. Ten epitopes of this allergen have been described.

Group 3 Allergens of this group show high homology to insect hemolymph proteins. Per a 3 induces IL-4 expression in PBMC from allergic patients and this correlates with skin reactivity and clinical symptoms (8).

Group 4 Bla g 4 is a lipocalin. These molecules are very stable and their structure consists of a $C$-terminal $\alpha$-helix and a $\beta$-barrel enclosing an internal hydrophobic cavity that binds small ligands such as retinoids, glucocorticoids and pheromones. Four epitopes of Bla g 4 have been described. The structure and possible epitopes of other homologous molecule (Per a 4) have been described.

Group 5 Bla g 5 is a sigma class glutathione S-transferase (GST), a major cockroach allergen which has a high IgE response in the cockroach sensitized individuals. Thirty nine epitopes of Bla g 5 have been described and cross reactivity with GSTs of several sources is known.

Group 6 The allergens of this group are homologues to insect troponin $\mathrm{C}$ and vertebrate calmodulins (61\% to $78 \%$ and $42 \%$ to $44 \%$ amino acid identity, respectively) and have 2 EF-hand calcium binding domains. Interestingly, IgE binding to Bla g 6 has proven to be calcium dependent indicating that IgE preferably binds to one of the conformers. 
Unusual asparctic preotease Midgut microvilli protein-homolog
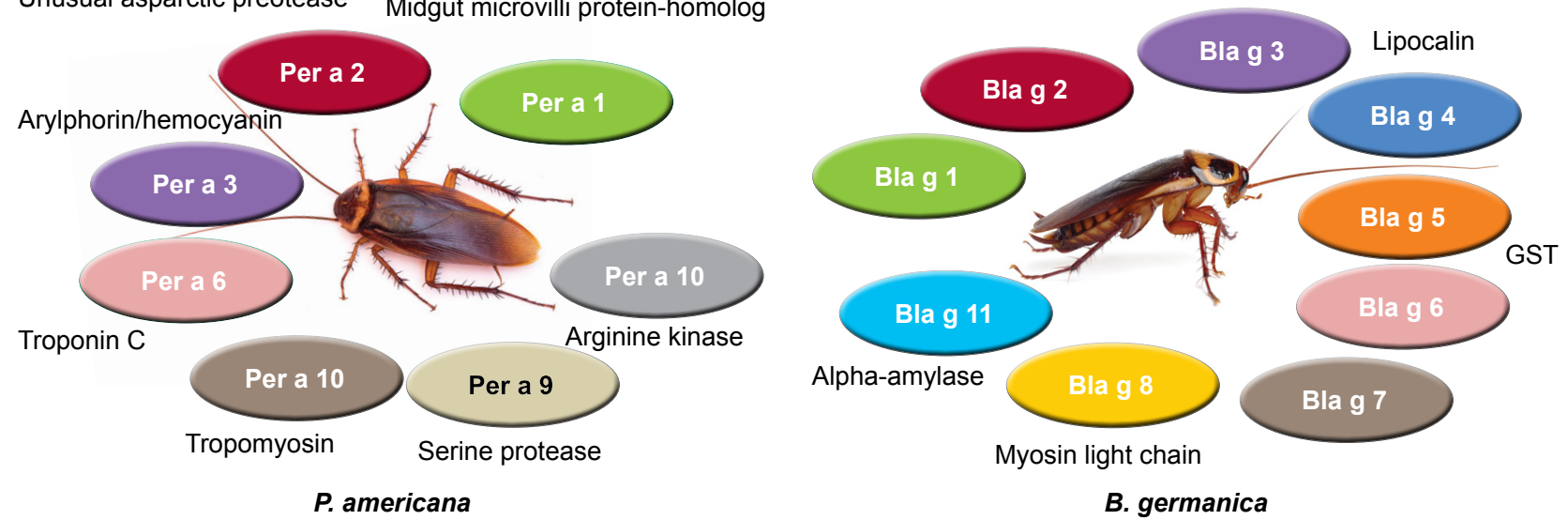

Figure 1

Allergen components from American and German Cockroaches. Homologous molecules have the same colour.

\section{Table 2}

Major and relevant minor Cockroach Allergens

\begin{tabular}{|llcc|}
\hline Allergenic molecule & Biochemical name & $\begin{array}{c}\text { Prevalence of Allergen-specific } \\
\text { IgE among patients (\%) }\end{array}$ & MW (kDa) \\
\hline B. germanica & & & \\
\hline Bla g 1 & Midgut microvilli protein-homolog & $20-50$ & $21-90$ \\
\hline Bla g 2 & Unusual aspartic protease & $40-70$ & 36 \\
\hline Bla g 3 & Arylphorin/hemocyanin & & 78.9 \\
\hline Bla g 4 & Lipocalin & $17-60$ & 21 \\
\hline Bla g 5 & Glutathione S-transferase & $35-73$ & 23 \\
\hline Bla g 6 & Troponin C & 14 & 17 \\
\hline Bla g 7 & Tropomyosin & 18 & 33 \\
\hline Bla g 8 & Myosin light chain & & 21 \\
\hline Bla g 11 & Alpha-amylase & & 57 \\
\hline P. americana & & $30-50,100$ & $26-51$ \\
\hline Per a 1 & & & 42 \\
\hline Per a 2 & Midgut microvilli protein-homolog & $26-95$ & $46-79$ \\
\hline Per a 3 & Aspartic protease-like & 17 & 17 \\
\hline Per a 6 & Arylphorin/hemocyanin & $13-54$ & 33 \\
\hline Per a 7 & Troponin C & $80-100$ & 43 \\
\hline Per a 9 & Tropomyosin & 80 & 28 \\
\hline Per a 10 & Arginine kinase & & 17 \\
\hline
\end{tabular}


Group 7 Invertebrate tropomyosins are important pan-allergens among dust mites, chironomids, silverfish, crustaceans, nematodes and mollusks. Tropomyosins from B. germanica and P. americana have been described. IgE binding frequency to cockroach tropomyosins are very different in some populations and this may reflect differences in environmental conditions.

Group 8 Bla g 8 shares $81-84 \%$ amino acid sequence identity with the myosin light chain of several insects and the shrimp Litopenaeus vannamei. The myosin regulatory light chains are small acidic polypeptides non-covalently bound to the neck region of the myosin head, which regulate the interaction of the myosin head with actin.

Group 9 Per a 9 was identified as a major allergen of $P$. americana in Thai patients (9). Arginine kinase homologues have also been reported in $B$. germanica, the shrimp Penaeus monodon (Pen $\mathrm{m}$ 2), D. pteronyssinus (Der p 20) and the Indian meal moth Plodia interpunctella (Plo i 1). There is evidence suggesting that arginine kinase is an invertebrate pan-allergen (10).

Group 10 Per a 10 isolated from P. americana is a major allergen in Indian allergic patients (11). Other important allergens are also serine protease (Der $f$ 3, Der p 3, Der p 6 and Der p 9). Cross reactivity is expected among these molecules.

Group 11 Bla g 11 shares $55.8 \%$ sequence identity with pig a-amylase and with group 4 mite allergens Blo t 4 (50.4\%), Der p 4 (49.8\%) and Eur m 4 (47.4\%). Bla g 11 seems to be an important novel allergen because the recombinant a-amylase inhibited 55\% of specific IgE of German cockroach extract.

\section{SENSITIZATION TO INDIVIDUAL MOLECULES AND ITS CLINICAL RELEVANCE}

Cockroach allergens are strong inducers of sensitization and asthma $(12,13)$ and cockroach allergy is an important risk factor for emergency room visits and hospital admissions. Special clinical characteristics have been described for cockroachinduced allergic asthma; it might be more severe and associated to higher total IgE levels than pollen induced asthma. Most characterized allergens are from B. germanica and P. americana, although homologous from other species have been purified.
Satinover S et al. found that Bla g 2 and Bla g 5 have the higher frequency of IgE positivity among cockroach allergens in US patients (4) but there are important differences in the profiles of IgE reactivity among individual patients and populations.

In a study performed in Taiwan to determine whether sensitization to different cockroach allergenic components correlates with different clinical manifestations and severities, eight $P$. americana allergens (Per a 1 through Per a 7 and Per a 9) were evaluated. IgE binding to Per a 2 was more frequent in patients with persistent asthma than in patients with rhinitis only, suggesting that this allergen could be a marker for more severe airway disease.

Also, IgE to Per a 9 was strongly associated with rhinitis (14). The availability of cloned, purified allergens will allow furthering investigating their particular effects on the immune responses and the possibilities to be used as reagents for Component resolved diagnosis (CRD) and markers of severity and response to treatment. For example, results of a study on cockroach allergen epitopes showed that $\mathrm{T}$ cell cytokine responses were different for Bla g 2, Bla g 5 and Bla g 6; in addition, analysis of the IgE response in the same patients suggested that $T$ cell responses to these allergens appear uncorrelated with IgE responses.

Groups 1 and 7, and possibly 6, are cross reactive. Some P. americana molecules share homology with mite allergens of group 2, 3 and 13. Since mite and cockroach co-exposure is common a differential CDR of sensitization might be necessary. In some populations there is cross reactivity between Bla $g$ 5 and other Glutathione-transferases (GST), such as Der $\mathrm{p} 8$ and Wuchereria bancrofti GST. In addition, the high correlation between IgE antibodies to Bla g 5 and Ascaris lumbricoides GST (Asc I 13) suggests the presence of cross reactivity between these molecules. However, the frequency of sensitization to Asc I 13 and Bla $g$ in a tropical Caribbean population is around $23 \%$ and, in comparison to that of the mite allergen Der $\mathrm{p} 2$, the strength of the IgE response to these allergens was low (15). The clinical importance of potential cross sensitization between helminth and cockroach GSTs should be further investigated.

Bla g 7 and Per a 7, two of the cockroaches' tropomyosins, are pan allergens and positive correlation between shrimp, cockroach, and dust mite IgE levels have been described. In this study, high exposure to cockroach in the home showed significant correlation to higher IgE levels to cockroach 


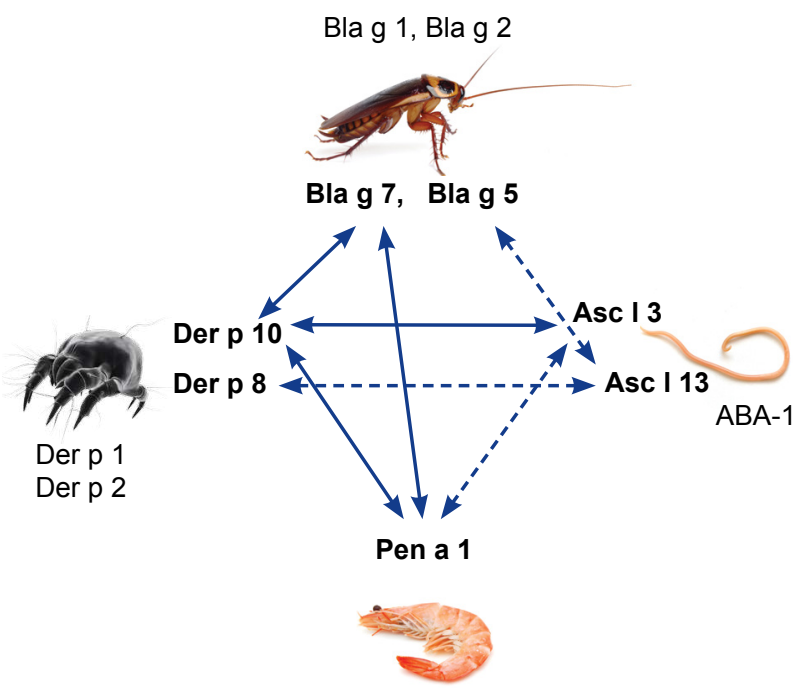

Figure 2

Clinically relevant cross-reactivity of B. germanica, D. pteronyssinus, shrimp and Ascaris allergens.

and shrimp, but no to mite. Sensitization rates to tropomyosins, including mite and cockroaches, are low in the US and Europe and high in tropical countries $(16,17)$, more likely because of cross reactivity with helminth tropomyosins $(2,18,19)$. Arginine kinases have been described as allergens not only in seafood and other sources but also in cockroaches (Per a 9) and mites (Der p 20). Fig. 2 shows predicted (dotted lines) and experimentally confirmed (solid lines) cross reactivity of $B$. germanica. Potential species specific components are also shown (no lines).

\section{CLINICAL MANAGEMENT}

Cockroach allergy should be investigated in all patients with respiratory allergy (Fig. 3). Diagnosis is performed by skin testing and/or measurement of specific IgE to cockroach, using crude extracts. However, inconsistent protein contents and relative potencies have been reported in the commercially available cockroach extracts. In vitro testing for sensitization to components (CRD) is commercially available for Bla g 1, Bla g 2, Bla g 5 and Bla g 7. Since Bla g 5 (GST) and Bla g 7 (tropomyosin) are cross reactive allergens, Bla g 1 and Bla g 2 are useful for detecting genuine sensitization to cockroaches in patients co exposed to mites and cockroaches. The effectiveness of recombinant Bla g 2, Bla g 4, Bla g 5, Per a 1 and Per a 7 for skin testing was evaluated
Textbox 1

Clinical relevance

- Cockroach allergens are strong inducers of sensitization and asthma

- Sensitization to cockroach allergens should be investigated in asthmatic patients

- In some places, co-exposure to cockroach and mite allergens occurs

- CRD could help to detect genuine sensitization to mite and cockroach allergens

in cockroach allergic patients living in Brazil (17). In this study, sensitization to Per a 7 was dominant with a frequency of $42 \%$, in contrast with results from other places where a heterogeneous IgE reactivity profile among cockroach-allergic patients has been found. In the US a panel of 5 recombinant allergens (rBla g 1, rBla g 2, rBla g 4, rBla g 5, and rPer a 7) could identify $64 \%$ of cockroach-allergic patients (4). A larger battery of recombinant allergens was tested in cockroach allergic patients in Taiwan showing that all patients reacted to at least one allergen and discovering that vitellogenin is an important allergen of $B$. germanica (20). Together, these studies suggest that a cocktail of five cockroach allergens Bla $\mathrm{g} 1$ and/or Per a 1, Bla g 2, Bla g 4, Bla g 5, Bla g 7, and/or Per a 7, would be expected to diagnose $50-64 \%$ of cockroach allergic patients worldwide (21).

Bla g 1 and Bla g 2 allergens are secreted in the digestive system and excreted in fecal particles, being good markers of cockroach allergen exposure. Threshold levels of exposure for sensitization and asthma symptoms in the susceptible population are 2 and $8 \mathrm{U} / \mathrm{g}$ of dust; however, sensitization by chronic exposure of very low levels $(1-10 \mu \mathrm{g} / \mathrm{g}$ of dust) of Bla g 2 is associated with asthma; also is a risk factor for wheezing in children (22). Reducing the environmental allergen exposure in homes of patients with cockroach-induced asthma, could lead to improvement of symptoms. However, cockroach allergens may persist for months following eradication of the insects. A controlled intervention including professional cleaning, bait traps, insecticides, and HEPA filters, decreased allergen levels, which correlated with decreased asthma symptoms, suggesting that allergen reduction is possible but difficult because continuous efforts and non-accessible equipment might be necessary; 


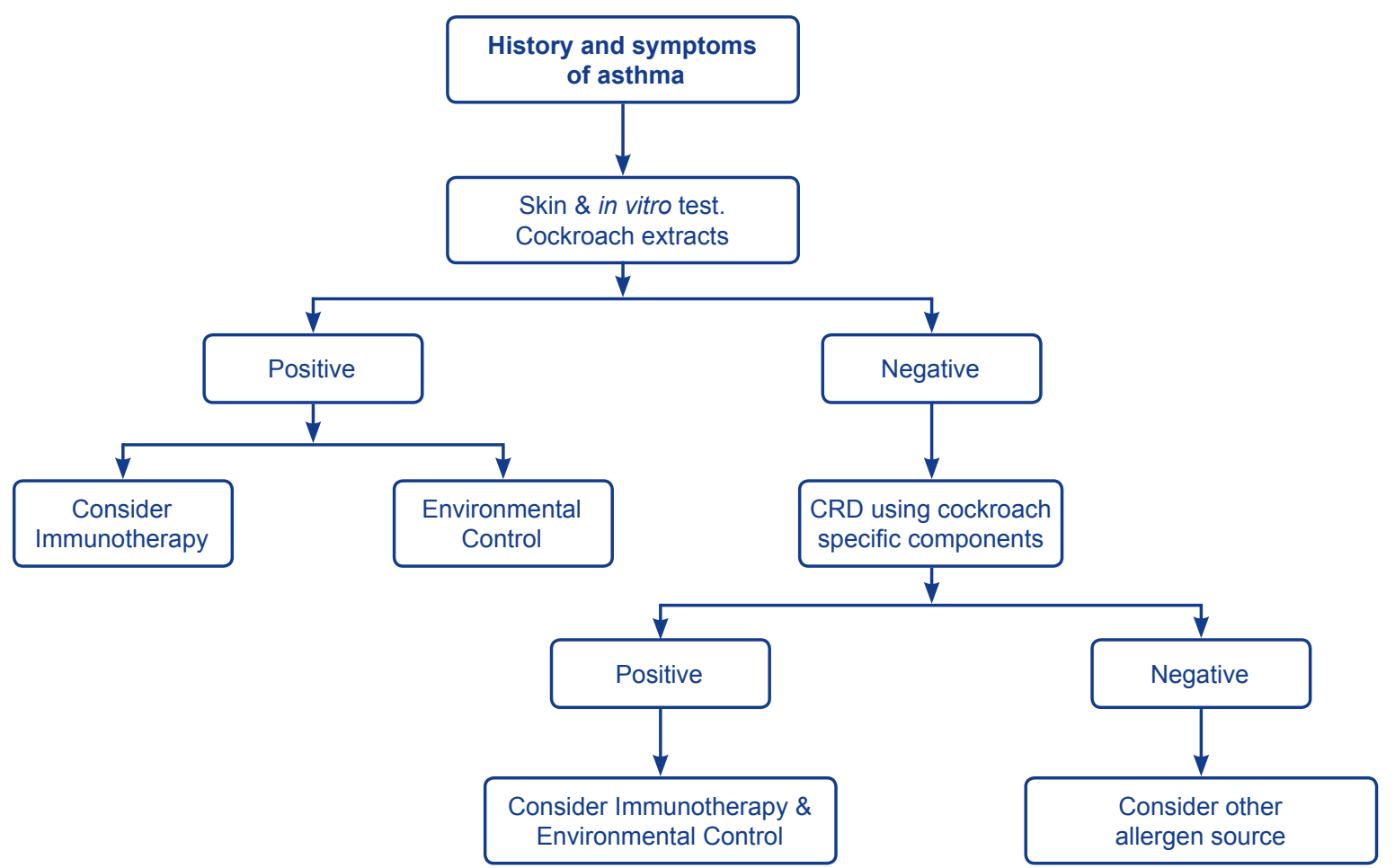

Figure 3

Decision algorithm for cockroach allergy.

also, the level of expertise that would be required to achieve significant cockroach extermination should be determined.

Immunotherapy (IT) is currently performed with crude extracts and there are reports supporting its effectiveness (23). In a work including four pilot studies of IT with $B$. germanica extract, subcutaneous IT was more effective modifying immune parameters than sublingual IT, although both types proved to be safe. Potential cockroach allergen immunotherapy has been tested in mouse models for prophylaxis (Bla g 2 DNA vaccine) or control (liposome-entrapped Per a 9) of airway inflammation (24).

\section{References}

1. Cochram D., Cockroaches: their biology, distribution and control 1999: Geneva, World Health Organization (document number:WHO/CDS/CPC/WHOPES/99.3).

2. Pomés, A, Arruda LK, Investigating cockroach allergens: aiming to improve diagnosis and treatment of cockroach allergic patients. Methods 2014;66:7585.
3. Pomés A, Melén E, Vailes LD, Retief JD, Arruda LK, Chapman MD. Novel allergen structures with tandem amino acid repeats derived from German and American cockroach. J Biol Chem, 1998;273:30801-30807.

4. Satinover SM, Reefer AJ, Pomes A, Chapman MD, Platts-Mills TA, Woodfolk JA. Specific IgE and IgG antibody-binding patterns to recombinant cockroach allergens. J Allergy Clin Immunol 2005;115:803-809.

5. Gustchina A, Li M, Wünschmann S, Chapman MD, Pomés A, Wlodawer A. Crystal structure of cockroach allergen Bla g 2, an unusual zinc binding aspartic protease with a novel mode of self-inhibition. J Mol Biol 2005;348:433-444.

6. Glesner J, Wüschmann S, Li M et al. Mechanisms of allergen-antibody interaction of cockroach allergen Bla g 2 with monoclonal antibodies that inhibit IgE antibody binding. PLoS One 2011;6:e22223.

7. Li M, Gustchina A, Alexandratos J, Wlodawer A, Wünschmann S, Kepley CL et al. Crystal structure of a dimerized cockroach allergen Bla g 2 complexed with a monoclonal antibody. J Biol Chem 2008;283:2280622814.

8. Jeng KC, Liu MT, Wu CH, Wong DW, Lan JL. American cockroach $\mathrm{Cr}$-PI allergen induces lymphocyte 
proliferation and cytokine production in atopic patients. Clin Exp Allergy 1996;26:349-356.

9. Sookrung N, Chaicumpa W, Tungtrongchitr A, Vichyanond P, Bunnag C, Ramasoota Pet al. Periplaneta americana arginine kinase as a major cockroach allergen among Thai patients with major cockroach allergies. Environ Health Perspect 2006;114:875-880.

10. Binder M, Mahler V, Hayek B, Sperr WR, Schöller $M$, Prozell $S$ et al. Molecular and immunological characterization of arginine kinase from the Indianmeal moth, Plodia interpunctella, a novel cross-reactive invertebrate pan-allergen. JImmunol 2001;167:54705477.

11. Sudha VT, Arora N, Gaur SN, Pasha S, Singh BP. Identification of a serine protease as a majorallergen (Per a 10) of Periplaneta americana. Allergy 2008;63:768776.

12. Bernton HS, Brown H. Insect Allergy--Preliminary Studies of the Cockroach. J Allergy 1964;35:506-513.

13. Kang B, Vellody D, Homburger H, Yunginger JW. Cockroach cause of allergic asthma. Its specificity and immunologic profile. J Allergy Clin Immunol 1979;63:80-86.

14. Lee MF, Song PP, Hwang GY, Lin SJ, Chen YH. Sensitization to Per a 2 of the American cockroach correlates with more clinical severity among airway allergic patients in Taiwan. Ann Allergy Asthma Immunol 2012;108:243-248.

15. Acevedo N, Mohr J, Zakzuk J, Samonig M, Briza P, Erler $A$ et al. Proteomic and immunochemical characterization of glutathione transferase as a new allergen of the nematode Ascaris lumbricoides. PLoS One 2013;8:e78353.

16. Westritschnig K, Sibanda E, Thomas W, Auer $H$, Aspöck $\mathrm{H}$, Pittner $\mathrm{G}$ et al. Analysis of the sensitization profile towards allergens in central Africa. Clin Exp Allergy 2003;33:22-27.
17. Barbosa MC, Santos $A B$, Ferriani VP, Pomés A, Chapman MD, Arruda LK. Efficacy of recombinant allergens for diagnosis of cockroach allergy in patients with asthma and/or rhinitis. Int Arch Allergy Immunol 2013;161:213-219.

18. Acevedo N, Erler A, Briza P, Puccio F, Ferreira F, Caraballo L. Allergenicity of Ascaris lumbricoides tropomyosin and IgE sensitization among asthmatic patients in a tropical environment. Int Arch Allergy Immunol 2011;154:195-206.

19. Ahumada V, García E, Dennis R, Rojas MX, Rondón MA, Pérez A et al. IgE responses to Ascaris and mite tropomyosins are risk factors for asthma. Clin Exp Allergy 2015;45:1189-1200.

20. Chuang JG, Su SN, Chiang BL, Lee HJ, Chow LP. Proteome mining for novel lgE-binding proteins from the German cockroach (Blattellagermanica) and allergen profiling of patients. Proteomics 2010;10:3854-3867.

21. Arruda LK, Barbosa MC, Santos AB, Moreno AS, Chapman MD, Pomés A. Recombinant allergens for diagnosis of cockroach allergy. Curr Allergy Asthma Rep 2014;14:428.

22. Finn PW, Boudreau JO, He H, Wang Y, Chapman MD, Vincent $C$ et al. Children at risk for asthma: home allergen levels, lymphocyte proliferation and wheeze. J Allergy Clin Immunol 2000;105:933-942.

23. Srivastava D, Gaur SN, Arora N, Singh BP. Clinicoimmunological changes post-immunotherapy with Periplaneta americana. Eur J Clin Invest 2011;41:879888.

24. Meechan P, Tungtrongchitr A, Chaisri U, Maklon $\mathrm{K}$, Indrawattana N, Chaicumpa $\mathrm{W}$ et al. Intranasal, liposome-adjuvanted cockroach allergy vaccines made of refined major allergen and whole-body extract of Periplaneta americana. Int Arch Allergy Immunol 2013;161:351-362. 



\section{PART B \\ USING \\ MOLECULAR \\ ALLERGOLOGY \\ IN THE CLINICAL \\ PRACTICE}

\section{ALLERGY \\ TO CAT, DOG AND HORSE}

\author{
Marianne van Hage, Christiane Hilger
}

\section{THE ALLERGEN SOURCES}

Mammalian furry animals are an important source of indoor allergens (1). They are considered as risk factors for the development of allergic rhinitis and asthma in the domestic and occupational environment. Pets are present in up to $60 \%$ of European and US households, with cats and dogs being the most popular pets. Horse riding is a favorite leisure activity among young people. Animal allergens are present in urine and saliva dander and above all, and are dispersed. They stick to animal hair and dander and are dispersed indoors (Fig. 1). They also adhere to human clothes and are easily transported to public places. Exposure measurement studies have shown their presence in schools, day-care centres, public transport and households of non-pet owners (2).

Allergic reaction have been described upon

- Inhalation by direct contact with the animal

- Inhalation by indirect contact in a contaminated environment

- Animal bites

- Ingestion of raw or medium cooked meat
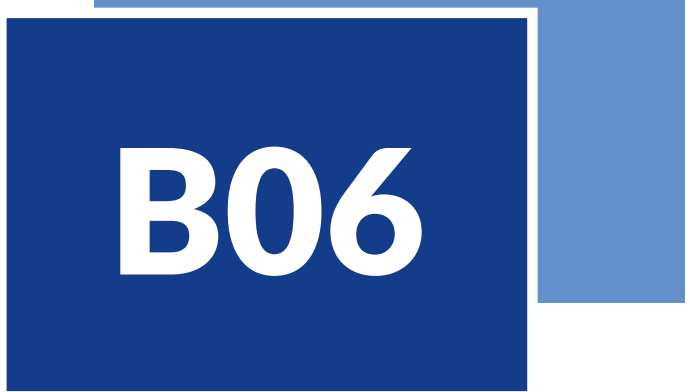

Mammalian furry animals are an important indoor allergen source

Sensitisation to mammalian furry animals is common and a risk factor for the development of allergic rhinitis and asthma

Cat, dog and horse contain cross-reactive molecules such as serum albumins and some lipocalins

Co-sensitization to furry animals can be distinguished from cross-sensitization by allergen component testing 


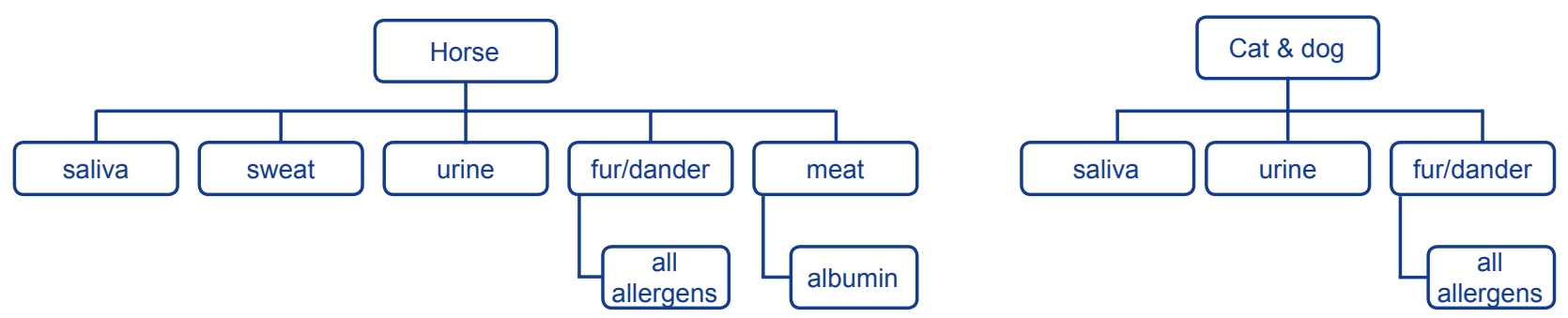

Figure 1

Animal allergen sources.

Most sensitised patients experience allergic symptoms like rhinits or asthma upon direct exposure to the animal. As animal allergens are easily transported by human clothes, they are ubiquitous. There is evidence that exposure to cat allergens in schools may lead to asthma exaberations in catsensitized students (3). In classrooms with a high number of cat-owners, allergen levels measured are considered to be high enough to induce sensitization to cat (4). Animal bites are also capable of provoking anaphylactic reactions. Several cases of anaphylaxis upon rodent bites have been described in the literature (5). Anaphylaxis to cat, dog or horse bites does not to seem common. Only one case of anaphylaxis to cat bite has been reported in the literature (6). Serum albumins present in meat are easily inactivated by heat, but they can induce symptoms in sensitized patients upon ingestion of raw meat such as ham or sausages (7).

\section{MAJOR AND RELEVANT MINOR ALLERGENIC MOLECULES}

A number of cat, dog and horse allergens have been described. Lipocalins constitute the most important allergen protein family (8). Most of them are major allergens: Equ c 1, Can f 1, Can f 6 and Fel d 4. Lipocalins are characterized by a common three-dimensional structure and a low sequence identity (see C07). They are synthezised in salivary glands and are dispersed into the environment by saliva and dander. Serum albumins are highly cross-reactive molecules generally considered as minor allergens. They are abundant in saliva and dander. Fel d 1, the major cat allergen, is an uteroglobin expressed in salivary glands and skin. The production of Fel $d 1$ is related to sexual homones (9). Three allergens are known to have surfactant properties, Equ c 1, Equ c 4 and Fel d 8. The latter two
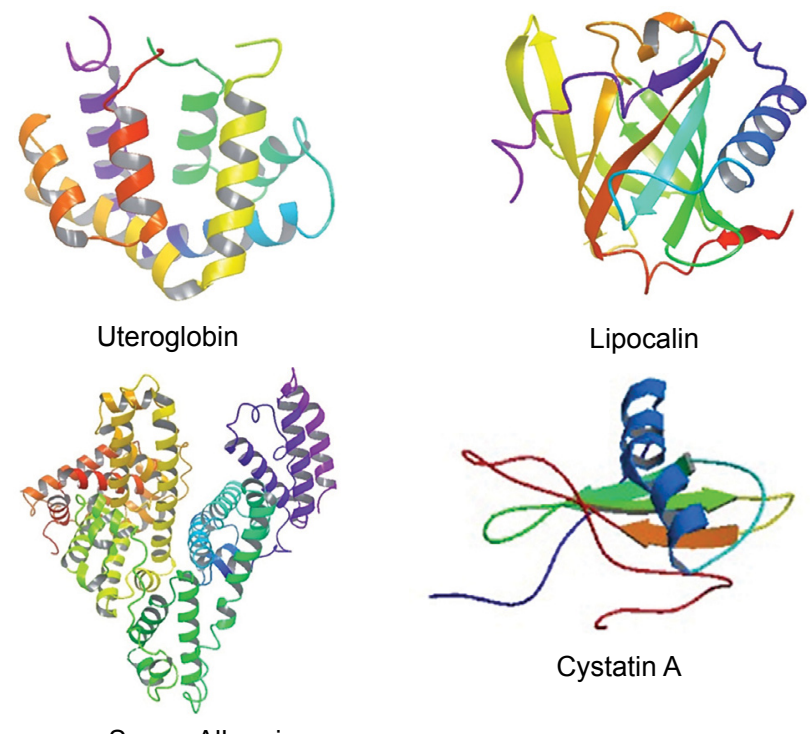

Serum Albumin

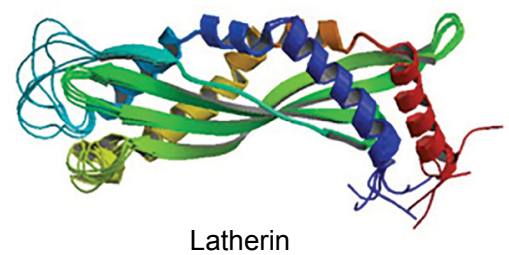

Figure 2

Molecular structures of animal allergens. The uteroglobin family is represented by Fel $\mathrm{d} 1$, lipocalins by Equ c 1, serum albumins by Equ c 3 , cystatins by human cystatin $A$ and latherins by Equ c 4. 
Table 1

Major and relevant minor allergenic molecules from dog, horse and cat

\begin{tabular}{|llccc|}
\hline Allergen & Biochemical name & MW & Glycosylation & Isoforms \\
\hline Dog (Canis familiaris) & & & \\
\hline Can f 1 & lipocalin & $23-25$ & yes & 1 \\
\hline Can f 2 & lipocalin & 19 & yes & 1 \\
\hline Can f 3 & serum albumin & 69 & no & 1 \\
\hline Can f 4 & lipocalin & 18 & no & 1 \\
\hline Can f 5 & kallikrein & 28 & yes & 1 \\
\hline Can f 6 & lipocalin & $27-29$ & yes & 1 \\
\hline Horse (Equus caballus) & & & \\
\hline Equ c 1 & lipocalin & 25 & yes & 1 \\
\hline Equ c 2 & lipocalin & 17 & no & 2 \\
\hline Equ c 3 & serum albumin & 67 & no & 1 \\
\hline Equ c 4 & latherin & $17,20.5$ & no & 1 \\
\hline Cat (Felis domesticus) & & & 1 \\
\hline Fel d 1 & uteroglobin & 18 & yes & 1 \\
\hline Fel d 2 & serum albumin & 69 & no & 1 \\
\hline Fel d 3 & cystatin & 11 & yes & 1 \\
\hline Fel d 4 & lipocalin & 22 & yes & 1 \\
\hline Fel d 5 & immunoglobulin A & 400 & yes & 1 \\
\hline Fel d 6 & immunoglobulin M & $800-1000$ & yes & 1 \\
\hline Fel d 7 & lipocalin & 17.5 & no & 1 \\
\hline Fel d 8 & latherin-like protein & 24 & no & 1 \\
\hline & & & & 1 \\
\hline
\end{tabular}

aPresence or absence of glycosylation deduced from sequence analysis, not based on experimental evidence.

are considered as latherins. Fel $\mathrm{d} 3$ is a minor allergen belonging to the cystatin protein family. Can $\mathrm{f} 5$, a prostatic kallikrein was isolated from urine of male dogs. It is considered as major allergen.

\section{SENSITIZATION TO INDIVIDUAL MOLECULES AND THEIR CLINICAL RELEVANCE}

Large epidemiology studies are based on skin prick test results and determination of specific IgE to animal dander. These studies are hampered by the fact that cross-reactive molecules such as serum albumins are present in the extracts used and this may lead to an overestimation of sensitzation rates to a particular animal. A recent German study analysed sensitization rates to 50 allergen sources in more than 7000 healthy adults (10) Seven percent were found to have specific IgE against cat and dog, $3.5 \%$ were sensitized against horse. Another study reported sensitization rates of $9.7 \%$ to dog, $8.1 \%$ to cat and $4.4 \%$ to horse dander in about 13000 German children and adolescents (11). Interestingly, animal sensitization prevalence raised from $5.7 \%$ in the age group 3-6 years to $17.2 \%$ in the $14-17$ years old adolescents. The Swedish BAMSE study, which 


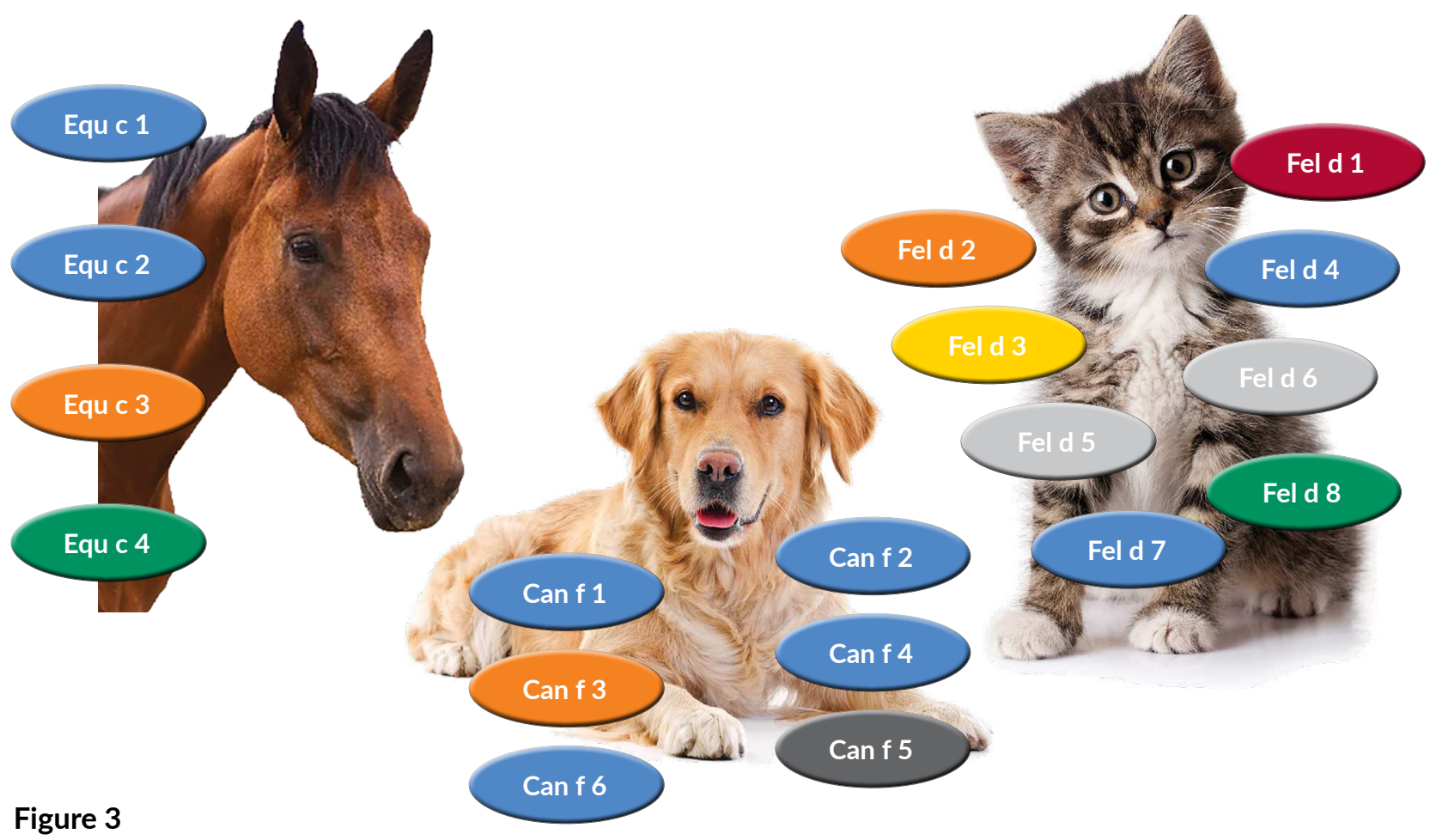

Known allergens of cat, dog and horse. Proteins belonging to the lipocalin family are depicted in blue, serum albumins are shown in orange, latherins in green, immunoglobulins in light grey, cystatin in light orange, uteroglobin in dark red and kallikrein in dark grey.

is an unselected population-based birth cohort of more than 4000 children with data from childhood up to adolescence, recently reported an increase in sensitization to cat (from $6.4 \%$ to $19.0 \%$ ), dog (from $4.8 \%$ to $22.6 \%$ ) and horse (from $3.1 \%-10.6 \%$ ) in 1699 children followed from 4 to 16 years (12). The $\mathrm{GA}^{2} \mathrm{LEN}$ skin test study revealed striking geographic sensitization pattern among 14 European countries (288). Among patients presenting at allergy centres with suspected allergic reaction to inhalant allergens, prevalence to cats and dogs was highest in Denmark and lowest in Austria. Sensitization to animals tended to be higher in Nordic countries which probably depends on the fact that e.g. cats are kept indoors in a higher frequency in the Northern part of Europe.

As not all pet allergen molecules are commercially available has only been adressed for specific IgE diagnosis, their clinical relevance have only been adressed in a few studies. Using an allergen chip based (MeDALL chip) containing several individual pet allergens (Table 2), sera from nearly 800 randomly collected children from the BAMSE birth cohort at 4,8 and 16 years were analyzed in relation to symptoms to these animals up to 16 yrs. The auhtors reported that IgE to Fel $d 1$ and Can $f 1$ in childhood and polysensitization to either cat or dog allergen molecules, are predicitve markers of allergy to cat or dog, respectively, at 16 years. IgE to Fel $d 1$ was as good as IgE to cat extract for diagnosing cat allergy. Furthermore, IgE to Can $\mathrm{f} 1$ was the most important prognostic marker of dog allergy and superior to IgE to dog allergen extract. IgE to Can $f 5$ was to a lower extent associated with allergy to dog than IgE to Can f 1 (14).

Furthermore, multiple sensitizations towards lipocalins, kallikrein and uteroglobin components have been associated with increased bronchial inflammation in severe asthmatics (15). In addition, in children with severe asthma and allergy towards furry animals sensitization to Can f 2 ( $22 \%$ vs. $0 \%$, $\mathrm{p}=0.009)$ and Equ c 1 (51\% vs. $25 \%, \mathrm{p}=0.03)$ was shown to be more common than in children with controlled asthma (16). Moreover, children with asthma due to cat have also been reported to have higher IgE antibody levels to Fel $d 1$ than compared to children with rhinoconjunctivitis (17). 
A single case of a cat-induced anaphylactic reaction in a child sensitized exclusively to Fel $d 1$ was recently reported (18). Elevated IgE levels of cat and dog-specific lgG4 antibodies seem to be protective as increasing ratios of specific IgG4/IgE have been associated with a lower likelihood of reporting allergic symptoms (19). However, a dissociation of allergenspecific IgE and IgG responses in animal allergy has also been reported, indicating that IgG can be directed against other allergens/epitopes than IgE, which may explain why naturally occurring allergenspecific lgG is not always protective (20). Commercial skin prick test extracts for dog allergy diagnosis have been evaluated for their content of major and minor allergens (21). The contents of Can $f 1,2$ and 3 showed marked variations between companies and this is likely to have an impact of patient diagnosis.

Epidemiological studies have shown that the presence of animal allergens in the indoor environment has been associated with an increased risk of developing allergic symptoms. Monitoring of allergen contamination allows to dermine allergen levels and to assess eviction strategies. Different methods of dust collection and antibody based allergen quantification assays allow to measure allergen levels of Fel $d$ 1, Can $f 1$ and Equ c 4 in settled dust and ambiant air (2). Currently, there are no detection systems available for other major and minor cat, dog and horse allergens.

\section{CLINICAL RELEVANCE, DIAGNOSIS AND MANAGEMENT}

A careful record of the clinical history such as the presence of pets at home or regular pet contact is of great value. Skin prick test or allergen-specific IgE using extracts from cat, dog or horse will confirm furry animal sensitization. As cat, dog and horse contain cross-reactive molecules such as serum albumins, some of the cross-reactive lipocalins and potentially other cross-reactive molecules, it is important to define the primary allergenic source, especially if a specific immunotherapy is intended. Co-sensitization has to be distinguished from cross-sensitization. It is important to acknowledge that IgE-cross-reactivity may not always imply clinical cross-reactivity. Exposure to furry animals can lead to different sensitization patterns with different clinical implication. In this context the dose of exposure is also of importance.

\section{Table 2}

Major and minor relevant allergenic molecules from dog, horse and cat

\begin{tabular}{|c|c|c|}
\hline Allergen & Degree of cross-reactivity & $\begin{array}{l}\text { Sensitiza- } \\
\text { tion rate }\end{array}$ \\
\hline Can $\mathrm{f} 1$ & $\begin{array}{l}\text { high risk of cross-reactivity } \\
\text { with Fel } d 7\end{array}$ & $50-76$ \\
\hline Can f 2 & & $22-35$ \\
\hline Can f 3 & $\begin{array}{l}\text { high risk of cross-reactivity } \\
\text { with other serum albumins }\end{array}$ & $25-59$ \\
\hline Can $\mathrm{f} 4$ & & $35-59$ \\
\hline Can f 5 & & 71 \\
\hline Can $\mathrm{f} 6$ & $\begin{array}{l}\text { moderate risk of cross-reactivity } \\
\text { with Fel d } 4 \text { and Equ c } 1\end{array}$ & $23-61$ \\
\hline Equ c 1 & $\begin{array}{l}\text { moderate risk of cross-reactivity } \\
\text { with Fel d } 4 \text { and Can } \mathrm{f} 6\end{array}$ & $27-100$ \\
\hline Equ c 2 & & 50 \\
\hline Equ c 3 & $\begin{array}{l}\text { high risk of cross-reactivity } \\
\text { with other serum albumins }\end{array}$ & 36 \\
\hline Equ c 4 & & 77 \\
\hline Fel d 1 & $\begin{array}{l}\text { response to Fel d } 1 \text { higher } \\
\text { among children with asthma }\end{array}$ & $60-100$ \\
\hline Fel d 2 & $\begin{array}{l}\text { high risk of cross-reactivity } \\
\text { with other serum albumins }\end{array}$ & $14-54$ \\
\hline Fel d 3 & & 10 \\
\hline Fel d 4 & $\begin{array}{l}\text { moderate risk of cross-reactivity } \\
\text { with Equ c } 1 \text { and Can f } 6\end{array}$ & 63 \\
\hline Fel d 5 & & 38 \\
\hline Fel d 6 & & $?$ \\
\hline Fel d 7 & $\begin{array}{l}\text { high risk of cross-reactivity } \\
\text { with Can } f 1\end{array}$ & 38 \\
\hline Fel d 8 & & 19 \\
\hline
\end{tabular}

A) Sensitization to major cat/dog/horse allergens (e.g. Fel d 1/Can f 1/Can f 2/Can f 5/Equ c 1) are specific markers of cat/dog/horse sensitization. Patients may experience symptoms from the upper and/or lower airways to cat/dog/horse. IgE to Fel $d 1$ and Can $f 1$ in childhood have shown to be predictive markers of cat or dog allergy in adolescence (14). For further information, please see section $\mathrm{CO} 7$ on Lipocalins. 
B) Sensitization to cross-reactive animal allergens with high sequence homology, e.g. serum albumins from cat/dog/horse. There is very limited data on sensitization to serum albumins and symptoms. High levels of IgE to Fel d 2 have been associated with atopic dermatitis in children with cat allergy (22). Serum albumins are involved in pork-cat syndrome, where sensitization to cat serum albumin represents the primary event in the development of the cross-reactive IgE (23). For further information, please see section C04 on Serum albumins.

If the cross reactive IgE is against allergens with low to moderate degree of sequence homology, which is the case for many of the lipocalins, the patient may not experience symptoms to these allergen sources. However, if the cross-reacting lipocalin allergens have high sequence homology, patients may experience symptoms to all these allergen sources. There are few data on symptoms clearly related to cross-reactive molecules, as monosensitization to these components seems to be rare.

\section{Clinical diagnosis}

Proper interpretation of sensitisation results and case history (which allergen source causes symptoms? Which organ/s are affected? Progression? In case of polyallergies investigation of genuine sensitization to the different furry animals is needed (please see Fig. 4-5 (C07). The description of the chronology of onset of symptoms to each animal may also help to identify the primary sensitization source. Additional atopic diseases (atopic eczema).

\section{Skin prick test (SPT)}

Commercial cat extract can be used, but dog extract has shown marked variations between companies in their content of major dog allergens. There is no data available on the usefulness of horse extract.

\section{IgE-Testing}

Total IgE has no added value in this context.

IgE to Fel $d 1$ is equally good as IgE to cat extract in predicting cat allergy

IgE to Can $\mathrm{f} 1$ has shown to be the most important prognostic marker of dog allergy and superior to dog allergen extract IgE (14)

Different sensitization patterns are discussed in the sections on Lipocalins and serum albumins.

\section{Challenge tests}

Challenge tests using natural cat extracts are usually not needed, but may be indicated in selected cases such as polysensitization or when discordances are observed between skin tests and IgE results. Challenge tests using a cat challenge chamber are only performed in clinical trials to evaluate efficacy of new molecules used for immunotherapy or pharmacological treatment.

\section{Clinical management}

Advices and avoidance

1. If the patient experiences asthma symptoms at exposure to dog or cat even after proper medication the patient should be informed that such direct and continous exposure may have detrimental effects on health

2. If the patient experiences asthma at indirect exposure to cat or dog, allergen-specific immunotherapy is recommended.

3. Patients who are sensitised to Can $f 5$ only may tolerate female or castrated dogs. Furthermore, patients sensitized to Can $f 5$ may show allergic reactions to seminal fluid (24).

\section{Pharmacotherapy (emergency kit)}

1. Symptomatic treatment as required.

\section{Allergen-specific immunotherapy}

Allergen-specific immunotherapy is especially recommended for cat if the patient experiences asthma at indirect cat exposure and is primarily sensitized to cat.

\section{Other domestic animals}

Bovine allergens are important inducers of occupational allergic airway diseases in cattle-exposed farmers (302). The European Farmers' Project Study Group has determined that the prevalence of work-related respiratory symptoms was $21.8 \%$ among cattle farmers. The main sources of bovine allergens are cow hair and dander, but allergens are also found in urine, saliva, milk and beef. Early investigations of bovine dander extracts have identified 17 different antigenic components. Three of these, having molecular weights of 24, 22 and $20 \mathrm{kDa}$, have been characterised as major allergens. Subsequent studies have shown that the 20-kDa protein, designated as Bos $\mathrm{d} 2$, is the most important allergen in cow antigen extracts and belongs to the lipocalin family of proteins (Section C07: Lipocalins). 
ELISA is available to quantify Bos d 2 or cow hair proteins (25) in the air and dust samples to monitor the allergen load in occupational and home environment.

\section{References}

1. Konradsen JR, Fujisawa T, van Hage M, Hedlin G, Hilger $\mathrm{C}$, Kleine-Tebbe $\mathrm{J}$ et al. Allergy to furry animals: New insights, diagnostic approaches, and challenges. J Allergy Clin Immunol 2015;135:616-625.

2. ZahradnikE, RaulfM.Animalallergensand theirpresence in the environment. Front Immunol 2014;5:76.

3. Almquist C, Wickman M, Perfetti L, Berglind N, Renström $A$, Hedrén $M$ et al. Worsening of asthma in children allergic to cats, after indirect exposure to cat at school. Am J Respir Crit Care Med 2001;163:694-698.

4. Ritz BR, Hoelscher B, Frye C, Meyer I, Heinrich J. Allergic sensitization owing to 'second-hand' cat exposure in schools. Allergy 2002;57:357-361.

5. Pecquet C. New pets and allergies. Eur J Dermatol 2012;22:14-22.

6. Maeda Y, Akiyama K. Anaphylaxis after a cat bite. Allergol Int 2012;61:511-512.

7. Hilger C, Kohnen M, Grigioni F, Lehners C, Hentges F. Allergic cross-reactions between cat and pig serum albumin. Study at the protein and DNA levels. Allergy 1997;52:179-187.

8. Hilger C, Kuehn A, Hentges F. Animal lipocalin allergens. Curr Allergy Asthma Rep 2012;12:438-447.

9. Charpin C, Zielonka TM, Charpin D, Ansaldi JL, Allasia C, Vervloet D. Effects of castration and testosterone on Fel d I production by sebaceous glands of male cats: II--Morphometric assessment. Clin Exp Allergy 1994;24:1174-1178.

10. Haftenberger M, Laußmann D, Ellert U, Kalcklösch $M$, Langen $U$ et al. Prevalence of sensitisation to aeraoallergens and food allergens: results of the German Health Interview and Examination Survey for Adults (DEGS1). BundesgesundheitsblattGesundheitsforschung Gesundheitsschutz 2013;56:687-697.

11. Schmitz R, Ellert U, Kalcklösch M, Dahm S, Thamm M. Patterns of Sensitization to Inhalant and Food Allergens - findings from the German Health Interview and Examination Survey for Children and Adolescents. Int Arch Allergy Immunol 2013;162:263-270.

12. Wickmann $M$, Asarnoj A, Tillander $H$, Andersson $\mathrm{N}$, Bergström A, Kull I et al. Childhood-toadolescence evolution of IgE antibodies to pollens and plant foods in the BAMSE cohort. J Allergy Clin Immunol 2014;133:580-582.

13. Heinzerling LM., Burbach GJ., Edenharter G, Bachert C, Bindslev-Jensen C, Bonini $S$ et al. GA(2)LEN skin test study I: GA(2)LEN harmonization of skin prick testing: novel sensitization patterns for inhalant allergens in
Europe. Allergy 2009;64:1498-1506.

14. Asarnoj A, Hamsten C, Wadén K, Andersson N, Lupinek $\mathrm{C}$, Anto $\mathrm{J}$ et al. Sensitization to cat and dog allergen molecules in childhood and prediction of symptoms of cat and dog allergy in adolescence: a BAMSE/MeDALL study. J Allergy Clin Immunol 2016;137:813-821.e7.

15. Nordlund B, Konradsen JR, Kull I, Borres MP, Önell A, Hedlin $G$ et al. IgE antibodies to animal-derived lipocalin, kallikrein and secretoglobin are markers of bronchial inflammation in severe childhood asthma. Allergy 2012;67:661-669.

16. Konradsen JR, Nordlund B, Onell A, Borres MP, Grönlund H, Hedlin G. Severe childhood asthma and allergy to furry animals: refined assessment using molecular-based allergy diagnostics. Pediatr Allergy Immunol 2014;25:187-192.

17. Grönlund H, Adédoyin J, Reininger R, Varga EM, Zach M, Fredriksson $\mathrm{M}$ et al. Higher immunoglobulin $\mathrm{E}$ antibody levels to recombinant Fel d 1 in cat-allergic children with asthma compared with rhinoconjunctivitis. Clin Exp Allergy 2008;38:1275-1281.

18. Casale TB, Cruz C. Anaphylaxis to cat in a child with exclusive sensitivity to Fel d 1. J Allergy Clin Immunol Pract 2013;1:416-417.

19. Burnett M, Wegienka G, Havstad S, Kim H, Johnson CC, Ownby D et al. Relationship of dog- and cat-specific lgE and lgG4 levels to allergic symptoms on pet exposure. J Allergy Clin Immunol Pract 2013;1:350-353.

20. Curin M, Swoboda I, Wollmann E, Lupinek C, Spitzauer $S$, van Hage $M$ et al. Microarrayed dog, cat, and horse allergens show weak correlation between allergen-specific IgE and IgG responses. J Allergy Clin Immunol 2014;133:918-921.

21. Curin M, Reininger R, Swoboda I, Focke M, Valenta $\mathrm{R}$, Spitzauer S. Skin prick test extracts for dog allergy diagnosis show considerable variations regarding the content of major and minor dog allergens. Int Arch Allergy Immunol 2011;154:258-263.

22. Wisniewski JA, Agrawal R, Minnicozzi S, Xin W, Patrie J, Heymann PW et al. Sensitization to food and inhalant allergens in relation to age and wheeze among children with atopic dermatitis. Clin Exp Allergy 2013;43:11601170.

23. Hilger C, Kohnen M, Grigioni F, Lehners C, Hentges F. Allergic cross-reactions between cat and pig serum albumin. Study at the protein and DNA levels. Allergy 1997;52:179-187.

24. Kofler L, Kofler H, Mattsson L, Lidholm J. A case of dog related human seminal plasma allergy. Eur Ann Allergy Clin Immunol 2012;44:89-92.

25. Zahradnik E, Sander I, Bruckmaier L, Flagge A, Fleischer C, Schierl R et al. Development of a sandwich ELISA to measure exposure to occupational cow hair allergens. Int Arch Allergy Immul 2011;155:225-233. 



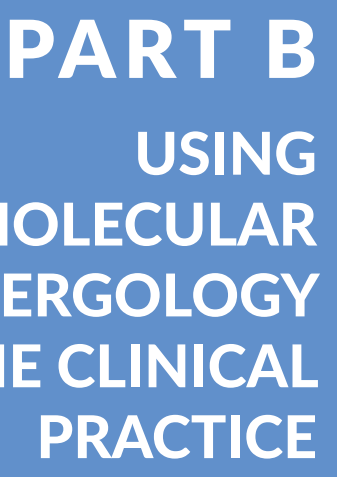

ALLERGIC

BRONCHO-

PULMONARY

ASPERGILLOSIS (ABPA)
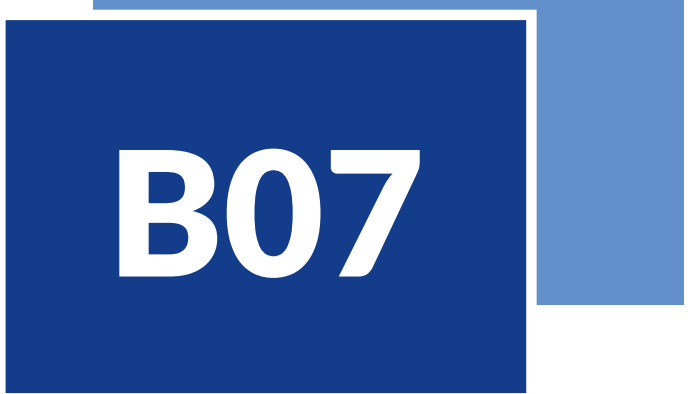

\author{
Reto Crameri
}

\section{THE ALLERGEN SOURCE}

Aspergillus fumigatus (A. fumigatus) is a ubiquitous mould perennially present in the indoor and outdoor environment (1) being both, a primary and opportunistic pathogen associated with many pathologic conditions (2) as well as a major allergenic source. With 23 cloned allergens officially approved by the World Health Organization and International Union of Immunological Species (WHO/IUIS) Allergen Nomenclature Subcommittee (3) and more than 80 putative IgE-binding proteins described (4). A. fumigatus represents the most complex allergenic source described so far.

\section{MAJOR AND RELEVANT MINOR ALLERGENIC MOLECULES}

The spectrum of allergens produced by the fungus spans a wide variety of different molecular structures covering enzymes, secreted, intracellular, and structural proteins (Table 1). A subdivision into major and minor allergens is difficult because the sensitization to a very variable pattern of allergens
Aspergillus fumigatus is the causative agent involved in allergic bronchopulmonary aspergillosis, an intense inflammatory response of the lung to $A$. fumigatus allergens.

Twenty-tree allergens from the fungus have been cloned, produced as recombinant proteins, and characterized.

Component resolved serologic investigations of patient's sera with the recombinant $A$. fumigatus allergens of the fungus can aid to confirm a diagnosis of ABPA suspected from clinical signs. 


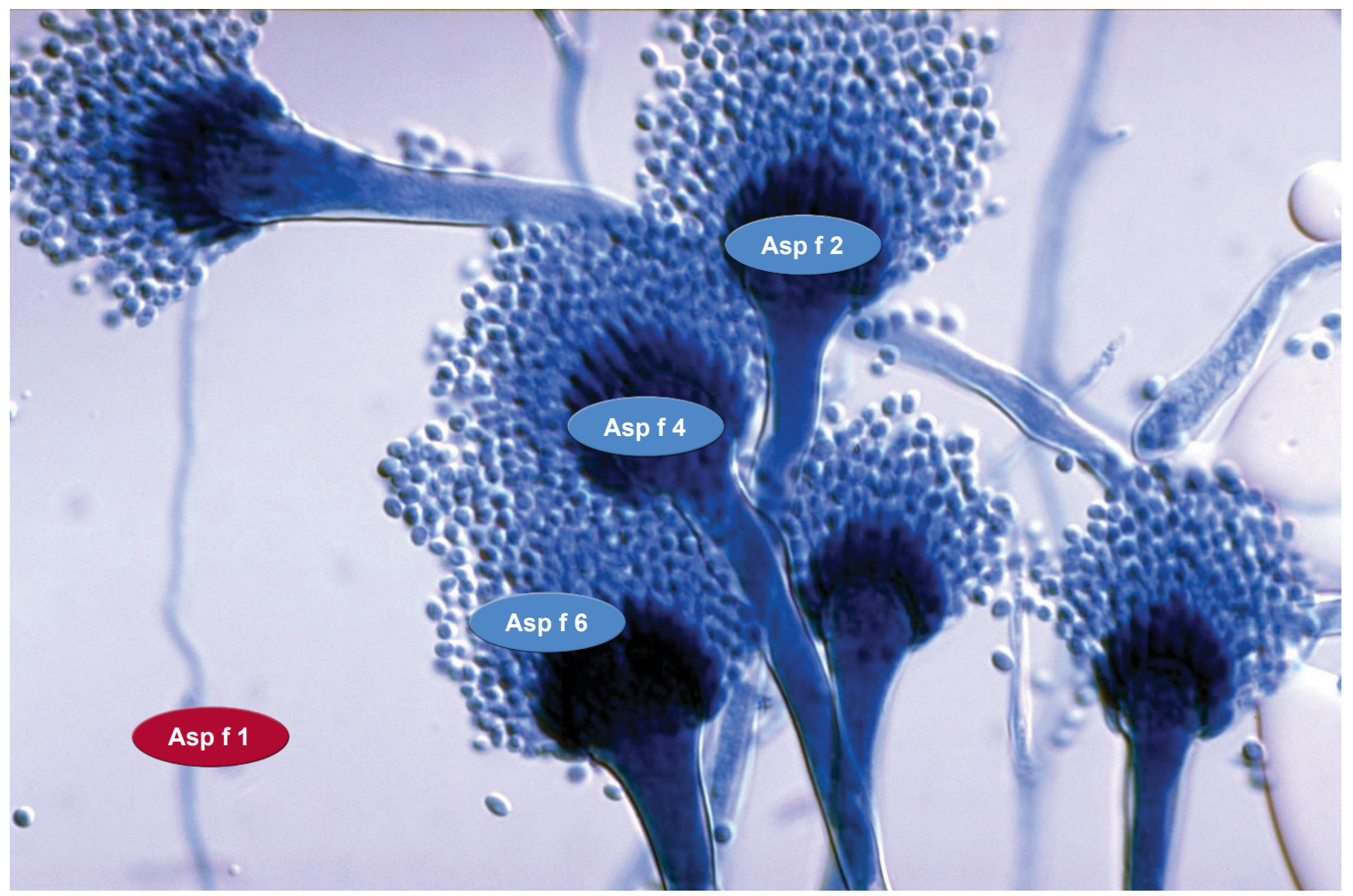

Figure 1

Picture of Aspergillus fumigatus showing the major secreted allergen Asp $f 1$ (red) and the ABPA-related intracellular allergen s Asp f 2, Asp f 4 and Asp f 6 (blue).

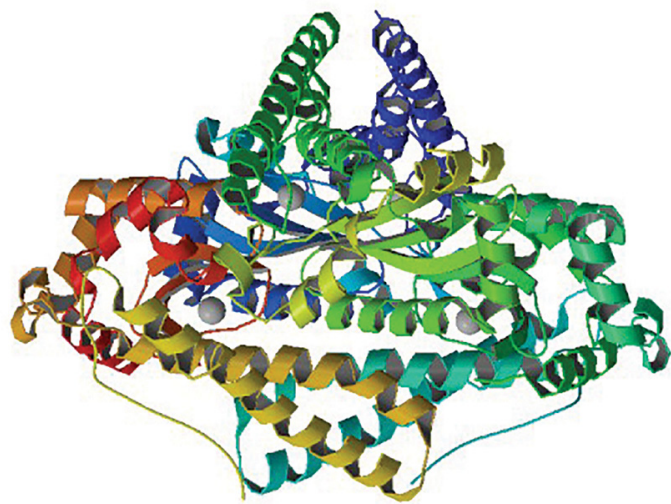

Figure 2

Ribbon diagram showing the assembly of the $A$. fumigatus MnSOD (Asp f 6). The four identical subunits are indicated by different colours.

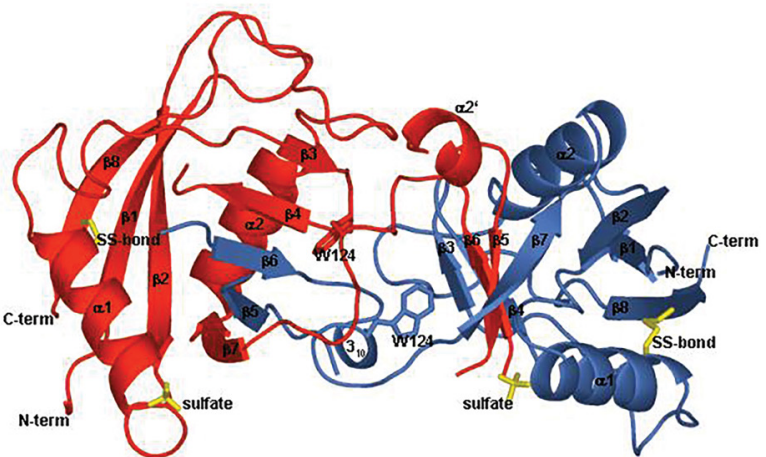

Figure 3

Ribbon diagram showing the dimer formation of A. fumigatus cyclophilin (Asp f 11). The two identical subunits form a dimer through swapping of a central domain involving the ?-sheets 5 and 6 of the cyclophilin monomeric unit. 


\section{Table 1}

Officially recognized Aspergillus fumigatus allergens

\begin{tabular}{|c|c|c|c|c|c|c|}
\hline \multirow{2}{*}{ Allergen } & \multirow{2}{*}{ Biochemical function } & \multirow{2}{*}{$\begin{array}{c}\mathrm{MW} \\
(\mathrm{kDa})\end{array}$} & \multirow{2}{*}{$\begin{array}{l}\text { Accession } \\
\text { Number }\end{array}$} & \multicolumn{2}{|c|}{$\begin{array}{l}\% \text { IgE-positive among } \\
\text { (n) patients tested with }\end{array}$} & \multirow{2}{*}{ Ref } \\
\hline & & & & ABPA & A. fumigatus Allergy & \\
\hline Asp f 1 & Ribotoxin & 18 & M83781 & $83 \%(60)$ & $45 \%(40)$ & 5 \\
\hline Asp f 2 & Unknown & 27 & U56938 & $100 \%(10)$ & $0 \%(10)$ & 6 \\
\hline Asp f 3 & Peroxisomal protein & 19 & U58050 & $88 \%(60)$ & $52 \%(40)$ & 5 \\
\hline Asp f 4 & Unknown & 30 & AJ001732 & $80 \%(60)$ & $0 \%(40)$ & 5 \\
\hline Asp f 5 & Metalloprotease & 40 & Z30424 & $93 \%(54)$ & $74 \%(35)$ & 7 \\
\hline Asp $f 6$ & MnSOD & 26.5 & U53561 & $55 \%(60)$ & $0 \%(40)$ & 5 \\
\hline Asp f 7 & Unknown & 12 & AJ223315 & $46 \%(64)$ & $29 \%(35)$ & 7 \\
\hline Asp f 8 & P2 ribosomal protein & 11 & AJ224333 & $15 \%(92)$ & $8 \%(75)$ & 8 \\
\hline Asp f 9 & Unknown & 34 & AJ223327 & $89 \%(54)$ & $31 \%(35)$ & 7 \\
\hline Asp f 10 & Aspartate protease & 34 & X85092 & $28 \%(54)$ & $3 \%(35)$ & 7 \\
\hline Asp $\mathrm{f} 11$ & Cyclophilin & 24 & AJ006689 & ND* & $90 \%(30)$ & 9 \\
\hline Asp $\mathrm{f} 12$ & Heat shock protein P90 & 90 & U92465 & ND & ND & \\
\hline Asp f 13 & Alkaline serine protease & 34 & Z11580 & ND & ND & \\
\hline Asp f 15 & Unknown & 16 & AJ002026 & ND & ND & \\
\hline Asp $f 16$ & Unknown & 43 & AF062651 & $70 \%(26)$ & ND & 10 \\
\hline Asp $\mathrm{f} 17$ & Unknown & & AJ224865 & ND & ND & \\
\hline Asp f 18 & Vacuolar serine protease & 34 & Y13338 & ND & $79 \%(33)$ & 11 \\
\hline Asp $f 22$ & Enolase & 46 & AF284645 & ND & $86 \%(7)$ & 12 \\
\hline Asp $\mathrm{f} 23$ & L3 ribosomal protein & 44 & AF464911 & ND & ND & \\
\hline Asp f 27 & Cyclophilin & 18 & AJ937743 & $75 \%(40)$ & ND & 13 \\
\hline Asp $f 28$ & Thioredoxin & 13 & AJ937744 & $30 \%(40)$ & ND & 14 \\
\hline Asp $\mathrm{f} 29$ & Thioredoxin & 13 & AJ937745 & $50 \%(40)$ & ND & 14 \\
\hline Asp f 34 & PhiA cell wall protein & 20 & AM496018 & $93 \%(40)$ & $46 \%(24)$ & 15 \\
\hline
\end{tabular}

*ND: not determined

strongly depends on the pathologic background of the single individual. Aspergillus allergy rarely affects atopic individuals without asthma or cystic fibrosis (2) which are as such heterogeneous diseases with many phenotypes.

Interestingly the phylogenetically highly conserved allergens Asp f 6 (MnSOD), Asp f 8 (P2 ribosomal protein), Asp f 11 and Asp f 27 (cyclophilins), as well as Asp f 28 and Asp f 29 (thioredoxins) show a high degree of in vitro and in vivo cross-reactivity with other fungal proteins belonging to the same families and also with the corresponding human proteins due to the presence of conserved epitopes on the surface of the proteins. The clinical relevance of these reactions is, however, still unclear. 


\section{SENSITIZATION TO INDIVIDUAL MOLECULES AND ITS CLINICAL RELEVANCE}

As shown in Table 1 the incidence of sensitization to individual $A$. fumigatus allergens strongly depends from the status of the patient. Asthmatic, as well as cystic fibrosis (CF) patients (16) suffering from ABPA, show a higher sensitization to all investigated allergens then asthmatic or CF patients suffering from a simple $A$. fumigatus allergy. Interestingly the allergens like Asp f 2, 4 and 6 seem to be exclusively recognized by patients with ABPA both, in asthma (5) and CF (17) although large confirmatory studies are still lacking (16). At cDNA level only two $A$. fumigatus allergens, Asp $f 1$ and Asp $f 5$ did not have homologues in both $A$. nidulans and $A$. oryzae although Asp $f 5$ did, and has a closely related homologue in $A$. oryzae indicating a high degree of cross-reactivity among Aspergillus species (19). The clinical significance of this observation awaits experimental elucidation.

\section{CLINICAL MANAGEMENT}

ABPA, an intense inflammatory reaction to Aspergillus in the lung, is considered a complex clinical syndrome with defined serological, pathological, radiological, and clinical features. The diagnosis of ABPA in asthmatic and CF patients sensitized to $A$. fumigatus is complicated by interfering laboratory and clinical findings shared between the diseases. The criteria for the diagnosis of ABPA are summarized in Table 2, and when all criteria are present the diagnosis is readily made.

However, all criteria are rarely present simultaneously even in classical ABPA patients because their presence depends on the stage of the disease (20), and therefore the correct diagnosis of ABPA suspected from clinical signs remains challenging. Nevertheless, because an untreated ABPA lead to a lethal end stage lung fibrosis, the disease should be ruled out in all patients sensitized to $A$. fumigatus.

Because $A$. fumigatus is ubiquitously present in our environment (1), allergen avoidance is not an option for the management of the disease, and allergenspecific immunotherapy with recombinant fungal allergens has never been reported.

The overall goals for the management of ABPA are: i) to control symptoms of asthma or $\mathrm{CF}$, ii) prevent or treat pulmonary exacerbations, iii) reduce or remit pulmonary inflammation, and iv) mitigating

\section{Table 2}

Criteria for a clinical diagnosis of classical ABPA

\begin{tabular}{|c|c|c|}
\hline Clinical and laboratory findings & Importance & Ref \\
\hline $\begin{array}{l}\text { Asthma or CF with deterioration } \\
\text { of the lung function }\end{array}$ & Required & 16,20 \\
\hline $\begin{array}{l}\text { Immediate cutaneous reactivity } \\
\text { to A. fumigatus extracts }\end{array}$ & Required & 16,20 \\
\hline $\begin{array}{l}\text { Elevated total serum IgE ( }>416 \\
\text { IU/mI) or Elevated serum IgE } \\
\text { and IgG to A. fumigatus }\end{array}$ & Required & 16,20 \\
\hline Chest graphic infiltrates & Required & 16,20 \\
\hline $\begin{array}{l}\text { Serum precipitating antibodies } \\
\text { to } A \text {. fumigatus }\end{array}$ & Additional & 16,20 \\
\hline $\begin{array}{l}\text { Central bronchiectasis on chest } \\
\mathrm{CT}\end{array}$ & Additional & 16,20 \\
\hline Peripheral blood eosinophilia & Additional & 16,20 \\
\hline $\begin{array}{l}\text { A. fumigatus containing mucus } \\
\text { plugs }\end{array}$ & Additional & 16,20 \\
\hline
\end{tabular}

or preventing progression to end-stage fibrotic lung diseases (20). The treatment of choice for ABPA still remains the administration of systemic corticosteroids, with itraconazole or omalizumab as adjunctive treatments in severe cases (18).

Despite the availability of the recombinant allergens Asp $f$ 1, 2, 3, 4, and 6 on ImmunoCAP's, the "gold standard" for the determination of allergen-specific IgE in serum and experimental evidence that these allergens could help to discriminate between $A$. fumigatus sensitization and ABPA, large multicentre studies $(5,6,17,21)$ validating the utility of these allergens for the discrimination between the two diseases are still lacking.

\section{References}

1. Horner WE, Helbling A, Salvaggio JE, Lehrer SB. Fungal allergens. Clin Microbiol Rev 1995;8:161-179.

2. Crameri R, Blaser K. Allergy and immunity to fungal infections and colonization. Eur Respir J 2002;19:151157.

3. http://www.allergen.org

4. Kodzius R, Rhyner C, Konthur Z, Buczek D, Lehrach $\mathrm{H}$, Walter $\mathrm{G}$ et al. Rapid identification of allergenencoding cDNA clones by phage display and 


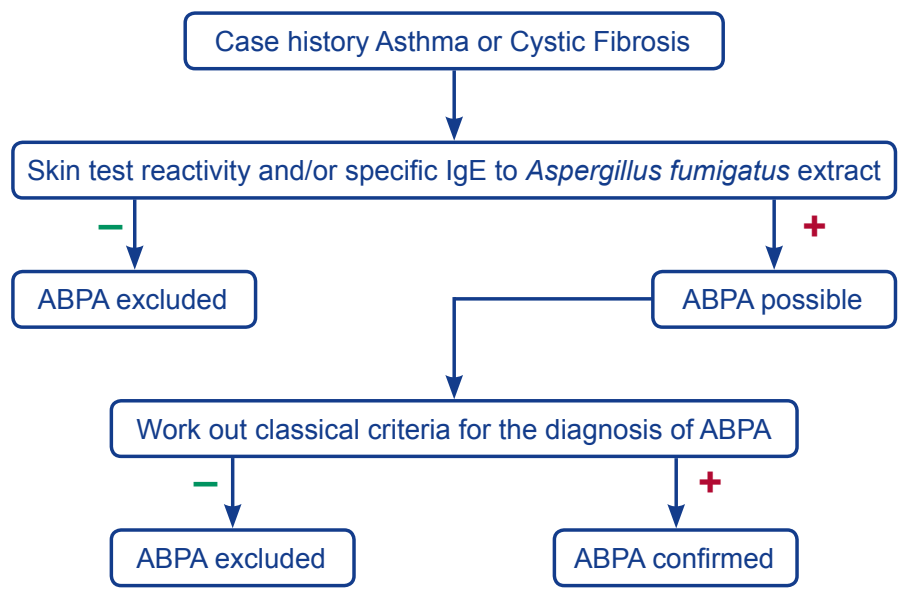

\section{Figure 4}

Decision algorithm for the diagnosis of ABPA. Allergen-specific lgE against rAsp $f 2$, rAsp $f$, and rAsp $f 6$ might be used to corroborate the diagnosis made based on clinical signs.

high-density arrays. Comb Chem High Throughput Screen 2003;6:147-154.

5. Crameri R, Hemmann S, Ismail C, Menz G, Blaser K. Disease-specific recombinant allergens for the diagnosis of allergic bronchopulmonary aspergillosis. Int Immunol 1998;10:1211-1216.

6. Banerjee B, Greenberger PA, Fink JN, Kurup VP. Immunological characterization of Asp $f$ 2, a major allergen of Aspergillus fumigatus associated with allergic bronchopulmonary aspergillosis. Infect Immun 1998;66:1575-1582.

7. Crameri R. Recombinant Aspergillus fumigatus allergens: from the nucleotide sequences to clinical applications. Int Arch Allergy Immunol 1998;115:99114.

8. Mayer C, Appenzeller U, Seelbach H, Achatz G, Oberkofler $\mathrm{H}$, Breitenbach $M$ et al. Humoral and cell-mediated autoimmune reactions to human acidic ribosomal P2 protein in individuals sensitized to Aspergillus fumigatus P2 protein. J Exp Med 1999;189:1507-1512.

9. Flückiger $\mathrm{S}$, Fijten $\mathrm{H}$, Whitley $\mathrm{P}$, Blaser $\mathrm{K}$, Crameri R. Cyclophilins, a new family of cross-reactive allergens. Eur J Immunol 2002;32:10-7.

10. Banerjee B, Kurup VP, Greenberger PA, Johnson $\mathrm{BD}$, Fink JN. Cloning and expression of Aspergillus fumigatus allergen Asp $\mathrm{f} 16$ mediating both humoral and cell-mediated immunity in allergic bronchopulmonary aspergillosis (ABPA). Clin Exp Allergy 2001;31:761-770.

11. Shen HD, Lin WL, Tam MF, Chou H, Wang CW, Tsai JJ et al. Identification of vacuolar serine protease as a major allergen of Aspergillus fumigatus by immunoblotting and $\mathrm{N}$-terminal amino acid sequence analysis. Clin Exp Allergy 2001;31:295-302.

12. Lai HY, Tam MF, Tang RB, Chou H, Chang CY, Tsai JJ et al. cDNA cloning and immunological characterization of a newly identified enolase allergen from Penicillium citrinum and Aspergillus fumigatus. Int Arch Allergy Immunol 2002;127:181-190.

13. Glaser AG, Limacher A, Flückiger $S$, Scheynius A, Scapozza L, Crameri R. Analysis of the crossreactivity and of the $1.5 \mathrm{~A}$ crystal structure of the Malassezia sympodialis Mala s 6 allergen, a member of the cyclophilin pan-allergen family. Biochem J 2006;396:41-49.

14. Glaser AG, Menz G, Kirsch Al, Zeller S, Crameri $\mathrm{R}$, Rhyner C. Auto- and cross-reactivity to thioredoxin allergens in allergic bronchopulmonary aspergillosis. Allergy 2008;63:1617-1623.

15. GlaserAG, KirschAI,Zeller S, Menz G, RhynerC, Crameri R. Molecular and immunological characterization of Asp $f 34$, a major cell wall allergen of Aspergillus fumigatus. Allergy 2009;64:1144-1151.

16. Stevens DA, Moss RB, Kurup VP, Knutsen $A P$, Greenberger $P$, Judson $M A$ et al. Allergic bronchopulmonary aspergillosis in cystic fibrosis - state of the art: cystic fibrosis consensus conference. Clin Infect Dis 2003;37:S225-264.

17. Hemmann S, Nikolaizik WH, Schöni MH, Blaser K, Crameri R. Differential IgE recognition of recombinant Aspergillus fumigatus allergens by cystic fibrosis patients with allergic bronchopulmonary aspergillosis of Aspergillus allergy. Eur J Immunol 1998;28:1155-1160.

18. Greenberger PA, Bush RK, Demain JG, Luong A, Slavin RG, Knutsen AP. Allergic bronchopulmonary 
aspergillosis. J Allergy Clin Immunol Pract 2014;2:703708.

19. Bowyer P, Fraczek M, Denning DW. Comparative genomics of fungall allergens and epitopes shows widespread distribution of closely related allergen and epitope orthologous. BMC Genomics 2006;7:251.

20. Patterson R, Greenberger PA, Radin RC, Roberts M.
Allergic bronchopulmonary aspergillosis: staging as an aid to management. Ann Intern Med 1982;96:286291.

21. Fricker-Hidalgo H, Coltey B, Llerena C, Renversez JC, Grillot R, Pin I et al. Recombinant allergens combined with biological markers in the diagnosis of allergic bronchopulmonary aspergillosis in cystic fibrosis patients. Clin Vaccine Immunol 2010;17:1330-1336. 


\title{
PART B \\ USING \\ MOLECULAR \\ ALLERGOLOGY \\ IN THE CLINICAL \\ PRACTICE
}

\section{ROLE OF MICROBIAL ALLERGENS IN ATOPIC DERMATITIS}

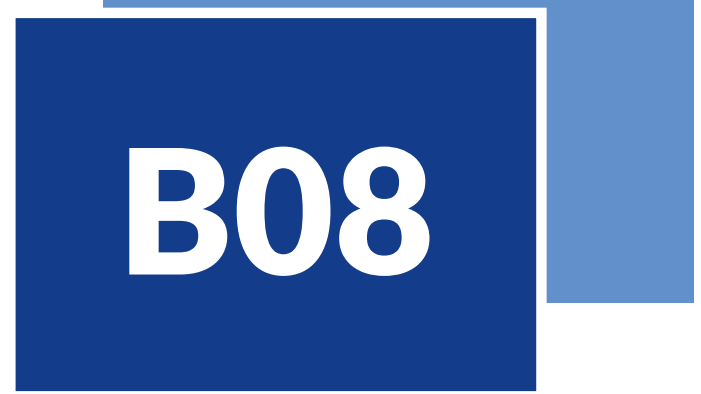

\author{
Martin Glatz, Philipp P. Bosshard, Reto \\ Crameri, Peter Schmid-Grendelmeier
}

\section{INTRODUCTION}

Atopic dermatitis (AD) is a chronic relapsing inflammatory skin disorder, characterized by intensely itchy skin eczema. The prevalence of $A D$ in industrialized countries has tripled during the past 30 years (1), affecting $15-30 \%$ of children and up to $10 \%$ of adults (2). The pathogenesis of $A D$ is not fully understood. Several factors seem to contribute to the development of $A D$ including an impaired barrier function of atopic skin with higher skin $\mathrm{pH}$ and higher transepidermal water loss than healthy skin (3), and an altered skin immune system with the altered production of antimicrobial peptides and cytokines (4). It can be speculated that the impaired barrier function and the altered immune system in atopic skin play intertwining roles and contribute to a colonization and growth of microorganisms on the skin of $A D$ patients (3).

The skin is a complex ecosystem that harbors diverse and body site-specific microbial communities, which have been termed skin microbiome. The phylogenetic profiling of the skin microbiome in healthy individuals revealed that most body sites predominantly harbor bacteria (5). Notably, fungi are part of the normal

The best-characterized molecular allergens in atopic dermatitis are fungal allergens of the skin-colonizing yeast Malassezia spp.

Fourteen allergens from 3 Malassezia species have been characterized to date.

The well characterized allergens Mala s 11 and Mala s13 from M. sympodialis can induce auto-reactive T cells against homologeous proteins in human skin.

Non-fungal allergens of supposed relevance in atopic dermatitis are Der $p 11$ from the mite Dermatophagoides pteronyssinus and enterotoxin $B$ from the bacterium Staphylococcus aureus. 
skin flora at all body sites and comprise 1-22\% of the phylogenetic composition of the skin microbiome (5). The fungal flora of healthy skin harbors almost exclusively Malassezia spp. (5) Recent investigations suggested a significant role of the skin microbiome in the development and course of $\operatorname{AD}(3,6)$. Some species of skin microbial communities or their immunogenic components such as allergens may interact with the altered immune system in atopic skin and so contribute to skin inflammation in AD. In the following we will discuss the significance of skin microbes in $A D$ from an immunologic point of view, and we will overview the possible interactions between microbial allergens with the immune system in atopic skin. We will focus on fungal allergens because recent investigations particularly suggested a possible role of Malassezia allergens in $A D$, but we will also briefly discuss the role of allergens from other microorganisms,

\section{THE ALLERGEN SOURCE}

Malassezia is a genus of lipophilic yeasts that belongs to the phylum Basidiomycota and currently encompasses 14 species. Nine of them are frequently isolated from human skin, while five are usually isolated from animal skin (Table 1). Most Malassezia species lack the genes for the fatty acid synthase and therefore rely on exogenous fatty acid sources, for example skin lipids, to satisfy their nutritive requirement (7). Their need for exogenous lipids explains the predilection of Malassezia spp. for seborrhoeic skin sites, such as the head and neck (8).

Several studies investigated the colonization of healthy and atopic skin with Malassezia spp. (Table 2). Interestingly, these studies did not reveal a consistent difference in the frequency of skin colonization with particular Malassezia spp. between healthy individuals and $A D$ patients. This inconsistency might be due to different methods used in these studies, for example the use of different culture media that might favor the growth of a particular species (9). Despite these methodical considerations, some epidemiological studies postulated a geographic variation in the distribution of particular Malassezia spp., presumably due to climate factors. However, no study directly compared the prevalence of Malassezia spp. in skin samples from different geographic areas.

\section{Table 1}

Currently identified Malassezia species

\begin{tabular}{|c|c|c|c|}
\hline $\begin{array}{l}\text { Malassezia } \\
\text { species }\end{array}$ & $\begin{array}{l}\text { Isolated } \\
\text { from } \\
\text { human skin }\end{array}$ & $\begin{array}{l}\text { Isolated } \\
\text { from } \\
\text { animal skin }\end{array}$ & $\begin{array}{l}\text { Description } \\
\text { as species } \\
\text { (year) }\end{array}$ \\
\hline M. caprae & & $x$ & 2007 \\
\hline M. cuniculi & & $x$ & 2011 \\
\hline M. dermatis & $x$ & & 2002 \\
\hline M. equina & & $x$ & 2007 \\
\hline M. furfur & $x$ & $x$ & 1889 \\
\hline M. globosa & $x$ & $x$ & 1996 \\
\hline M. japonica & $x$ & & 2003 \\
\hline M. nana & & $x$ & 2004 \\
\hline M. obtusa & $x$ & & 1996 \\
\hline M. pachydermatis & & $x$ & 1925 \\
\hline M. restricta & $x$ & & 1996 \\
\hline M. slooffiae & $x$ & $x$ & 1996 \\
\hline M. sympodialis & $x$ & $x$ & 1990 \\
\hline M. yamatoensis & $x$ & & 2004 \\
\hline
\end{tabular}

modified from (8)

\section{MAJOR AND RELEVANT MINOR ALLERGENIC MOLECULES}

Currently 14 immunogenic allergens are characterized that are produced by three Malassezia species, namely M. furfur, M. sympodialis and M. globosa (Table 3). The crystal structure of some of these allergens are known and two examples are shown in Fig. 1. Thirteen allergens, all of which are produced by M. furfur or M. sympodialis, are listed in the official allergen nomenclature list of the International Union of Immunological Societies (IUIS, http://www.allergen. org). Two Malassezia allergens have raised special attention, namely Mala s 11 and Mala s 13. Mala s 11 is $50 \%$ homologous in its amino acid sequence with human MnSOD (10) and 56\% homologous to the MnSOD from Aspergillus fumigatus (Asp f6) (11). Mala s 13 belongs to the family of thioredoxins. Both allergens can induce auto-reactive $T$ cells, which react against the human homologous proteins, hence contributing to skin inflammation in $A D(12,13)$. Recently, an allergen (MGL_1304) derived from M. globosa was described. In vitro experiments have shown that this 


\section{Table 2}

Frequency of skin colonization with Malassezia species

\begin{tabular}{|c|c|c|c|c|c|c|}
\hline \multirow{2}{*}{$\begin{array}{l}\text { Malassezia } \\
\text { species }\end{array}$} & \multicolumn{2}{|c|}{ Identification by PCR (\%) } & \multirow{2}{*}{$\begin{array}{l}\text { Geographic } \\
\text { region }\end{array}$} & \multicolumn{2}{|c|}{ Identification by culture (\%) } & \multirow{2}{*}{$\begin{array}{l}\text { Geographic } \\
\text { region }\end{array}$} \\
\hline & Healthy skin & Atopic skin & & Healthy skin & Atopic skin & \\
\hline M. dermatis & $4-34$ & $29-43$ & $\begin{array}{c}\text { Japan, } \\
\text { South Korea }\end{array}$ & & 7 & South Korea \\
\hline M. furfur & $5-27$ & $6-29$ & $\begin{array}{c}\text { Japan, } \\
\text { South Korea }\end{array}$ & $6-21$ & $4-21$ & $\begin{array}{c}\text { Iran, Bosnia, Canada, } \\
\text { Tunisia }\end{array}$ \\
\hline M. globosa & $28-100$ & 100 & $\begin{array}{c}\text { Japan, } \\
\text { South Korea }\end{array}$ & $32-78$ & $16-28$ & $\begin{array}{c}\text { Iran, Bosnia, Canada, } \\
\text { Tunisia, South Korea, } \\
\text { Sweden }\end{array}$ \\
\hline M. japonica & $10-13$ & $6-19$ & Japan & & & \\
\hline M. obtusa & $1-13$ & $12-24$ & $\begin{array}{c}\text { Japan, } \\
\text { South Korea }\end{array}$ & & $10-30$ & Canada, Sweden \\
\hline M. restricta & $32-100$ & 100 & $\begin{array}{c}\text { Japan, } \\
\text { South Korea }\end{array}$ & $1-8$ & $3-22$ & $\begin{array}{l}\text { Iran, Canada, Tunisia, } \\
\text { South Korea, Sweden }\end{array}$ \\
\hline M. slooffiae & $1-17$ & $12-24$ & $\begin{array}{c}\text { Japan, } \\
\text { South Korea }\end{array}$ & $2-3$ & $3-7$ & $\begin{array}{c}\text { Bosnia, Canada, } \\
\text { Tunisia, South Korea }\end{array}$ \\
\hline M. sympodialis & $29-63$ & $59-62$ & $\begin{array}{c}\text { Japan, } \\
\text { South Korea }\end{array}$ & $7-78$ & $32-100$ & $\begin{array}{l}\text { Iran, Bosnia, Canada, } \\
\text { Tunisia, Poland }\end{array}$ \\
\hline M. yamatoensis & $7-16$ & 24 & Japan & & & \\
\hline
\end{tabular}

references are with the authors
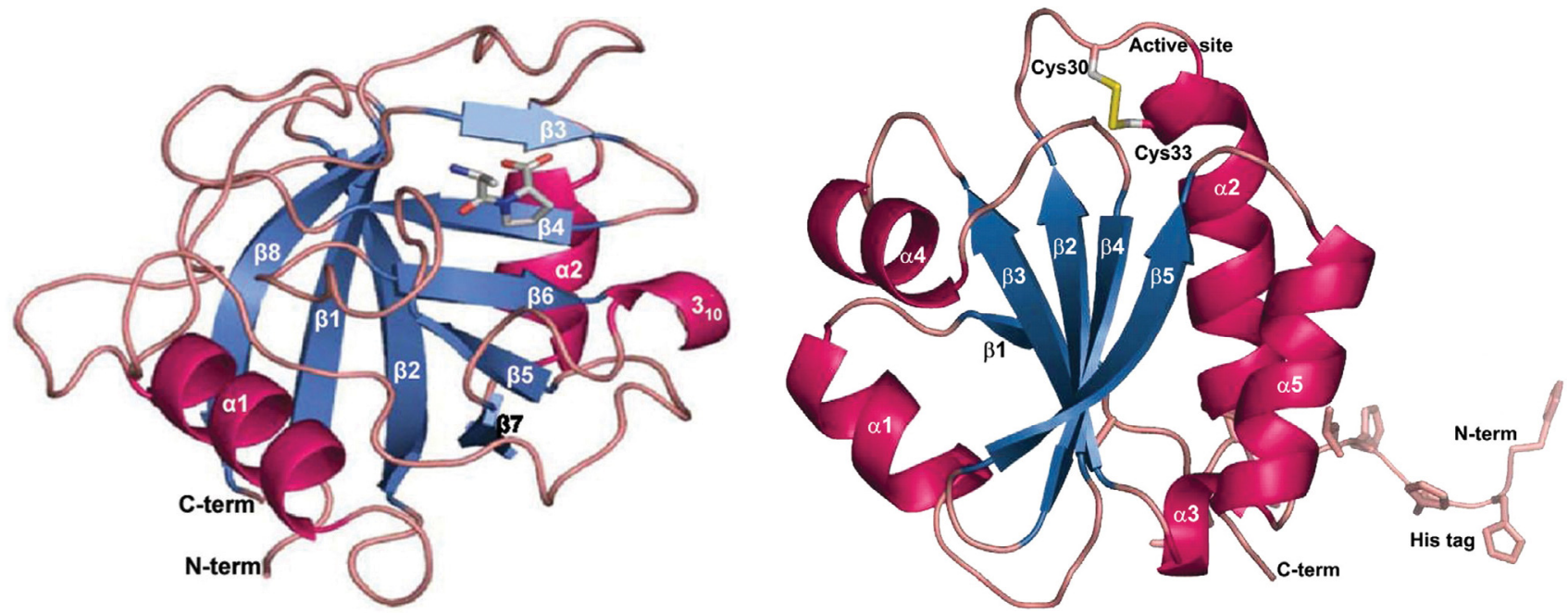

Figure 1

Crystal structure of two Malassezia spp. allergens. 
Table 3

Allergens from Malassezia species and their relevance in atopic dermatitis

\begin{tabular}{|c|c|c|c|c|c|}
\hline Allergen & Source & $\begin{array}{l}\text { Mass } \\
\text { (kDa) }\end{array}$ & Function & $\begin{array}{l}\text { Sensitization } \\
(\%)\end{array}$ & Comment \\
\hline Mala f 2 & M. furfur & 21 & $\begin{array}{l}\text { Peroxysomal } \\
\text { membrane protein }\end{array}$ & 72 & \\
\hline Mala f 3 & M. furfur & 20 & $\begin{array}{l}\text { Peroxysomal } \\
\text { membrane protein }\end{array}$ & 70 & \\
\hline Mala f 4 & M. furfur & 35 & $\begin{array}{l}\text { Mitochondrial malate } \\
\text { dehydrogenase }\end{array}$ & 83 & \\
\hline Mala s 1 & M. sympodialis & 36 & & unknown & \\
\hline Mala s 5 & M. sympodialis & 19 & & unknown & \\
\hline Mala s 6 & M. sympodialis & 18 & Cyclophilin & $92 \%$ & \\
\hline Mala s 7 & M. sympodialis & 22 & & $40-60$ & \\
\hline Mala s 8 & M. sympodialis & 16 & & $40-72$ & \\
\hline Mala s 9 & M. sympodialis & 11 & & $24-36$ & \\
\hline Mala s 10 & M. sympodialis & 86 & Heat shock protein70 & 69 & \\
\hline Mala s 11 & M. sympodialis & 23 & $\begin{array}{l}\text { Manganese superoxide } \\
\text { dismutase }\end{array}$ & $43-75$ & $\begin{array}{l}\text { Sensitization correlates to disease } \\
\text { severity } \\
\text { Induces dendritic cell maturation, } \\
\text { Induces release of IL-6, IL-8, IL- } \\
12 \mathrm{p} 70 \text {, TNF- } \alpha \text { by dendritic cells } \\
\text { Induces auto-reactive T cells against } \\
\text { human homologue }\end{array}$ \\
\hline Mala s 12 & M. sympodialis & 67 & $\begin{array}{l}\text { Glucose-methanol- } \\
\text { choline oxidoreduktase }\end{array}$ & 62 & $\begin{array}{l}\text { Increased production under higher } \\
\mathrm{pH} \text {, which is typical for atopic skin }\end{array}$ \\
\hline Mala s 13 & M. sympodialis & 13 & Thioredoxin & 50 & $\begin{array}{l}\text { Induces auto-reactive T cells against } \\
\text { human homologue }\end{array}$ \\
\hline MGL_1304 & M. globosa & 17 & & 62 & $\begin{array}{l}\text { Induces degranulation of mast cells } \\
\text { Induces IL- } 4 \text { release by basophils }\end{array}$ \\
\hline
\end{tabular}

references are with the authors

allergen induces mast cell degranulation and the release of IL-4 by basophils (14).

\section{SENSITIZATION TO INDIVIDUAL MOLECULES AND ITS CLINICAL RELEVANCE}

Malassezia spp. are part of the healthy skin flora and Malassezia-specific IgG and IgM antibodies are therefore regularly detected in healthy individuals (7). However, healthy individuals usually do not have detectable levels of Malassezia-specific lgE antibodies.
In contrast, $30-80 \%$ of adult AD patients are sensitized to Malassezia spp. as demonstrated by positive atopy patch tests, skin prick tests (SPT) or detectable serum levels of specific IgE antibodies $(15,16)$. Malasseziaspecific IgE are found in $5-27 \%$ of children and 29 $65 \%$ of adults with AD $(17,18)$, which is consistent to the rates found by SPT. The sensitization rate against particular allergens from Malassezia spp. (as far as known) is shown in Table 3.

\section{Clinical relevance of sensitization}

M. sympodialis produces higher amounts of the allergen Mala s 12 (Table 3) when cultured under 


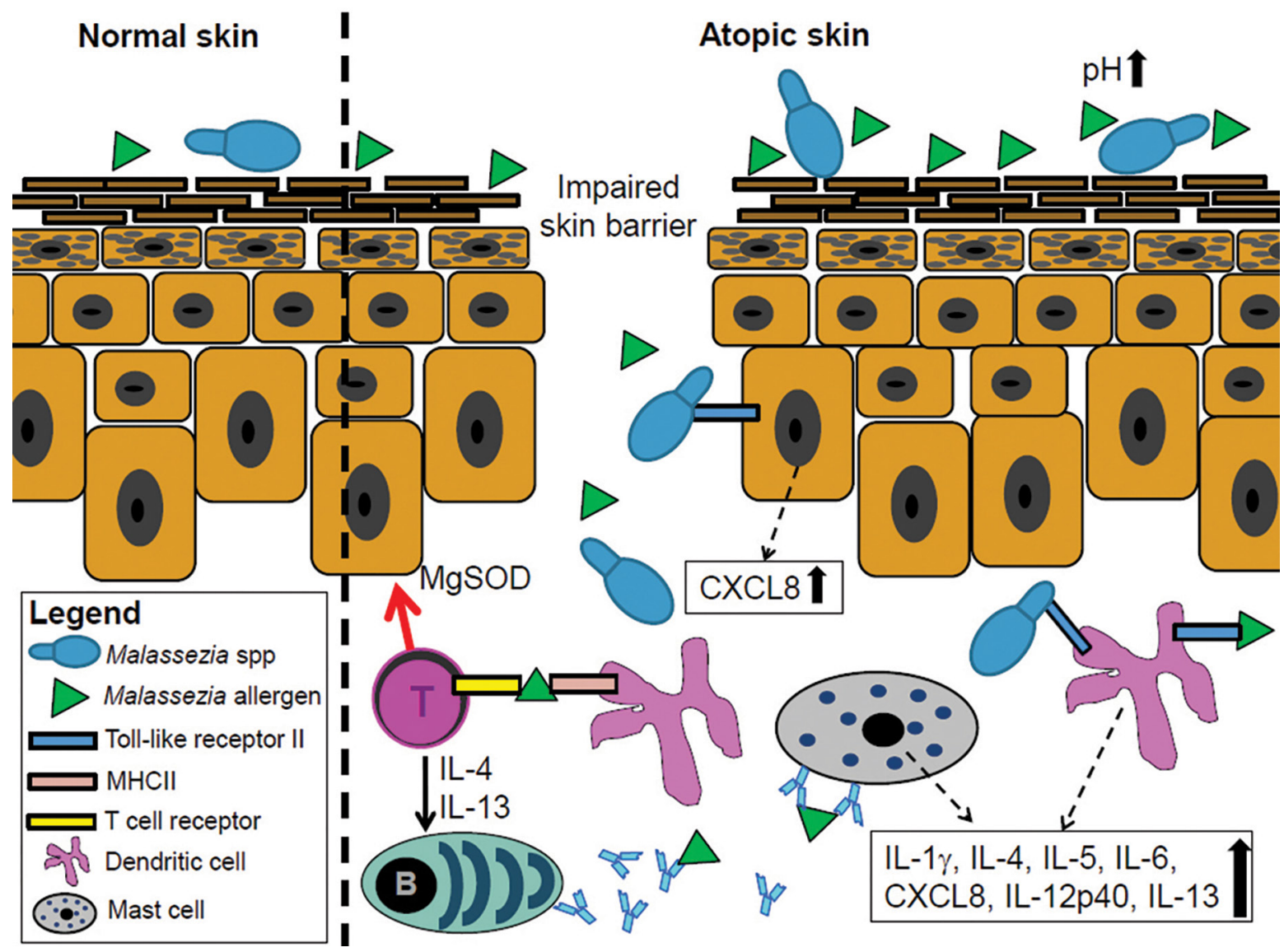

Figure 2

Proposed mechanisms of Malassezia spp. allergen-induced skin inflammation in atopic dermatitis (AD). Increased skin $\mathrm{pH}$ in $\mathrm{AD}$ induces increased allergen release by Malassezia spp. These allergens penetrate the epidermis through the disturbed skin barrier typically found in atopic skin. Allergens may be recognized by toll-like receptor 2 on keratinocytes and dendritic cells and elicit the release of pro-inflammatory cytokines by these cells, or induce the production of specific IgE antibodies. Autoreactive T cells can cross react between fungal and human manganese-dependent superoxide dismutase (MgSOD) or thioredoxin, hence sustaining skin inflammation.

high $\mathrm{pH}$ conditions reflecting the higher $\mathrm{pH}$ of atopic compared to normal skin. This might contribute to Malassezia-related skin inflammation in AD (19). The currently proposed role of Malassezia allergens in the pathogenesis of AD are depicted in Fig. 2.

Some studies found a significant correlation between the severity of $A D$ and serum levels of Malassezia spp.-specific IgE, usually in adults but not in children (18). The lower frequency of Malassezia sensitization in children compared to adults might be related to the poor growth conditions for Malassezia spp. in children compared to adults. The lipid content of sebum, which is a prerequisite for skin colonization for most Malassezia spp. is low in children but rises during puberty and is high until the age of 50 (20). Accordingly, sensitization to Malassezia spp. seems to occur preferably in adulthood, and therefore later than the sensitization to food allergens and aeroallergens, which frequently occurs during childhood (18). 


\section{CLINICAL MANAGEMENT}

\section{Diagnosis}

Standardized skin test extracts for Malassezia spp. are not yet commercially available. Malassezia -specific serum IgE can be measured using a commercially available standardized test (ImmunoCAP®) m70, Phadia, Thermo Scientific, Uppsala, Sweden; www. phadia.com) based on M. sympodialis (ATCC strain 42132) extracts. Recently, a new kit containing several species of Malassezia has been introduced (ImmunoCAP ${ }^{\circ} \mathrm{m} 227$ ) with a slightly increased sensitivity compared to the single species test according to our experience (17). Cultivation of Malassezia spp. from the skin is feasible but challenging, and this method is usually not implicated in the clinical routine care of AD patients.

Due to its sequence homology, Asp $f \quad 6$ is highly cross-reacting to Mala s 11 (11). It might therefore be hypothesized, that serum levels of Asp $\mathrm{f}$ 6-specific IgE are a marker for auto-reactivity. This could be of clinical relevance, because, in contrast to Mala $s$ 11, a test using recombinant Asp f 6 to determine allergen-specific IgE is available in clinical routine (ImmunoCAP® m222).

\section{Treatment}

The benefit of topical or systemic antifungal treatment for the clinical improvement of human $A D$ is still debated. Azole antifungals are the most common class of antifungal drugs prescribed for $A D$ patients. In vitro, azole antifungals are effective against Malassezia spp (20). They have anti-inflammatory properties through inhibition of the IL-4 and IL-5 production by $\mathrm{T}$ cells, which might reduce skin inflammation contributing to the improvement of $A D$ during antifungal treatment (21).

In clinical routine, the topical application of ketoconazole on the face of patients with $A D$ of the head and neck type frequently improves eczema, presumably due to a partial overlap with seborrhoeic dermatitis in some cases (personal communication and own experience). The benefit of a systemic antifungal treatment for $A D$ patients has been assessed in several randomized, placebo-controlled trials. One study compared $36 \mathrm{AD}$ patients treated with ketoconazole with $39 A D$ patients treated with placebo. AD severity improved significantly in the ketoconazole group but not in the placebo group (22). In another trial, a total of $53 \mathrm{AD}$ patients were treated with itraconazole or placebo. The improvement of $A D$ severity was significantly higher in itraconazole treated patients than in the placebo group (23).

A different therapeutic approach for AD patients specifically against Malassezia spp. might be photodynamic therapy. For example, irradiation with a 670-nm diode laser significantly reduces the viability of $M$. furfur cultures that were grown in the presence of a cationic photosensitizer (24). However, the relevance of these results in clinical routine still needs to be proved.

\section{OTHER MOLECULAR ALLERGENS RELEVANT IN ATOPIC DERMATITIS}

House dust mites are one of the most relevant allergen sources worldwide. About $50 \%$ of allergic individuals in Central Europe are sensitized to house dust mites (25) and the severity of $A D$ correlates with the indoor concentration of house dust mite allergens such as Der $f 1$ (26). Another AD-relevant allergen of house dust mite is the $104 \mathrm{kD}$ protein Der $p 11$ (27). Der p 11 is a paramyosin, a family of muscle-associated high molecular weight proteins in invertebrates such as ticks, lice and insects (28). The IgE-mediated sensitization to Der $\mathrm{p} 11$ is relatively low in house dust mite-allergic individuals in Europe (7-16\%), while the sensitization in AD patients is much higher (55-67\%) (28). Therefore, Der p 11 may be a marker allergen for house dust mite-allergic $A D$ patients (28). Another AD-relevant microbial allergen source may be cockroaches. Cockroach allergens, as well as house dust mite allergens, may exacerbate $A D$ via activation of the protease-activated receptor-2 (PAR-2) on human keratinocytes. This impairs the skin barrier function via delayed lamellar body secretion by keratinocytes and decreased neutral lipid deposition in the stratum corneum of the epidermis (29).

A pathogenic role in $A D$ is attributed to the Grampositive bacterium Staphylococcus aureus, because it more frequently colonizes lesional and nonlesional skin of $A D$ patients $(91-100 \%$ and $31-78 \%$, respectively) than the skin of healthy individuals (10\%) (30), and its relative abundance on the skin increases with the development of AD flares (6). S. aureus allergen-specific lgE might be relevant for the pathogenetic role of this bacterium in AD as $29-45 \%$ of $A D$ patients have specific IgE against $S$. aureus 
enterotoxin $B$, and $10-18 \%$ against enterotoxin A (31), although sensitization rates to $S$. aureus allergens appear lower than for Malassezia spp. allergens (18). Furthermore, the sensitization rate to $S$. aureus allergens correlates with the severity of $A D$ (31), and the serum levels of enterotoxin B-specific IgE correlate with serum IL-18 levels in $A D$ patients (32). S. aureus enterotoxin $B$ belongs to the family of superantigens, and therefore also induces an IgE-independent cytokine release from $T$ cells and macrophages via cross-linking the major histocompatibility complex II on antigen-presenting cells and the beta-chains of T cell receptors. Some S. aureus strains isolated from AD patients produce up to 23 superantigens, and the cytokine release induced by these antigens may contribute to skin inflammation in AD (33).

Also, autoreactivity induced by microbial sources other than Malassezia spp have been postulated as a pathogenic or exacerbating factor in AD (34). For instance, five IgE-reactive autoallergens from keratinocytes named Hom s 1 through Hom s 5 have officially been named by WHO-IUIS (www.allergen. org) and are supposed to trigger skin inflammation in $A D$, particularly as a late stage phenomenon in severe $\operatorname{AD}(35,36)$.

\section{CASE}

A 37-years-old men with long-lasting generalized AD predominantly on the head and neck presented with severe relapses of the eczema (maximum EASI score 51) due to unknown causes except worsening during strong sweating and physical exercise. No other trigger factors such as seasonal influences, food or topical contacts were identified. Topical treatment with steroids and emollients was not sufficient during $A D$ flares, therefore the patient sometimes received systemic steroids. Also phototherapy and short-time Cyclosporin A have been used.

Skin prick test with common inhalant and food allergens was negative, as well as patch testing with standard series, topical steroids and antibiotics/ disinfectants. Testing for fungi-specific IgE in serum and patch testing with Malassezia spp. extract and fungal allergens was positive (Table 4).

This patient demonstrated strong IgE-mediated sensitization to $M$. sympodialis and fungal MnSOD and also T-cell mediated reactivity and eczematous

\section{Table 4}

Test results of the patient

\begin{tabular}{|lcc|}
\hline Serum IgE titer & $\begin{array}{c}\text { Value } \\
\text { (kU/l) }\end{array}$ & Interpretation \\
\hline Total IgE & 523 & \multicolumn{1}{c|}{ Elevated } \\
\hline $\begin{array}{l}\text { Inhalant allergen } \\
\text { screening }\end{array}$ & 0.2 & CAP-Class 0 \\
\hline Food Screening (Fx5) & 0.3 & CAP-Class 0 \\
\hline IgE Malassezia spp (m227) & 53.2 & CAP-Class 5 \\
\hline $\begin{array}{l}\text { IgE Aspergillus fumigatus } \\
\text { (m3) }\end{array}$ & 12.4 & CAP-Class 3 \\
\hline IgE Asp f 6 (m222) & 22.4 & CAP-Class 4 \\
\hline IgE Mold mix (mx2) & 24.1 & CAP-Class 4 \\
\hline Patch testing (assessment after 48 hours) \\
\hline Malassezia spp. extract & +++ & Highly pos \\
\hline Fungal MnSOD (r Asp f 6) & +++ & Highly pos \\
\hline Human MnSOD & ++ & Highly pos \\
\hline
\end{tabular}

reaction to fungal and recombinant human MnSOD by patch testing. In addition to emollients and topical steroids the patient received a peroral anti-fungal treatment (itraconazole $100 \mathrm{mg}$ bid for two weeks) what led to a fast but only temporary improvement of his AD (EASI 23 after two weeks therapy, EASI 35 three weeks later). The patient currently uses topical antifungal agents in the face and neck region and an antifungal shampoo every six weeks resulting in significant improvement of eczema and decrease of $A D$ flare frequency.

This case demonstrates that $A D$ patients with a significant sensitization to Malassezia spp. may benefit from a systemic and topical anti-fungal treatment.

\section{TOOLS}

There is not enough data available for recommendations of molecular-based allergy diagnosis in the diagnostic work-up of AD. However, assessment of some allergen-specific IgE might be useful to determine the sensitization to fungal and human autologous antigens (Fig. 3). 


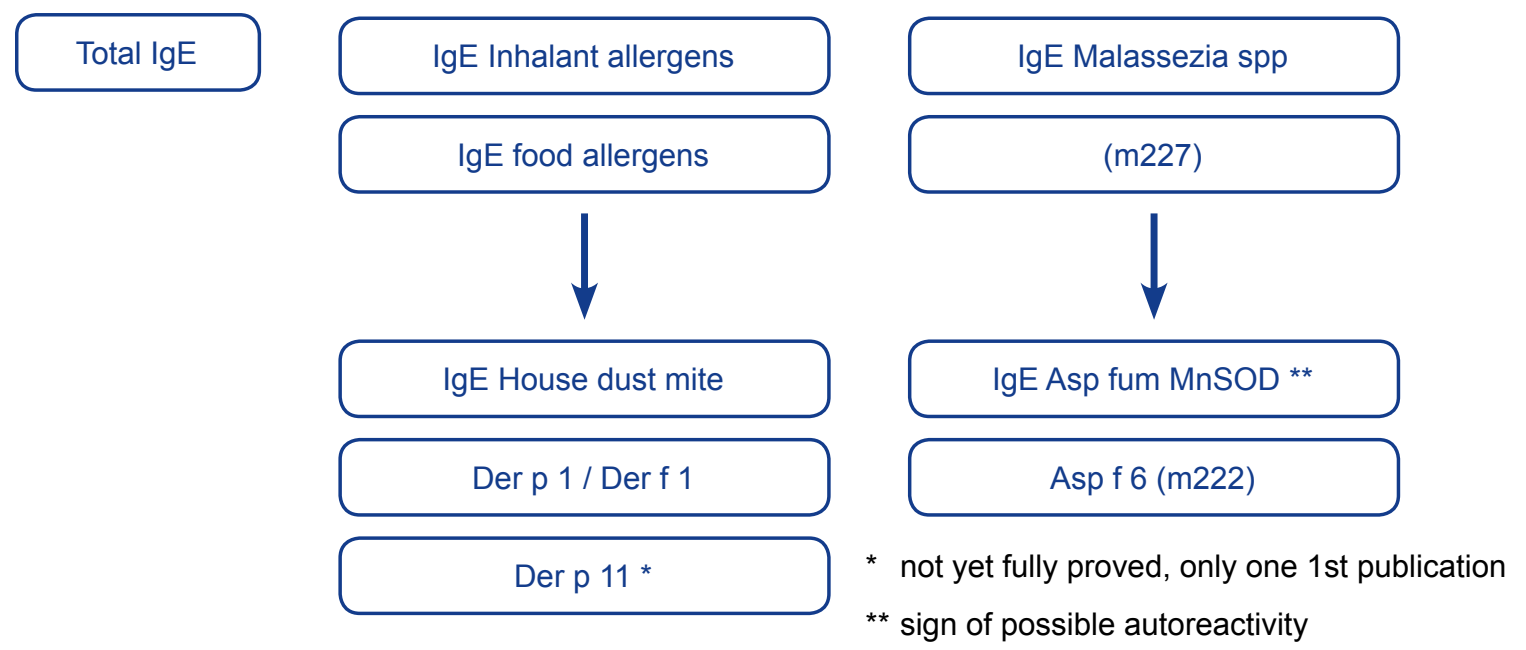

\section{Figure 3}

Possible use of molecular allergy diagnosis in atopic dermatitis.

\section{References}

1. Bieber T. Atopic dermatitis. N Engl J Med 2008;358: 1483-1494.

2. Williams $\mathrm{H}$, Flohr $\mathrm{C}$. How epidemiology has challenged 3 prevailing concepts about atopic dermatitis. J Allergy Clin Immunol 2006;118:209-213.

3. De Benedetto A, Kubo A, Beck LA. Skin barrier disruption: a requirement for allergen sensitization? J Invest Dermatol 2012;132:949-963.

4. Kuo IH, Yoshida T, De Benedetto A, Beck LA. The cutaneous innate immune response in patients with atopic dermatitis. J Allergy Clin Immunol 2013;131:266-278.

5. Oh J, Byrd AL, Deming C, Conlan S, Kong HH, Segre JA. Biogeography and individuality shape function in the human skin metagenome. Nature 2014;514:59-64.

6. Kong HH, Oh J, Deming C, Conlan S, Grice EA, Beatson MA et al. Temporal shifts in the skin microbiome associated with disease flares and treatment in children with atopic dermatitis. Genome Res 2012;22:850859.

7. Saunders CW, Scheynius A, Heitman J. Malassezia fungi are specialized to live on skin and associated with dandruff, eczema, and other skin diseases. PLoS Pathog 2012;8:e1002701.

8. Cabañes FJ. Malassezia yeasts: how many species infect humans and animals? PLoS Pathog 2014;10:e1003892.

9. Kaneko T, Makimura K, Abe M, Shiota R, Nakamura $\mathrm{Y}$, Kano $\mathrm{R}$ et al. Revised culture-based system for identification of Malassezia species. J Clin Microbiol 2007;45:3737-3742.

10. Vilhelmsson M, Glaser AG, Martinez DB, Schmidt $M$, Johansson $C$, Rhyner $C$ et al. Mutational analysis of amino acid residues involved in IgE-binding to the Malassezia sympodialis allergen Mala s 11 . Mol Immunol 2008;46:294-303.

11. Schwienbacher M, Israel L, Heesemann J, Ebel F. Asp f6, an Aspergillus allergen specifically recognized by IgE from patients with allergic bronchopulmonary aspergillosis, is differentially expressed during germination. Allergy 2005;60:1430-1435.

12. Schmid-Grendelmeier $P$, Fluckiger $S$, Disch $R$, Trautmann A, Wuthrich B, Blaser $\mathrm{K}$ et al. IgE-mediated and $T$ cell-mediated autoimmunity against manganese superoxide dismutase in atopic dermatitis. J Allergy Clin Immunol 2005;115:1068-1075.

13. Balaji $H$, Heratizadeh A, Wichmann K, Niebuhr $M$, Crameri R, Scheynius A et al. Malassezia sympodialis thioredoxin-specific $T$ cells are highly cross-reactive to human thioredoxin in atopic dermatitis. J Allergy Clin Immunol 2011;128:92-99.

14. Hiragun $T$, Ishii K, Hiragun M, Suzuki $H$, Kan T, Mihara $S$ et al. Fungal protein MGL_1304 in sweat is an allergen for atopic dermatitis patients. J Allergy Clin Immunol 2013;132:608-615.

15. Johansson C, Sandstrom MH, Bartosik J, Sarnhult T, Christiansen J, Zargari A et al. Atopy patch test reactions to Malassezia allergens differentiate subgroups of atopic dermatitis patients. $\mathrm{Br} J$ Dermatol 2003;148:479-488. 
16. Scheynius A, Johansson C, Buentke E, Zargari A, Linder MT. Atopic eczema/dermatitis syndrome and Malassezia. Int Arch Allergy Immunol 2002;127:161169.

17. Brodská P, Panzner P, Pizinger K, Schmid-Grendelmeier P. IgE-mediated sensitization to malassezia in atopic dermatitis: more common in male patients and in head and neck type. Dermatitis 2014;25:120-126.

18. Glatz M, Buchner M, von Bartenwerffer W, SchmidGrendelmeier P, Worm M, Hedderich J et al. Malassezia spp.-specific immunoglobulin $\mathrm{E}$ level is a marker for severity of atopic dermatitis in adults. Acta Derm Venereol 2015;95:191-196.

19. Selander C, Zargari A, Möllby R, Rasool O, Scheynius A. Higher $\mathrm{pH}$ level, corresponding to that on the skin of patients with atopic eczema, stimulates the release of Malassezia sympodialis allergens. Allergy 2006;61:1002-1008.

20. Darabi K, Hostetler SG, Bechtel MA, Zirwas M. The role of Malassezia in atopic dermatitis affecting the head and neck of adults. J Am Acad Dermatol 2009;60:125136.

21. Kanda N, Enomoto U, Watanabe S. Anti-mycotics suppress interleukin- 4 and interleukin-5 production in anti-CD3 plus anti-CD28-stimulated $T$ cells from patients with atopic dermatitis. J Invest Dermatol 2001;117:1635-1646.

22. Lintu $P$, Savolainen J, Kortekangas-Savolainen $O$, Kalimo K. Systemic ketoconazole is an effective treatment of atopic dermatitis with IgE-mediated hypersensitivity to yeasts. Allergy 2001;56:512-517.

23. Svejgaard E, Larsen $P \varnothing$, Deleuran M, Ternowitz T, Roed-Petersen J, Nilsson J. Treatment of head and neck dermatitis comparing itraconazole $200 \mathrm{mg}$ and $400 \mathrm{mg}$ daily for 1 week with placebo. J Eur Acad Dermatol Venereol 2004;18:445-449.

24. Takahashi H, Nakajima S, Sakata I, lizuka H. Antifungal effect of TONS504-photodynamic therapy on Malassezia furfur. J Dermatol 2014;41:895-897.

25. Thomas WR. The advent of recombinant allergens and allergen cloning. JAllergy Clin Immunol 2011;127:855859.

26. Kim J, Lee S, Woo SY, Han Y, Lee JH, Lee IY et al. The indoor level of house dust mite allergen is associated with severity of atopic dermatitis in children. J Korean Med Sci 2013;28:74-79.
27. Lee CS, Tsai LC, Chao PL, Lin CY, Hung MW, Chien $\mathrm{Al}$ et al. Protein sequence analysis of a novel 103kDa Dermatophagoides pteronyssinus mite allergen and prevalence of serum immunoglobulin $E$ reactivity to rDer p 11 in allergic adult patients. Clin Exp Allergy 2004;34:354-362.

28. Banerjee S, Resch Y, Chen KW, Swoboda I, FockeTejkl M, Blatt K, et al. Der $\mathrm{p} 11$ is a major allergen for house dust mite-allergic patients suffering from atopic dermatitis. J Invest Dermatol 2015;135:102-109.

29. Jeong SK, Kim HJ, Youm JK, Ahn SK, Choi EH, Sohn MH, et al. Mite and cockroach allergens activate proteaseactivated receptor 2 and delay epidermal permeability barrier recovery. J Invest Dermatol 2008;128:19301939.

30. Matsui K, Nishikawa A, Suto H, Tsuboi R, Ogawa H. Comparative study of Staphylococcus aureus isolated from lesional and non-lesional skin of atopic dermatitis patients. Microbiol Immunol 2000;44:945-947.

31. Bunikowski R, Mielke M, Skarabis H, Herz U, Bergmann $\mathrm{RL}$, Wahn $\mathrm{U}$ et al. Prevalence and role of serum IgE antibodies to the Staphylococcus aureus-derived superantigens SEA and SEB in children with atopic dermatitis. J Allergy Clin Immunol 1999;103:119-124.

32. Sonesson A, Bartosik J, Christiansen J, Roscher I, Nilsson F, Schmidtchen A et al. Sensitization to skinassociated microorganisms in adult patients with atopic dermatitis is of importance for disease severity. Acta Derm Venereol 2013;93:340-345.

33. Schlievert PM, Case LC, Strandberg KL, Abrams BB, Leung DY. Superantigen profile of Staphylococcus aureus isolates from patients with steroid-resistant atopic dermatitis. Clin Infect Dis 2008;46:1562-1567.

34. Tang TS, Bieber T, Williams HC. Does "autoreactivity" play a role in atopic dermatitis? J Allergy Clin Immunol 2012;129:1209-1215.

35. Valenta R, Natter S, Seiberler S, Wichlas S, Maurer $D$, Hess $M$ et al. Molecular characterization of an autoallergen, Hom $\mathrm{s} 1$, identified by serum IgE from atopic dermatitis patients. J Invest Dermatol 1998;111:1178-1183.

36. Hradetzky S, Roesner LM, Balaji H, Heratizadeh A, Mittermann I, Valenta R et al. Cytokine effects induced by the human autoallergen alpha-NAC. J Invest Dermatol 2014;134:1570-1578. 

MOLECULAR DIAGNOSTICS

\section{IN THE MANAGEMENT OF FOOD ALLERGY}

\author{
Helen A. Brough, Gideon Lack, Jörg Kleine- \\ Tebbe, Antonella Muraro
}

Food allergy has increased particularly in children and is an increasing public health concern (1). Clinical practice guidelines covering general diagnosis and management of food allergies have recently been published and disseminated by the EAACl Food Allergy Diagnosis Systematic Review and Guidelines $(2,3)$. Correct diagnosis of food allergy is of great importance so as to avoid falsely labelling the patient as food allergic or not allergic:

A false positive food allergy diagnosis will lead to unnecessary food exclusions and potential impact on nutrition and growth (4), heightened anxiety and inappropriate recommendation of emergency medication (5).

- A false negative food allergy diagnosis will lead to unexpected allergic reactions to the food in question and risk of potentially severe symptoms without the availability of rescue medication - thus the need to continuously improve and evaluate our approach on the diagnosis of food allergy.

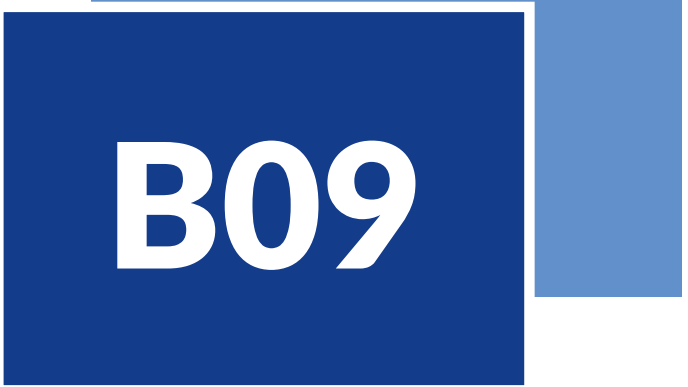

Molecular diagnosis (MD) provides a major step in improving the accuracy of diagnosing IgE-mediated food allergy.

Use of MD in combination with conventional sensitization testing improves analytical and diagnostic performance and can lead to a reduction in the need for diagnostic oral food challenges

New component allergen / pattern of component allergens for a food should be evaluated against conventional allergy testing against whole allergens (both SPT and slgE) as well as against the gold standard oral food challenge to determine their diagnostic performance. 
POTENTIAL ADVANTAGES AND LIMITATIONS OF MOLECULAR DIAGNOSIS

Molecular diagnosis (MD) provides a major step in improving the accuracy of diagnosing IgE-mediated sensitizations in food allergy. The ability to identify and characterize single allergens at a molecular level has added a significant body of understanding as to the mechanism of sensitization to foods. The increasing number of available single allergens, particularly for peanut protein allows for a comprehensive review of the pattern of sensitization (e.g. entire allergenspecific IgE repertoire) (see section A05). Research into the structural similarity between allergens and the amino acid sequence homology between food allergens also helps to explain cross-reactivity between allergens, which may be clinically relevant or irrelevant. Certain panallergen molecules can indicate broad cross-sensitization and unfold particular pollenfood or plant food syndromes (6) (see Sections on Cross-reactivity C01-C08).

Natural extracts used for conventional allergy testing (commercial extracts for skin test and serum specific IgE) contain a complex mixture of proteins. Some of these proteins are relevant for clinical reactivity and some are not but still result in IgE binding and thus positive allergy tests (e.g. positive IgE results due to cross-reactive carbohydrate determinants, (CCDs). Therefore it is assumed that conventional allergy tests (i.e. extract-based peanut $\operatorname{lgE}$ ) are more likely than molecular allergen testing to lead to clinically irrelevant results with the negative implications described above (see also Section B18 on peanut allergy).

The specific context in which MD can improve on total allergen IgE testing include where there are 1) low abundant and or labile food proteins in conventional allergy tests, 2) MD provides information on riskor severity associated molecules, 3) MD provides indicators of food-related cross-reactivity or 4) markers of genuine (species-specific) sensitization. These scenarios are discussed in further depth in Sections A04 and A05.

The performance of any test can be assessed based on metrics such as sensitivity, specificity and negative predictive value (NPV), positive predictive value (PPV), likelihood ratio (LR) with optimum cut-off values being determined on the receiver operating characteristics (ROC) curve. Using these metrics, predictions on the likelihood of clinical reactivity, severity and course provided by MD, go beyond the detection of allergen- specific IgE sensitization. However, these tests are still tests for IgE sensitization and are not synonymous with allergy. Following this line of reasoning one could argue that they should not be called "allergy tests", but rather viewed and termed as "sensitization tests" (see terminology in numerous chapters of this book as well as international guidelines on assays for human $\lg E$ ) (7).

Numerous clinical studies have produced variable performances of the above metrics for IgE assay results including MD testing. Thus, these tests require thorough individual clinical interpretation and the expectations from MD to predict clinical reactivity should be restrained and valued with caution. The strength of MD is related to the level of lgEsensitizations, providing more detailed and precise profiles of the allergen-specific IgE repertoire. In the end, the physician has to define the clinical relevance of the diagnostic test result, not the test by itself.

\section{ATTEMPTS TO PREDICT CLINICAL REACTIVITY WITH SENSITIZATION TESTS USING EXTRACTS OR MOLECULES}

Clinical allergist have always been interested in evaluating the potential of sensitization test outcomes i.e. skin prick test (SPT), allergen-specific lgE (slgE), basophil activation test (BAT) for the prediction of clinically relevant food allergic reactions.

In the Healthnuts Australian based study, peanut SPT had a similar area under the curve (AUC) on ROC curve analysis of 0.94 to Ara h 2 (AUC: 0.95) for predicting challenge proven peanut allergy (364). In a UK based study, peanut SPT had a similar AUC (0.93) than Ara h 2 (AUC: 0.91) using optimal cut-off points of $5 \mathrm{~mm}$ and $0.53 \mathrm{kUA} / \mathrm{I}$ respectively (9). Furthermore, SPT and slgE to whole allergens for cow's milk, egg and peanut have robust, but variable $95 \%$ PPV in the published literature $(8,10)$. The specific cut-offs for $95 \%$ PPV for cow's milk, egg and peanut usually vary between populations depending on the prevalence of disease in that population, inclusion criteria and study protocols. This is also the case for molecular allergens such as Ara $\mathrm{h} 2$ where the $90-95 \%$ cut-offs range from 0.35 $\mathrm{kUA} / \mathrm{I}$ to $42.2 \mathrm{kUA} / \mathrm{I}$ in the published literature (11, 12). Subsequently, no general recommended cut-off level is available for Ara h 2, indicating the difficulty to precisely predict clinical food reactions with pure sensitization test levels, regardless of using extracts or molecules. It is, common practice that as each new 
component allergen becomes available, its diagnostic performance is compared against SPT and slgE to the whole allergen.

\section{GENERAL VALUE OF DIAGNOSTIC TESTS IN FOOD ALLERGY, INDICATING IgE ALLERGY (SPT, BAT)}

Conventional sensitization tests with whole protein allergens have the advantage that they include the majority of relevant, mainly stable and highly abundant allergens, particularly where these molecular allergens are not yet commercially available (which is the case for the majority of foods). In this case SPTs with extracts are less likely to lead to false negative results. Exceptions are indeed allergens missing (i.e. lipophilic food allergens like oleosins in aqueous extracts or defensins) or of low abundance and stability (i.e. pollen-food-syndromes based on Bet $v$ 1-homologs or profilins). SPT confers the benefit of immediate interpretation allowing for a 'one-stopshop' approach to the allergy consultation, which has innumerable benefits for the patient and family (13) Additionally, the BAT is another diagnostic method, which is increasing in popularity and, although still mainly used as a research tool, its combination with MD could represent a powerful tool. Advantages and disadvantages of the various testing options are described in Table 1.

There is growing evidence that the use of MD in combination with conventional sensitization testing improves analytical and diagnostic performance and can lead to a reduction in the need for diagnostic oral food challenges in selected cases (8). Therefore, rather than replace conventional testing, $\mathrm{MD}$ should be added to our existing armamentarium of diagnostic testing.

The chapter below will summarize the following in the evaluation of patients with a suspected food allergy:

1. Considerations for the diagnostic work-up of patients with a suspected food allergy

2. Circumstances in which MD is not necessary and circumstances when it improves the diagnostic work-up and management of food allergy

3. At which stage in the diagnostic algorithm for food allergy does MD provide the most benefit

4. Future perspectives in the diagnosis and management of food allergy in the age of MD

\section{CONSIDERATIONS FOR THE DIAGNOSTIC WORK-UP OF PATIENTS WITH A SUSPECTED FOOD ALLERGY}

When evaluating a patient with a suspected food allergy, performing a wide range of allergy tests without corresponding history of symptoms is likely to lead to unnecessary food elimination due to clinically irrelevant tests. The 'molecules to symptoms' approach, where a battery of tests are performed without first assessing clinical symptoms (as described in Section A03 (Molecular allergy diagnostics in clinical practice) is therefore generally not recommended and at this point is mainly used for research purposes. Allergy testing should be performed where there is a clear history of a convincing lgE-mediated allergic reaction in relation to a particular food/substance. Anticipatory allergy testing may also be considered where there is a known risk of co-reactivity (for example testing for peanut allergy in a child with egg allergy and eczema as per the LEAP study findings) (14). In cases where the allergen is not clearly elucidated from the history, such as in the case of food dependent exercise induced anaphylaxis or idiopathic anaphylaxis, multiple allergen testing (both component and whole allergens) may elucidate the responsible allergen; often this will be omega-5-gliadin, LTPs (in the mediterranean) or tropomyosins (15), or may be due to other, sometimes unusual allergen sources (e.g. lupine or buckwheat).

Whatever diagnostic tests are performed, history forms the cornerstone of our diagnosis thus one must not forget the basic questions outlined in Table 2 .

\section{CIRCUMSTANCES IN WHICH MOLECULAR ALLERGOLOGY IS NOT NECESSARY AND CIRCUMSTANCES WHEN IT IMPROVES THE DIAGNOSTIC WORK-UP AND MANAGEMENT OF FOOD ALLERGY}

In patients where there is a convincing history of IgE-mediated allergy (not delayed gastrointestinal or eczematous reactions) a positive SPT or slgE to the relevant whole food allergen may be sufficient to confirm the diagnosis (Fig. 1). Ninety-five percent PPV values for SPT and slgE that have been documented in the past for peanut, cow's milk and egg, however they are highly dependent on the specific clinical 


\section{Table 1}

Testing modalities for a suspected food allergy (advantages and disadvantages)

\begin{tabular}{|c|c|c|}
\hline & Advantages & Disadvantages \\
\hline $\begin{array}{l}\text { Allergen } \\
\text { extracts } \\
\text { in SPT }\end{array}$ & $\begin{array}{l}\text { - Inexpensive } \\
\text { - Contain many allergenic proteins } \\
\text { - Immediate results in clinic (one-stop shop } \\
\text { clinic) } \\
\text { - Modified (prick to prick) SPT allows for any } \\
\text { allergen source to be evaluated }\end{array}$ & $\begin{array}{l}\text { - Patient is exposed to the allergen } \\
\text { - Difficult characterization and standardization } \\
\text { - Unknown allergen composition } \\
\text { - Contains undefined components, i.e. } \\
\text { endotoxins } \\
\text { - Low abundance or degradation may lead to } \\
\text { low analytic and diagnostic sensitivity } \\
\text { - Proteins mixture complexity may lead to low } \\
\text { specificity }\end{array}$ \\
\hline $\begin{array}{l}\text { Allergen } \\
\text { extracts } \\
\text { in slgE }\end{array}$ & $\begin{array}{l}\text { - Contain many allergenic proteins } \\
\text { - Patient not exposed to the allergen } \\
\text { - Large allergen source panel }\end{array}$ & $\begin{array}{l}\text { - Delay in obtaining results } \\
\text { - Higher costs } \\
\text { - Unknown allergen composition } \\
\text { - Contains undefined components, i.e. } \\
\text { endotoxins } \\
\text { - Low abundance may lead to low sensitivity } \\
\text { - Proteins mixture complexity may lead to low } \\
\text { specificity }\end{array}$ \\
\hline $\begin{array}{l}\text { Molecular } \\
\text { allergens } \\
\text { for slgE }\end{array}$ & $\begin{array}{l}\text { - Detection of sensitization to relevant allergens. } \\
\text { - } \text { Identification of cross reactivity as cause of a } \\
\text { - } \text {-lgE measurement } \\
\text { - Highly defined proteins } \\
\text { - Structure - amount of protein precisely known } \\
\text { - Patient not exposed to the allergen } \\
\text { - Number of allergen molecules can be tested } \\
\text { simultaneously }\end{array}$ & $\begin{array}{l}\text { - Results not immediately available during } \\
\text { consultation } \\
\text { - Technical challenges (first set up) } \\
\text { - Higher costs } \\
\text { - Identification of IgE only to a limited number } \\
\text { of component allergens }\end{array}$ \\
\hline $\begin{array}{l}\text { Basophil } \\
\text { activation } \\
\text { test }\end{array}$ & $\begin{array}{l}\text { - Reflects reactivity and sensitivity to allergen } \\
\text { - Will pick up sensitizations in case of extremely } \\
\text { - Maw total IgE }(<10 \mathrm{kU} / \mathrm{I}) \\
\text { - } \text { of reaction } \\
\text { - May use molecular allergens in culture } \\
\text { - Patient not exposed to the allergen } \\
\text { - Limited number of allergen molecules could be } \\
\text { tested simultaneously }\end{array}$ & $\begin{array}{l}\text { - Higher costs } \\
\text { - Significant manpower requirements } \\
\text { - Fresh blood sample must be used } \\
\text { - Results not available in clinic }\end{array}$ \\
\hline
\end{tabular}

setting (prevalence, geographical region, clinical phenotype, inclusion criteria, procedures, etc.) and cannot be extrapolated and transformed into general recommendations $(16,17)$. Due to a high NPV $(91 \%$ for SPT $<3 \mathrm{~mm}$ ) a negative SPT can be helpful in ruling out an IgE mediated egg allergy (18). Similarly fresh cow's milk SPT $<3 \mathrm{~mm}$ has been reported with a NPV of $98 \%$ (19). The NPV of peanut SPT is also good (29). Thus a negative SPT to the natural extract may be used to rule out IgE-sensitization without the need for MD. The NPV of slgE to whole allergen cow's milk, egg white and peanut have been shown reliable in excluding an IgE mediated food allergy. Whole peanut allergen slgE appears to be sometimes diagnostically more sensitive test than Ara h 2 depending on the population and clinical settings (21). In addition, new peanut allergens (www.allergen.org) indicate gaps in our present molecular peanut allergen panel, being likely available in the future as reagents for allergenspecific IgE assays. This will fill the gap, increasing 


\section{Table 2}

Questions and considerations in the evaluation of food allergy

Question Considerations

What is the suspected food allergy? $\begin{aligned} & \text { Consider whether the allergen is typical for the patient's age and } \\ & \text { population }\end{aligned}$

Was the suspected food allergen A proportion of patients have a reaction after inhalation of or contact ingested, inhaled or touched? with the allergen

Does the patient have an aversion to Generally patients dislike and refuse food containing the allergen the suspected food allergen?

How soon after exposure to the food IgE-mediated allergic reactions usually occur within 20 minutes and allergen did the symptoms occur? certainly within 2 hours

What are the specific symptoms and If the symptoms are not typical of IgE-mediated food allergy, consider a how severe are they? differential diagnosis (which may be delayed food allergy). If the symptoms are severe an emergency plan and rescue medication will be necessary

How long did it take for the The typical time to symptom resolution after reaction is 4-12 hours symptoms to resolve?

How reproducible are the symptoms with previous or subsequent ingestion

Does exercise precipitate the symptoms?
A patient is unlikely to have a reaction to a food on just a few occasions, although reactivity may vary depending on factors such as processing and heating and co-factors.

Exercise that precipitates symptoms may suggest a diagnosis such as food dependent, exercise-induced anaphylaxis (see chapter B16 on wheat dependent, exercise-induced anaphylaxis).

Children with early onset severe eczema have an increased risk of IgEmediated food allergy, particularly to milk, egg and peanut.

Children with birch or grass pollen allergy are more likely to demonstrate cross-reactivity with legumes, fruits, peanut and tree-nuts leading to false positive allergy testing.

See also: Chapter B16 on wheat wheat dependent, exercise induced anaphylaxis

the repertoire and general analytical sensitivity by applying MD-based allergy tests.

There are, however, other whole allergen tests with poor predictive values for clinical reactivity, such as soy, wheat and hazelnut (16). In these cases, MD may outperform whole allergen testing providing more detailed information on the sensitization profile and within limits on the clinical outcome. For example Cor a 9 and Cor a 14 have been shown to outperform hazelnut SPT and slgE tests for objective symptoms on oral food challenge $(12,22,23)$, predominantly due to hazelnut cross-reactivity with birch leading to frequently occurring and clinically irrelevant extractbased results. The component allergen Tri a 19 (omega-5 Gliadin) to diagnose IgE-mediated clinical reactivity to wheat has had encouraging results in
Japan (24), but these results were not reproduced in America/Germany (25). Focusing on the assay level, Tri a 19 has, however, dramatically increased analytical sensitivity and specificity compared to whole wheat extracts, providing a powerful tool to accurately identify omega-5 Gliadin sensitized individuals.

There are situations where MD can differentiate between severity of clinical phenotypes but its role in adding to the clinical history need to be carefully thought through. For example, if the patient has symptoms suggestive of oral allergy syndrome (OAS) after eating apple in the context of birch pollen allergy then doing SPT to a birch pollen extract or to raw and cooked apple is one way to confirm this diagnosis; molecular testing to the underlying primary sensitizer (e.g. Bet $v 1$ ) would be another option. If the patient 
has more ambiguous symptoms which could suggest a more severe clinical phenotype then molecular diagnosis to Mal d 1 (PR-10 protein) or Mal d 3 (LTP) will contribute significantly to the diagnostic algorithm (Fig. 58). This is particularly relevant for apple allergy in the case of a patient of Mediterranean origin where lipid transfer protein allergy (LTP) is more common. This scenario is also relevant for ambiguous histories related to raw or roasted hazelnut (see Section B19 on Tree Nuts and Seeds Allergy) and has important implications in management especially regarding the prescription of self-injectable adrenaline. Soy allergy is often also confusing due to the overlap between OAS symptoms versus more significant clinical reactions. Previously soy components Gly $\mathrm{m} 5$ and 6 were shown to predict more systemic allergic reactions to soy (with both positive Gly $\mathrm{m} 5$ and 6 giving an Odds Ratio of 12 for severe reactions) (26). More recently the $2 S$ albumin Gly $\mathrm{m} 8$ was found to be a better marker for systemic reactions to soy than Gly $\mathrm{m} 5$ and 6 (and soy extract) but it still misclassified many patients $(27,28)$. With MD testing for soya there is also the caveat that sole reactivity to the PR-10 protein Gly $\mathrm{m} 4$ has been responsible for anaphylaxis following consumption of unprocessed soya (29) (see Section B17).

In summary, can add value in cases where there is the possibility of clinically irrelevant sensitization (for example in children with moderate to severe eczema), sensitization due to cross-reactivity (for example in birch pollen allergic patients tested for peanut allergy) or symptoms consistent with either mild allergic phenomenon (OAS) versus potentially more severe allergic phenomenon (with LTP or seed storage protein allergy).

\section{AT WHICH STAGE IN THE DIAGNOSTIC ALGORITHM FOR FOOD ALLERGY DOES MOLECULAR DIAGNOSIS PROVIDES THE MOST BENEFIT?}

The classical top-down approach to testing in a patient with suspected food allergy (as discussed in section A04) combines extract based testing with subsequent targeted allergen specific molecule testing. This approach, from 'symptoms to molecules' provides a pragmatic, yet thorough approach to allergy testing in patients with a suspected food allergy. A proposed algorithm for the use of molecular diagnosis in combination with conventional testing is outlined in Fig. 1 and 2. For general recommendations about food allergy diagnosis, the reader may also refer to the "EAACI Food Allergy Guidelines" (2).

\section{FUTURE PERSPECTIVES IN THE DIAGNOSIS AND MANAGEMENT OF FOOD ALLERGY IN THE AGE OF MOLECULAR ALLERGOLOGY}

Only a small proportion of relevant molecular allergens to foods have been fully characterized. Such a process should involve:

- thorough technical evaluation (e.g. molecular characterization, appropriateness of an isoform, stability) of the chosen molecule/component

- scientific information on its analytical performance (assay features like analytical sensitivity, analytical specificity, potential as a marker for serological cross-reactivity or indicator for primary, genuine sensitization), and ideally

- additional information on diagnostic-clinical performance (diagnostic sensitivity, diagnostic specificity, PPV, NPV, etc).

In order to increase the performance characteristics of $\mathrm{MD}$ full characterization is required particularly for seed storage proteins for tree nuts, legumes and seeds. As with all diagnostic tests discussed MD require validation in large populations both in the hospital and at a community level using the doubleblind placebo-controlled food challenge (DBPCFC) as the gold standard for validation. The value of combination testing using all the tests that have been assessed also needs to be validated as has been performed for peanut component allergens $(8,9)$.

MD has become synonymous with slgE to clinical relevant food allergens but it could be used for SPT to the relevant allergen or BAT to the relevant allergen. Given the benefit of bed-side testing towards the running of a one-stop shop allergy clinic it would be of great value to have SPTs containing the major component allergens. Such SPT materials have been commercialized for certain allergens (e.g. LTP (Pru p 3), profilin (Pho d 2) and Bet v 1 homologs (Mal d 1) $(30,31)$ but not for seed storage proteins such as Ara h 2. Similarly, bed-side molecular allergen testing with quantitative assessments would improve the diagnostic experience for patients. Results of the LEAP study now recommend testing in high risk infants to peanut; having bed-side molecular testing would greatly improve the diagnostic efficiency and 


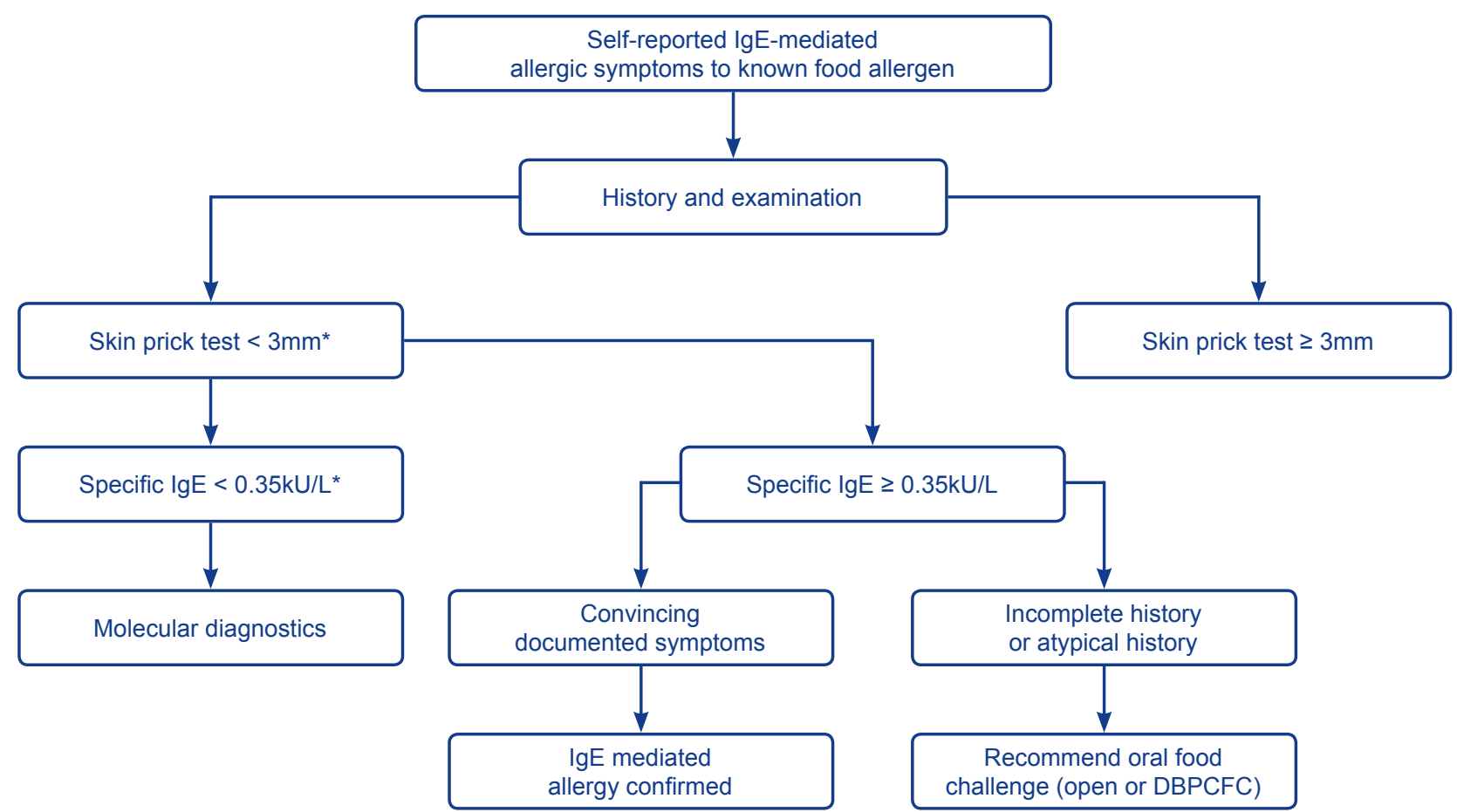

Figure 1

Simplified algorithm for diagnostic approach for lgE-mediated allergic symptoms to a known food allergen. *Exactly the same algorithm can be used using specific IgE first and SPT second depending on the practice of the allergy clinician.

facilitate the decision as to whether peanut can be safely introduced into the patient's diet (32).

A progression of MD could be linear epitope mapping which appears to have been useful in certain contexts such as determining transient versus persistent egg allergy at a research level (33). However, it is unclear whether this would be an improvement over MD, which relies on the 3 dimensional structure of the allergen. There should be emphasis in bringing both BAT using molecular allergen and epitope mapping assays into clinical practice by validating simple methods that could be applied to the laboratory equipment already in use to run slgE tests.

\section{CONCLUSIONS}

$M D$ is a very useful adjunct to conventional allergy testing with whole allergens (SPT and slgE testing). We are just at the beginning of getting comprehensive analysis of all food allergens but already at this early stage the fruits of this labour are apparent for primary food allergies such as peanut allergy and secondary food allergies due pollen-food or plant-food syndromes. Allergy testing should only be performed in the context of the clinical history as history provides the cornerstone of diagnosis. Multiple allergy tests (especially in children with eczema) often give rise to false positive results (this is more the case for slgE testing to whole allergens than SPT to whole allergens) and thus can lead to unnecessary avoidance or delay in introduction of foods. A 'molecule to symptom' approach is therefore not recommended as this will lead to confusion and potential avoidance of foods to which the child or adult is not allergic. However, the progress achieved so far is paving the way to exciting developments. A proper methodological approach should be implemented any time there is a new component allergen / pattern of component allergens available for a food. Evaluation against conventional allergy testing against whole allergens (both SPT and slgE) as well as validation against the gold standard DBPCFC to determine their diagnostic performance should be carried out for that component allergen before ultimately guidance is put in place for daily clinical practice. 
Clear OAS symptoms with no symptoms suggestive of a more severe clinical phenotype (e.g. patient tolerates cooked but not raw foods, with only local mild allergic symptoms)

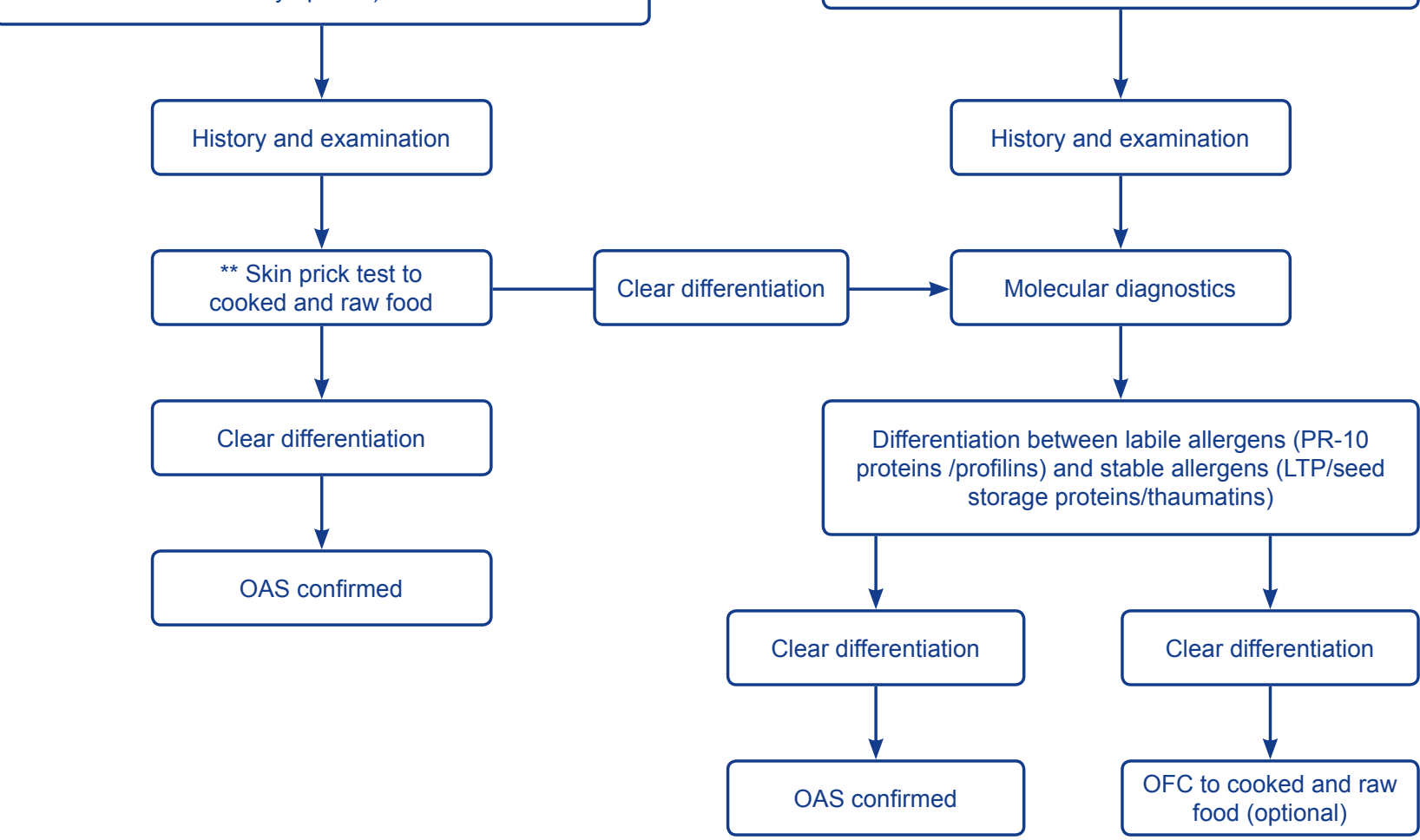

Ambiguous symptoms which could suggest a more severe clinical phenotype or patient with aversions to multiple fruits and vegetables

Figure 2

Algorithm for diagnostic approach for possible OAS / systemic reaction to known food allergen*.

*The gold standard for the evaluation of food allergy is the double blind placebo controlled food challenge.

** If no SPT facilities available further differentiation using MD for clinical relevance of other food stuffs

containing similar proteins Bet $v$ 1-homologs, profilins, thaumatins or LTPs (unlikely given history) may be

considered.

\section{References}

1. Venter C, Hasan AS, Grundy J, Pereira B, Bernie CC, Voigt $\mathrm{K}$ et al.Time trends in the prevalence of peanut allergy: three cohorts of children from the same geographical location in the UK. Allergy 2010;65:103108.

2. Muraro A, Werfel T, Hoffmann-Sommergruber K, Roberts G, Beyer K, Bindslev-Jensen C et al. EAACI food allergy and anaphylaxis guidelines: diagnosis and management of food allergy. Allergy 2014;69:10081025.

3. Soares-Weiser K, Takwoingi $\mathrm{Y}$, Panesar SS, Muraro A, Werfel T, Hoffmann-Sommergruber $K$ et al. The diagnosis of food allergy: a systematic review and meta-analysis. Allergy 2014;69:76-86.

4. Flammarion S, Santos C, Guimber D, Jouannic L, Thumerelle C, Gottrand F et al. Diet and nutritional status of children with food allergies. Pediatr Allergy Immunol 2011;22:161-165.

5. King RM, Knibb RC, Hourihane JO. Impact of peanut allergy on quality of life, stress and anxiety in the family. Allergy 2009;64:461-468.

6. Gonzalez-Mancebo E, Gonzalez-de-Olano D, Trujillo MJ, Santos S, Gandolfo-Cano M, Melendez A et al. Prevalence of sensitization to lipid transfer proteins and profilins in a population of 430 patients in the south of Madrid. J Investig Allergol Clin Immunol 2011;21:278282.

7. Hamilton R.G., Matsson P., Chan S., Van Cleve M., Hovanec D., Magnusson C. et al. Analytical performance characteristics, quality assurance and clinical utility of immunological assays for human immunoglobulin $E$ (IgE) antibodies of defined allergen specificities. Third edition[CLSI document I/LA20-A3]. 2015. Wayne: 
Clinical and Laboratory Standards Institute.

8. Dang TD, Tang M, Choo S, Licciardi PV, Koplin JJ, Martin PE et al.Increasing the accuracy of peanut allergy diagnosis by using Ara $\mathrm{h}$ 2. J Allergy Clin Immunol 2012;129:1056-1063.

9. Santos AF, Douiri A, Becares N, Wu SY, Stephens A, Radulovic $S$ et al. Basophil activation test discriminates between allergy and tolerance in peanut-sensitized children. J Allergy Clin Immunol 2014;134:645-652.

10. Roberts G, Lack G. Diagnosing peanut allergy with skin prick and specific IgE testing. J Allergy Clin Immunol 2005;115:1291-1296.

11. Nicolaou N, Poorafshar M, Murray C, Simpson A, Winell $\mathrm{H}$, Kerry $\mathrm{G}$ et al. Allergy or tolerance in children sensitized to peanut: prevalence and differentiation using component-resolved diagnostics. J Allergy Clin Immunol 2010;125:191-197.

12. Beyer K, Grabenhenrich L, Hartl M, Beder A, Kalb $B$, Ziegert $M$ et al. Predictive values of componentspecific lgE for the outcome of peanut and hazelnut food challenges in children. Allergy 2015;70:90-98.

13. Kapoor S, Roberts G, Bynoe Y, Gaughan M, Habibi P, Lack G. Influence of a multidisciplinary paediatric allergy clinic on parental knowledge and rate of subsequent allergic reactions. Allergy 2004;59:185191.

14. Du Toit G, Roberts G, Sayre P, Plaut M. Identifying infants at high risk of peanut allergy - The LEAP Screening Study. J Allergy Clin Immunol 2013;131:135-143.

15. Heaps A, Carter S, Selwood C, Moody M, Unsworth J, Deacock $S$ et al. The utility of the ISAC allergen array in the investigation of idiopathic anaphylaxis. Clin Exp Immunol 2014;177:483-490.

16. Sampson HA. Utility of food-specific lgE concentrations in prediciting symptomatic food allergy. J Allergy Clin Immunol 2001;107:891-896.

17. Sporik R, Hill DJ, Hosking CS. Specificity of allergen skin testing in predicting positive open food challenges to milk, egg, and peanut in children. Clin Exp Allergy 2000;30:1540-1546.

18. Caffarelli C, Cavagni G, Giordano S, Stapane I, Rossi C. Relationship between oral challenges with previously uningested egg and egg-specific IgE antibodies and skin prick tests in infants with food allergy. J Allergy Clin Immunol 1995;95:1215-1220.

19. Calvani M, Alessandri C, Frediani T, Lucarelli S, Miceli SS, Panetta $V$ et al. Correlation between skin prick test using commercial extract of cow's milk protein and fresh milk and food challenges. Pediatr Allergy Immunol 2007;18:583-588.

20. Rancé $F$, Abbal M, Lauwers-Cancès V. Improved screening for peanut allergy by the combined use of skin prick tests and specific IgE assays. J Allergy Clin Immunol 2002;109:1027-1033.
21. Lieberman JA, Glaumann S, Batelson S, Borres MP, Sampson HA, Nilsson C. The utility of peanut components in the diagnosis of IgE-mediated peanut allergy among distinct populations. J Allergy Clin Immunol Pract 2013;1:75-82.

22. Andrews T, Banks JR. Sensitization to cor a 9 and cor a 14 is highly specific for a hazelnut allergy with objective symptoms in dutch children and adults. Pediatrics 2014;134:s152.

23. Kattan JD, Sicherer SH, Sampson HA. Clinical reactivity to hazelnut may be better identified by component testing than traditional testing methods. J Allergy Clin Immunol Pract 2014;2:633-634.

24. Shibata R, Nishima S, Tanaka A, Borres MP, Morita E. Usefulness of specific IgE antibodies to omega-5 gliadin in the diagnosis and follow-up of Japanese children with wheat allergy. Ann Allergy Asthma Immunol 2011;107:337-343.

25. Beyer K, Chung D, Schulz G, Mishoe M, Niggemann $B$, Wahn $U$ et al. The role of wheat omega-5 gliadin IgE antibodies as a diagnostic tool for wheat allergy in childhood. J Allergy Clin Immunol 2008;122:419-421.

26. Holzhauser T, Wackermann O, Ballmer-Weber BK, Bindslev-Jensen C, Scibilia J, Perono-Garoffo $L$ et al. Soybean (Glycine max) allergy in Europe: Gly $\mathrm{m} 5$ (beta-conglycinin) and Gly $\mathrm{m} 6$ (glycinin) are potential diagnostic markers for severe allergic reactions to soy. J Allergy Clin Immunol 2009;123:452-458.

27. Ebisawa M, Brostedt P, Sjölander S, Sato S, Borres MP, Ito $\mathrm{K}$. Gly $\mathrm{m} 2 \mathrm{~S}$ albumin is a major allergen with a high diagnostic value in soybean-allergic children. J Allergy Clin Immunol 2013;132:976-978.

28. Kattan JD, Sampson HA. Clinical reactivity to soy is best identified by component testing to Gly $\mathrm{m} 8$. J Allergy Clin Immunol Pract 2015;3:970-972.

29. Kleine-Tebbe J, Vogel L, Crowell DN, Haustein UF, Vieths S. Severe oral allergy syndrome and anaphylactic reactions caused by a Bet $v$ 1- related PR-10 protein in soybean, SAM22. J Allergy Clin Immunol 2002;110:797-804.

30. Asero R, Jimeno L, Barber D. Component-resolved diagnosis of plant food allergy by SPT. Eur Ann Allergy Clin Immunol 2008;40:115-121.

31. Asero R, Monsalve R, Barber D. Profilin sensitization detected in the office by skin prick test: a study of prevalence and clinical relevance of profilin as a plant food allergen. Clin Exp Allergy 2008;38:1033-1037.

32. Du Toit G, Roberts G, Sayre PH et al. Randomized Trial of Peanut Consumption in Infants at Risk for Peanut Allergy. N Engl J Med 2015;372:803-813.

33. Jarvinen KM, Beyer K, Vila L, Bardina L, Mishoe M, Sampson HA. Specificity of IgE antibodies to sequential epitopes of hen's egg ovomucoid as a marker for persistence of egg allergy. Allergy 2007;62:758-765. 



\section{COW'S MILK ALLERGY}

Anna Nowak-Wegrzyn, Antonella Muraro

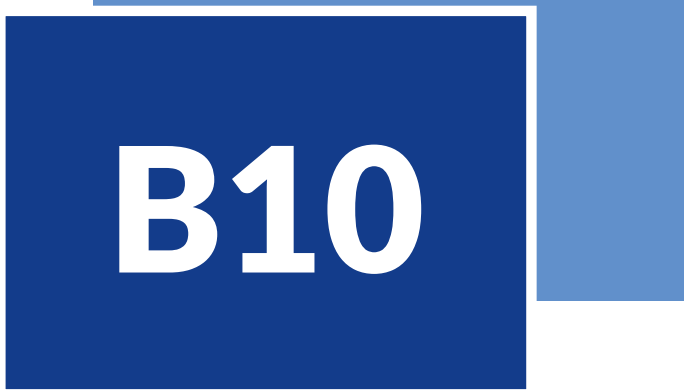

Cow milk caseins and whey proteins are type I food allergens inducing allergic sensitization via gastrointestinal tract.

The majority of cow milk allergic subjects are polysensitized to several casein and whey proteins.

Natural history is favorable with majority of the milk allergic children becoming tolerant with age; those with cow milk-specific IgE serum levels exceeding $60 \mathrm{kIU} / \mathrm{L}$ are more likely to have persistent milk allergy.

Extensive heating e.g., baking, affects the allergenicity of CM protein, with caseins being more resistant to heating compared to whey proteins that are susceptible to heating.

Majority of cow milk allergic children can tolerate extensively heated (baked) products with milk; reactivity to baked milk is a marker of a more severe and more persistent cow milk allergy.

CM proteins are classified as class I food allergens, due to their resistance to digestion and heating. They 
do induce sensitization via gastrointestinal tract. Proteins in CM have a high sequence homology (>80\%) with proteins from goat and sheep and are highly clinically cross-reactive (>90\%) with these species. In contrast, the laboratory and clinical crossreactivity is very low ( $<5 \%)$ with milks from donkey, mare, buffalo, or camel (7).

\section{MAJOR AND RELEVANT MINOR ALLERGENIC MOLECULES}

CM contains approximately 30 to $35 \mathrm{~g}$ of proteins per liter. Under the influence of rennin or upon acidification of the milk to $\mathrm{pH} 4.6$, proteins segregate into 2 fractions: curd (coagulum) which contains approximately $80 \%$ of the CM proteins and whey (lactoserum) which contains approximately 20\% of the CM proteins (Table 1). All of the proteins present in cow's milk are also present in human breast milk, with an exception of beta-lactoglobulin. Caseins, beta-lactoglobulin and alpha-lactalbumin are considered major allergens, i. e. more than $50 \%$ of the individuals with CMA are sensitized to these proteins. Most of the patients are polysensitized to several proteins $(8,9)$.

Casein supplies amino acids, carbohydrates, and the two inorganic elements calcium and phosphorus. Casein fraction is very resistant to high temperatures, retaining strong $\operatorname{lgE}$ binding after 90 minutes of boiling at $>90^{\circ} \mathrm{C}(10)$. Except for short alpha-helical regions, caseins have little secondary or tertiary structure. The caseins of milk exist in the form of colloidal complexes called micelles. The micelles contain an amorphous micellar calcium phosphate core, surrounded by a casein shell $(11,12)$.

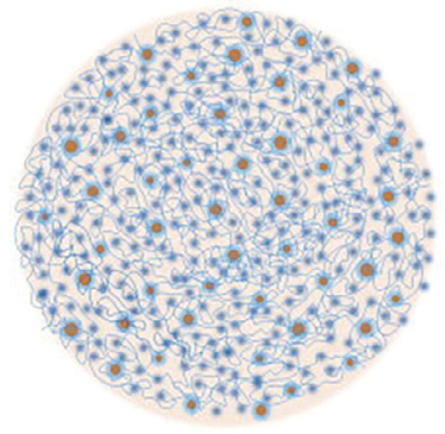

Figure 1

Casein micelles (from ref 10).
Alpha $_{\mathrm{s1}}$-casein is the most abundant protein of bovine milk. It exists as a major and minor form and is highly phosphorylated.

Alpha $_{\text {s2 }}$-casein is also highly phosphorylated and has four isoforms.

Beta-casein has one isoform. Limited hydrolysis of beta-casein by endogenous peptides (e.g., plasmin) present in milk produces gamma-caseins 1, 2, and 3.

Kappa-casein is the only casein soluble in the presence of calcium ions. It also has the smallest amount of phosphate, with phosphorylation sites being present only in the $\mathrm{C}$-terminal region. Kappacasein is the only casein to contain carbohydrate moieties.

Whey is a mixture of beta-lactoglobulin ( 65\%), alpha-lactalbumin ( 25\%), bovine serum albumin ( 8\%) and immunoglobulins (390). These are soluble in their native forms, independent of $\mathrm{pH}$. Whey proteins are more sensitive to heating than caseins and lose IgE binding following 15-20 minutes of boiling at $>90^{\circ} \mathrm{C}(10)$.

Alpha-lactalbumin is a protein present in the milk of almost all mammals. In primates, alpha-lactalbumin expression is upregulated in response to the hormone prolactin and increases the production of lactose (5). Alpha-lactalbumin forms the regulatory subunit of the lactose synthase (LS) heterodimer and beta-1,4galactosyltransferase forms the catalytic component. Together, these proteins enable LS to produce lactose by transferring galactose moieties to glucose. As a multimer, alpha-lactalbumin strongly binds calcium and zinc ions and may possess bactericidal and / or antitumor activity.

Beta-lactoglobulin under physiological conditions forms dimers but dissociates to a monomer below $\mathrm{pH}$ 3. Beta-lactoglobulin solutions form gels in various conditions, when the native structure is sufficiently destabilized to allow aggregation (6). No clear function has been identified for beta-lactoglobulin, although it binds to several hydrophobic molecules, suggesting potential role in their transport. Beta-lactoglobulin is the only CM protein that is not present in the human breast milk.

Bovine serum albumin (BSA) is a globular, watersoluble, un-glycosylated serum protein. Albumin functions primarily as a carrier protein for steroids, fatty acids, and thyroid hormones in the blood and plays a major role in stabilizing extracellular fluid 
Table 1

Allergens in Cow's Milk

\begin{tabular}{|c|c|c|c|c|}
\hline Protein name & Allergen name & $\begin{array}{l}\text { Molecular } \\
\text { mass (kDa) }\end{array}$ & AA\# & Tertiary structure \\
\hline \multicolumn{5}{|c|}{ Curd (coagulum)-Casein family } \\
\hline Caseins & Bos d 8 & $20-30$ & & \multirow{5}{*}{$\begin{array}{l}\text { Caseins don't have a } \\
\text { rigid tertiary structure } \\
\text { but develop a random } \\
\text { coil conformation } \\
\text { stabilized by } \\
\text { hydrophobic interactions }\end{array}$} \\
\hline Alpha $_{s 1}$-casein & Bos d 9 & 23.6 & 199 & \\
\hline Alpha $_{\text {s2 }}$-casein & Bos d 10 & 25.2 & 207 & \\
\hline Beta-casein & Bos d 11 & 24 & 209 & \\
\hline Kappa-casein & Bos d 12 & 19 & 169 & \\
\hline \multicolumn{5}{|c|}{ Whey (lactoserum) } \\
\hline $\begin{array}{l}\text { Alpha- } \\
\text { lactalbumin }\end{array}$ & Bos d 4 & 14.2 & $\begin{array}{l}\text { 123; } 4 \text { disulphide bridges, } \\
70 \% \text { homology with human } \\
\text { alpha-lactalbumin }\end{array}$ & \\
\hline $\begin{array}{l}\text { Beta- } \\
\text { lactoglobulin } \\
\text { Protein family: } \\
\text { lipocalins }\end{array}$ & Bos d 5 & $\begin{array}{l}18.3 \\
\text { exists as a } \\
\text { dimer }\end{array}$ & $\begin{array}{l}\text { 162; } 2 \text { disulphide bridges, } \\
\text { one free cysteine; exist as } \\
\text { isoforms } A \text { and B; binds and } \\
\text { carries hydrophobic molecules }\end{array}$ & \\
\hline $\begin{array}{l}\text { Bovine serum } \\
\text { albumin; } \\
\text { Family: Serum } \\
\text { albumins }\end{array}$ & Bos d 6 & 67 & 583 & \\
\hline $\begin{array}{l}\text { Immunoglobulins } \\
\text { (mostly IgG) } \\
\text { Family: } \\
\text { Immunoglobulins }\end{array}$ & Bos d 7 & 160 & & \\
\hline $\begin{array}{l}\text { Lactoferrin } \\
\text { Family: } \\
\text { Transferrins }\end{array}$ & & 800 & $\begin{array}{l}703 \text {; forms two homologous } \\
\text { globular domains named } \\
\mathrm{N} \text {-and C-lobes } \\
\text { Lactoferrin exists in various } \\
\text { polymeric forms: monomers } \\
\text { to tetramers }\end{array}$ & है? \\
\hline
\end{tabular}

source: IUIS Allergen Database, July 2015 


\section{Table 2}

Sensitization and cross-reactivity patterns of the CM proteins

\begin{tabular}{|c|c|c|c|c|}
\hline Allergen name & Allergenicity & $\begin{array}{l}\text { Sensitization rate } \\
\% \text { among those } \\
\text { reactive to } \mathrm{CM}\end{array}$ & $\begin{array}{l}\text { Laboratory } \\
\text { cross-reactivity }\end{array}$ & Clinical cross-reactivity \\
\hline \multicolumn{5}{|l|}{ Curd fraction (coagulum) } \\
\hline Caseins (Bos d 8) & Major & 63 & \multirow{5}{*}{$\begin{array}{c}>85 \% \text { with sheep } \\
\text { and goat milk } \\
\text { caseins }\end{array}$} & \multirow{5}{*}{$\begin{array}{c}>90 \% \text { with other } \\
\text { mammalian milks (27) } \\
20 \% \text { with mare's } \\
\text { milk29 and donkey } \\
\text { milk } 30\end{array}$} \\
\hline Alpha $_{\text {s1 }}$-casein (Bos d 9) & Major & $98^{*}$ & & \\
\hline Alpha $_{\mathrm{s} 2}$-casein (Bos d 10) & Major & $94^{*}$ & & \\
\hline Beta-casein (Bos d 11) & Major & $91^{*}$ & & \\
\hline Kappa-casein (Bos d12) & Major & $91 *$ & & \\
\hline \multicolumn{5}{|l|}{ Whey fraction (lactoserum) } \\
\hline Alpha-lactalbumin (Bos d 4) & Major & 51 & \multirow{5}{*}{$80 \%$ with beef } & \multirow{5}{*}{$15-20 \%$ with raw beef } \\
\hline Beta-lactoglobulin (Bos d 5) & Major & 61 & & \\
\hline Bovine serum albumin (Bos d 6) & Minor & 43 & & \\
\hline Immunoglobulins (Bos d 7) & Minor & 36 & & \\
\hline Lactoferrin & Minor & 35 & & \\
\hline
\end{tabular}

* Percentage of those sensitized to casein Bos $d 8$

volume by contributing to oncotic pressure of plasma. BSA is highly homologous with human serum albumin and albumins of other species, e.g. cow (beef), cat, and dog. BSA has been identified as one of the major beef allergens and is responsible for clinical cross reactivity between $\mathrm{CM}$ and raw beef (13-16).

Immunoglobulins present in CM are predominantly of the $\mathrm{G}$ class. Immunoglobulins may play a role in cross-reactivity with beef (14).

Lactoferrin is a multifunctional protein of the transferrin family. Lactoferrin is a globularglycoprotein with a molecular mass of about $80 \mathrm{kDa}$ that is widely represented in various secretory fluids, such as milk, saliva, tears, and nasal secretions. Lactoferrin is one of the transferrin proteins that transfer iron to the cells and control the level of free iron in the blood and external secretions. Lactoferrin is one of the components of the immune system of the body; it has antimicrobial activity (bactericide, fungicide) and is part of the innate immune defense, mainly at mucosal surfaces. In particular, lactoferrin provides antibacterial activity to human infants. Lactoferrin interacts with DNA and RNA, polysaccharides and heparin. Lactoferrin is a minor allergen in CM (9).

\section{SENSITIZATION TO INDIVIDUAL MOLECULES AND ITS CLINICAL RELEVANCE}

The patterns of sensitization to the individual CM proteins vary significantly by study population and age of the affected individuals. In general, the majority of the affected subjects are polysensitized to several casein and whey proteins (17-20). Caseins, beta-lactoglobulin and alpha-lactalbumin are the major allergens, with over $50 \%$ of CM-allergic subjects having evidence of IgE-antibodies directed at these proteins. IgE-sensitization to caseins, betalactoglobulin and alpha-lactalbumin is closely related, whereas IgE-sensitization to BSA is independent of other CM proteins, and may reflect cross-reactivity with beef (14) (Table 2).

Natural history of CMA: Molecular diagnosis may be useful for monitoring for natural spontaneous) tolerance development in CMA (20). In a prospective follow up study of 66 infants, lower serum levels of casein-lgE were associated with higher chances of resolution of CMA (21). In another prospective study in which CMA diagnosis was confirmed with DBPCFC to $C M$, children with lower serum levels of specific 
IgE to CM, alpha-lactalbumin, beta-lactoglobulin, kappa-casein and alpha s1 casein had better odds of outgrowing CMA over 80 month period (19). On an individual epitope levels, children with persistent milk allergy have been shown to predominantly generate IgE antibodies directed against sequential casein epitopes (22-24). IgE epitope-binding patterns were stable over time in patients with persisting CMA, whereas binding decreased in patients who recovered early. Binding patterns of IgE and IgG4 overlapped. Among patients who recovered early, the signal of IgG4 binding increased and that of lgE decreased over time. IgE and IgG4 binding to a panel of alpha(s1)-, alpha(s2)-, beta-, and kappa-casein regions predicted outcome with significant accuracy (25).

\section{Effect of heating on CM protein allergenicity}

CM proteins contain both conformational and sequential IgE-binding epitopes. Children with persistent milk allergy have been shown to predominantly generate IgE antibodies directed against sequential casein epitopes (22-24). Extensive heating e.g., baking, affects the allergenicity of CM protein, with caseins being more resistant to heating compared to whey proteins that are susceptible to heating. Heating of beta-lactoglobulin results in formation of the intermolecular disulphide bonds and binding to other food proteins that result in a reduced allergenicity of beta-lactoglobulin (30). The majority (70-80\%) of the CM-allergic children tolerate $\mathrm{CM}$ as an ingredient in the baked products $(31,32)$. Reactivity to baked milk is a marker of a more severe and more persistent CM allergy. Inclusion of the baked products containing CM into the diet of children with CM allergy appears to accelerate development of tolerance to unheated CM (33). High levels of specific IgE antibodies directed against casein are predictive of clinical reactivity to baked milk $(32,34)$. In a peptide microarray assay, subjects with persistent milk allergy had increased epitope diversity to caseins and beta-lactoglobulin compared with those who outgrew their CM allergy $(24,35)$. Baked milk-tolerant subjects had IgEbinding patterns similar to those who had outgrown CM allergy, but IgG4-binding patterns that were more similar to those of the allergic group. Binding to higher numbers of IgE peptides was associated with more severe allergic reactions during an oral CM challenge. There was no association between IgG4 peptides and clinical features of milk allergy. Using a competitive peptide microarray assay, $\mathrm{CM}$-allergic patients had a combination of high- and low-affinity IgE binding, whereas baked milk-tolerant subjects and those who had outgrown their CM allergy had primarily low-affinity binding.

\section{CLINICAL MANAGEMENT}

Diagnosis of CM allergy begins with an assessment of clinical history and an assessment of the potential immunologic mechanism involved in the reactions.

\section{Suspected IgE-mediated CM allergy}

Laboratory testing: Routine testing involves skin prick (SPT) and/or serologic testing with complete CM extract. Molecular diagnosis is not recommended for standard evaluation of suspected CM allergy. Diagnostic decision points have been proposed; they vary by population studied and age. Negative SPT and undetectable serum level of CM-specific IgE antibodies have a very high negative predictive value $>90 \%$ for IgE-mediated CM allergy. The positive predictive value of the test increases with an increased size of the wheal of the SPT and serum level of the specific CM-IgE antibody (Table 3).

Molecular diagnosis may be helpful for evaluation of reactivity to baked milk, based on the differential resistance to heating among the $\mathrm{CM}$ protein. As caseins are more resistant to extensive heating, higher levels of casein-specific IgE are associated with increased likelihood of reactivity to baked milk. Basophil activation test with CM proteins has been utilized in a research setting but it is not yet recommended for a routine diagnosis of CM allergy $(32,36)$. Several studies indicated that molecular diagnosis may be useful for monitoring and predicting the resolution of $\mathrm{CMA}(17,19,21,24,25)$.

Not recommended: Testing for CM- specific IgG / IgG4 antibodies is not recommended in the diagnosis of CM allergy as these antibodies reflect the presence of $\mathrm{CM}$ in the diet, not an allergy.

Elimination-Challenge testing: In general, the conclusive diagnosis of $\mathrm{CM}$ allergy requires elimination of CM proteins from the diet followed by a supervised oral food challenge. Double-blind placebo controlled oral food challenge (DBPCFC) remains the gold standard for food allergy diagnosis. Open controlled challenge can replace DBPCFC in the children younger than 2 years of age. The initial assessment of reactivity to baked milk is also 
recommended to be conducted under the physiciansupervised food challenge condition because children reactive to baked milk may experience anaphylaxis.

\section{Suspected non IgE-mediated CM allergy}

Laboratory testing: There is no reliable laboratory diagnostic testing for non-IgE mediated CM allergy (4, 9). Atopy patch testing may be considered in selected cases of Eosinophilic Esophagitis (EoE) but not as a routine diagnostic test(4). Lymphocyte transformation test, serum CM- specific IgG / IgG4 antibodies, or stool measurements of pro-inflammatory mediators (e.g., calprotectin, eosinophilic cationic protein, eosinophil-derived neurotoxin) are not recommended (1). As some non-IgE-mediated disorders may be associated with a concomitant IgE-mediated food allergy, testing for CM-specific IgE antibodies may be utilized in such cases, e.g., EoE and food proteininduced enterocolitis syndrome (FPIES) to diagnose IgE-mediated CM allergy. The ultimate confirmation of diagnosis in non-IgE-mediated $\mathrm{CM}$ allergy requires an elimination of CM proteins from the diet followed by an oral CM challenge.

\section{Management of CM allergy}

Management relies on dietary avoidance of CM proteins. In infants and young children, substituting alternative sources of protein, calories, and calcium with a specialized hypoallergenic formula may be necessary. The alternative formula choices include: soy-based, rice hydrolysate, casein-hydrolysate, whey-hydrolysate and amino-acid based formulas (37).

Nutritional consultation is recommended for those with severe form of CM allergy, multiple food allergies and poor growth. Education about recognition of allergic symptoms and prompt treatment of anaphylaxis is crucial in the patients at risk for anaphylaxis. As most children outgrow $\mathrm{CM}$ by school age, periodic re-evaluations every 6-12 months with laboratory testing and oral food challenges are recommended. A drop in the CM level by $50 \%$ or more over $12-24$ months is a favorable prognostic indicator of developing tolerance (38). Children with peak lifetime CM-lgE $>50 \mathrm{kUA} / \mathrm{L}$ are more likely to retain milk allergy until teenage years and may need less frequent testing (39). Introduction of baked products with CM should be attempted under physician supervision for patients with lgEmediated CMA. Baked milk products may be tolerated by a subset of patient with EoE (40). It is unknown if children with FPIES can tolerate baked milk products and therefore strict avoidance is recommended.

\section{Novel therapies for CM allergy}

Oral (OIT), sublingual (SLIT) and epicutaneous (EPIT) immunotherapy routes have been evaluated for CMA with promising results in clinical trials (41). OIT, SLIT, and EPIT utilize native CM proteins in a form of a CM powder. In a trial comparing CM OIT and SLIT, 10\% receiving SLIT (maintenance daily dose 7 mg CM) were desensitized, 60\% receiving SLIT/low dose OIT (maintenance daily dose $1000 \mathrm{mg}$ CM) were desensitized, and $80 \%$ receiving SLIT/high dose OIT (maintenance daily dose 2000 mg CM) were desensitized (42). In general, CM SLIT was associated with very mild side effects mostly oro-pharyngeal pruritus, whereas CM OIT was associated with more systemic side effects, involving gastrointestinal tract (nausea, vomiting, abdominal pain, diarrhoea), or respiratory system (rhinorrhea, sneezing, congestion, cough, wheezing). CM OIT has been associated with cases of EoE. More studies are needed to determine the potential of inducing permanent oral tolerance to milk with CM OIT.

In a small pilot study, after 90 days, CM EPIT treatment tended to increase the cumulative tolerated dose, from a mean $\pm \mathrm{SD}$ of $1.77 \pm 2.98 \mathrm{~mL}$ at day 0 to $23.61 \pm 28.61 \mathrm{~mL}$ at day 90 (43). Large international clinical trial of CM EPIT is ongoing and will provide more evidence regarding safety and efficacy of CM EPIT. Molecular diagnosis may be utilized to identify subjects at higher risk to experience side effects from milk OIT and to monitor evolution of immunologic parameters during milk OIT and to correlate it with desensitization and tolerance. Children with at high risk for adverse reactions to milk OIT recognized a statistically significant higher number of IgE peptides in caseins at all the time, before and during milk OIT (44). IgE binding to CM peptides decreased and IgG4 binding increased following the OIT in children who attained desensitization (45). Compared with children who successfully completed OIT, those who discontinued OIT due to adverse reactions developed increased quantities and affinity of epitope-specific IgE antibodies and a broader diversity of IgE and IgG4 binding, but less overlap in IgE and IgG4 binding to $C M$ peptides. Thus, detailed analysis of $\operatorname{IgE}$ and IgG4 binding to $\mathrm{CM}$ peptides may help in predicting whether CM OIT will be tolerated successfully and may thus improve the safety of milk OIT $(44,45)$. 
Table 3

Proposed specific IgE diagnostic decision points for CM allergy diagnosis derived from studies in children, majority of whom had atopic dermatitis.

\begin{tabular}{|lll|}
\hline \multicolumn{2}{|l}{ OFC to regular [non-heated milk] } & CM-lgE [kUA/L] \\
\hline $\begin{array}{lll}\text { Defer } \\
>95 \% \text { PPV }\end{array}$ & $>8{ }^{47}$ & $>15^{46}$ \\
$\begin{array}{ll}\text { Perform } \\
<50 \% \text { PPV }\end{array}$ & Not done & $>5$ if less than 1 year old ${ }^{17}$ \\
\hline
\end{tabular}

OFC to baked milk

\begin{tabular}{|c|c|c|}
\hline & SPT to CM, mean wheal diameter (mm) & CM-slgE [kUA/L] \\
\hline $\begin{array}{l}\text { Defer } \\
>95 \% \text { PPV }\end{array}$ & Not done & $>24.5^{34}$ \\
\hline \multirow[t]{2}{*}{$\begin{array}{l}\text { Perform } \\
>90 \text { NPV }\end{array}$} & $<12^{48}$ & $<9.97$ \\
\hline & SPT to Casein, mean wheal diameter (mm) & Casein-slgE [kUA/L] \\
\hline $\begin{array}{l}\text { Defer } \\
>95 \% \text { PPV }\end{array}$ & Not done & $>10^{34}$ \\
\hline $\begin{array}{l}\text { Perform } \\
>90 \text { NPV }\end{array}$ & $<948$ & \\
\hline$<50 \%$ PPV & & $<5^{34}$ \\
\hline
\end{tabular}

This might be a practical guidance for the clinical ambulatory setting, aiming to identify the optimal candidates for OFC and to limit unnecessary OFC. It is important to recognize that OFC can always be performed at the discretion of the treating physician despite the elevated results of the serologic tests. In the research setting, OFC are recommended regardless of the slgE level.

\section{CLINICAL CASES}

\section{Case 1}

Parents of a 2-year old child with atopic dermatitis and history of milk-induced generalized urticaria at the age 6 months inquire about the likelihood of their child outgrowing milk allergy. There are no additional allergic reactions to milk or milk products. Skin prick test with a commercial cow milk extract is positive at a mean wheal diameter at $10 \mathrm{~mm}$. Serum CM- specific IgE antibody level is $17 \mathrm{kIU} / \mathrm{L}$. Based on these results, the child has more than $95 \%$ chances of reacting to liquid milk. However, considering that about $70-80 \%$ of milk allergic children tolerate milk in the baked products, further diagnostic testing is performed. Serum specific IgE antibodies directed against casein level is $4.5 \mathrm{kIU} / \mathrm{L}$ and beta-lactoglobulin IgE is 25 $\mathrm{kIU} / \mathrm{L}$. Based on the level of the casein-specific IgE, it is estimated that the likelihood of tolerating baked milk products in form of a muffin is approximately $50 \%$. A physician-supervised oral challenge with baked milk in a form of a muffin is performed in the office and the child tolerates it without an adverse reaction. Baked milk products are incorporated into the diet.

\section{Case 2}

A 10-year-old asthmatic male has history of severe anaphylaxis to trace amounts of milk in a cookie. He wants to know what his chances of outgrowing his milk allergy are. His CM-lgE is $75 \mathrm{kUA} / \mathrm{L}$, casein $\lg \mathrm{E}$ is $90 \mathrm{kUA} / \mathrm{L}$; SPT to CM extract is $20 \mathrm{~mm}$ diameter. Based on his past history of anaphylaxis to baked milk and the current test results highly predictive of clinical reactivity to both baked and unheated milk, it is likely that he will remain-milk allergic until his teenage years. 


\section{References}

1. Muraro A, Werfel T, Hoffmann-Sommergruber $\mathrm{K}$, Roberts G, Beyer K, Bindslev-Jensen C et al. EAACl food allergy and anaphylaxis guidelines: diagnosis and management of food allergy. Allergy 2014 ;69:100825.

2. Boyce JA, Assa'ad A, Burks AW, Jones SM, Sampson HA, Wood RA et al. Guidelines for the Diagnosis and Management of Food Allergy in the United States: Summary of the NIAID-Sponsored Expert Panel Report. J Allergy Clin Immunol 2010;126:1105-1118.

3. Schoemaker AA, Sprikkelman AB, Grimshaw KE, Roberts G, Grabenhenrich L, Rosenfeld $L$ et al. Incidence and natural history of challenge-proven cow's milk allergy in European children - EuroPrevall birth cohort. Allergy 2015;70:963-972.

4. Sampson HA, Aceves S, Bock SA, James J, Jones $S$, Lang $D$ et al. Food allergy: a practice parameter update-2014. J Allergy Clin Immunol 2014;134:101625 e43.

5. Nwaru BI, Hickstein L, Panesar SS, Roberts G, Muraro A, Sheikh A et al. Prevalence of common food allergies in Europe: a systematic review and metaanalysis. Allergy 2014;69:992-1007.

6. Rona RJ, Keil T, Summers C, Gislason D, Zuidmeer L, Sodergren $\mathrm{E}$ et al. The prevalence of food allergy: a metaanalysis. J Allergy Clin Immunol 2007;120:638-646.

7. Restani P, Beretta B, Fiocchi A, Ballabio C, Galli CL. Cross-reactivity between mammalian proteins. Ann Allergy Asthma Immunol 2002;89:11-15.

8. Tsabouri S, Douros K, Priftis KN. Cow's milk allergenicity. Endocr metab immune disord drug targets 2014;14:16-26.

9. Fiocchi A, Dahdah L, Albarini M, Martelli A. Cow's Milk Allergy in Children and Adults. Chem Immunol Allergy 2015;101:114-123.

10. Bloom KA, Huang FR, Bencharitiwong R, Bardina L, Ross A, Sampson HA et al. Effect of Heat Treatment on Milk and Egg Proteins Allergenicity. Pediatr Allergy Immunol 2014;25:740-746.

11. Bijl E, van Valenberg HJ, Huppertz T, van Hooijdonk AC. Protein, casein, and micellar salts in milk: current content and historical perspectives. J Dairy Sci 2013;96:5455-5464.

12. de Kruif CG, Huppertz T, Urban VS, Petukhov AV. Casein micelles and their internal structure. Adv colloid interface sci 2012;171-172:36-52.

13. Werfel SJ, Cooke SK, Sampson HA. Clinical reactivity to beef in children allergic to cow's milk. J Allergy Clin Immunol 1997;99:293-300.

14. Restani P, Ballabio C, Tripodi S, Fiocchi A. Meat allergy. Curr Opin Allergy Clin Immunol 2009;9:265269.
15. Restani P, Ballabio C, Cattaneo A, Isoardi P, Terracciano L, Fiocchi A. Characterization of bovine serum albumin epitopes and their role in allergic reactions. Allergy 2004;59(Suppl 78):21-4.

16. Vicente-Serrano J, Caballero ML, Rodríguez-Pérez R, Carretero P, Pérez R, Blanco JG et al. Sensitization to serum albumins in children allergic to cow's milk and epithelia. Pediatr Allergy Immunol 2007;18:503-507.

17. García-Ara C, Boyano-Martínez T, Díaz-Pena JM, Martín-Muñoz F, Reche-Frutos M, Martín-Esteban $M$. Specific-IgE levels in the diagnosis of immediate hypersensitivity to cow's milk protein in the infant. $J$ Allergy Clin Immunol 2001;107:185-190.

18. Ott H, Baron JM, Heise R, Ocklenburg C, Stanzel $\mathrm{S}$, Merk HF et al. Clinical usefulness of microarraybased IgE detection in children with suspected food allergy. Allergy 2008;63:1521-1528.

19. Ahrens B, Lopes de Oliveira LC, Grabenhenrich L, Schulz G, Niggemann B, Wahn U et al. Individual cow's milk allergens as prognostic markers for tolerance development? Clin Exp Allergy 2012;42:1630-1637.

20. Martorell-Aragonés A, Echeverría-Zudaire L, Alonso-Lebrero E, Boné-Calvo J, Martín-Muñoz MF, Nevot-Falcó $S$ et al. Position document: IgEmediated cow's milk allergy. Allergol Immunopathol (Madr) 2015;43:507-526.

21. García-Ara MC, Boyano-Martinez MT, Díaz-Pena JM, Martín-Muñoz MF, Martín-Esteban M. Cow's milkspecific immunoglobulin $E$ levels as predictors of clinical reactivity in the follow-up of the cow's milk allergy infants. Clin Exp Allergy 2004;34:866-870.

22. Chatchatee $P$, Järvinen KM, Bardina L, Vila L, Beyer $\mathrm{K}$, Sampson HA. Identification of IgE and IgG binding epitopes on beta- and kappa-casein in cow's milk allergic patients. Clin Exp Allergy 2001;31:1256-62.

23. Chatchatee $P$, Järvinen $K M$, Bardina $L$, Beyer $K$, Sampson HA. Identification of IgE- and IgG-binding epitopes on alpha(s1)-casein: differences in patients with persistent and transient cow's milk allergy. J Allergy Clin Immunol 2001;107:379-83.

24. Cerecedo I, Zamora J, Shreffler WG, Lin J, Bardina $\mathrm{L}$, Dieguez MC et al. Mapping of the IgE and IgG4 sequential epitopes of milk allergens with a peptide microarray-based immunoassay. J Allergy Clin Immunol 2008;122:589-594.

25. Savilahti EM, Rantanen V, Lin JS, Karinen S, Saarinen $\mathrm{KM}$, Goldis M, Mäkelä MJ et al. Early recovery from cow's milk allergy is associated with decreasing IgE and increasing IgG4 binding to cow's milk epitopes. J Allergy Clin Immunol 2010;125:1315-1321.

26. Restani P, Gaiaschi A, Plebani A, Beretta B, Cavagni G, Fiocchi $A$ et al. Cross-reactivity between milk proteins from different animal species. Clin Exp Allergy 1999;29:997-1004. 
27. Bellioni-Businco B, Paganelli R, Lucenti P, Giampietro PG, Perborn H, Businco L. Allergenicity of goat's milk in children with cow's milk allergy. J Allergy Clin Immunol 1999;103:1191-1194.

28. Businco L, Giampietro PG, Lucenti P, Lucaroni F, Pini C, Di Felice $G$ et al. Allergenicity of mare's milk in children with cow's milk allergy. J Allergy Clin Immunol 2000;105:1031-1034.

29. Monti G, Bertino E, Muratore Cristina M, Coscia A, Cresi $\mathrm{F}$ et al. Efficacy of donkey's milk in treating highly problematic cow's milk allergic children: an in vivo and in vitro study. Pediatr Allergy Immunol 2008;19:90-91.

30. Lorenz AR, Scheurer S, Vieths S. Food allergens: molecular and immunological aspects, allergen databases and cross-reactivity. Chem Immunol Allergy 2015;101:18-29.

31. Nowak-Wegrzyn A, Bloom KA, Sicherer SH, Shreffler WG, Noone $\mathrm{S}$, Wanich $\mathrm{N}$ et al. Tolerance to extensively heated milk in children with cow's milk allergy. JAllergy Clin Immunol 2008;122:342-347.

32. Ford LS, Bloom KA, Nowak-Wegrzyn AH, Shreffler WG, Masilamani M, Sampson HA. Basophil reactivity, wheal size, and immunoglobulin levels distinguish degrees of cow's milk tolerance. J Allergy Clin Immunol 2013;131:180-186.

33. Kim JS, Nowak-Wegrzyn A, Sicherer SH, Noone $\mathrm{S}$, Moshier EL, Sampson HA. Dietary baked milk accelerates the resolution of cow's milk allergy in children. J Allergy Clin Immunol 2011;128:125-131.

34. Caubet JC, Nowak-Wegrzyn A, Moshier E, Godbold J, Wang J, Sampson HA. Utility of casein-specific IgE levels in predicting reactivity to baked milk. J Allergy Clin Immunol 2013;131:222-224.

35. Wang J, Lin J, Bardina L, Goldis M, Nowak-Wegrzyn A, Shreffler WG et al. Correlation of IgE/lgG4 milk epitopes and affinity of milk-specific IgE antibodies with different phenotypes of clinical milk allergy. J Allergy Clin Immunol 2010;125:695-702.

36. Wanich N, Nowak-Wegrzyn A, Sampson HA, Shreffler WG. Allergen-specific basophil suppression associated with clinical tolerance in patients with milk allergy. J Allergy Clin Immunol 2009;123:789-794.

37. Groetch M, Nowak-Wegrzyn A. Practical approach to nutrition and dietary intervention in pediatric food allergy. Pediatr Allergy Immunol 2013;24:212-221.

38. Shek LP, Soderstrom L, Ahlstedt S, Beyer K, Sampson HA. Determination of food specific IgE levels over time can predict the development of tolerance in cow's milk and hen's egg allergy. J Allergy Clin Immunol 2004;114:387-391.

39. Skripak JM, Matsui EC, Mudd K, Wood RA. The natural history of IgE-mediated cow's milk allergy. J Allergy Clin Immunol 2007;120:1172-1177.

40. Leung J, Hundal NV, Katz AJ, Shreffler WG, Yuan $\mathrm{Q}$, Butterworth CA et al. Tolerance of baked milk in patients with cow's milk-mediated eosinophilic esophagitis. J Allergy Clin Immunol 2013;132:12151216.

41. Albin S, Nowak-Wegrzyn A. Potential treatments for food allergy. Immunol Allergy Clin North Am 2015;35:77-100.

42. Keet CA, Frischmeyer-Guerrerio PA, Thyagarajan A, Schroeder JT, Hamilton RG, Boden S et al. The safety and efficacy of sublingual and oral immunotherapy for milk allergy. J Allergy Clin Immunol 2012;129:448455.

43. Dupont $\mathrm{C}$, Kalach N, Soulaines P, Legoué-Morillon S, Piloquet $\mathrm{H}$, Benhamou PH. Cow's milk epicutaneous immunotherapy in children: a pilot trial of safety, acceptability, and impact on allergic reactivity. J Allergy Clin Immunol 2010;125:1165-1167.

44. Martínez-Botas J, Rodríguez-Álvarez M, Cerecedo I, Vlaicu C, Diéguez MC, Gómez-Coronado D et al. Identification of novel peptide biomarkers to predict safety and efficacy of cow's milk oral immunotherapy by peptide microarray. Clin Exp Allergy 2015;45:10711084.

45. Savilahti EM, Kuitunen M, Valori M, Rantanen V, Bardina L, Gimenez G, et al. Use of IgE and IgG4 epitope binding to predict the outcome of oral immunotherapy in cow's milk allergy. Pediatr Allergy Immunol 2014;25:227-235.

46. Sampson HA. Utility of food-specific IgE concentrations in prediciting symptomatic food allergy. J Allergy Clin Immunol 2001;107:891-6.

47. Sporik R, Hill DJ, Hosking CS. Specificity of allergen skin testing in predicting positive open food challenges to milk, egg, and peanut in children. Clin Exp Allergy 2000;30:1540-6.

48. Bartnikas LM, Sheehan WJ, Hoffman EB, Permaul $\mathrm{P}$, Dioun $\mathrm{AF}$, Friedlander $\mathrm{J}$ et al. Predicting food challenge outcomes for baked milk: role of specific IgE and skin prick testing. Ann Allergy Asthma Immunol 2012;109:309-313. 



\section{ALLERGY TO EGG}

\author{
Philippe A. Eigenmann, Jean-Christoph \\ Caubet, and Antonella Muraro
}

\section{THE ALLERGEN SOURCES}

Hen's egg is an ubiquitous food eaten in most parts of the world. It is a cheap and easily accessible food source, used in many home-made dishes, but also widely used by the food industry in processed foods.

Individuals are mostly exposed to hen's egg proteins in foods. Nevertheless, hen's egg proteins can be found in aerosolized particles produced by cooking. In a clinical report, respiratory symptoms to aerosolized egg proteins have been reported in bakery workers (1). It can be suspected that these may also contribute to primary sensitization to egg, similarly to what has been shown for peanut proteins (2).

\section{MAJOR AND RELEVANT MINOR \\ ALLERGENIC MOLECULES AND THEIR CLINICAL RELEVANCE}

Five proteins most commonly involved in allergic reactions to hen's egg have been identified and characterized (Gal $d 1$ to 5 , see Table 1). Despite being present in a lower quantity in hen's egg white than
Egg allergy is one of the most frequent food allergies in children around the world

The use of egg white components is clinically helpful for distinguishing between sensitization and clinical allergy

The use of egg white components is clinically helpful for distinguishing between allergy to cooked and raw egg, or exclusively to raw egg

The result of an lgE test can only be validated when corresponding to the clinical history, if necessary after a standardized egg challenge 


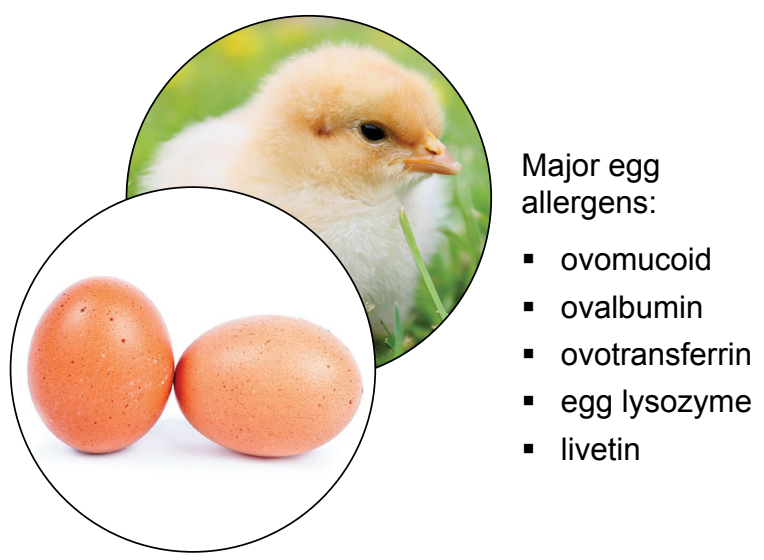

Figure 1

Major egg allergens.

ovalbumin, it has been shown that ovomucoid is most probably the immunodominant hen's egg allergen (3). Among the various physico-chemical characteristics, resistance to chemical denaturation has a direct clinical significance as structural modification of egg allergens can allow safe consumption of cooked/ baked egg containing foods.

\section{CLINICAL RELEVANCE, DIAGNOSIS AND MANAGEMENT}

According to the IgE sensitivity of a given patient, 3 different clinical scenarios should be distinguished in a patient with a positive test to egg proteins $(5,6)$.

1. Sensitized to hen's egg, but clinically tolerant. Can eat all forms of eggs. Such patients will present a positive serum IgE test to hen's egg white, in general in a low to mid range value, as well as a negative or low serum lgE test to ovomucoid.

2. Allergic to raw, or partially raw eggs only. Tolerant to cooked eggs, or processed foods containing cooked eggs. These patients will present similarly to scenario one, with a positive serum IgE test to hen's egg white, in general in a low to mid range value, as well as a negative or low serum lgE test to ovomucoid. Serum specific IgE to ovalbumin might be elevated in a similar range than the test to egg white.

3. Allergic to all forms of eggs. These patients have often serum specific IgE to egg white in the middle to upper range. They might also have elevated serum specific IgE to ovomucoid and well as to ovalbumin.

\section{Case history}

The case history is decisive. It needs to be assessed if the child has a concomitant atopic disease (e.g. atopic eczema), which might predispose to a positive test to egg white. If the child has a history of an allergic reaction after eating eggs, the history needs to specify to which form of egg the child reacted (cooked, partially cooked, or raw eggs).

\section{Skin prick testing (SPT)}

SPT can be done with commercial egg white extracts or with raw eggs. Both have a good accuracy for showing IgE sensitization. Extracts of major egg allergens (ovomucoid, ovalbumin or others) are not commercially available, and are not used in routine diagnostic testing. In absence of standardization of SPT procedure, cut-off values for prediction of clinical reactivity cannot be provided.

\section{IgE-testing}

Following tests are commercially available: Egg; Egg white; Egg yolk; Ovomucoid (Gal d 1); Ovalbumin (Gal d 2); Conalbumin (Gal d 3); Egg lysozyme (Gal d 4)

Egg white IgE testing is in general mostly recommended for primary diagnosis of egg allergy in children (most common age of sensitization and clinical allergy to eggs). In particular, egg white extract combines the most common major allergens recognized in egg allergy (ovomucoid and ovalbumin) and therefore constitutes the most accurate test for the initial diagnostic step (7). Egg white specific IgE levels have been studied in order to determine cut-off values indicative of true clinical egg allergy. Nevertheless, the published studies done in patient populations of different ages, or with various clinical pictures (e.g. presence or absence of eczema) have clearly shown cut-off value limitations to populations with similar characteristics (8-12). In addition, levels of specific IgE to egg white might be indicative of the severity of egg allergy.

Molecular diagnosis has been shown to be helpful in a more fine-tuned diagnosis of egg allergy, in particular for answering the following clinical questions: (1) sensitization vs clinical allergy to egg, (2) allergy to raw or partially cooked eggs, (3) allergy to all forms of egg (raw and cooked). 


\section{Table 1}

Allergenic molecules of hen's egg and clinical relevance of specific proteins

\begin{tabular}{|c|c|c|c|c|c|}
\hline Protein name & $\begin{array}{l}M_{r} \\
(k D a)\end{array}$ & protein family & biological function(s) & $\begin{array}{l}\text { Resistance } \\
\text { to heating } \\
\text { and chemical } \\
\text { denaturation }\end{array}$ & Clinical relevance \\
\hline \multicolumn{6}{|c|}{ Egg White Proteins } \\
\hline $\begin{array}{l}\text { ovomucoid } \\
\text { (Gal d 1) }\end{array}$ & 28 & $\begin{array}{l}\text { Kazal-type } \\
\text { serine protease } \\
\text { inhibitor }\end{array}$ & $\begin{array}{l}\text { serine protease } \\
\text { inhibition activity }\end{array}$ & high & $\begin{array}{l}\text { Heat-stable and highly } \\
\text { allergenic } \\
\text { Risk for reaction to all } \\
\text { forms of egg } \\
\text { High levels of specific lgE } \\
\text { may indicate sustained } \\
\text { egg allergy }\end{array}$ \\
\hline $\begin{array}{l}\text { ovalbumin } \\
\text { (Gal d 2) }\end{array}$ & 45 & $\begin{array}{l}\text { serine protease } \\
\text { inhibitor }\end{array}$ & storage protein? & low & $\begin{array}{l}\text { Heat-labile } \\
\text { Most abundant egg white } \\
\text { protein } \\
\text { Risk for clinical reaction to } \\
\text { raw or slightly heated egg } \\
\text { and certain vaccines }\end{array}$ \\
\hline $\begin{array}{l}\text { ovotransferrin or } \\
\text { conalbumin }\end{array}$ & & & & & $\begin{array}{l}\text { Heat-labile } \\
\text { Risk for clinical reaction to } \\
\text { raw or slightly heated egg }\end{array}$ \\
\hline (Gal d 3) & $76-77$ & transferrin & $\begin{array}{l}\text { iron-binding capacity } \\
\text { with antimicrobial } \\
\text { activity }\end{array}$ & low & $\begin{array}{l}\text { Risk for clinical reaction to } \\
\text { raw or slightly heated egg }\end{array}$ \\
\hline $\begin{array}{l}\text { egg lysozyme } \\
\text { (Gal d 4) }\end{array}$ & 14.3 & $\begin{array}{l}\text { glycoside } \\
\text { hydrolase family } \\
22\end{array}$ & antibacterial activity & moderate & \\
\hline ovomucin & 165 & $\begin{array}{l}\text { contains trypsin } \\
\text { inhibitor-like } \\
\text { domains }\end{array}$ & $\begin{array}{l}\text { heavily glycosylated } \\
\text { protein with potent } \\
\text { antiviral activities }\end{array}$ & n.a. & \\
\hline \multicolumn{6}{|l|}{ Egg Yolk Proteins } \\
\hline phosvitin & 35 & transferase? & metal-chelating agent & n.a. & n.a. \\
\hline $\begin{array}{l}\alpha \text {-livetin (Gal } \\
\text { d 5) }\end{array}$ & $65-70$ & serum albumin & $\begin{array}{l}\text { bind ions, fatty } \\
\text { acids, hormones } \\
\text { in physiological } \\
\text { conditions }\end{array}$ & n.a. & n.a. \\
\hline apovitellenins I & 9.5 & $\begin{array}{l}\text { very low density } \\
\text { lipoprotein }\end{array}$ & $\begin{array}{l}\text { potent lipoprotein } \\
\text { lipase inhibitor }\end{array}$ & n.a. & n.a. \\
\hline $\begin{array}{l}\text { apovitellenins VI } \\
\text { (orapoprotein B) }\end{array}$ & 170 & unknown & lipid-binding activity & n.a. & \\
\hline
\end{tabular}

Adapted from (4) 
Ando et al. have defined a positive decision point for 95\% diagnostic accuracy for allergy to heated egg at $10.8 \mathrm{kU} / \mathrm{L}$ for ovomucoid (13). In a similar study, but with different patient characteristics, we have found a cut-off value of $6.9 \mathrm{kU} / \mathrm{L}$ with a $95 \%$ specificity (14). Also in this study, a cut-off of $4.1 \mathrm{kU} / \mathrm{L}$ can be used for egg white in order to distinguish between allergy to all forms of egg, and sensitization in absence of allergy. Cut-off differences between different studies clearly limit the application of cut-off values to well defined patient populations (12).

Similarly, the heat-labile egg white allergen ovalbumin can help distinguishing between the various patterns of clinical reactivity to egg. We have shown that sequential testing starting with IgE measurement to egg white, followed by measuring IgE to ovomucoid will significantly increase the sensitivity of diagnostic testing compared to testing egg white only, although with a decrease in specificity (14).

We postulated that egg extracts modified by denaturation for mimicking heating of eggs, or egg digestion in the gut might provide more accurate proteins for clinical diagnosis. For distinguishing between egg sensitized subjects and patients allergic to all forms of eggs, native egg proteins provide reliable extracts for diagnosis as determined by ROC curves. For more fine tuned diagnosis, i.e. for distinguishing between patients allergic to eggs but tolerating cooked egg, denatured egg white or egg white allergens might be helpful (14). Nevertheless clinical utility of such tests need to be confirmed in various other patient populations.

\section{Food challenge tests}

Egg can be challenged in an open, or double-blind, placebo-controlled way. Patients with subjective symptoms or active atopic eczema should preferably be challenged with placebo phase. In addition, and according to the clinical question, egg might be given raw or in a cooked form. Routine testing does not include challenges with isolated egg proteins. Food challenges are useful in cases with a doubtful diagnosis, as well as for the follow-up of food allergy (at intervals defined according to the type of food, as well as the IgE test results, but not more frequently than every 1 to 2 years), in order to determine natural tolerance acquisition.

\section{Clinical management}

The food avoidance diet should be restricted only to the form of egg not tolerated by the patient. All other forms should be regularly consumed. The diagnosis should aim to properly identify forms of eggs to which the patient is tolerant.

\section{Pharmacotherapy}

Similarly to other foods, is not available for cure, but for treatment of acute symptoms due to accidental ingestions.

\section{Immunotherapy}

Various studies have shown a clinical efficacy for specific oral tolerance induction protocols (SOTI) (15). Nevertheless, this procedure is not yet routinely applicable.

\section{CLINICAL CASES}

\section{Case 1 (original)}

History: girl, 8 months old, severe atopic eczema. Allergy testing is done for ruling out food allergy as a triggering factor of her severe atopic eczema. Has never eaten eggs, neither isolated or in processed foods.

SPT: Positive to egg white, negative to milk, wheat, soy, fish, peanut and hazelnut.

In-vitro testing: Total lgE: $1825 \mathrm{kU} / \mathrm{l}$, specific IgE to hen's egg white: $5.02 \mathrm{kUA} / \mathrm{l}$, ovomucoid: $0.82 \mathrm{kU} / \mathrm{L}$.

Oral challenge: Egg is progressively introduced at home in baked goods as well as in pasta with eggs. Well tolerated, without immediate reactions or flaring of atopic eczema

Diagnosis: Sensitization to egg white in the context of moderate atopic eczema.

Recommendation: Continue eggs in cooked form, retesting and possibly a food challenge before introducing egg in partially cooked or raw forms.

\section{Case 2 (original)}

History: girl, 13 months old, in good health. Eats cooked eggs, either isolated or in processed foods without any symptoms since 8 months of age. Is given for the first time a chocolate mousse made with raw beaten egg white. Present within minutes a facial rash spreading to the upper thorax, a dry cough and several episodes of sneezing. The symptoms rapidly disappear after administration of an oral antihistamine. 
SPT: Positive to egg white.

In-vitro testing: specific IgE to hen's egg white 3.65 kUA/l, ovomucoid $0.78 \mathrm{kU} / \mathrm{L}$. Interpretation: a low ovomucoid allergen-specific IgE (relative to the specific IgE to hen's egg white) is indicative of probable tolerance to cooked eggs.

Diagnosis: Allergy to raw eggs only.

Recommendation: Eggs well tolerated in baked goods or hard boiled can be eaten. Elimination diet of incompletely cooked, or raw eggs in any form. Follow-up at 2 years of age with measurement of slgE to egg white and ovomucoid, assess clinical reactivity with oral food challenge if there is a reasonable chance of tolerance acquisition.

\section{Case 3 (original)}

History: boy, 9 months old, history of moderate atopic eczema. Eats for the first time a hardboiled egg. Present within minutes a urticarial rash over the thorax, followed by an episode of vomiting. The symptoms rapidly disappear after administration of an oral antihistamine.

SPT: Positive to egg white.

In-vitro testing: specific IgE to hen's egg white 18.23 $\mathrm{kUA} / \mathrm{l}$, ovomucoid $8.56 \mathrm{kU} / \mathrm{L}$.

Interpretation: a high ovomucoid-specific lgE concentration is indicative of probable allergy to all forms of eggs.

Diagnosis: Allergy to all forms of egg.

Recommendation: Eggs in all forms and foods containing eggs need to be avoided. Follow-up at 2 years of age with measurement of slgE to egg white and ovomucoid, assess clinical reactivity with oral food challenge first to cooked egg if there is a reasonable chance of tolerance acquisition. Not completely cooked eggs and raw egg will probably need to be continued to be avoided

\section{CONCLUSION}

At the present stage, the measurement of serum IgE or skin prick testing to egg white should represent the first diagnostic test which should be available also to primary care physician. Using tests with egg white components is most helpful for fine tuning of the diagnosis in order to define tolerance or not to cooked eggs, and the follow-up of egg allergy.
Nevertheless, the use of these tests is still subject of research and their interpretation might be subjected to knowledge by the allergy specialist. The definite diagnosis should always be done in relation to the clinical outcome, if necessary by a standardized food challenge. For general recommendations about food allergy diagnosis, the reader might also refer to the "EAACI Food Allergy Guidelines" (16).

\section{References}

1. Escudero $C$, Quirce $S$, Fernández-Nieto $M$, Miguel J, Cuesta J, Sastre J. Egg white proteins as inhalant allergens associated with baker's asthma. Allergy 2003;58:616-620.

2. Brough HA, Santos AF, Makinson K, Penagos M, Stephens AC, Douiri A et al. Peanut protein in household dust is related to household peanut consumption and is biologically active. J Allergy Clin Immunol 2013;132:630-638.

3. Cooke SK, Sampson HA. Allergenic properties of ovomucoid in man. J Immunol 1997;159:2026-2032.

4. Mine Y, Yang M. Recent Advances in the Understanding of Egg Allergens: Basic, Industrial, and Clinical Perspectives. J Agric Food Chem 2008;56:48744900.

5. Urisu A, Ando $H$, Morita $Y$, Wada E, Yasaki T, Yamada $\mathrm{K}$ et al. Allergenic activity of heated and ovomucoid-depleted egg white. J Allergy Clin Immunol 1997;100:171-6.

6. Eigenmann PA. Anaphylactic reactions to raw eggs after negative challenges with cooked eggs. J Allergy Clin Immunol 2000;105:587-588.

7. Benhamou $\mathrm{AH}$, Caubet JC, Eigenmann PA, NowakWegrzyn A, Marcos CP, Reche M et al. State of the art and new horizons in the diagnosis and management of egg allergy. Allergy 2010;65:283-289.

8. Sampson HA. Utility of food-specific lgE concentrations in predicting symptomatic food allergy. J Allergy Clin Immunol 2001;107:891-896.

9. Boyano Martínez T, García-Ara C, Díaz-Pena JM, Muñoz FM, García Sánchez G, Esteban MM. Validity of specific IgE antibodies in children with egg allergy. Clin Exp Allergy 2001;31:1464-1469.

10. Celik-Bilgili S, Mehl A, Verstege A, Staden U, Nocon $\mathrm{M}$, Beyer $\mathrm{K}$ et al. The predictive value of specific immunoglobulin E levels in serum for the outcome of oral food challenges. Clin Exp Allergy 2005;35:268273.

11. Benhamou AH, Zamora SA, Eigenmann PA. Correlation between specific immunoglobulin $E$ levels and the severity of reactions in egg allergic patients. Pediatr Allergy Immunol 2008;19:173-179. 
12. Eigenmann PA. Are specific immunoglobulin E titres reliable for prediction of food allergy? Clin Exp Allergy 2005;35:247-249.

13. Ando H, Moverare R, Kondo $\mathrm{Y}$, Tsuge I, Tanaka A, Borres MP et al. Utility of ovomucoid-specific IgE concentrations in predicting symptomatic egg allergy. J Allergy Clin Immunol 2008;122:583-588.

14. Benhamou Senouf AH, Borres MP, Eigenmann PA. Native and denatured egg white protein IgE tests discriminate hen's egg allergic from egg-tolerant children. Pediatr Allergy Immunol 2015;26:12-17.

15. Burks AW, Jones SM, Wood RA, Fleischer DM, Sicherer SH, Lindblad RW et al. Oral Immunotherapy for Treatment of Egg Allergy in Children. $N$ Engl J Med 2012;367:233-243.

16. Muraro A, Werfel T, Hoffmann-Sommergruber K, Roberts G, Beyer K, Bindslev-Jensen C, et al. EAACl food allergy and anaphylaxis guidelines: diagnosis and management of food allergy. Allergy 2014;69:10081025. 


\section{ALLERGY TO FISH}

\author{
Lars K. Poulsen, Martine Morisset, Annette \\ Kuehn
}

\section{THE ALLERGEN SOURCES}

Fish together with egg, milk and crustaceans represent the animal kingdom in the "big eight" group of food allergens, to which the majority of food allergic patients reacts. As fish is both an important food component and a potent source of food allergens, fish has been included in the European Union regulation of food labelling (1).

Despite the broad biodiversity among fishes (more than 30,000 individual species have been described), the most frequently consumed species belong to a limited number of orders, the salmonlike (Salmoniformes), cod-like (Gadiformes), perchlike (Perciformes), herring-like (Clupeiformes), carplike (Cypriniformes), catfish-like (Siluriformes) and platfishes (Pleuronectiformes) (Fig. 1).

Globally, a much higher number of fish species is commercially available (2). The market share of these species varies in different countries according to regional availability and eating habits. While cod and salmon are important food fishes in Europe, other low-value freshwater species are popular in Asia (e.g. grass carp and Asian carp). Fish allergens have been described in around 40 species but detailed analysis
Fish species may differ by their allergenic potency.

Allergy can be elicited by proteins present in fish muscle, roe, skin or blood.

Allergens from fish and shellfish (e.g. crustaceans, molluscs) are not the same.

Less than $1 \%$ of the general population suffer from a fish allergy.

Parvalbumin is the major fish allergen (prevalence rates 70-95\%).

Most patients have slgE to new fish allergens, enolases and aldolases. 


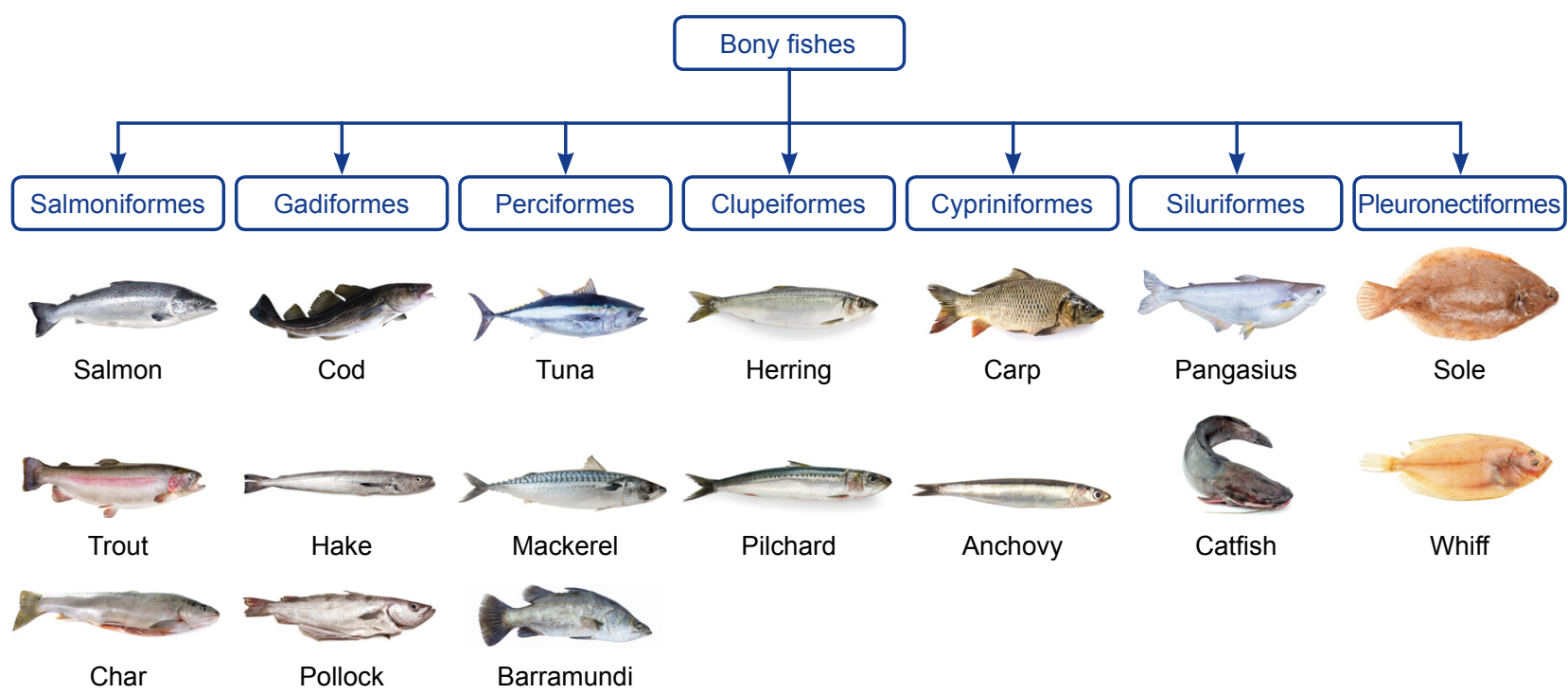

Figure 1

Most important food fishes are members of seven taxonomic orders.

of the allergy-eliciting molecules was performed mainly for fish which are commonly consumed in Europe such as carp, cod, salmon, trout and tuna. A large and clinically relevant cross-reactivity seems to exist between parvalbumins of different fish species (see chapter 'Parvalbumins'). This has probably limited the research and willingness to experiment with other species for both patients and doctors.

The following fish products can be elicitors of severe allergic reactions in sensitized patients.

- Fish meat - The largest allergenic activity resides in the muscle of the fish (2). Fish is consumed as a cooked, fried, pickled or raw food product. Food processing does not seem to affect the allergenic potency of the fish but rather the allergen content which varies in different species (3). Parvalbumins are the major fish allergens. With a serving size of $200 \mathrm{~g}$ carp or herring filet, the consumer ingests up to $1 \mathrm{~g}$ of parvalbumin per meal. Other fish allergens present in the muscle are enolases and aldolases. As a food ingredient, fish has to be listed specifically on a product label regardless of the percentage of content.

- Eggs, roe, caviar - There are case reports that caviar has elicited allergic reactions (4). Typically, roe is consumed in its raw form. Vitellogenin has been identified as an important fish egg allergen. This protein and its metabolites represent nearly the total protein content of roe. The knowledge about fish egg allergens is still limited. However, parvalbumins are not present in fish eggs.

- Fish gelatin, isinglass and similar products Recently, concern has been raised as to whether fish-derived products such as fish gelatin may contain allergenic activity. Fish gelatin, hydrolysed collagen, is made from fish skins and bones (5). Isinglass is derived from fish swim bladders and mainly contains collagen. Food (beverages, candy), pharmaceutical (gel capsules and coatings) or biological (vaccines, sublingual immunotherapy) products may contain these ingredients. Allergenicity might be inherent to collagen-like products or it might stem from contaminations by fish meat residues. Consumers are not aware of these fish-derived food ingredients as they are exempted from the food labelling regulation.

- Fish blood - Fish hemin (fish blood) or individual blood proteins have been used by the food industry as additives or processing aids but they seem to be relevant as an allergen source only in the fish-processing environment. Occupational asthma has been linked to the aerosolization of blood-derived allergens during processing of fish. Serum albumin has been suggested as a potential allergen but this could not be confirmed so far. 
The term seafood comprises both fish and shellfish (e.g. shrimps, crabs, lobsters, mussels, oysters, octopus, squid). Considering the large phylogenetic distance between fish and these other organisms it is not surprising that little cross-reactivity occurs. Therefore, seafood other than fish as allergenic food will not be discussed further in the context of this chapter (see Section B13).

An increasing number of studies reports on IgEmediated allergy which is caused by the parasite Anisakis simplex found in contaminated raw or undercooked fish (6). Since it is not a proper fish allergen, it will not be discussed further in this chapter. However, it is important to keep it in consideration when diagnosing patients experiencing allergic reactions following the ingestion of fish.

\section{PARVALBUMIN AND MINOR ALLERGENIC MOLECULES}

A search in the WHO/IUIS database reveals currently 29 entries while the database Allergen Online (www. allergenonline.org, version 15) comprises 65 fish allergens of known sequence. Nineteen and 55, respectively, of these belong to the parvalbumin family (Table 1$)$. The others are enolases $(n=4)$, aldolases $(n=4)$, tropomyosin $(n=1)$ and vitellogenin from salmon roe $(n=1)$. These are discussed further below.

- Fish meat - The dominating major allergen in fish muscle is parvalbumin of which the codfish Gad c 1 was the first to be identified (see chapter 'Parvalbumins') (2). Subsequently, studies were performed with a number of homologous proteins such as Gad m 1 from Atlantic cod, Cyp c 1 from Common carp and Sal s 1 from Atlantic salmon (Fig. 2). Parvalbumins are small muscle proteins (10-12 kDa) of remarkable stability towards physicochemical effects by food processing. During fish preparation, they can even become airborne. Because of specific characteristics of their protein structure, these calcium-binding allergens belong to the so-called EF-hand family (Fig. 3) (7). Parvalbumin levels vary considerably in different fish tissues and species $(3,8)$. Carp and herring muscle contain about 100-times more parvalbumin than mackerel and tuna. Most fish-allergic patients have specific IgE to these allergens (Table 1). Highly conserved parvalbumin epitopes have been used to explain not only lgE-

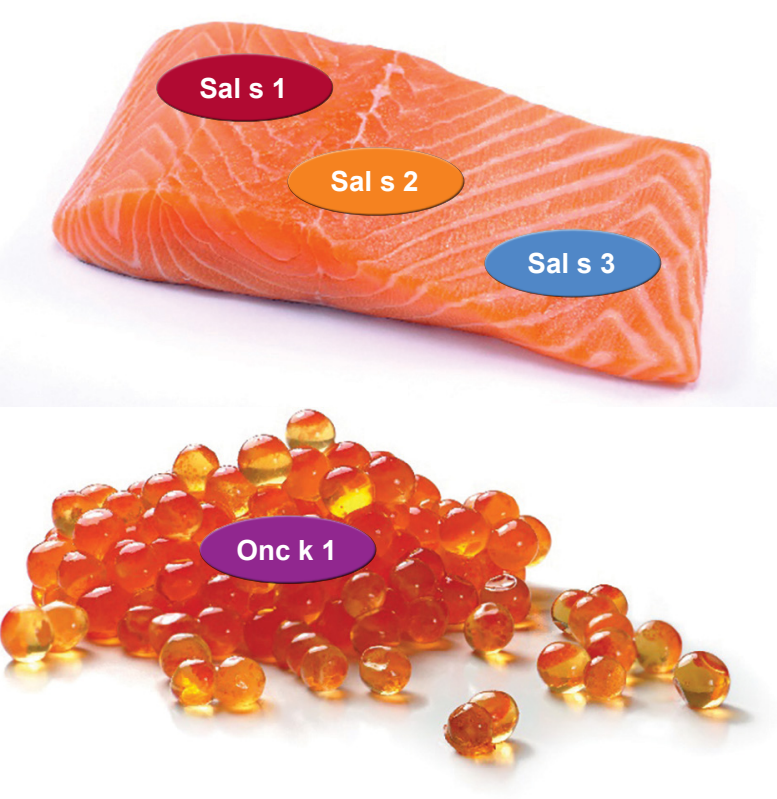

Figure 2

Most important allergens from Atlantic salmon muscle and fish roe.

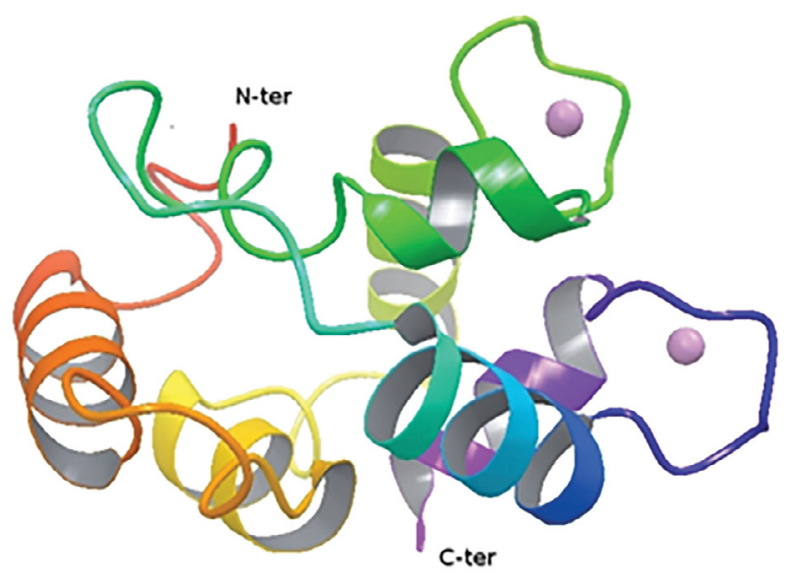

Figure 3

Protein structure of parvalbumin Cyp c 1 from carp (pdb 4CPV). Two EF-hand motifs each bind a Ca $a^{2+}$-ion. 


\section{Table 1}

Major and relevant minor fish allergenic molecules

\begin{tabular}{|c|c|c|c|c|c|c|}
\hline Order & English name (Species) & $\begin{array}{l}\text { Allergenic } \\
\text { molecule }\end{array}$ & $\begin{array}{l}\text { Biochemical } \\
\text { name }\end{array}$ & $\begin{array}{l}\text { IUIS rec- } \\
\text { ognized }\end{array}$ & $\begin{array}{l}\text { Preva- } \\
\text { lence (\%) }\end{array}$ & $\begin{array}{l}\mathrm{MW} \\
(\mathrm{kDa})\end{array}$ \\
\hline \multirow{2}{*}{ Clupeiformes } & Herring (Clupea harengus) & Clu h 1 & parvalbumin & X & 45 & 12 \\
\hline & Pilchard (Sardinops sagax) & Sar sa 1 & parvalbumin & $x$ & 80 & 12 \\
\hline Cypriniformes & Carp (Cyprinus carpio) & Сур с 1 & parvalbumin & X & 100 & 12 \\
\hline \multirow{4}{*}{ Gadiformes } & Baltic cod (Gadus callarias) & Gad c 1 & parvalbumin & $x$ & 100 & 12 \\
\hline & \multirow{3}{*}{ Atlantic cod (Gadus morhua) } & Gad m 1 & parvalbumin & $\mathrm{X}$ & 100 & 12 \\
\hline & & Gad m 2 & enolase & $x$ & 56 & 50 \\
\hline & & Gad m 3 & aldolase & $x$ & 37 & 40 \\
\hline \multirow{3}{*}{ Perciformes } & \multirow{3}{*}{ Tuna (Thunnus albacares) } & Thu a 1 & parvalbumin & $x$ & 95 & 11 \\
\hline & & Thu a 2 & enolase & $x$ & 19 & 50 \\
\hline & & Thu a 3 & aldolase & $\mathrm{X}$ & 13 & 40 \\
\hline $\begin{array}{l}\text { Pleuronecti- } \\
\text { formes }\end{array}$ & $\begin{array}{l}\text { Megrim, whiff (Lepidorhombus } \\
\text { whiffiagonis) }\end{array}$ & Lep w 1 & parvalbumin & $x$ & 100 & 11.5 \\
\hline \multirow{5}{*}{$\begin{array}{l}\text { Salmoni- } \\
\text { formes }\end{array}$} & Pacific salmon (Oncorhynchus keta) & Onc k 5 & vitellogenin & $x$ & nd & 18 \\
\hline & Rainbow trout (Oncorhynchus mykiss) & Onc m 1 & parvalbumin & $x$ & 95 & 12 \\
\hline & \multirow{3}{*}{ Salmon (Salmo salar) } & Sal s 1 & parvalbumin & $\mathrm{X}$ & 64 & 12 \\
\hline & & Sal s 2 & enolase & $x$ & 24 & 50 \\
\hline & & Sal s 3 & aldolase & $x$ & 16 & 40 \\
\hline $\begin{array}{l}\text { Scorpaeni- } \\
\text { formes }\end{array}$ & Redfish (Sebastes marinus) & Seb m 1 & parvalbumin & $x$ & 95 & 11 \\
\hline
\end{tabular}

Other parvalbumins: Sco s 1 (mackerel), Sol so 1 (sole), Lat c 1 (barramundi), Xip g 1 (swordfish); nd, not determined.

but also clinical cross-reactivity among various fish species. More recently, new fish allergens were identified, namely $50 \mathrm{kDa}$-enolases and $40 \mathrm{kDa}$-aldolases from cod, salmon and tuna (9). These glycolytic enzymes are highly expressed in the fish muscle. Their potency as food allergens still needs to be defined as they are less stable than parvalbumins. However, a considerable number of fish-allergic subjects seem to have lgE against these allergens (Table 1). In-vitro crossreactivity has been reported for homologues from cod, salmon and tuna.

- Fish gelatin - Collagen consists of three individual polypeptide chains corresponding to two alphasubunits $\left(\alpha_{1}, \alpha_{2}\right.$; each $\left.110 \mathrm{kDa}\right)$ and one betasubunit $(210 \mathrm{kDa})$. These chains are wound around one another in a confirmation of a triple helix. Fish gelatin is a heterogeneous product, which is obtained from acidic acid extraction of collagen followed by chemical hydrolysis. According to the molecular weight of fish gelatin components, it is available at different hydrolysation grades. The allergenic potency of fish gelatin is not yet well understood. IgE-binding to fish collagen has been shown using sera from fish-allergic patients. Skin testing was positive in two studies (10, 11). Severe anaphylaxis has been only reported in a single patient following ingestion of several grams of fish gelatin (12). Fish gelatin differs considerably by its amino acid composition from their mammalian counterparts so that there is no cross-reactivity among these molecules.

- Eggs, roe, caviar - The allergens of roe are different from those of fish meat. Patients with roe allergy often tolerate fish meat and vice versa. Vitellogenins are glycolipoproteins of high molecular weight 
(>150 kDa) belonging to the family of lipidtransport proteins. Studies of allergens from salmonid roe have led to the identification of a 35 $\mathrm{kDa}$ vitellogenin fragment consisting of two partly identical subunits (18 and $16 \mathrm{kDa}$ ) named Onc k 5 (Fig. 2) (13). Cross-reactivity has been proven for roe allergens from different fish species by lgEand skin testing. However, no cross-reactivity was found to homologues from chicken egg yolk.

\section{SENSITIZATION TO INDIVIDUAL}

\section{MOLECULES AND ITS CLINICAL RELEVANCE}

So far, epidemiological studies on fish allergy are missing to present consistent data of sensitization to fish and fish allergens. Prevalence rates to fish have been determined in studies of variable design and methodology $(2,14,15)$. Overall, it seems that less than $1 \%$ of the global population is affected by allergy to fish. A higher percentage is observed in paediatric cohorts (up to $10 \%$ ) and in countries with long coast lines which have a high fish consumption as well as in regions with fish-processing industries (up to $3 \%$ ) (2). Patients get sensitized to fish not only upon ingestion but also by skin contact and inhalation of fish steam during processing of this food. Occupational asthma has been reported in 7-36 \% of workers in industrial fish production lines.

Parvalbumins have been defined as the panallergens in fish. Sensitization rates for parvalbumins were determined based on studies during allergen characterization $(2,16-18)$. It was concluded that 90$95 \%$ of the patients had specific IgE to these muscle proteins. Recent studies showed that the fish-allergic population might be subdivided into the following clinical clusters, (i) highly sensitized patients reacting to all fish, (ii) oligo-sensitized patients reacting to several, specific fishes, and (iii) patients with 'selective reactions' to individual fish species only $(9,19,20)$. Patients of these clinical clusters vary by their IgE-recognition profiles. It was shown that the prevalence of IgE-binding to parvalbumin was lower than assumed. The sensitization rate to this major allergen might be rather around $70 \%$, which would need to be confirmed in future studies. A single study demonstrated that fish-allergic patients with specific IgE to cod parvalbumin might be cosensitized to cod enolases (81 \%) and aldolases (58 \%). The clinical origin and relevance of this co-sensitization is not yet resolved. However, specific parvalbuminnegative patients seem to develop IgE-antibodies to fish enolase (47\%) and aldolase (41\%) which is rather linked to species-specific fish allergies (20).

It is important to note that there are still limited data available to delineate how many patients can be categorized in each proposed clinical cluster. Also, it has to be taken into consideration that a geographical and/or a temporal gradient might be relevant for such a prevalence data collection.

\section{CLINICAL MANAGEMENT}

Diagnosis is mostly based on clinical history, skin tests and IgE tests, followed (if needed) by a an oral food challenge (ideally, double-blind placebo-controlled challenge) with the fish that has elicited the reaction $(15,21)$. Level of serum IgE antibodies have been correlated with the clinical reactivity to predict allergy to fish. In a US population, a diagnostic serum IgE level of $20 \mathrm{kUA} / \mathrm{L}$ to cod (ImmunoCAP, Thermo Fisher) has been established to predict an allergy to this fish with $95 \%$ certainty (22). However, clinical reactions have been indicated for patients with much lower IgE-titers. Therefore, the significance of this diagnostic indicator using fish extract is doubtful and needs to be further evaluated.

The availability of individual allergens for IgE-testing is still limited and thus, not of much help in predicting whether the patient is allergic to other fish species. However, an outline of the future diagnosis using single allergens is presented in the 'Clinical cases' and in the chapter 'Parvalbumins'.

Two important questions should be addressed if the initial suspicion of fish allergy is confirmed by the challenge procedure (Fig. 4): first, how sensitive is the patient? This can normally be deduced from the titrated challenge procedure, and the patient should be advised for future dietary precautions based on his or her individual threshold.

The second question relates to the degree of crossreactivity between fish species (Fig. 5). If the patient reacts with IgE of similar magnitude and reacts to a parvalbumin it is likely that there is a broad crossreactivity. If the sensitization pattern suggests a more "selective" reaction to a single fish species, an open challenge may be tried to confirm this tolerance.

\section{History of exposure}

As for diagnosis of fish-allergic patients, it should be remembered that most people are aware of ingesting 
Case history: Immediate reaction after potential consumption of fish (product)

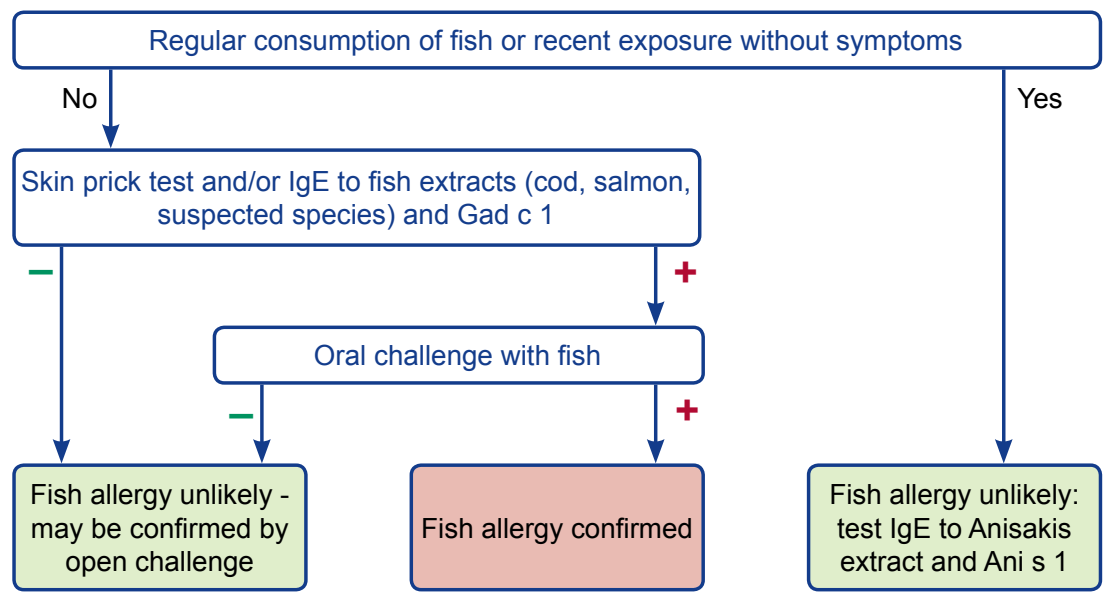

Figure 4

Diagnostic algorithm in patients with suspected fish allergy.

Case history: Immediate reaction after consumption of SPECIFIC fish

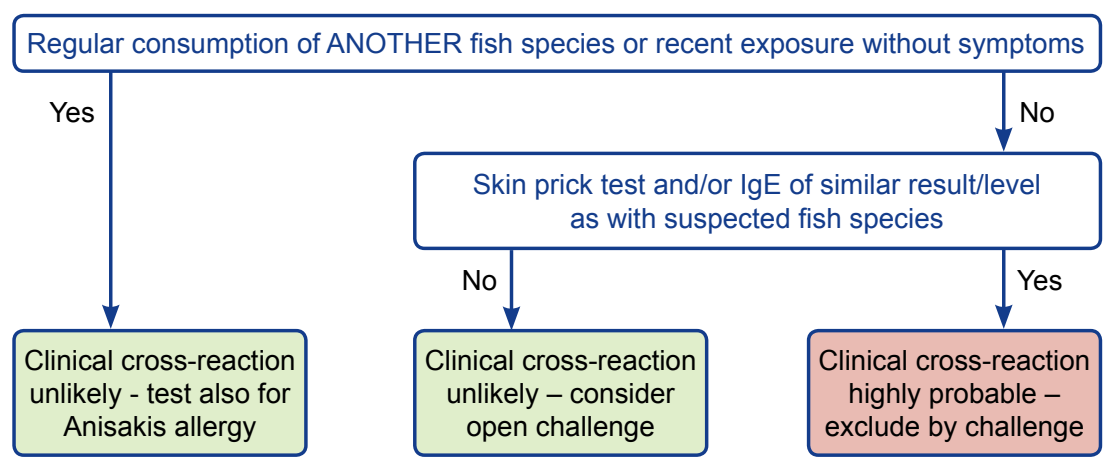

Figure 5

Diagnostic algorithm in patients with suspected allergy to single or specific fishes.

fish, and thus the patient history is often quite reliable as for the exposure (which does of course not exclude other ingredients in a meal). While probably extremely rare, there are examples of patients - mentioned above - reacting to fish allergens that are hidden in foods (23). It is obviously difficult to demonstrate a $100 \%$ safety level, but a number of studies have suggested that some fish-derived food additives seem to have a quite low risk of causing reactions in previously sensitized fish-allergic persons.

\section{Quantitative risk assessment}

As relating to the dose of fish producing food allergic symptoms only data for fish meat are available in the published literature. According to the literature the lowest provoking dose of fish is in the low milligram range (24). A larger population was tested in the EuroPrevall project, an EU-funded project addressing the prevalence, cost and basis of food allergy across Europe, and an ED10 of $27.3 \mathrm{mg}$ of protein was found (25). 


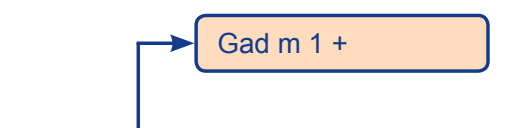

Potentially cross- $/$ co-sensitization to other fishes

Figure 6

\section{Diagnostic procedure Case 1.}

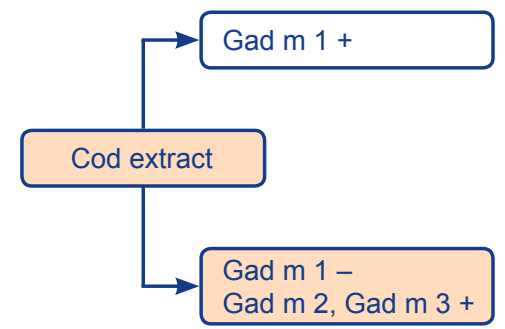

Primary sensitization and clinical monosensitivity to cod
Potentially cross-/

co-sensitization to other fishes

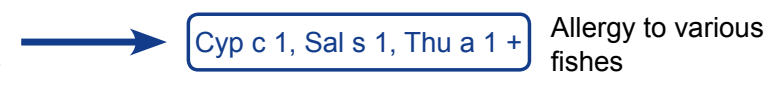

\section{Other risk factors}

It is important to notice that parvalbumins, the major fish allergens, are extremely heat-stable (26). Thus, their allergenicity cannot be expected to be reduced by food processing. However, its proteolytic resistance seem to be less, e.g. to pepsin at low $\mathrm{pH}$ (27). Accordingly, maintenance of a well-functioning digestive system with low ventricular $\mathrm{pH}$ may be of importance for avoiding fish allergy (28).

\section{CLINICAL CASES}

Most cases of fish allergy present with classical food allergic symptoms short after intake of fish. Symptoms may include oral allergy syndrome, rhinitis/conjunctivitis, bronchospasm, urticaria, gastro-intestinal symptoms and anaphylaxis.

\section{Case 1 (published (9))}

Clinical History: A woman, 21 years, with a clinical history of fish allergy presenting with angioedema and urticaria after eating fish (cod, salmon, herring) at several episodes.

Test with extracts: Skin testing was positive for cod and pollock extract. Specific IgE were positive for cod extract (7.3 kUA/L), salmon (5.7 kUA/L) and tuna (3.5 kUA/L).

Food challenge: The patient refused a food challenge.
Test with molecules: Specific lgE were found for cod, carp, salmon and tuna parvalbumins $(5.0 \mathrm{kUA} / \mathrm{L}$, $45.0 \mathrm{kUA} / \mathrm{L}, 5.1 \mathrm{kUA} / \mathrm{L}$ and $5.3 \mathrm{kUA} / \mathrm{L}$, respectively).

Conclusion: In this case, the clinical cross-reactivity to several fishes was confirmed by the detection of specific IgE to fish allergens Gad $m$ 1, Cyp c 1 , Sal s 1, and Thu a 1 (Fig. 6).

\section{Case 2 (published (20))}

Clinical History: A male child, 10 years, with a clinical history of fish allergy presenting with swelling of the lips and tongue, throat discomfort and conjunctivitis after eating cod.

Test with extracts: Skin testing was positive for cod extract only. Specific IgE were positive for cod extract $(0.4 \mathrm{kUA} / \mathrm{L})$ but negative for other extracts (salmon, tuna).

Food challenge: The parents of the child refused a food challenge.

Test with molecules: Cod, salmon and tuna parvalbumins were negative in IgE ELISA. Only new cod allergens, enolase and aldolase were positive (Gad m 2, 0.5 kUA/L; Gad m 3, 0.4 kUA/L). No cross-inhibition was performed because IgEtesting was negative for other fish parvalbumins.

Conclusion: In this case, the monosensitivity to cod was confirmed by the detection of specific IgE recognizing the newly identified allergens Gad $m$ 2 and Gad $m 3$ (Fig. 7). 


\section{References}

1. Taylor SL, Baumert JL. Worldwide food allergy labeling and detection of allergens in processed foods. Chem Immunol Allergy 2015;101:227-234.

2. Sharp MF, Lopata AL. Fish allergy: in review. Clin Rev Allergy Immunol 2014;46:258-271.

3. Kuehn A, Scheuermann T, Hilger C, Hentges F. Important variations in parvalbumin content in common fish species: a factor possibly contributing to variable allergenicity. Int Arch Allergy Immunol 2010;153:359-366.

4. Untersmayr E, Focke M, Kinaciyan T, Poulsen LK, BoltzNitulescu G, Scheiner O et al. Anaphylaxis to Russian Beluga caviar. J Allergy Clin Immunol 2002;109:1034-1035.

5. Boran G, Regenstein JM. Fish gelatin. Adv Food Nutr Res 2010;60:119-143.

6. Nieuwenhuizen NE, Lopata AL. Allergic reactions to Anisakis found in fish. Curr Allergy Asthma Rep 2014;14:455.

7. Radauer C, Bublin M, Wagner S, Mari A, Breiteneder $\mathrm{H}$. Allergens are distributed into few protein families and possess a restricted number of biochemical functions. J Allergy Clin Immunol 2008;121:847-852.

8. Griesmeier $U$, Vázquez-Cortés $S$, Bublin $M$, Radauer C, Ma Y, Briza P et al. Expression levels of parvalbumins determine allergenicity of fish species. Allergy 2010;65:191-198.

9. Kuehn A, Hilger C, Lehners-Weber C, Codreanu-Morel F, Morisset M, Metz-Favre C et al. Identification of enolases and aldolases as important fish allergens in cod, salmon and tuna: component resolved diagnosis using parvalbumin and the new allergens. Clin Exp Allergy 2013;43:811-822.

10. André F, CavagnaS,André C. Gelatin prepared from tuna skin: a risk factor for fish allergy or sensitization? Int Arch Allergy Immunol 2003;130:17-24.

11. Hansen TK, Poulsen LK, Stahl Skov P, Hefle SL, Hlywka JJ, Taylor SL et al. A randomized, double-blinded, placebo-controlled oral challenge study to evaluate the allergenicity of commercial, food-grade fish gelatin. Food Chem Toxicol 2004;42:2037-2044.

12. Kuehn A, Hilger C, Hentges F. Anaphylaxis provoked by ingestion of marshmallows containing fish gelatin. J Allergy Clin Immunol 2009;123:708-709.

13. Perez-Gordo M, Sanchez-Garcia S, Cases B, Pastor C, Vivanco F, Cuesta-Herranz J. Identification of vitellogenin as an allergen in Beluga caviar allergy. Allergy 2008;63:479-480.

14. Sicherer SH, Muñoz-Furlong A, Sampson HA. Prevalence of seafood allergy in the United States determined by a random telephone survey. J Allergy Clin Immunol 2004;114:159-165.

15. Worm M, Reese I, Ballmer-Weber B, Beyer K, Bischof SC, Claßen $M$ et al. Guidelines on the management of IgE-mediated food allergies. Allergo J Int 2015;24:256-293.

16. Swoboda I, Bugajska-Schretter A, Verdino P, Keller W, Sperr WR, Valent $P$ et al. Recombinant carp parvalbumin, the major cross-reactive fish allergen: a tool for diagnosis and therapy of fish allergy. J Immunol 2002;168:4576-4584.

17. Van Do T, Elsayed S, Florvaag E, Hordvik I, Endresen C. Allergy to fish parvalbumins: studies on the crossreactivity of allergens from 9 commonly consumed fish. J Allergy Clin Immunol 2005;116:1314-1320.

18. Sten E, Hansen TK, Stahl Skov P, Andersen SB, Torp A, Bindslev-Jensen $U$ et al. Cross-reactivity to eel, eelpout and ocean pout in codfish-allergic patients. Allergy 2004;59:1173-1180.

19. Kuehn A, Metz-Favre C, Pauli G, Lehners-Weber C, Codreanu-Morel F, Hentges F et al. Comparison of fish allergy symptoms and patient-specific IgE profiles to parvalbumin, enolase, aldolase and fish gelatin. $\mathrm{Rev} \mathrm{Fr}$ Allergol 2014;54:51-60.

20. Kuehn A, Fischer J, Hilger C, Sparla C, Biedermann T, Hentges F. Correlation of clinical monosensitivity to cod with specific IgE to enolase and aldolase. Ann Allergy Asthma Immunol 2014;113:670-671.

21. Muraro A, Werfel T, Hoffmann-Sommergruber K, Roberts G, Beyer K, Bindslev-Jensen C et al. EAACl food allergy and anaphylaxis guidelines: diagnosis and management of food allergy. Allergy. 2014;69(8):1008-25.

22. Sampson HA, Ho DG. Relationship between foodspecific IgE concentrations and the risk of positive food challenges in children and adolescents. J Allergy Clin Immunol 1997;100:444-451.

23. Añíbarro B, Seoane FJ, Múgica MV. Involvement of hidden allergens in food allergic reactions. J Investig Allergol Clin Immunol 2007;17:168-172.

24. Helbling A, Haydel R Jr, McCants ML, Musmand JJ, ElDahr J, Lehrer SB. Fish allergy: is cross-reactivity among fish species relevant? Double-blind placebo-controlled food challenge studies of fish allergic adults. Ann Allergy Asthma Immunol 1999;83:517-523.

25. Ballmer-Weber BK, Fernandez-Rivas $M$, Beyer $K$, Defernez M, Sperrin M, Mackie AR et al. How much is too much? Threshold dose distributions for 5 food allergens. J Allergy Clin Immunol 2015;135:964-971.

26. Poulsen LK, Hansen TK, Nørgaard A, Vestergaard H, Stahl Skov P, Bindslev-Jensen C. Allergens from fish and egg. Allergy 2001;56(Suppl 67):39-42.

27. Untersmayr E, Poulsen LK, Platzer $\mathrm{MH}$, Pedersen $\mathrm{MH}$, Boltz-Nitulescu G, Skov PS et al. The effects of gastric digestion on codfish allergenicity. J Allergy Clin Immunol 2005;115:377-382.

28. Untersmayr E, Poulsen LK, Platzer MH, Pedersen MH, Boltz-Nitulescu G, Skov PS et al. Incomplete digestion of codfish represents a risk factor for anaphylaxis in patients with allergy. J Allergy Clin Immunol 2007;119:711-717. 
ALLERGY TO

CRUSTACEAN

\section{AND MOLLUSKS \\ (SHELLFISH)}

\author{
Andreas L. Lopata, Sandip D. Kamath
}

\section{THE ALLERGEN SOURCES}

The shellfish group is included among the "Big Eight" food groups, which are responsible for more than $90 \%$ of all food allergy cases. In general, $2 \%$ of the population are affected by food allergy to shellfish, including the crustacean and mollusk groups. Shellfish allergy, particularly to prawns, has one of the highest rates of food-induced anaphylaxis with nearly $42 \%$ among affected adults and $12 \%$ in children (1). It is noteworthy that although shellfish, along with fish are commonly termed as seafood, these two groups are very distinct in evolutionary terms and contain different molecular repertoires of food allergens. All shellfish species are invertebrate animals, in comparison to fish, which are lower vertebrates. Comparing evolutionary distance, crustaceans are placed closer to insects and arachnids, and this seems to be the major factor for molecular crosssensitization and clinical cross-reactivity between crustacean, house dust-mites and insects.

Edible shellfish consists of more than 300 different species. These should be further grouped into crustaceans (prawns, crabs, lobsters, krill etc.) and mollusks (mussels, oysters, abalone, squid, octopus,
Over $2 \%$ of the world population are affected by allergy to shellish (crustaceans and molluscs), with one of the highest rates of food-induced anaphylaxis.

Food allergy to shellfish may cause crosssensitisation and clinical reactivity to dust mites, insects and arachnids.

The major shellfish allergens are highly heatstable proteins, which also cause inhalational exposure and sensitisation among workers in the shellfish processing industry leading to asthma and subsequently food-induced allergy. 


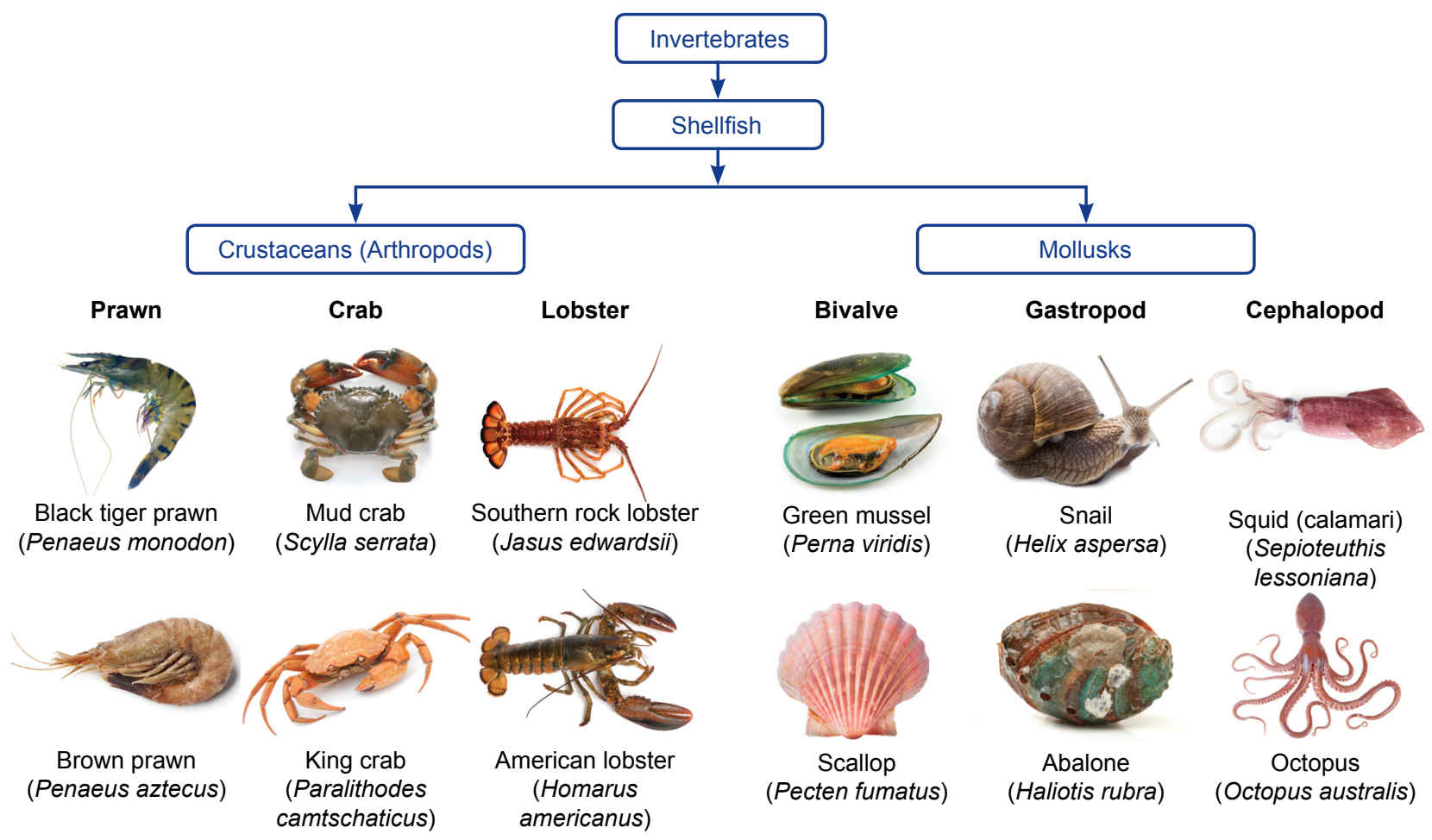

Figure 1

Classification of crustacean and mollusk species, comprising the shellfish group.

snail, limpets etc.). This classification is important from the clinical and immunological cross-reactivity point-of-view. The allergenic proteins present in these shellfish species have variable primary structures and often present a challenge in allergen detection as well as accurate allergy diagnosis and management. In addition, the availability and consumption of different shellfish species varies to a high degree in different parts of the world.

Shellfish are a rich source of proteins and omega-3 fatty acids. An increased awareness of the nutritional value has resulted in increased consumption of shellfish, and this has been accompanied by more frequent reporting of allergic health problems. The following types of consumption and exposure can be potent elicitors of severe allergic reactions to shellfish.

A) Fresh and cooked meat - Allergenic proteins are found in high concentrations in the edible muscle regions of the shellfish. In crustaceans, it is found in the abdominal, tail and pincer meat. Food processing methods such as thermal or pressure treatment does not seem to destroy most of the allergens, but may enhance their allergenic activity $(2,3)$. The tropomyosin family represent the major heat-stable allergen present in all shellfish species, and may constitute up to $20 \%$ of the total protein content. For food safety, products containing shellfish have to be indicated on the label. Moreover, the European Union has mandated separate food allergen labelling for crustaceans and mollusks, however appropriate tests for mollusks are not yet available (4). A recent study has demonstrated that at least 0.1-1.0 g of meat has to be ingested to trigger an allergic response (5).

B) Food additives - Shellfish products such as dried shrimp or shrimp paste are widely used as flavouring agents in various packaged and processed food products such as instant noodles and soups. This may be a potential source and cause of accidental consumption and exposure to shellfish allergens (see Table 1 for food products indicating the presence of shellfish groups).

C) Occupational exposure - In the seafood industry, workers are constantly exposed to air-borne 
shellfish particulate matter arising from different processing activities that results in the inhalation of air-borne allergens and cooking fumes (6). Such occupational exposure to shellfish allergens elicit upper and lower respiratory tract symptoms such as asthma, rhinitis and even result in skin symptoms (7). Workers with occupational asthma have been shown to develop ingestion induced food allergies to the same shellfish species.

D) Fish parasites -The food-borne parasite Anisakis or herring worm is an important food allergen. Anisakis is a parasitic nematode which mainly infects fish, but has also been reported for crustacean and squid, and the ingestion of contaminated fish or shellfish can result in severe allergic reactions (8). More importantly, the tropomyosin family of allergens are thought to be primarily responsible for cross-reactivity between anisakis and other invertebrates such as insects, mites and crustaceans. However, it is difficult to estimate the prevalence data of anisakis and shellfish cross-reactivity due to the lack of population-based studies.

\section{MAJOR AND RELEVANT MINOR ALLERGENIC MOLECULES}

At least 6 shellfish allergens have been identified displaying mainly metabolic or structural functions. They belong to diverse protein families characterized by conserved three-dimensional structures leading to possible broad immunochemical IgE mediated cross-reactions among different members of the crustacean or mollusk group. Due to complexity and heterogeneity of proteins among crustaceans and mollusks the relationship between their structure and subsequent allergenicity has only partly been addressed. In the past, mainly tropomyosin from many crustacean and few mollusks had been characterized in detail. In the past 10 years five additional allergenic proteins have been identified to induce lgE-mediated hypersensitivity through ingestion and inhalation and are officially accepted by the IUIS Allergen Nomenclature Sub-Committee (Table 2 and 4). The major allergen, tropomyosin was first identified in 1993 as the major shrimp allergen (9). Subsequently, arginine kinase (10) myosin light chain (11) and sarcoplasmic calcium binding protein (12) were identified as minor crustacean allergens.

\section{Table 1}

List of ingredients, which may contain Crustacean or Mollusk proteins

Crustaceans species that may be included as food ingredients

Barnacle, Crab, Crawfish, Krill, Lobster, Moreton bay bugs, Prawns, Shrimp (Crevette, Scampi)

Mollusk species that may be included as food ingredients

Abalone, Clam, Cockle, Cuttlefish, Limpet, Mussel, Octopus, Oyster, Periwinkle, Sea cucumber, Sea urchin, Scallop, Snail, Squid (calamari), Whelk

Food preparations that may contain shellfish

Bouillabaisse, Cuttlefish Ink, Glucosamine, Fish stock, Seafood flavouring, Surimi, Condiments and spices, Fish stock (shells of shrimp), Clam broth base powder, Crab extract powder, Shrimp powder, Scallop extract powder, Oyster juice powder, Lobster extract powder

\section{SENSITIZATION TO INDIVIDUAL MOLECULES AND ITS CLINICAL RELEVANCE}

\section{Epidemiology and sensitization/cross reactivity rates}

Previous sensitization rates were mainly based on skin or lgE testing to whole shellfish extracts $(13,14)$. Previous studies have identified the prevalence of shellfish allergy to be $2 \%$ for the general population and $0.1 \%-0.9 \%$ for children (15-17).

Allergen-specific IgE sensitization to various shellfish allergens have been demonstrated (Table 4). On an average, $60 \%$ of individuals with confirmed allergy to shellfish elicit specific lgE binding to tropomyosin. More importantly, it has been demonstrated that serumspecific lgE to tropomyosin is a better predictor of shrimp allergy than shrimp SPT or IgE to whole shrimp extract. Tropomyosin (Pen $\mathrm{m}$ 1) and sarcoplasmic calcium binding protein (Pen $\mathrm{m} 4$ ) sensitization has been associated with clinical reactivity to shrimp allergy (18).

Notably, conclusions on true sensitization rates are hampered due to the highly cross-reactive nature of some shellfish allergens. The allergen group of tropomyosin among the crustacean group seems to demonstrate very strong clinical cross-reactivity (Fig. 3), possible due to the high amino acid homology with 

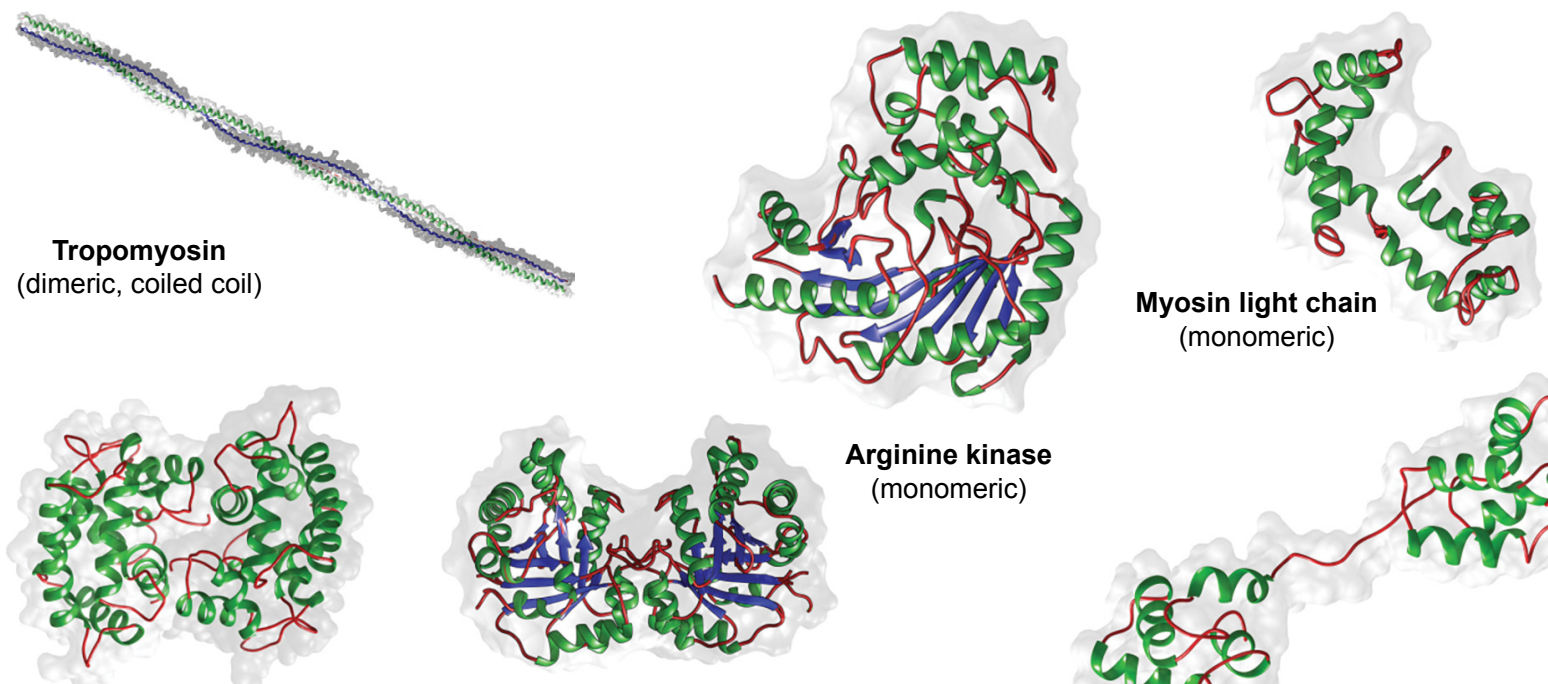

Sarcoplasmic calcium binding protein (dimeric)

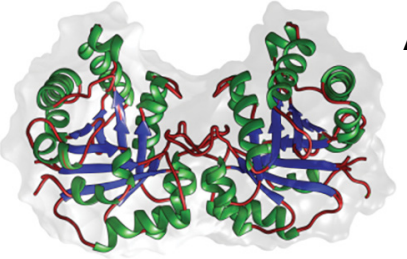

Myosin light chain (monomeric)

\section{Arginine kinase} (monomeric)

\section{Figure 2}

Three dimensional ribbon models of major and minor shellfish allergens in native conformation.

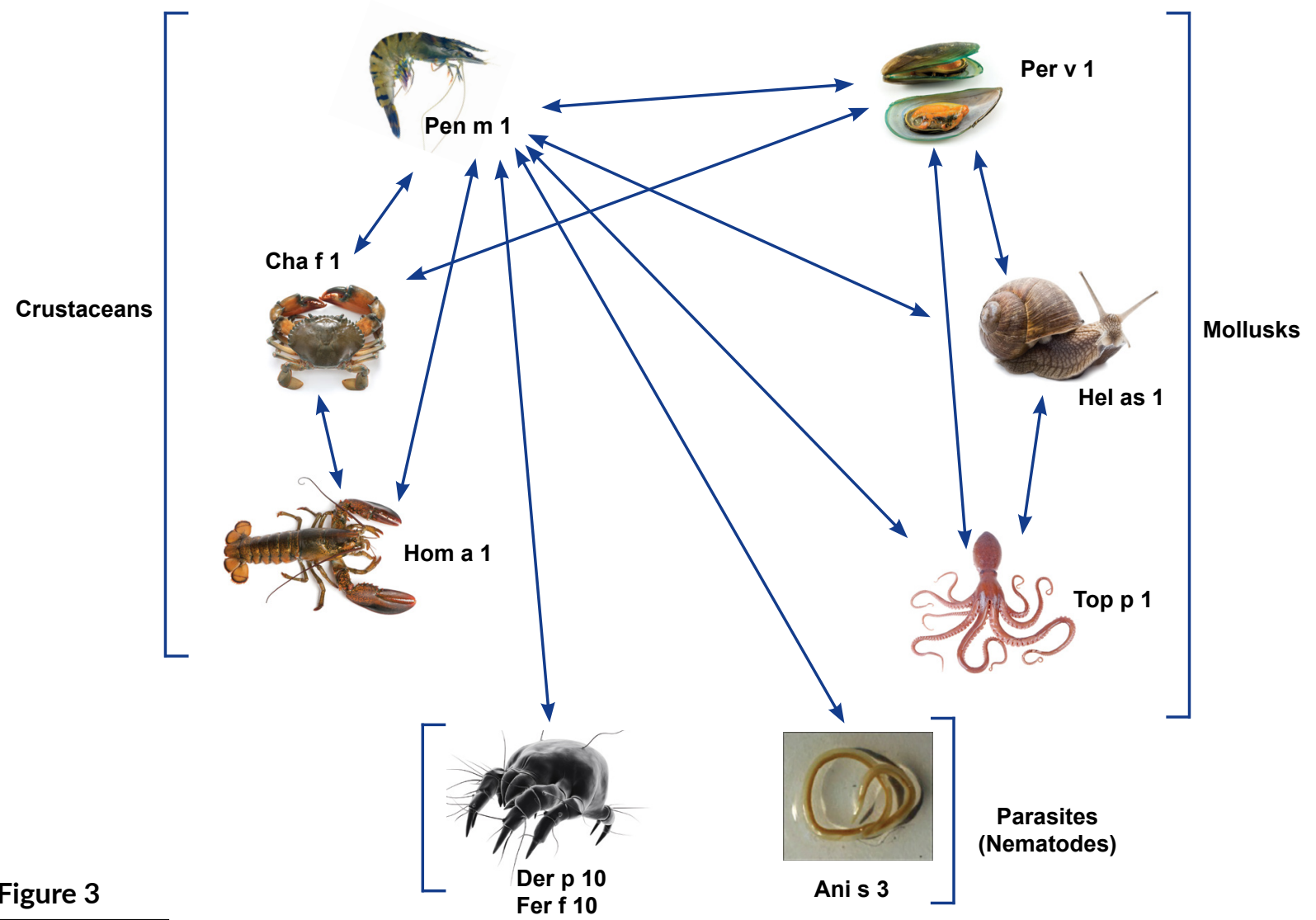

Clinically relevant cross-reactivity between shellfish and invertebrate allergens. 


\section{Table 2}

Allergenic molecules present in crustacean species

\begin{tabular}{|c|c|c|c|c|c|c|}
\hline $\begin{array}{l}\text { Species frequently } \\
\text { implicated }\end{array}$ & $\begin{array}{l}\text { Allergen name } \\
\text { (IUIS) }\end{array}$ & $\begin{array}{l}\text { Biochemical } \\
\text { name }\end{array}$ & $\begin{array}{l}\text { Molecular } \\
\text { weight kDa }\end{array}$ & $\begin{array}{l}\text { Heat } \\
\text { stability }\end{array}$ & $\begin{array}{l}\text { Physiological } \\
\text { function }\end{array}$ & $\begin{array}{l}\text { Route of } \\
\text { exposure }\end{array}$ \\
\hline $\begin{array}{l}\text { Penaeus monodon } \\
\text { (Black tiger prawn) }\end{array}$ & $\begin{array}{l}\text { Cha f } 1 \text {, Cra c } \\
1 \text {, Hom a 1, Lit } \\
\vee 1, \text { Mel I } 1\end{array}$ & Tropomyosin & $34-38$ & $\begin{array}{l}\text { Highly } \\
\text { heat } \\
\text { stable }\end{array}$ & $\begin{array}{c}\text { Muscle } \\
\text { contraction }\end{array}$ & $\begin{array}{l}\text { Ingestion } \\
\text { Inhalation }\end{array}$ \\
\hline $\begin{array}{l}\text { Litopenaeus vannamei } \\
\text { (White leg shrimp) }\end{array}$ & $\begin{array}{l}\text { Met e 1, Pan b 1, } \\
\text { Pen } \mathrm{m} 1 \text {, Por } \mathrm{p} 1\end{array}$ & & & & & \\
\hline $\begin{array}{l}\text { Penaeus aztecus } \\
\text { (Brown shrimp) }\end{array}$ & $\begin{array}{l}\text { Cra c 2, Lit v 2, } \\
\text { Pen } \mathrm{m} 2\end{array}$ & Arginine kinase & $40-45$ & Stable & $\begin{array}{l}\text { Energy metabolism } \\
\text { in muscles }\end{array}$ & $\begin{array}{l}\text { Ingestion } \\
\text { Inhalation }\end{array}$ \\
\hline $\begin{array}{l}\text { Homarusamericanus } \\
\text { (American lobster) }\end{array}$ & $\begin{array}{l}\text { Hom a 3, Lit v } \\
\text { 3, Pen } \mathrm{m} 3\end{array}$ & $\begin{array}{l}\text { Myosin light } \\
\text { chain }\end{array}$ & $17-20$ & Stable & $\begin{array}{c}\text { Muscle } \\
\text { contraction }\end{array}$ & Ingestion \\
\hline $\begin{array}{l}\text { Crangoncrangon } \\
\text { (Sand shrimp) }\end{array}$ & $\begin{array}{l}\text { Cra c 4, Lit v 4, } \\
\text { Pen m 4, Pon } \\
\text { I } 4\end{array}$ & $\begin{array}{l}\text { Sarcoplasmic } \\
\text { calcium binding } \\
\text { protein }\end{array}$ & $20-25$ & Stable & $\begin{array}{l}\text { Muscle contrac- } \\
\text { tion regulation }\end{array}$ & Ingestion \\
\hline $\begin{array}{l}\text { Charybdis feriatus } \\
\text { (Crucifix crab) }\end{array}$ & $\begin{array}{l}\text { Cra c 6, Hom a } \\
\text { 6, Pen m } 6\end{array}$ & Troponin C & $20-21$ & Unknown & $\begin{array}{l}\text { Calcium depen- } \\
\text { dent activation of } \\
\text { muscle contraction }\end{array}$ & Ingestion \\
\hline $\begin{array}{l}\text { Portunuspelagicus } \\
\text { (Blueswimmer crab) }\end{array}$ & Arc s 8, Cra c 8 & $\begin{array}{l}\text { Triose-Phosphate } \\
\text { isomerase }\end{array}$ & 28 & Labile & $\begin{array}{l}\text { Glycolysis (energy } \\
\text { metabolism) }\end{array}$ & $\begin{array}{l}\text { Ingestion } \\
\text { Inhalation }\end{array}$ \\
\hline
\end{tabular}

Registered allergen names are stated in accordance with WHO/IUIS Allergen Nomenclature

\section{Table 3}

Allergenic molecules present in mollusk species.

\begin{tabular}{|c|c|c|c|c|c|c|}
\hline $\begin{array}{l}\text { Species frequently } \\
\text { implicated }\end{array}$ & $\begin{array}{l}\text { Allergen name } \\
\text { (IUIS) }\end{array}$ & $\begin{array}{l}\text { Biochemical } \\
\text { name }\end{array}$ & $\begin{array}{l}\text { Molecular } \\
\text { weight kDa }\end{array}$ & $\begin{array}{l}\text { Heat } \\
\text { stability }\end{array}$ & $\begin{array}{l}\text { Physiological } \\
\text { function }\end{array}$ & $\begin{array}{l}\text { Route of } \\
\text { exposure }\end{array}$ \\
\hline Haliotismidae (Abalone) & Hal m1, Tod p 1 & Tropomyosin & 34-49 kDa & $\begin{array}{l}\text { Highly } \\
\text { heat } \\
\text { stable }\end{array}$ & $\begin{array}{l}\text { Muscle } \\
\text { contraction }\end{array}$ & $\begin{array}{l}\text { Ingestion } \\
\text { Inhalation }\end{array}$ \\
\hline $\begin{array}{l}\text { Helix aspersa (Garden } \\
\text { snail) }\end{array}$ & Hel as 1 & $\begin{array}{l}\text { Arginine } \\
\text { kinase }\end{array}$ & $40-45 \mathrm{kDa}$ & Stable & $\begin{array}{l}\text { Energy } \\
\text { metabolism in } \\
\text { muscles }\end{array}$ & $\begin{array}{l}\text { Ingestion } \\
\text { Inhalation }\end{array}$ \\
\hline $\begin{array}{l}\text { Todarodespaci ficus } \\
\text { (Flying squid) } \\
\text { Amphioctopus fangsiao } \\
\text { (Octopus) } \\
\text { Crassostreagig as } \\
\text { (Pacific oyster) } \\
\text { Fulviamutica (Cockle) }\end{array}$ & Un-assigned & Actin & $42 \mathrm{kDa}$ & $\begin{array}{l}\text { Heat- } \\
\text { labile }\end{array}$ & $\begin{array}{l}\text { Muscle } \\
\text { contraction }\end{array}$ & Ingestion \\
\hline
\end{tabular}

Registered allergen names are stated in accordance with WHO/IUIS Allergen Nomenclature 


\section{Table 4}

Clinical relevance and IgE sensitisation to allergenic molecules in crustaceans.

\begin{tabular}{|c|c|c|c|c|}
\hline & Allergen & IgE Sensitisation & $\begin{array}{l}\text { Allergenicity (FC and } \\
\text { SPT) }\end{array}$ & Risk factors \\
\hline \multirow{5}{*}{ Tropomyosin (TM) } & Pen a 1 & $51 \%$ (total 45 subjects) & \multirow{5}{*}{$\begin{array}{l}61 \% \text { ( } 24 \text { subjects) } \\
37 \% \text { ( } 45 \text { subjects) } \\
\text { slgE to tropomyosin is } \\
\text { a better predictor of } \\
\text { shrimp allergy than } \\
\text { shrimp SPT or slgE to } \\
\text { whole shrimp }\end{array}$} & \multirow{5}{*}{$\begin{array}{l}\text { Ingestion } \\
\text { Inhalation } \\
\text { Occupational } \\
\text { exposure }\end{array}$} \\
\hline & Lit v 1 & $\begin{array}{l}\text { 94\% (34 children) } \\
61 \% \text { (19 adults) }\end{array}$ & & \\
\hline & Pen m 1 & $62 \%$ (16 subjects) & & \\
\hline & \multirow{2}{*}{ Cra c 1} & $68 \%$ (31 subjects) & & \\
\hline & & 71\% (35 subjects) & & \\
\hline \multirow{3}{*}{$\begin{array}{l}\text { Arginine kinase } \\
\text { (AK) }\end{array}$} & Pen $\mathrm{m} 2$ & $50 \%$ (16 subjects) & \multirow{3}{*}{ Not known } & \multirow{3}{*}{$\begin{array}{l}\text { Ingestion } \\
\text { Inhalation } \\
\text { Occupational } \\
\text { exposure }\end{array}$} \\
\hline & Lit $\vee 2$ & $\begin{array}{l}67 \% \text { (34 children) } \\
21 \% \text { (19 adults) }\end{array}$ & & \\
\hline & Cra c 2 & 29\% (31 subjects) & & \\
\hline \multirow{3}{*}{$\begin{array}{l}\text { Myosin light chain } \\
\text { (MLC) }\end{array}$} & Pen $\mathrm{m} 3$ & $31 \%$ (16 subjects) & \multirow{3}{*}{ Not known } & \multirow{3}{*}{ Ingestion } \\
\hline & Lit v 3 & $\begin{array}{l}\text { 70\% (34 children) } \\
31 \% \text { (19 adults) }\end{array}$ & & \\
\hline & Crac 3 & 19\% (31 subjects) & & \\
\hline \multirow{3}{*}{$\begin{array}{l}\text { Sarcoplasmic } \\
\text { calcium binding } \\
\text { protein (SCBP) }\end{array}$} & Pen $\mathrm{m} 4$ & $19 \%$ (16 subjects) & \multirow{3}{*}{$\begin{array}{l}\text { slgE to SCBP may be } \\
\text { a better predictor of } \\
\text { shrimp allergy in children }\end{array}$} & \multirow{3}{*}{ Ingestion } \\
\hline & Lit v 4 & $\begin{array}{l}59 \% \text { (34 children) } \\
21 \% \text { (19 adults) }\end{array}$ & & \\
\hline & Cra c 4 & $35 \%$ (31 subjects) & & \\
\hline Troponin C (TnC) & Crac 6 & 29\% (31 subjects) & Not known & Ingestion \\
\hline \multirow{2}{*}{$\begin{array}{l}\text { Triose phosphate } \\
\text { isomerase (TIM) }\end{array}$} & Pen $\mathrm{m} 8$ & 19\% (16 subjects) & \multirow{2}{*}{ Not known } & \multirow{2}{*}{$\begin{array}{l}\text { Ingestion } \\
\text { Inhalation }\end{array}$} \\
\hline & Cra c 8 & 23\% (31 subjects) & & \\
\hline
\end{tabular}

SPT - skin prick test, FC - Food challenge

over $95 \%$ among all currently analyzed prawns, crabs and lobsters. For example $75 \%$ of shrimp allergic patients elicited immunological IgE cross-reactivity to crab tropomyosin Por p 1 (19).

In contrast, there is very limited information about tropomyosin among the mollusk group. The major allergens from various mollusk species such as abalone, mussel, oysters, squid and cockle share rather low amino acid homology between 65-99\%. This can result in limited clinical cross-reactivity of patients as demonstrated in a study where $54 \%$ of the recruited patients with anaphylaxis to crustaceans were tolerant to mollusks (20). Sensitization rates to recently identified allergens including triose phosphate and troponin c are still lacking.

\section{CLINICAL RELEVANCE, DIAGNOSIS AND MANAGEMENT}

\section{Clinical relevance and clinical pattern}

Due to different routes and exposure to different allergen quantities, degree of shellfish processing and physicochemical properties of the involved allergenic shellfish proteins, three distinct developments (Table 5) can prompt lgE-mediated sensitizations and clinical symptoms of mainly immediate Type I hypersensitivity:

A) Early and presumably gastro-intestinal sensitization to rather stable shellfish allergens (e.g. tropomyosin and arginine kinase) in highly atopic individuals are the basis of subsequent 
severe systemic reactions after ingestion of small amounts of (processed) shellfish products. IgE-sensitizations (e.g to structural protein tropomyosin and enzyme arginine kinase) could also evolve from exposure to different crustacean or mollusk species (e.g. prawn and lobster or abalone and squid) with various degrees of crossreactivity, prompting occasionally severe clinical symptoms after ingestion of shellfish products. These reactions have mainly been described in adults but also reported for children.

B) Exposure to inhaled shellfish allergens is frequently reported in atopic individuals working in the seafood processing industry. Sensitizing allergens have to be heat stable (e.g. tropomyosin and arginine kinase) to endure the heat processing. Cross-reactivity among members of the crustacean group is more common than among mollusks. Reactions to inhaled shellfish allergens subsequent to ingestion sensitization have also been reported.

C) Skin exposure to heated as well as unprocessed shellfish induce IgE-mediated sensitization to shellfish allergens with subsequent inhalant and ingestion allergies in exposed individuals.

\section{CLINICAL MANAGEMENT}

Specific questions, interpretation of sensitization tests (e.g. SPT, IgE) and optional food challenges help to establish the diagnosis of shellfish allergy. Following work-up (Fig. 4) might facilitate correct diagnosis.

\section{Case history}

Previous reaction(s) to crustacean or mollusk, symptoms, affected organ system(s), onset and course (progression), shellfish containing food items (highly-, mildly-, non-processed grade), co-factors (exercise, NSAID, alcohol etc.), previous reactions after shellfish allergen sources, additional (allergic) features, e.g.house dust-mite or insect (e.g cockroach, moth) allergy, reactions to seafood poisoning (e.g. histamine from Scombroid poisoning (fish) or marine biotoxins from filter feeders (mussels), additional atopic diseases, i.e. atopic eczema, asthma.

\section{Skin prick test (SPT)}

- Commercial shellfish extracts (reasonable results in case of highlyabundant allergens (e.g tropomyosin), but limited value due to false negative responses in case of heat sensitive allergens (e.g. MLC) or weak- or non-cross-reactive allergens (e.g. arginine kinase) or commercial extracts do not represent the specific shellfish species consumed by the patient (e.g. Southern hemisphere).

- Prick-prick test with offending shellfish product (in case of severe anaphylactic reaction, preferably titrated testing with diluted shellfish product; primary lgE-testing before SPT; potentially false negative, depending on the abundance and stability of the shellfish allergen in question (e.g. MLC).

\section{IgE-Testing}

\section{Allergen-specific IgE}

- Shellfish extract (potentially false negative or low titers in case of low abundant allergens or extract do not represent the specific shellfish species consumed by the patient (e.g. Southern hemisphere)

- Purified allergen (tropomyosin) from shrimps, house dust-mite and anisakis are available on the allergen microchip (ISAC, Thermo Fisher Scientific) and used to quantify allergen-specific lgE

Interpretation of SPT and IgE (sensitization tests) outcomes see also Fig. 4 and Table 5. For examples of currently available slgE and SPT kits, see Table 6.

Results only clinically relevant in case of corresponding symptoms after shellfish ingestion or contact via inhalation or skin.

\section{Challenge tests}

(in case of doubtful relationship between shellfish ingestion and allergic reaction):

Oral double-blind, placebo-controlled food challenge in patients without moderate or severe anaphylactic reactions:

1. Preparing meal with whole seafood extract or the ground boiled of the offending seafood species (masked in fruit juice, chocolate or vanilla ice cream, or in a burger)

2. Accumulated doses of up to $24 \mathrm{~g}$ of seafood (e.g. 1-2 large prawns)

\section{Clinical management}

\section{Advices and avoidance}

- Strict avoidance of all shellfish containing products, even with small amounts, regardless of the grade of shellfish processing. 


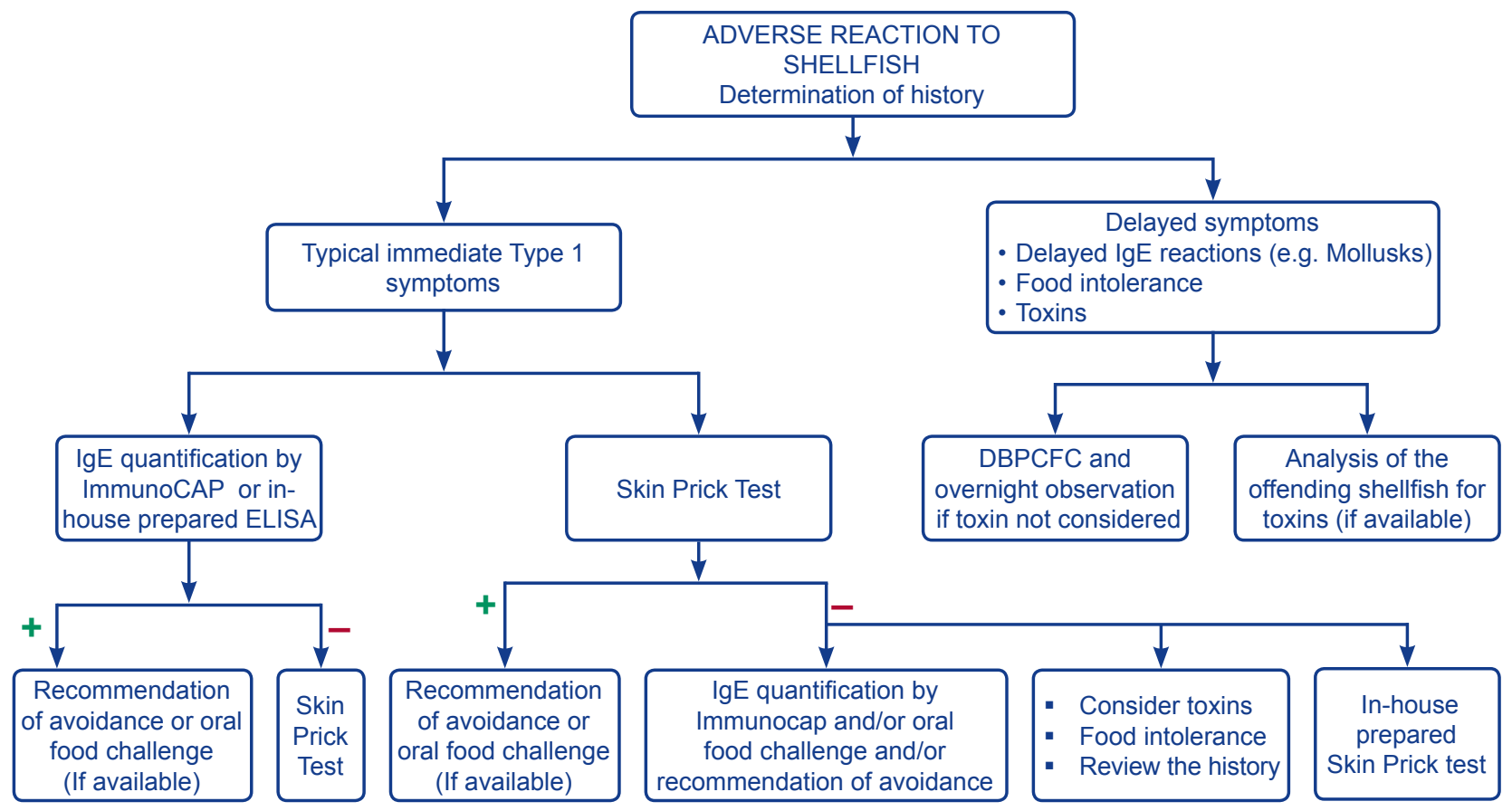

\section{Figure 4}

Diagnostic algorithm for shellfish allergy.

- Avoidance of inhalation of shellfish containing protein vapours

- Avoidance of touching or handling shellfish

Pharmacotherapy (emergency kit)

- Due to the risk of severe reactions after unintentional ingestion of shellfish containing products, emergency medication for selfadministered drug application required

- Symptomatic treatment is required

- Emergency medication optional (not mandatory)

\section{Allergen-specific immunotherapy}

At present commercially available products for allergen-specific immunotherapy of shellfish protein allergy are not available.

\section{CLINICAL CASES}

\section{Case 1 (published (21))}

History: 19-year-old Chinese male, developed hives, circumoral swelling and systemic symptoms - faintness, vomiting, diarrhea and abdominal cramp after running. He was bought into the Emergency Department (EMD). He had consumed a homecooked meal containing prawns prior to exercise. The patient also had rashes when ingesting clams.

SPT: Negative to extensive list of foods, including wheat, prawn but positive to Der p, Der f, Blo t

Diagnosis: Food-dependent exercise-induced gastrointestinal reactions and anaphylaxis to shellfish allergens

Recommendation: Oral administration of chlorpheniramine

\section{Case 2 (published (22))}

History: 22-months old Latin-American male with history of milk allergy, reactive airway disease, and eczema and no history of allergic rhinitis symptoms. On ingestion of one shrimp, there was immediate swelling of eyes and hives on his face without difficulty in breathing. Symptoms subsided within 24 hours on administration of diphenhydramine.

In-vitro testing: Serum-specific IgE showed levels of $12.6 \mathrm{kU} / \mathrm{L}$ to shrimp, $12.3 \mathrm{kU} / \mathrm{L}$ to milk, $0.55 \mathrm{kU} / \mathrm{L}$ 


\section{Table 5}

Features and clinical pattern of three different developments of shellfish allergy

\begin{tabular}{|c|c|c|c|}
\hline & Ingestion & Inhalation & Contact \\
\hline $\begin{array}{l}\text { Routes of } \\
\text { sensitiza- } \\
\text { tion } \\
\text { (IgE specific } \\
\text { response) }\end{array}$ & $\begin{array}{l}\text { 1. Gastro-intestinal uptake and } \\
\text { subsequent IgE sensitization to } \\
\text { stable shellfish allergens } \\
\text { 2. Gastro-intestinal uptake of shell- } \\
\text { fish allergens and subsequent IgE } \\
\text { cross-reactivity to shellish or fish } \\
\text { parasite allergens }\end{array}$ & $\begin{array}{l}\text { 1. Inhalation of air-borne par- } \\
\text { ticulate shellfish matter and } \\
\text { subsequent lgE sensitization } \\
\text { to allergens tropomyosin and } \\
\text { arginine kinase } \\
\text { 2. Ingestion-induced IgE sensitiza- } \\
\text { tion and subsequent inhalation } \\
\text { related cross-reactivity to dust } \\
\text { mite or insect tropomyosin }\end{array}$ & $\begin{array}{l}\text { 1. Primary uptake and IgE sensi- } \\
\text { tization to shellfish allergens } \\
\text { through IgE receptors on epi- } \\
\text { dermal Langerhans' (dendritic) } \\
\text { cells } \\
\text { 2. IgE sensitization to shellfish pro- } \\
\text { teins via gastro-intestinal or in- } \\
\text { halational route and subsequent } \\
\text { IgE reactivity on skin contact }\end{array}$ \\
\hline $\begin{array}{l}\text { Affected } \\
\text { age group }\end{array}$ & children/adolescents/ adults & $\begin{array}{l}\text { adolescents / seafood processing } \\
\text { workers/restaurant workers }\end{array}$ & $\begin{array}{l}\text { children/ adolescents/ adults/ } \\
\text { seafood processing workers/ } \\
\text { restaurant workers }\end{array}$ \\
\hline $\begin{array}{l}\text { Shellfish } \\
\text { allergens } \\
\text { involved }\end{array}$ & $\begin{array}{l}\text { TM (Pen m 1), AK (Pen m 2), MLC } \\
\text { (Pen m 3), SCP (Pen m 4), TnC (Pen } \\
\text { m 6), TIM (Pen m 8) }\end{array}$ & Tropomyosin, Arginine kinase & Not known \\
\hline $\begin{array}{l}\text { Allergen } \\
\text { abundance }\end{array}$ & $\begin{array}{l}\text { High in fresh and cooked meat and } \\
\text { related products }\end{array}$ & $\begin{array}{l}\text { Moderate to high in aerosol aller- } \\
\text { gen content of Pen } \mathrm{m} 1 \text { and Pen } \\
\mathrm{m} 2 \text { near cooking stations }\end{array}$ & Not known \\
\hline $\begin{array}{l}\text { Thermal } \\
\text { stability }\end{array}$ & Very high stability & High & High \\
\hline Prevalence & $\begin{array}{l}2 \% \text { adults } \\
0.9 \% \text { children }\end{array}$ & $\begin{array}{l}4-36 \% \text { among shellfish process } \\
\text { workers }\end{array}$ & $\begin{array}{l}65 \% \text { among shrimp workers (irri- } \\
\text { tant of allergic origin) }\end{array}$ \\
\hline Elicitors & $\begin{array}{l}\text { Fresh or cooked shellfish meat. } \\
\text { Processed foods containing shell- } \\
\text { fish }\end{array}$ & $\begin{array}{l}\text { Air-borne shellfish bio-matter and } \\
\text { cooking vapours }\end{array}$ & $\begin{array}{l}\text { Wet aerosols, splash on hands } \\
\text { and face }\end{array}$ \\
\hline Symptoms & $\begin{array}{l}\text { Generalised reactions (anaphylax- } \\
\text { is), cutaneous (urticaria, angiode- } \\
\text { ma, atopic dermatitis), gastroin- } \\
\text { testinal (pain, nausea, diarrhoea, } \\
\text { vomiting), oral allergy syndrome }\end{array}$ & $\begin{array}{l}\text { Upper and lower respiratory tract } \\
\text { symptoms: asthma. Ocular-nasal } \\
\text { symptoms: rhinitis, conjunctivitis }\end{array}$ & $\begin{array}{l}\text { Contact dermatitis, urticaria, } \\
\text { eczema }\end{array}$ \\
\hline $\begin{array}{l}\text { Medical } \\
\text { diagnosis }\end{array}$ & $\begin{array}{l}\text { Moderate to severe food allergic } \\
\text { reaction to shellfish proteins }\end{array}$ & $\begin{array}{l}\text { Moderate to severe respiratory } \\
\text { symptoms which may lead to } \\
\text { ingestion induced food allergy to } \\
\text { shellfish proteins }\end{array}$ & $\begin{array}{l}\text { Contact dermatitis and urticaria } \\
\text { due to primary sensitization to } \\
\text { shellfish proteins }\end{array}$ \\
\hline $\begin{array}{l}\text { Type of } \\
\text { food allergy }\end{array}$ & Type I - immediate onset & Type I - immediate onset & Type IV - delayed onset \\
\hline $\begin{array}{l}\text { Avoidance/ } \\
\text { Technical } \\
\text { solution }\end{array}$ & $\begin{array}{l}\text { No oral intake of crustacean or } \\
\text { mollusk products, even small } \\
\text { amounts }\end{array}$ & $\begin{array}{l}\text { Protection of nose and mouth } \\
\text { using appropriate facemask, good } \\
\text { ventilation in shellfish processing } \\
\text { workplace, minimize aerosoliza- } \\
\text { tion of shellfish proteins }\end{array}$ & $\begin{array}{l}\text { Use of hand gloves and facemask } \\
\text { for protection }\end{array}$ \\
\hline $\begin{array}{l}\text { Product } \\
\text { declara- } \\
\text { tion }\end{array}$ & $\begin{array}{l}\text { Mandatory on each shellfish } \\
\text { containing protein. Separate for } \\
\text { crustaceans and mollusks (EU } \\
\text { legislation) }\end{array}$ & - & - \\
\hline
\end{tabular}


Table 6

Currently available in vitro and skin prick tests for diagnosis of crustacean or mollusk allergy

\begin{tabular}{|c|c|c|c|}
\hline \multirow{2}{*}{ Group } & \multirow{2}{*}{ Sub-group } & In vitro tests & Skin Prick Tests \\
\hline & & ImmunoCAP (test code) & SPT (test code) \\
\hline \multirow[t]{3}{*}{ Crustaceans } & $\begin{array}{l}\text { Prawns, } \\
\text { shrimps }\end{array}$ & $\begin{array}{l}\text { Shrimps (f24) } \\
\text { Black tiger prawn (Penaeus monodon) } \\
\text { Northern shrimp (Pandalus borealis) } \\
\text { Velvet prawn (Metapenaeopsisbarbata) } \\
\text { Shiba shrimp (Metapenaeusjoyneri) }\end{array}$ & $\begin{array}{l}\text { Shrimp (6.89) ALK-Abello, Soluprick } \\
\text { (Pandalus borealis) } \\
\text { Shrimp (SHRI) ALK-Abello } \\
\text { (Penaeus spp.) } \\
\text { Shrimp (120) Stallergenes }\end{array}$ \\
\hline & Crabs & $\begin{array}{l}\text { Crab (f23) } \\
\text { Edible crab (Cancer pagurus) }\end{array}$ & $\begin{array}{l}\text { Crab (6.9) ALK-Abello, Soluprick } \\
\text { (Cancer pagurus) } \\
\text { Crab (CRAB) ALK-Abello } \\
\text { (Paralithodescamtschaticus) }\end{array}$ \\
\hline & Lobsters & $\begin{array}{l}\text { European Lobster (f80) (Homarusgammarus) } \\
\text { Spiny Lobster (f304) (Panulirus vulgaris) } \\
\text { Crayfish (f320) (Astacusastacus) }\end{array}$ & $\begin{array}{l}\text { Lobster (LOBS) ALK-Abello } \\
\text { (Panulirus spp.) } \\
\text { Spiny lobster (131) Stallergenes }\end{array}$ \\
\hline \multirow[t]{3}{*}{ Mollusks } & Bivalves & $\begin{array}{l}\text { Blue mussel (f37) (Mytilusedulis) } \\
\text { Oyster (f290) (Ostreaedulis) } \\
\text { Clam (f207) (Ruditapes spp.) } \\
\text { Scallop (f338) (Pecten spp.) }\end{array}$ & $\begin{array}{l}\text { Oyster (OYST), ALK-Abello } \\
\text { Mussel (139), Stallergenes } \\
\text { Oyster (131), Stallergenes }\end{array}$ \\
\hline & Gastropods & $\begin{array}{l}\text { Abalone (f346) (Haliotis spp.) } \\
\text { Snail (f314) (Helix aspersa) }\end{array}$ & \\
\hline & Cephalopods & $\begin{array}{l}\text { Squid (f258) (Loligo vulgaris, Loligoedulis) } \\
\text { Pacific squid (f58) (Todarodespacificus) } \\
\text { Octopus (f59) (Octopus vulgaris) }\end{array}$ & \\
\hline $\begin{array}{l}\text { Allergen } \\
\text { components } \\
\text { or Allergen } \\
\text { mix }\end{array}$ & Shrimp & Brown Shrimp (f351) (Penaeus aztecus) & $\begin{array}{l}\text { Shellfish, mix (MISH) (crab, shrimp, } \\
\text { lobster, oyster) ALK-Abello } \\
\text { Shellfish Mix } 4 \text { (SHM4) (crab, clam, } \\
\text { lobster, shrimp) }\end{array}$ \\
\hline
\end{tabular}

to egg white, $0.72 \mathrm{kU} / \mathrm{L}$ to wheat, $0.41 \mathrm{kU} / \mathrm{L}$ to soybean, $0.61 \mathrm{kU} / \mathrm{L}$ to peanut, and $<0.35 \mathrm{kU} / \mathrm{L}$ to codfish.

Recommendations: Strict avoidance of shrimp and other shellfish in his diet. Autoinjector epinephrine was prescribed. No further episodes of angioedema or hives were observed.

\section{References}

1. Chokshi NY, Maskatia Z, Miller S, Guffey D, Minard CG, Davis CM. Risk factors in pediatric shrimp allergy. Allergy Asthma Proc 2015;36:65-71.

2. Yu HL, Cao MJ, Cai QF, Weng WY, Su WJ, Liu GM. Effects of different processing methods on digestibility of Scylla paramamosain allergen (tropomyosin). Food Chem Toxicol 2011;49:791-798.
3. Kamath SD, Rahman AM, Voskamp A, Komoda T, Rolland JM, O'Hehir RE etal. Effect of heat processing on antibody reactivity to allergen variants and fragments of black tiger prawn: A comprehensive allergenomic approach. Mol Nutr Food Res 2014;58:1144-1155.

4. Koeberl M, Clarke D, Lopata AL. Next generation of food allergen quantification using mass spectrometric systems. J Proteome Res 2014;13:3499-3509.

5. Ballmer-Weber BK, Fernandez-Rivas M, Beyer K, Defernez M, Sperrin M, Mackie AR et al. How much is too much?: Threshold dose distributions for 5 food allergens. J Allergy Clin Immunol 2015;135:964-971.

6. Kamath SD, Thomassen MR, Saptarshi SR, Nguyen HM, Aasmoe L, Bang BE et al. Molecular and immunological approaches in quantifying the air-borne food allergen tropomyosin in crab processing facilities. Int J Hyg Environ Health 2014;217:740-750. 
7. Lopata AL, Jeebhay MF. Airborne seafood allergens as a cause of occupational allergy and asthma. Curr Allergy Asthma Rep 2013;13:288-297.

8. Nieuwenhuizen NE, Lopata AL. Anisakis--a food-borne parasite that triggers allergic host defences. Int $J$ Parasitol 2013;43:1047-1057.

9. Shanti KN, Martin BM, Nagpal S, Metcalfe DD, Rao PVS. Identification of tropomyosin as the major shrimp allergen and characterization of its IgE-binding epitopes. J Immunol 1993;151:5354-5363.

10. Yu CJ, Lin YF, Chiang BL, Chow LP. Proteomics and immunological analysis of a novel shrimp allergen, Pen m 2. J Immunol 2003;170:445-453.

11. Ayuso R, Grishina G, Bardina L, Carrillo T, Blanco C, Ibanez MD et al. Myosin light chain is a novel shrimp allergen, Lit v 3. J Allergy Clin Immunol 2008;122:795802.

12. Shiomi K, Sato Y, Hamamoto S, Mita H, Shimakura K. Sarcoplasmic calcium-binding protein: identification as a new allergen of the black tiger shrimp Penaeus monodon. Int Arch Allergy Immunol 2008;146:91-98.

13. Lopata AL, O'Hehir RE, Lehrer SB. Shellfish allergy. Clin Exp Allergy 2010;40:850-858.

14. Rona RJ, Keil T, Summers C, Gislason D, Zuidmeer L, Sodergren $\mathrm{E}$ et al. The prevalence of food allergy: a meta-analysis. J Allergy Clin Immunol 2007;120:638646.

15. Osborne NJ, Koplin JJ, Martin PE, Gurrin LC, Lowe AJ, Matheson $M C$ et al. Prevalence of challenge-proven IgE-mediated food allergy using population-based sampling and predetermined challenge criteria in infants. J Allergy Clin Immunol 2011;127:668-676.e12.

16. Sicherer SH, Munoz-Furlong A, Sampson HA. Prevalence of seafood allergy in the United States determined by a random telephone survey. J Allergy Clin Immunol 2004;114:159-165.

17. Nwaru BI, Hickstein L, Panesar SS, Roberts G, Muraro A, Sheikh A. Prevalence of common food allergies in Europe: a systematic review and metaanalysis. Allergy 2014;69:992-1007.

18. Pascal M, Grishina G, Yang AC, Sanchez-Garcia S, Lin J, Towle D, et al. Molecular Diagnosis of Shrimp Allergy: Efficiency of Several Allergens to Predict Clinical Reactivity. J Allergy Clin Immunol Pract 2015;3:521529.e10.

19. Abramovitch JB, Kamath S, Varese N, Zubrinich C, Lopata AL, O'Hehir RE et al. IgE Reactivity of Blue Swimmer Crab Portunus pelagicus Tropomyosin, Por p 1, and Other Allergens; Cross-Reactivity with Black Tiger Prawn and Effects of Heating. PLoS One 2013;8: e67487.

20. Vidal C, Bartolome B, Rodriguez V, Armisen M, Linneberg A, Gonzalez-Quintela A. Sensitization pattern of crustacean-allergic individuals can indicate allergy to molluscs. Allergy 2015;70:1493-1496.

21. Teo SL, Gerez IF, Ang EY, Shek LP. Food-dependent exercise-induced anaphylaxis - a review of 5 cases. Ann Acad Med Singapore 2009;38:905-909.

22. Kandyil RM, Davis CM. Shellfish allergy in children. Pediatr Allergy Immunol 2009;20:408-414. 



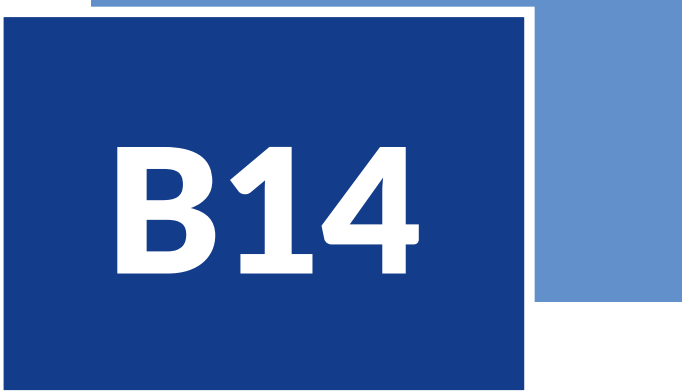

\author{
Marianne van Hage, Tilo Biedermann, \\ Thomas A.E. Platts-Mills
}

\title{
THE ALLERGEN SOURCES
}

Meat derived from domesticated mammals has been an important part of the human diet for at least ten thousand years. The animal tissue sold and eaten as meat includes: blood, fat, innards and tendons as well as muscles. Sensitisation to serum proteins and milk proteins from the same animal is inevitable due to cross-reactivity. Milk is a relevant allergen source only for goats, sheep, and cows in the USA and Europe, but it is important to remember that camels and other animals are milked in some parts of the world. Further, although there is a limited range of animals that are sold commercially in the west, a much larger variety of wild animals may be hunted and eaten in rural communities worldwide.

Farm animals provide a major part of the diet in western societies and in many other parts of the world. These animals are prized for their meat and organs (Fig. 1). In addition, there are hundreds of forms of processed meat, including sausages, salami, bacon, etc. Meat also incorporates significant quantities of protein derived from serum, which includes many proteins that are recognized as allergens in cow's milk. Other products derived from these mammals

Farm animals provide a major part of the diet in many parts of the world

Sensitization to meat can be acquired through different routes (inhaled, oral, skin)

New forms of allergic reactions to meat have been recognized (cat-pork and red meat)

IgE assays including meat allergen sources and components will help identifying the patients

The only effective treatment is avoidance of relevant meat sources 


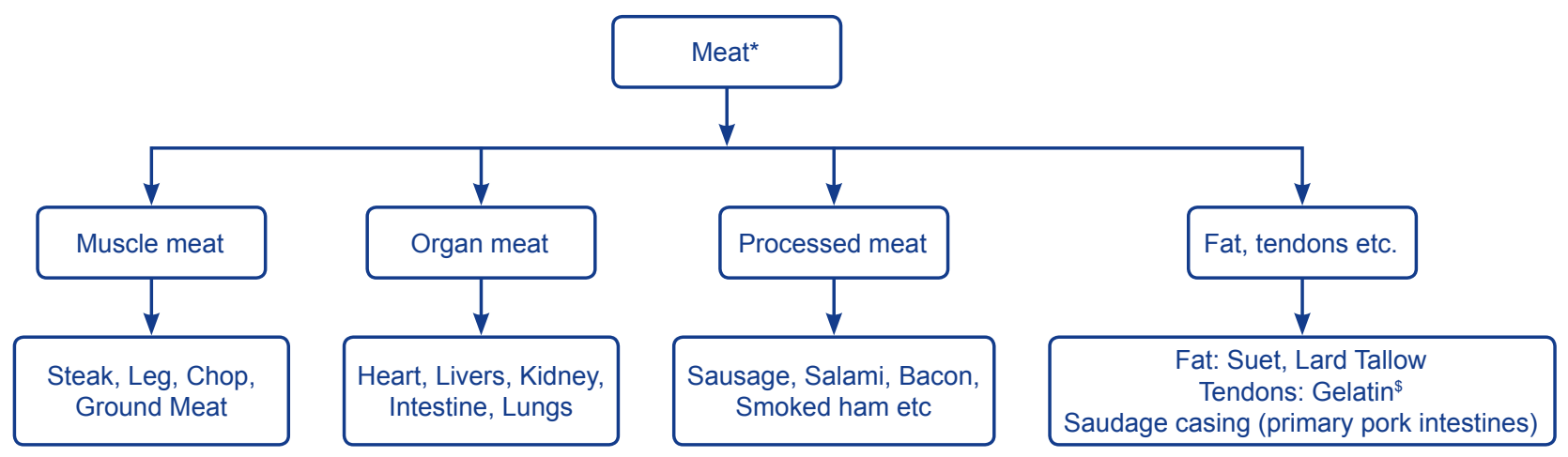

\section{Figure 1}

*Any of these products can include serum proteins including proteins present in cow's milk (e.g., albumins, globulins and the mammalian oligosaccharide galactose-alpha-1,3-galactose (alpha-gal)).

$\$$ Gelatin is made from tendons, cartilage or skin and is a protein with varying quantities of glycosylation, used widely in food.

include different forms of fat, paté, as well as gelatin, which is derived from tendons, cartilage, or skin.

\section{MAJOR AND RELEVANT MINOR ALLERGENIC MOLECULES}

Initially the identification of meat derived allergens focused on protein antigens recognized by patients who reported allergic reactions that occurred rapidly after exposure. Most of these cases presented in childhood and many of the allergens were speciesspecific proteins $(1,2)$. However, it was already clear that some mammalian proteins showed crossreactivity between species, and this included both immunoglobulins and albumins $(3,4)$ (see also chapter A04). The major allergens from beef, Bos taurus, are serum albumin (Bos d 6) and immunoglobulin IgG (Bos d 7) (Table 1) (1, 3-5). Beef-allergic children have been reported to react to bovine serum albumin on SPT, but only some of them do so during challenge (1). Myoglobin has been identified as a clinically relevant and heat-resistant allergen in one case report (6). Actin was shown to bind IgE of meat-allergic patients, but skin prick tests were negative (4). More studies are needed to evaluate the importance of these allergens.

Despite the truly enormous quantities of meat eaten worldwide there are only a limited number of allergens defined (Table 1). Indeed despite the extensive consumption of meat from sheep, goats
Table 1

Major and minor meat allergenic molecules

\begin{tabular}{|c|c|c|c|}
\hline Animals & Milk & $\begin{array}{l}\text { Allergen } \\
\text { molecules* \# }\end{array}$ & $\begin{array}{l}\text { Alpha-gal } \\
\text { Reactions to } \\
\text { meat intake }\end{array}$ \\
\hline $\begin{array}{l}\text { Bos domesticus } \\
\text { (cow) }\end{array}$ & +++ & $\begin{array}{l}\text { eleven (9) } \\
\text { Bos d 2-12 }\end{array}$ & Yes \\
\hline $\begin{array}{l}\text { Sus domesticus } \\
\text { (pig) }\end{array}$ & NA & $\begin{array}{l}\text { albumin (1) } \\
\text { Sus s }\end{array}$ & Yes \\
\hline $\begin{array}{l}\text { Capra Aegagrus } \\
\text { (goat) }\end{array}$ & ++ & 0 & Yes \\
\hline $\begin{array}{l}\text { Ovis Aries } \\
\text { (sheep) }\end{array}$ & ++ & 0 & Yes \\
\hline $\begin{array}{l}\text { Odocoileus } \\
\text { virginianus (deer) }\end{array}$ & NA & 0 & Yes \\
\hline Equus caballus & NA & $\begin{array}{c}\text { four (1) } \\
\text { Equ c } 1-4\end{array}$ & Yes \\
\hline $\begin{array}{l}\text { Oryctolagus } \\
\text { cuniculus (rabbit) }\end{array}$ & NA & $\begin{array}{c}\text { three (0) } \\
\text { Ory c 1, 3-4 }\end{array}$ & $?$ \\
\hline $\begin{array}{l}\text { Cavia porcellus } \\
\text { (guinea pig) }\end{array}$ & NA & $\begin{array}{c}\text { five (0) } \\
\text { Cav p 1-4, } 6\end{array}$ & $?$ \\
\hline
\end{tabular}

NA=not applicable

*IUIS Allergen Nomenclature Sub-Committee

\#Number in brackets indicates the number of allergens defined as food allergen. 
and deer as well as horses, there are no relevant allergens included in the IUIS database from these sources. Even for rabbit and guinea-pig, where there are 3 and 5 allergens respectively in the database, all of the defined allergens were recognized on the basis of inhalant symptoms. Single case reports show that serum albumin is responsible for clinical symptoms to rabbit or horsemeat $(7,8)$. Thus the only species with a significant number of food allergens recognized is the cow where nine of the allergenic proteins are recognized as food allergens. However, even in that case, most of the allergens were initially identified as allergens in cow's milk. Indeed, the majority of reported reactions to beef in childhood have occurred in cow's milk allergic children $(1,5,9)$. The same can occur with reactions to goat or sheep meat, with goat's milk or sheep milk as the primary sensitizer but this is much less common. It is difficult to access the world literature on reactions to meat, because a large proportion of the populations eating goat and sheep have only limited medical care. However, it is likely that the main meat proteins taken orally have very little allergenicity in man (10).

\section{SENSITIZATION TO INDIVIDUAL MOLECULES AND ITS CLINICAL RELEVANCE}

In the last fifteen years, two new forms of allergic reactions to meat have been recognized. In both of these syndromes, presentation is most often in adult life and in both cases the relevant antigens are characterized by extensive cross-reactivity between different mammals. First, it was recognized that some patients who had allergic reactions to pork, were reacting because of pre-existing IgE antibodies to cat albumin that cross-react with pork albumin (11, 12). Secondly, an enigmatic allergic reaction to the monoclonal antibody cetuximab led to the recognition that a surprisingly large number of individuals in the South East of the United States had IgE antibodies to the oligosaccharide galactose alpha-1, 3-galactose (alpha-gal) (13). This oligosaccharide is a blood group substance of the non-primate mammals and is present on all forms of tissue including red meat $(14,15)$, organs such as kidney (16), gelatin (17) and cat IgA (18). These patients also present with a novel form of delayed food allergy where in general they feel no symptoms for 2-6 hours after eating meat (16, 19-22).

\section{Table 2}

Routes of Sensitization for Allergens Related to Allergic Reactions to Meat

\begin{tabular}{|ll|}
\hline Inhaled: & $\begin{array}{l}\text { Cat albumins related to systemic rapid } \\
\text { reactions to pork (cat pork syndrome) }\end{array}$ \\
\hline Oral: & $\begin{array}{l}\text { Cow's milk allergens related to allergic } \\
\text { reactions to beef }\end{array}$ \\
\hline Skin: & $\begin{array}{l}\text { Tick bites lead to alpha-gal sensitization, } \\
\text { and ingestion of red meat may later trigger } \\
\text { reactions }\end{array}$ \\
\hline
\end{tabular}

\section{Routes of sensitization}

Prior to the year 2000, it was generally assumed that sensitization to food antigens was induced by oral exposure. However, we now have at least two alternative routes of exposure (Table 2).

Sensitization to cat albumin occurs predominantly in patients who own cats and is assumed to occur by inhaling dander particles carrying this protein. Thus the route of sensitization to the crossreacting pork albumin reflects a sensitization that was initially established by inhaled exposure. The second alternative route is through the skin. For the oligosaccharide alpha-gal, the only established route for sensitization is by tick bites. Strikingly there are three different tick species that have been implicated: Amblyomma americanum in the USA; Ixodes holocyclus in Australia; and Ixodes ricinus in Europe. Moreover, Ixodes ricinus has been shown to contain alpha-gal (23). While it is certainly possible that other parasites can induce this response there is no clear evidence for that at present. On the other hand, there is good evidence that eating meat carrying this oligosaccharide does not induce IgE-mediated sensitization in children or adults to alpha-gal since these lgE antibodies are not present in the serum of children raised in arctic areas where the ticks are not present (24).

\section{CLINICAL RELEVANCE, DIGANOSIS AND MANAGEMENT}

The only effective form of treatment for allergic reactions to meat is to avoid the relevant source or sources. There are no consistent studies using immunotherapy for meat allergy, nor are there studies using anti-lgE as therapy. Thus, the main 
parts of management are accurate diagnosis and education. Cofactors (for example, alcohol, ASA, physical exercise) can increase intestinal absorption and substitute for higher sensitivity to red meat (16).

\section{Clinical diagnosis}

Diagnosis is based on i) history, ii) skin test and/or IgE antibody assays, and iii) challenge protocols (Table 3 ).

The diagnosis of alpha-gal sensitivity may be obvious from the history and skin tests. However, it may be useful to have a panel of serum IgE assays to establish the diagnosis of meat reactions. This will need to include IgE to alpha-gal, beef and pork, as well as IgE antibodies to milk, cat and porcine albumin, and chicken, turkey and cod can be used as negative controls.

\section{Clinical management}

Discussion of avoidance may require written protocols, as well as discussion of the many forms in which proteins derived from meat are eaten. With meat sensitization in childhood it may be necessary to go dairy-free to establish a symptom-free condition. After that it should be possible to carry out challenge tests if necessary and to progressively modify details of the diet. For pork-cat syndrome it is usually sufficient to avoid pork and pork products. In some cases, the cross-reactivity with beef albumin is strong enough to give symptoms with beef products. As albumins are thermolabile proteins, well-cooked meat is often tolerated whereas ham and sausage are not. Challenges may be useful to assess the tolerance of well cooked beef in children.

Full avoidance of all products containing alpha-gal is not easy because this includes all products derived from mammals. However, most cases require a significant dose of red meat (i.e., $\geq 20 \mathrm{~g}$ of meat) to cause reactions, and over $80 \%$ of cases can tolerate milk and milk products. In many cases, the patients have already recognized what they can tolerate before they present to physicians. As in other IgE-

\section{Table 3}

Diagnosis of Meat Reactions
History
SPT
IgE Challenge

Primary meat sensitivity in childhood

Immediate reactions to meat often with pre- existing sensitivity to cow's milk.

Milk and relevant meat.

Milk and meat.
Graded meat challenges in cases where doubt remains.

Pork-Cat Syndrome

Reactions to pork within one hour In some case with additional reactions to beef and in most cases pre-existing sensitization to cats.

$\begin{array}{ccc}\text { Pork, } & \text { Graded challenge tests may } \\ \text { cat, beef, } & \text { be necessary in some cases } \\ \text { porcine } & \text { and cat } & \text { where diagnosis remains } \\ \text { albumin } & \text { unclear. }\end{array}$

Pork, porcine and cat albumin unclear.

Delayed Anaphylaxis to Red Meat or the Alpha-gal syndrome

Beef, lamb, pork, and cow's milk negative or 2-4 $\mathrm{mm}$ in diameter. Intradermal skin tests give much clearer results.

Urticaria and/or anaphylaxis occurring 3-6 hours after eating beef,
Gelatin-derived colloids can be used as alternative where test solutions for intracutaneous testing with meat-extracts is not available, some used cetuximab however with less sensitivity.
Challenge tests have been carried out in experimental protocols.
Alpha-gal, beef, lamb, pork, and be necessary in some cases where diagnosis remains unclear, co-factors may need to be included to elucidate clinically overt reactions. 
mediated food allergies, mastocytosis increases the risk for severe reactions also for alpha-gal allergic individuals. Thus, measurement of serum tryptase in the investigation of red meat allergy is recommended.

\section{CLINICAL CASES}

\section{Case 1}

Clinical history: Patient, 56-year-old female, who previously had ingested red meat without experiencing symptoms, visited in early autumn the ER due to urticaria. The case history revealed that she had eaten a sausage five hours earlier. In November the same year she had an anaphylactic reaction six hours after eating a hamburger. She was referred to the Allergy Clinic for investigation of food allergy. The examination revealed that she had obtained several tick bites during the summer and noted prolonged redness and itching at the site of the tick bite.

In vitro testing: IgE negative to soybean, peanut, cod and wheat. IgE against beef was $14 \mathrm{kU} / \mathrm{L}$, pork 7.6 $\mathrm{kU} / \mathrm{L}$, milk $1.7 \mathrm{kU} / \mathrm{L}$ and alpha-gal $80 \mathrm{kU} / \mathrm{L}$.

In vivo tests: SPT $3+$ against birch. SPT negative to beef, lamb, pork and cow's milk.

Diagnosis: Red meat allergy.

Recommendation: She was recommended to avoid red meat and became symptom-free.

\section{Case 2}

Clinical history: An 18-year-old male patient describes five, mostly nocturnal, anaphylactic reactions with hives, nausea, abdominal pain and dyspnea in the last ten years with unknown trigger. In four of five episodes, allergic reactions occurred 3-5 hours after a meal containing pork meat, once with physical exercise (cycling) where cutaneous reactions occurred shortly (1h) after. The patient is otherwise healthy and has no history of atopy or other (food) allergies. No further episodes occurred since the patient had become a vegetarian. There is a history of tick bites in the patient's childhood.

In vitro testing: Specific IgE to alpha-gal was highly positive $(29.6 \mathrm{kU} / \mathrm{l})$ and negative to pork $(<0.1$ $\mathrm{kU} / \mathrm{l})$, beef $(<0.1 \mathrm{kU} / \mathrm{l})$, chicken $(<0.1 \mathrm{kU} / \mathrm{l})$ and omega-5-gliadin $(<0.1 \mathrm{kU} / \mathrm{l})$. The tryptase level was $2.13 \mu \mathrm{g} / \mathrm{l}(<11.4 \mu \mathrm{g} / \mathrm{l})$.
In vivo tests: Skin prick test was negative to beef, lamb, pork and cow's milk, but prick-to-prick test revealed positive reactions to raw and cooked pork kidney. Intradermal testing was positive to Gelafundin ${ }^{\circledR} 4 \%$ (gelatin polysuccinate) diluted 1:100.

An oral challenge was performed with cooked pork kidney $(17 \mathrm{~g})$ under careful monitoring. The patient developed urticaria approx. 3 hours after challenge and was treated with antihistamines and corticosteroids.

Diagnosis: Late-onset anaphylaxis to red meat based on IgE recognizing alpha-gal

Recommendation: The patient was informed to avoid red meat especially in combination with co-factors such as alcohol, acetylsalicylic acid or exercise as well as oral ingestion of large amounts of gelatin. Furthermore, we handed out an allergy pass listing Cetuximab and gelatin-colloid plasma expander Gelafundin ${ }^{\circledR}$.

It should be emphasised that skin prick test responses to beef, lamb, pork and cow's milk can be very small in size or negative. In adults, intradermal skin tests may give much clearer results (Table 3).

\section{References}

1. Werfel S, Cooke S, Sampson H. Clinical reactivity to beef in children allergic to cow's milk. J Allergy Clin Immunol 1997; 99:293-300.

2. Fiocchi A, Restani P, Riva E. Beef allergy in children. Nutrition 2000;16:454-457.

3. Ayuso R, Lehrer S, Lopez M, Reese G, Ibanez $M$, Esteban $M$ et al. Identification of bovine IgG as a major cross-reactive vertebrate meat allergen. Allergy 2000;55:348-354.

4. Restani P, Fiocchi $A$, Beretta B, Velonà T, Giovannini M, Galli CL. Meat allergy: III - Proteins involved and crossreactivity between different animal species. J Am Coll Nutr 1997;16:383-389.

5. Martelli A, De Chiara A, Corvo M, Restani P, Fiocchi A. Beef allergy in children with cow's milk allergy; cow's milk allergy in children with beef allergy. Ann Allergy Asthma Immunol 2002;89:38-43.

6. Fuentes $M M$, Palacios $R$, Garcés $M$, Caballero $M L$, Moneo I. Isolation and characterization of a heatresistant beef allergen: myoglobin. Allergy 2004;59: 327-331.

7. Martorell Calatayud C, Morales Rubio C, Bartolomé Zavala B, Ortega Sanchis S, Raducan I, Pélaez Hernández A. Allergy to rabbit meat after 
sensitization by inhalation. J Investig Allergol Clin Immunol 2014;24:455-456.

8. Restani P, Ballabio C, Tripodi S, Fiocchi A. Meat allergy. Curr Opin Allergy Clin Immunol 2009;9:265269.

9. Chandra RK, Puri S, Suraiya C, Cheema PS. Influence of maternal food antigen avoidance during pregnancy and lactation on incidence of atopic eczema in infants. Clin Allergy 1986;16:563-569.

10. Spitzauer S. Allergy to mammalian proteins: at the borderline between foreign and self? Int Arch Allergy Immunol 1999;120:259-269.

11. Hilger C, Kohnen M, Grigioni F, Lehners C, Hentges F. Allergic cross-reactions between cat and pig serum albumin. Study at the protein and DNA levels. Allergy 1997;52:179-87.

12. Posthumus J, James HR, Lane CJ, Matos LA, PlattsMills TA, Commins SP. Initial description of porkcat syndrome in the United States. J Allergy Clin Immunol 2013;131:923-925.

13. Chung $\mathrm{CH}$, Mirakhur B, Chan E, Le QT, Berlin J, Morse $M$ et al. Cetuximab- induced anaphylaxis and IgE specific for galactose-alpha-1,3-galactose. $N$ Engl J Med 2008;358:1109-1117.

14. Apostolovic D, Tran TA, Hamsten C, Starkhammar $M$, Cirkovic Velickovic $T$, van Hage $M$. Immunoproteomics of processed beef proteins reveal novel galactose-alpha-1, 3-galactose- containing allergens. Allergy 2014;69:1308-1315.

15. Takahashi H, Chinuki Y, Tanaka A, Morita E. Laminin gamma-1 and collagen -1 (VI) chain are galactose-alpha-1, 3-galactose-bound allergens in beef. Allergy 2014;69:199-207.

16. Fischer J, Hebsaker J, Caponetto P, Platts-Mills TA, Biedermann T. Galactose-alpha-1,3- galactose sensitization is a prerequisite for pork-kidney allergy and cofactor-related mammalian meat anaphylaxis. J Allergy Clin Immunol 2014;134:755-759.
17. Mullins RJ, James H, Platts-Mills TA, Commins S. Relationship between red meat allergy and sensitization to gelatin and galactose-alpha-1,3-galactose. J Allergy Clin Immunol 2012;129:1334-1342.

18. Grönlund H, Adédoyin J, Commins SP, Platts-Mills TA, van Hage $M$. The carbohydrate galactose-alpha-1,3galactose is a major IgE-binding epitope on cat IgA. J Allergy Clin Immunol 2009;123:1189-1191.

19. Commins SP, Satinover SM, Hosen J, Mozena J, Borish L, Lewis BD et al. Delayed anaphylaxis, angioedema, or urticaria after consumption of red meat in patients with IgE antibodies specific for galactose-alpha-1,3galactose. J Allergy Clin Immunol 2009;123:426-433.

20. Commins SP, James H, Stevens W, Pochan S, Land $M$, King $C$ et al. Delayed clinical and ex vivo response to mammalian meat in patients with IgE to galactose- alpha-1, 3-galactose. J Allergy Clin Immunol 2014;134:180-215.

21. Van Nunen SA, O'Connor KS, Clarke LR, Boyle RX, Fernando SL. An association between tick bite reactions and red meat allergy in humans. Med $J$ Aust 2009;190:510-511.

22. Hamsten C, Tran TAT, Starkhammar M, Brauner A, Commins SP, Platts-Mills TAE et al. Red meat allergy in Sweden - association with tick sensitization and B-negative blood groups. J Allergy Clin Immunol 2013;132:1431-1434.

23. Hamsten C, Starkhammar M, Tran TA, Johansson $M$, Bengtsson $U$, Ahlen $G$ et al. Identification of galactose1alpha-1,3-galactose in the gastrointestinal tract of the tick Ixodes ricinus; possible relationship with red meat allergy. Allergy 2013;68:549-552.

24. Commins S, James H, Kelly E, Pochan S, Workman L, Perzanowski $M$ et al. The relevance of tick bites to the production of IgE antibodies to the mammalian oligosaccharide galactose-a-1,3-galactose. J Allergy Clin Immunol 2011;127:1286-1293. 


\section{ALLERGY \\ TO FRUITS \\ AND VEGETABLES}

\author{
Barbara Ballmer-Weber, Karin Hoffmann- \\ Sommergruber
}

\section{THE ALLERGEN SOURCES}

Plant food, especially fruits and vegetables are part of a healthy diet and their consumption is recommended for prevention of cardiovascular and metabolic disorders. However, in predisposed individuals food allergic reactions are caused/induced upon uptake of a range of fruits and vegetables.

\section{Fruits}

While the range of allergenic fruits is broad, the majority of frequent inducers of allergic reactions belong to the Rosaceae family. Therefore, this botanical family will be described in more detail. Among those, pyrenocarps and stone fruits but also nuts (almond) are able to induce food allergic symptoms in atopic patients.

Fruits are consumed raw or processed, and both, peel and pulp contain allergens. For example the non-specific lipid transfer proteins (nsLTPs) are accumulated in the outer layer of fruits, and by removing the peel the allergen exposure can be reduced. Also, certain apple cultivars are known to be low in their allergen content for the Pathogenesisrelated $10(P R-10)$ proteins and nsLTPs, while others
Allergies to fruits and vegetables can either be due to cross sensitization with pollen allergens or are due to "true" food allergens

The majority of plant food allergens can be assigned to a restricted number of protein families

Frequently observed IgE cross reactivity does not always coincide with clinical relevance

Prick to prick testing using raw plant foods is often superior to extract based testing

Food challenges are the method of choice to rule out clinically silent IgE cross reactivity 
are expressing higher amounts of these allergens (1, 2). Unfortunately, the reduced levels of Mal d 1 do not always coincide with low levels of Mal d 3, thus an overall hypoallergenic apple is so far not available. Also postharvest treatment processes may impact on allergen levels, as storage under defined conditions has suggested for Mal d 1 and Mal d 3 levels (3).

Furthermore, fruits can be eaten in cakes, desserts, jams and jellies, as ingredients of dishes and as fruits juices either after pasteurization or without heat treatment.

In "biological cosmetics" fruit extracts are also used and may pose an unexpected risk in highly sensitized patients.

In addition, the kiwifruit will be presented as an example of a novel food, entering the European market in the late 70s of the 20th century, and thus eventually developing into a relevant allergen source, previously not anticipated as such.

In addition, citrus fruits, banana, melon, and grapes represent plant food allergen sources, affecting mostly Southern European patients/consumers.

The well-known latex fruit syndrome, comprising allergic reactions against latex products and fruits and vegetables containing cross reactive allergens will not be considered in this chapter, since this topic is covered in Section B22 by M. Raulf.

\section{Vegetables}

Also vegetables present a relevant source of allergens, with celery being an allergen food to be labelled on food products according to the EU allergen legislation (European Directive 2007/68/EC).

Celery and carrot, both belonging to the botanical Apiaceae family can be consumed raw as well as cooked, and several studies have investigated the impact on heat treatment on individual allergens, thus up- or downregulating their allergenic capacity. Especially in celery the bulb (tuber; celeriac) as well as the green parts (stalks) are eaten raw as well as cooked. In addition, celery seeds can be used as a spice as well and are offered either as "celery salt" alone or as a spice mixture to be used for various dishes. In the latter case the presence of celery derived proteins may not be that evident and may lead to unexpected reactions in predisposed individuals. Also celery seed oil is sometimes used as a food ingredient or in cosmetics.
In addition to celery and carrot also tomato and bell pepper are well known allergenic foods. In the recent past additional tomato allergens have been identified belonging to the seed storage proteins present in the seeds of the tomato fruit (4).

\section{MAJOR AND RELEVANT MINOR ALLERGENIC MOLECULES}

\section{Fruits}

Within the Rosaceae PR-10 protein family members have been identified as allergens in apple, peach, apricot, pear, raspberry, and strawberry. These proteins are major allergens in apple and peach, and are located in the pulp and skin of the fruits. In general, PR-10 proteins are labile proteins at extreme $\mathrm{pH}$ conditions and their structure is affected upon heat treatment. They are constitutively expressed in plant tissues. In addition they are upregulated upon environmental stress and pathogen attack. They are supposed to act as plant steroid carriers. PR-10 proteins in fruits are supposed to induce mild local reactions in patients. Also heat treatment of fruits (e.g. pasteurized fruit juices and jams) affects PR-10 allergenicity.

The nsLTPs are small proteins with a rigid tertiary structure formed by 4 disulfide bridges. Their function is to transport lipids across cell membranes. Allergens from this protein family are identified from apple, peach, apricot, cherry, plum, pear, raspberry, strawberry, and mulberry. They are major allergens and primarily located in the outer tissue layers (peel) of fruits. Upon pathogen attack they are upregulated and therefore classified as PR-14. In contrast to PR10 allergens they are stable proteins not affected by low $\mathrm{pH}$ environment and heat treatment. However, at neutral $\mathrm{pH}$ their resistance to heat treatment is much lower as compared at acidic $\mathrm{pH}$ (5). In general severe, generalized allergic symptoms are correlated with nsLTPs intake.

Profilins are small proteins with an ubiquitous expression throughout the plant kingdom. They are functional in various important cell-signaling pathways and bind actin. These small proteins are of intermediate to low stability when subjected to heat treatment. Sensitization to profilin is frequently observed in patients, however it often lacks clinical relevance. Allergens from the profilin family have been identified in apple, peach, pear, and strawberry. 
Pru p 1

\section{Pru p 3}
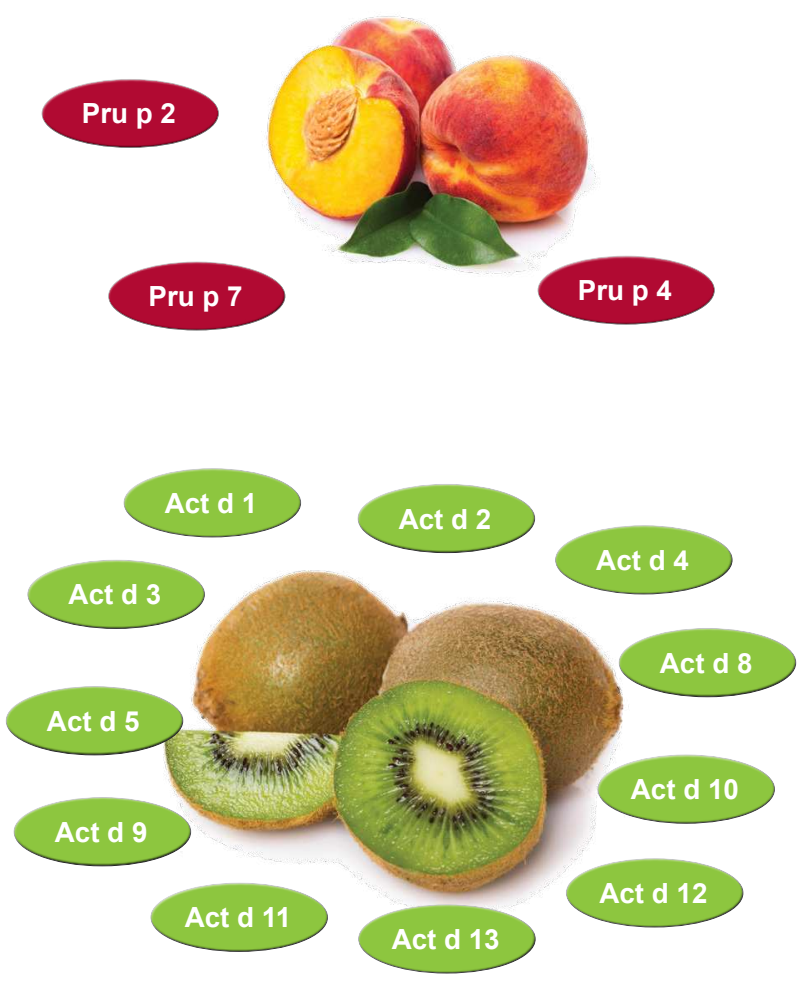

Figure 1

Peach and kiwifruit as selected examples of fruit allergen sources.

Thaumatin-like proteins share a common 3 dimensional rigid structure defined by conserved cysteine residues forming 8 disulfide bridges. These proteins are expressed in ripening fruits and are upregulated upon biotic and abiotic stress (PR-5). They are regarded as minor allergens, based on data obtained from apple, peach, and cherry.

The gibberellin-regulated protein, peamaclein, was identified from peach. This is a small protein upregulated upon biotic stress and located in the peach peel (6).

In pear another allergen, an isoflavone reductase related protein was identified, which showed allergenic activity in a small group of patients allergic to pear (7).

The green kiwifruit contains PR-10, nsLTP, profilin, and thaumatin-like proteins with allergenic activity. The cysteine protease, actinidin, enzymatically degrades seed storage proteins and is upregulated in blossoms and fruits. In kiwifruit monosensitized allergic patients it is a major allergen. In addition, a number of minor allergens have been identified such as phytocystatin, kiwellin, pectin-methylesterase and its inhibitor and a major latex-protein, which belongs to the Bet $v 1$ superfamily. $2 S$ albumins and $11 \mathrm{~S}$ globulins localized in the seeds were also characterized as allergens.

While actinidin is abundantly expressed in green kiwifruits its expression level and allergenic activity is much lower in golden kiwifruits (8) and in certain kiwifruit cultivars (8).

Banana contains profilin, nsLTP and thaumatin-like proteins with allergenic activity. In addition, beta-1,3 glucanase (PR-2) and class I chitinase (PR-3), both degrading fungal cell walls and the exoskeleton of insects, are banana allergens and contribute to the cross-reactivity with latex allergens.

In citrus fruits nsLTPs type 1 were identified, and germin-like proteins, as relevant allergens. In contrast to other fruits, profilins are regarded as major allergens in citrus fruits with clinical relevance (10).

From melon profilin, cucumisin, an alkaline serine protease, and a member of the PR-1 family are identified as allergens (Table 1 and Fig. 1).

\section{Vegetables}

In celery, the PR-10 protein is a major allergen, especially in Central Europe. Also, profilin is supposed to sensitize a relevant number of celeriac allergic patients. Less frequently, sensitization to the FADcontaining oxidase, a glycoprotein, is observed. In this case, the carbohydrate moieties of this enzyme seem to be relevant for the IgE binding capacity (11). In the recent past, nsLTPs have been identified from celery. While the nsLTP type 1 is expressed in the stalks, the nsLTP type 2 is found in the tuber (12, 13). Only limited IgE-cross-reactivity is observed between those two different proteins. Similarly to celery, the PR-10 protein is a major allergen in carrot. At least 2 isoforms of Dau c 1 seem to be responsible for sensitization and provide only partial crossreactivity. In addition, profilin has been identified as a minor allergen. Finally, the isoflavone-reductaselike protein is the most recently characterized food allergen. However, neither data on the prevalence of sensitization are available nor the clinical relevance of this allergen is known to date. 


\section{Table 1}

Allergens in Fruits

\section{Biochemical name \\ Molecular \\ mass $(\mathrm{kDa})$ \\ Allergen}

Pathogenesis related protein 10 (PR 10)

Profilin

14

3 (strawberry),

nsLTP (type 1)

Thaumatin-like protein

Rosales: Mal d 2 (apple), Pru p 2 (peach), Pru av 2 (cherry);

\begin{tabular}{|l|}
\hline Giberellin regulated protein \\
\hline $\begin{array}{l}\text { Isoflavone reductase related } \\
\text { protein }\end{array}$
\end{tabular}

Class I chitinase

Ericales: Act d 2 (green kiwifruit);

Zingiberales: Mus a 4 (banana);

\section{Sapindales: Cit I 3 (lemon), Cit $r 3$ (tangerine), Cit s 3 (sweet orange);}

: Cit 3 (lemon), Cit 3 ( Vitales: Vit $v 1$ (grape);

Beta 1,3 Glucanase

Germin like protein

23 Sapindales: Cit s 1 (sweet orange);

Alkaline serine protease

67 Cucurbitales: Cuc $\mathrm{m} 1$ (muskmelon);

Pathogenesis-related protein $1 \quad 17$ Cucurbitales: Cuc m 3 (muskmelon);

Actinidin $\quad 30 \quad$ Ericales: Act $d 1$ (green kiwifruit);

Phytocystatin $10 \quad$ Ericales: Act $d 4$ (green kiwifruit);

Kiwellin

26 Ericales: Act d 5 (green kiwifruit); Act c 5 (golden kiwifruit);

Major latex protein/ ripening related protein

17 Ericales: Act d 11 (green kiwifruit);

Cupin (11S globulin) $50 \quad$ Ericales: Act d 12 (green kiwifruit);

$2 S$ albumin

11 Ericales: Act d 13 (green kiwifruit); 


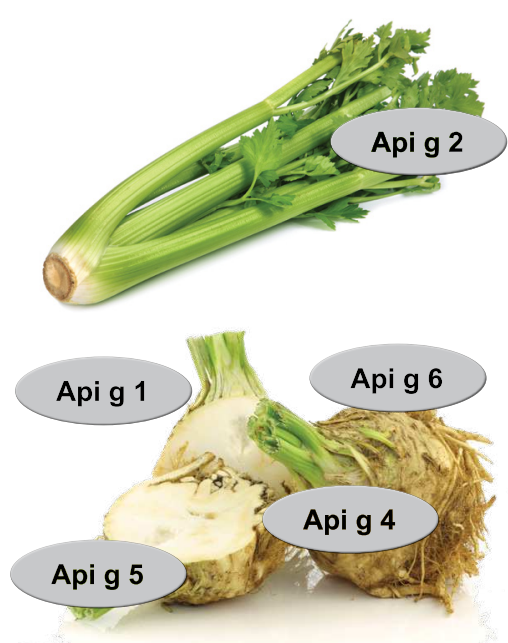

Table 2

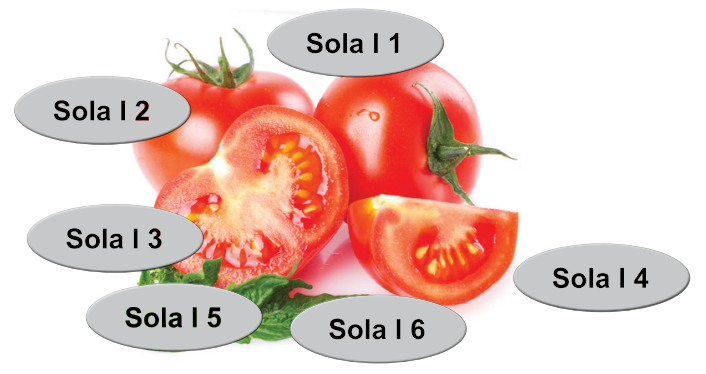

\section{Figure 2}

Celery and tomato as selected examples of vegeta-

ble allergen sources.

\begin{tabular}{|c|c|c|}
\hline Biochemical name & $\begin{array}{l}\text { Molecular } \\
\text { mass (KDa) }\end{array}$ & Allergen \\
\hline $\begin{array}{l}\text { Pathogenesis related protein } \\
\text { PR- } 10\end{array}$ & 15 & Apiales: Api g 1 (celery), Dau c 1 (carrot); \\
\hline Profilin & 14 & $\begin{array}{l}\text { Apiales: Api g } 4 \text { (celery), Dau c } 4 \text { (carrot); } \\
\text { Solanales: Cap a } 2 \text { (bell pepper), Sola I } 1 \text { (tomato); }\end{array}$ \\
\hline nsLTP type 1 & 9 & $\begin{array}{l}\text { Apiales: Api g } 2 \text { (celery); } \\
\text { Solanales: Sola I } 3 \text { (tomato); }\end{array}$ \\
\hline nsLTP type 2 & $6-7$ & $\begin{array}{l}\text { Apiales: Api g } 6 \text { (celery); } \\
\text { Solanales: Sola I } 6 \text { (tomato); }\end{array}$ \\
\hline $\begin{array}{l}\text { Osmotin-like protein } \\
\text { (thaumatin like protein) }\end{array}$ & 23 & Solanales: Cap a 1 (bell pepper); \\
\hline $\begin{array}{l}\text { Isoflavone reductase-like } \\
\text { protein }\end{array}$ & 33 & Apiales: Dau c 5 (carrot); \\
\hline Beta-fructofuranosidase & 50 & Solanales: Sola I 2 (tomato); \\
\hline PR protein, TSI-1 & 18 & Solanales: Sola I 4 (tomato); \\
\hline Cyclophilin & 19 & Solanales: Sola I 5 (tomato); \\
\hline FAD containing oxidase & 58 & Apiales: Api g 5 (celery); \\
\hline
\end{tabular}

source: IUIS Allergen Database, March 2015

From tomato, profilin was identified as a minor allergen. Additionally, beta-fructofuranosidase and cyclophilin are minor allergens and a member of the Bet $v 1$ superfamily. Recently nsLTPs, both, type 1 and type 2 were characterized, however, little is known about their prevalence in sensitization. For bell pepper profilin and the thaumatin-like protein - called osmotin-like protein - have been identified as allergens. However, data about their relevance for diagnosis is rather limited (Table 2 and Fig. 2). 
Table 3

Sensitization to individual fruit allergens

\begin{tabular}{|c|c|c|c|}
\hline Allergen & Allergenicity & Risk Factor & Sensitization rate \\
\hline Mal d 1 & $\begin{array}{l}\text { High in BP allergic } \\
\text { patients }\end{array}$ & $\begin{array}{l}\text { Birch pollen, Bet v 1-related } \\
\text { sensitization; Consumption of raw fruits; } \\
\text { Living in Central and Northern Europe; }\end{array}$ & $15-70 \%$ of apple allergic patients ${ }^{17}$ \\
\hline Mal d 2 & no data & $\begin{array}{l}\text { Consumption of raw and processed } \\
\text { fruits? }\end{array}$ & $5-18 \%$ of apple allergic patients ${ }^{17}$ \\
\hline Mal d 3 & $\begin{array}{l}\text { High in Southern } \\
\text { Europe }\end{array}$ & $\begin{array}{l}\text { Consumption of raw and processed } \\
\text { fruits, Southern Europe, multiple } \\
\text { Rosaceae sensitization }\end{array}$ & $1-50 \%$ of apple allergic patients ${ }^{17}$ \\
\hline Mal d 4 & $\begin{array}{l}\text { Predominantly GP } \\
\text { and BP allergic } \\
\text { patients }\end{array}$ & $\begin{array}{l}\text { Grass pollen, profilin-related sensitiz } \\
\text { sensitization }\end{array}$ & $10-40 \%$ apple allergic patients ${ }^{17}$ \\
\hline Pru p 1 & $\begin{array}{l}\text { High in BP allergic } \\
\text { patients }\end{array}$ & $\begin{array}{l}\text { Birch pollen, Bet } v \text { 1-related sensitization } \\
\text { Consumption of raw fruits; Living in } \\
\text { Central and Northern Europe }\end{array}$ & $\begin{array}{l}11 \% \text { in Spanish peach allergic } \\
\text { children } \\
7-31 \% \text { in adults (ES, IT) }{ }^{18-20}\end{array}$ \\
\hline Pru p 3 & $\begin{array}{l}\text { High in Southern } \\
\text { Europe }\end{array}$ & $\begin{array}{l}\text { Consumption of raw and processed } \\
\text { fruits, Southern Europe, multiple } \\
\text { Rosaceae sensitization }\end{array}$ & $\begin{array}{l}96 \% \text { peach allergic children (ES) }{ }^{19} \\
62-96 \% \text { peach allergic adults (IT, } \\
\text { ES) }\end{array}$ \\
\hline Pru p 4 & GP-allergic patients & Consumption of fruits & $\begin{array}{l}10 \% \text { peach allergic children ( } 7-34 \% \\
\text { adults }(E S, I T){ }^{19,20}\end{array}$ \\
\hline \multicolumn{4}{|c|}{ No data available for Pru p 2 and Pru p 7} \\
\hline Act d 1 & & Kiwifruit monosensitization & $\begin{array}{l}5-32 \% \text { (Central Europe - } \\
\text { Iceland) })^{11,22}\end{array}$ \\
\hline Act d 2 & & & $2-18 \%^{21}$ \\
\hline Act d 5 & & & $2-18 \%^{21}$ \\
\hline Act d 8 & BP allergy & Birch pollen, Bet v 1-related sensitization & $7-58 \%^{21,34}$ \\
\hline Act d 9 & BP and GP allergy & Birch pollen; Bet v 2-related sensitization & $31-7 \%^{21}$ \\
\hline Act d 10 & & & $3-22 \%{ }^{22}$ \\
\hline
\end{tabular}

SENSITIZATION TO INDIVIDUAL

MOLECULES AND THEIR CLINICAL RELEVANCE

\section{Epidemiology and sensitization/cross reactivity rates}

Prevalence data for plant food allergies are scarce and the available data so far originate from a few studies. In a systematic review by Zuidmeer et al. the overall prevalence for fruits ranged from 0.1 to $4.3 \%$ (14). Within a European Community Respiratory Health
Survey (ECRHS) overall sensitization rates for fruits were assessed by Burney and colleagues in 2010 and 2014. Peach was the most frequent inducer of sensitization increasing from $5.4 \%$ to $7.9 \%$ in 2014 . Apple ranked second with a sensitization rate of $4.2 \%$ and $6.5 \%$ followed by kiwifruit with $3.6 \%$ and $5.2 \%$ sensitizing capacity $(15,16)$. Prevalence data on allergen specific sensitization have been generated by a few European wide studies. For apple the SAFE study provided data on Mal d 1- Mal d 4 (17), while the peach allergens were investigated in Spanish and Italian studies (18-20) reflecting the clear cut 


\section{Table 4}

Sensitization to individual vegetable allergens

\begin{tabular}{|c|c|c|c|}
\hline Allergen & Allergenicity & Risk Factor & Sensitization rate \\
\hline Api g 1 & BP allergy & Birch pollen, Bet v 1-related sensitization & $75 \% 23,37$ \\
\hline Api g 4 & BP allergy & & $42 \%{ }^{23,37}$ \\
\hline Api g 5 & & & $45 \%{ }^{23}$ \\
\hline \multicolumn{4}{|c|}{ No data available for Api g 2 and Api g 6} \\
\hline Dau c 1 & BP allergy & Birch pollen, Bet v 1-related sensitization & $58-100 \%{ }^{24}$ \\
\hline Dau c 4 & GP and BP allergy & Pollen Profilin-related sensitization & $18 \%{ }^{24}$ \\
\hline
\end{tabular}

difference in the frequency of LTP-sensitization predominant in the Southern European areas as compared to the PR-10 sensitization detected in areas with Fagales pollen exposure. Several kiwifruit studies were performed including a multicenter, within the Europrevall project and single center studies $(21,22)$ (Table 3$)$.

\section{Vegetables}

In the systematic review the overall prevalence of food allergies caused by vegetables is around $1.4 \%$ (14). The sensitization rates for vegetables were assessed by Burney et al. within the ECRHS study. Sensitization to carrot was determined of $3.6 \%$ and increased in 2014 to $5.0 \%$ while celeriac sensitization was observed in $3.5 \%$ and $6,3 \%$ of the general population, respectively $(15,16)$. In celeriac allergy allergen-specific sensitization prevalence was investigated within the EuroPrevall project (23). For carrot allergy one study investigated the sensitization rate in Switzerland, Denmark and Spain (24) (Table 4).

\section{CLINICAL RELEVANCE, DIAGNOSIS AND MANAGEMENT}

To date limited components are available to facilitate the in vitro diagnosis in fruit and vegetable allergies (25). This comprises cross-reactive allergens derived from inhalant allergenic sources (Bet $v 1$ and Bet $v$ 2 from birch pollen and the latex allergens Hev b 6.01,6.02 and 11) as well as food components from few Rosaceae fruits (Mal d 1,3; Prup 1,3,4) (17, 19, 20), kiwifruit allergens (Act d 1,2,5,8) and some Apiaceae allergens (Dau c 1, Api g 1). The following section therefore concentrates on Rosaceae, Apiaceae, kiwifruit allergy and the latex-fruit-syndrome.

\section{Clinical relevance and clinical pattern}

\section{Rosaceae fruit allergy}

The Rosaceae family includes many edible fruits. Apple, cherry and peach are the best-studied species from an allergy point of view. Allergen components are available to date just from peach and apple. The allergens identified in the fruits of the Rosaceae family are 1) PR-10, (Bet $\vee 1$ family member), 2) profilin, 3), nsLTP type 1, and 4) thaumatin like protein. Additionally in peach a gibberellin-regulated protein has been detected. Due to high cross-reactivity between the PR-10 proteins, the profilins and the nsLTPs, the corresponding allergens derived from peach are usually applied for diagnostic approaches in all types of Rosaceae fruit allergies.

The sensitization pattern to these allergens is geographically influenced. Sensitization rates to the Bet $v 1$ homologous proteins are significantly higher in countries with high pollen exposure of the Fagales trees (birch, alder, hazel; see Sections B1 and C2), whereas sensitization to nsLTP is significantly higher in Mediterranean countries (see section C3) $(16,17$, 26). Sensitization to Rosaceae fruit profilins (see Section (1) is more evenly distributed but most likely higher in the Mediterranean area (17). Knowledge on sensitization to Thaumatin-like fruit proteins or gibberellin-regulated protein is restricted. The Bet $v$ 1-related food proteins, the profilin and the nsLTP are panallergens, depicting a high cross-reactivity across the plant kingdom and sensitization to these molecules is often not accompanied by clinical symptoms $(27,28)$. Therefore, determination of slgE 
to these molecules should not be used as a screening tool (no prophetic testing!) and sensitization without convincing case history should always be validated by food challenges (Fig. 3). All three protein families have been associated with the various types of clinical manifestation in Rosaceae fruit allergies ranging from contact urticaria of the oral mucosa (so called Oral Allergy Syndrome, OAS) up to anaphylaxis. The prevalence of systemic reactions in those patients with a confirmed fruit allergy is higher in nsLTP mediated fruit allergies than in the Bet $v 1$ or profilin mediated ones $(17,26,29)$. In the following three typical patterns of Rosaceae fruit allergy are outlined.

A) Patient with a sensitization to Fagales tree pollen and IgE to Bet $v 1$ may develop cross-sensitisation to Bet $v 1$ homologous proteins from different Rosaceae fruits. Symptoms are elicited by unprocessed fruits. The usual manifestations are local oropharyngeal symptoms (OAS).

Cave: in selected cases Bet $v 1$ related fruit allergy can be associated with systemic reaction (30), i.e. in conjunction with co-factors (alcohol, exercise, ingestion into an empty stomach (31), high quantity of the ingested Bet $v 1$ homologues)

B) Patient with a sensitization to nsLTP mainly derived from peach (Pru p 3) may develop crosssensitization to other fruit nsLTPs. The clinical manifestations vary from local oropharyngeal symptoms up to anaphylaxis. The clinical pattern is influenced by co-factors (see under A, so called LTP syndrome (32). Symptoms are elicited by unprocessed and processed fruits.

C) Patients with a sensitization to profilin, frequently acquired via sensitization to grass pollen, might develop a cross-sensitisation to profilin in Rosaceae fruits (17). The usual manifestations are local oropharyngeal symptoms (OAS). The risk for a systemic reaction is low.

Cave: sensitisation to profilin is highly likely to be clinically silent (33).

\section{Kiwifruit allergy}

Allergy to kiwifruit is one of the most frequently observed fruit allergies in Europe (22). Thirteen kiwifruit allergens have been identified to date (Table 1 and 3, Fig. 1). An allergy to kiwifruit can be acquired via gastrointestinal tract (primary food allergy) or via crosssensitization to birch or grass pollen and latex allergens. The allergic symptoms range from mild oropharyngeal symptoms to severe, generalized reactions.
Actinidin, Act $d$ 1, is the major allergen of kiwifruit and correlates significantly with a kiwifruit monosensitization (21). Sensitization to Act d 8 and Act $d 9$ is specific for patients with pollen-kiwifruit allergies $(21,34)$. The sequence homology between kiwifruit nsLTP (Act d 10) and other nsLTP, particularly Pru $\mathrm{p} 3$ from peach, is small and therefore there is a limited danger of cross-reactivity (35). Additionally, cross-reactivity between Hev b 11, a chitinase from latex, and a protein in kiwifruit has been identified. The sensitivity of IgE measurement to kiwifruit extract is low (17\%) but could be increased by including different kiwifruit components (Act d 1-Act d 5 and Act d 8-Act d 9) to 77\% (21). Sensitization to Act d 1 was associated with the severity of the reaction (22) in a pan-European study and sensitizations to Act $d 1$ and Act $d 3$ were significantly correlated with anaphylactic reactions of patients from Spain (36).

In the following four typical patterns of kiwifriut allergy are outlined.

a. Patient with a sensitization to Fagales tree pollen and IgE to Bet $v 1$ may develop cross-sensitisation to the Bet $v 1$ homologous protein Act $d$ 8. The usual manifestations are local oropharyngeal symptoms (OAS).

b. Patient with a sensitization to profilin particularly from grass pollen may develop cross-sensitization to profilin in kiwifruit. The usual manifestations are local oropharyngeal symptoms (OAS)

c. Patient with a sensitization to latex proteins (i.e. Hev b 6 and 11) may develop cross-sensitisation to homologous proteins in kiwifruit. The clinical pattern varies from mild oropharyngeal symptoms up to anaphylaxis.

d. Patients with a sensitization (usually monosensitization) to Act d 1 may develop a primary kiwifruit allergy. The risk to develop systemic reaction up to anaphylaxis is increased.

\section{Apiaceae vegetable allergy}

The major representatives of the Apiaceae family in terms of food allergy are celeriac (Apium graveolens) and carrot. Celeriac allergy is highly associated with birch pollen and mugwort pollen sensitization referred to as birch-mugwort-celery-syndrome.

To date, five celeriac allergens have been identified in celeriac tuber Api g 1 (Bet $v 1$ homologue), Api g 6 (nsLTP type 2), Api g 3 (chlorophyll a-b binding protein), Api g 4 (profilin), Api g 5 (flavoprotein) and in celery stalk, additionally Api g 2 (nsLTP type 1). 
Use of Api g 1, 4, 5 increased the sensitivity from approximately $70 \%$ to $88 \%$ (75\% rApi g 1, 42\% rApi g 4 and $42 \%$ to nApi g 5) (23).

Celeriac induced symptoms range from mild oropharyngeal symptoms (OAS) up to anaphylaxis (37). No marker allergen for prediction of severe reactions has yet been identified. Particular severe reactions to celeriac occur in mugwort-sensitised patients $(23,37)$. The culprit cross-reactive allergens between mugwort and celeriac have not been identified so far. The clinical significance of the nsLTPs derived either from celeriac tuber (Api g 6) or from celery stalk (Api g 2) has not been confirmed to date $(12,13)$.

Also carrot allergy is highly associated with a sensitization to birch and mugwort pollen (24). Allergens identified in carrot are Dau c 1 (Bet v 1 homologue), Dau c 4 (profilin), Dau c 5 (isoflavone reductase), Dau c CyP (cyclophyllin) and Dau c 3 (nsLTP). The diagnostic relevance of Dau c 5 and Dau c CyP has not been investigated and it is not clear whether Dau c 3 is indeed present in the edible parts of carrots. As for celeriac allergy carrot allergy induced symptoms range from mild oropharyngeal symptoms (OAS) up to anaphylaxis (24).

In the following two typical patterns of celeriac/ carrot allergy are outlined.

A) Patient with a sensitization to Fagales tree pollen and IgE to Bet $v 1$ may develop cross-sensitisation to Bet $v 1$ homologous proteins in Apiaceae vegetables such as carrot and celeriac. Symptoms are often elicited by unprocessed foods. The usual manifestations are local oropharyngeal symptoms (OAS).

Cave: Bet $v 1$ related allergy to carrot and celeriac can be associated with systemic reaction. Systemic reactions are more frequently observed in Bet $v 1$ related celeriac and carrot allergy than in Bet $v 1$ related allergy to Rosaceae fruits (37) and might be elicited also by processed foods (particularly in celeriac allergy) (26).

B. Patient with a sensitization to mugwort pollen may develop cross-sensitization to not yet defined allergens in celeriac and carrot. The clinical manifestation varies from local oropharyngeal symptoms up to anaphylaxis. Symptoms are elicited by unprocessed and processed vegetables. IgE determination and skin testing particularly to celeriac extract are often negative. Typical pattern:
Sensitization to mugwort pollen, positive prickprick test with native food, negative testing using celeriac extract.

\section{Latex-fruit-syndrome}

In $30-70 \%$ of patients with a latex allergy associated food allergies have been observed, particularly to banana, avocado, chestnut, kiwifruit (see kiwifruit allergy) and many more (38). $11 \%$ of patients with a fruit allergy showed symptoms after latex challenge (39). Oropharyngeal symptoms are frequently observed, but in about $10 \%$ of latex associated food allergies anaphylactic reactions have been observed. As cross-reactive allergens beta-1,3-glucanase (Hev b 2), hevein (Hev b 6.02) and the hevein like domain of class I chitinases (Hev b 11) have been identified. However, further studies are needed since in a recent study the pathogenic role of Hev b 6 and Hev b 11 have been questionned (40).

\section{Clinical diagnosis of fruit and vegetable allergies}

Allergies to fruits and vegetables are often initiated by a primary sensitization to pollen. Since the majority of fruit and vegetable allergens are part of few protein families and characterized by a high cross-reactivity the clinical relevance of a sensitization needs to be often established by food challenges.

\section{Case history}

Previous reaction(s) to the incriminated fruit/ vegetable or fruits and vegetables from the same plant family. Symptoms onset and course, elicitation by raw or processed food, co-factors (exercise, NSAID, alcohol etc.), allergies to pollen, latex.

\section{Skin prick test (SPT)}

- commercial fruit and vegetable extracts limited due to false negative results as a consequence of underrepresentation of Bet $v 1$ homologous proteins and in part also nsLTPs

- prick-prick test with offending fruits and vegetables increased sensitivity but limited to false positive results (irritation of the skin)

\section{IgE-Testing}

- Due to the low stability of the Bet $v 1$ homologous proteins, these allergens are in some but not all diagnostic food extracts underrepresented (low sensitivity) leading to false negative test results.

- IgE Bet $v 1$ or Bet $v 1$ homologous proteins in reaction pattern A (Pru p 1, Mal d 1, Act d 8, Api $\mathrm{g} 1$, Dau c 1) indicative for a Bet $v 1$ related fruit/ 
vegetable allergy but limited by low specificity.

- IgE to nsLTP (Pru p 3, Mal d 3) in reaction pattern $\mathrm{B}$, might be associated with systemic reactions, limited by low specificity.

- IgE to Act d 1 risk factor for monosensitization and for systemic reactions to kiwifruit.

- IgE Hev b 6.02 and Hev b 11 hint for possible latex induced fruit allergy.

\section{Challenge tests}

- A/C: often not indicated if symptoms are limited to the oropharyngeal area, challenge with processed foods indicated in case of "unclear" history in terms of tolerance, i.e. in celeriac allergy

- B/D/E/F: titrated challenge indicated in cases where allergy is not supported by clear-cut case history

\section{Clinical management}

\section{Advices and avoidance}

- A/C: Avoidance of symptom eliciting raw fruits and raw vegetables (avoidance of processed foods only in patients with positive food challenges with the respective processed food)

- B/D/E/F: Avoidance of symptom eliciting raw and processed fruits and vegetables, for celeriac allergy also traces

\section{Pharmacotherapy (emergency kit)}

- A/C: Due to the small risk of systemic reactions or severe local reaction (angioedema lips, swelling oral mucosa) emergency medication for p.o. selfadministration (antihistamines, steroids)

- B/D/E/F: Emergency medication for p.o. selfadministration (antihistamines, steroids) and in case of systemic reaction epinephrine for selfadministration (pen)

\section{Allergen-specific immunotherapy}

- Immunotherapy with birch pollen extract in Bet v 1mediated fruit allergy showed contradictory results $(41,42)$.

- Oral tolerance induction was observed in Bet $v 1$ related apple allergy (43) but results need to be confirmed

- Sublingual immunotherapy for patients with a nsLTP induced peach allergy using a Pru $\mathrm{p} 3$ quantified peach extract has shown promising results, but the beneficial effect needs to be confirmed in the future (44).

\section{CLINICAL CASES}

\section{Case 1}

History: 28-year-old female patient, with recurrent anaphylactic reactions. First anaphylaxis with four years after plum, in the following after apple, with 9 years after peach.

Microarrayed specific lgE: Bet $\vee 1$ negative, Bet $\vee 2$ 1.2 ISU-E, Ara h 92.9 ISU-E, Cor a 8 3.6 ISU-E, Jug r 38.8 ISU-E, Pru p 312 ISU-E

Diagnosis: LTP syndrome with recurrent anaphylactic reactions after plum, peach, apple

Recommendation: strict elimination of symptom inducing fruits in raw and processed form. Emergency kit with antihistamines, steroids and adrenaline pen (SLIT with Pru p 3 quantified extract has been considered, but denied by the patient).

\section{Case 2}

History: Male, 48 years old. Rhinoconjunctivitis to birch pollen and oropharyngeal itch to raw apples since school age. Regular ingestion of apples despite oral symptoms. He gets up in the night 3 am, ingests three apples into empty stomach and develops oral itch, slight swelling of the lips and collapses. Two weeks later he gets up again in the night, ingests several apples into empty stomach, develops severe itch of the oral mucosa, swelling of the lips and loses consciousness for few minutes. After 1 hour spontaneous regression of symptoms.

In-vitro testing: specific IgE to Bet $v$ 1: $88 \mathrm{kUA} /$, $\operatorname{lgE}$ to Bet $\vee 2<0.35 \mathrm{kUA} / \mathrm{l}$, IgE to Pru p $3<0.35$ kUA/l.

SPT: Birch pollen, raw apple strongly positive

Food provocation: No symptoms on provocation with cooked apple puree, contact urticarial with blisters of the oral mucosa and slight angioedema of the lips after one quarter of a raw apple

Diagnosis: Anaphylactic reaction with oral contact urticaria, angioedema of lips and collapse after ingestion of apples into empty stomach due to Bet $\checkmark 1$ related apple allergy

Recommendations: Strict avoidance of raw apples; cooked apples without dietary restriction (due to thermal instability of Bet $v$ 1-related allergens). 


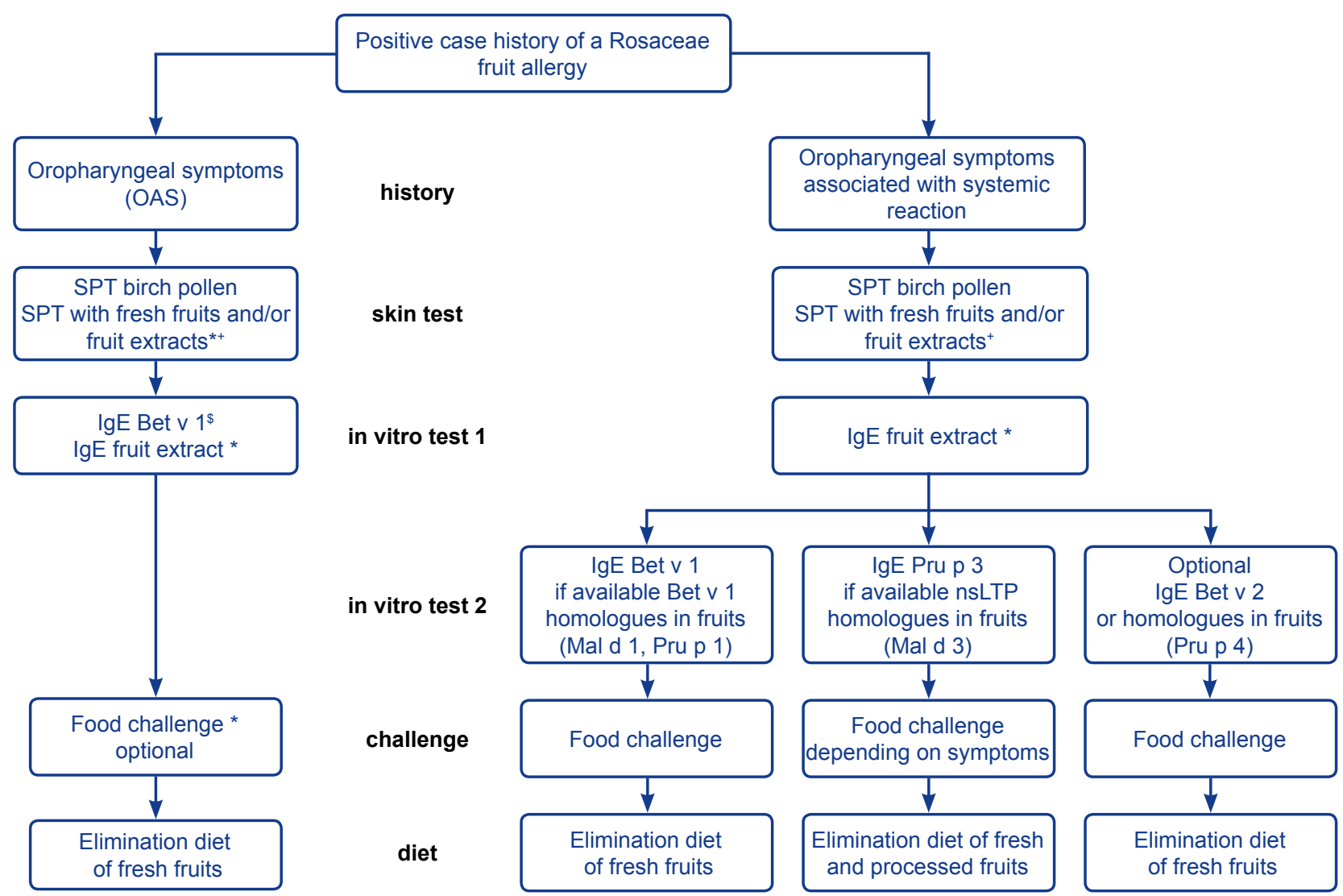

Figure 3

Diagnostic work-up in Rosaceae fruit allergy.

* Patients with sensitization to birch pollen or other Fagales tree pollen and history of Rosaceae fruit induced oropharyngeal symptoms usually do not need further investigation;

+ Sensitivity of skin test or in vitro IgE determination using fruit extracts might be low due to underrepresentation of Bet $\vee 1$ homologues;

$\$$ In patients from Mediterranean countries: OAS can also be linked to LTP or profilin sensitization. In case of LTP mediated OAS strict elimination of fresh and processed food is recommended.

\section{Case 3}

History: Young female patient, 23 years old, no atopic background. Ingestion of kiwifruit, after 15 minutes nausea, abdominal cramps, emesis, diarrhea, drop of blood pressure. Emergency treatment.

SPT: negative skin test with birch and grass pollen, latex, isolated positive skin test with raw kiwifruit

In-vitro testing (ImmunoCAP): specific IgE Bet $v 1<$ $0.35 \mathrm{kUA} / \mathrm{l}$, IgE to Bet $v 2<0.35 \mathrm{kUA} / \mathrm{l}$, IgE to latex $<0.35 \mathrm{kUA} /$; IgE to kiwi extract $<0.35 \mathrm{kUA} / \mathrm{l}$
Microarrayed specific IgE: positive to Act d 1 and Act d 2

Oral challenge: mucosa challenge with kiwifruit: oral contact urticaria, flush face and nausea

Diagnosis: primary kiwifruit allergy with sensitization to Act d 1 and Act d 2-

Recommendation: strict elimination diet for raw and processed kiwifruit, emergency kit with antihistamines, steroids and adrenaline pen. 


\section{References}

1. Bolhaar ST, van de Weg WE, van Ree R, GonzalezMancebo E, Zuidmeer L, Bruijnzeel-Koomen CA, et al. In vivo assessment with prick-to-prick testing and double-blind, placebo-controlled food challenge of allergenicity of apple cultivars. J Allergy Clin Immunol 2005;116:1080-1086.

2. Sancho Al, van Ree R, van Leeuwen A, Meulenbroek BJ, van de Weg EW, Gilissen LJ, et al. Measurement of lipid transfer protein in 88 apple cultivars. Int Arch Allergy Immunol 2008;146:19-26.

3. Sancho Al, Foxall R, Browne T, Dey R, Zuidmeer L, Marzban G, et al. Effect of postharvest storage on the expression of the apple allergen Mal d 1. Agric Food Chem 2006;54:5917-5923.

4. Bassler OY, Weiss J, Wienkoop S, Lehmann K, Scheler $\mathrm{C}$, Dolle $\mathrm{S}$, et al. Evidence for novel tomato seed allergens: IgE-reactive legumin and vicilin proteins identified by multidimensional protein fractionationmass spectrometry and in silico epitope modeling. J Proteome Res 2009;8:1111-1122.

5. Gaier S, Marsh J, Oberhuber C, Rigby NM, Lovegrove A, Alessandri S, et al. Purification and structural stability of the peach allergens Pru p 1 and Pru p 3. Mol Nutr Food Res 2008;52(Suppl 2):S220-229.

6. Tuppo L, Alessandri C, Pomponi D, Picone D, Tamburrini M, Ferrara R, et al. Peamaclein--a new peach allergenic protein: similarities, differences and misleading features compared to Pru p 3. Clin Exp Allergy 2013;43:128-140.

7. Karamloo F, Wangorsch A, Kasahara H, Davin LB, Haustein D, Lewis NG, et al. Phenylcoumaran benzylic ether and isoflavonoid reductases are a new class of cross-reactive allergens in birch pollen, fruits and vegetables. FEBS 2001;268:5310-5320.

8. Bublin M, Mari A, Ebner C, Knulst A, Scheiner O, Hoffmann-Sommergruber $K$, et al. IgE sensitization profiles toward green and gold kiwifruits differ among patients allergic to kiwifruit from 3 European countries. J Allergy Clin Immunol 2004;114:11691175.

9. Le TM, Fritsche $P$, Bublin $M$, Oberhuber $C$, Bulley S, van Hoffen $E$, et al. Differences in the allergenicity of 6 different kiwifruit cultivars analyzed by prick-to-prick testing, open food challenges, and ELISA. J Allergy Clin Immunol 2011;127:677-679 e1-2.

10. Asero R, Mistrello G, Roncarolo D, Amato S, Zanoni $D$, Barocci $F$, et al. Detection of clinical markers of sensitization to profilin in patients allergic to plantderived foods. J Allergy Clin Immunol 2003;112:427432.

11. Bublin M, Radauer C, Wilson IB, Kraft D, Scheiner O, Breiteneder $\mathrm{H}$, et al. Cross-reactive $\mathrm{N}$-glycans of Api g 5, a high molecular weight glycoprotein allergen from celery, are required for immunoglobulin $E$ binding and activation of effector cells from allergic patients. FASEB J 2003;17:1697-1699.

12. Gadermaier G, Hauser M, Egger M, Ferrara R, Briza P, Santos KS, et al. Sensitization prevalence, antibody cross-reactivity and immunogenic peptide profile of Api g 2, the non-specific lipid transfer protein 1 of celery. PLoS One 2011;6:e24150.

13. Vejvar E, Himly M, Briza P, Eichhorn S, Ebner C, Hemmer $W$, et al. Allergenic relevance of nonspecific lipid transfer proteins 2: Identification and characterization of Api g 6 from celery tuber as representative of a novel IgE-binding protein family. Mol Nutr Food Res 2013;57:2061-2070.

14. Zuidmeer L, Goldhahn K, Rona RJ, Gislason D, Madsen C, Summers C, et al. The prevalence of plant food allergies: a systematic review. J Allergy Clin Immunol 2008;121:1210-1218 e4.

15. Burney P, Summers C, Chinn S, Hooper R, van Ree R, Lidholm J. Prevalence and distribution of sensitization to foods in the European Community Respiratory Health Survey: a EuroPrevall analysis. Allergy 2010;65:1182-1188.

16. Burney PG, Potts J, Kummeling I, Mills EN, Clausen M, Dubakiene R, et al. The prevalence and distribution of food sensitization in European adults. Allergy 2014;69:365-371.

17. Fernandez-Rivas M, Bolhaar S, Gonzalez-Mancebo E, Asero R, van Leeuwen A, Bohle B, et al. Apple allergy across Europe: how allergen sensitization profiles determine the clinical expression of allergies to plant foods. J Allergy Clin Immunol 2006;118:481-488.

18. Fernandez-Rivas M, Gonzalez-Mancebo E, RodriguezPerez R, Benito C, Sanchez-Monge R, Salcedo G, et al. Clinically relevant peach allergy is related to peach lipid transfer protein, Pru p 3, in the Spanish population. J Allergy Clin Immunol 2003;112:789-795.

19. Boyano-Martinez T, Pedrosa M, Belver T, Quirce S, Garcia-Ara C. Peach allergy in Spanish children: tolerance to the pulp and molecular sensitization profile. Ped Allergy Immunol 2013;24:168-172.

20. Pastorello EA, Farioli L, Stafylaraki C, Mascheri A, Scibilia J, Pravettoni V, et al. Anti-rPru p 3 IgE levels are inversely related to the age at onset of peachinduced severe symptoms reported by peach-allergic adults. Int Arch Allergy Immunol 2013;162:45-49.

21. Bublin M, Pfister M, Radauer C, Oberhuber C, Bulley S, Dewitt AM, et al. Component-resolved diagnosis of kiwifruit allergy with purified natural and recombinant kiwifruit allergens. J Allergy Clin Immunol 2011;125:687-694, 94 e1.

22. Le TM, Bublin $M$, Breiteneder $H$, Fernandez-Rivas $M$, Asero R, Ballmer-Weber $B$, et al. Kiwifruit allergy across Europe: clinical manifestation and IgE 
recognition patterns to kiwifruit allergens. J Allergy Clin Immunol 2013;131:164-171.

23. Bauermeister K, Ballmer-Weber BK, Bublin M, Fritsche $P$, Hanschmann KM, Hoffmann-Sommergruber $\mathrm{K}$, et al. Assessment of component-resolved in vitro diagnosis of celeriac allergy. J Allergy Clin Immunol 2009;124:1273-1281 e2.

24. Ballmer-Weber BK, Skamstrup Hansen K, Sastre J, Andersson K, Batscher I, Ostling J, et al. Componentresolved in vitro diagnosis of carrot allergy in three different regions of Europe. Allergy 2012;67:758766.

25. Ballmer-Weber BK, Hoffmann-Sommergruber K. Molecular diagnosis of fruit and vegetable allergy. Curr Opin Allergy Clin Immunol 2011;11:229-235.

26. Ballmer-Weber BK, Scheurer $S$, Fritsche P, Enrique E, Cistero-Bahima A, Haase T, et al. Component-resolved diagnosis with recombinant allergens in patients with cherry allergy. J Allergy Clin Immunol 2002;110:167173.

27. Lidholm J, Ballmer-Weber BK, Mari A, Vieths S. Component-resolved diagnostics in food allergy. Curr Opin Allergy Clin Immunol 2006;6:234-240.

28. Gonzalez-Mancebo E, Gonzalez-de-Olano D, Trujillo MJ, Santos S, Gandolfo-Cano M, Melendez A, et al. Prevalence of sensitization to lipid transfer proteins and profilins in a population of 430 patients in the south of Madrid. J Invest Allergol Clin Immunol 2011;21:278-282.

29. Reuter A, Lidholm J, Andersson K, Ostling J, Lundberg $\mathrm{M}$, Scheurer $\mathrm{S}$, et al. A critical assessment of allergen component-based in vitro diagnosis in cherry allergy across Europe. Clin Exp Allergy 2006;36:815-823.

30. Le TM, van Hoffen E, Lebens AF, Bruijnzeel-Koomen CA, Knulst AC. Anaphylactic versus mild reactions to hazelnut and apple in a birch-endemic area: different sensitization profiles? Int Arch Allergy Immunol 2013;160:56-62.

31. Schulten V, Lauer I, Scheurer S, Thalhammer T, Bohle B. A food matrix reduces digestion and absorption of food allergens in vivo. Mol Nutr Food Res 2011;55:14841491.

32. Romano A, Scala E, Rumi G, Gaeta F, Caruso C, Alonzi C, et al. Lipid transfer proteins: the most frequent sensitizer in Italian subjects with fooddependent exercise-induced anaphylaxis. Clin Exp Allergy 2012;42:1643-1653.

33. Santos A, Van Ree R. Profilins: mimickers of allergy or relevant allergens? Int Arch Allergy Immunol 2011;155:191-204.

34. Oberhuber C, Bulley SM, Ballmer-Weber BK, Bublin M, Gaier S, DeWitt AM, et al. Characterization of Bet v 1-related allergens from kiwifruit relevant for patients with combined kiwifruit and birch pollen allergy. $\mathrm{Mol}$ Nutr Food Res 2008;52(Suppl 2):S230-240.

35. Bernardi ML, Giangrieco I, Camardella L, Ferrara $\mathrm{R}$, Palazzo P, Panico MR, et al. Allergenic lipid transfer proteins from plant-derived foods do not immunologically and clinically behave homogeneously: the kiwifruit LTP as a model. PloS One 2011;6:e27856.

36. Palacin A, Rodriguez J, Blanco C, Lopez-Torrejon G, Sanchez-Monge R, Varela J, et al. Immunoglobulin $\mathrm{E}$ recognition patterns to purified Kiwifruit (Actinidinia deliciosa) allergens in patients sensitized to Kiwi with different clinical symptoms. Clin Exp Allergy 2008;38:1220-1228.

37. Ballmer-Weber BK, Vieths $S$, Luttkopf $D$, Heuschmann $\mathrm{P}$, Wuthrich B. Celery allergy confirmed by double-blind, placebo-controlled food challenge: a clinical study in 32 subjects with a history of adverse reactions to celery root. J Allergy Clin Immunol 2000;106:373-378.

38. Brehler R, Theissen U, Mohr C, Luger T. "Latex-fruit syndrome": frequency of cross-reacting IgE antibodies. Allergy 1997;52:404-410.

39. Garcia Ortiz JC, Moyano JC, Alvarez M, Bellido J. Latex allergy in fruit-allergic patients. Allergy 1998;53:532536.

40. Radauer C, Adhami F, Furtler I, Wagner S, Allwardt $D$, Scala E, et al. Latex-allergic patients sensitized to the major allergen hevein and hevein-like domains of class I chitinases show no increased frequency of latex-associated plant food allergy. Mol Immunol 2011;48:600-609.

41. Bolhaar ST, Tiemessen MM, Zuidmeer $L$, van Leeuwen A, Hoffmann-Sommergruber $\mathrm{K}$, Bruijnzeel-Koomen $\mathrm{CA}$, et al. Efficacy of birch-pollen immunotherapy on cross-reactive food allergy confirmed by skin tests and double-blind food challenges. Clin Exp Allergy 2004;34:761-769.

42. Hansen KS, Khinchi MS, Skov PS, Bindslev-Jensen C, Poulsen LK, Malling HJ. Food allergy to apple and specific immunotherapy with birch pollen. Mol Nutr Food Res 2004;48:441-448.

43. Kopac P, Rudin M, Gentinetta T, Gerber R, Pichler C, Hausmann $\mathrm{O}$, et al. Continuous apple consumption induces oral tolerance in birch-pollen-associated apple allergy. Allergy 2012;67:280-285.

44. Fernandez-Rivas M, Garrido Fernandez S, Nadal JA, Diaz de Durana MD, Garcia BE, Gonzalez-Mancebo $\mathrm{E}$, et al. Randomized double-blind, placebo-controlled trial of sublingual immunotherapy with a Pru $\mathrm{p} 3$ quantified peach extract. Allergy 2009;64:876-883. 



\title{
WHEAT ALLERGY
}

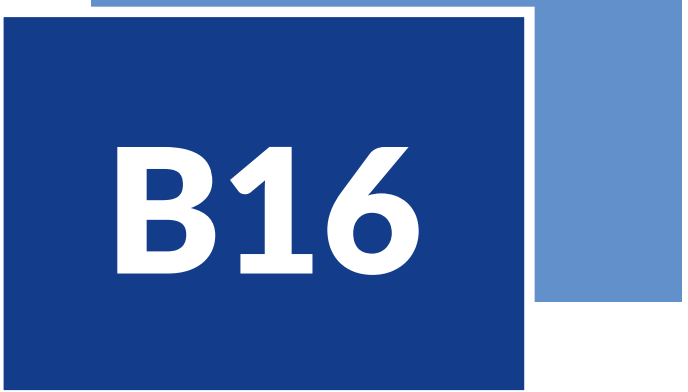

\author{
Mika Mäkelä
}

\section{THE ALLERGEN SOURCES}

Wheat (Triticum aestivum) may be considered the most important source of food globally. For example, world trade in wheat is greater than for all other crops combined. Most food cultures serve wheat as an important part of daily meals, including bread, pasta, breakfast cereal, semolina, bulgur and couscous, to name a few. Wheat has more vegetable protein than other two worldwide important cereals, corn or rice. There are several different classifications of wheat and a number of different species and sub-species have been described, not to mention more than 25000 cultivars (1). However, there seem to be no clinically significant differences in allergenicity.

In most countries, allergy to milk and egg are the two most single-common allergies but wheat comes as third at least in Germany, Japan and Finland (2). Wheat allergy prevalence varies depending on the age and region from $0.4 \%$ to $4 \%(2,3)$. The most typical clinical manifestations of wheat-induced food allergy include IgE-mediated food allergy and celiac disease. The latter is more an autoimmune disease, it is not included in allergy treatment algorithms in most countries and is often treated by gastroenterologists
Wheat is related to several clinically different allergic diseases even in different organs including food allergy, wheat-dependent exercise-induced anaphylaxis, respiratory allergy and contact urticarial.

due to cross-reactivity with other allergens including grasses, IgE measurement to whole wheat extract gives unreliable results with low specificity in diagnostics

there are several well-characterized allergenic molecules such as gliadins glutenins and alpha-amylase inhibitors but it has been difficult to name single major allergens

the sensitization to individual proteins is associated to disease manifestations but with a significant overlap 


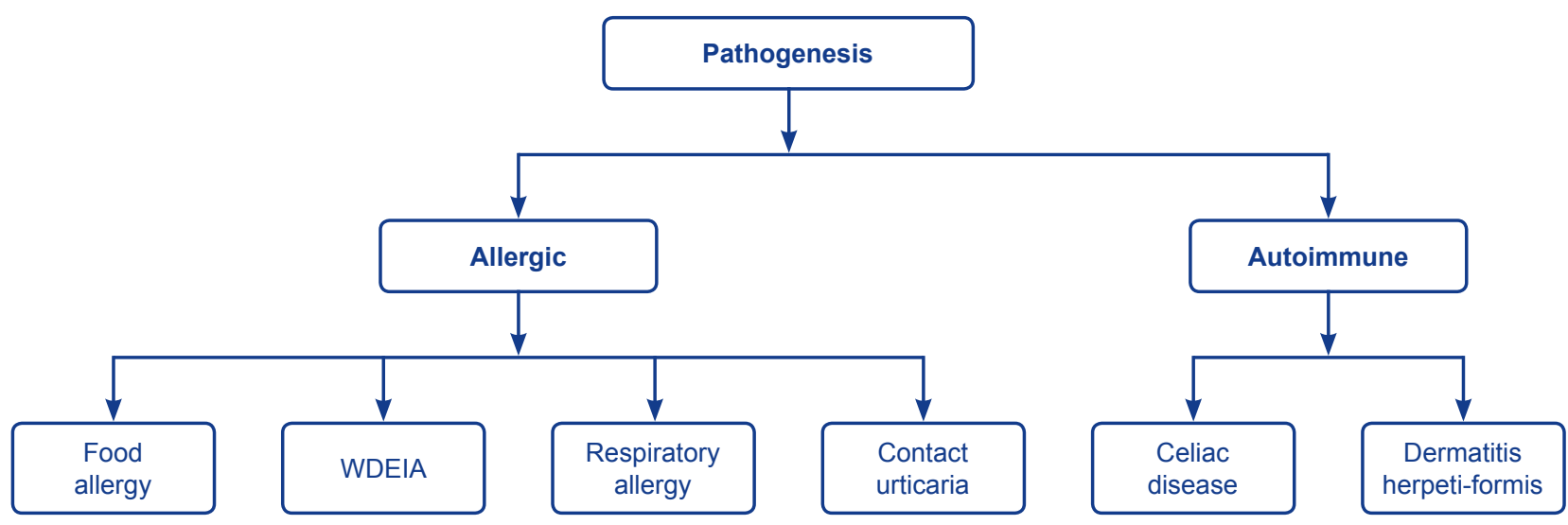

Figure 1

Classification of wheat-related allergic diseases. Modified from 1.

rather than allergists. Therefore, celiac disease is not covered in this chapter other than in the classification as shown in Fig. 1.

\section{MAJOR AND RELEVANT MINOR ALLERGENIC MOLECULES}

Basically, wheat is a grass from the Poaceae family. The measurement of wheat-specific lgE and its use for clinical diagnosis is problematic due to the low specificity when using whole wheat extract as a test allergen either in SPTs or in serum assays. Wheatspecific IgE is common among atopic children at all ages without true food allergies--up to $65 \%$ of the patients with grass pollen allergy had false positive IgE-ab test results to wheat extracts (4). On the other hand, some allergens are underrepresented in whole wheat extract based tests due to their relative insolubility.

The list of the World Health Organization/ International Union of Immunological Societies Allergen Nomenclature Sub-committee lists 27 wheat allergens (www.allergen.org). Wheat (Triticum aestivum) allergen numbers start with Tri a 12 (profilin) and end with Tri a 45 (Elongation factor 1). Many of the not yet clinically well-studied allergens are homologous to characterized grass pollen allergens or seedallergens from related cereals.

Wheat proteins have been broadly divided into watersoluble albumins, salt-soluble globulins, and insoluble prolamins, including the gliadins which are soluble in aqueous alcohols, and the glutenins (5) (Fig. 1). The gliadins and glutenins arethe major storage proteins in the wheat grain, also making wheat flour suitable for baking. Each of these fractions contains allergenic proteins which have been associated with clinical symptoms but as yet, there is no concensus definition of major and minor allergens of wheat. Little is known also of the allergenicity as what comes to heating and processing of the fractions.

The best-characterized single component of wheat is omega-5-gliadin. Gliadin is a major allergen for wheatdependent, exercise-induced anaphylaxis (WDEIA, later in this document clinical form B) but also for the early childhood wheat allergy with symptoms of type I reaction and atopic eczema (later A type) (5-8) and baker's asthma/allergy (type C) (10). Other relatively well-documented allergens include alpha-amylase inhibitors (AAl), the response to which is associated with both baker's allergy and food allergy $(5,9,10)$, wheat LTP has also obvious clinical relevance and it has been associated with Baker's asthma (11) and food allergy (12).

So far, attempts to find single components predicting clinical reactivity have produced at best high sensitivity with the expense of low specificity. Although an early study showed up to $100 \%$ specificity for clinical response with sensitization to omega-5-gliadin (6), later larger studies recruiting more heterogeneous patient groups have produced much lower rates for both sensitivity and specificity $(5,8)$. 


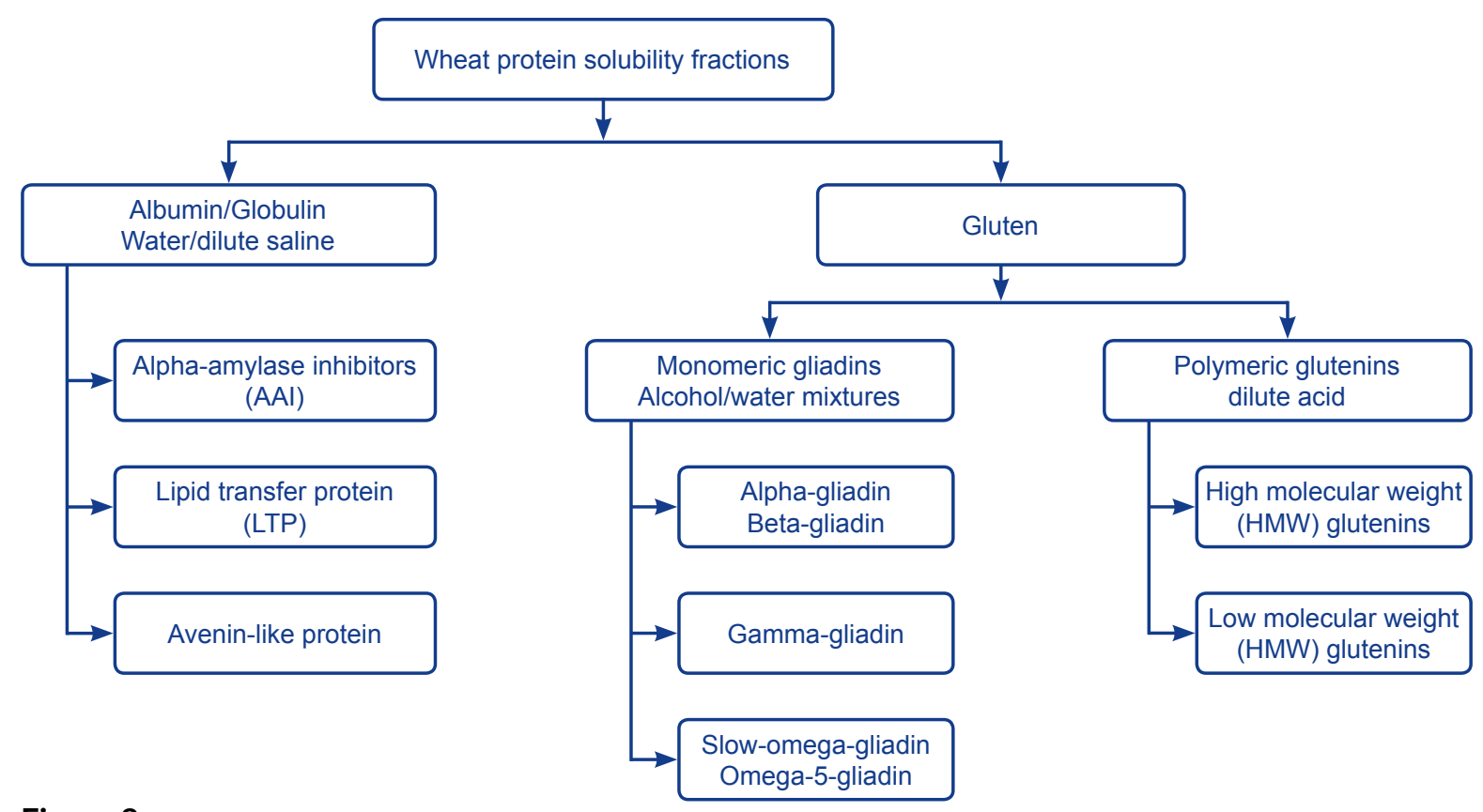

Figure 2

Allergenic molecules of wheat grouped according to their solubility.

\section{SENSITIZATION TO INDIVIDUAL MOLECULES AND THEIR CLINICAL RELEVANCE}

\section{Epidemiology and sensitization/cross reactivity rates}

Sensitization to wheat among children was $<1 \%$ in a systematic review taking into account a number of studies around the world (13). In a population-based study amongst six-year-old children in Britain, wheat sensitization rate was $0.4 \%$ and most of this was concluded to be result from grass sensitization based on the food challenges (14). No studies have examined response to single proteins at population level.

Cross-reactivity of wheat comes at least from sensitization to grass pollen. It varies, however, how much of the sensitization can be explained on this at different age groups and in different disease conditions. Wheat is also highly cross-reactive with other cereals, mainly rye and barley (10). Early studies showed that prolamins like gamma-70 and gamma-35 secalins in rye and gamma-3 hordein in barley cross-react with omega-5 gliadin (15) and there are several other proteins among these three cereals, which are highly crossreactive. Moreover, there is high sequence identity among many other proteins such as a-purothionins from wheat, rye, and barley ( $>80 \%$ ) (16). In a large study of baker's allergy, rye flour inhibited binding of IgE to most wheat allergens at a significant level (9).

There are many more relevant sensitizations to single proteins other than gliadins, AAI or LTP. Two recent studies have demonstrated the role of sensitization to both low-molecular-weight (LMW) Tri a 36 and the high-molecular-weight (HMW) glutenin, Tri a 26 with most typical type of childhood wheat allergy $(5,8,13$, 17). Moreover, omega-5-gliadin is not the only gliadin that seems to be of significance. Also alpha- betaand gamma-gliadins present as important allergens in several studies $(5,8)$.

Recently, based on construction of T. aestivum cDNA library and screening it with serum IgE from patients suffering from respiratory wheat allergy five novel wheat allergens were characterized: a thioredoxin $\mathrm{h}$ isoform, glutathione transferase, 1-Cysperoxiredoxin, profilin and dehydrin (18). Particularly of these, a potential and emerging food allergen is alpha-purothionin Tri a 37 (16).

In the largest study of any wheat allergic patients, 19 recombinant wheat flour proteins and 2 cross-reactive carbohydrate determinants were tested in sera of 101 bakers from several European countries with wheat flour allergy. Not a single allergen emerged as major 
Table 1

Allergenic molecules of wheat

\begin{tabular}{|c|c|c|c|}
\hline $\begin{array}{l}\text { Allergen/ } \\
\text { IUIS code }\end{array}$ & Biochemical name & $\begin{array}{l}\mathrm{MW} \\
\text { (kDa) }\end{array}$ & $\begin{array}{c}\text { heat } \\
\text { stability }\end{array}$ \\
\hline Tri a 12 & Profilin & 14 & low \\
\hline Tri a 14 & Non-specific lipid transfer protein & 9 & high \\
\hline Tri a 15 & Monomeric alpha-amylase inhibitor 0.29 & & high \\
\hline Tri a 18 & Agglutinin isolectin 1 & & \\
\hline Tri a 19 & Omega-5-gliadin, seed storage protein & 65 & high \\
\hline Tri a 20 & Gamma gliadin & $35-38$ & \\
\hline Tri a 21 & Alpha-beta-gliadin & $30-45$ & low \\
\hline Tri a 25 & Thioredoxin & 13 & \\
\hline Tri a 26 & High molecular weight glutenin & 88 & \\
\hline Tri a 27 & Thiol reductase homologue & 27 & \\
\hline Tri a 28 & Dimeric alpha-amylase inhibitor 0.19 & 13 & high \\
\hline Tri a 29 & Tetrameric alpha-amylase inhibitor CM1/CM2 & 13 & high \\
\hline Tri a 30 & Tetrameric alpha-amylase inhibitor CM3 & 16 & high \\
\hline Tri a 31 & Triosepohosphate-isomerase & 26 & \\
\hline Tri a 32 & 1-cys-peroxiredoxin & & \\
\hline Tri a 33 & Serpin & 40 & \\
\hline Tri a 34 & Glyceraldehyde-3-phosphate-dehydrogenase & $40-42$ & \\
\hline Tri a 35 & Dehydrin & & \\
\hline Tri a 36 & Low molecular weight glutenin GluB3-23 & 40 & \\
\hline Tri a 37 & Alpha purothionin & 12 & high \\
\hline Tri a 39 & Serine protease inhibitor-like protein & & \\
\hline
\end{tabular}

based on www.allergen.org

one and each baker showed an individual lgE-binding profile with great interindividual variation (9).

Taken altogether, there is generally a significant overlap of the responses to individual proteins in different disease conditions.

\section{CLINICAL RELEVANCE, DIAGNOSIS AND MANAGEMENT}

\section{Clinical relevance and clinical pattern}

Due to different routes and amounts of exposure four scenarios can prompt lgE-mediated sensitizations and clinical symptoms of immediate hypersensitivity (1) (Table 3, A - D).

A) Typically, IgE-mediated form of food allergy to wheat is analogous to the symptoms seen in milk or egg allergy. Allergic individuals develop symptoms within minutes to $1-2 \mathrm{~h}$ after ingestion of a wheat. The symptoms include urticaria, angioedema, erythema, pruritus, vomiting, abdominal pain, persistent cough, hoarse voice, wheeze, stridor, respiratory distress, nasal congestion and, in most severe case, anaphylaxis. These may be associated also with delayed-type symptoms which include the worsening of atopic dermatitis and gastrointestinal symptoms such as 


\section{Table 2}

Allergenic molecules of wheat

\begin{tabular}{|c|c|c|c|c|}
\hline Allergen & & Allergenicity & Risk factor & Clinical response rate \\
\hline Tri a 12 & Profilin & & inhaled flour & $\begin{array}{l}\text { baker's allergy, not } \\
\text { significant in food allergy }\end{array}$ \\
\hline Tri a 14 & $\begin{array}{l}\text { Non-specific lipid } \\
\text { transfer protein }\end{array}$ & $\begin{array}{l}\text { not cross-reactive } \\
\text { with grass pollen }\end{array}$ & $\begin{array}{l}\text { the allergenicity strengthened } \\
\text { by activated tissue } \\
\text { transglutaminase, }\end{array}$ & $\begin{array}{l}\text { mayh be important both in } \\
\text { WDEIA and in some cases } \\
\text { food allergy }\end{array}$ \\
\hline Tri a 15 & $\begin{array}{l}\text { Monomeric } \\
\text { alpha-amylase } \\
\text { inhibitor } 0.29\end{array}$ & $\begin{array}{l}60 \% \text { of the } A \text { type } \\
\text { patients positive }\end{array}$ & & \\
\hline Tri a 18 & $\begin{array}{l}\text { Agglutinin } \\
\text { isolectin } 1\end{array}$ & $?$ & $\begin{array}{l}\text { elevated general atopic risk } \\
\text { for childhood food allergies, } \\
\text { atopic eczema and exposure } \\
\text { to wheat }\end{array}$ & $\begin{array}{l}\text { mainly recognized by } \\
\text { patients with baker's } \\
\text { asthma? }\end{array}$ \\
\hline Tri a 19 & Omega-5-gliadin & $\begin{array}{l}50 \%-70 \% \text { of wheat } \\
\text { allergic patients }\end{array}$ & $\begin{array}{l}\text { elevated general atopic risk } \\
\text { for childhood food allergies, } \\
\text { atopic eczema and exposure } \\
\text { to wheat }\end{array}$ & $\begin{array}{l}>80 \% ?, \text { marker for severity } \\
\text { and persistence? }\end{array}$ \\
\hline Tri a 20 & Gamma gliadin & $\begin{array}{l}50 \%-70 \% \text { of wheat } \\
\text { allergic patients, }\end{array}$ & $\begin{array}{l}\text { elevated general atopic risk } \\
\text { for childhood food allergies, } \\
\text { atopic eczema and exposure } \\
\text { to wheat }\end{array}$ & $\begin{array}{l}\text { possibly best severity } \\
\text { marker }\end{array}$ \\
\hline Tri a 21 & $\begin{array}{l}\text { Alpha-beta- } \\
\text { gliadin }\end{array}$ & $\begin{array}{l}>50 \% \text { of wheat } \\
\text { allergic patients? }\end{array}$ & & $>50 \% ?$ \\
\hline Tri a 25 & Thioredoxin & grass cross-reactivity & inhaled flour & \\
\hline Tri a 26 & $\begin{array}{l}\text { High molecular } \\
\text { weight glutenin }\end{array}$ & $\begin{array}{l}50 \%-70 \% \text { of wheat } \\
\text { allergic patients, all } \\
\text { those with severe } \\
\text { reaction }\end{array}$ & & $\begin{array}{l}\text { significant, testing in } \\
\text { combination with other } \\
\text { gluten-derived proteins }\end{array}$ \\
\hline Tri a 27 & $\begin{array}{l}\text { Thiol reductase } \\
\text { homologue }\end{array}$ & $\begin{array}{l}\text { frequently detected } \\
\text { allergen among } \\
\text { bakers }\end{array}$ & inhaled flour & $\begin{array}{l}\text { not significant in food } \\
\text { allergy? }\end{array}$ \\
\hline Tri a 28 & $\begin{array}{l}\text { Dimeric alpha- } \\
\text { amylase inhibitor } \\
0.19\end{array}$ & $\begin{array}{l}50 \%-70 \% \text { of wheat } \\
\text { allergic patients }\end{array}$ & & $\begin{array}{l}\text { as a single allergen, optimal } \\
\text { combination for sensitivity } \\
\text { and specificity }\end{array}$ \\
\hline Tri a 29 & $\begin{array}{l}\text { Tetrameric alpha- } \\
\text { amylase inhibitor } \\
\text { CM1/CM2 }\end{array}$ & $\begin{array}{l}37 \% \text { of food allergic } \\
\text { positive }\end{array}$ & & \\
\hline Tri a 30 & $\begin{array}{l}\text { Tetrameric alpha- } \\
\text { amylase inhibitor } \\
\text { CM3 }\end{array}$ & & & $\begin{array}{l}\text { high specificity for baker's } \\
\text { asthma }\end{array}$ \\
\hline Tri a 31 & $\begin{array}{l}\text { Triosephosphate- } \\
\text { isomerase }\end{array}$ & & & $\begin{array}{l}\text { rarely recognized by patients } \\
\text { with baker's asthma, no data } \\
\text { on food allergy }\end{array}$ \\
\hline
\end{tabular}


Table 2 (continued)

Allergenic molecules of wheat

\begin{tabular}{|c|c|c|c|c|}
\hline \multicolumn{2}{|l|}{ Allergen } & \multirow{2}{*}{$\begin{array}{l}\text { Allergenicity } \\
? ?\end{array}$} & \multirow{2}{*}{$\frac{\text { Risk factor }}{\text { inhaled flour }}$} & \multirow{2}{*}{$\begin{array}{l}\text { Clinical response rate } \\
\text { recognized by patients with } \\
\text { baker's asthma }\end{array}$} \\
\hline Tri a 32 & $\begin{array}{l}\text { 1-cys- } \\
\text { peroxiredoxin }\end{array}$ & & & \\
\hline Tri a 33 & Serpin & & inhaled flour & $\begin{array}{l}\text { recognized by patients with } \\
\text { baker's asthma, no data on } \\
\text { food allergy }\end{array}$ \\
\hline Tri a 34 & $\begin{array}{l}\text { Glyceraldehyde- } \\
\text { 3-phosphate- } \\
\text { dehydrogenase }\end{array}$ & low & & $\begin{array}{l}\text { rarely recognized by patients } \\
\text { with baker's asthma }\end{array}$ \\
\hline Tri a 35 & Dehydrin & $?$ & inhaled flour & $\begin{array}{l}\text { mainly recognized by patients } \\
\text { with baker's asthma }\end{array}$ \\
\hline Tri a 36 & $\begin{array}{l}\text { Low molecular } \\
\text { weight glutenin } \\
\text { GluB3-23 }\end{array}$ & $\begin{array}{l}60 \%-80 \% \text { of wheat } \\
\text { allergic patients }\end{array}$ & & $\begin{array}{l}>50 \% \text {, testing in combination } \\
\text { with other gluten-derived } \\
\text { proteins }\end{array}$ \\
\hline Tri a 37 & Alpha purothionin & $\begin{array}{l}16 \% \text { of allergic } \\
\text { patients with food } \\
\text { allergy, patients with } \\
\text { baker's asthma not } \\
\text { sensitized }\end{array}$ & & $\begin{array}{l}\text { marker for severity with food } \\
\text { allergy? }\end{array}$ \\
\hline
\end{tabular}

\section{Table 3}

Clinical pattern of different types of wheat allergy

\begin{tabular}{|c|c|c|c|c|}
\hline & A & B & C & D \\
\hline $\begin{array}{l}\text { Routes of sensitiza- } \\
\text { tion (development } \\
\text { of an aller-gen-spe- } \\
\text { cific IgE immune } \\
\text { response) }\end{array}$ & $\begin{array}{l}\text { gastro-intestinal (or cuta- } \\
\text { neous) uptake of wheat } \\
\text { proteins with subsequent or } \\
\text { concomitant IgE- sensitiza- } \\
\text { tion to stable pro-teins }\end{array}$ & $\begin{array}{l}\text { gastro-intestinal } \\
\text { uptake of wheat } \\
\text { proteins with sensi- } \\
\text { tization to especial- } \\
\text { ly omega-5-gliadin }\end{array}$ & $\begin{array}{l}\text { inhalation of wheat flour } \\
\text { and dust during grain } \\
\text { processing and subse- } \\
\text { quent sensitization to } \\
\text { water-soluble allergens }\end{array}$ & $\begin{array}{l}\text { use of local } \\
\text { cosmetics on } \\
\text { the skin }\end{array}$ \\
\hline Affected (age) group & $\begin{array}{l}\text { infants/children/rarely } \\
\text { adults }\end{array}$ & adults/adolescents & $\begin{array}{l}\text { Exposed work-ers, typi- } \\
\text { cally bakers }\end{array}$ & $\begin{array}{l}\text { adolescents/ } \\
\text { adults }\end{array}$ \\
\hline \multirow[t]{5}{*}{$\begin{array}{l}\text { Wheat allergens } \\
\text { involved }\end{array}$} & $\begin{array}{l}\text { gliadins ( } \omega \text {-5-gliadin most } \\
\text { important (?) }\end{array}$ & $\begin{array}{l}\omega \text {-5-gliadin, LTP (Tri } \\
\text { a 14) }\end{array}$ & $\begin{array}{l}\text { combination of Tri a } \\
27,28,29,39,32 \text { gives } \\
\text { highest sensitivity and } \\
\text { specificity }\end{array}$ & $\begin{array}{l}\text { hydrolyzed } \\
\text { wheat pro- } \\
\text { tein (HWP)/ } \\
\text { gluten }\end{array}$ \\
\hline & \multicolumn{4}{|l|}{ HMW glutenins } \\
\hline & \multicolumn{4}{|l|}{ LMW-glutenins } \\
\hline & AAl's & & $\begin{array}{l}\text { sensitization to several } \\
\text { other al-lergens includ- } \\
\text { ing AAl's (particularly } \\
\text { Tri a } 15 \text { and 30), Tri a } 21 \\
\text { and } 33 \text { common }\end{array}$ & \\
\hline & $\begin{array}{l}\text { several others with varying } \\
\text { sensitization rate }\end{array}$ & & & \\
\hline
\end{tabular}




\section{Table 3 (continued)}

Clinical pattern of different types of wheat allergy

\begin{tabular}{|c|c|c|c|c|}
\hline & A & B & C & D \\
\hline Allergen abundance & & & Not known & common in cosmetics \\
\hline Thermal stability & high & high & low? & high? \\
\hline Digestive stability & high & high & low? & \\
\hline $\begin{array}{l}\text { Prevalence (North } \\
\text { and Middle Europe) }\end{array}$ & high & low & low? & low \\
\hline $\begin{array}{l}\text { Prevalence (South- } \\
\text { ern Europe) }\end{array}$ & moderate & low & low? & low \\
\hline $\begin{array}{l}\text { Elicitators (prod- } \\
\text { ucts) }\end{array}$ & all wheat products & $\begin{array}{l}\text { ingested wheat } \\
\text { prior to exercise }\end{array}$ & $\begin{array}{l}\text { wheat flour and dust } \\
\text { in the air }\end{array}$ & $\begin{array}{l}\text { hydrolyzed gluten in cos- } \\
\text { metics such as facial soap }\end{array}$ \\
\hline \multirow[t]{2}{*}{ Symptoms } & $\begin{array}{l}\text { quick onset (minutes to } \\
2 \text { hours) of potentially } \\
\text { severy systemic reactions } \\
\text { with various symptoms of } \\
\text { anaphylaxis: }\end{array}$ & $\begin{array}{l}\text { quick onset urti- } \\
\text { caria, angioedema, } \\
\text { and/or systemic } \\
\text { symptoms of ana- } \\
\text { phylaxis }\end{array}$ & $\begin{array}{l}\text { airway symptoms } \\
\text { (i.e. allergic rhinocon- } \\
\text { junctivis and asthma } \\
\text { symptoms) within few } \\
\text { hours of exposure }\end{array}$ & \\
\hline & $\begin{array}{l}\text { mucosal (i.e. oro-pharyn- } \\
\text { geal), cutaneous (urticar- } \\
\text { ia, angioedema, eczema } \\
\text { flaring), airway-related } \\
\text { (upper and lower air- } \\
\text { ways), gastrointestinal } \\
\text { and/or cardiovascular } \\
\text { symptoms }\end{array}$ & & & $\begin{array}{l}\text { typically urticaria after } \\
\text { application of the cream/ } \\
\text { soap. Ingestion of foods } \\
\text { containing deami-dated } \\
\text { gluten can cause systemic } \\
\text { reactions including ana- } \\
\text { phylaxis }\end{array}$ \\
\hline $\begin{array}{l}\text { Additional clinical } \\
\text { features }\end{array}$ & $\begin{array}{l}\text { atopic eczema ( infants } \\
\text { with wheat allergy), reac- } \\
\text { tions after other cereals } \\
\text { including rye and barley, } \\
\text { rarely oats }\end{array}$ & $\begin{array}{l}\text { alcohol consump- } \\
\text { tion enhances } \\
\text { responsiveness }\end{array}$ & & $\begin{array}{l}\text { usually patients do not } \\
\text { have other types of wheat } \\
\text { allergy }\end{array}$ \\
\hline Medical diagnosis & $\begin{array}{l}\text { obvious and repeata-ble } \\
\text { food allergic reaction to } \\
\text { wheat (porridge, bread, } \\
\text { pasta etc.) }\end{array}$ & $\begin{array}{l}\text { symptoms and his- } \\
\text { tory, sensitiza-tion } \\
\text { to omega-5-gliadin, } \\
\text { in some cases chal- } \\
\text { lenge test }\end{array}$ & $\begin{array}{l}\text { Allergic asthma and } \\
\text { rhinoconjunctivitis } \\
\text { due to wheat protein } \\
\text { inhalation }\end{array}$ & $\begin{array}{l}\text { obvious contact urticaria } \\
\text { when using HWP con- } \\
\text { tain-ing products } \\
\text { Patients can also develop } \\
\text { type A food allergy. }\end{array}$ \\
\hline Type of food allergy & class 1 & class 1 & $\begin{array}{l}\text { primary inhalant } \\
\text { allergy }\end{array}$ & class I \\
\hline Avoidance & $\begin{array}{l}\text { No oral intake of wheat } \\
\text { at significant amounts } \\
\text { (milligram levels) }\end{array}$ & $\begin{array}{l}\text { No ingestion of } \\
\text { wheat }\end{array}$ & $\begin{array}{l}\text { occupational avoid- } \\
\text { ance strategies in sit- } \\
\text { uations of large wheat } \\
\text { protein exposure }\end{array}$ & $\begin{array}{l}\text { cosmetics containing } \\
\text { HWP, in those with } \\
\text { sys-temic reactions avoid } \\
\text { ance of HWP in food }\end{array}$ \\
\hline Product declaration & $\begin{array}{l}\text { mandatory on each } \\
\text { wheat-containing prod- } \\
\text { uct (EU law) }\end{array}$ & $\begin{array}{l}\text { mandatory on each } \\
\text { wheat-containing } \\
\text { product (EU law) }\end{array}$ & - & $?$ \\
\hline Technical solution & $\begin{array}{l}\text { no general technical } \\
\text { solution available }\end{array}$ & $\begin{array}{l}\text { no technical solu- } \\
\text { tions }\end{array}$ & $\begin{array}{l}\text { dust extractor in oc- } \\
\text { cupational settings }\end{array}$ & $?$ \\
\hline
\end{tabular}


stomach pain and diarrhea or loose stools.

Early presumably gastro-intestinal or cutaneous sensitization to rather stable wheat allergens (for example, omega-5-gliadin, HMW glutenin, LMW glutenin, alpha-amylase inhibitor) in often atopic infants is the basis of most typical IgE-mediated wheat allergy, extending often until school-age and in rare cases, up to adulthood.

B) wheat-dependent, exercise-induced anaphylaxis (WDEIA) means the appearance of severe symptoms after ingestion of wheat followed by physical exercise typically among young adults and adult individuals. Symptoms vary from generalized urticaria to severe anaphylactic reaction. Sensitization to omega-5-gliadin is the most specific marker for the disease but the patients are sensitized also to a number of other wheat allergens. This is an important albeit not highly prevalent form of wheatallergy.

C) baker's allergy or asthma comes from inhalation of the wheat flour. At the moment, the test allergen with most sensitivity but low specificity is whole-wheat flour (including all allergens). The specificity of testing can be improved by component-specific analysis (Table 3 ).

D) contact urticaria associated with use of cosmetics and also sometimes together with food allergy. Hydrolysis of wheat is carried out to overcome its insolubility in cosmetics and exposure to hydrolyzed wheat protein (HWP) can cause either contact urticaria or even anaphylaxis when consuming deamidated gluten containing food

\section{Clinical diagnosis}

Typical case history, careful anamnesis, proper interpretation of sensitization tests (i.e. SPT, $\operatorname{lgE}$ ) and as the only golden standard, food challenge, form the basis of diagnosis for wheat allergy.

\section{Case history}

The most typical clinical picture of wheat-induced food allergy is similar to the other early childhood food allergies. The symptoms vary from eczema flares to anaphylaxis and the association of ingested food possibly containing wheat sometimes leads to the right direction. When the symptoms are vague and delayed the best way to proceed is to put the child on elimination diet for 1-2 weeks and monitor the response. It is, however, not recommended to test children with mild-to-moderate atopic eczema without

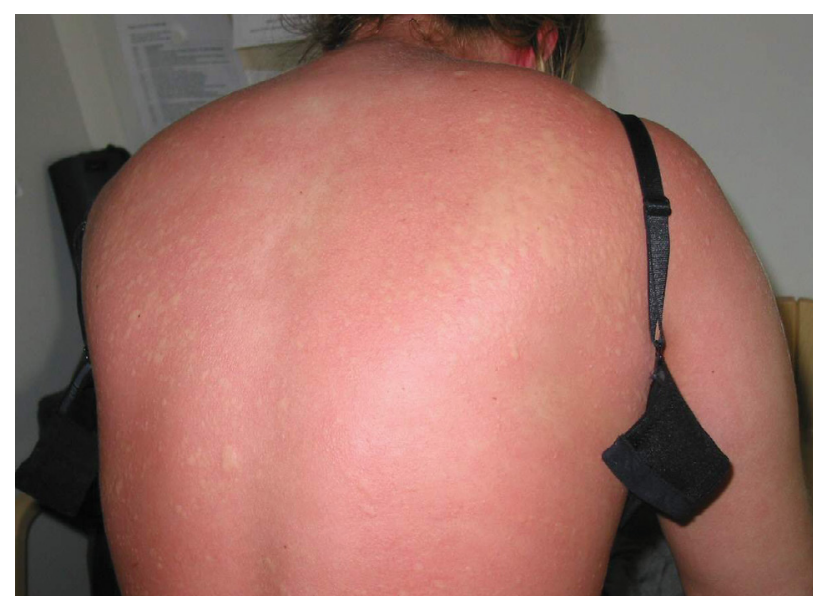

\section{Figure 3}

Severe urticaria on the whole body seen in a young woman after wheat ingestion and exercise challenge.

other symptoms for wheat due to common skin prick test positivity at this age without clinical relevance.

WDEIA should be suspected when a patient has experienced severe urticaria attacks or even anaphylaxis associated recurrently with exercise and eating preceded the exercise (Fig. 3). Sometimes the level of exercise can be relatively light such as brisk walking. It is also important to inquire possible use of alcohol and/or of acetylsalicylic acid (ASA) at the time of the reaction (19).

Baker's allergy is mainly seen in occupational settings and it should be suspected with patients suffering from chronic severe rhinitis and/or asthma symptoms.

Contact urticaria with use of cosmetics is relatively common and hydrolyzed wheat should always be thought as one possible inducer.

\section{Skin prick test (SPT)}

- comercial wheat extract or in-house solution employing wheat flour. Some authorities have claimed that this should not be used at all due to the very low specificity for all types of wheat allergy. Specificity can be improved by additional testing to omega-5 gliadin (dissolved in ethanol, in-house preparation) or other gliadins. For clinical pattern D, hydrolyzed wheat protein should be tested. There is little experience on SPT testing for other single proteins. 


\section{IgE-Testing}

allergen-specific IgE (commercially available are whole wheat extract, Tri a 14, Tri a 19, gliadins)

- wheat extract (low specificity although high sensitivity) to clinical patterns A-C

- omega-5-gliadinand gliadins (alpha, beta, gamma) for clinical patterns $A$ and $B$

- lipid transfer proteins (Tri a 14) for A and B, probably no cross-reactivity with grass pollen although there are not enough data to exclude this. Measuring sensitization may help in differentiating wheat sensitization from pollen allergy in patients with high levels of grass pollenspecific IgE, not very sensitive

- AAl's, particularly dimeric 0.19, LMW and HMW glutenins, Tri a 37 for $A$ and $C$

- combination of Tri a 27,28,29,39,32 for C

\section{Challenge tests}

Clinical pattern A mainly: various protocols with whole wheat can be used, for example, challenge in children with wheat flakes containing porridge or bread (5). Start with low dose (1-50 mg) of wheatspecific protein. A suitable time interval between the increasing doses should be an hour (digestion of wheat may be slower than milk and egg). Continue up to $500 \mathrm{mg}-1 \mathrm{~g}$ of cumulative dose of wheat protein. Also double-blind placebo-controlled protocols have been published both for children and adults $(5,20)$.

Clinical patterns B and C: usually case history and IgE testing is enough for diagnosis. In uncertain cases, carefully monitored exercise challenge with high readiness for anaphylaxis after wheat ingestion may be considered. Some centers have used ASA or alcohol as additional provocating factor instead of exercise (19) (Fig. 3).

Pattern D: challenge on the skin with HWP containing cream

\section{Clinical management}

\section{Advices and avoidance}

Patients with severe wheat allergy should be discouraged to try different forms of wheat. There is no evidence of reduced allergenicity between different species of wheat such as spelt. Little is known of changes in allergenicity during processing.

A: Avoidance of all wheat containing products, the level of avoidance can be titrated according to symptoms. Those with anaphylaxis should avoid products even with small amounts of wheat. Those with delayed symptoms and IgE-negative to wheat should be encouraged to use maximal dose not eliciting symptoms.

B: Avoidance of all gluten containing wheat

C: Avoidance of inhalation of wheat containing proteins

D: Avoidance of cosmetics with hydrolyzed wheat protein

\section{Pharmacotherapy (emergency kit)}

A: For those with delayed reactions or mild systemic reactions, antihistamine at age-dependent dosagesis enough. Because of therapidabsorption of cetirizine as compared to other antihistamines such as loratadine or desloratadine, it may be the antihistamine-of-choice. For those with a history of severe reactions in food challenge or after unintentional ingestion of wheat containing products, use of adrenaline autoinjector should be instructed carefully

B: Adrenaline autoinjector

C: Symptomatic treatment as required for rhinitis/ asthma

\section{Allergen-specific immunotherapy}

At present there are no commercially available products. Clinical studies are being done on oral immunotherapy for clinical pattern $A$ but, as to date, there are not enough published data to draw conclusions about proper product to use or adequate protocols.

\section{Prognosis}

The studies on prognosis of wheat allergy have demonstrated a high rate of spontaneous resolution of the symptoms in children similar to that of milk or egg allergy $(8,21,22,23)$. In one study, sensitization to gliadins correlated best with persistent wheat hypersensitivity and the development of asthma in children (22). Generally, high levels of wheat-specific IgE predict slower resolution and those with IgE negative allergy are clinically tolerant by age three. Taken altogether, children should be challenged at certain intervals, in early childhood yearly, to test for development of tolerance. 


\section{Textbox 1}

\section{Highlights}

- wheat allergy can manifest as different clinical conditions including typical childhood food allergy, wheat-dependent exercise-induced food allergy and baker's allergy/asthma

- The IgE response is diverse among patients and is directed against a number of allergens in all clinical conditions

- specific IgE to whole wheat extract is often positive without clinical significance in all age groups, not only due to heavy crossreactivity with grass pollen

- component-specific measurement of the response improves specificity but at the moment the only golden standard remains food challenge

\section{CLINICAL CASES}

\section{Case 1 (original)}

History: 9-year old boy. Atopic dermatitis since early infancy. First obvious reactions to wheat (skin flares with eczema, urticaria) soon after start of wheat at 6 months. First anaphylactic reaction to wheat at 2 years of age with generalized urticaria, bronchial obstruction and vomiting. Strict avoidance and adrenalin autoinjector continued until now. Developed also birch allergy at 2 years of age and later other pollen sensitizations. Avoids some fresh vegetables such as cucumber, pea, nuts and banana due to oral allergy syndrome symptoms. The family wants to know whether to continue avoiding wheat or not.

SPT: whole wheat extract $9 \mathrm{~mm}$, omega-5-gliadin 7 $\mathrm{mm}$, rye 5 , barley 5 , oat 0 . Birch $7 \mathrm{~mm}$, timothy grass $5 \mathrm{~mm}$.

Specific serum IgE: wheat $390 \mathrm{kU} / \mathrm{l}$, omega-5-gliadin $18 \mathrm{kU} / \mathrm{l}$, timothy grass $40 \mathrm{kU} / \mathrm{l}$, birch $100 \mathrm{kU} / \mathrm{l}$.

Oral challenge: wheat porridge $1 \mathrm{ml}(=13 \mathrm{mg}$ wheat protein) as a starting dose: mild tickling in the mouth which resolves spontaneously. With doubling the dose, the boy starts vomiting, complains of nasal obstruction.

Diagnosis: wheat allergy.
Recommendation: complete avoidance of wheat, adrenalin autoinjector guidance for the boy and all caretakers.

\section{Case 2 (original)}

History: 30-year-old female nurse. Generally healthy and mild pollen allergy. During the last few years occasional urticaria which the patient has sometimes linked to wheat ingestion (bread, pasta). Occasionally urticarial appearance after brisk walk or jogging.

SPT: whole wheat extract $5 \mathrm{~mm}$, omega-5-gliadin 7 $\mathrm{mm}$, rye 3 , barley 3 , oat 0 .

Specific serum IgE: wheat $20 \mathrm{kU} / \mathrm{l}$, omega-5-gliadin $10 \mathrm{kU} / \mathrm{l}$.

Challenge: large amount of pasta ingested. One hour later a nurse-controlled free field running test for 6 minutes was performed. During the last 2 minutes patient starts complaining severe itching and develops rapidly massive generalized urticaria, no signs of bronchial obstruction or severe gastrointestinal symptoms. Receives adrenalin, which relieves urticaria (see Figure 3 after one adrenalin injection). The urticaria starts increasing again in 15 minutes, and the patient receives another adrenalin shot. After this, the urticaria is resolved, and the patient feels fine.

Diagnosis: wheat-dependent exercise-induced urticaria.

Recommendation: 4 hours after ingestion of wheat no exercise including brisk walking.

\section{Case 3 (original)}

History: 37-year-old female cook who later studied to become pastry chef/ baker. After 2 years of working as a baker the patient started experiencing nasal symptoms first from rye flour and later from wheat and malt flours. No asthmatic symptoms at any time.

SPT: whole wheat extract $0 \mathrm{~mm}$ as were the other cereals

specific serum IgE: in-house immunoassay with the working place dust from wheat flour and rye flour positive. Wheat IgE $2.1 \mathrm{kU} / \mathrm{l}$, rye $0.8 \mathrm{kU} / \mathrm{l}$.

Challenge: chamber challenge with wheat flour induced nasal symptoms and also increase in nasal resistance (acoustic rhinomanometry). 
Diagnosis: occupational allergic rhinitis due to wheat and rye (baker's allergy)

Recommendation: primarily a respiration filter and change of the station in the kitchen. This did not help this patient enough so she is considering to learn a new profession.

\section{References}

1. Sapone A, Bai JC, Ciacci C, Dolinsek J, Green PH, Hadjivassiliou $M$ et al. Spectrum of gluten-related disorders: consensus on new nomenclature and classification. BMC Med 2012;10:13.

2. LongoG,Bertil,BurksAW,KraussB,BarbiE.IgE-mediated food allergy in children. Lancet 2013;382:16561664.

3. Ostblom E, Lilja G, Pershagen G, van Hage M, Wickman M. Phenotypes of food hypersensitivity and development of allergic diseases during the first 8 years of life. Clin Exp Allergy 2008;38:1325-1332.

4. Constantin C, Quirce S, Poorafshar M, Touraev A, Niggemann B, Mari A, et al. Micro-arrayed wheat seed and grass pollen allergens for component-resolved diagnosis. Allergy 2009;64: 1030-1037.

5. Mäkelä MJ, Eriksson $C$, Kotaniemi-Syrjänen $A$, Palosuo K, Marsh J, Borres $M$ et al. Wheat allergy in children - new tools for diagnostics. Clin Exp Allergy 2014;44:1420-1430.

6. Palosuo K, Varjonen E, Kekki OM, Klemola T, Kalkkinen N, Alenius $\mathrm{H}$ et al. Wheat omega-5 gliadin is a majorallergen in children with immediate allergy to ingested wheat. J Allergy Clin Immunol 2001;108:634638.

7. Ito K, Futamura M, Borres MP, Takaoka Y, Dahlstrom J, Sakamoto T et al. IgE antibodies to $\omega$ - 5 gliadin associate with immediate symptoms on oral wheat challenge in Japanese children. Allergy 2008;63:1536-1542.

8. Nilsson N, Sjölander S, Baar A, Berthold M, Pahr S, Vrtala S et al. Wheat allergy in children evaluated with challenge and IgE antibodies to wheat components. Pediatr Allergy Immunol 2015;26:119-125.

9. Sander I, Rihs H-P, Doekes G, Quirce S, Krop E, Rozynek $P$ et al. Component-resolved diagnosis of baker's allergy based on specific IgE to recombinant wheat flour proteins. J Allergy Clin Immunol 2015;135:152937.

10. Tatham AS, Shewry PR. Allergens to wheat and related cereals. Clin Exp Allergy 2008;38:1712-1726.

11. Palacin A, Quirce $S$, Armentia A, Fernández-Nieto $M$, Pacios LF, Asensio T et al. Wheat lipid transfer protein is a major allergen associated with baker's asthma. J Allergy Clin Immunol 2007;120:1132-1138.
12. Pastorello EA, Farioli L, Conti A, Pravettoni V, Bonomi $S$, lametti $S$ et al. Wheat IgE-mediated food allergy in European patients: alpha-amylase inhibitors, lipid transfer proteins and low-molecular-weight glutenins. Allergenic molecules recognized by double-blind, placebo-controlled food challenge. Int Arch Allergy Immunol 2007;144:10-22.

13. Zuidmeer L, Goldhahn K, Rona RJ, Gislason D, Madsen C, Summers $C$ et al. The prevalence of plant food allergies: a systematic review. J Allergy Clinlmmunol 2008;121:1210-1218.

14. Venter C, Pereira B, Grundy J, Clayton CB, Arshad $\mathrm{SH}$, Dean T. Prevalence of sensitization reported and objectively assessed food hypersensitivity amongst sixyear-old children: a population-based study. Pediatr Allergy Immunol 2006;17:356-363.

15. Palosuo K, Alenius H, Varjonen E, Kalkkinen N, Reunala T. Rye gamma-70 and gamma-35 secalins and barley gamma-3 hordein cross-react with omega- 5 gliadin, a major allergen in wheat-dependent, exercise-induced anaphylaxis. Clin Exp Allergy 2001;31:466-473.

16. Pahr S, Constantin C, Papadopoulos NG, Giavi S, Mäkelä $\mathrm{M}$, Pelkonen $\mathrm{A}$ et al. a-Purothionin, a new wheat allergen associated with severe allergy. J Allergy Clin Immunol 2013;132:1000-1003.

17. Baar A, Pahr S, Constantin C et al. The high molecular weightgluteninsubunitBx7allergenfromwheatcontains repetitive IgE epitopes. Allergy 2014;69:1316-1323.

18. Pahr S, Constantin C, Mari A et al. Molecular characterization of wheat allergens specifically recognized by patients suffering from wheat-induced respiratory allergy. Clin Exp Allergy 2012;42:597-609.

19. Brockow K, KneissI D, Valentini L, Zelger O, Grosber $\mathrm{M}$, Kugler $\mathrm{C}$ et al. Using a gluten oral food challenge protocol to improve diagnosis of wheat-dependent exercise-induced anaphylaxis. J Allergy Clin Immunol 2015;135:977-984.

20. Scibilia J, Pastorello EA, Zisa G, Ottolenghi A, BindslevJensen $C$, Pravettoni $V$ et al. Wheat allergy: a doubleblind, placebo-controlled study in adults. J Allergy Clin Immunol 2006;117:433-439.

21. Kotaniemi-Syrjänen $A$, Palosuo $K$, Jartti T, Kuitunen M, Pelkonen AS, Mäkelä MJ. The prognosis of wheat hypersensitivity in children. Pediatr Allergy and Immunol 2010;21(2p2):e421-e428.

22. Keet CA, Matsui EC, Dhillon G, Lenehan P, Paterakis M, Wood RA. The natural history of wheat allergy. Ann Allergy Asthma Immunol 2009;102:410-415.

23. Czaja-Bulsa G, Bulsa M. The natural history of IgE mediated wheat allergy in children with dominant gastrointestinal symptoms. Allergy Asthma Clin Immunol 2014;10:12. 



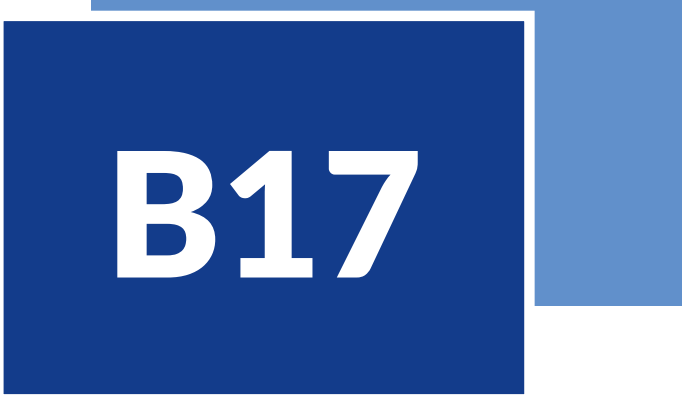

Jörg Kleine-Tebbe, Kirsten Beyer, Motohiro

\section{Ebisawa}

\section{THE ALLERGEN SOURCES}

Soybeans, a legume species native to East Asia, represent a rich and inexpensive source of proteins for animal feeds and foods, including textured vegetable protein (TVP) in many meat and diary analogues (Fig. 1).

Soybean is grouped within the "big eight" foods being responsible for $90 \%$ of all allergic reactions and an important allergen source due to the wide use in processed foods presenting a hidden allergen carrier (1). Fermented soy products such as soy sauce and miso are much less allergenic compared with tofu and soymilk (2, 3). Advising complete avoidance of all soy products impairs quality of life for soybean allergy patients.

Allergic reactions have been described after exposure to

A) highly processed soy (containing) products, including TVP (for review see $(1,4)$.

B) hardly processed (5-7) as well as

C) unprocessed soy beans (8-11).

Soy allergens can induce food allergic reactions and occupational inhalant allergies

IgE sensitizations to stable seed storage proteins Gly $m$ 5, 6 and 8, more common in infants, are associated with severe allergic reactions

In regions with birch pollen exposure Bet $v$ 1-crossreactive soy allergen Gly $m 4$ induces the most common soy allergy

Oropharyngeal and sometimes severe reactions to Gly $m 4$ are limited to fresh, hardly processed soy protein containing products 


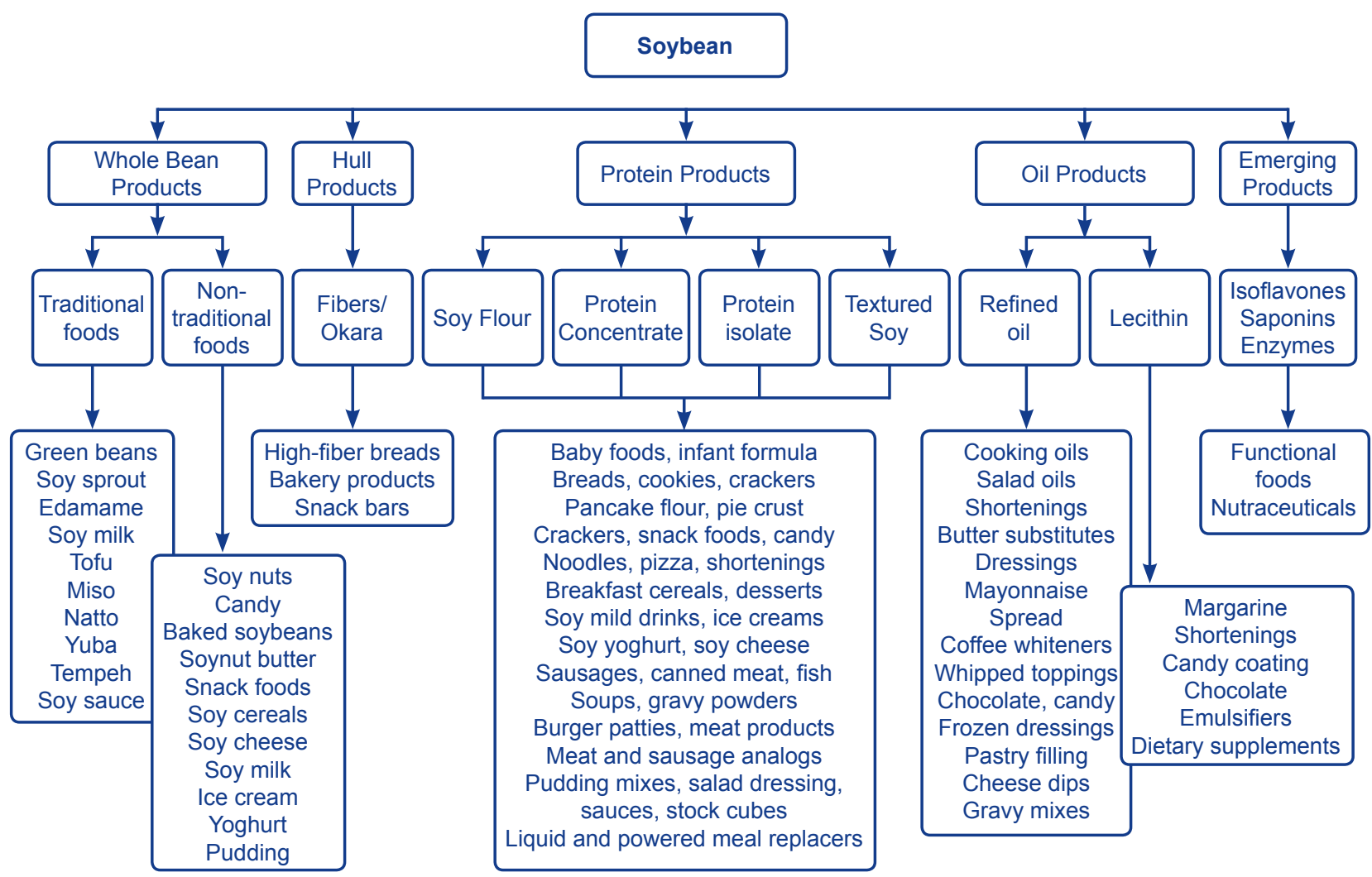

Figure 1

Soybean foods and ingredients (figure modified from (1)).

A) Since many decades, occasionally severe allergic reactions primarily in children have been attributed to processed soy containing foods (12), based on IgE to stabile, officially recognized soybean allergens (see Table 53, www.allergen. org) and some suggested soy proteins (see www. allergome.org; search for Gly $\mathrm{m}$ ).

B) Since 2002 allergic reactions have increasingly been recognized in birch pollen allergic individuals after ingestion of hardly processed dietary soy powder containing or soy drink products $(5,6$, 13). These often oro-pharyngeal symptoms due to IgE-related cross reactivity present the most common type of soy allergy in the northern hemisphere, particularly in regions with relevant birch pollen exposure (13).

C) High exposure and inhalation of raw unprocessed soybeans during unloading of freight ships caused epidemic outbreaks of inhalant allergies in harbor workers as well as citizens in close vicinity (8-11).

\section{MAJOR AND RELEVANT MINOR ALLERGENIC MOLECULES}

At least 16 soybean allergens have been identified displaying metabolic, storage or protective functions (for review see (1). ). They belong to diverse protein families characterized by conserved three-dimensional structures leading to broad immunochemical lgE mediated cross-reactions among different members of the legume families or other plant foods. Due to complexity and heterogeneity of soybean proteins the relationship between their structure and subsequent allergenicity has only partly been addressed.

In the past numerous fractions of soybeans and various soy proteins with partial characterization were proposed to induce IgE-mediated hypersensitivity food reactions (for extensive review of older literature on soy allergy until 2000 see online repository http://www. food-allergens.de and (4), newer literature until 2007 see (1) or inhalant allergies (i.e. Gly $\mathrm{m} 1$, Gly $\mathrm{m} 2$ ). 


\section{Table 1}

Allergenic molecules of soy

\begin{tabular}{|c|c|c|c|c|}
\hline Allergen & Biochemical name & MW & $\begin{array}{l}\text { Heat } \\
\text { stability }\end{array}$ & Isoforms \\
\hline Gly m 1 & hydrophobic protein (shell), LTP & 7 & $?$ & 2 \\
\hline Gly m 2 & defensin & 8 & low? & \\
\hline Gly m 3 & profilin & 14 & low & 2 \\
\hline Gly m 4 & $\begin{array}{l}\text { pathogenesis-related protein, PR-10, Bet v } 1 \\
\text { family member }\end{array}$ & 17 & low & 1 \\
\hline Gly m 5 & "beta-conglycinin (vicilin, 7S globulin)" & 63-65 ( $\alpha-$-s.u.) & & \\
\hline 48 (beta-subunit) & high & 2 ( $\alpha-$ s.u.) & & \\
\hline \multicolumn{5}{|l|}{2 (beta-subunit) } \\
\hline Gly m 6 & glycinin (legumin, 11S globulin) & $52-61$ (s.u.) & high & 5 (s.u.) \\
\hline Gly m 7 & seed biotinylated protein & 76 & $?$ & 1 \\
\hline Gly m 8 & $2 \mathrm{~S}$ albumin & 28 & high & 1 \\
\hline \multicolumn{5}{|l|}{ Gly m 39kD, P39 } \\
\hline Gly m Agglutinin & Agglutinin, Lectin & & & \\
\hline Gly m Bd28K & 7S vicilin-like globulins & & & \\
\hline Gly m 30kD & cysteine protease & & & \\
\hline Gly m CPI & cystatin & & & \\
\hline Gly m TI & trypsin inhibitor & & & \\
\hline
\end{tabular}

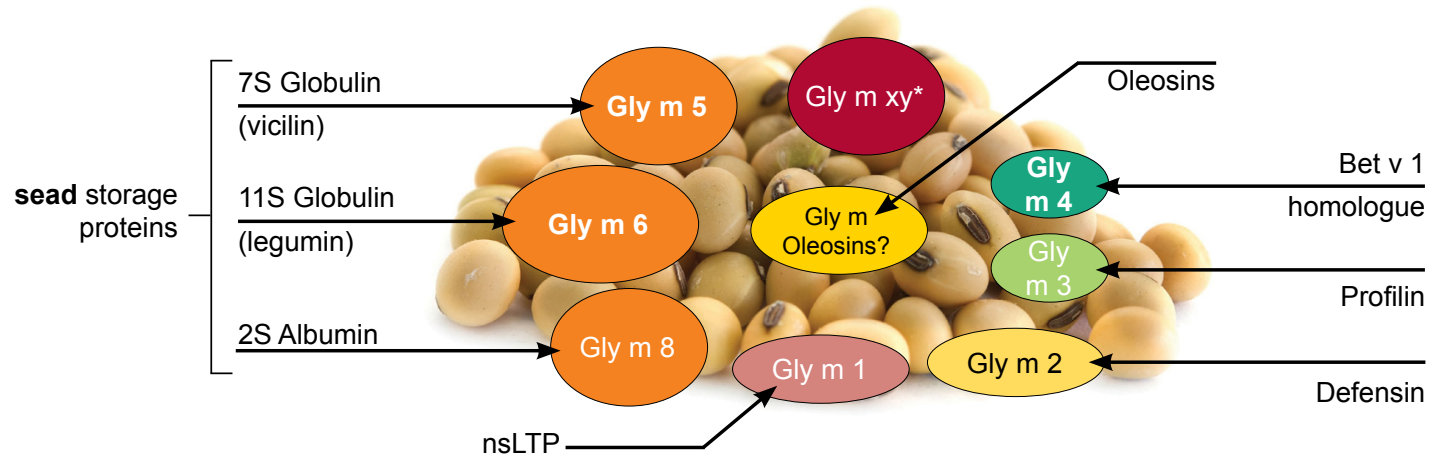

\section{Figure 2}

Major and relevant minor allergenic molecules of soy. Gly m xy: Gly m 39kD (P39), Gly m Agglutinin, Gly m Bd28K, Gly m 30kD, Gly m CPI, Gly m TI (soybean allergens without official designation, www.allergome. org), boldface: available for allergen-specific IgE diagnostics. 
During the last 15 years additional soy allergens (Gly m 4 - 8) have been identified and officially accepted by the IUIS Allergen Nomenclature Sub-Committee (Table 1).

\section{SENSITIZATION TO INDIVIDUAL MOLECULES AND THEIR CLINICAL RELEVANCE}

Reliable data on sensitization rates are available only for Gly $m$ 4, 5 and 6.

\section{Epidemiology and sensitization/cross reactivity rates}

Previous sensitization rates were mainly based on skin or IgE testing to whole soybean extracts (for recent review and summarized analysis of sensitization rates in children see (12): From 40 studies identified weighted prevalence of soy allergy in children was reported, providing ranges and total means (in brackets) between 0 and $0.5 \%(0.27 \%)$ for the general population, 0.4 to $3.1 \%(1.9 \%)$ for the referred population, and 0 to $12.9 \%$ (2.7\%) for lgEsensitized children (12).

IgE testing in a large cohort of approx. 13,000 young German subjects between 3 to 17 years revealed allergen-specific IgE to soybean extract in $6.3 \%$ of the cohort (14). A similar approach in approx. 7,000 German adults revealed sensitization rates of $3.7 \%$ to soybean extract and $10.3 \%$ to soybean allergen Gly m 4 (15).

Notably, conclusions on primary sensitization rates are hampered due to highly cross reactive, labile soybean allergens of low abundance:

- Bet v 1-homologue Gly m 4 and

- Soybean profilin Gly $\mathrm{m} 3$ (i.e. presumably in regions with high grass pollen exposure and subsequent sensitization to i.e. grass profilin).

Gly $\mathrm{m} 4$ (and possibly Gly $\mathrm{m} 3$ ) are underrepresented in diagnostic soybean extracts leading to vast differences between extract and single allergen based IgE results (15). In addition, the (low) presence of Gly $m 4$ (and Gly $\mathrm{m} 3$ ) in soybean extracts will obscure a differentiation of food reactions to stabile allergens (i.e. Gly $5(16,17)$, $6(16,17)$, and $8(18,19)$, likely to be well represented in soybean extracts) and cross reactions to the Bet v 1-homologue in soy, as demonstrated in cohort studies with atopic children (20).
Sensitization rates to Gly $\mathrm{m} 1$ - 3 and Gly $\mathrm{m} 5$ - 8 are still lacking.

\section{CLINICAL RELEVANCE, DIAGNOSIS AND MANAGEMENT}

\section{Clinical relevance and clinical pattern}

Due to different routes and amounts of exposure, degree of soybean processing and physicochemical properties of the involved soybean protein allergens, three distinct scenarios (Table 3, A - C) can prompt IgE-mediated sensitizations and clinical symptoms of immediate hypersensitivity:

A) (Early) presumably gastro-intestinal (or cutaneous?) sensitization to rather stable soybean allergens (i.e. Gly $5(16,17), 6(16,17)$, and $8(18$, 19 ), and many others) in atopic individuals are the basis of subsequent severe systemic reactions after ingestion of small amounts of (processed) soy products $(1,4)$, IgE-sensitizations (i.e. to seed storage proteins such as $7 \mathrm{~S}$ globulins, $11 \mathrm{~S}$ globulins and $2 S$ albumins) could also evolve from exposure and subsequent IgE-sensitization to more than one legume (i.e. peanut and soy) prompting symptoms occasionally after ingestion of peanut as well as soy products. These reactions - considered rather rare - have mainly described in young infants $(16,18)$, and are hardly reported in adults $(8,17)$

B) Exposure to fagales pollen in atopic individuals developing Bet $v$ 1-specific IgE with variable degree of cross reactivity to soybean PR-10 protein Gly $\mathrm{m} 4$ (in $>70 \%$ of Bet $v$ 1-sensitized individuals), potentially inducing mainly oropharyngeal and sometimes systemic allergic symptoms after consumption of mildly processed soy products (i.e. soy protein powder, soy drinks etc.) in approx. $10 \%$ of birch pollen sensitized subjects (5-7). These individuals are likely to have very high IgE to birch pollen $(6,21)$.

This type of soy allergy due to Bet $v$ 1-crossreactions is considered the most prevalent soy allergy in Northern and Middle Europe, presumably also in Northern parts of Asia, as well as in North America (Canada, Northern states of the US), depending on the degree of birch pollen exposure.

C) Massive exposure to unprocessed soybeans inducing IgE-mediated sensitizations to hull 
Table 2

Clinical relevance of IgE sensitization to soy allergenic molecules

\begin{tabular}{|c|c|c|c|c|}
\hline Allergen & Allergenicity & Risk factor & $\begin{array}{l}\text { Sensitization } \\
\text { rate }\end{array}$ & $\begin{array}{l}\text { Clinical } \\
\text { response rate }\end{array}$ \\
\hline Gly m 1 & $\begin{array}{l}90 \% \text { of exposed } \\
\text { harbor workers }\end{array}$ & occupational massive exposure to soy beans & $?$ & $?$ \\
\hline Gly m 2 & $?$ & $?$ & $?$ & $?$ \\
\hline Gly m 3 & $?$ & $\begin{array}{l}\text { high grass pollen exposure, individuals with } \\
\text { broad pollen-specific IgE repertoire? }\end{array}$ & $?$ & $?$ \\
\hline Gly m 4 & $\begin{array}{l}70 \% \text { serological } \\
\text { Gly } \mathrm{m} 4 \text { cross } \\
\text { reactivity in Bet } \\
\vee 1 \text {-sensitized } \\
\text { individuals }\end{array}$ & $\begin{array}{l}\text { a) Birch pollen Bet v 1-related sensitization } \\
\text { b) high native Gly m } 4 \text { content in less } \\
\text { processed soy products " }\end{array}$ & $\begin{array}{c}10.3 \% \text { in a } \\
\text { representative } \\
\text { cohort of }>7000 \\
\text { German adults } \\
\text { (583) }\end{array}$ & $\begin{array}{l}\text { 10(-20?)\% clinical } \\
\text { Gly m } 4 \text { cross } \\
\text { reactivity in Bet } \\
\text { v } 1 \text {-sensitized } \\
\text { individuals (575) }\end{array}$ \\
\hline Gly m 5 & $?$ & $\begin{array}{l}\text { elevated general atopic risk for infant (adult) } \\
\text { food allergies, atopic eczema and exposure } \\
\text { to seed storage proteins from soy }\end{array}$ & $?$ & $?$ \\
\hline Gly m 6 & $?$ & $\begin{array}{l}\text { elevated general atopic risk for infant (adult) } \\
\text { food allergies, atopic eczema and exposure } \\
\text { to seed storage proteins from soy }\end{array}$ & $?$ & $?$ \\
\hline Gly m 7 & $?$ & $?$ & $?$ & $?$ \\
\hline Gly m 8 & $?$ & $\begin{array}{l}\text { elevated general atopic risk for infant (adult) } \\
\text { food allergies, atopic eczema and exposure } \\
\text { to seed storage proteins from soy }\end{array}$ & $?$ & $?$ \\
\hline
\end{tabular}

allergens (Gly m 1, Gly m 2) with subsequent inhalant allergies in exposed (newly or formerly nsLTP-sensitized) individuals (8-11).

\section{Clinical diagnosis}

Specific questions, proper interpretation of sensitization tests (i.e. SPT, IgE) and optional food challenges help to establish the diagnosis of soy allergy. Following work-up (Fig. 3) might facilitate proper diagnosis.

\section{Case history (anamnesis)}

Previous reaction(s) to soy, symptoms, affected organ system(s), onset and course (progression), soy containing food items (highly-, mildly-, nonprocessed grade), co-factors (exercise, NSAID, alcohol etc.), previous reactions after other allergen sources (i.e. peanuts or Bet $\vee$ 1-related plant foods in table 3, see Section C02 on Bet v 1-related cross reactivity), additional (allergic) features, i.e. birch pollen allergy, LTP sensitization (see Section C03 on LTP allergy syndrome), additional atopic diseases, i.e. atopic eczema, asthma.
Working hypothesis: based on soy-related clinical pattern A - C (Table 3).

\section{Skin prick test (SPT)}

- commercial soy extract (reasonable results in case of highly abundant allergens, i.e. seed storage proteins, limited value due to false negative responses in case of cross reactive labile soy allergen, i.e. Gly $\mathrm{m} 4$ as elicitators),

- prick-prick test with offending soy product (in case of severe anaphylactic reaction, preferably titrated testing with diluted soy product or primary IgE-testing before SPT; potentially false negative, depending on the abundance and stability of the soy allergen in question, i.e. Gly $\mathrm{m} 4$ )

- prick-prick test with mildly processed soy drink or soy powder (if Gly m 4 is suspected)

\section{IgE-Testing}

total total IgE (for appropriate interpretation of allergen-specific IgE) 


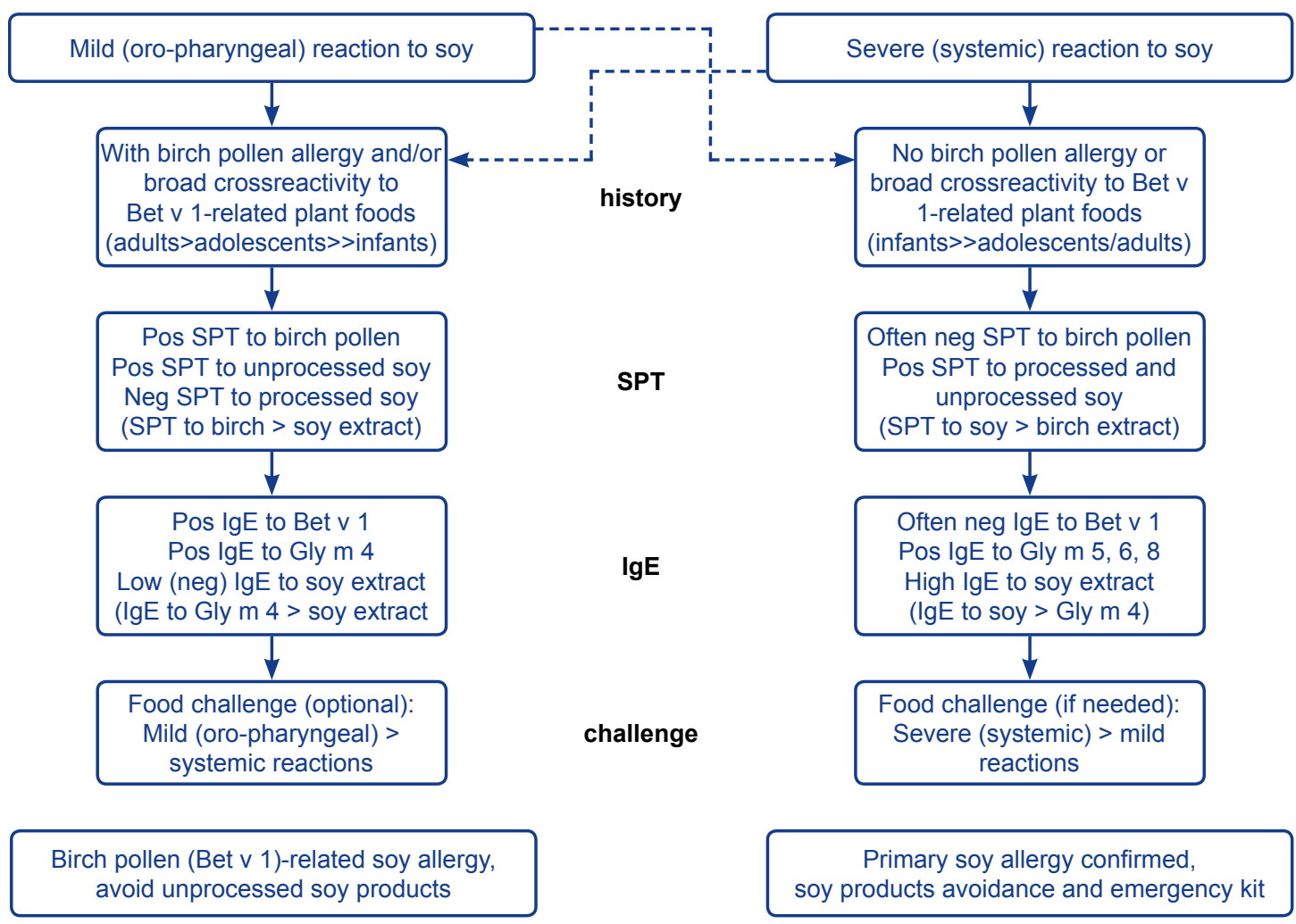

\section{Figure 3}

Diagnostic work-up in soy-related allergic reactions (representing food allergy class 2 in left column and class 1 in right column). Arrows indicate potential diagnostic steps; dashed arrows indicate that mild as well as severe reactions can be associated with different clinical features (information from the allergy history).

\section{Table 3}

Features and clinical pattern of three different types of soy allergy

\begin{tabular}{|c|c|c|c|}
\hline & $\mathbf{A}$ & B & C \\
\hline \multirow{3}{*}{$\begin{array}{l}\text { Routes of } \\
\text { sensitization } \\
\text { (development of } \\
\text { an allergen-spe- } \\
\text { cific lgE immune } \\
\text { response) }\end{array}$} & $\begin{array}{l}\text { 1. gastro-intestinal (or cuta- } \\
\text { neous) uptake of soybean } \\
\text { proteins with subsequent } \\
\text { lgE- sensitization to stable } \\
\text { soybean proteins }\end{array}$ & $\begin{array}{l}\text { 1. inhalation of birch pollen } \\
\text { allergens and subsequent } \\
\text { IgE-sensitization to major } \\
\text { allergen Bet } v 1\end{array}$ & $\begin{array}{l}\text { 1.inhalation of (unprocessed) } \\
\text { soybeans and subsequent } \\
\text { sensitization to hull aller- } \\
\text { gens }\end{array}$ \\
\hline & or & or & or \\
\hline & $\begin{array}{l}\text { 2. gastro-intestinal (or cu- } \\
\text { taneous) sensitization of } \\
\text { other legume proteins (i.e. } \\
\text { peanut) with subsequent } \\
\text { cross-reactivity to i.e. stable } \\
\text { soybean proteins }\end{array}$ & $\begin{array}{l}\text { 2. presumably inhalation of } \\
\text { (grass) pollen allergens and } \\
\text { subsequent sensitization to } \\
\text { pollen profilin (i.e. from grass- } \\
\text { es, Phl p } 12 \text { from timothy)? }\end{array}$ & $\begin{array}{l}\text { 2. previous sensitization to } \\
\text { LTP-containing plant foods } \\
\text { (i.e. peach) or LTP-contain- } \\
\text { ing pollen (mugwort, pari- } \\
\text { etaria, plane tree) with cross } \\
\text { reactivity to soy LTP? }\end{array}$ \\
\hline $\begin{array}{l}\text { Affected (age) } \\
\text { group }\end{array}$ & $\begin{array}{l}\text { infants/children } \\
>\text { adults }\end{array}$ & $\begin{array}{l}\text { adults/adolescents } \\
>\text { children (occasoinally) }\end{array}$ & $\begin{array}{l}\text { Exposed workers and citizens } \\
\text { in close vincinity }\end{array}$ \\
\hline
\end{tabular}


Table 3 (continued)

Features and clinical pattern of three different types of soy allergy

\begin{tabular}{|c|c|c|c|}
\hline & A & B & C \\
\hline $\begin{array}{l}\text { Soybean allergens } \\
\text { involved }\end{array}$ & $\begin{array}{l}\text { Gly } \mathrm{m} 5 \text {; Gly } \mathrm{m} 6 \text {; Gly } \mathrm{m} 7 \text { ? ;Gly } \mathrm{m} 8 \text { ?? } \\
\text { and others (without official IUIS- } \\
\text { name, see table 1) }\end{array}$ & Gly m 4; Gly m 3 (presumably) & $\begin{array}{l}\text { Gly m } 1 \text { (LTP); Gly m } 2 \\
\text { (defensin) }\end{array}$ \\
\hline $\begin{array}{l}\text { Allergen abun- } \\
\text { dance }\end{array}$ & $\begin{array}{l}\text { moderate to high content (i.e. seed } \\
\text { storage proteins Gly } \mathrm{m} 5,6 \text { and } 8 \text { ) in } \\
\text { soy beans and related products }\end{array}$ & $\begin{array}{l}\text { 1. low content of Gly m } 4 \text { in soy } \\
\text { beans and soy protein ( } 0.01- \\
0.1 \%) \text { and related, less pro- } \\
\text { cessed soy products } \\
2 . \text { Gly } m 3 \text { content not known }\end{array}$ & Not known \\
\hline Thermal stability & high & low & moderate \\
\hline Digestive stability & high & low & moderate \\
\hline $\begin{array}{l}\text { Prevalence (North } \\
\text { and Middle Eu- } \\
\text { rope) }\end{array}$ & low & high & $?$ \\
\hline $\begin{array}{l}\text { Prevalence } \\
\text { (Southern Europe) }\end{array}$ & low & low & $\begin{array}{l}\text { occasionally asthma } \\
\text { outbreaks in the past }\end{array}$ \\
\hline $\begin{array}{l}\text { Elicitators (prod- } \\
\text { ucts) }\end{array}$ & $\begin{array}{l}\text { Many soy products (including highly } \\
\text { processed or refined soy products) }\end{array}$ & $\begin{array}{l}\text { Hardly processed soy products } \\
\text { (soy drinks, soy protein powder, } \\
\text { even after 1st consumption }\end{array}$ & $\begin{array}{l}\text { Unprocessed soybeans } \\
\text { (i.e. during unloading) }\end{array}$ \\
\hline Symptoms & $\begin{array}{l}\text { quick onset (minutes to } 2 \text { hours) of } \\
\text { potentially severy systemic reactions } \\
\text { with various symptoms of anaphy- } \\
\text { laxis: mucosal (i.e. oro-pharyngeal), } \\
\text { cutaneous (urticaria, angioedema, } \\
\text { eczema flaring), airway-related (up- } \\
\text { per and lower airways), gastrointesti- } \\
\text { nal and/or cardiovascular symptoms }\end{array}$ & $\begin{array}{l}\text { quick onset ( } 2 \text { - } 30 \text { minutes) } \\
\text { mostly mild mucosal symptoms } \\
\text { (oro-pharyngeal), occasionally } \\
\text { severe reactions (angioedema, } \\
\text { i.e. eyelid-, lip-, ear- and/or } \\
\text { throat swelling) and/or rare sys- } \\
\text { temic symptoms of anaphylaxis } \\
\text { (see left column) }\end{array}$ & $\begin{array}{l}\text { airwary symptoms (i.e. } \\
\text { allergic rhinoconjunctiv- } \\
\text { is and asthma symp- } \\
\text { toms) within few hours } \\
\text { of exposure }\end{array}$ \\
\hline $\begin{array}{l}\text { Additional clinical } \\
\text { features }\end{array}$ & $\begin{array}{l}\text { atopic eczema (i.e. in infants with } \\
\text { soy allergy), sometimes also (poten- } \\
\text { tially severe) reactions after other } \\
\text { legumes, i.e. peanut, lupine (flour), } \\
\text { seeds or tree nuts }\end{array}$ & $\begin{array}{l}\text { commonly additional (multiple) } \\
\text { clinical cross reactivities to oth- } \\
\text { er Bev v 1-related plant foods, } \\
\text { i.e. apple, hazelnut, cherry, } \\
\text { plum, peach, carrot, celery }\end{array}$ & \\
\hline Medical diagnosis & $\begin{array}{l}\text { Risk of systemic food allergic reac- } \\
\text { tion to stable soybean proteins }\end{array}$ & $\begin{array}{l}\text { Birch pollen/Bet } v \text { 1-related } \\
\text { food allergy to cross reactive } \\
\text { soy allergen Gly } \mathrm{m} 4\end{array}$ & $\begin{array}{l}\text { Allergic asthma and rhi- } \\
\text { noconjunctivitis due to } \\
\text { soy protein inhalation }\end{array}$ \\
\hline $\begin{array}{l}\text { Type of food } \\
\text { allergy }\end{array}$ & class $\mathrm{A}$ & class B & primary inhalant allergy \\
\hline Avoidance & $\begin{array}{l}\text { Advising avoidance of allergenic soy } \\
\text { products, in most cases soy sauce } \\
\text { and miso can be consumed }\end{array}$ & $\begin{array}{l}\text { No ingestion of large amounts } \\
\text { of unprocessed soybean } \\
\text { products }\end{array}$ & $\begin{array}{l}\text { occupational avoidance } \\
\text { strategies in situations } \\
\text { of large soy protein } \\
\text { exposure (unloading of } \\
\text { soy shipments) }\end{array}$ \\
\hline $\begin{array}{l}\text { Product } \\
\text { declaration }\end{array}$ & $\begin{array}{l}\text { mandatory on each soy-containing } \\
\text { product (EU law) }\end{array}$ & $\begin{array}{l}\text { (voluntary) additional warning } \\
\text { on hardly processed soy } \\
\text { products particularly for birch } \\
\text { pollen allergic individuals }\end{array}$ & - \\
\hline $\begin{array}{l}\text { Technical } \\
\text { solution }\end{array}$ & $\begin{array}{l}\text { no general technical solution } \\
\text { available }\end{array}$ & $\begin{array}{l}\text { thermic or pressure processing } \\
\text { of soy to reduce content in } \\
\text { labile soy proteins }\end{array}$ & $\begin{array}{l}\text { dust extractor in } \\
\text { occupational settings }\end{array}$ \\
\hline
\end{tabular}


allergen-specific lgE:

- soy extract (22) (IgE potentially false negative or low titers in case of clinical pattern B),

- Gly m $5(16,17), 6(16,17)$ and $8(18,19)$ (if soyrelated clinical pattern A, Table 55)

- Gly $\mathrm{m} 4$ and/or Bet $\vee 1$ (if soy-related clinical pattern B, Table 3)

Interpretation of SPT and IgE (sensitization tests) outcome see also Fig. 81:

Results only clinically relevant in case of corresponding symptoms after soy ingestion.

\section{Challenge tests}

(in case of doubtful relationship between soy ingestion and allergic reaction):

Depending on the clinical pattern $(A-C)$ titrated (oral) provocation with

A) offending or another appropriate soy protein containing product (soy powder, tofu, soy drink)

B) preferably mildly processed soy protein containing product with soy allergens of low abundance (i.e. Gly $\mathrm{m}$ 4)

C) if needed, nasal/bronchial challenge with titrated soy extracts or the offending (unprocessed) soy products

\section{Clinical management}

\section{Advices and avoidance}

A) Avoidance of soy containing products depending on the symptom-eliciting-dose and the severity of the event (rule by thumb): in case of severe reactions after a small dose avoidance of small amounts, regardless of the grade of soy processing; in case of a mild reaction after a large dose less strict avoidance needed. Fermented soy products such as soy sauce and miso are much less allergenic compared with tofu and soymilk $(2,3)$ Advising complete avoidance of all soy products impairs quality of life for soybean allergy patients.

B) Avoidance of larger amounts of soy products, particularly if hardly or mildly processed (i.e. due to thermal processing, heating, like soy drinks, soy powders).

C) Avoidance of inhalation of soy containing (hull) proteins

\section{Pharmacotherapy (emergency kit)}

A) Due to the risk of severe reactions after unintentional ingestion of soy containing products emergency medication including selfadministered adrenaline required

B) Emergency medication optional (not mandatory)

C) Symptomatic treatment as required

\section{Allergen-specific immunotherapy}

At present commercially available products for allergen-specific immunotherapy of soybean protein allergy are not available.

\section{CLINICAL CASES}

\section{Case 1 (published (23))}

History: girl, 16 years old, atopic dermatitis: After $20 \mathrm{~min}$. bike riding anaphylactic reaction (abdominal pain, dyspnea, semiconsciousness, generalized flushing and swelling, low blood pressure), subsequent emergency treatment and hospitalization ( 2 days). Two similar reactions during bike riding before. No food allergy to soy, peanut or other food item known so far.

SPT: Tofu, soy milk, boiled green soybean, soybean flour weakly positive (half histamine-equivalent), miso, soy sauce and soybean fibers negative.

In-vitro testing: Total IgE $542 \mathrm{kU} / \mathrm{l}$, specific IgE to soybean $34 \mathrm{kUA} / \mathrm{l}$, peanut $1.3 \mathrm{kUA} / \mathrm{l}$, wheat, Omega-5-Gliadin, various pollen including birch pollen and Gly $\mathrm{m} 4$ negative.

Microarrayed specific IgE: high to Gly $m 5$ (ß-conglycinin); Gly m 6 (glycinin), Gly m 3, CCD negative.

Oral challenge: 30 minutes exercise after ingestion of $200 \mathrm{~g}$ tofu development of severe urticaria and face swelling. No reactions after boiled green soybeans, soy milk, without or with exercise.

Diagnosis: Food-dependent exercise-induced anaphylaxis (FDEIA) to soybean allergen Gly $\mathrm{m} 5$.

Recommendation: 4 hours after ingestion of soybean products (particularly tofu) no exercise.

\section{Case 2 (original)}

History: Male, born 1954: Since 1996 severe birch pollen induced rhinoconjunctivitis; after ingestion 
of raw apples, hazelnuts or strawberries oral itch. Adverse event after SCIT with non-modified birch pollen extract with severe systemic reaction.

In 2006 after soy dessert increasing local itch (after 5 min: mouth, palate; after 15 min: eyes) and complete eyelid swelling within $20-30 \mathrm{~min}$; subsequent emergency treatment and hospitalization overnight. In 2012 after 3 fresh apple pieces local itch (3 min: mouth, palate) and burning throat, after $15 \mathrm{~min}$. almost complete eye lid swelling, itchy eyes, stuffy nose and after 30 min itchy hives at upper limbs; subsequent emergency treatment.

In-vitro testing: 2007: Total IgE $37 \mathrm{kU} / \mathrm{l}$, specific IgE to Gly m 4: 2.3 kUA/l. 2012: Total IgE $48.5 \mathrm{kU} / \mathrm{l}$, specific IgE to Bet $\vee$ 1: $24 \mathrm{kUA} / \mathrm{I}$ (almost 50\% of total IgE specific for Bet $v 1$ indicating a strong sensitization to Bet $\vee 1$ with cross reactivity to structurally related allergens).

Diagnosis: Angioedema after soymilk ingestion due to Gly $\mathrm{m} 4$ (Bet v 1-cross reactive soy allergen)

Recommendations: Strict avoidance of mildly processed soy protein products and raw apples; great care with large amounts of raw, Bet $v 1$-cross reactive pome and stone fruits, tree nuts and legumes. Cooked plant products without dietary restriction (due to thermal instability of Bet $v$ 1-related allergens).

\section{Case 3 (primary sensitization (18))}

\section{History: Male (2y 9m)}

He had started to develop atopic eczema around his mouth at 4 months after birth. He first received blood examination at the age of 8 months and was already sensitized to egg white, wheat, and soybean (total lgE $250 \mathrm{IU} / \mathrm{ml}$, egg white $21.40 \mathrm{kU} /$ $\mathrm{ml}$, wheat $3.19 \mathrm{kU} / \mathrm{ml}$, and soybean $0.99 \mathrm{kU} / \mathrm{ml}$ ). He was advised to avoid those foods from diet by the doctor. He then visited a hospital at the age of $2 y 5 \mathrm{~m}$ to receive oral food challenges.

In-vitro testing: His laboratory finding at the first visit was as follows; total IgE $5650 \mathrm{IU} / \mathrm{ml}$, egg white 70 $\mathrm{kU} / \mathrm{ml}$, wheat $3 \mathrm{kU} / \mathrm{ml}$, and soybean $17.1 \mathrm{kUml}$ (Gly m 8: 37.6 kU/ml, Gly m 5: 4.9 kU/ml, Gly m 6: $1.1 \mathrm{kU} / \mathrm{ml})$.

Diagnosis: He then received soy product (Tofu) challenge at the age of $2 \mathrm{y} 9 \mathrm{~m}$, and developed skin rash, and sneezing + coughing at the dose of $9 \mathrm{~g}$ of tofu.
Recommendations: Avoidance of tofu, soymilk but not soy sauce, miso, and natto was advised.

Prognosis: He has naturally outgrown from soybean allergy 1 year later with soybean IgE $1.90 \mathrm{kU} / \mathrm{ml}$.

\section{References}

1. L'Hocine L, Boye Jl. Allergenicity of soybean: new developments in identification of allergenic proteins, cross-reactivities and hypoallergenization technologies. Crit Rev Food Sci Nutr 2007;47:127143.

2. Moriyama T, Yano E, Suemori Y, Nakano K, Zaima $\mathrm{N}$, Kawamura $\mathrm{Y}$. Hypoallergenicity of various miso pastes manufactured in Japan. J Nutr Sci Vitaminol (Tokyo) 2013;59:462-469.

3. Kobayashi M, Hashimoto Y, Taniuchi S, Tanabe S. Degradation of wheat allergen in Japanese soy sauce. Int J Mol Med 2004;13:821-827.

4. Besler M, Helm KM, Ogawa T. Allergen Data Collection - Update: Soybean (Glycine max). Internet Symposium on Food Allergens 2000;2 (Suppl.3):1-35 (http://www. food-allergens.de).

5. Kleine-Tebbe J, Vogel L, Crowell DN, Haustein UF, Vieths S. Severe oral allergy syndrome and anaphylactic reactions caused by a Bet $v$ 1- related PR-10 protein in soybean, SAM22. J Allergy Clin Immunol 2002;110:797-804.

6. Mittag D1, Vieths S, Vogel L, Becker WM, Rihs HP, Helbling A et al. Soybean allergy in patients allergic to birch pollen: clinical investigation and molecular characterization of allergens. J Allergy Clin Immunol 2004;113:148-154.

7. Ballmer-Weber BK, Vieths S. Soy allergy in perspective. Curr Opin Allergy Clin Immunol 2008;8:270-275.

8. Antó JM, Sunyer J, Rodriguez-Roisin R, SuarezCervera M, Vazquez L. Community outbreaks of asthma associated with inhalation of soybean dust. Toxicoepidemiological Committee. N Engl J Med 1989;320:1097-1102.

9. Rodrigo MJ, Morell F, Helm RM, Swanson M, Greife A, Anto JM et al. Identification and partial characterization of the soybean-dust allergens involved in the Barcelona asthma epidemic. J Allergy Clin Immunol 1990;85:778784.

10. Codina R, Lockey RF, Fernández CE, Rama R. Identification of the soybean hull allergens responsible for the Barcelona asthma outbreaks. Int Arch Allergy Immunol 1999;119:69-71.

11. Antó JM, Soriano JB, Sunyer J, Rodrigo MJ, Morell $\mathrm{F}$, Roca $J$ et al. Long term outcome of soybean epidemic asthma after an allergen reduction intervention. Thorax 1999;54:670-674. 
12. Katz Y, Gutierrez-Castrellon P, González MG, Rivas $\mathrm{R}$, Lee BW, Alarcon P. A comprehensive review of sensitization and allergy to soy-based products. Clinical Rev All Immunol 2014;46:272-281.

13. Ballmer-Weber BK, Holzhauser T, Scibilia J, Mittag D, Zisa G, Ortolani C et al. Clinical characteristics of soybean allergy in Europe: a double-blind, placebocontrolled food challenge study. J Allergy Clin Immunol 2007;119:1489-1496.

14. Schmitz R, Ellert U, Kalcklösch M, Dahm S, Thamm M. Patterns of sensitization to inhalant and food allergens - findings from the German Health Interview and Examination Survey for Children and Adolescents. Int Arch Allergy Immunol 2013;162:263-270.

15. Haftenberger M, Laußmann D, Ellert U, Kalcklösch $M$, Langen U, Schlaud $M$ et al. Prevalence of sensitisation to aeraoallergens and food allergens: results of the German Health Interview and Examination Survey for Adults (DEGS1). Bundesgesundheitsblatt, Gesundheitsforschung, Gesundheitsschutz 2013;56: 687-697.

16. Ito K, Sjölander $S$, Sato $S$, Movérare R, Tanaka A, Söderström Letal. IgE to Glym 5 and Glym 6 is associated with severe allergic reactions to soybean in Japanese children. J Allergy Clin Immunol 2011;128:673-675.

17. Holzhauser T, Wackermann O, Ballmer-Weber BK, et al. Soybean (Glycine max) allergy in Europe: Gly $\mathrm{m} 5$ (beta-conglycinin) and Gly $\mathrm{m} 6$ (glycinin) are potential diagnostic markers for severe allergic reactions to soy. J Allergy Clin Immunol 2009;123:452-458.

18. Ebisawa M, Brostedt P, Sjölander S, Sato S, Borres MP, Ito K. Gly $\mathrm{m} 2 \mathrm{~S}$ albumin is a major allergen with a high diagnostic value in soybean-allergic children. J Allergy Clin Immunol 2013;132:976-978 e1-5.

19. Klemans RJ, Knol EF, Michelsen-Huisman A et al. Components in soy allergy diagnostics: Gly $\mathrm{m} 2 \mathrm{~S}$ albumin has the best diagnostic value in adults. Allergy 2013;68:1396-1402.

20. Matricardi PM, Bockelbrink A, Beyer K, Keil T, Niggemann B, Grüber C et al. Primary versus secondary immunoglobulin $E$ sensitization to soy and wheat in the Multi-Centre Allergy Study cohort. Clin Exp Allergy 2008;38:493-500.

21. Westman M, Lupinek C, Bousquet J, Andersson N, Pahr S, Baar A et al. Early childhood IgE reactivity to pathogenesis-related class 10 proteins predicts allergic rhinitis in adolescence. $J$ Allergy Clin Immunol 2015;135:1199-1206.

22. Komata T, Söderström L, Borres MP, Tachimoto $H$, Ebisawa $M$. Usefulness of wheat and soybean specific IgE antibody titers for the diagnosis of food allergy. Allergol Int 2009;58:599-603.

23. Adachi A, Horikawa T, Shimizu H, Sarayama Y, Ogawa T, Sjolander $\mathrm{S}$ et al. Soybean beta-conglycinin as the main allergen in a patient with food-dependent exerciseinduced anaphylaxis by tofu: food processing alters pepsin resistance. Clin Exp Allergy 2009;39:167-173. 


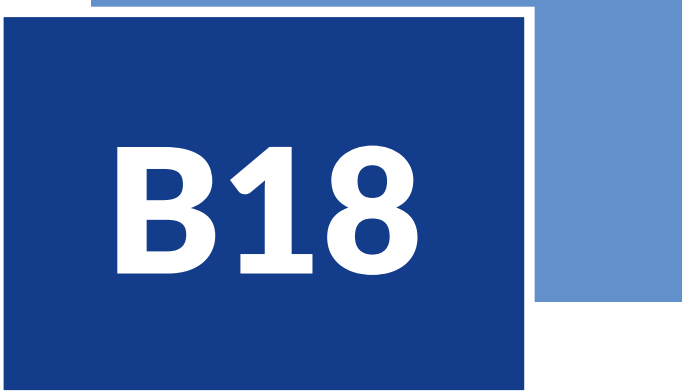

Jörg Kleine-Tebbe, Kirsten Beyer, Motohiro Ebisawa

\section{THE ALLERGEN SOURCES}

The peanut (Arachis hypogaea) belongs to the legume family (Leguminosae). Peanuts are most commonly grown in China, followed by India and the United States of America. Many different cultivars are known. Peanuts are a common trigger of foodinduced anaphylaxis. In many parts of the world such as Europe or the US peanuts are primarily consumed in roasted form. They might be eaten as whole peanuts, peanut butter, peanut flips or as an ingredient in many products. Peanuts can be roasted in the shell and sold like this or they are shelled, blanched, oven roasted either dry or in oil, and ground for the production of peanut butter or to be sprayed on peanut flips. In other parts of the world such as Asia and Africa, raw peanuts are used more commonly as a cooking ingredient. Peanuts have a high protein content of $24 \%-29 \%$ and contain various allergens. The processing of peanuts seems to be important in regard to their allergenicity as roasting at high temperatures likely promotes the formation of compact globular protein aggregates that can increase the allergenicity of Ara h 1 and 2 (1), whereas cooking might reduce their allergenicity. In addition peanut oil is commonly used and in its
Peanut allergens can induce severe food allergic reactions

IgE sensitizations to stable seed storage proteins (Ara h 2, less to Ara 1, 3 and 6), mainly developing in infancy and early childhood, are associated with severe allergic reactions

Depending on regional pollen or allergen exposures IgE sensitization to cross-reactive (pollen) allergens can occur in adolescents and adults being rarely responsible for severe reactions

Due to the incomplete panel of identified peanut allergens and the difficulties interpreting sensitization results diagnostics with peanut extract and oral peanut challenges are integral part of current allergy work-up 
unrefined form, may contain sufficient amounts of allergens to trigger allergic reactions.

\section{MAJOR AND RELEVANT MINOR ALLERGENS}

A number of peanut allergens have been identified. Many of them have protective functions or are seed storage proteins. The peanut allergens belong to diverse protein families leading to immunochemical IgE-mediated cross-reactions among different members of the legume families but also other plant foods such as tree nuts (2). The identified peanut allergens are shown in Table 1.

\section{SENSITIZATION TO INDIVIDUAL MOLECULES AND THEIR CLINICAL RELEVANCE}

\section{Epidemiology and sensitization/cross reactivity rates}

Sensitization rates are mainly based on skin prick test or specific IgE measurement to whole peanut extracts. The sensitization rates vary widely depending on the age and co-morbidities and other (inhalant) allergies of the tested population. Irrelevant sensitization seems to be especially high in subjects with coexisting pollen allergy whereas clinically relevant sensitization is much higher in children with eczema. In an Australian study it has been shown that infants with eczema were 11-times more likely to develop peanut allergy than infants without eczema (3). Screening a non-selected cohort of 13,100 German children of the general population aged 3-17 years demonstrated that almost $11 \%$ of the children were sensitized to peanuts (4). However, this high prevalence of peanut sensitization predominantly results from cross-reactivity to pollen and does not reflect the rate of primary peanut allergy.

In a multicentre, multinational study on prevalence of sensitizations to foods in adults in Europe (EuroPrevall) (5), peanut extract sensitization rates showed high variability between $0.5 \%$ in Reykjavik, $5 \%$ in Zurich, $1.6 \%$ in Utrecht und $7.2 \%$ in Madrid. Prevalence of IgE sensitization to seed storage proteins of peanut showed lower rates (6): No sensitizations were found in Sofia and Lodz, 0.1 \% in Utrecht, 0.4 \% in Zurich und $0.5 \%$ in Madrid.
Interestingly, sensitizations to seed storage proteins seem to be only present, if peanut allergy started in childhood and therefore might serve as a marker for early onset peanut allergy. Data from the multicentre study on children and adults (EuroPrevall) indicate, that allergic subjects possess allergen-specific IgE to seed storage proteins, only if the allergy manifested before the age of 14 years (6).

In patients with clinically relevant peanut allergy it has been shown that $76-96 \%$ of peanut-allergic children and adolescents in the US and central and northern Europe possess specific IgE to Ara h 2 and Ara h 6, compared with only $42 \%$ in Spain $(7,8)$. The sensitization rates for Ara h 1 are between $63 \%$ and $80 \%$ and for Ara h 3 somewhat lower, whilst the rate for Ara $\mathrm{h} 7$ is only $43 \%$ $(7,8)$. Ara $\mathrm{h} 9$ is considered a secondary food allergen particularly in Mediterranean countries. This secondary sensitization/cross-reaction is probably due to other nsLTPs (e.g., Pru p 3 in peach). Sensitizations to the Bet v 1-homologous PR-10 protein Ara $h$ 8, the profilin Ara $\mathrm{h} 5$ and glycoproteins (CCD) are usually caused by cross-reactions to pollen allergens. Sensitization rates vary depending on regional pollen exposure but also eating habits. The prevalence of sensitization to Ara $h$ 10/11 and Ara h 14/15 is not known and likely affects only a small number of peanut allergic sufferers. The fact that oleosins may be underrepresented or absent in aqueous peanut extracts represents a diagnostic gap hampering the identification of affected patients (9).

\section{CLINICAL RELEVANCE, DIAGNOSIS AND MANAGEMENT}

\section{Clinical relevance and clinical pattern}

Distinct scenarios can prompt sensitizations and potential clinical symptoms depending on different routes of exposure and physicochemical properties of the involved peanut proteins:

A) Early, presumably cutaneous sensitization to rather stable peanut allergens (i.e. Ara h 1, 2, 3, 6, 7) in children with eczema (10) and an impaired skin barrier function is the basis of subsequent severe systemic reactions after ingestion of peanut products. Patients frequently react with immediate type symptoms involving the skin (e.g. urticaria), the gastrointestinal tract (e.g. vomiting), the respiratory system (e.g. wheezing) and/or the cardiovascular system (e.g. drop in blood pressure). This hazard is probably linked to 
Table 1

Peanut allergens

\begin{tabular}{|c|c|c|c|}
\hline Allergen & Biochemical name & MW & $\begin{array}{c}\text { Heat } \\
\text { stability }\end{array}$ \\
\hline Ara h 1 & Cupin (Vicillin-type, 7S globulin) & 64 & Yes \\
\hline Ara h 2 & Conglutin ( $2 \mathrm{~S}$ albumin) & 17 & Yes \\
\hline Ara h 3 & Cupin (Legumin-type, 11S globulin, Glycinin) & 60,37 (fragment) & Yes \\
\hline Ara h 5 & Profilin & 15 & No \\
\hline Ara h 6 & Conglutin (2S albumin) & 15 & Yes \\
\hline Ara h 7 & Conglutin ( $2 \mathrm{~S}$ albumin) & 15 & Yes \\
\hline Ara h 8 & $\begin{array}{l}\text { Pathogenesis-related protein, PR-10, Bet } v 1 \text { family } \\
\text { member }\end{array}$ & 17 & No \\
\hline Ara h 9 & Nonspecific lipid-transfer protein type 1 & 9.8 & Yes \\
\hline Ara h 10 & Oleosin & 16 & Yes \\
\hline Ara h 11 & Oleosin & 14 & Yes \\
\hline Ara h 12 & Defensins & 8 & \\
\hline Ara h 13 & Defensins & 8 & \\
\hline Ara h 14 & Oleosin & 17.5 & Yes \\
\hline Ara h 15 & Oleosin & 17 & Yes \\
\hline Ara h 16 & non-specific Lipid Transfer Protein 2 & 8.5 & Yes \\
\hline Ara h 17 & non-specific Lipid Transfer Protein 1 & 11 & Yes \\
\hline
\end{tabular}

www.allergen.org, 27-09-2015

\section{Figure 1}

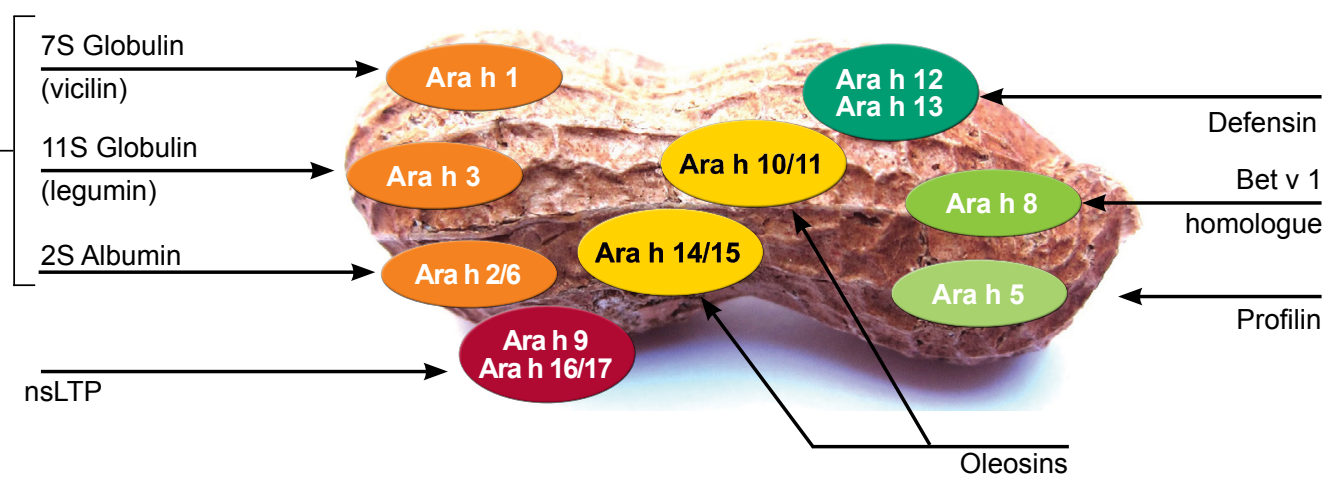

Identified peanut allergens. Bold letters: allergens available for allergen-specific IgE testing (modified and updated from (13) with kind permission of Springer-Verlag, Heidelberg Berlin, Germany). 


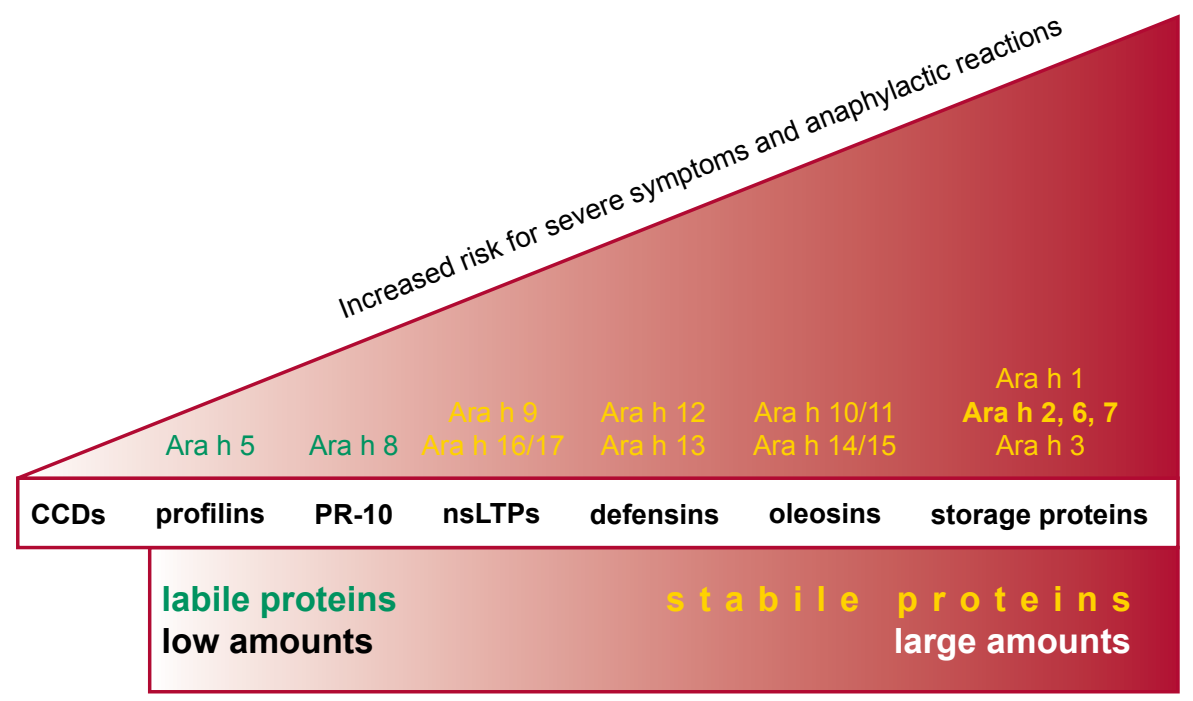

\section{Figure 2}

Risk ramp for peanut allergens: increased risk for severe symptoms and anaphylactic reactions from left to right. Green: Pollen-related highly cross-reactive allergens, Yellow: Food allergens with increased thermal stability and digestive resistance.

the high stability of the allergens and their high proportion of the total protein content.

B) Sensitizations to the Bet $v$ 1-homologous PR10 protein Ara $\mathrm{h}$ 8, the profilin Ara $\mathrm{h} 5$ and glycoproteins (CCD) are usually caused by crossreactions to pollen allergens. Birch trees are responsible for a considerable north-south gradient in Europe in terms of cross-reactions to Ara h 8; in regions of higher grass pollen exposure, increased cross-reactive lgE to Ara h 5 and CCD-containing peanut extracts can be expected. The involved proteins are largely labile to heat and digestion. Since peanuts are generally not consumed raw but roasted or cooked, only mild and predominantly oropharyngeal symptoms develop.

C) Ara h 9 is considered a secondary food allergen particularly in Mediterranean countries. This secondary sensitization/cross-reaction is probably due to other nsLTPs (e.g., Pru p 3 in peach). Since Ara h 9 possesses thermal and digestive stability, affected patients can develop systemic symptoms (11).

\section{Clinical diagnosis}

Specific questions, proper interpretation of sensitization tests (i.e. SPT, IgE) and optional food challenges help to establish the diagnosis of peanut allergy.

\section{Case history (anamnesis)}

Previous reaction(s) to peanut, symptoms, affected organ system(s), onset and course (progression), co-factors (exercise, NSAID, alcohol etc.), previous reactions to other allergen sources (i.e. peach), additional (allergic) features, i.e. birch pollen allergy, additional atopic diseases (i.e. atopic eczema, asthma).

Working hypothesis: based on peanut-related clinical pattern A - C

\section{Skin prick test (SPT)}

- Commercial peanut extract (reasonable results in case of highly abundant allergens, i.e. seed storage proteins),

- Prick-prick test with peanuts or offending peanut product (in case of severe anaphylactic reaction primary IgE-testing before SPT)

\section{IgE-Testing}

Allergen-specific IgE:

- Peanut extract,

- Ara h 2 (Fig. 3) 


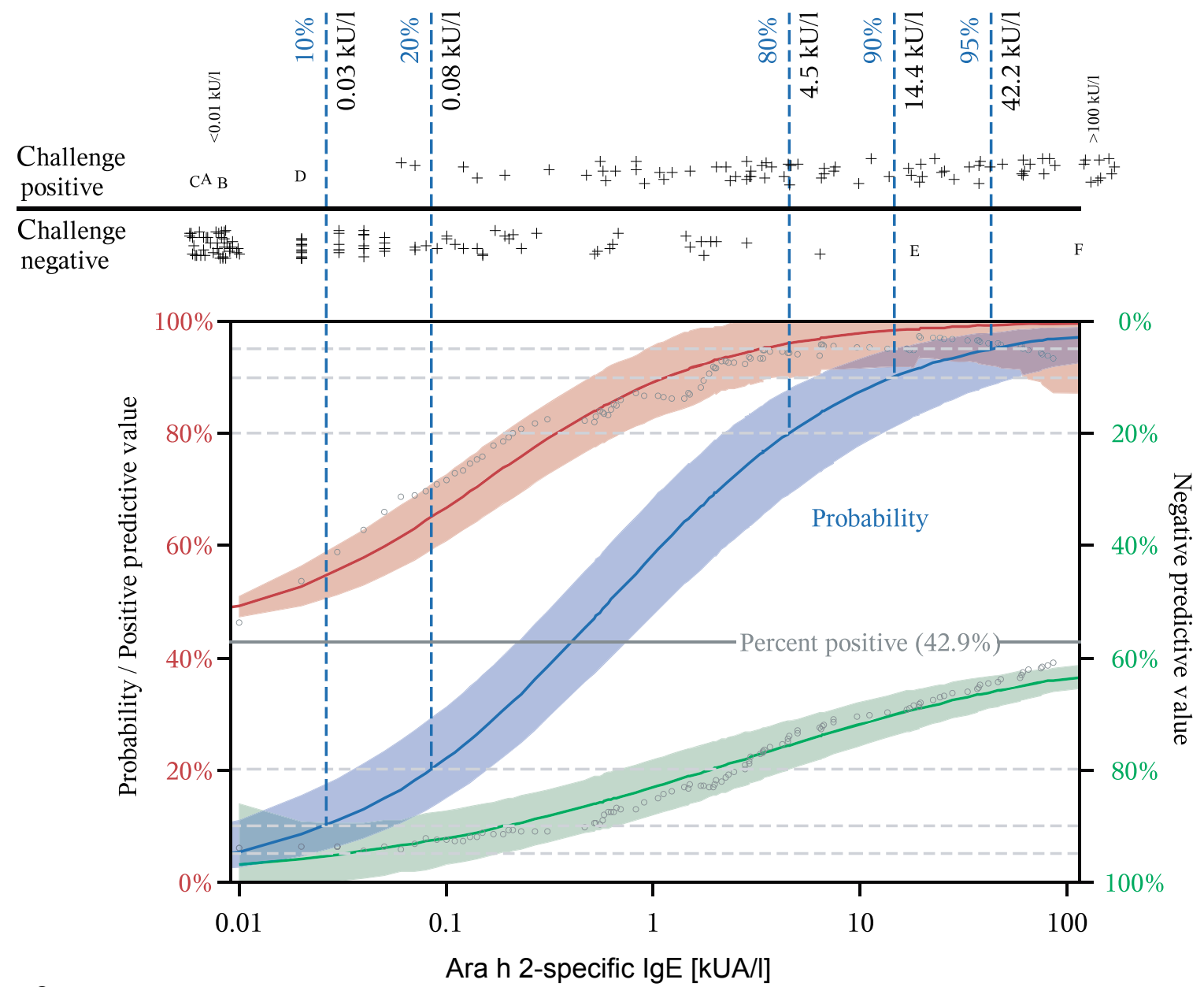

Figure 3

Study results regarding the risk of peanut allergy associated with allergen-specific IgE to the 2S-albumin seed storage protein Ara h 2. Shown is the S-shaped calculated probability for a positive oral peanut challenge in case of Ara h 2-specific serum IgE (blue line with confidence intervals). Resulting IgE concentration with predefined probabilities $(5,10,20 \%$ and $80,90,95 \%)$ for a positive challenge above the figure. Calculated positive (left y-axis, red line with range) and negative (right y-axis, green line with range) prediction (PPV, NPV) with single values (empty dots). All single lgE measurements $(+)$ depending on oral challenge outcome on top of the figure. Capitalized letters mark unexpected positive peanut challenges in a few cases (A - D) despite low Ara h 2-specific IgE-concentrations $<0.03 \mathrm{kUA} / \mathrm{I}$ (see $<10 \%$ probability range) or unexpected negative peanut challenges (cases E and F) despite high Ara h 2-specific IgE-concentrations $>14.4 \mathrm{kUA} / \mathrm{I}$ (see $>90 \%$ probability range). (reproduced from (12) with kind permission of John Wiley \& Sons, Inc).

\section{Comments:}

a) Until now Ara h 2 and Ara h 6 specific IgE demonstrates the best association with systemic reactions to peanut based on controlled oral challenges $(12,14)$.

b) To predict a positive peanut challenge with $95 \%$ probability, Ara h 2-specific IgE had extensively been tested in controlled studies with different designs involving oral food challenges to a variable degree (15-18). Predictive values from $<1$ to $42 \mathrm{kUA} / \mathrm{I}$ demonstrate the broad range and subsequent limitations to use predefined cut-offs (Ara h 2-specific lgE) for the ultimate prediction of clinically relevant reactions to peanut. Based on vigorous challenges in all patients with suspected 
peanut allergy (12), Ara h 2-specific IgE has to exceed levels of $42 \mathrm{kUA} / \mathrm{l}$ - a rare finding and therefore only useful for clinical decisions in case of similar high IgE levels.

c) To predict a negative peanut challenge with $90 \%$ probability, Ara h 2-specific IgE has to be $<0.03$ $\mathrm{kUA} / \mathrm{l}$ - with some discordant exceptions (12).

d) An absolute (100\%) prediction cannot be achieved with measurements of Ara h 2-specific IgE due to the before mentioned exceptions. Subsequently, the individual clinical relevance of allergenspecific lgE concentrations (i.e. to single allergens of legumes) has to be determined on a case-bycase basis by the physician in charge (13).

\section{Challenge tests}

(in case of a doubtful relationship between peanut ingestion and allergic reaction):

Depending on the patient's history, titrated oral provocation with peanuts (e. g. whole peanuts or lightly roasted peanut flour) should be performed.

The following procedures (Fig. 4-6) might facilitate proper diagnosis.

Measurement of peanut-specific IgE is a good screening parameter in patients at risk for peanut allergy such as patients with atopic dermatitis (Fig. 4). However, the risk of peanut allergy in children with atopic dermatitis is not the same in all geographical areas: it is high in i.e. the USA, UK, Australia and low in the Mediterranean area for instance. Therefore, the need to test for peanut sensitisation is strikingly different depending on the country and global region. It is not needed in children with regular consumption of peanut showing general tolerance to peanut.

The absence of peanut-specific IgE has a high negative predictive value. Elevated peanut-specific IgE is only clinically relevant in the presence of corresponding symptoms. In the case of no detectable peanutspecific lgE, an additional skin prick test with peanut serves to detect or exclude sensitization. If the screening is positive, an oral peanut challenge test should be considered.

Measurement of peanut- and Ara h 2-specific IgE plays an essential role in case of suspected primary peanut allergy. Elevated peanut- and Ara h 2-specific IgE and a clearly positive patient history are highly suggestive of a clinically relevant allergy (Fig. 5) Oral challenge is often not needed.
Peanut-specific IgE is detectable in around $10 \%$ of the population, especially in birch pollen areas. Therefore, unexpected findings of elevated IgE to peanut are often seen in the clinical practice. A stepwise approach (Fig. 6) takes the potential consequences into consideration. The most important initial question relates to the frequency (e.g., more than once a month) and time course (e.g., within the previous 6 weeks) of peanut consumption.

In general, the measurement of Ara $\mathrm{h}$ 1- and Ara $\mathrm{h}$ 3-specific IgE is often not necessary, since there is high cross-reactivity between these seed storage proteins (19) and mono-sensitizations to Ara h 1 and/or 3 are rare. In doubt, an oral food challenge test can clarify cases of negative or low lgE to Ara h 2. If no specific lgE to any seed storage protein can be identified, a clinically relevant peanut allergy is unlikely, although it cannot be ruled out completely in the presence of sufficient clinical suspicion (diagnostic gap due to e.g., oleosins (Ara $h$ 10/11, 14/15(20)) and defensins (Ara h 12/13(21)). Specific IgE to the nsLTP Ara $\mathrm{h} 9$ should be additionally determined in patients with systemic reactions but negative to the storage proteins of peanut.

\section{Clinical management}

\section{Advices and avoidance}

Strict avoidance of all peanut-containing products. Within the EU peanuts are required to be labeled in all pre-packed and non-pre-packed foodstuff. A great problem exists still with the "may contain" labels.

\section{Pharmacotherapy (emergency kit)}

Due to the risk of severe reactions after unintentional ingestion of peanut containing products emergency medication for self-administered drug application are recommended for all peanut allergic patients.

\section{Allergen-specific immunotherapy}

At present, commercial products for allergen-specific immunotherapy of peanut protein allergy are not available.

\section{CLINICAL CASES}

\section{Case 1A}

History: boy, 13 months of age with moderate atopic dermatitis. So far never eaten peanut or peanut containing products (Fig. 1). 


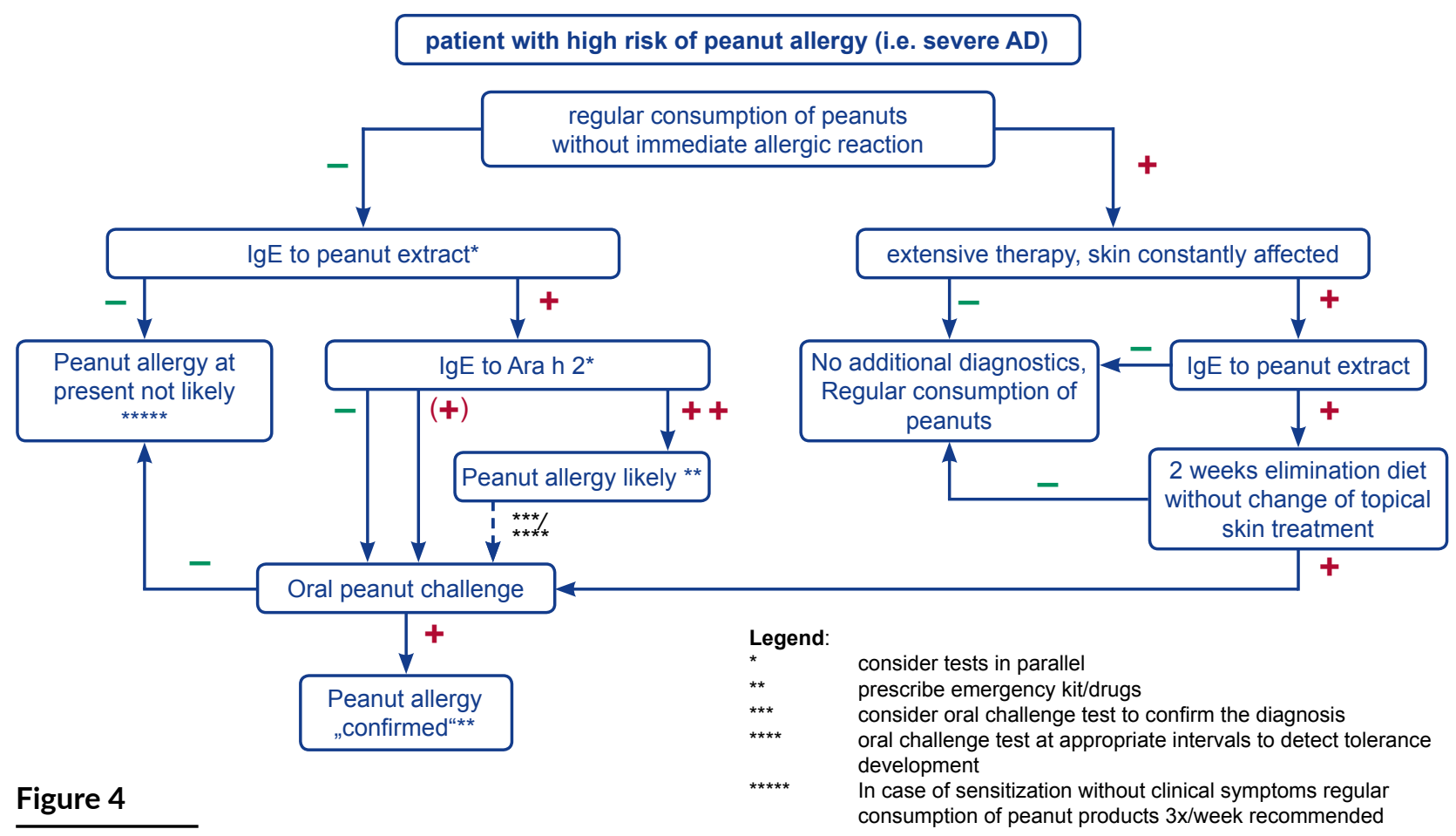

Patients with atopic dermatitis or other food allergies have a high risk for peanut allergy and sensitization might be excluded prior to the consumption of peanut-containing products. Depending on the context, the signs plus (+) or minus (-) carry different meanings: either positive/negative, yes/no or observed effect/no effect. (modified from (13)).

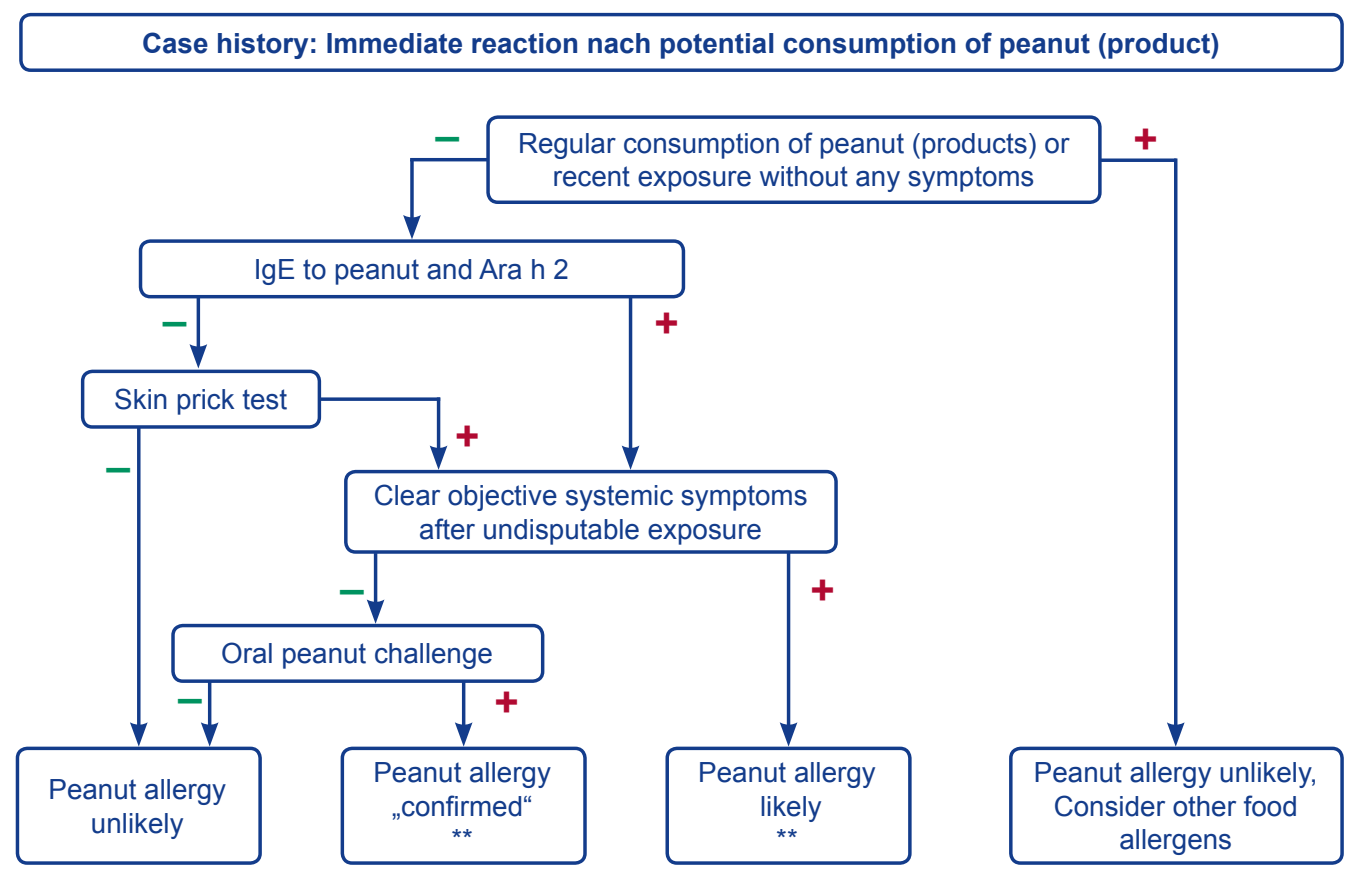

Figure 5

Allergic reaction following consumption of peanut. (modified from (13)). 


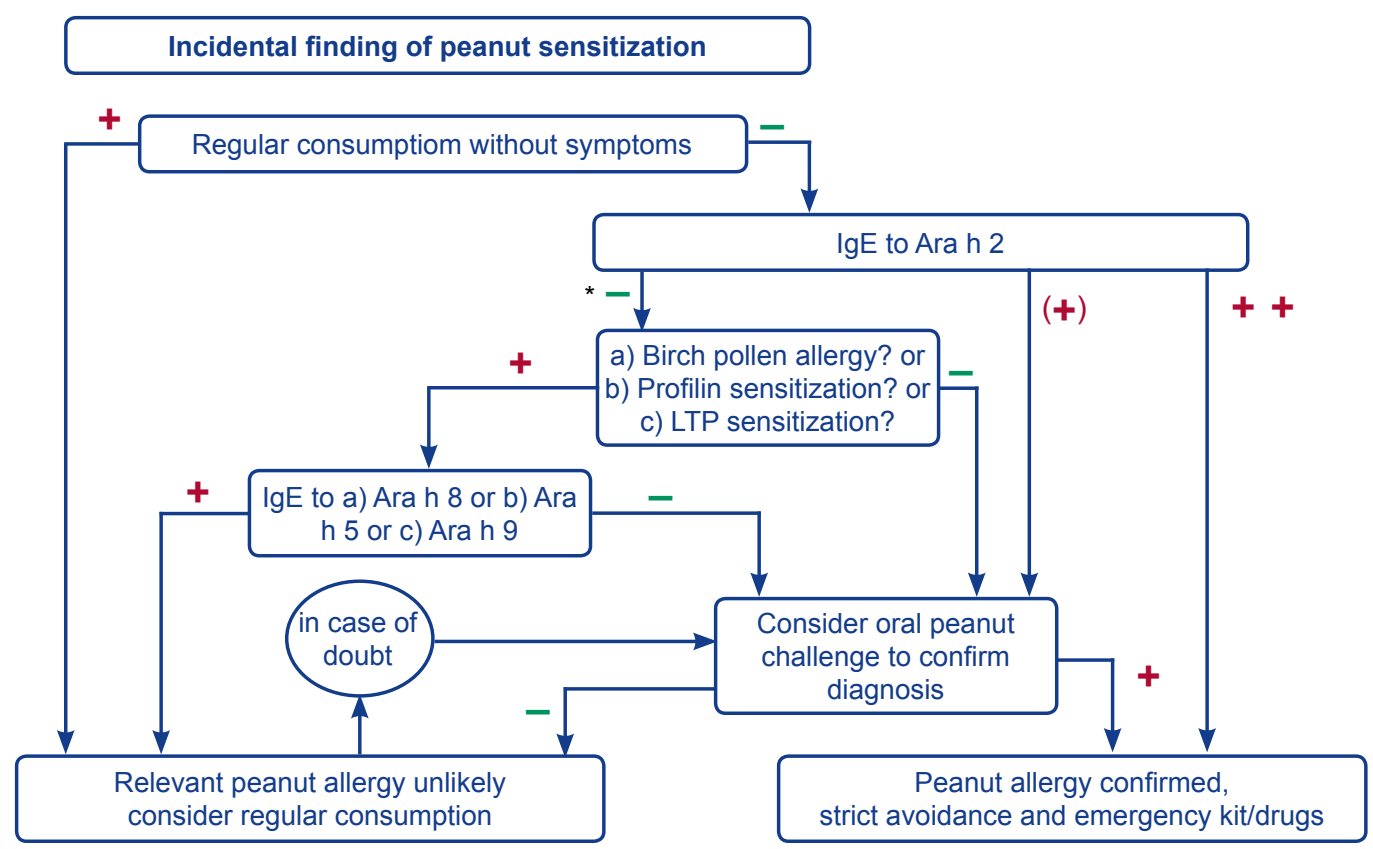

\section{Figure 6}

Incidental finding of sensitization (e.g., elevated IgE to peanut in panel or screening tests). The algorithm refers to different geographical regions i.e. a) areas with many birch/fagales trees and prevalent pollen allergies initiated by Bet $\vee 1$, b) areas with high grass or weed pollen exposure and subsequent sensitizations to profilins, and c) the Mediterranean with prevalent LTP sensitizations. Clinicians should consider appropriate IgE testing depending on the geographical region where they are working.

In-vitro testing: peanut- specific IgE $3.4 \mathrm{kUA} / \mathrm{l}$, Ara h 2-specific lgE 0.9 kUA/l.

Oral challenge: a titrated peanut challenge was performed up to $3 \mathrm{~g}$ of peanut protein. He passed the peanut challenge without any clinical symptoms.

Diagnosis: peanut sensitized but clinical tolerant.

Recommendation: Regular peanut product consumption suitable for young children (e.g. peanut butter).

\section{Case 1B}

History: girl, 11 months of age with moderate atopic dermatitis. So far never eaten peanut or peanut containing products (Figure 1).

In-vitro testing: peanut- specific IgE $89.1 \mathrm{kUA} / \mathrm{l}$, Ara h 2-specific IgE $62.6 \mathrm{kUA} / \mathrm{l}$.

Oral challenge: a titrated peanut challenge was not recommended as the predicted probability for peanut allergy was $95 \%$.
Diagnosis: peanut allergy

Recommendation: Peanut avoidance, education from dietitian, emergency medication including adrenaline auto injector.

\section{Case 2A}

History: boy, 2 years of age. He ate a peanut snack at a friend's house. He had never eaten peanuts before. After 30 minutes he developed urticaria and coughing, after 40 minutes wheezing (Figure 2).

In-vitro testing: peanut- specific lgE $5.2 \mathrm{kUA} / \mathrm{l}$, Ara h 2-specific IgE $3.1 \mathrm{kUA} /$.

Diagnosis: peanut allergy, an oral peanut challenge is not necessary.

Recommendation: Peanut avoidance, education from dietitian, emergency medication including adrenaline auto injector. 


\section{Case 2B}

History: boy, 9 years of age. He ate with his parents at a restaurant. His dish contained peanut sauce and the dessert various tree nuts. After 20 minutes he vomited, developed hives and wheezing. He had eaten peanuts in the past but infrequently and the last time he remembered was about 7 months ago (Figure 2).

In-vitro testing: peanut- specific lgE $4.1 \mathrm{kUA} / \mathrm{l}$, Ara h 2-specific IgE $1.1 \mathrm{kUA} / \mathrm{l}$, and cashew nut-specific IgE $11.6 \mathrm{kUA} / \mathrm{l}$.

Oral challenge: a titrated peanut challenge was performed up to $3 \mathrm{~g}$ of peanut protein. He passed the peanut challenge without any clinical symptoms but showed clear reaction to cashew upon food challenge

Diagnosis: cashew nut allergy, peanut sensitized but clinical tolerant.

Recommendation: Cashew avoidance, education from dietitian, emergency medication including adrenaline auto injector AND regular peanut product consumption

\section{Case 3}

History: boy, 14 years of age with allergic rhinoconjunctivitis in spring. On a panel test he showed sensitization against birch and peanut. He had eaten peanuts in the past without symptoms but infrequently (Figure 3).

In-vitro testing: birch-specific lgE $>100 \mathrm{kUA} / \mathrm{l}$, peanut-specific IgE $20.1 \mathrm{kUA} / \mathrm{l}$, Ara h 2-specific IgE $0.1 \mathrm{kUA} / \mathrm{l}$.

Diagnosis: Allergic rhinoconjunctivitis, relevant peanut allergy unlikely.

Recommendation: Consider regular consumption

\section{References}

1. Vissers YM, Blanc F, Skov PS, Johnson PE, Rigby NM, Przybylski-Nicaise Let al. Effect of heating and glycation on the allergenicity of $2 S$ albumins (Ara $h$ 2/6) from peanut. PLoS One 2011;6:e23998.

2. Bublin $\mathrm{M}$, Breiteneder $\mathrm{H}$. Cross-reactivity of peanut allergens. Curr Allergy Asthma Rep 2014;14:426.

3. Martin PE, Eckert JK, Koplin JJ, Lowe AJ, Gurrin LC, Dharmage SC, et al. Which infants with eczema are at risk of food allergy? Results from a population-based cohort. Clin Exp Allergy 2015;45:255-264.
4. Niggemann B, Schmitz R, Schlaud M. The high prevalence of peanut sensitization in childhood is due to cross-reactivity to pollen. Allergy 2011;66:980-981.

5. Burney PG, Potts J, Kummeling I, Mills EN, Clausen M, Dubakiene Ret al. The prevalence and distribution of food sensitization in European adults. Allergy 2014;69:365-371.

6. Ballmer-Weber BK, Lidholm J, Fernández-Rivas $M$, Seneviratne S, Hanschmann KM, Vogel $L$ et al. IgE recognition patterns in peanut allergy are age dependent: perspectives of the EuroPrevall study. Allergy 2015;70:391-407.

7. Codreanu F, Collignon O, Roitel O, Thouvenot B, Sauvage $C$, Vilain AC et al. A novel immunoassay using recombinant allergens simplifies peanut allergy diagnosis. Int Arch Allergy Immunol 2011;154:216226.

8. Vereda $A$, van Hage $M$, Ahlstedt S, Ibañez MD, Cuesta-Herranz J, van Odijk J et al. Peanut allergy: Clinical and immunologic differences among patients from 3 different geographic regions. J Allergy Clin Immunol 2011;127:603-607.

9. Aalberse JA, Meijer Y, Derksen N, van der PalenMerkus T, Knol E, Aalberse RC. Moving from peanut extract to peanut components: towards validation of component-resolved IgE tests. Allergy 2013;68:748756.

10. Trendelenburg V, Rohrbach A, Schulz G, Schwarz V, Beyer K. Molecular slgE profile in infants and young children with peanut sensitization and eczema. Allergo J Int 2014;23:152-157.

11. Romano A, Scala E, Rumi G, Gaeta F, Caruso C, Alonzi $C$ et al. Lipid transfer proteins: the most frequent sensitizer in Italian subjects with fooddependent exercise-induced anaphylaxis. Clin Exp Allergy 2012;42:1643-1653.

12. Beyer K, Grabenhenrich L, Härtl M, Beder A, Kalb $B$, Ziegert $\mathrm{M}$ et al. Predictive values of componentspecific IgE for the outcome of peanut and hazelnut food challenges in children. Allergy 2015;70:90-98.

13. Lange L, Beyer K, Kleine-Tebbe J. Benefits and limitations of molecular diagnostics in peanut allergy. Allergo J Int 2014;23:158-163.

14. Kukkonen AK, Pelkonen AS, Mäkinen-Kiljunen S, Voutilainen H, Mäkelä MJ. Ara h 2 and Ara 6 are the best predictors of severe peanut allergy: a double-blind placebo-controlled study. Allergy 2015;70:12391245.

15. Klemans RJ, Broekman HC, Knol EF, BruijnzeelKoomen CA, Otten HG, Pasmans SG et al. Ara h 2 is the best predictor for peanut allergy in adults. J Allergy Clin Immunol Pract 2013;1:632-638.

16. Klemans RJ, Otte D, Knol M, Knol EF, Meijer Y, GmeligMeyling $\mathrm{FH}$ et al. The diagnostic value of specific IgE to 
Arah 2 to predictpeanutallergyin children is comparable to a validated and updated diagnostic prediction model. J Allergy Clin Immunol 2013;131:157-163.

17. Lieberman JA, Glaumann S, Batelson S, Borres MP, Sampson HA, Nilsson C. The utility of peanut components in the diagnosis of IgE-mediated peanut allergy among distinct populations. J Allergy Clin Immunol Pract 2013;1:75-82.

18. Dang TD, Tang M, Choo S, Licciardi PV, Koplin JJ, Martin PE et al. Increasing the accuracy of peanut allergy diagnosis by using Ara h 2. J Allergy Clin Immunol 2012;129:1056-1063.
19. Bublin M, Kostadinova M, Radauer C, Hafner C, Szépfalusi Z, Varga EMet al. IgE cross-reactivity between the major peanut allergen Ara $\mathrm{h} 2$ and the nonhomologous allergens Ara h 1 and Ara h 3. J Allergy Clin Immunol 2013;132:118-124.

20. Schwager C, Kull S, Krause S, Schocker F, Petersen A, Becker WM et al. Development of a novel strategy to isolate lipophilic allergens from peanuts. Plos One 2015:10;10:e0123419

21. Petersen A, Kull S, Rennert S, Becker WM, Krause $S$, Ernst $M$ al. Peanut defensins: novel allergens isolated from lipophilic peanut extract. J Allergol Clin Immunol 2015;136:1295-1301. 


\title{
TREE NUT AND SEED ALLERGY
}

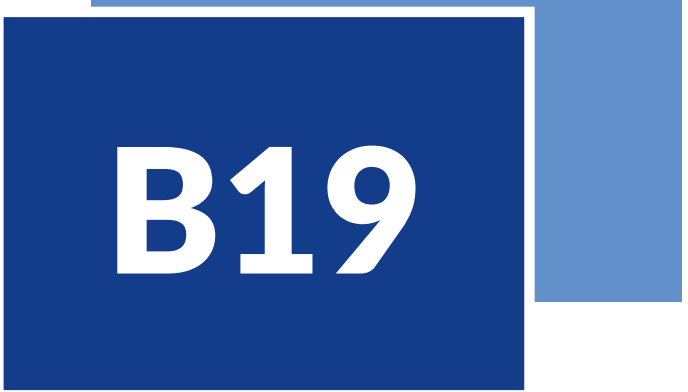

\author{
Edward Knol, Magnus Wickman
}

\section{THE ALLERGEN SOURCES}

Tree nuts, and seeds, but actually also legumes are in fact all seeds. Therefore, allergenic proteins in these food items are often shared. Such proteins belong to the families of seed storage proteins ( $2 \mathrm{~S}$ albumins, legumins - 7S globulins and vicilins - 11 S globulin), non-specific (ns) lipid transfer protein (nsLTP) and oilbody associated oleosins. The degree of sequence homology of the same protein family between tree nuts and between seeds is largely dependent on the botanical relationship.

Consumption of tree nuts and seeds in individuals allergic to these food items is related to the majority of allergic reactions to foods. Such reactions may be fatal or almost fatal. Some nuts and seeds were uncommon in the diet some decades ago in many countries in the Western world. However, tree nuts and seeds have become increasingly popular in health foods, as single food items such as snacks, as ingredients in dishes such as in ragouts and salads, as well as in pastries, chocolate and candies. The current pattern of increased exposure to, and consumption of nuts and seeds is a likely explanation for the suggested increase of reported reactions to such food items (Fig.
Tree nut and seed allergens largely belong to the pathogenesis-related, seed storage, lipid transfer and oleosin protein families

Sensitization for tree nut and seed allergens is largely independent of birch pollen, with the exception of hazelnut

Analysis of lgE to molecular components in suspected nut or seed allergy may disentangle harmless allergy due to crossreactivity to pollens from potentially harmful allergy. 
Food and products that contain or often contain tree nuts and seeds

Chocolate and pralines

Nougat (sugar paste made with nuts), for example, Torrone

Dishes such as almond chicken, pad thai, chili and trout amandine

Gianduja and giandula (chocolate blended with hazel nuts)

Marzipan (almond paste)

Almond milk

Nut milk

Tree nut oils

Spreads, for example, almond paste-based spreads, cheese spreads, chocolate nut spreads

Nutella (hazelnut)

Tahini, halva (sesame)

Vegetarian dishes

Various Indian curries and Asian dishes

Industrial made Pesto

Industrial made tomato sauce with cheese Industrial made pasta dishes

Cereals
Other possible sources of tree nuts

Baked goods

Baking mixes, cereals, crackers and muesli

Barbecue and pesto sauces

Dressings and gravies

Flavoured coffees

Frozen desserts

Liqueurs, for example, amaretto,

Frangelico

Natural flavourings and extracts, for

example, pure almond extract

Salads, for example, Waldorf salad

Snack foods, for example, trail mix

\section{Non-food sources of tree nuts}

Bean bags, kick sacks/hacky sacks

Bird seed

Cosmetics, hair care products, sun

screens

Massage oils

Pet food

\section{Figure 1}

Foods and ingredients containing tree nuts and seeds. Note: These lists are not complete and may change. Food and food products purchased from other countries, through mail-order or the Internet, are not always produced using the same manufacturing and labeling standards as in Europe.

1) in addition to the general increased incidence of food allergies as such. In this chapter, we will discuss allergens of hazelnut, almond, cashew/pistachio, walnut/pecan nut and Brazil nut, as well as to lesser extent allergens of pine nut, coconut and the seeds of sunflower, pumpkin, poppy, mustard and buckwheat. Nuts or seeds not discussed herein have been excluded due to a lack of solid data.

In Europe, Canada and the United States, tree nuts, peanuts and seeds are listed as priority food allergens. Tree nuts and seeds are found in many food products, as listed below (Fig. 1). Today, many food items are labelled with "may contain (nuts/peanuts/seeds)" which causes substantial problems for tree nut or seed allergic individuals.

Allergic reactions have been described after exposure to all food items containing nuts or seeds. Oil made from these nuts and seeds have been found to contain potential allergenic proteins from these sources. However, the concentrations of these proteins are very low and should not present a risk of allergic reactions in the vast majority of allergic people (1). As the allergenic proteins, with the exception of profilin and pathogenesis-related 10 (PR-10) allergens, in tree nuts and seeds are heat stable, processing does not impact the allergenicity of such food products.

\section{MAJOR AND RELEVANT MINOR ALLERGENIC MOLECULES}

An overview of the allergenic proteins, described until May 2015, in tree nuts and seeds is depicted in Tables 1 and 2 . With regard to the tree nuts, it is clear that the allergens belong to a limited number of protein families. As discussed before, these protein families resemble digestion and heat stable proteins. In tree nuts, Table 1 , the $2 \mathrm{~S}$ albumin proteins allergens are well represented. Recent information has indicated that these can be potent allergens and are related to the more severe forms of allergy, such as Cor a 14 in hazelnut (2). The molecular mass of the $2 \mathrm{~S}$ albumins ranges around $10-16 \mathrm{kDa}$, while the other prominent protein families $11 \mathrm{~S}$ and $7 \mathrm{~S}$ globulins have higher molecular mass of about around $50 \mathrm{kDa}$. In the tree nuts it is remarkable that the prominent PR-10/Bet $v 1$ family is only described in the hazelnut as Cor a 1, but this allergen has not been described (yet) in the other tree nuts. Oral cavity symptoms to almond and walnut are often described by birch pollen allergic patients.

In nuts, most allergens have been described for hazelnut, which is most probably the direct result of more research in this food. In addition to the allergen families described above, hazelnut also contains 


\section{Table 1}

Tree nuts allergens. Overview of the different proteins per protein families for the tree nut allergens

\begin{tabular}{|c|c|c|c|c|c|c|c|c|}
\hline \multirow{2}{*}{ Source } & \multicolumn{3}{|c|}{ Storage proteins } & \multicolumn{2}{|c|}{$\begin{array}{l}\text { Pathogenesis- } \\
\text { related proteins }\end{array}$} & \multirow{2}{*}{$\begin{array}{l}\text { Oleo- } \\
\text { sin }\end{array}$} & \multirow{2}{*}{$\begin{array}{l}\text { Profi- } \\
\text { lin }\end{array}$} & \multirow{2}{*}{$? ?$} \\
\hline & $\begin{array}{c}11 S \\
\text { globulin }\end{array}$ & $\begin{array}{c}7 S \\
\text { globulin }\end{array}$ & $\begin{array}{l}2 S \\
\text { albumin }\end{array}$ & $\begin{array}{l}\text { PR10 } \\
\text { Bet v 1-like }\end{array}$ & $\begin{array}{l}\text { PR-14 } \\
\text { nsLTP }\end{array}$ & & & \\
\hline $\begin{array}{l}\text { Hazelnut } \\
\text { Corylus avellana }\end{array}$ & Cor a 9 & Cor a 11 & Cor a 14 & Cor a 1 & Cor a 8 & $\begin{array}{l}\text { Cor a } 12 \\
\text { Cor a } 13\end{array}$ & Cor a 2 & \\
\hline $\begin{array}{l}\text { Almond } \\
\text { Prunus dulcis }\end{array}$ & Pru du 6 & & & & $\begin{array}{l}\text { Pru du } \\
\quad 3\end{array}$ & & Pru du 4 & \\
\hline $\begin{array}{l}\text { Cashew } \\
\text { Anacardium occidentale }\end{array}$ & Ana o 2 & Ana o 1 & Ana o 3 & & & & & \\
\hline $\begin{array}{l}\text { Pistachio } \\
\text { Pistacia vera }\end{array}$ & $\begin{array}{l}\text { Pis v } 2 \\
\text { Pis v } 5 ?\end{array}$ & Pis $\vee 3$ & Pis v 1 & & & & & \\
\hline $\begin{array}{l}\text { Walnut } \\
\text { Juglans regia }\end{array}$ & Jug r 4 & Jug r 2 & Jug r 1 & & Jug r 3 & & Jug r $5 ?$ & \\
\hline $\begin{array}{l}\text { Pecan } \\
\text { Carya illinoinensis }\end{array}$ & Car i 2 & & Car i 1 & & & & & \\
\hline $\begin{array}{l}\text { Brazil nut } \\
\text { Bertholletia excelsa }\end{array}$ & Ber e 2 & & Ber e 1 & & & & & \\
\hline $\begin{array}{l}\text { Pine nut } \\
\text { Pinus pinea }\end{array}$ & & Pin pi 1 & Pin pi 6 kD? & & & & & Pin pi 17 kD \\
\hline $\begin{array}{l}\text { Coconut } \\
\text { Cocos nucifera }\end{array}$ & Cocn 4 & Coc n 2 & & & & & Coc n 5 & \\
\hline
\end{tabular}

Bold font indicates that these are available on commercial diagnostic platforms. LTP: Non-specific lipid transfer protein (3).

"?" = not yet established

allergens of the oleosin family and the LTPs. LTPs have also been described in almond (Pru du 3) and walnut (Jug $r$ 3).

For the seed allergens, Table 2, the same protein families have been described like for tree nuts, with $2 \mathrm{~S}$ albumins and $7 \mathrm{~S}$ and $11 \mathrm{~S}$ globulins as the most frequently described. Many of the seed allergens show cross reactivity with their protein family counterparts in tree nuts and peanut (4). In sesame two proteins per protein family are demonstrated in $11 \mathrm{~S}$ globulin, $2 \mathrm{~S}$ albumin and oleosin (5). Due to the fact that these three proteins show a high degree of heterogeneity, it is expected that there is more than one form present in the nut or seed. It has to be taken in account that the absence of some protein families in specific seeds might be due to the fact that many of these need to be discovered.

\section{SENSITIZATION TO INDIVIDUAL MOLECULES AND THEIR CLINICAL RELEVANCE}

\section{Epidemiology and sensitization/cross reactivity rates}

We have limited knowledge of sensitisation to tree nuts and seeds in the general population. Most reports on tree nut and seed allergy are based on clinical cases. However in a recent paper (6), the prevalence rates of sensitisation to two tree nuts and five seeds in an adult population from 13 different countries were presented (Table 3). Importantly, the authors reported prevalence rates of sensitisation to tree nuts and seeds irrespective of sensitisation to birch pollen, as well as sensitisation rates for tree nuts and seeds after excluding individuals sensitized to birch pollen. This is important because pathogenesis-related 10 


\section{Table 2}

Seed allergens Overview of the different proteins per protein families for the seed allergens

\begin{tabular}{|c|c|c|c|c|c|c|c|c|}
\hline \multirow{2}{*}{ Source } & \multicolumn{3}{|c|}{ Storage proteins } & \multicolumn{2}{|c|}{$\begin{array}{c}\text { Pathogenesis-relat- } \\
\text { ed proteins }\end{array}$} & \multirow{2}{*}{$\begin{array}{l}\text { Oleo- } \\
\sin \end{array}$} & \multirow{2}{*}{$\begin{array}{l}\text { Profi- } \\
\text { lin }\end{array}$} & \multirow{2}{*}{$? ?$} \\
\hline & $\begin{array}{c}11 S \\
\text { globulin }\end{array}$ & $\begin{array}{c}\text { 7S } \\
\text { globulin }\end{array}$ & $\begin{array}{l}2 S \\
\text { albumin }\end{array}$ & $\begin{array}{c}\text { PR10 } \\
\text { Bet v 1-like }\end{array}$ & $\begin{array}{l}\text { PR-14 } \\
\text { nsLTP }\end{array}$ & & & \\
\hline $\begin{array}{l}\text { Sesame } \\
\text { Sesamum indicum }\end{array}$ & $\begin{array}{l}\text { Ses i } 6 \\
\text { Ses i } 7\end{array}$ & Ses i 3 & $\begin{array}{l}\text { Ses i } 1 \\
\text { Ses i } 2\end{array}$ & & & $\begin{array}{l}\text { Ses i } 4 \\
\text { Ses i } 5\end{array}$ & & Ses i 8? \\
\hline $\begin{array}{l}\text { Mustard seed } \\
\text { Sinapis alba }\end{array}$ & Sin a 2 & & Sin a 1 & & Sin a 3 & & Sin a 4 & \\
\hline $\begin{array}{l}\text { Sunflower seed } \\
\text { Helianthus annuus }\end{array}$ & & & Hel a $2 \mathrm{~S}$ & & Hel a 3 & & Hel a 2 & $\begin{array}{l}\text { Hel a 4, } \\
\text { defensin }\end{array}$ \\
\hline $\begin{array}{l}\text { Pumpkin seeds } \\
\text { Cucurbita maxima }\end{array}$ & & & & & & & & $\begin{array}{c}\text { Cuc ma } \\
\text { Cuc ma } 2 \\
\text { Cuc ma Cyp }\end{array}$ \\
\hline $\begin{array}{l}\text { Poppy seed } \\
\text { Papaver somniferum }\end{array}$ & & & & Pop s 1 & & & Pop s 2 & Pop s 34 kD \\
\hline $\begin{array}{l}\text { Buckwheat } \\
\text { Fagopyrum } \\
\text { esculentum }\end{array}$ & Fag e 1 & Fag e 3 & Fag e 2 & & & & & \\
\hline
\end{tabular}

Bold font indicates that these are available on commercial diagnostic platforms. LTP: Non-specific lipid transfer protein (3).

"?" = not yet established

(PR-10) allergens may interfere with sensitization of tree nuts and seeds amongst birch allergic individuals. Interestingly, sensitisation to buckwheat was more commonly found than sensitisation to peanut (Table 3).

In an American paper from 2010, self-reported tree nut allergy in 1997, 2002 and 2008 was found in $0.5 \%, 0.7 \%$ and $0.6 \%$ respectively, of the population. In contrast, only $0.1 \%$ reported sesame allergy (602). In the HealthNuts study from Melbourne it was recently shown that sesame allergy occurred in $0.7 \%$ of infants (8). Reports of hazelnut allergy have largely been limited to European countries. Notably, there is some geographical variation, with the hazelnut allergen, Cor a 1, and the homologous birch pollen, Bet $v 1$, protein being the targets of focus in Northern and Central Europe, and the hazelnut allergen Cor a 8 sensitisation being the target of focus in reports in peach allergic individuals from Southern Europe. During the last decade, several papers have appeared on allergic reactions to cashew. In a recent review on cashew nut allergy (9), the authors concluded that an increase in cashew allergy in recent decades could not be clearly documented, despite the impression that this has been the case, particularly as exposure to cashew has increased in the population. Cashew is now used in industrial food as a replacement for more expensive pine nuts and for its properties of improving texture. In a recent population-based study of children admitted to emergency rooms due to reaction to foods in Stockholm during 2007, 5\% had reacted to cashew, $3 \%$ to hazelnut, $2 \%$ to almond, walnut or pistachio, whereas $0.3-0.5 \%$ reported reactions to pecans, Brazils nut or coconut (10).

\section{Cross reactivity rates of sensitization}

As homologous proteins, such as the $2 \mathrm{~S}$ albumins, $7 \mathrm{~S}$ globulins, $11 \mathrm{~S}$ globulins, oil-body associated oleosins and ns LTPs are all prevalent in nuts and seeds and have common epitopes within the same protein family, Immunoglobulin E (IgE) cross-reactivity is to be expected. This is particularly true if the amino acid sequence is similar, and the biological function and three-dimensional (3D) structure are the same, which usually is the case in botanically closely related plants. The (primary) structural similarity between different proteins of tree nuts and seeds, expressed 
Table 3

Prevalence of sensitisation to tree nuts, peanut and seeds in adults of mostly Western Europe, Australia and the United States

\begin{tabular}{|llc|} 
& Overall (\%) & $\begin{array}{c}\text { Overall, excluding birch } \\
\text { pollen positive (\%) }\end{array}$ \\
\hline Hazelnut & 7.2 & 3.1 \\
\hline Sesame & 3.7 & 2.8 \\
\hline Buckwheat & 2.8 & 2.2 \\
\hline Peanut & 2.6 & 1.8 \\
\hline Walnut & 2.2 & 1.8 \\
\hline Sunflower & 2.1 & 1.8 \\
\hline Poppy seed & 1.8 & 1.4 \\
\hline Mustard & 0.9 & 0.8 \\
\hline
\end{tabular}

Modified from (517)

as a percentage of amino acid sequence identity or by another measure, is a molecular basis for antibody or clinical cross-reactivity, but not at all a demonstration of such cross-reactivity. Generally speaking, the higher the structural similarity between two allergens, the higher is the probability of cross-reactivity between the two. However, as antibodies may bind to specific epitopes residing in regions that are more conserved (less variable) than the protein as a whole, crossreactivity may also occur to a higher extent than indicated by the overall percentage sequence identity.

Furthermore, patients' specific sensitization profiles need to be taken into account when considering possible cross-reactions. For example, $11 \mathrm{~S}$ globulin mediated IgE cross-reactivity between peanut and hazelnut cannot occur unless the patient displays IgE sensitization to Ara h 3 and Cor a 11 (11). In a very interesting paper by Maloney et al. (12), the use of serum-specific IgE measurements for the diagnosis of peanut, tree nut, and seed allergy serum IgE levels were investigated. The correlation of IgE levels between cashew and pistachio, and between walnut and pecan were 0.98 and 0.96 , respectively. The corresponding correlations of IgE levels between hazelnut, peanut, almond and sesame were between 0.6 and 0.7. Between cashew/pistachio and other nuts, the IgE level correlation ranged from 0.16 to 0.55 . The high correlations noted between $\operatorname{lgE}$ of cashew and pistachio and the high correlations between walnut and pecan are explained by very homologous proteins in botanically related plants. Cashew and pistachio, both belong to the Anacardiaceae family (Sumak trees) and walnut and pecan nut both to the Juglandaceae family (Walnut trees). Due to the close correlation between cashew and pistachio, an individual who reacts to cashew may have a similar reaction to pistachio or vice versa on equivalent doses. The same can be expected for an individual who is allergic to walnuts and who eats pecans, or vice versa.

\section{CLINICAL RELEVANCE, DIAGNOSIS AND MANAGEMENT}

\section{Clinical pattern and relevance}

Five clear patterns types of clinical patterns and relevance have been described amongst individuals sensitised or allergic to tree nuts, and seeds.

A) Primary sensitization to one tree nut or seed allergen

B) Co-sensitization to at least two primary tree nut and/or seed allergens

C) Primary sensitization and allergy to at least one tree nut or seed and cross reactive IgE to another botanically-related tree nut or seed (high degree of sequence homology)

D) Primary sensitization and allergy to at least one tree nut or seed and cross reactive IgE to another botanically not closely related tree nut or seed (low to moderate degree of sequence homology)

E) Primary sensitization to pollen and cross-reactive IgE between PR-10 and LTP allergenic proteins in tree nuts and seeds.

- $\operatorname{ad} \mathrm{A}$. The patient is sensitized only to one tree nut or seed out of several tested. Irrespective of symptoms the IgE is relatively low. This patient is in general at younger age. At a very low age lgE levels below $0.35 \mathrm{kU} / \mathrm{l}$ can be found in children reacting to nuts, in particular cashew nut. This patient should avoid the culprit nut or seed but nothing else.

- $\quad$ ad B. This patient is often poly-sensitized to nuts and/or seeds with relatively high IgE levels to all tested relevant allergens. For a patient with nutseed allergy and polysensitised with high IgE in general, total restriction of tree nuts and avoidance of the seeds causing symptoms is recommended. 
- ad C. The patient is sensitized to both, cashew and pistachio, or to walnut and pecan with rather equal IgE levels between the botanically related groups of nuts. Patients sensitized to only cashew and pistachio should only avoid theses nuts. No other restrictions should be made. The same recommendation would be appropriate for patients sensitised and allergic to only walnut and pecan.

- For patients sensitized to walnut, clinical crossreaction to hazelnut may occur and vice versa. Such cross-reactions usually result in milder reactions as compared to reactions attributed to the primary sensitizing nut.

- $\quad \operatorname{ad} \mathrm{D}$. This is the most common patient with nut allergy: allergic to a few nuts with rather high IgE and with IgE to other nuts 10 times less or more. Usually those patients tolerate well other nuts.

- ad E. This patient is most likely birch pollen allergic (Bet $\vee 1$ ) if (s)he is resident in Northern Europe. If resident in Southern Europe, positive IgE to Artemisia, Parietaria or plane tree (LTP) may be present, but considered as markers of cross-reactivity between LTPs. Approximately $80 \%$ of this Northern European population will experience "birch pollen related food allergy" to other PR-10 allergens. The PR-10 allergen, Cor a 1, is unstable to gastric digestion and is heat labile. Symptoms in the oral cavity may be unpleasant, but will not cause systemic reactions under normal circumstances. In most tree nuts and seeds PR-10 proteins are likely to be present, most notably in Fagales trees.

\section{Clinical diagnosis}

Specific questions, appropriate interpretation of sensitization test results and, under certain conditions, open- or blinded airborne or oral food challenges will help to establish the diagnosis of tree nut and seed allergy and the grade of severity. The following work-up might facilitate proper diagnosis, which will be beneficial for the patient (Fig. 2).

\section{Detailed patient history}

The following are examples of questions that will help establish a detailed patient history, followed by the questions in a bulleted list.

- Achieve information on previous reaction(s) to tree nuts and/or seeds or if this is first time of reaction?
- Did the patient previously tolerate the offending food?

- What were the symptoms and which were the affected organs?

- Were multiple foods ingested or multiple nuts?

- What was the dose causing the symptoms?

- What was the time for onset of symptoms after ingestion?

- What is the estimated duration of administration of the adrenalin autoinjector (AAI).

- What was the progression despite injection of adrenalin?

- Were one, two or more AAI used?

- Did the patient engage in concomitant exercise, take non-steroidal anti-inflammatories (NSAID), consume alcohol or other potentially aggravating factors?

- Is the patient birch pollen allergic or allergic to plants with pollen containing LTP?

Working hypothesis: based on tree nut-seed related clinical pattern A - E

Appropriate interpretation of sensitisation results

\section{Skin prick test (SPT)}

Commercial extracts of nuts or prick-to-prick test with fresh nuts has a very limited value due to false positive responses in case of cross-reactive labile nut allergen proteins, such as Cor a 1.

\section{IgE-Testing}

Total IgE has no added value in this context.

In clear cases, with exposure to a single tree nut or seed followed by a systemic reaction, one could ask if IgE testing is needed. However, the rationale for testing of sensitization is to examine the possibility of co-sensitisation or cross-reactive IgE to other nuts or seeds and assess the risk of reaction at exposure:

- in hazelnut or walnut allergic individuals Cor a 14 and Jug $r$, both $2 S$ albumins and sequence homology of $66 \%$ could be tested (clinical pattern A, B or D)

- for Cor a 9 and Cor a 14 at reaction to hazelnut and to disentangle from sensitisation to solely Cor a 1 (if nut-related clinical pattern A-D)

- for nut or seed extracts containing PR-10 protein or LTP: risk of false positive results (clinical pattern E), 


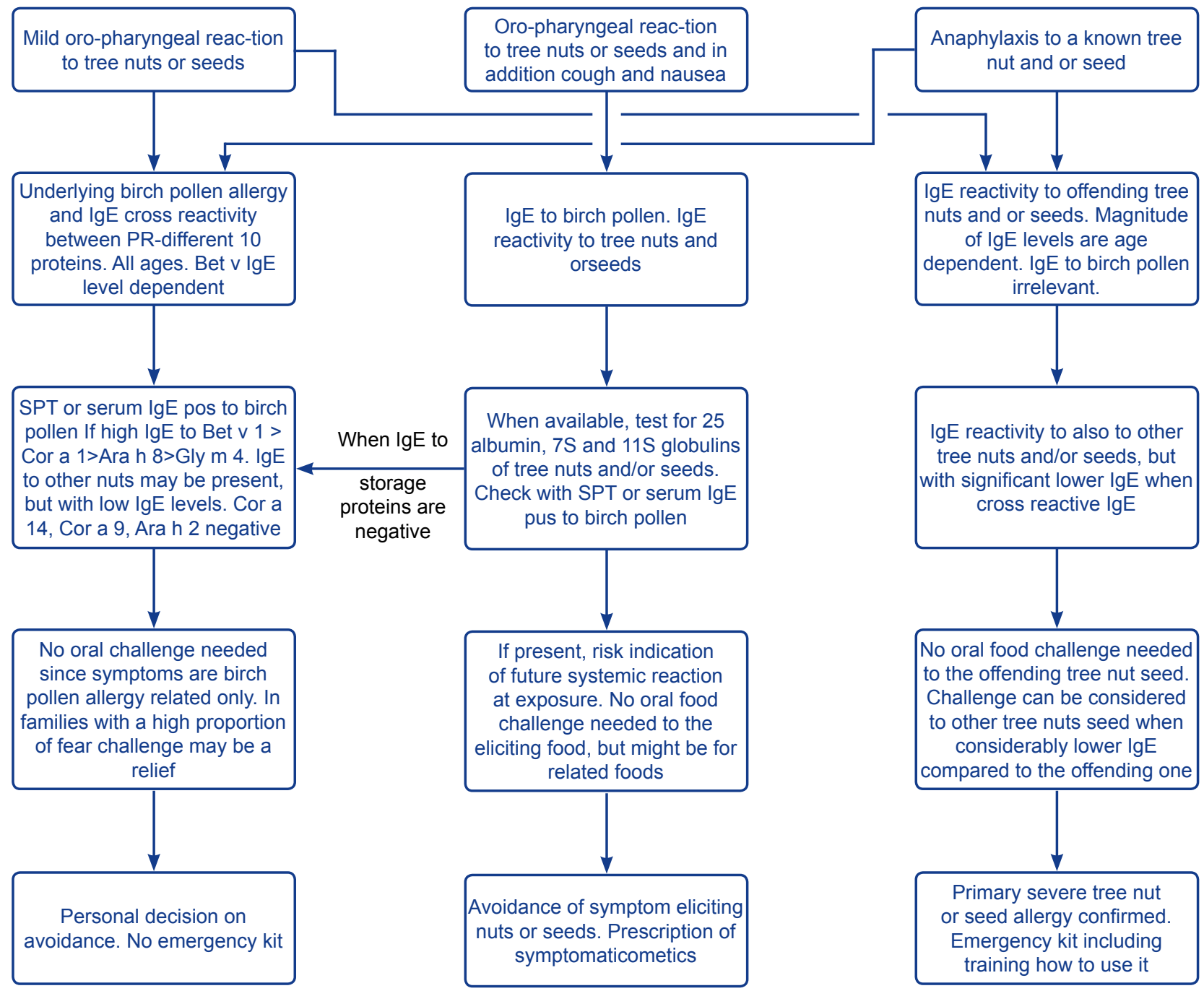

\section{Figure 2}

Diagnostic work-up in tree-nut, peanut and/or seed-related allergic reactions. Arrows indicate potential diagnostic steps; dashed arrows indicate that mild as well as severe reactions can be associated with different clinical features (based on information from the detailed patient history).

- for Cor a 1 and/or Bet v 1 or Cor a 8 and LTP containing pollens (if hazelnut-related clinical pattern E).

- for other tree nut and seed (sesame) allergen components there is to date little experience in clinical practice

For interpretation of sensitization test results see also Fig. 2.

Results only clinically relevant in case of corresponding symptoms after tree nut or seed ingestion.

\section{Oral food challenges}

Oral food challenges should be performed in cases of doubtful relationship between reported symptoms following ingestion and IgE test results and in patients who have avoided certain tree nuts, legumes/peanuts or seeds due to a previous reaction to these kind of foods and sensitisation to that particular food can be demonstrated:

- Depending on the clinical pattern (A - E), an oral food challenge with standardised increasing 


\section{Textbox 1}

\section{Oral food challenges}

An oral challenge can tell what the patient should not eat, not what the patient can eat in the future without any risk

doses of the offending food may be performed. It is important to not stop the challenge at a too low dose. Five to 10 nuts or an equivalent dose of seeds is mostly required for judgement of little risk at further exposure on not too high doses.

- Low dose challenge in nut/seed allergic individuals to reduce fear of products labelled with "may contain"

- Airborne blinded challenges for those with a fear being in an environment where nuts/seeds are present (restaurants, cafés, parties, travelling by air).

\section{Clinical management}

\section{Advice and avoidance}

1. Balanced avoidance of the offending tree nut, and/or seeds. If a patient is likely to experience a systemic reaction on a minor dose, a high degree of pre-cautious is required.

2. When a patient is likely to experience a mild reaction on relatively large dose, avoidance of larger doses should be avoided, especially when aggravating factors are present.

3. If reactions are due to PR-10 allergy, it is up to the patient to decide on amount of exposure.

\section{Pharmacotherapy (emergency kit)}

1. In patients with previously anaphylaxis or systemic reactions on a minor dose, emergency medication including AAI for self-administration is required accompanied with a personalized emergency treatment plan.

2. Tablets of corticosteroids and non-sedating antihistamines are optional, but not mandatory.

3. Symptomatic treatment as required.

\section{Allergen-specific immunotherapy}

At present, commercially available products for allergen-specific immunotherapy of tree nut or seed protein allergy are not available.

\section{CLINICAL CASES}

\section{Case 1 (original)}

Birch pollen allergy with symptoms to several PR-10 protein family containing fruits

History: Boy, 13 years: Since he was 6 years old, birch pollen induced rhinoconjunctivitis; after having had consumed pieces of several raw fruits, but in particular raw apples, plums, cherries, peaches, pears, carrot or strawberries oral itch, feeling of swelling in the throat and breathing difficulties. Several emergency visits because of this. Does not dare to drink apple juice or eat an apple cake. He experiences oral itch after eating hazelnut and almond. He has been prescribed an adrenalin auto-injector which has been frequently used due to suspected anaphylaxis, but never urticarial, never facial oedema. Despite dyspnoea, rhonchi have never been verified and oxygen saturation has always been within normal range.

Micro array in-vitro testing: Most allergen molecules in the PR-10 family positive. All LTPs and storage proteins negative.

Interpretation: PR-10 family related symptoms only?

Oral challenge: Due to disabling anxiety an open oral challenge to apple juice is performed which he passes. At challenge with raw apple at the day care unit oral itch and dyspnoea, but not rhonchi, is demonstrated and oxygen saturation is within normal range. He is required to calm down and breathing gets back to normal.

Diagnosis: PR-10 induced OAS symptoms due to underlying birch pollen allergy. At follow up both mother and son confirm panic attacks of the son at start of OAS symptoms after having taken pieces of a fruit containing the PR-10 protein family.

Recommendations: Only avoidance of what he thinks is relevant for him. No need for adrenalin auto-injector. Since this challenge he has had no more emergency visits.

\section{Case 2 (original)}

\section{Combined PR-10 and tree nut storage protein allergy}

History: 7-year old boy with rather severe rhinoconjunctivitis both, to birch and timothy pollen. Eats Nutella and after some minutes gets oral itch, blisters on the tongue and lip oedema. Some swallowing difficulties and hyper salivation. 
Can eat raw fruits including apples and peaches. Is this a systemic reaction or just local reaction due to $\mathrm{PR}-10$ protein family allergy?

In-vitro testing ImmunoCAP (kU/l): Birch, 33; Timothy, 14; Hazelnut, 22; Cor a 1, 38; Cor a 8, 1.2; Cor a 9, 9; Cor a 14, 6.6; Almond, 3.5; Cashew, 0.97; Walnut, 7.4; Jug r 1, <0.1; Jug r 3, 1.7; Peanut, 4.5 , but all peanut storage proteins negative

Interpretation: PR-10 driven nut allergy, but also combination with IgE to hazelnut storage protein IgE including nut LTP (Cor a 8 and Jug $r$ 3).

Rational for oral challenge: What not to avoid

Oral challenge (peanut, cashew nut and walnut): Tolerates the maximum dose of 10 cashew nuts and 10 peanuts at 2 different occasions. At walnut challenge oral cavity symptoms which can be verified at 1 walnut.

Interpretation of challenge: Challenge to hazelnut is not necessary. Tolerates both, cashew and peanut, but reacts to walnut.

Diagnosis: Besides PR-10 protein family allergy also storage protein family allergy to hazelnut. Sensitisation to walnut and reaction to walnut indicates either co-sensitisation to walnut or serological and clinical cross reactivity between hazelnut and walnut proteins. To test for Jug $r 4$ (11S globulin) had been of great value.

Recommendation: Since he tolerates cashew nuts he will also tolerate pistachio (close relationship). $\mathrm{He}$ is eating marzipan without problems. Thus, he tolerates almonds. He should maintain avoiding hazelnut and also to avoid walnut (see case below).

\section{Case 3 (original)}

\section{Primary hazel nut allergy with clinical cross-reactions to walnut and buckwheat}

History: Woman 20 years old with moderate asthma and mild eczema. No known pollen or food allergy. Eating chocolate with hazelnuts which she has done before. After some bites (5-10 minutes) she gets tingling feeling in her mouth. After an additional 5 minutes abdominal cramping followed by breathing difficulties and dizziness. At the emergency ward low blood pressure. Low oxygen saturation at arrival. Anaphylaxis grade II.

In-vitro testing, ImmunoCAP positives (kU/l): Hazelnut, 260; Cor a 8, 2.4; Cor a 9, 172; Cor a 14, 60; Walnut, 72; Jug r 1, 2.8; Jug r 3, 15; Cashew,
44; Almond, 2.3; pine nuts, 1.4; Brazil nut <0.1; Ara h 1, Ara h 2 and Ara h 3 all between 2.4-0.4.

Micro arrayed specific IgE: relatively high to all available $11 \mathrm{~S}$ globulins, but low to $2 \mathrm{~S}$ albumins.

Rational for oral challenge: what not to avoid

Oral challenge with cashew nut: Tolerates the maximum dose of 10 cashew nuts.

Interpretation of challenge: The sequence homology between the $2 \mathrm{~S}$ albumin and $7 \mathrm{~S}$ and $11 \mathrm{~S}$ globulins vary from between $47 \%-60 \%$.

Oral challenge with walnut: After 1 walnut she gets itch in her mouth and irritation in the throat. After 30 minutes she receives 1 more walnut. Now dermal itch and itch in the palms and scalp. Interpretation of challenge: in this patient it is obvious that walnut molecules for IgE testing are missing, which is likely to be the $11 \mathrm{~S}$ globulin Jug $r$ 4 molecule. This molecule has a sequence identity to Cor a 9 of $76 \%$. Hazelnut and walnut are from an evolutionary point of view located on sister branches.

Continued challenge at home: Peanut, almond and pine nut - without symptoms

Diagnosis: Multiple tree-nut allergy with anaphylaxis to hazelnut at a moderate dose. After three months anaphylaxis to buckwheat most likely $11 \mathrm{~S}$ globulin driven. IgE buckwheat 29.

Recommendation: Avoidance of hazelnut, walnut, pecan nut and buckwheat.

\section{References}

1. Crevel RW, Kerkhoff MA, Koning MM. Allergenicity of refined vegetable oils. Food Chem Toxicol 2000;38:385-393.

2. Masthoff LJ, Mattsson L, Zuidmeer-Jongejan L, Lidholm J, Andersson K, Akkerdaas JH et al. Sensitization to Cor a 9 and Cor a 14 is highly specific for a hazelnut allergy with objective symptoms in Dutch children and adults. J Allergy Clin Immunol 2013;132:393-399.

3. http://www.allergome.org/, visited May 2015

4. Beyer K, Grishina G, Bardina L, Sampson HA. Identification of 2 new sesame seed allergens: Ses $i$ 6 and Ses i 7. J Allergy Clin Immunol 2007;119:15541556.

5. Sirvent $S$, Akotenou M, Cuesta-Herranz J, Vereda A, Rodríguez R, Villalba M, et al. The $11 \mathrm{~S}$ globulin Sin a 2 from yellow mustard seeds shows IgE cross-reactivity with homologous counterparts from tree nuts and 
peanut. Clin Transl Allergy 2012;2:23.

6. Burney P, Summers C, Chinn S, Hooper R, van Ree R, Lidholm J. Prevalence and distribution of sensitization to foods in the European Community Respiratory Health Survey: a EuroPrevall analysis. Allergy 2010;65:11821188.

7. Sicherer SH, Muñoz-Furlong A, Godbold JH, Sampson HA. US prevalence of self-reported peanut, tree nut, and sesame allergy: 11-year follow-up. J Allergy Clin Immunol 2010;125:1322-1326.

8. Peters RL, Allen KJ, Dharmage SC, Lodge CJ, Koplin $J J$, Ponsonby AL et al. Differential factors associated with challenge-proven food allergy phenotypes in a population cohort of infants: a latent class analysis. Clin Exp Allergy 2015;45:953-963.

9. van der Valk JP, Dubois AE, Gerth van Wijk R, Wichers
$\mathrm{HJ}$, de Jong NW. Systematic review on cashew nut allergy. Allergy 2014;69:692-698.

10. Vetander M, Helander D, Flodström C, Ostblom E, Alfvén $\mathrm{T}$, Ly $\mathrm{DH}$ et al. Anaphylaxis and reactions to foods in children--a population-based case study of emergency department visits. Clin Exp Allergy 2012;42:568-577.

11. Masthoff LJ, van Hoffen E, Mattsson L, Lidholm J, Andersson K, Zuidmeer-Jongejan L et al. Peanut allergy is common among hazelnut-sensitized subjects but is not primarily the result of IgE crossreactivity. Allergy 2015;70:265-274.

12. Maloney JM, Rudengren $M$, Ahlstedt S, Bock SA, Sampson HA. The use of serum-specific IgE measurements for the diagnosis of peanut, tree nut, and seed allergy. JAllergy Clin Immunol 2008;122:145151. 


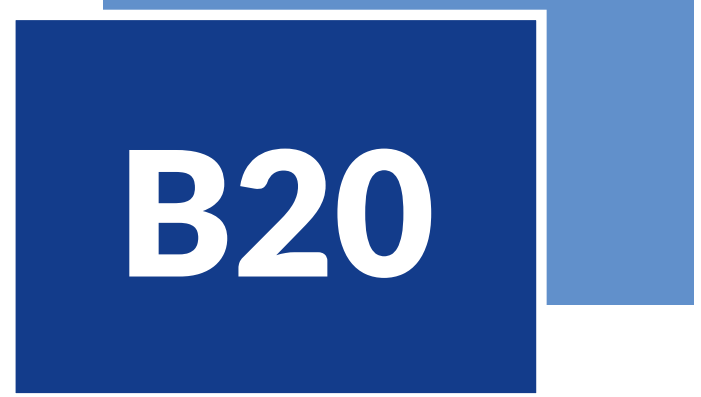

\author{
Markus Ollert, Simon Blank
}

\section{THE ALLERGEN SOURCES}

Bees are flying insects of the order Hymenoptera with approximately 20000 known species closely related to wasps and ants. The most common elicitors of bee venom allergy are honeybees which are known for their outstanding role in pollination and for producing honey and beeswax. Only two honeybee species have been truly domesticated, whereby Apis mellifera (European, western or common honeybee) (Fig. 1) is the most common domesticated species and shows a world-wide distribution due to human-induced global dispersal. The other domesticated species Apis cerana (eastern honeybee) is native to southern and eastern Asia. Nowadays, also bumblebees (Bombus spp.) (Figure 1), the close relatives of honeybees, have gained some importance as elicitors of work related venom allergy since they are increasingly used for pollination in greenhouses (1).

Sensitization to bee venom occurs after a sting, whereby honeybees are the only stinging Hymenoptera that nearly always leave their stinger with adherent venom sac in the skin of the victim. Meanwhile the venom is continuously pumped into the skin until the venom sac is exhausted
Bee venom allergy can cause severe and even fatal anaphylaxis

Specific immunotherapy is effective in inducing immunological tolerance and, therefore, protection in bee venom-allergic patients

Component-resolved diagnosis is able to discriminate between true sensitization and cross-reactivity and facilitates the choice of correct immunotherapy

Molecular analyses offer the potential for novel ways of therapy monitoring and prediction of therapy outcome 


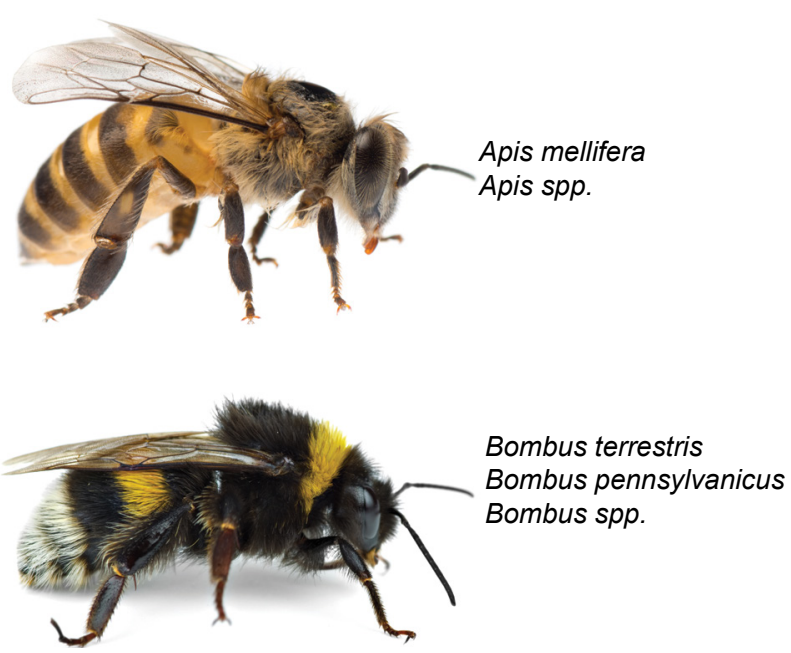

Figure 1

The honeybee and the bumblebee.

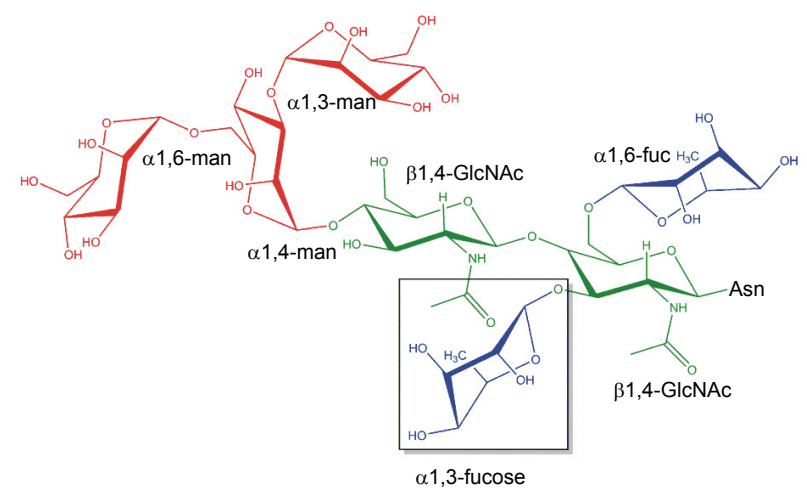

Figure 2

Schematic representation of the core glycosylation of insects. The glycan carries an alpha-1,3-fucose residue which is not present in human carbohydrate structures and therefore is highly immunogenic. It can induce the generation of cross-reactive human IgE antibodies (GlcNAc, N-acetylglucosamine; man, mannose; fuc, fucose).

or the stinger removed. Bee venom is a complex mixture of low molecular weight substances such as biogenic amines, basic peptides, toxins and of higher molecular weight proteins, many of them with enzymatic activity. The so far identified honeybee venom allergens are proteins or glycoproteins with a molecular mass ranging from 3 to $200 \mathrm{kDa}$ (Table 1).
Venom allergy is one of the most frequent triggers of severe anaphylactic reactions in adults. Approximately 9.2 to $28.7 \%$ of the adult population shows a sensitization to Hymenoptera venom (including bees and vespids) and the prevalence of systemic sting reactions among adults ranges between 0.3 and 7.5 $\%(2,3)$. The prevalence of sensitization to honeybee venom is related to the degree of exposure. Thus, the frequency of honeybee venom allergy is higher in rural than in urban populations and especially beekeepers and their family members are at a high risk for honeybee venom allergy (4).

The venom allergens of different honeybee species are highly similar and also bumblebee venom closely resembles honeybee venom. Both venoms are reported to be highly cross-reactive. Cross-reactivity of honeybee and vespid venom at protein level is limited and largely contributed to homologous allergens present in both venoms such as hyaluronidases, dipeptidylpepdidases and vitellogenins. However, the majority of crossreactivities can be attributed to clinically irrelevant IgE antibodies that are directed against cross-reactive carbohydrate determinants (CCDs) (5) (Fig. 2). This is of particular importance, since most Hymenoptera venom allergens are glycoproteins with one or more of such carbohydrate structures (Table 1). In insects the relevant CCD epitope is defined by an alpha-1,3-linked fucose residue at the innermost $\mathrm{N}$-acetylglucosamine of the carbohydrate core structure. Since both glycan modifications are not present on human carbohydrate structures, they are highly immunogenic and can induce the production of specific IgG and IgE antibodies. IgE antibodies with specificity for the alpha-1,3-fucose epitope are responsible for a significant proportion of slgE double sensitizations to honeybee and wasp (known as yellow jacket in the Unites States) venom (6).

\section{MAJOR AND RELEVANT MINOR ALLERGENIC MOLECULES}

The venom of the honeybee Apis mellifera is certainly the best characterized Hymenoptera venom (for detailed reviews see Ollert and Blank 2015 (7) and Spillner et al., 2014 (8). Mainly by proteomic approaches in the last years much progress has been made in identifying important allergens of low abundance. Moreover, recombinant strategies have helped to characterize the allergenic potential of 


\section{Table 1}

Important allergenic molecules of honeybee and bumblebee venom

\begin{tabular}{|c|c|c|c|c|c|}
\hline $\begin{array}{l}\text { Allergenic } \\
\text { molecule }\end{array}$ & Biochemical name & IUIS code & $\begin{array}{l}\text { MW } \\
\text { (kDa) }\end{array}$ & $\begin{array}{c}\text { Native aller- } \\
\text { gen contains } \\
\text { CCDs }\end{array}$ & Prevalence among patients \\
\hline \multicolumn{6}{|c|}{ Apis cerana (eastern honeybee) } \\
\hline Api c 1 & Phospholipase A2 & 58 & 16 & Yes & $?$ \\
\hline \multicolumn{6}{|c|}{ Apis mellifera (European, western or common honeybee) } \\
\hline Api m 1 & Phospholipase A2 & 65 & 16 & Yes & $57-97 \%^{1-7}$ \\
\hline Api m 2 & Hyaluronidase & 67 & 45 & Yes & $46.3-52.2 \%^{2,6-7}$ \\
\hline Api m 3 & Acid phosphatase & 68 & 49 & Yes & $50 \%^{7}$ \\
\hline Api m 4 & Melittin & 69 & 3 & No & $22.9-42.5 \% \%^{2,6-7}$ \\
\hline Api m 5 & Allergen C/DPP IV & 70 & 100 & Yes & $58.3-60 \%{ }^{7-8}$ \\
\hline Api m 10 & CRP / Icarapin & 66 & 55 & Yes & $51.5-61.8 \%^{7,9}$ \\
\hline Api $m 12$ & Vitellogenin & 735 & 200 & Yes & $50 \%^{10}$ \\
\hline \multicolumn{6}{|c|}{ Bombus pennsylvanicus (American bumblebee) } \\
\hline Bom p 1 & Phospholipase A2 & 156 & 16 & Yes & $?$ \\
\hline Bom p 4 & Protease & 157 & 27 & No & $?$ \\
\hline \multicolumn{6}{|c|}{ Bombus terrestris (Large earth bumblebee) } \\
\hline Bom t 1 & Phospholipase A2 & 158 & 16 & Yes & $?$ \\
\hline Bom t 4 & Protease & 159 & 27 & Yes & $?$ \\
\hline
\end{tabular}

CRP: carbohydrate-rich protein; DPP IV: dipeptidyIpeptidase IV

${ }^{1}$ Jakob T et al. J Allergy Clin Immunol; 130:276-278; ${ }^{2}$ Hofmann SC et al. J Allergy Clin Immunol; 128:248; ${ }^{3}$ Hofmann SC et al. J Allergy Clin Immunol; 127:265-267; ${ }^{4}$ Korosec P et al. J Allergy Clin Immunol; 128:671-673; ${ }^{5}$ Müller UR et al. Allergy; 64:543-548; ' 'Sturm GJ et al. J Allergy Clin Immunol; 128:247-248; ${ }^{7}$ Köhler J et al. J Allergy Clin Immunol; 133:1383-1389; ${ }^{8}$ Blank S et al. J Immunol; 184:5403-5413; ${ }^{\circ}$ Blank S et al. Allergy; 66:1322-1329; ${ }^{10}$ Blank S et al. PLoS One; 8:e62009.

several individual allergens in detail (Table 1). The allergenicity of most of the honeybee venom allergens seems to depend on correct threedimensional folding of the molecules (Figure 3). The role as major allergens to which more than $50 \%$ of patients show IgE reactivity to, so far was demonstrated for the honeybee venom allergens Api m 1, Api m 2, Api m 3, Api m 5 and Api m 10 (for references see Table 1).

Although, in recent years much progress has been made in the identification and characterization of honeybee venom allergens, the picture might be much more complex since more than 100proteins and peptides were identified in honeybee venom (9) Moreover, the complexity is increased by different glycosylation patterns and protein heterogeneity and even seasonal effects seem to influence the venom composition. Thus, the season of venom collection might also influence the composition of venom extracts for specific immunotherapy.

\section{SENSITIZATION TO INDIVIDUAL MOLECULES AND ITS CLINICAL RELEVANCE}

Previous analyses of the sensitization to individual allergens using native purified allergens or immunoblots with venom extracts are only partially reliable due to the interference of IgE antibodies 


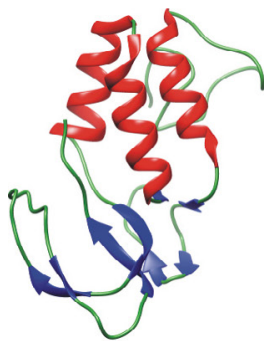

Api $\mathrm{m} 1$

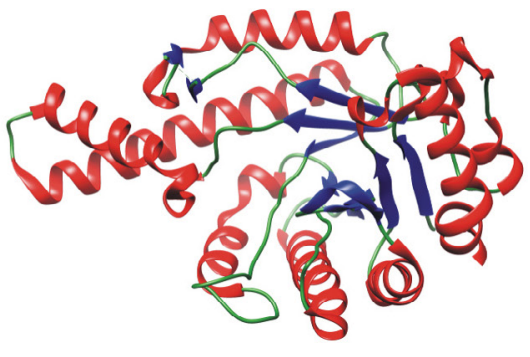

Api $\mathrm{m} 2$

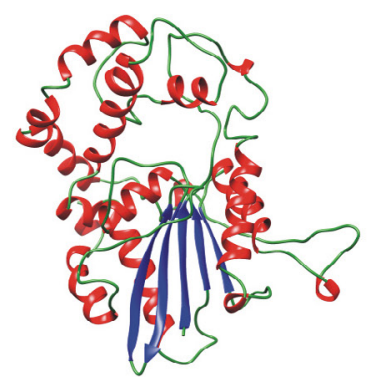

Api $m 3$

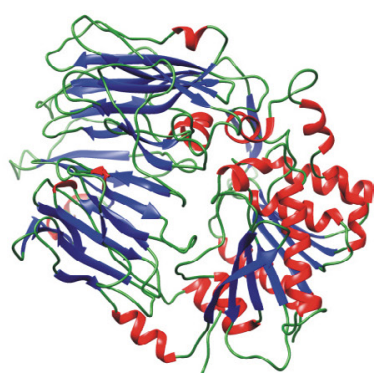

Api $\mathrm{m} 5$

Figure 3

Structures of selected honeybee venom allergens. Structures were generated by either X-ray diffraction $(10,11)$ or structural modelling.

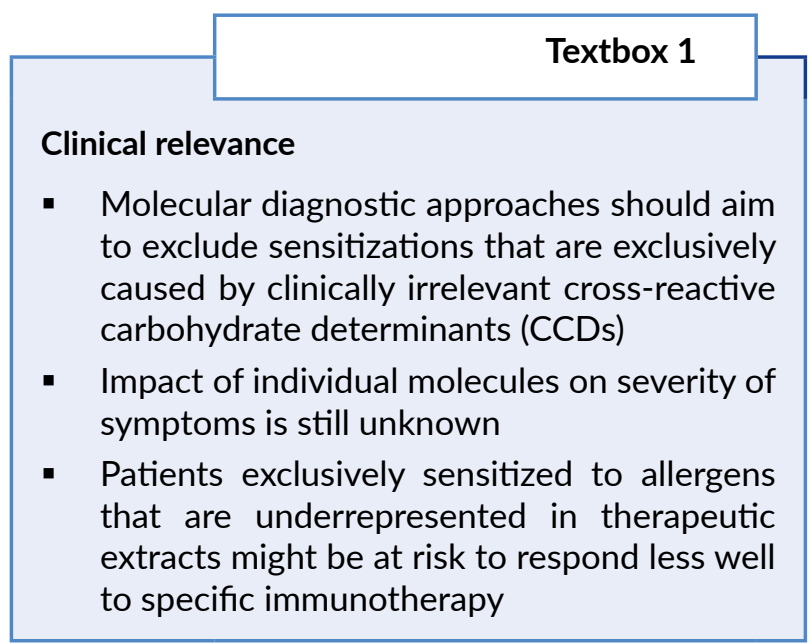

directed against cross-reactive carbohydrate determinants (CCDs). Nowadays, advanced recombinant strategies allow the production of correctly folded allergens, devoid of carbohydratebased cross-reactivity, which allow the elucidation of the role of particular allergens beyond clinically irrelevant cross-reactivity (12). Thus, reliable data on sensitization rates are available for several honeybee venom allergens (Table 1). Major differences in sensitization rates have been observed for Api $m 1$ in different studies. These differences are partially attributable to differential inclusion criteria of the patient population and partially to the preparation of the allergen Api $m$ 1, which had been used in those studies in various ways - as natural purified allergen or as recombinant allergen from bacterial or insect cell expression systems.
Recently, it was demonstrated that not only Api $m$ 1, but also Api m 2, Api m 3, Api m 5 and Api m 10 are major allergens, thus indicating that honeybee venom contains a higher number of clinically important allergens than formerly anticipated (13). Moreover, the combination of 6 allergens (Api m 1-5, 10) showed a diagnostic sensitivity of approximately $95 \%$ for honeybee venom-allergic patients, whereby $74 \%$ of patients were sensitized to more than one allergen (39 different sensitization profiles).

It is not known whether certain sensitization profiles exist that are linked to the severity of the disease or that are predictive for the outcome of immunotherapy. Recently, it was shown that the allergens Api m 3 and Api $\mathrm{m} 10$ (68\% of patients show slgE reactivity with Api $\mathrm{m} 3$ and/or Api $\mathrm{m} 10$ and $4.8 \%$ are sensitized to Api $\mathrm{m} 3$ and/or Api m 10 exclusively (13) are underrepresented or missing in several of the licensed preparations routinely used for honeybee venom immunotherapy (14), and moreover, that during immunotherapy there is minimal lgG4 induction against these two allergens in contrast to those that are present in high amounts in the extracts (13). It will remain an important topic of future clinical research to analyze the relationship between slgE specific sensitization profiles and unwanted side effects and/or treatment failure during honeybee venom immunotherapy.

\section{CLINICAL MANAGEMENT}

\section{Clinical diagnosis}

The diagnosis of honeybee venom allergy comprises the patient history of a systemic sting reaction, a 
positive skin test response and/or the detection of venom-specific IgE antibodies. Especially, when the patient was not able to definitely identify the culprit insect, the correct diagnosis is not always straightforward in clinical practice due to inherent problems and limitations of both diagnostic tests when using extract.

\section{Patient history}

One focus of taking the patient history should be the identification of the culprit insect. An important factor for the identification of honeybees is that they are the only stinging Hymenoptera species that nearly always leaves their stinger with adherent venom sac in the skin of the victim. However, several patients are not able to discriminate between honeybee and vespid stings, so that the results of patient's history often remain inconclusive. Moreover, patient history should consider information on number and date of sting reactions, severity of symptoms and the time between sting and the onset of symptoms as well as the assessment of potential risk factors (such as medication, cardiovascular risks and other diseases).

\section{Skin tests}

Skin tests are performed as prick test or intradermal test with commercial honeybee venom and vespid venom extract at least 2 weeks after the sting reaction to avoid possible false-negative results during the refractory period (15). For more detailed information please refer to the vespid venom chapter.

\section{Baseline serum tryptase}

It is recommended to determine the tryptase concentration in all patients with a history of a severe reaction after a Hymenoptera sting. Adult patients with mastocytosis and/or elevated baseline serum tryptase are at risk formore-severe reactions following stings. In addition, proper diagnosis of venom allergy in mastocytosis patients can be affected since slgE might be more often negative compared to venom allergic patients without mastocytosis.

\section{IgE testing}

Total lgE (tlgE):

Several studies investigated the specific/total lgE ratio in the context of atopy and of allergen-specific immunotherapy (reviewed in Hamilton et al. (16). In $54 \%$ of Hymenoptera venom-sensitized individuals, the ratio of slgE/tlgE was $>4 \%$ (16). Thus, in the clinical management of bee venom allergy, the measurement of tlgE can provide guidance to the clinician in the context of the ratio slgE/tlgE, although it is not generally recommended in the guidelines.

Allergen-specific IgE:

Honeybee venom and vespid venom extract: Specific IgE measurements to venom extracts might show multiple positive test results due to sensitization to multiple venoms or to cross-reactivity of cross-reactive carbohydrate determinants (CCD) or homologous allergens present in different venoms. Results might be negative due to the underrepresentation of particular allergens in the extract (for schematic representation of diagnostic problems associated with venom extracts see (Fig. 4).

Bumblebee venom extract: Although, allergy to bumblebee venom is rare, slgE detection to bumblebee venom could be useful in patients heavily exposed to bumblebee stings since bumblebee venom contains proteins not found in honeybee venom. Although major allergens of bumblebee and honeybee venom are partially cross-reactive, additional species-specific epitopes are present due to an incomplete sequence identity.

Allergens Api m 1, Api m 2, Api m 10, Ves v 1, Ves v 5 , Pol d 5 (and others not available yet for routine slgE testing): A) In case of multiple positive test results with different venoms to discriminate between true sensitization and cross-reactivity. B) For differential diagnosis in patients with inconclusive patient history to identify the culprit insect(s). C) In case of negative test results with different venoms despite a convincing clinical history due to enhanced sensitivity of the component-resolved diagnostic approach. D) In patients with mastocytosis.

Although Api $\mathrm{m} 2$ seems to be an important allergen to diagnose honeybee venom allergy in certain patients, it might show cross-reactivity with vespid allergen Ves $\vee 2$, and thus is no specific marker allergen.

CCD markers (MUXF3, horseradish peroxidase, bromelain, ascorbate oxidase): To confirm the presence of CCD-specific IgE antibodies as reason of multiple positive test results. However, since specific IgE directed against both, CCD and protein epitopes might be present, the detection of CCD-specific IgE alone does not allow the exclusion of sensitization to protein epitopes of multiple venoms.

For the interpretation of specific IgE outcome see also (Fig. 4). 
venom extract

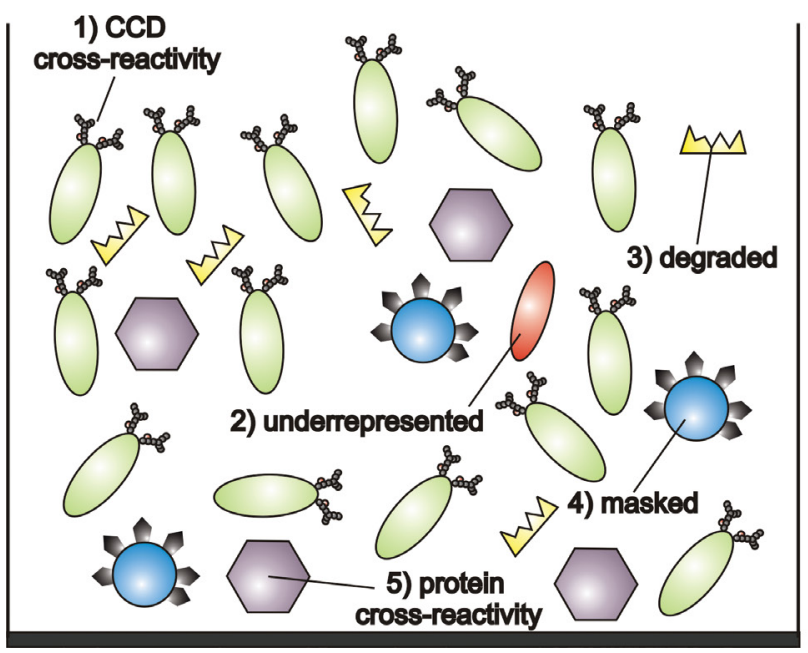

recombinant marker allergens
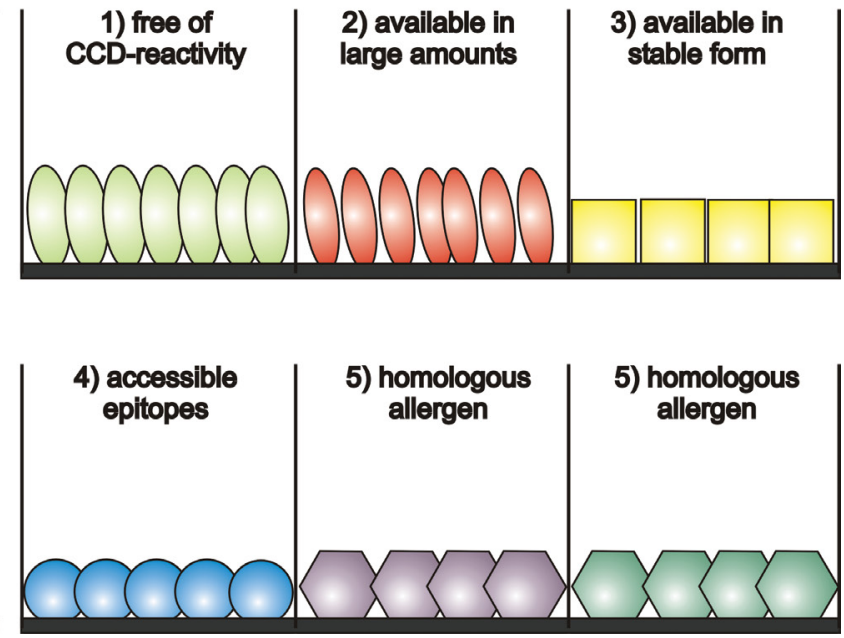

Figure 4

Molecular slgE diagnostics in venom allergy. Molecular diagnostics with recombinant marker allergens is able to exclude "false-positive" test results due to IgE directed against CCDs or homologous allergens present in venom extracts and to uncover lgE sensitizations to allergens that are underrepresented, labile, degraded or masked in venom extracts.

\section{Cellular tests}

When skin tests and specific IgE measurements yield negative results in patients with a systemic anaphylactic reaction, additional cellular tests, such as basophil activation, are recommended and have shown additional benefits when used together with allergen components (for more detailed information about cellular tests please refer to the chapter about vespid venom allergy).

\section{Sting challenge}

A sting challenge with a live insect is not recommended as diagnostic tool in untreated patients and should serve only as control of success of venom immunotherapy. For more detailed information refer to the vespid venom chapter.

\section{Prevention and Therapy}

\section{Avoidance of honeybee stings}

- Avoidance of perfumes.

- Avoidance of floral or bright colored clothing.

- Careful outdoor eating and drinking.

- Wearing shoes outside.
- Avoidance of swatting to bees.

- Keeping windows of the vehicle closed.

- Staying away from beehives.

Pharmacotherapy (emergency kit)

Due to the risk of severe reactions patients allergic to bee venom should carry an emergency kit including an adrenaline autoinjector for self-administration, especially during the bee season. Although, this is a highly debated issue, according to current guidelines, also patients who have successfully undergone immunotherapy are recommended to carry an emergency kit to eliminate a remaining risk.

\section{Specific immunotherapy}

Honeybee venom immunotherapy is indicated both in children and adults with a history of a severe systemic reaction including respiratory and cardiovascular symptoms and documented sensitization to honeybee venom with either skin test and/or specific serum IgE tests. Immunotherapy is not indicated when neither skin testing nor serum specific lgE indicate a sensitization as well as for large local reactions or unusual reactions (17). 


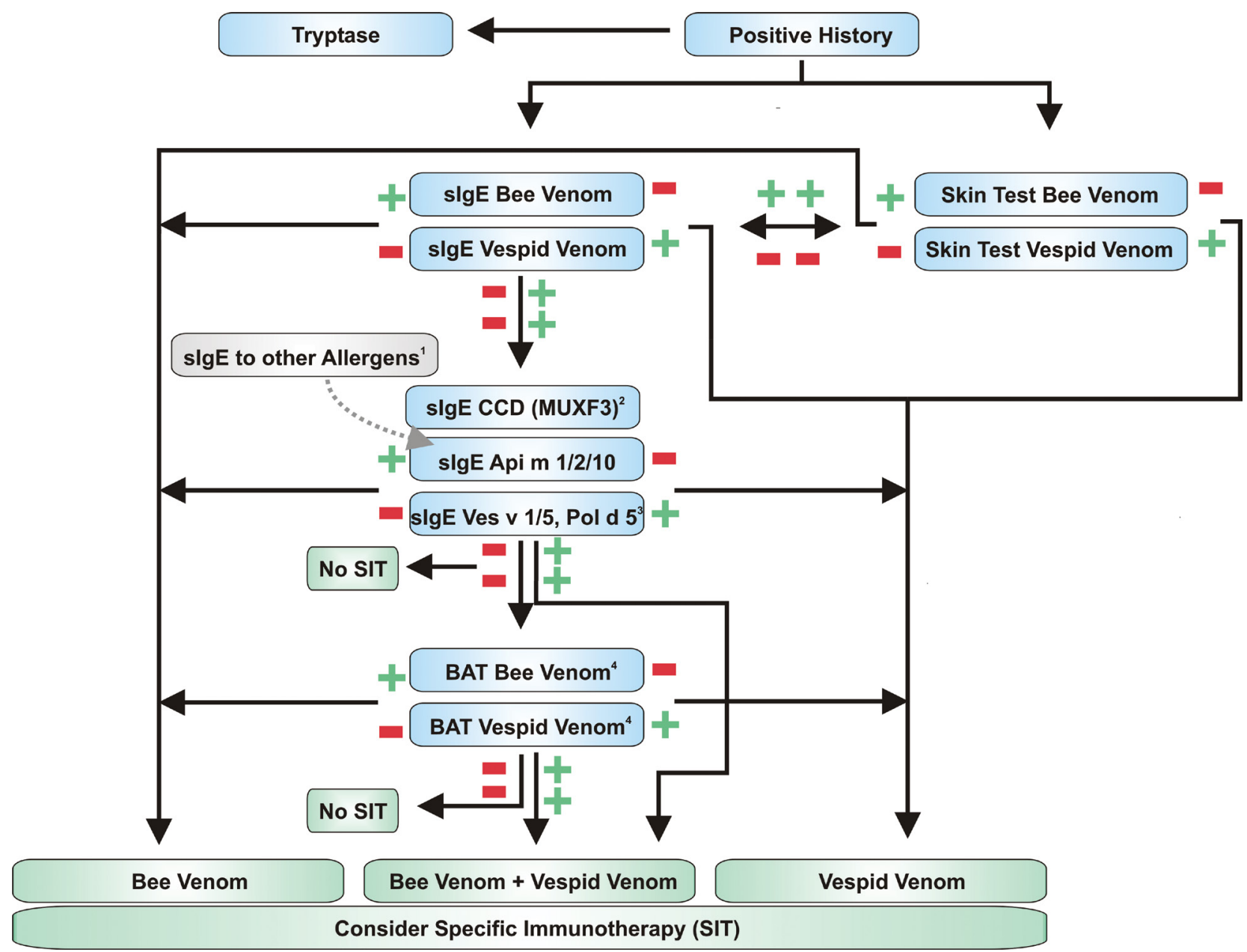

Figure 5

Diagnostic algorithm in honeybee and vespid venom allergy. A red minus indicates a negative and a green plus a positive test result. Two red minus and two green plus indicate double-negative and double-positive test results, respectively. Some centers additionally use CAP inhibition tests. However, due to the availability of the growing number of molecular components CAP inhibition becomes less and less important for the discrimination between bee and vespid venom allergy. Nevertheless, due to the lack of proper marker allergens, CAP inhibition can be useful to differentiate between Polistes and Vespula venom allergy. 1 Not yet available for routine diagnosis. 2For individual patients perhaps low sensitivity. 3For discrimination between Vespula and Polistes venom allergy refer to the vespid venom chapter. 4The basophil activation test (BAT) is not available for routine diagnosis in every clinic.

Venom immunotherapy with honeybee venom also seems to be sufficient in nonprofessionally exposed bumblebee-allergic patients who most likely react on the basis of cross-reactivity and a primary sensitization to honeybee venom. Indeed, in heavily exposed greenhouse workers who are frequently stung by bumblebees an immunotherapy with bumblebee venom would be preferable. However, bumblebee venom for routine therapeutic approaches is commercially not generally available and such approaches have only been reported in case reports.

The success of specific immunotherapy may be monitored by a sting challenge test with a live insect (see also chapter about vespid venom allergy). 


\section{Textbox 2}

Pay attention

- slgE to CCDs does not exclude clinical relevant sensitization to different venoms

- Missing slgE to so far available allergens does not necessarily exclude clinical relevant sensitization to the allergen source

- In mastocytosis patients slgE measurement might be more often "false-negative" compared to patients without mastocytosis

- The documentation of a sensitization for consideration of venom immunotherapy may be performed with either skin test and/or specific serum IgE tests or cellular tests

\section{CLINICAL CASES}

\section{Case 1 (original)}

History: Female, 40 years old, stung by unidentified insect in tip of the middle finger of the right hand. Within minutes generalized itching and urticaria, dyspnea and a feeling of tightness in the throat. Care by an emergency physician.

Skin prick test: Honeybee venom $(100 \mu \mathrm{g} / \mathrm{mL})$ and wasp (yellow jacket) venom $(300 \mu \mathrm{g} / \mathrm{mL})$ positive.

In vitro testing (conventional): tlgE $18.6 \mathrm{kU} / \mathrm{L}$, slgE to honeybee venom $6.9 \mathrm{kUA} / \mathrm{L}$, slgE to wasp (yellow jacket) venom $1.3 \mathrm{kUA} / \mathrm{L}$, baseline tryptase $6.3 \mu \mathrm{g} / \mathrm{L}$.

In vitro testing (molecular): slgE to rApi $m 15.7$ $\mathrm{kUA} / \mathrm{L}$, slgE to $\mathrm{rVes} \vee 1<0.1 \mathrm{kUA} / \mathrm{L}$, slgE to $\mathrm{rVes}$ v $5<0.1 \mathrm{kUA} / \mathrm{L}$, slgE to CCD (MUXF3) $2.4 \mathrm{kUA} / \mathrm{L}$.

Diagnosis: Honeybee venom allergy, cross-reactivity to wasp (yellow jacket) venom due to CCD-specific IgE.

Recommendation: Venom immunotherapy with honeybee venom extract, emergency kit with adrenaline autoinjector.

\section{Case 2 (original)}

History: Male, 52 years old, history of 3 episodes of severe anaphylactic reactions ( 1 after a honeybee sting and 2 after wasp/yellow jacket stings).

Intradermal skin test: Honeybee venom $(0.001 \mu \mathrm{g} /$ $\mathrm{mL}$ ) and wasp (yellow jacket) venom $(0.01 \mu \mathrm{g} / \mathrm{mL})$ positive.
In vitro testing (conventional): tIgE $15 \mathrm{kU} / \mathrm{L}$, slgE to honeybee venom $<0.1 \mathrm{kUA} / \mathrm{L}$, slgE to wasp (yellow jacket) venom $1.1 \mathrm{kUA} / \mathrm{L}$, baseline tryptase 18.9 $\mu \mathrm{g} / \mathrm{L}$.

In vitro testing (molecular): slgE to rApi m $1<0.1$ $\mathrm{kUA} / \mathrm{L}$, slgE to rApi m $2<0.1 \mathrm{kUA} / \mathrm{L}$, slgE to rApi m $31.28 \mathrm{kUA} / \mathrm{L}$, slgE to sApi m $4<0.1 \mathrm{kUA} / \mathrm{L}$, slgE to $\mathrm{rVes} \vee 11.99 \mathrm{kUA} / \mathrm{L}$, slgE to $\mathrm{rVes} \vee 5$ $1.53 \mathrm{kUA} / \mathrm{L}$, slgE to CCD (MUXF3) <0.1 kUA/L (For the measurement of slgE to rApi $\mathrm{m} 3$ and rApi $m 4$ research prototype allergens were used which are not yet available for routine diagnosis, demonstrating the usefulness of additional allergens for diagnosis.)

Diagnosis: Honeybee and wasp (yellow jacket) venom allergy, mastocytosis.

Recommendation: Venom immunotherapy with honeybee venom and wasp (yellow jacket) venom extracts, emergency kit with adrenaline autoinjector.

\section{Case 3 (original)}

History: Male, 45 years old, stung by an unidentified insect in the neck, within minutes generalized itching, dyspnea, loss of consciousness. Care by an emergency physician.

Skin prick test: Honeybee venom $(100 \mu \mathrm{g} / \mathrm{mL})$ and wasp (yellow jacket) venom $(100 \mu \mathrm{g} / \mathrm{mL})$ positive.

In vitro testing (conventional): tlgE $360 \mathrm{kU} / \mathrm{L}$, slgE to honeybee venom $23.6 \mathrm{kUA} / \mathrm{L}$, slgE to wasp (yellow jacket) venom $4.3 \mathrm{kUA} / \mathrm{L}$, baseline tryptase 3.1 $\mu \mathrm{g} / \mathrm{L}$.

In vitro testing (molecular): slgE to rApi m 110.9 $\mathrm{kUA} / \mathrm{L}$, slgE to $\mathrm{rVes} \vee 1<0.1 \mathrm{kUA} / \mathrm{L}$, slgE to $\mathrm{rVes}$ v $57.4 \mathrm{kUA} / \mathrm{L}$, slgE to CCD (MUXF3) <0.1 kUA/L.

Diagnosis: Honeybee and wasp (yellow jacket) venom allergy.

Recommendation: Venom immunotherapy with honeybee venom and wasp (yellow jacket) venom extracts, emergency kit with adrenaline autoinjector.

\section{References}

1. de Groot H, de Graaf-in 't Veld C, van Wijk RG. Allergy to bumblebee venom. I. Occupational anaphylaxis to bumblebee venom: diagnosis and treatment. Allergy 1995;50:581-584. 
2. Bilo BM, Rueff F, Mosbech H, Bonifazi F, OudeElberink JN, Hypersensitivity EIGolV. Diagnosis of Hymenoptera venom allergy. Allergy 2005;60:13391349.

3. Golden DB. Insect sting allergy and venom immunotherapy: a model and a mystery. J Allergy Clin Immunol 2005;115:439-447; quiz 48.

4. Antonicelli L, Bilo MB, Bonifazi F. Epidemiology of Hymenoptera allergy. Curr Opin Allergy Clin Immunol 2002;2:341-346.

5. Aalberse RC, Akkerdaas J, van Ree R. Cross-reactivity of IgE antibodies to allergens. Allergy 2001;56:478490.

6. Jappe U, Raulf-Heimsoth M, Hoffmann M, Burow G, Hubsch-Muller C, Enk A. In vitro hymenoptera venom allergy diagnosis: improved by screening for crossreactive carbohydrate determinants and reciprocal inhibition. Allergy 2006;61:1220-1229.

7. Ollert M, Blank S. Anaphylaxis to Insect Venom Allergens: Role of Molecular Diagnostics. Curr Allergy Asthma Rep 2015;15:527.

8. Spillner E, Blank S, Jakob T. Hymenoptera allergens: from venom to "venome". Front Immunol 2014;5:77.

9. Van Vaerenbergh M, Debyser G, Devreese B, de Graaf DC. Exploring the hidden honeybee (Apis mellifera) venom proteome by integrating a combinatorial peptide ligand library approach with FTMS. J Proteomics 2014;99:169-178.

10. Markovic-Housley Z, Miglierini G, Soldatova L, Rizkallah PJ, Muller U, Schirmer T. Crystal structure of hyaluronidase, a major allergen of bee venom. Structure 2000;8:1025-1035.
11. Scott DL, Otwinowski Z, Gelb MH, Sigler PB. Crystal structure of bee-venom phospholipase $\mathrm{A} 2$ in a complex with a transition-state analogue. Science 1990;250: 1563-1566.

12. Seismann H, Blank S, Braren I, Greunke K, Cifuentes $\mathrm{L}$, Grunwald $\mathrm{T}$ et al. Dissecting cross-reactivity in hymenoptera venom allergy by circumvention of alpha1,3-core fucosylation. Mol Immunol 2010;47:799808.

13. Kohler J, Blank S, Muller S, Bantleon F, Frick M, HussMarp J et al. Component resolution reveals additional major allergens in patients with honeybee venom allergy. J Allergy Clin Immunol 2014;133:1383-1389, 9 e1-6.

14. Blank S, Seismann H, Michel Y, McIntyre M, Cifuentes $L$, Braren I et al. Api m 10, a genuine A. mellifera venom allergen, is clinically relevant but underrepresented in therapeutic extracts. Allergy 2011;66:1322-1329.

15. Position paper: Allergen standardization and skin tests. The European Academy of Allergology and Clinical Immunology. Allergy 1993;48:48-82.

16. Hamilton RG, Williams PB, Specific IgE Testing Task Force of the American Academy of Allergy A, Immunology, American College of Allergy A, Immunology. Human IgE antibody serology: a primer for the practicing North American allergist/ immunologist. JAllergy Clin Immunol 2010;126:33-38.

17. Bonifazi F, Jutel M, Bilo BM, Birnbaum J, Muller U, Hypersensitivity EIGolV. Prevention and treatment of hymenoptera venom allergy: guidelines for clinical practice. Allergy 2005;60:1459-70. 



\section{VESPID \\ ALLERGY}

\author{
M. Beatrice Bilò, Markus Ollert
}

\section{THE ALLERGEN SOURCES}

The family Vespidae is divided into the Vespinae and Polistinae subfamilies. Vespula, Dolichovespula and Vespa make up the three genera of the Vespinae. Vespula (called wasps in Europe, yellow jackets in the USA) being the most important species in Europe.

The species Polistes dominula and Polistes gallicus are European paper wasps; P. dominula has also spread to the northeastern United States and also been reported in Australia. The species Polistes exclamans, Polistes annularis and Polistes fuscatus are indigenous to North America and not present in Europe.

Several of the European species of the vespid family differ from those found in the USA. Furthermore, popular names for vespids in the USA and Europe are different and may lead to confusion (Table 1) (1).

\section{MAJOR AND RELEVANT MINOR ALLERGENS}

Hymenoptera venoms are complex cocktails of low molecular weight substances such as biogenic amines, basic peptides, toxins and of
In wasp venom allergy CRD is a valuable tool since both $r$ ves $v 5$ and $r$ Ves $v 1$ are commercially available.

There are currently no marker recombinant allergens available that allow a definite discrimination between Vespula and Polistes venom sensitization.

It is recommended to determine the baseline tryptase concentration in all patients with a history of a severe reaction after $a$ Hymenoptera sting. 
Table 1

Vespid species in the USA and Europe

\begin{tabular}{|c|c|c|c|c|}
\hline \multirow[t]{2}{*}{ Genus } & \multicolumn{2}{|c|}{ Europe } & \multicolumn{2}{|c|}{ USA } \\
\hline & Species & Popular name & Species & Popular name \\
\hline Polistes & $\begin{array}{c}\text { gallicus } \\
\text { dominula }\end{array}$ & Wasp & $\begin{array}{l}\text { annularis } \\
\text { fuscatus } \\
\text { exclamans }\end{array}$ & Paper wasp \\
\hline Vespula & $\begin{array}{c}\text { vulgaris } \\
\text { germanica } \\
\text { rufa }\end{array}$ & Wasp & $\begin{array}{c}\text { vulgaris } \\
\text { germanica } \\
\text { maculifronts }\end{array}$ & Yellow jacket \\
\hline Dolichovespula & $\begin{array}{c}\text { media } \\
\text { saxonica }\end{array}$ & Wasp & $\begin{array}{c}\text { maculata } \\
\text { arenaria }\end{array}$ & Hornet \\
\hline Vespa & $\begin{array}{c}\text { crabro } \\
\text { orientalis }\end{array}$ & Hornet & crabro & European hornet \\
\hline
\end{tabular}

higher molecular weight proteins, many of them with enzymatic activity, together with a variety of other components, all of which may contribute to sensitization, allergic symptoms and success of immunotherapy. The certainly best characterized venom is that of the honeybee Apis mellifera, which is due to the outstanding importance of beekeeping and thus of the honeybee venom (HBV) as elicitor of venom allergy all over the world and moreover, to the availability of detailed proteomic data of pure venom (2) and genomic information of the honeybee (3). Additionally, in the last years much progress has been made in the identification of new allergens of the common wasp (in USA known as yellow jacket) Vespula vulgaris. Prominent wasp/vespid venom (VV) allergens include phospholipase A1 (Ves $\vee 1$ ), hyaluronidase (Ves $\vee 2.0101$ ) and antigen 5 (Ves $\vee 5$ ), a protein of unknown function but high abundance in the venom (Table 2; Fig. 1) $(4,5)$. Recently, a second inactive hyaluronidase (Ves $\vee 2.0201$ ), carrying an inactivating mutation in the active site of the enzyme, was identified in $\mathrm{V} V$ which interestingly seems to be the predominant allergen $(6,7)$. In the last years significant progress has been achieved mainly by proteomic approaches in identifying important allergens of low abundance. The genes of the 100 $\mathrm{kDa}$ dipeptidylpeptidases IV (DPP IV) from HBV (Api $m 5$ ) and YJV (Ves $\vee 3)$, a new class of homologous and cross-reactive Hymenoptera venom enzymes, were identified (8). Additionally, the $200 \mathrm{kDa}$ vitellogenins Api $m 12$ and Ves $v 6$ were described as novel pair of cross-reactive panallergens of HBV and VV (9). So far, only the major allergens Ves $\vee 1$ (phospholipase A1) and Ves $v 5$ (antigen 5) of VV and Pol d 5 (antigen 5) of Polistes dominula venom are available for routine molecular diagnostics.

\section{SENSITIZATION TO INDIVIDUAL MOLECULES AND ITS CLINICAL RELEVANCE (VESPULA SPP. AND POLISTES SPP.)}

Asymptomatic sensitization: The prevalence of sensitization (indicated by a positive skin test and/or the detection of serum specific IgE to whole venom extract in patients with no previous case history) is estimated at between $9.3 \%$ and $28.7 \%$ (12) in the adult population which is related to the degree of exposure to stings and to the insect distribution and behavior. Reliable data on asymptomatic sensitization rate are not available for Vespid venom recombinant allergens.

However, the risk for sensitized asymptomatic patients of developing a systemic reaction (SR) has been demonstrated to be low $15.3 \%$ in a recent study (13) and $10-15 \%$ in old studies (14). Moreover, an increase in serum specific IgE levels after a sting is not an indicator for conversion into a clinically relevant hypersensitivity (13).

\section{Sensitization rate to individual molecules and clinical relevance}

Reliable data on sensitization rates are available for many vespid allergens (Table 3 and 4). 


\section{Table 2}

Overview of the vespid venom allergens which are presently listed in the WHO/IUIS Allergen Nomenclature official database.

\section{Allergen \\ Name/Function \\ $\mathrm{MW}(\mathrm{kDa})$ \\ Potential N-glycosylation}

American paper wasps (Polistes annularis, P. exclamans, P. fuscatus, P. metricus)

\begin{tabular}{|l|l|c|c|}
\hline Pol a 1, Pol e 1 & Phospholipase A1 & 34 & 0 \\
\hline Pol a 2 & Hyaluronidase & 38 & \\
\hline Pol e 4 & Protease & $?$ & 0 \\
\hline Pol a 5, Pol e 5, Pol f 5, Pol m 5 & Antigen 5 & 23 & 0 \\
\hline
\end{tabular}

European paper wasps (Polistes dominula, P. gallicus)

\begin{tabular}{|l|l|l|l}
\hline Pol d 1, Pol g 1 & Phospholipase A1 & 34 & 1 \\
\hline Pol d 4 & Protease & 33 & 6 \\
\hline Pol d 5, Pol g 5 & Antigen 5 & 23 & 0
\end{tabular}

Hornets (Vespa crabro, V. magnifica, V. mandarinia)

\begin{tabular}{|l|l|l|l}
\hline Vesp c 1, Vesp m 1 & Phospholipase A1 & 34 & 0 \\
\hline Vesp ma 2 & Hyaluronidase & 35 & 4 \\
\hline Vesp c 5, Vesp ma 5, Vesp m 5 & Antigen 5 & 23 & 0
\end{tabular}

Polybia wasp (Polybia paulista, P. scutellaris)

\begin{tabular}{|l|l|l|l|}
\hline Poly p 1 & Phospholipase A1 & 34 & 0 \\
\hline Poly s 5 & Antigen 5 & 23 & 0 \\
\hline
\end{tabular}

White-faced hornet, yellow hornet (Dolichovespula maculate, D. arenaria)

\begin{tabular}{|l|l|l|l|}
\hline Dol $m 1$ & Phospholipase A1 & 34 & 2 \\
\hline Dol m 2 & Hyaluronidase & 42 & 2 \\
\hline Dol m 5, Dol a 5 & Antigen 5 & 23 & 0 \\
\hline
\end{tabular}

Yellow jackets (Vespula vulgaris, V. flavopilosa, V. germanica, V. maculifrons, V. pensylvanica, V. squamosa, V. vidua)

\begin{tabular}{|c|c|c|c|}
\hline Ves v $1^{*}$, Ves $\mathrm{m} 1$, Ves s 1 & Phospholipase A1 & 35 & $0,0,2$ \\
\hline Ves v 2.0101, Ves m 2 & Hyaluronidase & 45 & 4 \\
\hline Ves v 2.0201 & $\begin{array}{l}\text { Hyaluronidase } \\
\text { (inactive) }\end{array}$ & 45 & 2 \\
\hline Ves v 3 & DPP IV & 100 & 6 \\
\hline $\begin{array}{l}\text { Ves v } 5^{*} \text {, Ves } f 5 \text {, Ves g } 5 \text {, Ves m } 5 \text {, } \\
\text { Ves p } 5 \text {, Ves s } 5 \text {, Ves vi } 5\end{array}$ & Antigen 5 & 25 & 0 \\
\hline Ves v 6 & Vitellogenin & 200 & 4 \\
\hline
\end{tabular}

Allergens which are available for routine molecular diagnostics in 2015 are printed bold.

*Marker allergens with experimental evidence to be able to discriminate each by itself between honeybee and yellow jacket venom allergy. 


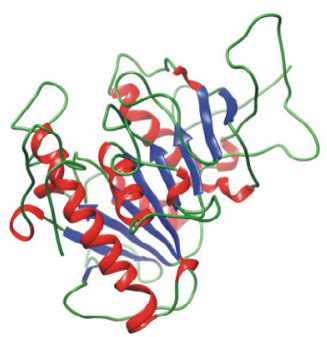

Ves $\vee 1$

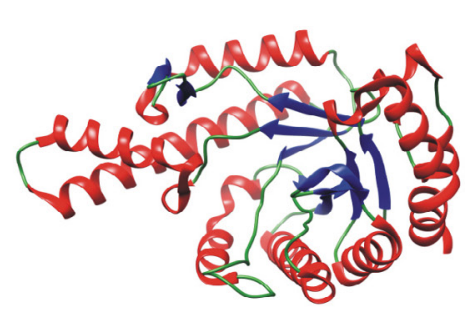

Ves $\vee 2$

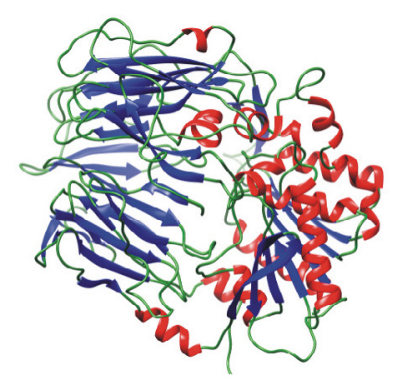

Ves $\vee 3$

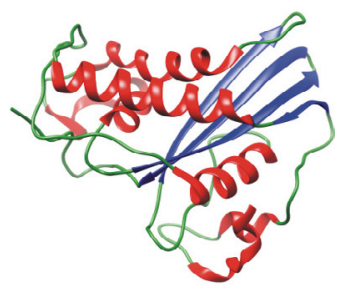

Ves $\vee 5$

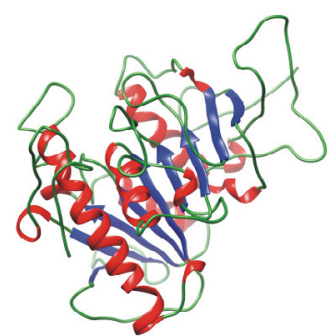

Pol d 1

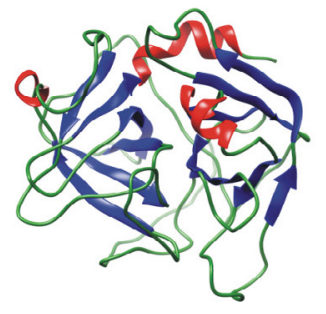

Pol d 4

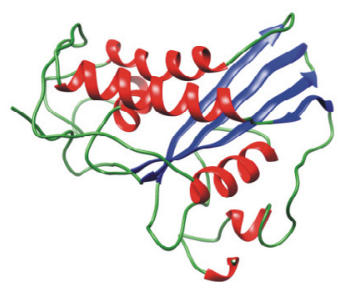

Pol d 5

\section{Figure 1}

Structures of selected vespid venom allergens. Structures were generated by either X-ray diffraction (10) or structural modeling (11).

\section{Table 3}

Sensitization rates to individual yellow jacket venom allergens

\begin{tabular}{|llll|}
\hline Allergen & Name/Function & Sensitization Rate & References \\
\hline Ves v 1 & Phospholipase A1B & $33.3-54 \%$ & $638,639,640,641,642$ \\
\hline Ves v 2a (0101 & Hyaluronidase & $5 \%$ & 618 \\
Ves v 2b (0201) & Hyaluronidase* & $20-25 \%$ & 618 \\
\hline Ves v 3 & Dipeptidylpeptidase IV & $50-62.8 \%$ & 630.640 \\
\hline Ves v 4 & CUB-protease & $?$ & \\
\hline Ves v 5 & Antigen 5 & $84.5-100 \%$ & $636,637,638,639,640$ \\
\hline Ves v 6 & Vitellogenin & $39 \%$ & 631 \\
\hline
\end{tabular}

* Inactive isoform of Ves $v 2 a$ without enzymatic activity

Specific IgE determination against non-glycosylated recombinant species-specific major allergen Ves $\vee 5$ alone showed a sensitivity between $84.5 \%$ and $100 \%$ (15-20).

Sensitization to rVes $v 1$ was found in $33.3-54 \%$ of yellow jacket venom (YJV) allergic patients (17-22). Sensitization to $r$ Ves $\vee 2$ was reported in $5-25 \%$ of
YJV allergic patients, while sensitization to rVes $v 3$ in $50-62.8 \%$ of YJV allergic patients $(8,20)$.

Wasp and bee vitellogenins (rApi m 12 and rVes $v$ 6) seem to play a relevant role as sensitizing venom components and as a novel cross-reactive class of homologous allergens responsible for double positive results with bee and $\mathrm{YJ}$ venoms apart from 


\section{Table 4}

Sensitization rates to individual Polistes dominula/annularis venom allergens

\begin{tabular}{|llll|}
\hline Allergen & Name/Function & Sensitization Rate & References \\
\hline Pol d 1 & Phospholipase A1 & $87 \%(\mathrm{nPol} d \mathrm{l})$ & 650 \\
\hline Pol d 4 & Serine protease & $?$ & \\
\hline Pol d 5 & Antigen 5 & $69-72 \%(\mathrm{nPol} d \mathrm{~s})$ & 650 \\
\hline Pol a 5 & Antigen 5 & $44 \%(\mathrm{r}$ Pol a 5$)$ & 650 \\
\hline
\end{tabular}

cross-reactive carbohydrate determinants (CCDs). In particular rVes $\vee 6$ was recognized by $39 \%$ of YJV allergic patients (9).

The diagnostic sensitivity of a combination of the YJV recombinant allergens $r$ Ves $v 5$ and $r V e s ~ v 1$ has been reported to be as high as $92-98 \%(17,19,21,23-25)$. Since both $r$ Ves $\vee 5$ and $r$ Ves $\vee 1$ are commercially available, in wasp venom allergy CRD is valuable and can be used to exclude unspecific sensitization due to CCDs.

Nevertheless, rVes $v 1$ and 5 failed to diagnose $2-8 \%$ of subjects with established allergy, thus indicating the need to add more allergens (17).

Indeed, one recent study using a combination of ELISA assays and routine ImmunoCAPs suggested that the measurement of the combination of recombinant antigens ( $r$ es $\vee 1,2,3,5)$ may further increase the sensitivity of routine assays to wasp venom up to $100 \%$ (20). In contrast, a following study using ImmunoCAP system for all allergens showed that none out of 27 patients with a convincing history of YJV anaphylaxis but slgE level below $0.35 \mathrm{kUA} / \mathrm{L}$ to the currently available YJV ImmunoCAP (spiked with rVes $\vee 5$ ) displayed IgE reactivity above $0.35 \mathrm{kUA} / \mathrm{L}$ to rVes $\vee 1,2$, or 5, and only one displayed IgE reactivity to rVes $\vee 3$, most likely due to cross reactivity to Api m 5 (26).

In patients with a documented history but negative tests results, several studies each collecting a limited number of patients demonstrated the chance to diagnose these cases by using recombinant Ves $v 5$ as allergen (from $42.1 \%$ up to $84 \%)(16,19,20,21$, $24,25)$. Consequently the conventional extract was spiked with Ves $\vee 5$ which led to a higher sensitivity $(19,21)$. This $r$ Ves $\vee 5$ spiked YJV ImmunoCAP was introduced for routine diagnostics in autumn 2012 and has replaced the previous YJV ImmunoCAP since then.
In the case of double positivity to honeybee (HB) and wasp venom extracts, IgE detection to recombinant allergens Api m 1, Ves $\vee 1$ and Ves $\vee 5$ is able to discriminate double sensitization from CCD related cross-reaction in venom allergic patients $(15,16)$. However, the discrimination between HBV and YJV sensitization is still difficult due to the limited sensitivity of the only available HBV allergen Api m 1. While Ves $\vee 1$ and Ves $\vee 5$ negative results exclude YJV sensitization with a high likelihood, Api $\mathrm{m} 1$ negative results do not exclude HBV sensitization. Here additional HBV marker allergens are needed to optimize the diagnostic precision (see chapter on bee venom).

In the south of Europe double sensitization to either Vespula or Polistes species is more frequent than that of Vespula and honeybee (27-29). According to a recent study, the major allergens of Polistes dominula and Vespula vulgaris venoms, namely phopholipase A1 and antigen 5 , are required to identify the probable sensitizing species in vespid-allergic patients, while Vespula hyaluronidase was shown to have no additional value as regards the specificity of the assay (30).

Unlike YJV and HBV, venoms of different Polistes species have recently been demonstrated to be devoid of any a1,3-core-fucosylation and hence allow CCD interference free diagnostics (30).

The importance of rPol $\mathrm{d} 1$ for a CRD of Polistes venom allergy in Europe was also underlined by a recent case report. One Spanish patient on immunotherapy with the American Polistes species venom mixture developed anaphylaxis following a sting challenge with Polistes dominula However, no reaction occurred with the same sting challenge when the treatment was switched to the Polistes dominula extract after a more sensitive diagnosis using rPol d 1 specific lgE (32).

So far, no data are available on the correlation between certain molecular sensitization profiles to 
vespid allergens and severity of the sting reaction. In addition, there are currently no marker allergens available that allow a definite discrimination between YJ and Polistes venom sensitization.

\section{CLINICAL MANAGEMENT}

\section{Clinical Diagnosis}

The goal of vespid allergy diagnostics is to classify the type of reaction, to identify the culprit insect and to explain the pathomechanism. Currently it is based on clinical history, skin tests and measurement of specific IgE antibodies to vespid venom (33).

Since in vitro tests to whole venom extracts are negative in approximately $20 \%$ of positive skin tests and approximately $10 \%$ of negative skin tests yield a positive in vitro result, the European guidelines published ten years ago recommends skin testing as well as evaluation of venom-specific IgE as a standard procedure in all patients with a history of SRs (33).

Moreover, as venom sensitization is found in about $10-30 \%$ of history-negative persons, only those with a history of a previous SR are in general eligible for diagnostic testing.

\section{Patient history}

The patient is asked to describe his/her symptoms and the course of the sting reaction, number of stings, clues as to the type of insect involved and individual risk factors for anaphylaxis. Uncertainty regarding the nature of the stinging insect (bee or wasp) is a common clinical conundrum except in the case of beekeepers, and sometimes gardeners and farmers.

Vespinae and Polistinae subfamilies are very similar, with differences at the junction of the thorax and abdomen. Vespinae have a truncated junction while Polistinae are more oval in shape. Vespidae are almost hairless and have black and yellow striped abdomens (33). Thus it is understandable that the laypersons often cannot differentiate between members of the Vespinae and Polistinae subfamily.

In patients with a history of severe systemic reaction dermatological evaluation is recommended to rule out a possible diagnosis of cutaneous mastocytosis. In patients with an unclear or suggestive psychosomatic reaction, scrutiny of emergency room and ambulance records is recommended.

\section{Skin tests}

The sensitivity of the skin prick test is lower than that of the intradermal test (up to $1.0 \mu \mathrm{g} / \mathrm{ml}$ ), which has to be used in order to confirm the negative result (609). In a recent series of 301 patients with YJV allergy, the titrated skin prick correctly identified $49 \%$ and the combination of prick test and intradermal tests $94 \%$ of the cases (19).

Standardised hymenoptera venom products, including YJ and Polistes wasp venoms, are commercially available in many countries, being mixtures of the clinically relevant species for YJ (Vespula vulgaris, V. flavopilosa, V. germanica, V. maculifrons, V. pensylvania, $V$, squamosa) as well as American Polistes (Polistes annularis, $P$. exclamans, $P$. fuscatus, $P$. metricus) venom extracts.

Contrary to the USA, dialyzed bee and yellow jacket venoms are used for diagnosis and therapy in some European countries, allowing different diagnostic accuracy with respect to the use of un-dialyzed extracts (34). In dialyzed venom, low molecular weight components (like histamine and components with histamine-releasing activity) smaller than 1000-3000 Dalton are removed. In view of lower cross-reactivity between venoms of the European and American species of Polistes (35), commercial preparations of European Polistes dominula venom are now available (29).

No recombinant venom allergens are commercially available for skin testing.

\section{Skin test results and severity of sting reaction}

There is no correlation between the severity of sting reactions and skin-test reactivity to whole venom extracts (36). Indeed, the most positive skin tests occur in patients with LLRs only, while almost $25 \%$ of patients who are referred for evaluation of a stinginduced SR have a negative SPT and show a positive intradermal test only at the highest concentration (1 $\mu \mathrm{g} / \mathrm{ml})(33)$.

\section{IgE-testing}

Total lgE (tlgE)

Determination of tlgE may be useful for appropriate interpretation of allergen-specific IgE, especially in the case of very low level of slgE.

Specific antibodies to hymenoptera venoms are frequently seen in asymptomatic individuals with high total IgE (37), and correlate with atopy status 
(38), but appear to be largely irrelevant in clinical terms (37).

\section{Allergen specific IgE}

Venom specific IgE can be detected immediately after the sting, but the optimal time point will be 1-4 weeks later (33), since the sting most likely will have induced a boost of the lgE production.

Sensitivity for YJV is usually lower than for HB venom. According to some recent studies, the conventional YJV slgE assay using the whole extract yielded a sensitivity between $83.4 \%$ and $91 \%(15,19,21)$.

In some studies $(19,21)$ it has been demonstrated that the newly developed YJV solid-phase assay complemented with $\mathrm{rVes} v 5$ has a higher sensitivity than the traditional YJV test.

Honeybee venom and vespid venom extract: Specific IgE measurements to venom extracts might show multiple positive test results due to sensitization to multiple venoms or to cross-reactivity of CCDs or homologous allergens present in different venoms. Results might be negative due to the underrepresentation of particular allergens in the extract (for schematic representation of diagnostic problems associated with venom extracts see Fig. 93 in the chapter on bee venom). Polistes species venom has been demonstrated to be devoid of CCD reactivity, thus avoiding the potential for $C C D$ related cross-reactivity (31).

Vespula spp. and Polistes spp. / P. dominulavenom extracts: A double in vitro positivity to $\mathrm{YJ}$ and Polistes venom is common in Mediterranean countries (2729), where the species Polistes dominula and Polistes gallicus are the most common species. A incomplete cross-reactivity between European and American paper wasps was demonstrated $(29,35)$, leading to the need to introduce, at least in Europe, the Polistes gallicus or dominulus extract (the latter only being available in some European countries) into clinical practice for diagnostic and therapeutic purposes.

Total IgE, allergen specific IgE results and severity of sting reaction: There is no correlation between the severity of sting reactions and the concentration of venom slgE to whole venom extracts $(33,39,40)$, as some patients with minimal or absent venom-specific IgE antibodies can develop severe anaphylaxis (41). The influence of total IgE and venom specific IgE on the severity of hymenoptera sting reactions has been evaluated, with conflicting results. In a prospective study on a large population of patients with history of sting anaphylaxis, neither skin tests to insect venom nor venom specific IgE were significantly correlated with the reaction's severity after in-hospital sting challenge, in accordance to previous prospective and retrospective studies (36). In another study, high specific IgE levels were related to high total IgE levels, but not to severe sting reactions, which occurred more frequently in patients with low total IgE (42). In a more recent study, an influence of total and specific IgE on severity of sting reactions could not be shown, except for honey bee venom specific IgE, which were significantly lower in patients with severe anaphylaxis (43).

For the use of species-specific marker allergens that help to differentiate between honeybee venom and vespid venom allergy, please refer to the chapter on bee venom allergy and the diagnostic algorithm depicted in (Fig. 2).

\section{Cellular tests}

Among cellular tests, basophil activation test (BAT) is the most useful one and can be used as a diagnostic tool in some specific cases. BAT allows the identification of approximately two thirds of patients with systemic sting reactions but negative venomspecific IgE and skin test results (44).

BAT is also recommended in double positive patients with inconclusive recombinant or skin test double positive results, especially if the patient has had an anaphylactic reaction to only one insect45. Since results of BAT are influenced by the presence of CCDs in venoms, BAT together with a component-resolved diagnosis by means of CCDs-free recombinant allergens, may be useful to a better diagnostic approach to hymenoptera venom allergy $(45,46)$.

\section{IgE-inhibition test}

The lgE-inhibition test is of use when distinguishing between cross-reactivity and double sensitization. However, it is costly and results are sometimes difficult to interpret $(33,40)$.

\section{Baseline serum tryptase}

As outlined in the chapter on bee venom allergy, it is recommended to determine the baseline tryptase concentration in all patients with a history of a severe reaction after a Hymenoptera sting. For more details refer to the bee venom chapter. 


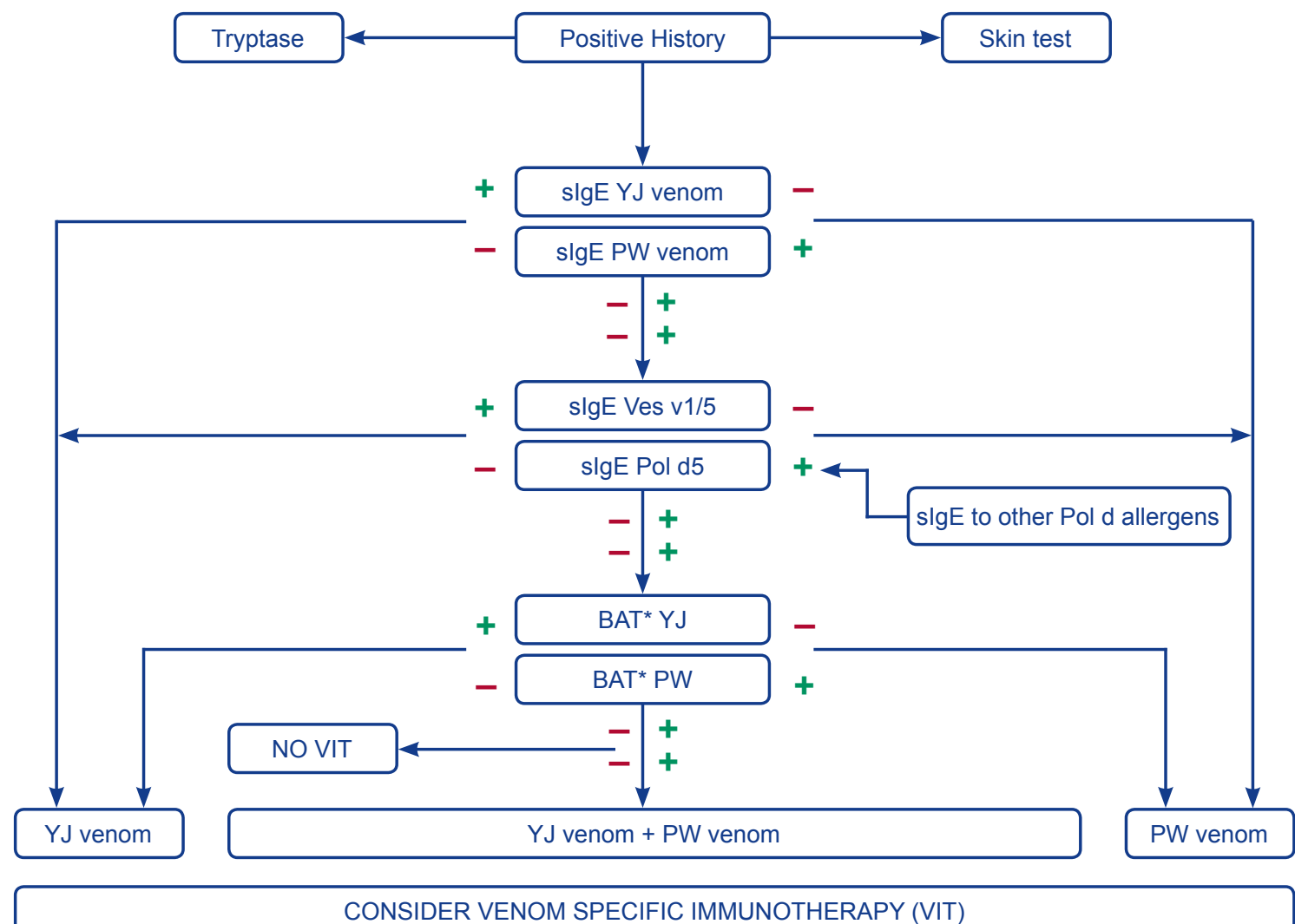

* BAT is not available for routine diagnosis in every clinic

Yellow jacket: YJ - Paper wasp: PW

Figure 2

Diagnostic algorithm in yellow jacket and paper wasp venom allergy. A red minus indicates a negative and a green plus a positive test results.

\section{Sting challenge}

As challenge test with subcutaneously or intracutaneously administered venom is not reliable, it should be performed using live insects (47). The outcome of sting challenge is influenced by a number of factors, including insect biology, indicating that bees yield more reliable sting challenge results than do vespids. However, sting challenge should not be used as a diagnostic tool in untreated patients, as a tolerated sting challenge does not fully predict the outcome of future stings in an individual patient and as untreated patients may develop very severe reactions to a sting challenge (47).

\section{Prevention and Therapy}

\section{Preventive measures}

A series of recommendations have been formulated aimed at substantially minimizing the risk of field re-sting, although as yet no evidence-based studies have been performed to support this.

Untreated patients with anaphylaxis should not be given $\beta$-blockers, except when the administration of these drugs is urgently required as in the case of certain cardiac arrhythmias. If possible, angiotensin converting enzyme inhibitors (ACEI) should also be avoided.

\section{Pharmacotherapy (emergency kit)}

A) Hymenoptera venom allergic patients should carry an emergency kit for self-administration at all times, especially during the insect season.

B) Self-injectable adrenaline should be considered for all patients with a history of a SR, particularly those who have experienced 'moderate-severe' 
episodes or those with increased risk of future exposure to stings (beekeepers, gardeners, waste management workers etc.), underlying mast cell disorders or raised baseline serum tryptase or other co-morbidities (48).

\section{Venom specific immunotherapy (VIT)}

The efficacy of subcutaneous venom immunotherapy has been confirmed by both sting challenge and infield sting in prospective controlled and uncontrolled studies, in one meta-analysis and systematic reviews demonstrating that the protection rate of vespid VIT is greater than that of honeybee VIT $(39,40,48-50)$.

The repeatedly observed difference in the success rates in bee and vespid venom-allergic patients is not completely clear. The fact that the amount of venom delivered by a bee sting during a sting challenge is much larger and more consistent may partially explain this difference in the reaction rate to sting challenges, which has also been observed in untreated patients (668). The composition of bee venom, which is a mixture of proteins and other pharmacologically active molecules, including melittin, and/or the absence or underrepresentation of major allergens in commercially available venom preparations may be another alternative explanation (52).

Finally, venom-allergic patients with mast cell diseases will benefit from VIT, albeit to a lesser extent than patients without mastocytosis (53).

\section{References}

1. Bilò BM, Bonifazi F. Advances in hymenoptera venom immunotherapy. Curr Opin Allergy Clin Immunol 2007;7:567-573.

2. Peiren N, Vanrobaeys F, de Graaf DC, Devreese B, Van Beeumen J, Jacobs FJ. The protein composition of honeybee venom reconsidered by a proteomic approach. Biochim Biophys Acta 2005;1752:1-5.

3. Honeybee Genome Sequencing Consortium. Insights into social insects from the genome of the honeybee Apis mellifera. Nature 2006;443:931-949.

4. King TP, Spangfort MD. Structure and biology of stinging insect venom allergens. Int Arch Allergy Immunol 2000;123:99-106.

5. Müller UR. Recombinant Hymenoptera venom allergens. Allergy 2002;57:570-576.

6. Kolarich D, Léonard R, Hemmer W, Altmann F. The $\mathrm{N}$-glycans of yellow jacket venom hyaluronidases and the protein sequence of its major isoform in Vespula vulgaris. FEBS J 2005;272:5182-5190.
7. Seismann H, Blank S, Braren I, Greunke K, Cifuentes L, Grunwald T et al. Dissecting cross-reactivity in hymenoptera venom allergy by circumvention of alpha-1,3-core fucosylation. Mol Immunol 2010; 47:799-808.

8. Blank S, Seismann H, Bockisch B, Braren I, Cifuentes $L$, Mclntyre $M$ et al. Identification, recombinant expression, and characterization of the $100 \mathrm{kDa}$ high molecular weight Hymenoptera venom allergens Api m 5 and Ves v 3. J Immunol 2010;184:5403-5413.

9. Blank S, Seismann H, Mclntyre M, Ollert M,Wolf S, Bantleon Fl et al. Vitellogenins are new high molecular weight components and allergens (Api m 12 and Ves v 6) of Apis mellifera and Vespula vulgaris venom. PLoS One 2013;8:e62009.

10. Markovic-Housley Z, Miglierini G, Soldatova L, Rizkallah PJ, Muller U, Schirmer T. Crystal structure of hyaluronidase, a major allergen of bee venom. Structure 2000;8:1025-1035.

11. Biasini M, Bienert S, Waterhouse A, Arnold K, Studer G, Schmidt T et al. SWISS-MODEL: modelling protein tertiary and quaternary structure using evolutionary information. Nucleic Acids Res 2014;42:W252-258.

12. Bilò $M B$, Bonifazi $F$. The natural history and epidemiology of insect venom allergy: clinical implications. Clin Exp Allergy 2009;39:1467-1476.

13. Sturm GJ, Kranzelbinder B, Schuster C, Sturm EM, Bokanovic D, Vollmann J et al. Sensitization to Hymenoptera venoms is common, but systemic sting reactions are rare. J Allergy Clin Immunol 2014;133:1635-1643.

14. Golden DB. New directions in diagnostic evaluation of insect allergy. Curr Opin Allergy Clin Immunol 2014;14:334-339.

15. Müller UR, Johansen N, Petersen AB, Fromberg-Nielsen J, Haeberli G. Hymenoptera venom allergy: analysis of double positivity to honey bee and Vespula venom by estimation of IgE antibodies to species-specific major allergens Api $\mathrm{m} 1$ and Ves v5. Allergy 2009;64:543548.

16. Hofmann SC, Pfender N, Weckesser S, Huss-Marp J, Jakob T. Added value of IgE detection to rApi $\mathrm{m} 1$ and $\mathrm{rVes} \vee 5$ in patients with Hymenoptera venom allergy. J Allergy Clin Immunol 2011;127:265-267.

17. Korošec P, Valenta R, Mittermann I, Celesnik N, Silar M, Zidarn $M$ et al. High sensitivity of CAP-FEIA rVes $v 5$ and $\mathrm{rVes} v 1$ for diagnosis of Vespula venom allergy. $J$ Allergy Clin Immunol 2012;129:1406-1408.

18. Mittermann I, Zidarn M, Silar M, Markovic-Housley Z, Aberer W, Korosec P et al. Recombinant allergenbased IgE testing to distinguish bee and wasp allergy. J Allergy Clin Immunol 2010;125:1300-1307.

19. Vos B, Köhler J, Müller S, Stretz E, Ruëff F, Jakob T. Spiking venom with rVes $v 5$ improves sensitivity of IgE 
detection in patients with allergy to Vespula venom. J Allergy Clin Immunol 2013;131:1225-1227.

20. Cifuentes L, Vosseler S, Blank S, Seismann H, Pennino $D$, Darsow $U$ et al. Identification of Hymenoptera venom-allergic patients with negative specific IgE to venom extract by using recombinant allergens. J Allergy Clin Immunol 2014;133:909-910.

21. Ebo DG, Faber M, Sabato V, Leysen J, Bridts CH, De Clerck LS. Component-resolved diagnosis of wasp (yellow jacket) venom allergy. Clin Exp Allergy 2013;43:255-261.

22. Seismann H, Blank S, Cifuentes L, Braren I, Bredehorst R, Grunwald T et al. Recombinant phospholipase A1 (Ves $v$ 1) from yellow jacket venom for improved diagnosis of hymenoptera venom hypersensitivity. Clin Mol Allergy 2010;8:7.

23. Jin C, Focke $M$, Léonard R, Jarisch R, Altmann F, Hemmer W. Reassessing the role of hyaluronidase in yellow jacket venom allergy. J Allergy Clin Immunol 2010;125:184-190.

24. Sturm GJ, Bilò MB, Bonadonna P, Hemmer W, Caruso $B$, Bokanovic $D$ et al. Ves $\vee 5$ can establish the diagnosis in patients without detectable specific lgE to wasp venom and a possible north-south difference in Api $\mathrm{m} 1$ sensitization in Europe. J Allergy Clin Immunol 2012;130:817.

25. Bokanovic D, Schwarz I, Wutte N, Komericki P, Aberer W, Sturm GJ. Specificity of conventional and Ves $v$ 5 -spiked venom decreases with increasing total lgE. J Allergy Clin Immunol 2014;134:739-741.

26. Rafei-Shamsabadi D, Müller S, Pfützner W, Spillner E, Ruëff $F$, Jakob $T$. Recombinant allergens rarely allow identification of Hymenoptera venom-allergic patients with negative specific lgE to whole venom preparations. J Allergy Clin Immunol 2014;134:493-494.

27. Grant JA, Rahr R, Thueson DO, Lett-Brown MA, Hokanson JA, Yunginger JW. Diagnosis of Polistes wasp hypersensitivity. J Allergy Clin Immunol 1983;72:399406.

28. Blanca M, Garcia F, Miranda A, Carmona MJ, Garcis J, Fernandez J et al. Determination of IgE antibodies to Polistes dominulus, Vespula germanica and Vespa crabro in sera of patients allergic to vespids. Allergy 1991;46:109-114.

29. Severino MG, Campi P, Macchia D, Manfredi M, Turillazzi S, Spadolini I et al. European Polistes venom allergy. Allergy 2006;61:860-863.

30. Monsalve RI, Vega A, Marqués L, Miranda A, Fernandez $\mathrm{J}$, Soriano $\mathrm{V}$ et al. Component-resolved diagnosis of vespid venom-allergic individuals: phospholipases and antigen $5 \mathrm{~s}$ are necessary to identify Vespula or Polistes sensitization. Allergy 2012;67:528-536.

31. Blank S, Neu C, Hasche D, Bantleon FI, Jakob T, Spillner E. Polistes species venom is devoid of carbohydrate- based cross-reactivity and allows interference-free diagnostics. J Allergy Clin Immunol 2013;131:12391242.

32. Dalmau Duch G, Gázquez García V, Gaig Jané P, Galán Nieto A, Monsalve Clemente RI. Importance of controlled sting challenge and component-resolved diagnosis in the success of venom immunotherapy. $J$ Investig Allergol Clin Immunol 2012;22:135-136.

33. Bilò BM, Rueff $F$, Mosbech $H$, Bonifazi F, OudeElberink JN, EAACl Interest Group on Insect Venom Hypersensitivity: Diagnosis of Hymenoptera venom allergy. Allergy 2005;60:1339-1349.

34. Golden DB, Kelly D, Hamilton RG, Wang NY, Kagey-Sobotka A. Dialyzed venom skin testsfor identifying yellow jacket-allergic patients not detected using standard venom. Ann Allergy Asthma Immunol 2009;102:47-50.

35. Pantera B, Hoffman DR, Carresi L, Cappugi G, Turillazzi $S$, Manao G, et al.Characterization of the major allergens purified from the venom of the paper wasp Polistes gallicus. Biochim Biophys Acta 2003;1623:7281.

36. van der Linden PW, Hack CE, Struyvenberg A, van der Zwan JK. Insect-sting challenge in324 subjects with a previous anaphylactic reaction: current criteria for insect-venomhypersensitivity do not predict the occurrence and the severity of anaphylaxis. J Allergy Clinlmmunol 1994;94:151-159.

37. Sturm GJ, Schuster C, Kranzelbinder B, Wiednig M, Groselj-Strele A, Aberer W.Asymptomatic sensitization to hymenoptera venom is related to total immunoglobulin Elevels. Int Arch Allergy Immunol 2009;148:261-264.

38. Schafer T, Przybilla B. IgE antibodies to Hymenoptera venoms in the serum are common inthe general population and are related to indications of atopy. Allergy 1996;51:372-377.

39. Golden DB, Moffitt J, Nicklas RA, Freeman T, Graft DF, Reisman RE, et al. Stinging insecthypersensitivity: a practice parameter update 2011. J Allergy Clin Immunol 2011;127:852-854 e851-823.

40. Krishna MT, Ewan PW, Diwakar L, Durham SR, Frew AJ, Leech SC, et al. Diagnosis andmanagement of hymenoptera venom allergy: British Society for Allergy and Clinicallmmunology (BSACI) guidelines. Clin Exp Allergy 2011;41:1201-1220.

41. Golden DB, Kagey-Sobotka A, Norman PS, Hamilton RG, Lichtenstein LM. Insect stingallergy with negative venom skin test responses. J Allergy Clin Immunol 2001;107:897-901.

42. Sturm GJ, Heinemann A, Schuster $C$, Wiednig $M$, Groselj-Strele A, Sturm EM, et al.Influence of total IgE levels on the severity of sting reactions in Hymenoptera venomallergy. Allergy 2007;62:884-889. 
43. Blum S, Gunzinger A, Muller UR, Helbling A. Influence of total and specific IgE, serumtryptase, and age on severity of allergic reactions to Hymenoptera stings. Allergy 2011;66:222-228.

44. Korosec P, Silar M, Erzen R, Celesnik N, Bajrovic N, Zidarn $\mathrm{M}$, et al. Clinical routine utilityof basophil activation testing for diagnosis of hymenopteraallergic patients with emphasison individuals with negative venom-specific IgE antibodies. Int Arch Allergy Immunol 2013;161:363-368.

45. Eberlein B, Krischan L, Darsow U, Ollert M, Ring J. Double positivity to bee and waspvenom: improved diagnostic procedure by recombinant allergen-based IgE testing andbasophil activation test including data about cross-reactive carbohydrate determinants. J Allergy Clin Immunol 2012;130:155-161.

46. Balzer L, Pennino D, Blank S, Seismann H, Darsow $\mathrm{U}$, Schnedler $\mathrm{M}$ et al. Basophilactivation test using recombinant allergens: highly specific diagnostic methodcomplementing routine tests in wasp venom allergy. PLoS One 2014;9:e108619.

47. Ruëff F, Przybilla B, Müller U, Mosbech H. The sting challenge test in hymenoptera venomallergy. Position paper of the Subcommittee on Insect Venom Allergy of the EuropeanAcademy of Allergology and Clinical Immunology. Allergy 1996;51:216-225.
48. Bonifazi F, Jutel M, Bilo BM, Birnbaum J, Muller U. EAACI Interest Group on Insect VenomHypersensitivity: Prevention and treatment of hymenoptera venom allergy: guidelines forclinical practice. Allergy 2005;60:1459-1470.

49. Hockenhull J, Elremeli M, Cherry MG, Mahon J, Lai $M$, Darroch J, Oyee J, et al. Asystematic review of the clinical effectiveness and cost-effectiveness of Pharmalgen ${ }^{\circledR}$ forthe treatment of bee and wasp venom allergy. Health Technol Assess 2012;16:III-IV, 1-110.

50. Boyle RJ, Elremeli M, Hockenhull J, Cherry MG, Bulsara MK, Daniels M, Oude Elberink JN.Venom immunotherapy for preventing allergic reactions to insect stings. CochraneDatabase Syst Rev 2012;10:CD008838.

51. Bilò MB, Antonicelli L, Bonifazi F. Honeybee venom immunotherapy: certainties and pitfalls. Immunotherapy 2012;4:1153-1166.

52. Blank S, Seismann H, Michel Y, Mclntyre M, Cifuentes L, Braren I, et al. Api m 10, agenuine A. mellifera venom allergen, is clinically relevant but underrepresented intherapeutic extracts. Allergy 2011;66:1322-1329.

53. González-de-Olano D, Alvarez-Twose I, Vega A, Orfao A, Escribano L. Venom immunotherapy in patients with mastocytosis and hymenoptera venom an. Immunotherapy 2011;3:637-651. 



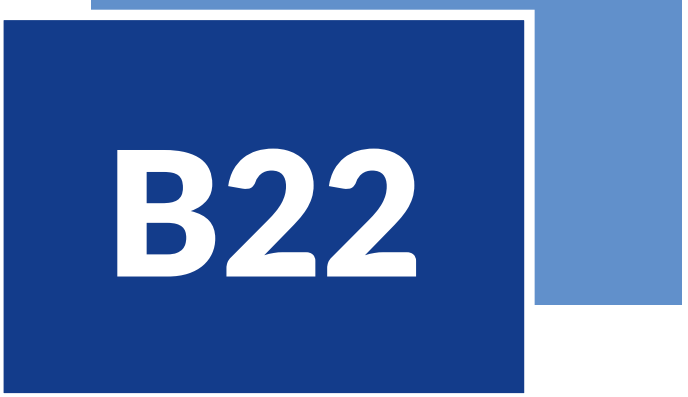

\author{
Monika Raulf
}

\section{INTRODUCTION}

Adverse reactions to latex include non-allergic contact dermatitis, delayed type IV and immediate type I hypersensitivity. Irritant contact dermatitis and type IV hypersensitivity are immediate or delayed (24-96 hours) responses to chemicals and additives in latex products. Type I hypersensitivity is the most serious response to latex proteins occurring minutes to hours after exposure and ranging from mild irritation to loss of life. Here the term "latex allergy" covers latex protein induced IgE-mediated hypersensitivity reactions.

\section{THE ALLERGEN SOURCES}

During the late 1980s and early 1990s natural rubber latex (NRL) allergy has become an important occupational health concern, particularly among hospital personnel especially health-care workers (HCW) and medical doctors and in dental clinics. Additionally, in children with spina bifida also a high prevalence of latex sensitization (up to $72 \%$ ) was observed. The milky sap of the rubber tree
Implementation of low-allergen, powder-free latex gloves or latex-free devices decrease the prevalence of latex allergy

Several recombinant latex allergens (Hev b 1 -15) are available and useful for the determination of sensitization profiles and exclusion of glyco-epitopes (with low clinical relevance) responsible for lgE-binding to latex; the serological work-up for the diagnosis of latex type I-allergy is highly recommended;

With respect of the sensitivity of the lgE assays, the improved natural latex extract spiked with the recombinant Hev b 5 is a sensitive tool for the diagnosis of latex sensitization and superior compared to the available panel of recombinant latex allergens. 

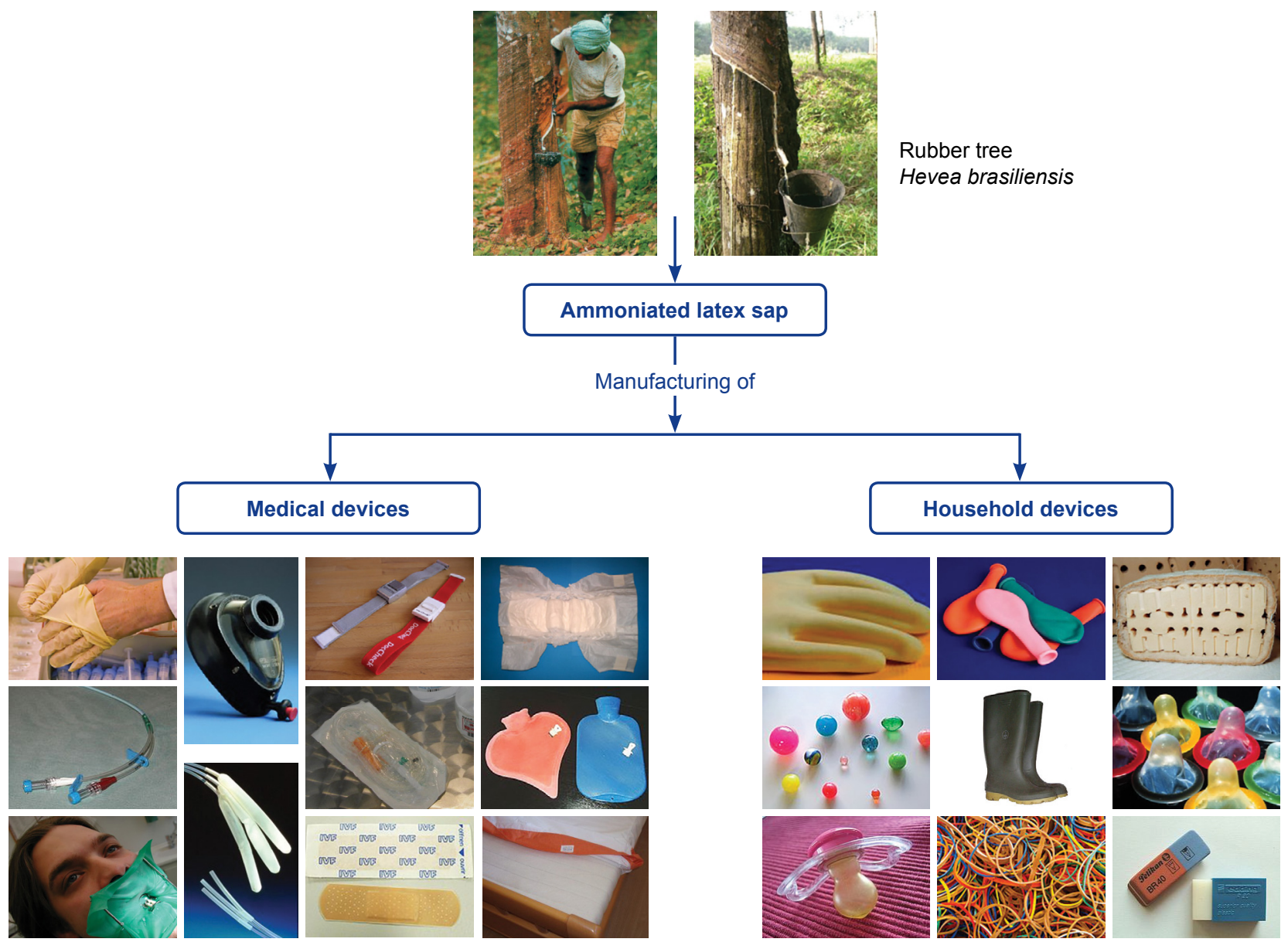

Figure 1

From the sap of the latex tree Hevea brasiliensis to multiple latex devices.

Hevea brasiliensis is the source for the production of commercial natural rubber latex (NRL) devices and represents also a source of potent allergenic proteins (1, 2) (Fig. 1).

Most of Hevea brasiliensis grows commercially in a number of tropical countries, mainly in Thailand, Indochina, Malaysia and India. The milky sap is synthesized by specialized laticifer cells and collection of the latex is possible by scarifying the trunk of the Hevea brasiliensis tree. Ammonia treatment prevents coagulation resulting in hydrolysis of the latex proteins. The main constituent of Hevea latex is the polymeric hydrocarbon 1,4 cis-poly-isoprene and only $1-2 \%$ of the fresh milky sap is made up of proteins. The proteins are heterogeneously distributed in the latex sap and they are involved in the biosynthesis of the polyisoprene, associated with the coagulation of latex and in the defense of the plant against various diseases. After ultra-centrifugation of the fresh latex sap basically three main fractions (rubber phase, the $\mathrm{C}$-serum and the bottom fraction (B-serum) are easily discerned (Fig. 2).

The rubber phase comprises the rubber particles and two main, insoluble proteins, which are extractable from the surface of the rubber particles. Most of the $\mathrm{C}$-serum and $\mathrm{B}$-serum proteins are water-soluble. Latex $\mathrm{C}$-serum contains various proteins (more than 200 polypeptides) and some of them are enzymes associated with the rubber biosynthesis (3).

Allergic reactions have been described through direct skin/mucus membrane contact or via inhalation. For HCWs, there are numerous potential routes of exposure to NRL allergens. Skin exposure may 


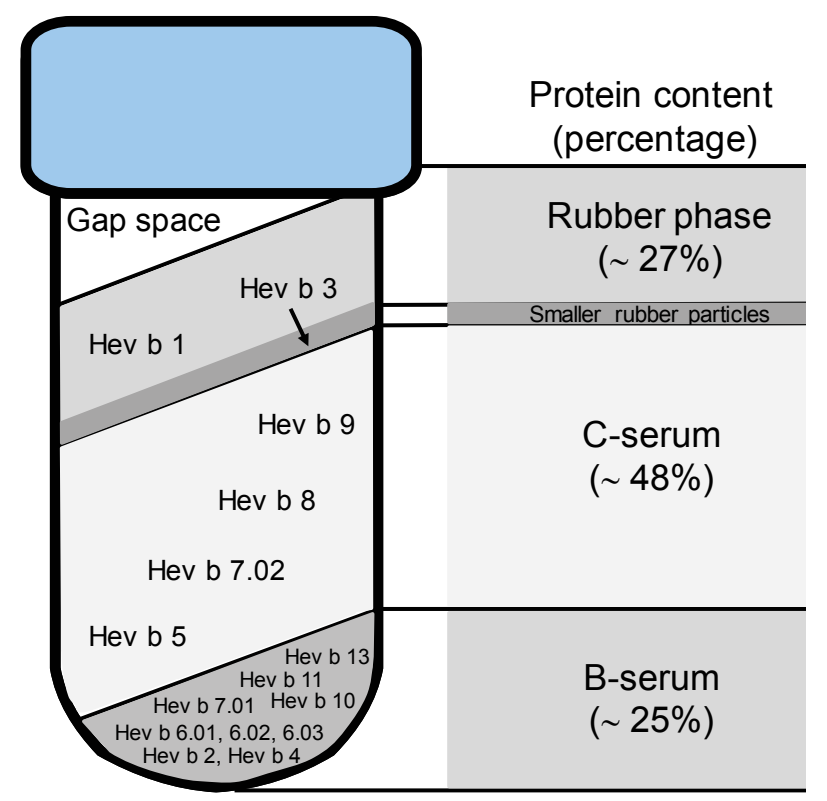

Figure 2

Separation of the latex sap into three main fractions after centrifugation and distribution of the latex allergens.

occur from direct contact with many NRL products, such as latex gloves. In the case of latex gloves, lubricants that facilitate donning and removal, such as cornstarch, may be added by dipping the gloves into a powder-slurry. Proteins, liberated from the latex, attach to the powder as the slurry dries onto the gloves. This powder can act as a carrier for latex proteins and protein-loaded powder particles can become aeroallergens during donning and removal of the gloves. Inhalational exposure may occur when aerosolized glove powder coated with NRL allergens comes into contact with the mucous membranes of the nose, throat and airways of the lung.

Before initiating a primary NRL prophylaxis around and during surgery and anesthesia of all spina bifida patients and the introduction of powder-free gloves with reduced protein levels in hospitals, children with spina bifida, health care workers ( $\mathrm{HCW}$ ), and persons with a history of multiple surgeries belonged to a group with a higher risk to develop a NRL allergy. In addition, high risks were also described for nonhealth care workers with exposure to latex such as hairdressers, cleaners and food-service workers, as well as workers in rubber industrial companies or subjects with food allergy and atopy.

\section{MAJOR AND RELEVANT MINOR ALLERGENIC MOLECULES}

Up to now about 250 different NRL polypeptides were identified. About 60 are capable to bind human IgE (3) and currently 15 allergens have been included in the latest nomenclature list of the International Nomenclature Committee of Allergens (IUIS) and assigned official numbers (Hev b 1-15) (http://www. allergen.org) (Table 1, Fig. 2).

Hev b 1 together with Hev b 3 are rubber particle proteins. Hev b 5 is an acidic (pl 3.5) and heatstable 16-24 kDa protein, rich of glutamic acid as well as of proline residues. The first recombinant Hev b 5 (rHev b 5) was described by Slater et al. (4). Prohevein, assigned by the WHO/IUIS as Hev b 6.01, was isolated from the $\mathrm{B}$-serum and posttranslational cleavage proceeds two further proteins, the $4.7 \mathrm{kDa}$ hevein (Hev b 6.02) and the $14 \mathrm{kDa} C$-terminal domain Hev b 6.03 (5). All three allergens additionally exist in the plant and the ratio between Hev b 6.01 and Hev b 6.03 is about 30:1. Hev b 6.02 (hevein) comprises the most important part of IgE-binding epitopes in the prohevein molecule. In addition, hevein shows homology to several chitin-binding lectin domains (4) and may be responsible for certain cross-reactivity to several other plants and food. Most of the Hev b-proteins have been cloned and expressed as recombinant proteins. Sequencing demonstrated both unique epitopes and sequences commonly found in other plant proteins. Sequence homology helps to explain the cross-reactivity to a variety of foods experienced by latex allergic individuals.

\section{SENSITIZATION TO INDIVIDUAL MOLECULES AND THEIR CLINICAL RELEVANCE}

Depending on the definition of the study group and the methods for assessment of latex sensitization and/or allergy (mainly based on skin or IgE testing with latex extracts), the observed prevalence among health care workers ranges between 0-30\%, whereas the prevalence among children with spina bifida ranges between $25-72 \%$ (5). Among adult blood donors showing latex-specific IgE antibodies two separate studies reported a sensitization frequency of about $6 \%(6)$. In contrast, the prevalence in the general population is between 0 and $2.3 \%$ (7). Individuals who are allergic to latex products may 


\section{Table 1}

Immunological and clinical properties of characterized latex allergens of the rubber tree Hevea brasiliensis

\begin{tabular}{|c|c|c|c|}
\hline Allergenic molecule & Biochemical name & Prevalence among patients* & MW (in kDa) \\
\hline Hev b 1 & Rubber Elongation Factor (REF) & $81 \%^{1}$ & 14 \\
\hline Hev b 2 & $\beta$-1,3-Glucanase & $70 \%^{1,2,3}$ & 34 \\
\hline Hev b 3 & Small rubber particle proteins & $58 \%^{1}$ & 24 \\
\hline Hev b 4 & Lecithinase homologue & - & $53-55$ \\
\hline Hev b 5 & Acidic structural protein & up to $60 \%{ }^{2}$ & 16 \\
\hline Hev b 6 & Prohevein (hevein precursor) & up to $70 \%^{2}$ & 20 \\
\hline Hev b 7 & $\begin{array}{l}\text { Patatin-like protein (esterase) } \\
\text { from latex-B- and C-serum }\end{array}$ & $25 \%^{1,2}$ & 44 \\
\hline Hev b 8 & Profilin (actin-binding protein) & $10 \%^{1,2}$ & 14 \\
\hline Hev b 9 & Enolase & up to $3 \%^{2}$ & 51 \\
\hline Hev b 10 & $\begin{array}{l}\text { Manganese superoxide } \\
\text { dismutase (MnSOD) }\end{array}$ & up to $3 \%^{2}$ & 26 \\
\hline Hev b 11 & Chitinase class I & up to $18 \%^{1,2}$ & 30 \\
\hline Hev b 12 & $\begin{array}{l}\text { Non-specific Lipid Transfer } \\
\text { Protein type } 1 \text { (nsLTP1) }\end{array}$ & - & 9 \\
\hline Hev b 13 & Esterase & up to $80 \% 1,2,3$ & 42 \\
\hline Hev b 14 & Hevamine & - & 30 \\
\hline Hev b 15 & Serine protease inhibitor & - & 7.5 \\
\hline
\end{tabular}

${ }^{1}$ among spina bifida patients, ${ }^{2}$ among health care workers, ${ }^{3}$ only testing with native Hev b 2 or Hev b 13 , respectively, ${ }^{*}$ all data published in (8)

experience allergic reactions due to cross-reactivity from a variety of fresh fruits, vegetables and nuts.

Latex allergy shows distinct patterns that can be predicted through component-resolved diagnosis including cross-reactive carbohydrate determinants to clarify if protein epitopes (with clinical relevance) or the glyco-epitopes (with low clinical relevance) are responsible for the IgE-binding to latex. Based on different exposure routes, different allergens are dominantly recognized by IgE of latex-sensitized patients. The recombinantly available latex allergens Hev b 5 and Hev b 6.01 are the major allergens involved in sensitizing HCW (8).

Hev b 1 and Hev b 3 are of minor relevance since $<20 \%$ of the HCW have positive IgE antibody responses to these insoluble particle-bound allergens. Based on this insolubility, Hev b 1 and Hev b 3 are much harder to aerosolize, and their sensitization process thus seems to require contact to latex with blood or mucosal surfaces, which occurs during surgery. Hev b 7.02 appears to be of an intermediate relevance and showed no association to the atopic status or the presence of latex-fruit syndrome. Hev b 11.0102, the Hevea latex class I chitinase derived from Hevea leaves displays minor relevance as allergen in $\mathrm{HCW}$ and is only recognized in sera with slgE responses to $\mathrm{rHev} b$ 6.01. Lee et al. described that hevamine (Hev b 14) and Hev b 1 are the major allergens in Taiwanese medical workers. Major allergens in spina bifida patients are Hev b 1, Hev b 3, Hev b 5 and Hev b 6.01 (8).

\section{CLINICAL RELEVANCE, DIAGNOSIS AND MANAGEMENT}

The latex epidemic hit its peak in the mid-1990s. Since then, the apparent prevalence of latex allergy has been steadily decreasing. The implementation of 


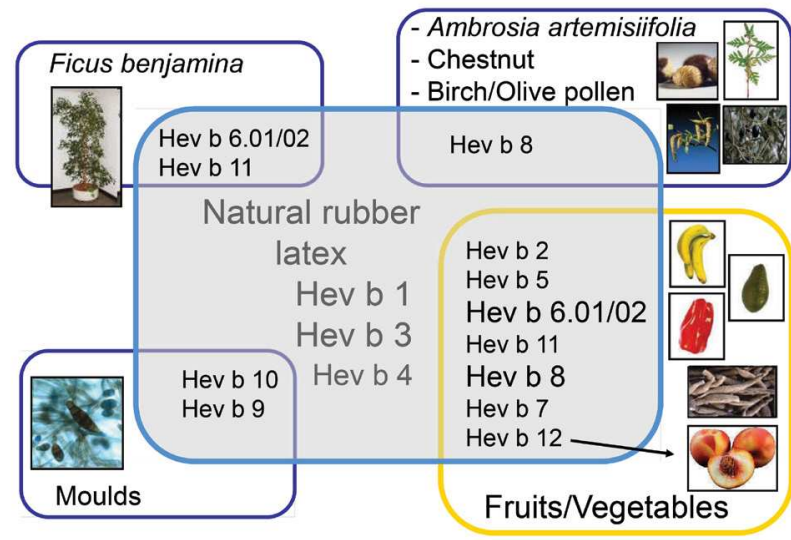

Figure 3

Molecular background and association of latex-fruit/vegetable syndrome -allergens with potential importance for cross-reactivity. (modified according to (26)).

low-allergen, powder-free latex gloves in hospitals had shown a dramatic decrease in the prevalence of latex allergy.

About $30-50 \%$ of latex-allergic patients show allergic symptoms to plant-derived foods, especially fresh fruits (9). The association was called latex-fruit syndrome [review in (10), (11) and huge amounts of relevant fruits, constantly increasing, are described and the most commonly involved are avocado, banana, chestnut, and kiwi. Several latex allergens were discussed as responsible for the latex-fruit cross-reactivity (Fig. 3), such as Hev b 2 (12), Hev b 6.02 (13-15) Hev b 7 (16), Hev b 8 (17) and Hev b 12 (18). The time course of sensitization is a topic of discussion. In the literature there are some reports describing a symptomatic fruit allergy (banana) before symptoms of NRL allergy were noted (19) as well as NRL allergic symptoms preceded a banana allergy. In some cases the use of recombinant single latex allergens for latex-specific IgE mapping is helpful to discriminate between cross-reactivity and co-sensitization to latex and fruits $(15,17)$.

\section{Diagnosis}

The diagnostic approach for latex allergy starts with obtaining a detailed clinical history, including the history of risk factors and the temporal relationship between exposure to NRL devices and the symptoms. Specific questions concerning symptoms at the workplace, use of latex devices etc. proper interpretation of sensitization tests (i.e. skin prick test, lgE) and optional challenge test with powdered gloves and glove-wearing test help to establish the diagnosis of type I- latex allergy. In the past the diagnostic of a latex sensitization was rather difficult, because several studies demonstrated that IgE test systems gave false-negative results in more than $25 \%$ of patients with allergic reactions based on NRL and skin test positive subjects (20). One reason for these discrepancies was a missing allergen. Indeed, by addition of rHev b 5 to the diagnostic repertoire of latex allergens, 8 out of 16 subjects with clear latex allergy and negative latex-specific IgE value using Thermo Fisher (Phadia) CAP system showed significantly elevated IgE levels to Hev b 5 (21). These findings demonstrate that latex allergen $\mathrm{Hev} b$ 5 may play a special role in diagnostic tests showing false-negative IgE results. The addition of $\mathrm{rHev} b 5$ in sufficient amounts improved the ImmunoCAP used for serological testing significantly $(22,23)$. These results indicate a new approach: if relevant allergens are too labile to survive all steps required for the production of a standardized allergen preparation they can be added as a stable recombinant protein to the allergen preparation during the production.

In contrast, false-positive latex-specific IgE results in subjects without clinical symptoms to latex and negative skin prick test most often base on IgE-binding to $\mathrm{N}$-glycans and/or O-glycans which are often part of allergens of plant origin, like latex. In these cases e.g. application of CCD tools (like horseradish peroxidase (HRP) and/or inhibition studies with "cross-reactive carbohydrate determinants" (CCD) to clarify if protein epitopes (with clinical relevance) or the glyco-epitopes (with low clinical relevance) are responsible for the IgE-binding to latex (8) are helpful. In addition, component-resolved diagnosis using microarray (24) or single placed ImmunoCAP coupled with recombinant or native allergens (8) are reliable tools for diagnosing latex allergy. Following serological work-up (Fig. 4) might support proper diagnosis.

\section{Clinical management}

Studies have shown that economically feasible interventions to reduce NRL exposure can successfully allow latex-allergic individuals to continue working. Implementation of powder-free gloves with low or undetectable NRL allergen was a successful strategy to reduce the latex allergen concentration at the work site and allowed most 


\section{Table 2}

Improvement of NRL allergy diagnostics

\begin{tabular}{|c|c|c|c|c|c|}
\hline Method & Sensitivity & Specificity & $\begin{array}{l}\text { Positive predic- } \\
\text { tive value (PPV) }\end{array}$ & $\begin{array}{l}\text { Negative predic- } \\
\text { tive value (NPV) }\end{array}$ & Test efficacy \\
\hline NRL (k82) & $76 \%$ & $98.3 \%$ & $98.1 \%$ & $78.7 \%$ & $86.7 \%$ \\
\hline $\mathrm{k} 82+{ }_{\text {„rHev b } 5 “}$ & $90 \%$ & $98.3 \%$ & $98.4 \%$ & $89.4 \%$ & $93.75 \%$ \\
\hline rHev b-mix* & $83.6 \%$ & $98.3 \%$ & $98.2 \%$ & $84.3 \%$ & $90.6 \%$ \\
\hline
\end{tabular}

Spiking of the NRL extract with the rHev b 5 was a suitable method to improve the in vitro latex allergy diagnostic; ${ }^{*} r H e v b$-mix include $r$ Hev $b 1$, $r \mathrm{Hev} b 5, r \mathrm{Hev} b 6.01$ and $r \mathrm{Hev} b$ 8; data published in (8)

HCWs to remain on the job, and decreased the number of new cases of occupational latex allergy. The complete cessation of exposure was associated with a greater rate of asthma-related disability and loss of income; therefore, reduction of exposure to latex is safe and more economical alternative to complete removal from the workplace. The risk of re-sensitization by re-exposure to NRL has to be minimized. In some cases, when avoidance measures are not feasible or effective, specific immunotherapy with latex extracts can be offered (25).

\section{CLINICAL CASE}

\section{Case 1 (published (15))}

History: man, 37 years old, developed urticaria with skin redness, itching, dyspnea and tachycardia 5 minutes after drinking a glass of apple juice supplemented with acerola (Malpighia glabra; Barbados cherry); no allergy to apple and apple juice was well tolerated; in the past seasonal hay fever symptoms caused by grass pollen and wild herbal pollen; since childhood, a significant contact urticaria induced by natural rubber latex products was well known; OAS after ingesting avocado, celery, walnut, and curry during pollen season.

SPT: wheal size same as for histamine - grass pollen mixture, latex, rye; weak reactions to plantain, hazel, birch pollen; limited reaction to mugwort and ragweed pollen and to curry; intracutaneous skin reaction with acerola pulp and with acerolacontaining apple juice; apple juice without acerola negative.

In-vitro testing: Total IgE $145 \mathrm{kU} / \mathrm{L}$, specific IgE to latex $24.7 \mathrm{kU} / \mathrm{L}$; acerola (RAST) $1.5 \mathrm{kU} / \mathrm{L}$; CAP class 3 to grass pollen, CAP class 2 to plantain, peanut, tomato soy bean, CAP class 1 to hazelnut pollen, ragweed pollen, banana, green apple, herbal mixture and nut mixture; single recombinant latex allergens: Hev b 6.01, $17.1 \mathrm{kU} / \mathrm{L}$; Hev b 6.02, 18.7 kU/L; Hev b 8, 0.7 kU/L; Hev b 1, 3, 5, $10<0.35$ $\mathrm{kU} / \mathrm{L}$. Inhibition studies showed that IgE binding to acerola allergens was completely inhibited by latex and acerola extract, whereas preincubation of the patient's serum with acerola showed nearly complete inhibition to latex (79\%) and rHev b 6.01 (85\%). rHev b 8 as a solid-phase allergen and acerola as an inhibitor revealed no significant inhibition.

Oral challenge: open oral challenge tests with apple juice with and without acerola and diluted acerola pulp; negative for apple juice; exposure to acerola pulp with a latency period of 5 minutes induced itching and swelling lips

Diagnosis: Latex-acerola cross-reactivity based on Hev b 6.01/Hev b 6.02; Primary sensitization to latex led to cross-reactivity to acerola; (Pro-) hevein is the important allergen and responsible for the cross-reactivity;

Allergologists should include acerola on the growing list of latex-cross-reactive food allergens and inform latex-allergic patients about cross-reacting allergens as the traces of acerola in apple juice.

\section{TOOLS}

For latex allergy diagnosis an improved latex ImmunoCAP spiked with rHev b 5 (ThermoFisher Scientific) is available and useful. In addition, recombinant single latex allergens are also 


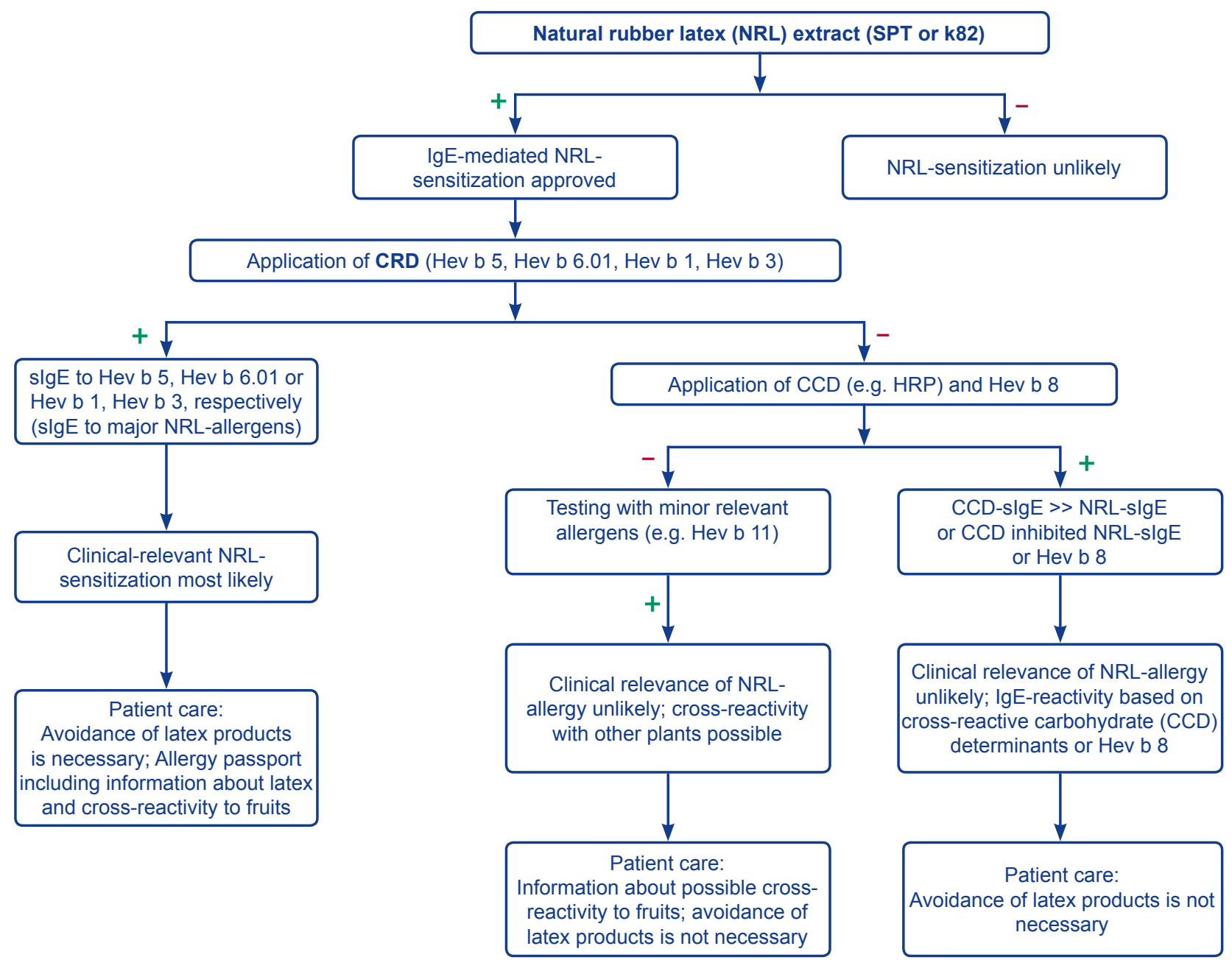

\section{Figure 4}

Serological work-up for the diagnosis of latex type I-allergy; This approach is not useful in patients with contact dermatitis and or protein contact dermatitis, where additional patch tests including other rubber components (e.g. chemical additives) might be needed (modified according to (26)).

commercially available (rHev b $1, \mathrm{rHev}$ b 3 , rHev b 5 , rHevb 6.01, rHevb 6.02, rHevb 8, rHevb 9, rHevb 11) for single testing (ImmunoCAP) and for screening in a multiplex system (ISAC allergen chip; ThermoFisher Scientific) and are useful for the determination of individual sensitization profiles, but in comparison to the rHev b 5-spiked latex extract their application achieves no better diagnostic sensitivity. Currently no latex skin test extract is commercially available e.g. in Germany.

\section{References}

1. Ownby DR. A history of latex allergy. J Allergy Clin Immunol 2002;110:S27-32.
2. Raulf M. The latex story. Chem Immunol Allergy 2014;100:248-255.

3. Posch A, Chen Z, Wheeler C, Dunn MJ, Raulf-Heimsoth $M$, Baur $X$. Characterization and identification of latex allergens by two-dimensional electrophoresis and protein microsequencing. I Allergy Clin Immunol 1997;99:385-395.

4. Slater JE, Vedvick T, Arthur-Smith A, Trybul DE, Kekwick RG. Identification, cloning, and sequence of a major allergen (Hev b 5) from natural rubber latex (Hevea brasiliensis). J Biol Chem 1996;271:2539425399.

5. Posch A, Chen Z, Raulf-Heimsoth M, Baur X. Latex allergens. Clin Exp Allergy 1998;28:134-140.

6. Ownby DR, Ownby HE, McCullough J, Shafer 
AW. The prevalence of anti-latex IgE antibodies in 1000 volunteer blood donors. J Allergy Clin Immunol 1996;97:1188-1192.

7. Turjanmaa $\mathrm{K}$, Alenius $\mathrm{H}$, Makinen Kiljunen S, Reunala T, Palosuo T. Natural rubber latex allergy. Allergy 1996;51:593-602.

8. Raulf-Heimsoth $M$, Rihs HP, Rozynek P, Cremer R, Gaspar A, Pires G et al. Quantitative analysis of immunoglobulin $E$ reactivity profiles in patients allergic or sensitized to natural rubber latex (Hevea brasiliensis). Clin Exp Allergy 2007;37:1657-1667.

9. Radauer C, Adhami F, Fürtler I, Wagner S, Allwardt $D$, Scala $E$ et al. Latex-allergic patients sensitized to the major allergen hevein and hevein-like domains of class I chitinases show no increased frequency of latex-associated plant food allergy. Mol Immunol 2011;48:600-609.

10. Blanco C. Latex-fruit syndrome. Curr Allergy Asthma Rep 2003;3:47-53.

11. Wagner $\mathrm{S}$, Breiteneder $\mathrm{H}$. The latex-fruit syndrome. Biochem Soc Trans 2002;30:935-940.

12. Barre A, Culerrier R, Granier C, Selman L, Peumans WJ, Van Damme, Els J.M. et al. Mapping of IgE-binding epitopes on the major latex allergen Hev b 2 and the cross-reacting $\beta$-1,3-glucanase fruit allergens as a molecular basis for the latex-fruit syndrome. Mol Immunol 2009;46:1595-1604.

13. Chen Z, Posch A, Cremer R, Raulf-Heimsoth M, Baur $X$. Identification of hevein ( Hev b 6.02) in Hevea latex as a major cross-reacting allergen with avocado fruit in patients with latex allergy. J Allergy Clin Immunol 1998;102:476-481.

14. Posch A, Wheeler $\mathrm{CH}$, Chen Z, Flagge A, Dunn MJ, Papenfuss $F$ et al. Class I endochitinase containing a hevein domain is the causative allergen in latex-associated avocado allergy. Clin Exp Allergy 1999;29:667-672.

15. Raulf-Heimsoth M, Stark R, Sander I, Maryska S, Rihs $\mathrm{H}$, Brüning $\mathrm{T}$ et al. Anaphylactic reaction to apple juice containing acerola: cross-reactivity to latex due to prohevein. J Allergy Clin Immunol 2002;109:715-716.

16. Schmidt, Mirko H H, Raulf-Heimsoth M, Posch A. Evaluation of patatin as a major cross-reactive allergen in latex-induced potato allergy. Ann Allergy Asthma Immunol 2002;89:613-618.

17. Raulf-Heimsoth M, Kespohl S, Crespo JF, Rodriguez J, FeliuA, Bruning Tetal. Natural rubber latex and chestnut allergy: cross-reactivity or co-sensitization. Allergy 2007;62:1277-1281.

18. Beezhold DH, Hickey VL, Kostyal DA, Puhl H, Zuidmeer $L$, van Ree $R$ et al. Lipid transfer protein from Hevea brasiliensis (Hev b 12), a cross-reactive latex protein. Ann Allergy Asthma Immunol 2003;90:439445.

19. M'Raihi L, Charpin D, Pons A, Bongrand P, Vervloet D. Cross-reactivity between latex and banana. J Allergy Clin Immunol 1991;87:129-130.

20. Hamilton RG, Adkinson NF. Diagnosis of natural rubber latex allergy: multicenter latex skin testing efficacy study. Multicenter Latex Skin Testing Study Task Force. J Allergy Clin Immunol 1998;102:482-490.

21. Chen Z, Rihs HP, Slater JE, Paupore EJ, Schneider $E M$, Baur $X$. The absence of Hev b 5 in capture antigen may cause false-negative results in serologic assays for latex-specific IgE antibodies. J Allergy Clin Immunol 2000;105:S83.

22. Lundberg M, Chen Z, Rihs HP, Wrangsjo K. Recombinant spiked allergen extract. Allergy 2001;56:794-795.

23. Huss-Marp J, RaulfM, Jakob T. Spiking with recombinant allergens to improve allergen extracts: benefits and limitations for the use in routine diagnostics. Part 19 of the Series Molecular Allergology. Allergo J Int. 2015;24:236-243.

24. Ebo DG, Hagendorens MM, De Knop, KJ, Verweij MM, Bridts $\mathrm{CH}$, De Clerck, $\mathrm{L} S$ et al. Component-resolved diagnosis from latex allergy by microarray. Clin Exp Allergy 2010;40:348-358.

25. Nettis E, Delle Donne P, Di Leo E, Fantini P, Passalacqua $\mathrm{G}$, Bernardini $\mathrm{R}$ et al. Latex immunotherapy: state of the art. Ann Allergy Asthma Immunol 2012;109:160165.

26. Raulf-Heimsoth, M, Rihs HP. Latexallergene: Sensibilisierungsquellen und Einzelallergenprofile erkennen. Allergo J Int 2011;20:241-243. 


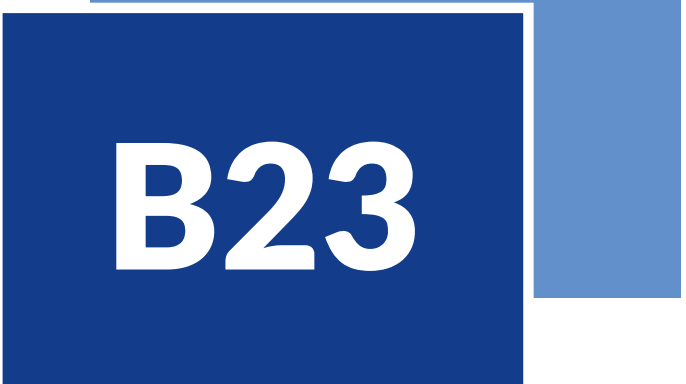

\author{
Monika Raulf
}

\section{THE ALLERGEN SOURCES}

Laboratory animal allergy (LAA) is an important occupational disease (1). Rodents like mice and rats frequently used in animal research are the most common causes of LAA. Also rabbits, hamsters and guinea pigs are used for research. A study from Japan (2) showed that the frequencies of symptoms against different laboratory animals were similar and that mice and rats are not more allergenic than other animals, but they are used more often in research facilities. LAA is commonly observed among technicians, animal caretakers, physicians, and scientists who work in pharmaceutical industries, university laboratories, and animal breeding facilities (3). In occupational settings, the prevalence rates of rodent allergies vary from 11 to $44 \%$ depending on the diagnostic methods (questionnaire or laboratory testing) used (2). The prevalence of mouse and rat allergy is very similar. Sensitization and work-related symptoms occur at the latest, 2-3 years after the initial exposure to laboratory animals (4). Besides an atopic background, the most important risk factor for the development of an allergy to rodents is the level of exposure to laboratory animal allergens. Symptoms of occupational allergy against mice and rats are
Laboratory animal allergy (LAA) is an important occupational disease and rodents like mice and rats frequently used in animal research are the most common causes of LAA.

Rodent allergens can be found in dander, hair, urine, saliva, and serum. Urine is the main source of allergenic proteins in both mice and rats.

As common for the most mammalian inhalant allergens, the major allergens from mouse, rat, guinea pig, hamster and rabbit are lipocalins.

Controlling of the airborne allergen exposure in animal facilities is possible by using commercially available sensitive immunoassays for mouse and rat allergens.

Specific lgE determination in the case of LAA based on extracts prepared from epithelia, serum-/urine protein as mixture or alone and is useful. Single LAA allergens are not commercially available so far for routine testing. 


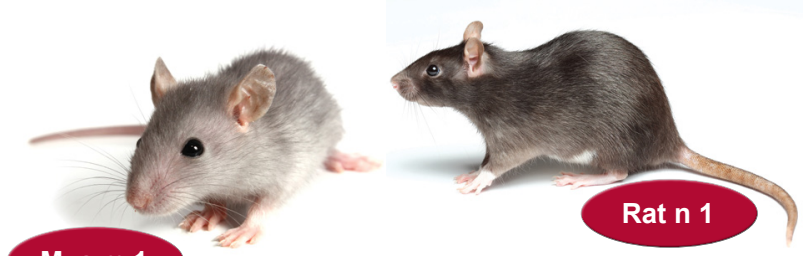

Mus $\mathbf{m} 1$
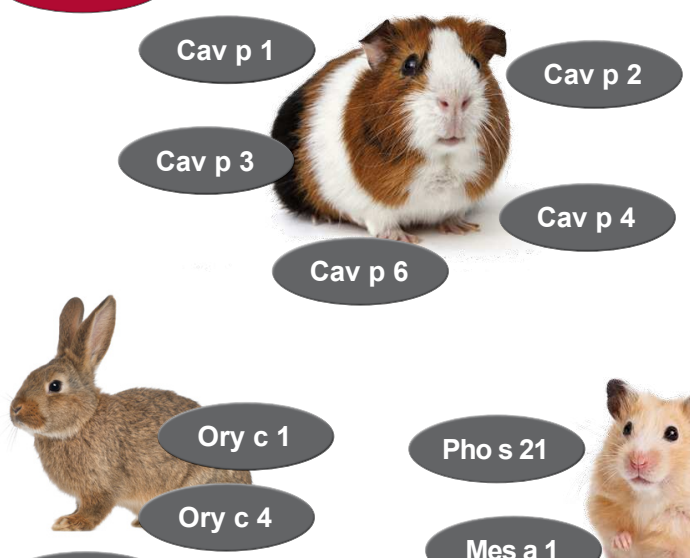

Ory c 3

Figure 1

Characterized allergens in mice, rats, rabbits, guinea pig and hamster.

comparable to symptoms induced by environmental allergens and consist of allergic rhinoconjunctivitis as well as allergic asthma.

Laboratory animal workers may also be exposed to other types of allergens in the workplace. These include allergens in animal or fish food (e.g. mealworm or corn cob), natural rubber latex (via using gloves), moulds, storage pests, mites, pollen, enzymes, antibiotics and several sensitizing chemicals.

\section{MAJOR AND RELEVANT MINOR ALLERGENIC MOLECULES}

Rodent allergens can be found in dander, hair, urine, saliva, and serum. Urine is the main source of allergenic proteins in both mice and rats. As common for the most mammalian inhalant allergens, the major allergens from mouse, rat, guinea pig, hamster and rabbit are lipocalins (Fig. 1, Table 1). Lipocalins (see chapter 'lipocalin') have a common tertiary structure composed of a central $\beta$-barrel formed of eight anti- parallel $\beta$-strands (5). The major mouse (Mus musculus) allergen, Mus $\mathrm{m} 1$ (about $17 \mathrm{kDa}$ ) is a prealbumin and lipocalin-odorant binding protein (6) belonging to the rodent family of major urinary proteins (MUP) (7) (Table 1). MUPs are produced in the liver and other exocrine glands under hormonal control and transported via blood and secreted in urine. Mouse MUPs are encoded by 35 genes, with 15 forms detectable in urine. The expression of MUPs varies according to species, strains, sexes, and individuals. MUPs seem to play a complex role in chemosensory signaling among rodents.

Analogous to mouse allergens, the major rat (Rattus norvegicus) allergen Rat $\mathrm{n} 1$ (about $17 \mathrm{kDa}$ ) is a prealbumin or alpha-2u-globulin that belongs to the lipocalin group and to the family of MUPs (3, 5, 7, 8). The amino acid identity between mouse and rat MUPs is approximately 65\%. In contrast to the MUPs from mice, the MUPs from rats are glycosylated. Urine collected from male rats contains much larger quantities of Rat $\mathrm{n} 1$ than urine from female rats.

Studies of particle size distribution revealed that airborne Mus $\mathrm{m} 1$ is carried on particles with aerodynamic diameter ranging from 0.4 to $>10 \mu \mathrm{m}$, with the majority on particles between 3.3 and $10 \mu \mathrm{m}$ and that airborne Rat $\mathrm{n} 1$ was detected on particles ranging from $<0.5$ to $20 \mu \mathrm{m}$ with the majority on particles larger than $8 \mu \mathrm{m}$ (9).

In addition to mice and rats, rabbits (Oryctolagus cuniculus) are also frequently used as laboratory animals or kept as domestic pets. Rabbit serum albumin, two lipocalins (Ory c 1 and Ory c 4) and a secretoglobin (Ory c 3 ) are described as allergens in saliva and dander (10). Members of the lipocalin-superfamily were also characterized as relevant allergens in guinea pig (Cavia porcellus) (5). Cav p 1 was identified in hair extracts and urine and can form both monomers (20 kDa) and dimers (40-42 kDa) (5). Cav p 2, a non-glycosylated 17 kDa lipocalin, has $39 \%$ sequence identity with Bos d 2 , the major allergenic protein from cow dander. Cav p 3 and 6 are also lipocalins and Cav p 4 is the $66 \mathrm{kDa}$ serum albumin.

Hamsters (Cricetinae) also belong to the group of domestic pets as well as to laboratory animals. So far allergic/anaphylactic reactions are described as case report. Although the Siberian or Djungarian hamsters (Phodopus sungorus) arebit easy to distinguish by a layperson from common hamsters such as the European (Cricetus cricetus) and Golden (Mesocricetus auratus) they belong to a different genus. The 


\section{Table 1}

Characterized inhalant animal allergens

\begin{tabular}{|c|c|c|c|c|c|}
\hline Animal species & Allergen & Protein family & $\begin{array}{l}\mathrm{MW} \\
(\mathrm{kDa})\end{array}$ & Allergen source & $\begin{array}{l}\text { Sensitization } \\
\text { rate }(\%)^{1}\end{array}$ \\
\hline $\begin{array}{l}\text { Mouse } \\
\text { (Mus musculus) }\end{array}$ & Mus m 1 & $\begin{array}{l}\text { Lipocalin/ urinary } \\
\text { prealbumin }\end{array}$ & 17 & Urine, Hair Dander & 66 \\
\hline $\begin{array}{l}\text { Rat } \\
\text { (Rattus norvegicus) }\end{array}$ & Rat n 1 & Lipocalin & 17 & Urine & $73-87$ \\
\hline \multirow{3}{*}{$\begin{array}{l}\text { Rabbit } \\
\text { (Oryctolagus cuniculus) }\end{array}$} & Ory c 1 & Lipocalin & $17-18$ & Saliva, Dander & - \\
\hline & Ory c 3 & Secretoglobin & $19-21$ & Saliva, Dander & 77 \\
\hline & Ory c 4 & Lipocalin & 24 & Saliva & 46 \\
\hline \multirow{5}{*}{$\begin{array}{l}\text { Guinea pig } \\
\text { (Cavia porcellus) }\end{array}$} & Cavp 1 & Lipocalin & 20 & Dander, Urine & 70 \\
\hline & Cavp 2 & Lipocalin & 17 & Saliva, Dander, Tears & 65 \\
\hline & Cavp 3 & Lipocalin & 18 & Saliva, Dander & 54 \\
\hline & Cav p 4 & Serum albumin & 66 & Serum & 52 \\
\hline & Cavp 6 & Lipocalin & 18 & Saliva & - \\
\hline $\begin{array}{l}\text { Golden or Syrian hamster, } \\
\text { (Mesocricetus auratus) }\end{array}$ & Mes a 1 & Lipocalin & $20-30$ & $\begin{array}{l}\text { Saliva, Tears, Urine } \\
\text { Dander }\end{array}$ & - \\
\hline $\begin{array}{l}\text { Siberian or Djungarian hamster } \\
\text { (Phodopus roborovskii) }\end{array}$ & $\begin{array}{l}\text { Pho s } \\
21 k D 2\end{array}$ & Lipocalin & $\begin{array}{c}18 \\
21,23\end{array}$ & Saliva, Dander & 100 \\
\hline
\end{tabular}

All allergens (with the exception of Phos s $21 \mathrm{kDa}$ ) are listed in the WHO/IUIS allergen data bank (www.allergen.org)

${ }^{1}$ Sensitization rates base on different studies with different patient groups and test systems (ELISA, Immunoblot, ImmunoCAP). They served as orientation points, no absolute numbers.

${ }^{2}$ Allergen listed in Allergome; this lipocalin had no cross-reactivity with common and golden hamster; MW: Molecular weight

Golden hamster is the main species used in animal research experiments. In the Golden hamster, malespecific submaxillary gland protein (MSP), a lipocalin expressed in a sex- and tissue-specific manner in the submaxillary and lacrimal glands, is secreted in the saliva, tears and urine. The major allergen from the Siberian hamster, Pho s $21 \mathrm{kDa}$, is also a lipocalin secreted by the submaxillary gland (11). It has a sequence identity of $40 \%$ with Mes a 1.

\section{CLINICAL RELEVANCE, DIAGNOSIS AND MANAGEMENT}

The major determinant of LAA is the exposure to rodent allergens but the exposure-response appears to be complex since at highest exposures to rats, there is attenuation of both sensitization and work-related symptoms. Additionally the effects of exposure seem to be modified importantly by individual susceptibility. A study of Jeal et al. (12) showed that high exposure to rats is associated with lower rates of specific IgE and symptoms but an increased frequency of high specific $\lg G$ and IgG4 production. Specific IgG4 produced together with specific IgE may reduce the risk of developing work-related chest symptoms compared with when specific IgE is produced alone (12-14).

\section{Diagnosis}

Specific occupational-related questions (working in laboratory animal facilities), proper interpretation of sensitization tests (i.e. SPT, IgE) and optional workplace-related, inhalant challenge tests help to establish the diagnosis of LAA.

\section{Skin prick testing}

Commercial allergen extracts of hair, epithelia and/ or dander from mouse, rat, rabbit and guinea pig are 


\section{Textbox 1}

\section{Clinical relevance}

- Mice, rats, rabbits, hamsters and guinea pigs are used in animal research; mice and rats are the most common causes of LAA

- LAA is commonly observed among technicians, animal caretakers, physicians, and scientists who work in pharmaceutical industries, university laboratories, and animal breeding facilities

- Sensitization and work-related symptoms occur at the latest, 2-3 years after the initial exposure to laboratory animals

- Laboratory animals are risk factors for respiratory symptoms and asthma

- Impact of individual molecules on severity of symptoms is still unknown

available and often used in the diagnosis procedure. Skin prick test extracts from hamster are based on Golden or Syrian hamster and sensitizations to Siberian or Djungarian hamster are not possible to determine accurately due to the lack of crossreactivity between the lipocalins Mes a 1 and Pho $s$ $21 \mathrm{kD}$.

\section{IgE-Testing}

Total IgE (for appropriate interpretation of allergenspecific lgE)

Allergen-specific IgE to the suspected animal allergen extracts is the common and recommended step. Specific IgE determination in the case of LAA is based on extracts prepared from epithelia, serum-/urine protein as mixture or alone (e71 = mice epithelia; e88 $=$ mice epithelia, serum/urine proteins; $\mathrm{e} 76=$ mice serum proteins; $\mathrm{e} 76$ = mice urine proteins; e73 = rat epithelia; e87 = rat epithelia, rat serum/urine proteins; e75 = rat serum proteins; $\mathrm{e} 74$ = rat urine proteins; $\mathrm{e} 84$ = hamster epithelia; e6 = guinea pig epithelia; e82 = rabbit epithelia; e206 rabbit serum proteins; e211 $=$ rabbit urine proteins). It has to be considered that dual sensitization to rat and mouse urinary allergens reflects cross-reactive molecules rather than atopy (15) and therefore the determination of the primary sensitizer is difficult. In this case, information from the exposure assessment and the clinical history are helpful. There is not enough evidence to advice the use of single molecules for in-vitro diagnosis. Single animal allergens, relevant for LAA, are not commercially available so far for routine testing.

\section{Clinical management}

Results of the study published by Schmid et al. (16) confirmed the necessity of regular medical checkups for employees with contact to laboratory animal dust. The authors recommended that medical checkups must be part of a prevention strategy including education, engineering controls, administrative controls, use of personal protective equipment and vocational integration. Controlling of the airborne allergen exposure in animal facilities is possible by using commercially available sensitive immunoassays for mouse and rat allergens (3).

In the case of individuals with LAA and asthmatic symptoms (occupational asthma) there is good evidence that removal from exposure within the first year of work-related symptoms leads to a better prognosis than that of those who remain exposed. In general the aim of management of individuals with occupational asthma is to preserve their health and wealth, preferably to leave their employer in business; it is necessary also to prevent other workers from developing occupational asthma (17).

Although atopy is strongly associated with the development of LAA, its predictive value is low and most employees with atopy will not develop a specific sensitization. Thus, the exclusion of atopic subjects from working with laboratory animals seems to be insufficiently discriminatory when screening for susceptible individuals (1). Laboratory animal research shows no signs of becoming less common, and an increasingly susceptible (atopic) population is likely to be recruited into such work.

An essential prerequisite for the formulation of allergy vaccines for specific immunotherapy is the knowledge about and the good characterization of the relevant allergens. Production of recombinant allergens from the small mammalian species responsible for the most cases of LAA is also insufficient so far. Therefore, it is a broad range of these unmet needs in the case of LAA to improve diagnosis and therapy.

\section{References}

1. Jeal $\mathrm{H}$, Jones $\mathrm{M}$. Allergy to rodents: an update. Clin Exp Allergy 2010;40:1593-1601.

2. Aoyama K, Ueda A, Manda F, Matsushita T, Ueda T, Yamauchi C. Allergy to laboratory animals: an 
epidemiological study. Br J Ind Med 1992;49:41-47.

3. ZahradnikE,RaulfM.Animalallergensandtheirpresence in the environment. Front Immunol 2014;5:76.

4. Gautrin D, Ghezzo H, Infante-Rivard C, Malo $\mathrm{JL}$. Incidence and determinants of IgE-mediated sensitization in apprentices. A prospective study. Am J Respir Crit Care Med 2000;162:1222-1228.

5. Hilger C, Kuehn A, Hentges F. Animal lipocalin allergens. Curr Allergy Asthma Rep 2012;12:438-447.

6. Lorusso JR, Moffat S, Ohman JL. Immunologic and biochemical properties of the major mouse urinary allergen (Mus $m$ 1). J Allergy Clin Immunol 1986;78:928-937.

7. Cavaggioni A, Mucignat-Caretta C. Major urinary proteins, alpha(2U)-globulins and aphrodisin. Biochim Biophys Acta 2000;1482:218-228.

8. Bayard C, Holmquist L, Vesterberg O. Purification and identification of allergenic alpha (2u)-globulin species of rat urine. Biochim Biophys Acta 1996;1290:129134.

9. Gordon S, Tee RD, Lowson D, Wallace J, Newman Taylor, A J. Reduction of airborne allergenic urinary proteins from laboratory rats. Br J Ind Med 1992;49:416-422.

10. Hentges F, Léonard C, Arumugam K, Hilger C. Immune responses to inhalant Mammalian allergens. Front Immunol 2014;5:234.

11. Torres JA, de Las Heras, Manuel, Maroto AS, Vivanco F, Sastre J, Pastor-Vargas C. Molecular and immunological characterization of the first allergenic lipocalin in hamster: the major allergen from Siberian hamster (Phodopus sungorus). J Biol Chem 2014;289:2338223388.

12. Jeal H, Draper A, Harris J, Taylor AN, Cullinan P, Jones M. Modified Th2 responses at high-dose exposures to allergen: using an occupational model. Am J Respir Crit Care Med 2006;174:21-25.

13. Jones $\mathrm{M}$, Jeal $\mathrm{H}$. IgG4 antibodies against rodents in laboratory animal workers. Allergy 2011;66:1249; author reply 1249-51.

14. Jones M, Jeal H, Schofield S, Harris JM, Shamji MH, Francis JN et al. Rat-specific IgG and IgG- antibodies associated with inhibition of IgE-allergen complex binding in laboratory animal workers. Occup Environ Med 2014;71:619-623.

15. Jeal H, Harris J, Draper A, Taylor AN, Cullinan P, Jones $M$. Dual sensitization to rat and mouse urinary allergens reflects cross-reactive molecules rather than atopy. Allergy 2009;64:855-861.

16. Schmid $K$, Jüngert $B$, Hager $M$, Drexler $H$. Is there a need for special preventive medical check-ups in employees exposed to experimental animal dust. Int Arch Occup Environ Health 2009;82:319-327.

17. Sherwood Burge P. New guidelines for the management of occupational asthma in primary care and occupational health. Prim Care Respir J 2004;13:131132. 



\section{PART C}

\section{CROSS-REACTIVE MOLECULES \& THEIR CLINICAL RELEVANCE}





\section{PART C CROSS-REACTIVE MOLECULES \& THEIR CLINICAL RELEVANCE}

\section{PROFILINS}

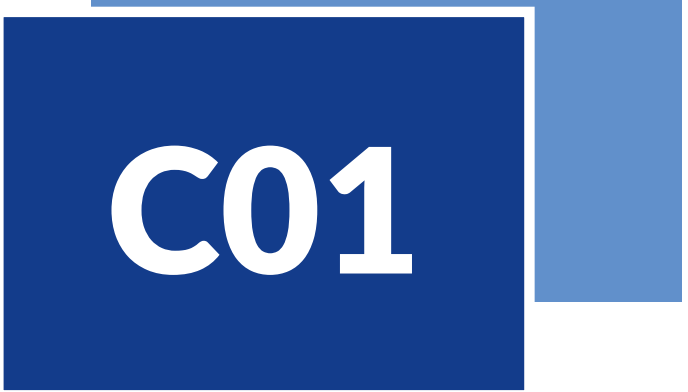

\section{Riccardo Asero, Domingo Barber}

\section{THE PROTEIN}

Profilin is a protein of $12-15 \mathrm{kDa}$ in size present in all eukaryotic cells and involved in the organization of cytoskeleton as well as in signal transduction. It is a monomeric actin-binding protein and a key regulator of actin-filament dynamics during processes such as cells movement, cytokinesis, and signaling (1). In higher plants, it is identified as an allergen in monocot and dicot angiosperms.

\section{THE PROTEIN FAMILY}

Profilins from higher plants constitute a family of highly conserved proteins showing sequence identities of at least $75 \%$ even between members from distantly related organisms. In view of the high sequence homology, cross-reactivity between profilins is extremely common and involves virtually every plant source. Thus, profilin can be considered the archetypal pan-allergen (2).
Up to $50 \%$ of pollen allergic patients are sensitized to profilin.

Sensitization virtually always follows primary sensitization to a specific pollen source.

On SPT most pollen sources score positive.

Clinical relevance is variable but potentially present.

Up to $50 \%$ of sensitized patients may have food allergy, oral allergy syndrome in most cases

Raw tomato, melon, watermelon, and citrus fruits are typically associated with profilin.

Patients tolerate processed foods.

The spectrum of offending plant foods sometimes is very large. 


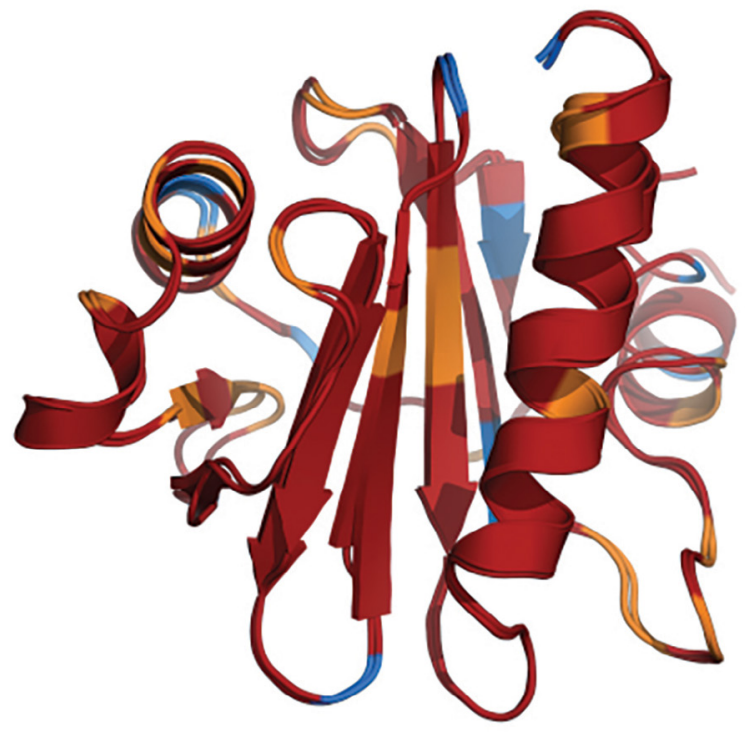

Figure 1

Three-dimensional structures of Phl p 12 (grass pollen) and Cuc m 2 (melon). Identical amino acid residues are in red, conservative changes in orange and unrelated amino acids are in blue. The structure is highly conserved in all profilins, consisting of a central 6-stranded antiparallel $\beta$-sheet and two a-helices situated at the $\mathrm{N}$ and C terminal sides. The 3-D structure of Cuc $m 2$ was modeled by using the Swiss-Model Protein Modelling Server,21 taking as templates the following crystal structures of plant profilins: (1) A thaliana pollen, Ara t 8, PDB code 1AOK (76.2\% of sequence identity); (2) birch pollen, Bet $\vee 2$, PDB code 1CQA (78.9\% identity); and (3) H brasiliensis, Hev b 8.0204, PDB code 1G5U (83.1\% identity). Secondary structure was identified with the DSSP program.

\section{CLINICAL RELEVANCE OF PROFILIN}

\section{As an airborne allergen}

Profilins are able to elicit IgE responses in 10-60\% of pollen-allergic patients $(2,3)$. However, the sensitization prevalence seems to increase, as more and more allergic patients seen at allergy departments show sensitization to a large number of botanically unrelated plants (4).

As a rule, profilin sensitization follows sensitization to a primary, major allergenic pollen source. In most cases, grass pollen is responsible for profilin
Table 1

Basic protein characteristics of Phl p 12

\begin{tabular}{|ll|}
\hline Protein characteristics & $\begin{array}{l}\text { Phleum pratense, } \\
\text { Timothy grass pollen }\end{array}$ \\
\hline Allergen source & Profilin \\
\hline Protein family & No \\
\hline UniProtKB accession No. & P35079 \\
\hline $\begin{array}{l}\text { Three dimensional } \\
\text { structure available }\end{array}$ & Central 6-stranded $\beta$ sheet \\
\hline Molecular structure & and two a-helices \\
\hline Molecular weight & 14,235 kDa \\
\hline Length & 131 amino acids \\
\hline Ligand binding & Actin, poly-L-proline \\
\hline Dimerization & No \\
\hline Glycosylation & No \\
\hline Disulfide bonds & Yes \\
\hline Isoelectric point & 5.07 \\
\hline Distribution & Every plant cell \\
\hline
\end{tabular}

hypersensitivity but, depending on geographical differences, also birch pollen, ragweed pollen and mugwort pollen may act as primary sensitizers $(3,5)$.

Being a minor pollen allergen, profilin sensitization is almost always associated with the sensitization to major pollen allergens. Assessing the clinical relevance of profilin as an airborne allergen is quite complicated, and in effect it was seldom investigated. In a Spanish study, only profilin-sensitized, pollenallergic patients scored positive on a conjunctival provocation test with date palm profilin, thus suggesting that profilin may act as an aeroallergen (6). Another study based on nasal/bronchial provocation with date palm profilin in sensitized subjects confirmed this finding (7). However, in a field study the clinical impact of profilin hypersensitivity turned out to be rather limited, as most sensitized patients reported symptoms only in the specific season of the primary sensitizing pollen source (8). Nonetheless, for the only case of primary sensitization to profilin reported so far, the patient also suffered from long lasting seasonal symptoms (9). 
Table 2

A selection of profilins from different pollen sources

\begin{tabular}{|c|c|c|}
\hline $\begin{array}{l}\text { Botanical } \\
\text { family }\end{array}$ & Allergen source & Allergen \\
\hline \multirow{6}{*}{ Fagales } & Birch (Betula pendula) & Bet $v 2$ \\
\hline & Hazel tree (Corylus avellana) & Cor a 2 \\
\hline & Alder (Alnus glutinosa) & Aln g 2 \\
\hline & Hornbeam (Carpinus betulus) & Car b 2 \\
\hline & Oak (Quercus alba) & Que a 2 \\
\hline & Beech (Fagus sylvatica) & Fag s 2 \\
\hline \multirow[b]{2}{*}{ Graminae } & Timothy (Phleum pratense) & Phl p 12 \\
\hline & $\begin{array}{l}\text { All other grass pollen } \\
\text { species }\end{array}$ & $\begin{array}{l}\text { Allergen } \\
12\end{array}$ \\
\hline \multirow[b]{2}{*}{ Asteraceae } & Mugwort (Artemisia vulgaris) & Art v 4 \\
\hline & $\begin{array}{l}\text { Ragweed (Ambrosia } \\
\text { artemisiifolia) }\end{array}$ & Amb a 8 \\
\hline Urticaceae & Pellitory (Parietaria judaica) & Par j 3 \\
\hline \multirow{2}{*}{ Oleaceae } & Olive (Olea europaea) & Ole e 2 \\
\hline & Ash (Fraxinus excelsior) & Fra e 2 \\
\hline Cupressaceae & $\begin{array}{l}\text { Cypress (Cupressus } \\
\text { sempervirens) }\end{array}$ & Cup s 8 \\
\hline \multirow{2}{*}{ Euphorbiaceae } & $\begin{array}{l}\text { Annual mercury (Mercurialis } \\
\text { annua) }\end{array}$ & Mer a 1 \\
\hline & $\begin{array}{l}\text { Date palm (Phoenix } \\
\text { dactylifera) }\end{array}$ & Pho d 2 \\
\hline
\end{tabular}

\section{As a plant food allergen}

Although profilin is present in every plant-derived food, its relevance as a food allergen has long been underrated $(10,11)$. Nonetheless, its role as a plant food allergen in about $50 \%$ of sensitized subjects has recently emerged (12), with clinical allergy to certain foods such as melon, watermelon, citrus fruits, banana, pineapple, persimmon, zucchini, and tomato being characteristically associated with profilin hypersensitivity (12-20). Being highly pepsin-sensitive (14), the clinical expression of profilin-induced food allergy is in most cases the oral allergy syndrome, although cases of systemic allergic reactions have been recently reported as well in specific areas in Spain where the levels of grass pollen allergy are extremely high (Fig. 3). Oral provocation of food allergic patients with low doses of purified profilin has proven to induce severe reactions in grass
Table 3

Profilins from some plant foods

\begin{tabular}{|c|c|c|}
\hline $\begin{array}{l}\text { Botanical } \\
\text { family }\end{array}$ & Allergen source & Allergen \\
\hline \multirow{3}{*}{ Rosaceae } & Apple (Malus domestica) & Mal d 4 \\
\hline & Peach (Prunus persica) & Pru p 4 \\
\hline & Pear (Pyrus communis) & Pyr p 4 \\
\hline Cucurbitaceae & Melon (Cucumis melo) & Cuc $\mathrm{m} 2$ \\
\hline Actinidiaceae & Kiwi (Actinidia deliciosa) & Act d 9 \\
\hline \multirow{2}{*}{ Apiaceae } & Celery (Apium graveolens) & Api g 4 \\
\hline & Carrot (Daucus carota) & Dau c 4 \\
\hline Rutaceae & Orange (Citrus sinensis) & Cit s 2 \\
\hline \multirow{2}{*}{ Leguminosae } & Peanut (Arachis hypogaea) & Ara h 5 \\
\hline & Soybean (Glycine maxima) & Gly $\mathrm{m} 3$ \\
\hline Solanaceae & $\begin{array}{l}\text { Tomato (Solanum } \\
\text { lycopersicum) }\end{array}$ & Sola I 1 \\
\hline Bromeliaceae & Pineapple (Ananas comosus) & Ana c 1 \\
\hline Corylaceae & Hazelnut (Corylus avellana) & Cor a 2 \\
\hline Brassicaceae & Yellow mustard (Sinapis alba) & Sin a 4 \\
\hline Asteraceae & $\begin{array}{l}\text { Sunflower (Helianthus } \\
\text { annuus) }\end{array}$ & Hel a 2 \\
\hline Moraceae & Fig (Ficus carica) & Fic c 4 \\
\hline
\end{tabular}

pollen (GP) allergic patients resident in areas with high grass pollen exposure (21). This fact should be taken into account when evaluating severe food allergic reactions in areas where GP allergy is dominant.

\section{Profilin and natural rubber latex allergy}

The end of the last century and the beginning of the current one have been characterized by an impressive increase in the prevalence of allergy to natural rubber latex. NRL contains many allergenic proteins, including profilin (Hev b 8). Therefore, the crude extract of Hevea brasiliensis latex scores often positive in patients with multiple pollen sensitizations. This has frequently caused concern for the risk of intraoperative anaphylactic reactions. However, most latex products have been replaced by synthetic products and therefore, the risk of allergic reactions due to latex allergens including profiling is no longer a health issue. Thus, patients who show uniquely IgE reactivity to profilin in NRL can undergo surgery and other medical procedures without any risk $(22,23)$. 


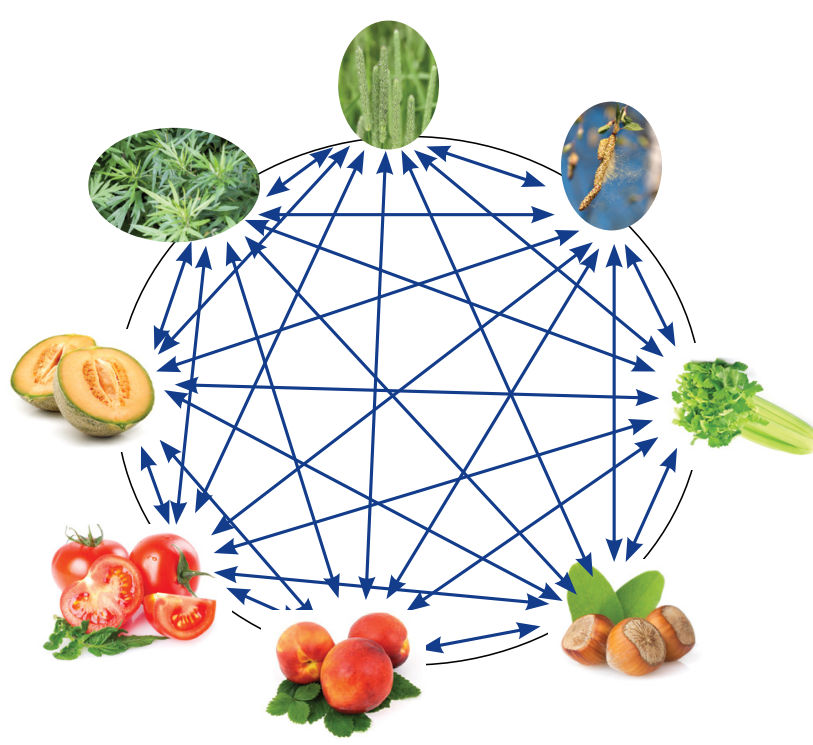

Figure 2

Cross-reactivity between profilins from different pollens and plant foods.
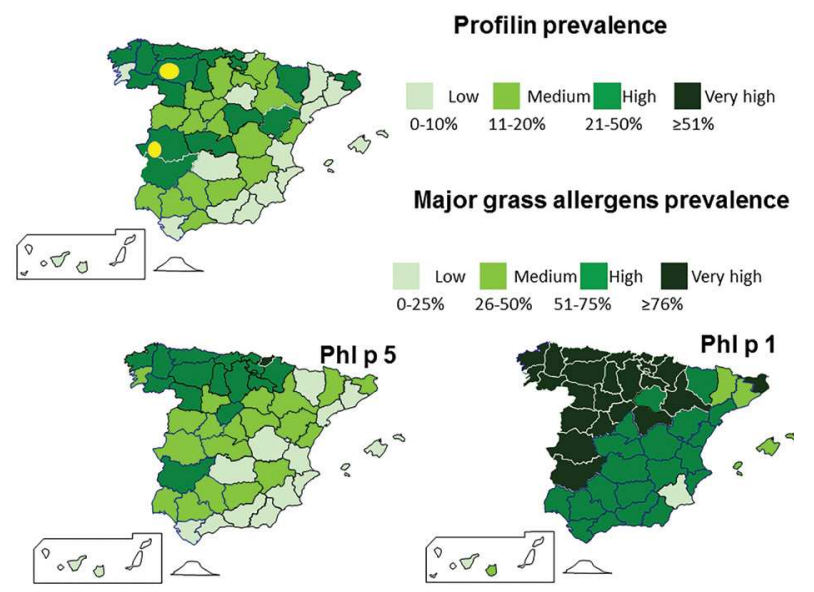

Figure 3

Areas of Spain characterized by a high prevalence of profilin sensitization (dots) where some cases of severe profilin-induced food allergy have been recorded.

\section{CLINICAL MANAGEMENT}

Profilin hypersensitivity can be diagnosed in-vivo by SPT using a commercial profilin-enriched date palm pollen extract currently available only in Italy, Spain and Austria (24) such extract for skin testing shows a sensitivity and specificity that is very close to that of the recombinant grass pollen profilin for in-vitro use (Phl p 12) (25).

Several recombinant profilins are currently available for the routine in-vitro diagnosis of $\lg \mathrm{E}$ hypersensitivity. In the ImmunoCAP assay (Thermo Fischer Scientific) Phl p 12, Bet v 2, Pru p 4, and Hev b 8 , the profilins from grass pollen, birch pollen, peach, and natural rubber latex are the 4 profilins currently available. In the ImmunoCAP ISAC multiplex assay, Mer a 1 (from annual mercury) is present as well (in a former version of the assay Ole e 2, the olive pollen profilin has also been present, but is currently withdrawn due to its low sensitivity (26)). Regarding the clinical significance of profilin-specific IgE levels, their value in predicting an oral allergy syndrome seems extremely limited or absent (27).

Since profilins are extremely well conserved and cross-reacting and show an equivalent IgE binding potency $(28,29)$, one wonders if a representative marker allergen is sufficient to detect hypersensitive patients. In this sense, a recent study showed that one single marker protein is sufficient to diagnose or exclude sensitization (26). As for the homology between profilins from different sources, extracts from two pollens, namely pellitory and cypress, score frequently negative in profilin hypersensitive patients (18). Whether this depends on a more limited cross reactivity of profilins from these two plants with the other profilins $(30,31)$, or on a lower concentration of the protein in the extract (32) is still a matter of debate; further studies are needed to clarify this point.

Profilin hypersensitivity does not normally require special clinicalmeasures.Althoughmostpollensources are probably able to induce profilin hypersensitivity (33), from a respiratory point of view the clinical impact of the inhaled protein is in most cases quite limited (8). Some studies have demonstrated the disappearance of profilin-associated food allergy in subjects submitted to injection immunotherapy with different pollen extracts $(34,35)$. 


\section{Case 1}

Clinical History - A 32-year-old woman resident in Extremadura, a heavily grass-exposed area, with pollen allergy and clinical history of oral allergy syndrome, urticaria, and asthma following the ingestion of Melon, Watermelon, Banana, Peach and Orange. Open challenge with melon was positive.

Tests with extracts - On SPT, with exception of cypress all pollens scored positive, and no reactivity to peach LTP was detected.

Tests with molecules - ISAC microarray scored positive for: Cyn d 1: 76.7, Phl p 1: 75, Phl p 2: 21 , Phl p 4: 1.0, Phl p 5: 51.5, Phl p 6: 2.6, Phl p 11: 0.73, Bet v 2: 6.39, Hev b 8: 5.14, Mer a 1: 8.94, Phl p 12: 1.64, Mux F3: 0.67, Ole e 1: 0.37.

Oral challenges - On DBPCFC, the administration of $74 \mu \mathrm{g}$ of pure date palm pollen profilin induced OAS and FEV1 decline of $20 \%$ that were treated with antihistamine and bronchodilators.

Diagnosis - Grass pollen allergy with severe profilinmediated food allergy was eventually diagnosed.

\section{Case 2}

Clinical history - A 38-year-old man living in the surroundings of Milan, Italy. At the age of 32 starts having severe rhino-conjunctivitis from midAugust to the end of September when he returns home from the summer holidays at the sea (where he is well). After 3 years, oral itching following the ingestion of melon, watermelon, tomato, banana, orange and peach appears.

Tests with extracts - SPT with pollen allergens score positive for grass, mugwort, ragweed, plantain, birch, hazel, plane and olive, and negative for pellitory and cypress. A SPT with profilin-enriched date palm pollen extracts scores intensely positive.

Tests with molecules - The ImmunoCAP assay scores strongly positive for Amb a 1 and $\mathrm{Phl} \mathrm{p} \mathrm{12,} \mathrm{and}$ negative for Phl p 1, Phl p 5, Phl p 7, Art v 1, Pla a 1, Pla I 1, Bet v 1, Cor a 1, Ole e 1, Par j 2, and Pru p 3.

Diagnosis - Respiratory allergy to ragweed and profilin-induced food allergy are eventually diagnosed.

\section{References}

1. Witke W. The role of profilin complexes in cell motility and other cellular processes. Trends Cell Biol 2004;14:461-469.

2. Valenta R, Duchene M, Ebner C, Valent P, Sillaber $C$, Deviller $P$ et al. Profilins constitute a novel family of functional plant pan-allergens. J Exp Med 1992;175:377-385.

3. Barber D, de la Torre F, Feo F, Florido F, Guardia P, Moreno $\mathrm{C}$, et al. Understanding patient sensitization profiles in complex pollen areas: a molecular epidemiological study. Allergy 2008;63:1550-58.

4. Mari A. Multiple pollen sensitization: a molecular approach to the diagnosis. Int Arch Allergy Immunol 2001;125:57-65.

5. Villalta $D$, Asero R. Analysis of the allergenic profile of patients hypersensitive to pollen pan-allergens living in two distinct areas of Northern Italy. Eur Ann Allergy Clin Immunol 2011;43:54-57.

6. Nunez R, Carballada F, Lombardero M, Jimeno L, Boquete M. Profilin as an aeroallergen by means of conjunctival allergen challenge with purified date palm profilin. Int Arch Allergy Immunol 2012;158:115-119.

7. Ruiz-García M, García del Potro M, Fernànzez-Nieto $M$, Barber D, Jimeno-Nogales L, Sastre J. Profilin: a relevant aeroallergen? J Allergy Clin Immunol 2011;128:416-418.

8. Asero R, Jimeno L, Barber D. Preliminary results of a skin prick test-based study of the prevalence and clinical impact of hypersensitivity to pollen panallergens (polcalcin and profilin). J Investig Allergol Clin Immunol 2010; 20: 35-38.

9. Asero R, Villalta D. Profilin may be a primary airborne sensitizer: a case report. J Investig Allergol Clin Immunol 2013;23:134-135.

10. Wensing $M$, Akkerdaas $\mathrm{JH}$, van Leeuwen WA, Stapel SO, Bruijnzeel-Koomen CA, Aalberse RC et al. IgE to Bet $v 1$ and profilin: cross-reactivity patterns and clinical relevance. J Allergy Clin Immunol 2002;110:435-442.

11. Santos A, van Ree R. Profilins: mimickers of allergy or relevant allergens? Int Arch Allergy Immunol 2011; 155:191-204.

12. Asero R, Mistrello G, Roncarolo D, Amato S, Zanoni $D$, Barocci $F$, et al. Detection of clinical markers of sensitization to profilin in patients allergic to plantderived foods. J Allergy Clin Immunol 2003; 112: 427-32

13. Reindl J, Rihs HP, Scheurer S, Wnagorsch A, Haustein D, Vieths S. IgE reactivity toprofilin in pollen-sensitized subjects with adverse reactions to banana and pineapple. Int Arch Allergy Immunol 2002;128:105-114.

14. Rodriguez-Perez R, Crespo JF, Rodriguez J, Salcedo G. Profilin is a relevant melon allergen susceptible to pepsin digestion in patients with oral allergy syndrome. J Allergy Clin Immunol 2003;111:634-639. 
15. Westphal S, Kempf W, Foetisch K, Retzek M, Vieths S, Scheurer S. Tomato profilin Lyc e 1: IgE cross-reactivity and allergenic potency. Allergy 2004;59:526-532.

16. Naliker MD, Reindl J, Vieths $S$, Wüthrich B. Allety caused by ingestion of persimmon (Diospyros kaki): detection of specific IgE, and cross-reactivity to profilin and carbohydrate determinants. J Allergy Clin Immunol 2001;107:718-723.

17. Reindl J, Anliker MD, Karamloo F, Vieths S, Wüthrich B. Allergy caused by ingestion of zucchini (Cucurbita pepo): characterization of allergens and crossreactivity to pollen and other foods. J Allergy Clin Immunol 2000;106:379-385.

18. Asero R, Monsalve R, Barber D. Profilin sensitization detected in the office by skin prick test: a study of prevalence and clinical relevance of profilin as a plant food allergen. Clin Exp Allergy 2008;38:1033-1037.

19. Asero R, Mistrello $G$, Amato $S$. The nature of melon allergy in ragweed-allergic subjects. A study of 1000 patients. Allergy Asthma Proc 2011;32:64-67.

20. Palacin A, Quirce S, Sanchez-Monge R, Bobolea I, Diazperales A, Martin Munoz F, et al. Sensitization profiles to purified plant food allergens among pediatric patients with allergy to banana. Pediatr Allergy Immunol 2011;22:186-195.

21. Alvarado MI, Jimeno L, De La Torre F, Boissy $P$, Rivas B, Lazaro $M$, et al. Profilin as a severe food allergen in allergic patients overexposed to grass pollen. Allergy 2014;69:1610-1616.

22. Quercia O, Stefanini GF, Scardovi A, Asero R. Patients monosensitized to Hev b 8 (Hevea brasiliensis latex profilin) may safely undergo major surgery in a normal (non-latex-safe) environment. Eur Ann Allergy Clin Immunol 2009;41:112-116.

23. Schuler S, Ferrari G, Schmid-Grendelmeier P, Harr T. Microarray-based component-resolved diagnosis of latex allergy: isolated IgE-mediated sensitization to latexprofilin Hev b8 may act as confounder. Clin Transl Allergy 2013;3:11.

24. Barber D, de la Torre F, Lombardero M, Antepara I, Colas C, Davila I, et al. Component-resolved diagnosis of pollen allergy based on skin testing with profilin, polcalcin and lipid transfer protein pan-allergens. Clin Exp Allergy 2009;39:1764-1773.

25. Asero R. Hypersensitivity to pollen pan allergens (profilin and polcalcin) detected in vitro and in- vivo: a comparative analysis. J Investig Allergol Clin Immunol 2011;21:323-324.

26. Villalta D, Asero R. Sensitization to the pollen panallergen profilin. Is the detection of IgE to multiple homologous protein from different sources clinically useful? J Investig Allergol Clin Immunol 2010;20:59195.

27. Asero R, Villalta D. Are anti-Phl p 12 lgE levels predictive of oral allergy syndrome in profilin hypersensitive patients? Eur Ann Allergy Clin Immunol 2011;43:184187.

28. Ganglberger E, Radauer C, Wagner S, Riordian G, Beezhold DH, Brehler R, et al. Hev b 8, the Hevea brasiliensis latex profilin, is a cross-reactive allergen of latex, plant foods, and pollen. Int Arch Allergy Immunol 2001;125:216-227.

29. Sirvent S, Tordesillas L, Villalba M, Diaz-Perales A, Cuesta-Herranz J, Salcedo G, et al. Pollen and plant food profilin allergens show equivalent IgE reactivity. Ann Allergy Asthma Immunol 2011;106:429-435.

30. Asero R, Mistrello G, Roncarolo D, Amato S. Parietaria profilin shows only limited cross-reactivity with birch and grass profilins. Int Arch Allergy Immunol 2004;133:121-124.

31. Asturias JA, Ibarola I, Eseverri JL, Arilla MC, Gonzàlez-Rioja R, Martinez A. PCR-based cloning and immunological characterization of parientaria judaica pollen profilin. J Investig Allergol Clin Immunol 2004;14:43-48.

32. Bonura A, Trapani A, Gulino L, Longo V, Valenta R, Asero $\mathrm{R}$, et al. Cloning, expression in E Coli and immunological characterization of Par j 3.0201, a Parietaria pollen profilin variant. Mol Immunol 2014;57:220-225.

33. Villalta $D$, Asero R. Analysis of the allergenic profile of patients hypersensitive to pollen pan-allergens living in two distinct areas of Northern Italy. Eur Ann Allergy Clin Immunol 2011;43:54-57.

34. Kelso JM, Jones RT, Tellez R, Yunginger JW. Oral allergy syndrome successfully treated with pollen immunotherapy. Ann Allergy Asthma Immunol 1995;74:391-396.

35. Asero R. Fennel, cucumber, and melon allergy successfully treated with pollen-specific injection immunotherapy. Ann Allergy Asthma Immunol 2000; 84:460-462. 


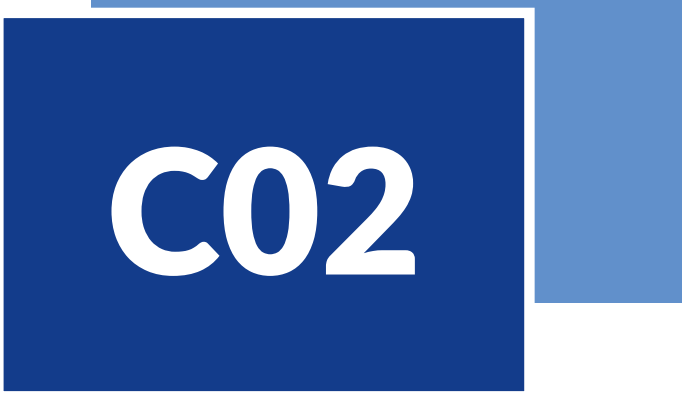

\section{Heimo Breiteneder, Jörg Kleine-Tebbe}

\section{THE PR-10 PROTEIN ARCHITECTURE}

Bet $v 1$, the major allergen of birch pollen, was the first plant allergen and the first allergenic PR-10-like protein to be cloned and characterized (Table 1) (1).

The protein architecture of Bet $v 1$ comprises a highly curved seven-stranded anti-parallel beta-sheet that embraces a 25 residue-long $C$-terminal alpha-helix (2). The beta-sheet and the C-terminal part of the long alpha-helix are separated by two consecutive alpha helices that connect the beta1- and beta2-strands. All these structural elements contribute to the formation of a large hydrophobic cavity that penetrates the entire protein and whose presence suggests that Bet v 1 might function as a carrier for presumably multiple hydrophobic ligands. Structural information is available for various Bet $v 1$-homologous allergens from plant foods (Fig. 1 shows some examples) and their overall similarity clearly illustrates the molecular basis for the cross-reactivity of these proteins.

Although Bet $v 1$ contains a variety of different $T$ cell epitopes, a major $\mathrm{T}$ cell epitope located at the C-terminal amino acid residue positions $142-156$ was recognized by $\mathrm{T}$ cells from $61 \%$ birch pollen allergic individuals studied (3). This part of the molecule shares
The major birch pollen allergen, Bet $v 1$, represents the prototype of all PR-10-like allergens and is the primary sensitizer in regions with birch pollen exposure

The presence of homologous allergens in Fagales tree pollen explains IgE crossreactivity between pollen from hazel, alder, beech, oak and hornbeam.

Minute amounts of PR-10-like allergens in raw fruits, nuts, vegetable and legumes can induce individual patterns of oropharyngeal symptoms and sometimes severe allergic reactions in Bet $v$ 1 -sensitized individuals.

Testing for Bet $v$ 1-specific lgE is sufficient. The relevance of cross-reacting pollens or foods can be clinically clarified by seasonal and food related symptoms without further IgE-testing of Bet v 1-homologues. 


\section{Table 1}

Bet v 1.0101 datasheet

\section{Protein characteristics}

\begin{tabular}{|c|c|}
\hline Allergen source & $\begin{array}{l}\text { Betula pendula (syn. } B \text {. } \\
\text { verrucosa, B. alba), birch }\end{array}$ \\
\hline Protein family & Bet $v 1$ \\
\hline Protein subfamily & PR-10 \\
\hline $\begin{array}{l}\text { UniProt accession } \\
\text { number }\end{array}$ & P15494 \\
\hline $\begin{array}{l}\text { Three dimensional } \\
\text { structure available }\end{array}$ & Yes \\
\hline Molecular structure & $\begin{array}{l}\text { Seven-stranded anti-parallel } \\
\beta \text {-sheet flanked by three } \\
\text { a-helices }\end{array}$ \\
\hline $\begin{array}{l}\text { Theoretical molecular } \\
\text { weight }\end{array}$ & 17439.63 Da \\
\hline $\begin{array}{l}\text { Molecular weight } \\
\text { measured by MS }\end{array}$ & $17439.6+/-0.3 \mathrm{Da}$ \\
\hline Length & 159 amino acid residues \\
\hline Ligand binding & Yes \\
\hline Oligomerization state & Monomeric \\
\hline Glycosylation & No \\
\hline Disulfide bonds & 0 \\
\hline Isoelectric point & 5.39 \\
\hline Synthesis & $\begin{array}{l}\text { In the cytoplasm of birch } \\
\text { pollen }\end{array}$ \\
\hline Distribution & $\begin{array}{l}\text { Pollen is distributed intro } \\
\text { the environment by wind } \\
\text { dispersal and can enter the } \\
\text { indoor environment. }\end{array}$ \\
\hline
\end{tabular}

high sequence similarities with various Bet $v$ 1-related tree pollen allergens. The extent of $T$ cell cross-reactivity with Bet $\vee$ 1-related food allergens also corresponded to the degree of sequence similarity of the food allergens' $\mathrm{C}$-termini to the Bet $\mathrm{v} 1$ amino acid residues 142-156 (3). The sensitizing capacity of different Bet $\checkmark 1$ homologues from plant foods seems to correlate with the presence of immunodominant $T$ cell epitopes (4). The Bet v 1-specific IgE response is polyclonal, and epitopes are spread across the entire Bet $v 1$ surface. Furthermore, the IgE recognition profile of Bet $v 1$ is variable and highly patient specific (5).
Table 2

Characteristics of the PR-10 like family of allergenic proteins

Common tertiary structure with seven-stranded antiparallel $\beta$-sheet with long $\mathrm{C}$-terminal $\alpha$-helix and two short $\alpha$-helices

Sequences with high identities

Small cross-reactive molecules of around $17 \mathrm{kDa}$

Present in early flowering Fagales trees, fruits, vegetables, nuts and seeds

\section{THE PR-10-LIKE FAMILY OF ALLERGENIC PROTEINS}

In 1980, pathogenesis-related proteins (PR proteins) were defined as "proteins encoded by the host plant but induced only in pathological or related situations" and subsequently grouped into families (6). Today, the list of PR proteins comprises 14 families (7). When the sequence of Bet $v 1$ was discovered in 1989, the PR10 family had not been defined yet but it was noted that Bet $v 1$ was homologous to a PR protein from pea (1). Bet $\vee 1$ is constitutively expressed in pollen at rather high concentrations. Hence, the term PR-10 for the Bet $v 1$ homologous allergens is not entirely correct. These constitutively expressed proteins are referred to as PR-10 like proteins.

The cDNA sequence coding for Bet $v 1.0101$ was discovered on July 3, 1988, and published in 1989 (1). Since then the number of sequences related to the Bet $v 1$ sequence has grown steadily and rapidly. At present, the version 29.0 of the Pfam database attributes 14,065 sequences from 1,452 species to the Bet $v$ 1-like superfamily (http://pfam.xfam.org/ clan/CL0209). These proteins are found in all three domains of life, i.e. archaea, bacteria and eukaryotes, and all share the Bet $v 1$ architecture (8). The Bet $v$ 1-like superfamily of proteins comprises 14 families, one of which is the Bet $v 1$ family. The Bet $v 1$ family in turn is composed of 11 subfamilies. One of these subfamilies is the PR-10 group of proteins to which almost all of the Bet $v 1$ homologous allergens belong. There are only two examples of allergens that are members of other subfamilies, the kiwi allergen Act d 11 (9) from the RRP/MLP (= ripening related proteins/major latex proteins) subfamily, and the 

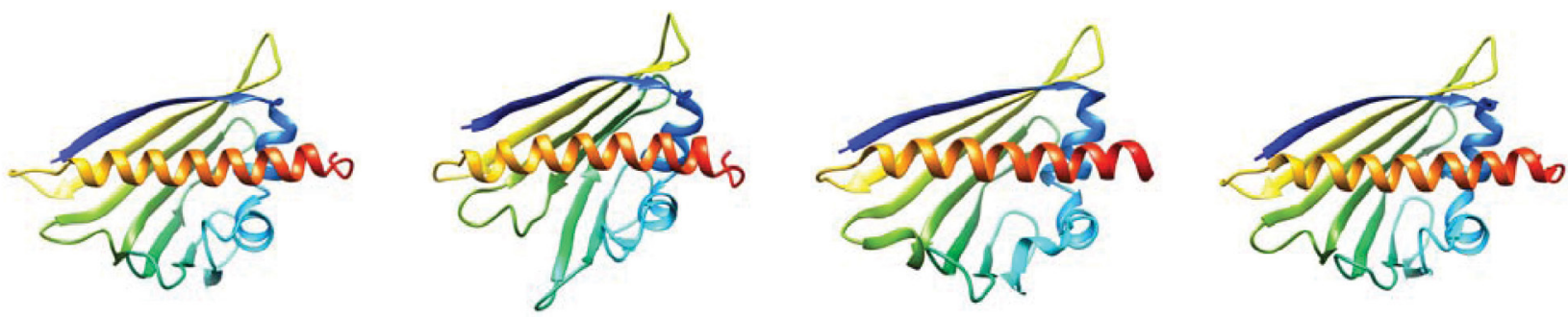

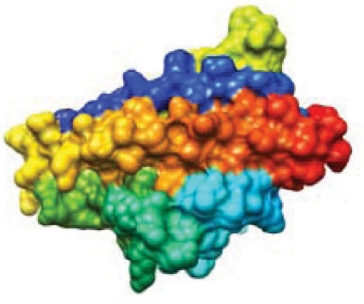

Bet v1

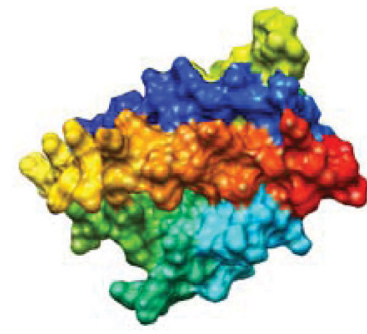

Pru av 1

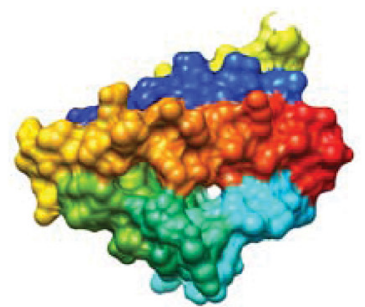

Api g 1

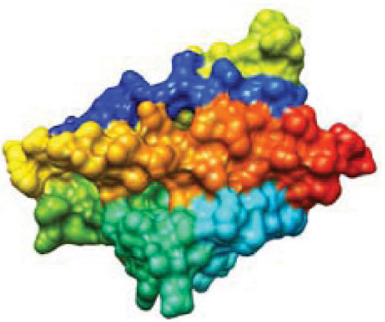

Ara h 8

Figure 1

Ribbon and solid surface representations of birch pollen Bet $\vee 1$ (PDB 1BV1) and homologues from cherry (Pru av 1; PDB 1E09), celeriac (Api g 1; PDB 2BKO) and peanut (Ara h 8; PDB 4M9B), rainbow-coloured from blue at the $\mathrm{N}$-terminus to red at the $\mathrm{C}$-terminus. The 3 - $\mathrm{D}$ images were created with the molecular modelling system UCSF Chimera (http://www.cgl.ucsf.edu/chimera).

mung bean allergen Vig $r 6$ (10) from the CSBP (= cytokinin specific binding proteins) subfamily.

Birch pollen is one of the most common causes of IgE-mediated allergy in Northern and Central Europe as well as in North America. The major sensitizing allergen present in birch pollen is Bet $v 1$ to which $93 \%$ of individuals with birch pollen allergy produce specific IgE (11). Birch belongs to the botanical order Fagales, which comprises eight families. Allergies have been strongly associated with pollen produced by the early flowering trees of the families Betulaceae and the Fagaceae (Table 3).

In general, allergic reactions to Fagales pollen are initiated by independent sensitization to members of the Betuloideae or Coryloideae subfamilies. However, $25 \%$ of the IgE epitopes of the Betuloideae and the Coryloideae pollen allergens are unique for the respective subfamily, whereas pollen allergens from the Fagaceae are generally cross-reactive (12).

Homologues of Bet $v 1$ have also been identified in a wide range of plant foods (13). The most frequently observed clinical entity is caused by IgE antibodies that cross-react between Bet $v 1$ and its homologues in fruits, nuts, seeds and vegetables. They induce predominantly oropharyngeal symptoms, coined oral allergy syndrome (OAS) (14). Severe reactions to Gly $\mathrm{m} 4$, the Bet $\mathrm{v} 1$ homologue from soybean (see Section B17), have been observed in a subpopulation of Bet v 1-allergic individuals (15). Bet v 1-allergic patients are at risk to acquire various plant food allergies, and even to react to novel foods without prior exposure. In contrast to Bet v 1, Bet v 1-related food allergens are regarded as incapable of sensitizing predisposed individuals. The few exceptions that were described include Dau c 1 from carrot (16-18), and Cor a 1 from hazelnut (19).

The list of plant food allergens to which Bet $v$ 1-allergic individuals may react is quite varied and most likely connected to the variation in the individual $\lg \mathrm{E}$ epitope patterns described (5). Interestingly, most of the Bet $v 1$ cross-reactive allergens are found in fruits of the Rosaceae, vegetables of the Apiaceae, or seeds of the Fabaceae (Table 4).

Amino acid sequence identities between selected PR10 pollen and plant food allergens fall between 38 and $88 \%$ (Table 5). There is also a range of plant foods that contain cross-reactive Bet $v 1$ homologues that have not yet received an official allergen nomenclature designation including plum, nectarine, fig, mango, persimmon, jackfruit, walnut, and chickpea. 
Table 3

Bet $v$ 1-homologous pollen allergens of Fagales pollen

\begin{tabular}{|c|c|c|c|}
\hline $\begin{array}{l}\text { Botanical } \\
\text { family }\end{array}$ & $\begin{array}{l}\text { Botanical } \\
\text { subfamily }\end{array}$ & Allergen source & Allergen \\
\hline \multirow{5}{*}{ Betulaceae } & \multirow{2}{*}{ Betuloideae } & Birch (Betula pendula) & Bet v 1 \\
\hline & & Alder (Alnus glutinosa) & Aln g 1 \\
\hline & \multirow{3}{*}{ Coryloideae } & Hazel (Corylus avellana) & Cor a 1 \\
\hline & & Hornbeam (Carpinus betulus) & Car b 1 \\
\hline & & Hop-hornbeam (Ostrya carpinifolia) & Ost c 1 \\
\hline \multirow{3}{*}{ Fagaceae } & Fagoideae & Beech (Fagus silvatica) & Fag s 1 \\
\hline & Quercoideae & Oak (Quercus alba) & Que a 1 \\
\hline & Castaneoideae & Chestnut (Castanea sativa) & Cas s 1 \\
\hline
\end{tabular}

Table 4

Bet $v 1$-homologous allergens from plant foods

\begin{tabular}{|c|c|c|}
\hline Botanical family & Allergen source & Allergen \\
\hline \multirow[t]{7}{*}{ Rosaceae } & Strawberry (Fragaria ananassa) & Fra a 1 \\
\hline & Apple (Malus domestica) & Mal d 1 \\
\hline & Apricot (Prunus armeniaca) & Pru ar 1 \\
\hline & Cherry (Prunus avium) & Pru av 1 \\
\hline & Peach (Prunus persica) & Pru p 1 \\
\hline & Pear (Pyrus communis) & Pyr c 1 \\
\hline & Raspberry (Rubus idaeus) & Rub i 1 \\
\hline \multirow[t]{2}{*}{ Actinidiaceae } & Golden kiwifruit (Actinidia chinensis) & Act c 8 \\
\hline & Green kiwifruit (Actinidia deliciosa) & Act d 8 \\
\hline \multirow[t]{2}{*}{ Apiaceae } & Celery (Apium graveolens) & Api g 1 \\
\hline & Carrot (Daucus carota) & Dau c 1 \\
\hline \multirow[t]{3}{*}{ Fabaceae } & Peanut (Arachis hypogaea) & Ara h 8 \\
\hline & Soybean (Glycine max) & Gly m 4 \\
\hline & Mung bean (Vigna radiata) & Vig $r 1$ \\
\hline Corylaceae & Hazelnut (Corylus avellana) & Cor a 1.04 \\
\hline Fagaceae & Chestnut (Castanea sativa) & Cas s 1 \\
\hline Solanaceae & Tomato (Solanum lycopersicum) & Sola | 4 \\
\hline
\end{tabular}




\section{CLINICAL RELEVANCE}

\section{General importance}

Bet $\mathrm{v} 1$ is regarded as (i) a marker allergen for a primary sensitization to pollen of birch and other Fagales trees (e.g. alder, hazel, hornbeam, beech), and (ii) an indicator of cross-reactivity to a number of related major pollen and plant food allergens.

Clinically, Bet v 1 and its homologues (iii) represent important pollen allergens (after inhalant exposure), and (iv) are considered important inducers of birch pollen-associated plant food allergies.

\section{Symptoms (inhalant allergy)}

Typical mucosal symptoms of tree pollen allergy in Middle Europe occur during spring between February and May with maximums depending on the current climate and the region:

- itchiness, redness, tearing of the eyes

- itch in the nose, (repeated) sneezing, runny and/ or stuffy nose

- optional dry cough (particularly during or shortly after exercise), dyspnea, pressure sensation on the chest, wheezing, bronchial secretion and difficulty in breathing as indicators for increasingly affected lower airways (asthmatic lower airway inflammation)

Clinical diagnoses of intermittent (seasonal) rhinoconjunctivitis and allergic asthma due to birch (Fagales) pollen become very likely in case of repeated, sometimes increasing mucosal symptoms during the same season in subsequent years.

Despite being indirect, additional oropharyngeal symptoms that occur in approximately $2 / 3$ of birch pollen allergic individuals after consumption of certain raw plant foods (Table 3 ) indirectly confirm a suspected birch pollen allergy. In a minority of birch pollen allergic individuals, ingestion of roasted hazelnuts or products containing roasted hazelnuts can induce OAS.

\section{Epidemiology}

According to the European Respiratory Health Survey, the prevalence of sensitization to birch pollen was on average $6.4 \%$ with a maximum of $22.4 \%$ in northern Europe (20). Sensitization was generally high in northern and mid-Europe and low in the south of Europe. A large nationwide study in Germany on a representative sample of children and adolescents revealed the presence of IgE specific for birch pollen allergens in $15 \%$ of the individuals in the age group of 3 to 17 years (21). In the age group of 13 to 17 years, $15.7 \%$ of the girls and $21.7 \%$ of the boys had IgE specific for birch pollen allergens. IgE-sensitization to birch pollen allergens in adults (age 19 to 79 years) was found to be $17.4 \%$ and sensitization to Bet v 1 15.2\% (22). Approximately half of all sensitized individuals will develop symptoms such as allergic rhinoconjunctivitis or allergic asthma.

Table 5

Amino acid identities (\%) between PR-10 allergens

\begin{tabular}{|c|c|c|c|c|c|c|c|c|c|}
\hline \multicolumn{10}{|l|}{ Bet v 1} \\
\hline Mal d 1 & 57 & & & & & & & & \\
\hline Pru av 1 & 60 & 88 & & & & & & & \\
\hline Act d 8 & 49 & 50 & 53 & & & & & & \\
\hline Api g 1 & 39 & 39 & 40 & 47 & & & & & \\
\hline Ara h 8 & 46 & 49 & 50 & 38 & 39 & & & & \\
\hline Gly m 4 & 48 & 53 & 54 & 44 & 38 & 70 & & & \\
\hline Cor a 1.04 & 67 & 62 & 65 & 52 & 43 & 53 & 57 & & \\
\hline \multirow[t]{2}{*}{ Que a 1} & 58 & 59 & 60 & 52 & 44 & 44 & 50 & 59 & \\
\hline & Bet $v 1$ & Mal d 1 & Pru av 1 & Act d 8 & Api g 1 & Ara h 8 & Gly m 4 & Cor a 1.04 & Que a 1 \\
\hline
\end{tabular}




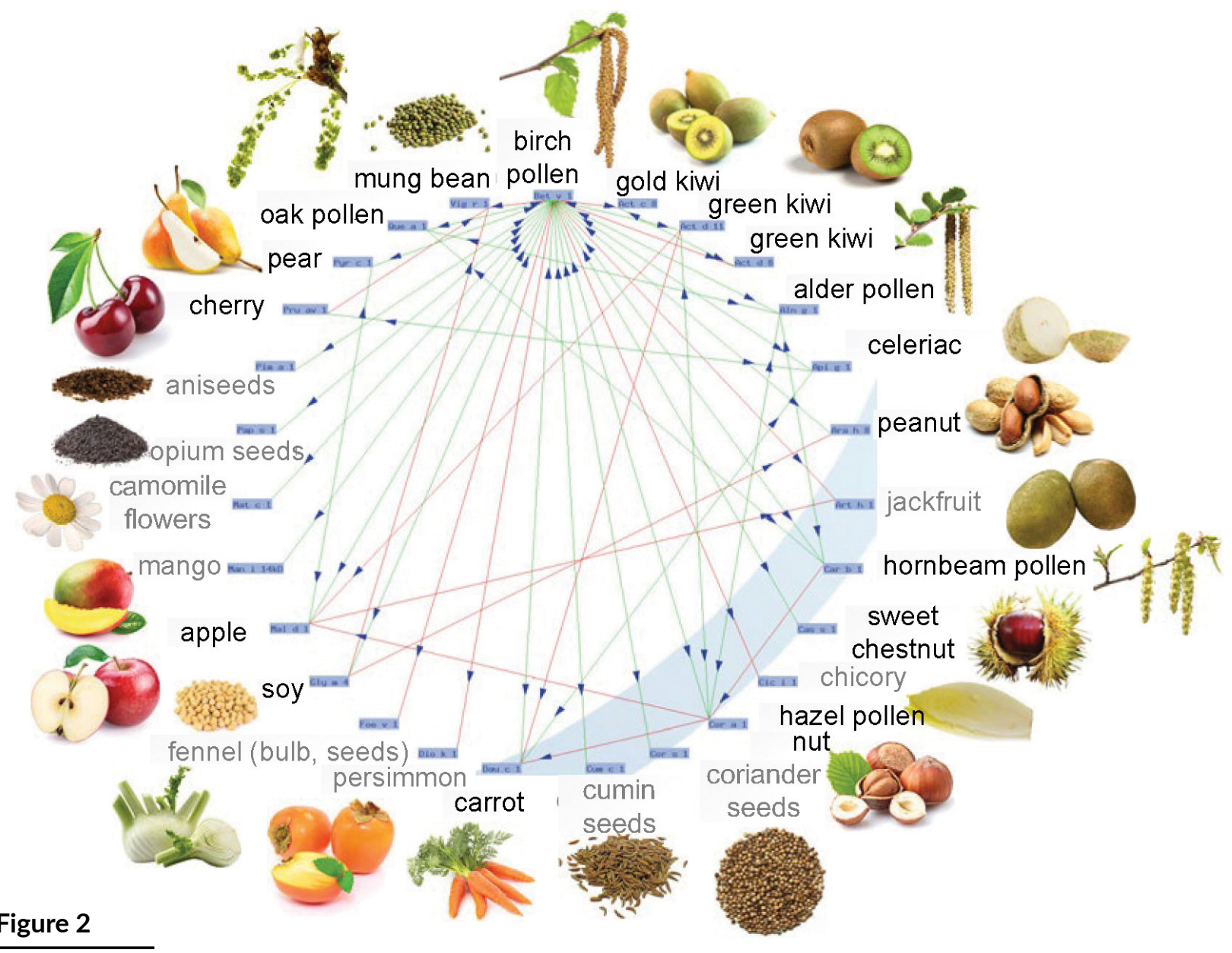

IgE Cross-reactivity between the birch pollen major allergen Bet $\vee 1$ (at 12 o'clock of the circle) and related allergens in tree pollen of the order Fagales, in pome and stone fruit, tree nuts, vegetables, and legumes. Additional Bet $\vee 1$ homologues are present in plum, peach, apricot, nectarine, strawberries, fig, mango, persimmon, jackfruit, walnut, chickpea, potatoes, tomatoes and parsley. Bidirectional cross reactivity is illustrated by red double arrows and unidirectional cross reactivity with green arrows. Allergen sources and corresponding without listing in the IUIS data bank are given in grey letters (Figure: modified from http://www.allergome.org; O-ring dynamically created on March 3, 2015), with kind permission of Springer Verlag, Heidelberg, Berlin.

\section{Symptoms (oral exposure)}

Minute amounts of Bet $v$ 1-homologous proteins are able to induce various, transient, predominantly oropharyngeal symptoms (Table 5) with a quick onset (sometimes immediately and often within minutes) after consumption of raw plant foods (Table 3 ) in approximately $2 / 3$ of birch pollen allergic individuals. These symptoms are often referred to as "oral allergy syndrome" (OAS), implicating a particular clinical entity. This is not the case, since

- oropharyngeal symptoms can occur quite variably including different degrees of severity (Table 6, symptom complex A)
- diffusion of inflammatory mediators (i.e. histamine) and/or neurogenic reflexes can prompt additional severe symptoms in the head area (Table 6, symptom complex B) or

- systemic, sometimes anaphylactic symptoms can occur in rare cases (Table 6, symptom complex C)

Noteworthy, oropharyngeal symptoms are not unique or specific for Bet $v$ 1-induced cross reactions or certain food items, since other food allergens are able to induce similar symptoms:

- profilin-containing plant foods (see Section C01)

- nsLTP-containing plant foods (see Section C03) 
- animal derived food allergens (see Section C05, C08)

Thus, the so-called OAS does not represent a defined clinical entity (syndrome), but a rathervariable symptom complex. The occurrence of only oropharyngeal symptoms reflects the physicochemical properties of the particular food allergens which in the case of the Bet v 1-homologues PR-10-like proteins are

- instability (rarely substantial intestinal absorption) and

- excellent aqueous solubility (quick onset of symptoms after mucosal exposure)

From a clinical point of view, a large and over the years increasing number of reported Bet $v$ 1-related cross-reactive plant foods

- indicates a high level of Bet $v$ 1-specific lgE based on a broad polyclonal IgE-repertoire

- is associated with more severe clinical reactions due to foods of the Bet $v 1$ cluster

The following variables are presumably involved in rare, severe clinical reactions to foods containing Bet v 1-homologues:

- strong Bet $v$ 1-specific lgE response (high specific IgE/total lgE ratio)

- broad Bet v 1-specific IgE repertoire (indirectly reflected in a large panel of implicated plant foods)

- ingested amount of particular Bet v 1-homologue containing food

- differences in stability of Bet $v 1$-homologue in the respective food items (more often systemic reactions due to hazelnuts, soy, carrots and celeriac in comparison to e.g. apples)

- presumably matrix effects of certain food items (e.g. soy) with high protein content, protecting the immediate degradation of a Bet $v$ 1-homologue containing food.

An increase of oropharyngeal symptoms during or shortly after the birch pollen season is generally observed. Natural birch pollen exposure might booster the Bet $v$ 1-specific IgE response with a possibly broadened IgE repertoire.

Occasionally, subjects experience oropharyngeal symptoms (Table 6) after consumption of typical Bet $v$ 1-related plant foods without suffering from inhalant symptoms during the tree pollen season. This observation, a (so far) clinically "silent" Bet v 1 sensitization, can still prompt unexpected allergic reactions after the first consumption of Bet $v$ 1-related plant foods. Without knowledge of the molecular relationship both, the diagnosis of a birch pollen (Bet $\vee 1$ )-associated plant food allergy, and proper consultation of the affected individuals might be unnecessarily delayed.

The most frequently observed allergy to soy in Central Europe is based on the serological cross-reactivity of Bet v 1-specific IgE (70\%) with its homologue Gly $m$ 4 from soybean (Section B17). In general, reactions occur following the ingestion of large amounts of soy-based products that did not undergo major processing steps (e.g. soymilk, soy-based protein powders). Reactions can be systemic and severe and have been observed in about $10 \%$ of birch-pollen allergic individuals (15).

\section{Conclusions on clinical relevance}

- 6.4 to $22.4 \%$ of the European population is sensitized to birch pollen

- Around half of all sensitized individuals will develop symptoms

- Around $70 \%$ of birch pollen allergic individuals suffer from associated plant food allergy

- Impact of IgE cross-reactivity on clinical symptoms of food allergy is still unknown

- Molecule-based therapeutic approaches are under investigation

\section{CLINICAL MANAGEMENT}

\section{Diagnostic testing of Bet $v$ 1-related allergies}

IgE-sensitization to Bet $\vee 1$ can be assessed (Fig. 3) directly by allergen-specific IgE testing to Bet $v 1$, or indirectly by SPT with birch pollen extract (wheal $>3$ $\mathrm{mm}$ or $>5 \mathrm{~mm}$ ) or allergen-specific IgE testing with birch pollen extract.

Convincing analytical specificity after birch pollen extract testing is only obtained in case of a limited sensitization profile (i.e. if only positive to pollen of Fagales trees). If a positive SPT result was already obtained with a birch pollen extract, allergen-specific IgE should rather be tested to Bet $v 1$, providing more (analytical) specificity than another birch pollen extract for in vitro diagnosis.

Commercial plant food extracts (for SPT as well as IgEtesting) often show false negative results and should be avoided due to a low abundance and a lack of stability 
Table 6

Potential symptoms due to Bet v 1-associated lgE cross-reactivity to plant foods

\begin{tabular}{|c|c|c|}
\hline Symptom complex & Symptoms & Organ/localisation \\
\hline \multirow{3}{*}{$\begin{array}{l}\text { A. Limited } \\
\text { oropharyngeal } \\
\text { symptoms } \\
\text { (frequent) }\end{array}$} & $\begin{array}{l}\text { itch } \\
\text { ("tingling", "tickling", "prickle") }\end{array}$ & lip mucosa, oral mucosa, palate \\
\hline & burning, stinging & palate, throat \\
\hline & mild mucosal swelling & lip mucosa, oral mucosa, palate, throat \\
\hline \multirow{5}{*}{$\begin{array}{l}\text { B. Additional } \\
\text { symptoms in } \\
\text { the head area } \\
\text { (isolated or with } \\
\text { symptoms from } \\
\text { A) (rare events) }\end{array}$} & itch, redness, tearing & conjunctiva \\
\hline & itch, sneezing, runny nose, stuffy nose & nose \\
\hline & itch & ears (internally, Eustachian tubes) \\
\hline & tissue (skin) swelling (angioedema) & eye lids, lips, cheeks, ears, face \\
\hline & $\begin{array}{l}\text { severe (internal) mucosal swelling, } \\
\text { globus sensation, difficulty swallowing, } \\
\text { hoarseness (indicating vocal cord or larynx } \\
\text { edema), shortness of breath, stridor }\end{array}$ & palate, throat, larynx \\
\hline \multirow{4}{*}{$\begin{array}{l}\text { C. systemic } \\
\text { symptoms } \\
\text { (extremely rare) }\end{array}$} & itch, redness, hives, swelling (angioedema) & localized, multifocal or generalized at the skin \\
\hline & nausea, vomiting, abdominal pain, diarrhea & stomach, intestine \\
\hline & $\begin{array}{l}\text { breathing difficulties, chest pressure, chest } \\
\text { tightness, shortness of breath, wheezing, } \\
\text { cough, sputum (optional) }\end{array}$ & bronchi \\
\hline & $\begin{array}{l}\text { dizziness, general weakness, } \\
\text { unconsciousness, circulatory collapse }\end{array}$ & heart and circulation \\
\hline
\end{tabular}

of Bet $v 1$ homologous food allergens. Instead, Prickto-Prick tests can be performed with suspected, fresh, raw plant foods. Despite a lack of standardization, this approach can provide a qualitative, indirect demonstration of IgE-sensitization/cross reactivity.

\section{No value of broad molecular testing of Bet $\mathbf{v}$ 1-sensitized subjects}

Is it worth it to demonstrate additional IgEsensitizations/cross reactions to Bet $v$ 1-homologues such as allergen-specific IgE to Mal d 1, Cor a 1.04, Pru p 1 and many others? Presumably not, since numerous positive serological cross-reactions are to be expected when Bet v 1-specific IgE is present (23). Thus, such test results are not informative in terms of their clinical relevance without further clinical information. Only a clear-cut negative IgE result against a Bet v 1-homologues single allergen (i.e. Dau c 1, Gly m 4, Pru p 1), obtained with a sensitive lgE test (cut-off at 0.1 kUA/l; singleplex assay) could reliably rule out an IgEsensitization/cross-reactivity and subsequent clinically relevant food allergy due to Bet $v 1$-cross-reactivity. Unfortunately, this scenario does hardly exist in reality. Instead, rational and targeted testing (i.e. allergen- specific IgE only to Bet $v 1$ ) is recommended. As a rule of thumb sensitization tests in case of Bet $v 1$-associated cross-reactions are only meaningful, if the results will potentially lead to clinical consequences.

\section{Interpretation of Bet $v$ 1-related IgE- sensitizations}

Interpretation of a test addressing the clinical relevance of a previously demonstrated Bet $v$ 1-sensitization can only be obtained with clinical information. Thus, a detailed anamnesis is so important in case of a birch pollen-associated food allergy in question. Following diagnostic work-up can facilitate a final interpretation of the clinical relevance of a sensitization test (i.e. positive birch pollen SPT, positive Bet $v$ 1-specific $\lg \mathrm{E})$ :

- The subject should be systematically asked again, whether oropharyngeal (or other) allergic symptoms (Table 6) occurred and after which ingested, raw plant foods they appeared or not. The list of foods should not only address the most common ones as apples and hazelnuts, but the whole panel of potentially Bet $v$ 1-cross-reactive plant foods (Table 4). 


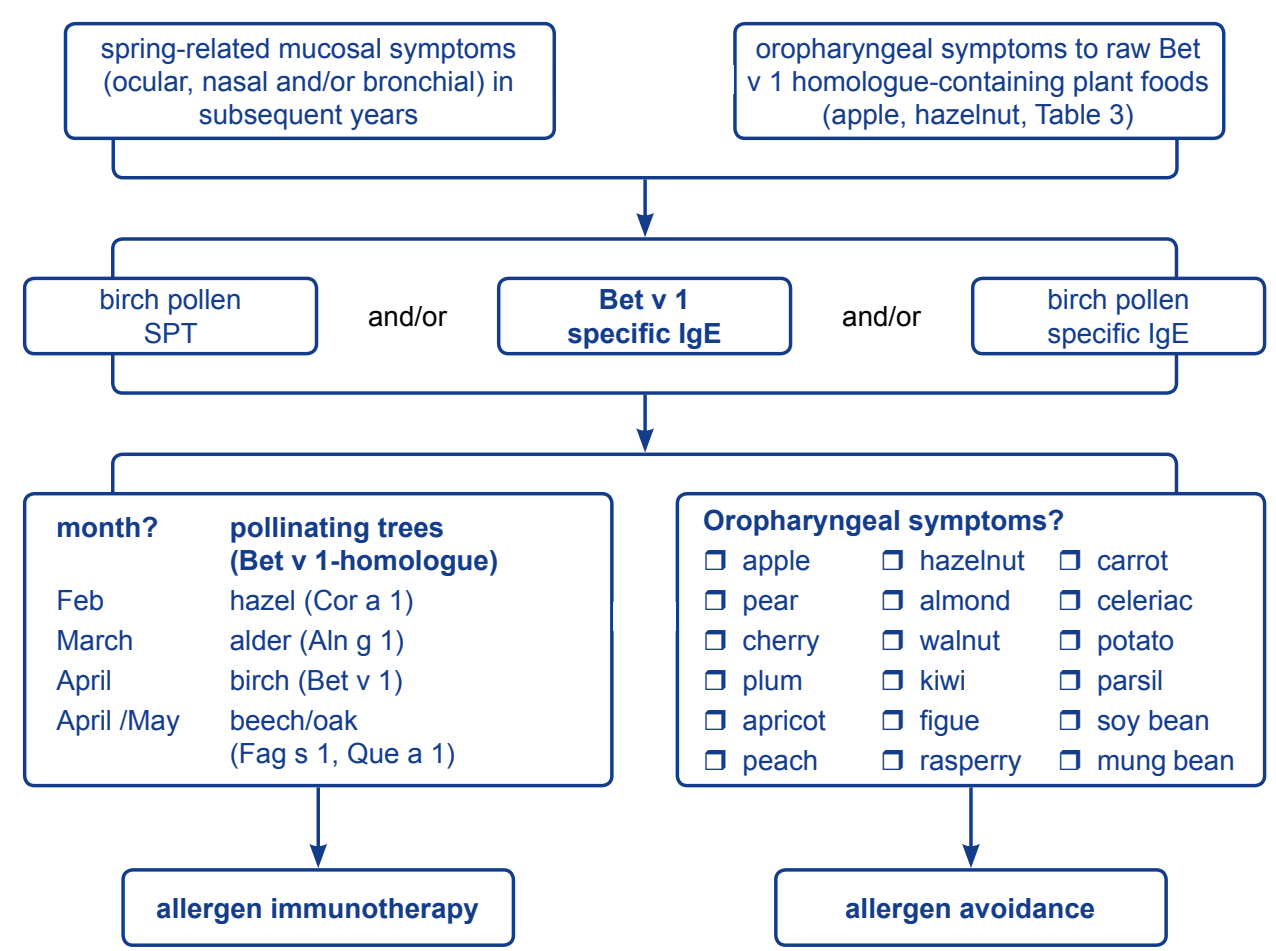

allergy management (consequences, advice)

molecular-concept- based interpretation(anamnesis, optionalchallenge) aggreement with history? clinically relevant?

\section{Figure 3}

\section{Diagnostic algorithm in case of Fagales tree pollen and/or Bet $\vee$ 1-homologue related food allergy to raw} plant foods

- Oral challenge tests can help - in case of missing clinical information or vague anamnesis - to prove or rule out clinical cross-reactions. They can also serve as a prospective test of a potentially crossreactive, but so far never eaten plant food.

Oral food challenges, however, are rarely routinely performed in case of Bet v 1-associated food allergies for following reasons:

- They are tedious to perform considering the number of potentially cross-reactive foods.

- There is no urgent indication in case of solely oropharyngeal symptoms.

- They are not easy to interpret due to predominantly subjective symptoms.

- There are almost no validated dose-dependent tests.

- There are only few proven published protocols for oral challenge procedures with Bet v 1-associated plant food $(24,25)$.

Finally, only those Bet $\vee$ 1-related foods should be avoided in their raw form, which have induced typical allergic symptoms. Avoiding every potentially cross- reactive food item of the Bet $v$ 1-cluster would be exaggerated and clinically not justified. The same is true for plant foods, showing indirectly after Prick-toPrick tests or directly after serological analysis positive IgE-sensitizations: Even the entire panel of Bet $v$ 1-homologues employed for diagnostic purposes would not be able to separate silent from clinically relevant sensitizations in case of positive test results.

\section{Diagnostic recommendations for Bet v 1-related allergy}

- Bet $v$ 1-homologues in tree pollen extracts for diagnostic purposes will induce positive SPT and IgE reactions to a number of Fagales trees (Table 3 ), not necessarily being clinically relevant.

- Prick-to-Prick tests with fresh (raw) foods are superior diagnostic tools compared to commercial food extracts in case of birch pollen-associated plant food allergies due to low stability of Bet $v$ 1-homomlogues.

- After being spiked with recombinant Bet $v$ 1-homologues (e.g. Cor a 1) birch pollen- 
associated plant food extracts (i.e. hazelnut extract) can bind more IgE, increase assay sensitivity (lowered "limit of quantitation", LoQ) and provide elevated allergen-specific IgE-values.

- At the same time "spiking" will unfold more positive (potentially clinically irrelevant) sensitizations, pointing to general drawbacks of extract-based diagnostics (i.e. high sensitization rates to peanut in Middle Europe due to cross-reactive natural Bet v 1-homologue Ara h 8 in peanut extracts).

- Bet v 1-specific IgE serves as a reliable marker for potential, serological cross-reactions to a number of plant foods (Table 3). Actual clinical relevance of potential cross-reactions is systematically addressed by the physician together with the patient based on the subject's individual symptoms after consumption of raw foods containing Bet v 1-homologues.

- Positive specific IgE to Bet $v$ 1-homologues plant food allergens (i.e. Pru $p 1$ from peach) demonstrates allergic sensitization, being clinically relevant in case of corresponding symptoms.

- A negative IgE results (i.e. to Gly $m 4$ from soy, approx. in only $25 \%$ of Bet $v 1$-sensitized subjects) would exclude serological cross-reactivity with certainty and clinically relevant cross-reactions as well.

The rule-of-thumb for the diagnostic work-up of Bet $v$ 1-associated allergic reactions concludes: "The physician's interpretation, based on the patient's individual symptoms, and not the outcome of a sensitization test will establish the decision about the clinical relevance of previous diagnostic findings."

Sensitization can be tested by skin prick testing, or in vitro extract- or molecule-based assays. IgE test results always have to be interpreted in the context of the anamnesis. At present, all Bet $v$ 1-related allergens that are of relevance in Europe are available as recombinant proteins, but only few for diagnostic purposes. However, the Bet $\vee 1$-related allergens from mango, persimmon or jackfruit - all of them have been described as causing symptoms in birch pollen allergic individuals - are not yet available as recombinant proteins.

\section{Extract composition for allergen-specific immunotherapy}

Due to the high cross-reactivity of the major allergens Bet $\vee 1$, Cor a 1 and Aln $g 1$ of birch, hazel and alder pollen, respectively, birch pollen monoextracts as well as combined extracts from hazel, alder and birch pollen are suitable for specific immunotherapy of a tree pollen allergy. The selection will mainly depend on the time when symptoms occur (Fig. 3).

Whether immunotherapy with tree pollen extracts has a beneficial influence on associated plant food allergies is still under discussion. Most studies were performed on birch pollen associated apple allergy. As the apple allergen Mal $d 1$ has the highest sequence identity to Bet $v 1$, one might expect the best results from a birch pollen based immunotherapy. However, the results from several studies on birch pollen associated apple allergy are controversial (26-28). No clinical effect on a birch pollen associated hazelnut allergy was observed after one year of specific immunotherapy with a birch pollen extract (29).

Therefore, oral allergy syndrome to birch pollen associated plant foods in the absence of pollen induced respiratory symptoms should not be considered as a main criterion for selecting patients for birch pollen immunotherapy. Recombinant Bet $v 1$ and variations of the molecule were tested in clinical trials for their efficacy as immunotherapy reagents but are not yet available on the market $(30,31)$.

\section{CLINICAL CASES}

\section{Case 1 (original)}

History: Female, 39 yrs: Since 2015 for the first time during spring time eye itch, tearing, swelling, sneezing, runny and blocked nose, later chest tightness, wheezing, coughing and white sputum. In addition, since spring 2015 itchy throat after eating raw fruits (apples, cherries, peaches).

In-vivo testing: SPT (wheal diameter [mm]): hazel 12, alder 5, birch 4, oak 6 .

In-vitro testing: Total lgE $190 \mathrm{kU} / \mathrm{l}$, specific IgE to Bet $v 1: 91 \mathrm{kUA} / \mathrm{I}$ (>47\% of total IgE indicating a strong sensitization).

Diagnosis: A) Allergic rhinoconjunctivitis due to Fagales tree pollen; B) Bet $\vee$ 1-associated foodallergy (oropharyngeal symptoms to certain raw Rosaceae fruits)

Recommendations: Allergen-specific immunotherapy with birch pollen extract with caution during the escalation phase (high relative specific IgE to Bet 
v 1 is associated with potential adverse events). Avoidance of raw Bet $v 1$-cross-reactive pome and stone fruits (see history); cooked, baked or roasted plant products without dietary restriction (due to thermal instability of Bet $v$ 1-related allergens).

Comments: a) Strong Bet v 1-sensitization based on high absolute IgE values and high ratio between Bet $\vee$ 1-specific IgE and total IgE; b) despite the short allergy history (1st allergy season!) strong indication for AIT due to the brisk disease development including lower airways and crossreactive oropharyngeal symptoms.

\section{Case 2 (original)}

History: Male, 35 yrs: Since 10 years Fagales tree pollen induced rhinoconjunctivitis with itchy eyes, sneezing, runny nose, sore throat, itch in the ears, general fatigue. After ingestion of raw apples, hazelnuts, cherries, peaches, apricots, strawberries, blueberries*, grapes* within 5 minutes itchy and sore throat, itchy eyes and ears for 15 minutes, after soy products loose stool.

In-vivo testing: SPT (wheal diameter [mm]): hazel 6, alder 5 , birch 10 , oak 3 , grass-mix 3 , mugwort 3 .

In-vitro testing: Total IgE $10 \mathrm{kUA} / \mathrm{l}$, specific IgE to Bet $v$ 1: $3.4 \mathrm{kUA} / \mathrm{I}(>1 / 3$ of total IgE indicating strong sensitization), $\mathrm{Phl} p$ 1: $2.8 \mathrm{kUA} / \mathrm{l}$, other allergen specificities Phl p 12 (profilin), Art $\vee 1$, mugwort, Pru p 3 (nsLTP) negative (<0.1 kUA/I).

Diagnosis: A) Allergic rhinoconjunctivitis due to Fagales tree pollen; B) Bet $v$ 1-associated foodallergy (oropharyngeal symptoms to raw foods)

Recommendations: Allergen-specific immunotherapy with birch or hazel-alder-birch extract. Avoidance of raw Bet $v$ 1-cross-reactive pome and stone fruits (see subject's history).

Comments: a) absolute IgE values (total and allergenspecific) are both low; b) relative relationship between Bet v 1-specific lgE and total IgE is high (pointing to the need to consider both, total and specific lgE, for proper interpretation); c) ${ }^{*}$ certain fruits are not indicative as containing Bet v 1-homologues, but are nsLTP-containing foods; thus, due to negative IgE to Pru $\mathrm{p} 3$ and profilin these reported reactions cannot be easily explained, i.e. Pru $\mathrm{p} 7$ homologues.

\section{Case 3 (original)}

History: Female, 47 yrs: Since 15 years after consumption of raw plant foods like apples, cherries, hazelnuts, walnuts within 1 minute mild sore throat for 5 minutes. So far no allergic symptoms during spring.

In-vitro testing: Total IgE $174 \mathrm{kUA} / \mathrm{l}$, specific IgE to Bet $\vee$ 1: 34 kUA/l.

Diagnosis: Bet $v$ 1-associated food allergy (oropharyngeal symptoms to raw foods) without allergic airway disease.

Recommendations: Avoidance of raw Bet $v 1$ 1-crossreactive pome and stone fruits (see subject's history). No indication for AIT due to missing Fagales tree pollen induced airway symptoms

Comments: Rare cases are suffering from oropharyngeal allergy symptoms due to plant foods containing Bet $v$ 1-homologues without any allergic airway symptoms during the birch pollen season. Diagnosis is established by IgE-testing to Bet $\vee 1$. Potential clinical consequences are addressed "clinically" (without further IgE-testing to other Bet v 1-homologues).

\section{References}

1. Breiteneder $\mathrm{H}$, Pettenburger K, Bito, Valenta R, Kraft $\mathrm{D}$, Rumpold $\mathrm{H}, \mathrm{A}$ et al. The gene coding for the major birch pollen allergen Betv1, is highly homologous to a pea disease resistance response gene. EMBO J 1989;8:1935-1938.

2. Gajhede M, Osmark P, Poulsen FM, Ipsen H, Larsen JN, Joost van Neerven RJ, et al. X-ray and NMR structure of Bet $v 1$, the origin of birch pollen allergy. Nat Struct Biol 1996;3:1040-1045.

3. Jahn-Schmid B, Radakovics A, Lüttkopf D, Scheurer S, Vieths S, Ebner C, et al. Bet v 1142-156 is the dominant T-cell epitope of the major birch pollen allergen and important for cross-reactivity with Bet v 1-related food allergens. J Allergy Clin Immunol 2005;116:213-219.

4. Kitzmüller C, Zulehner N, Roulias A, , Briza P, Ferreira F, Faé I, et al. Correlation of sensitizing capacity and T-cell recognition within the Bet $\vee 1$ family. J Allergy Clin Immunol 2015;136:151-158.

5. Gepp B, Lengger N, Bublin M, Hemmer W, Breiteneder $\mathrm{H}$, Radauer $\mathrm{C}$. Chimeras of Bet $\vee 1$ and Api g 1 reveal heterogeneous IgE responses in patients with birch pollen allergy. J Allergy Clin Immunol 2014;134:188-194.

6. Antoniw JF, Ritter CE, Pierpoint WS, van Loon LC. Comparison of three pathogenesis-related proteins from plants of two cultivars of tobacco infected with TMV. J Gen Virol 1980;47:79-87.

7. van Loon LC, van Strien EA. The families of pathogenesis-related proteins, their activities, and comparative analysis of PR-1 type proteins. Physiol 
Mol Plant Pathol 1999;55:85-97.

8. Radauer $\mathrm{C}$, Lackner $\mathrm{P}$, Breiteneder $\mathrm{H}$. The Bet $\vee 1$ fold: an ancient, versatile scaffold for binding of large, hydrophobic ligands. BMC Evol Biol 2008;8:286.

9. D'Avino R, Bernardi ML, Wallner M, Palazzo $P$, Camardella L, Tuppo L, et al. Kiwifruit Act d 11 is the first member of the ripening-related protein family identified as an allergen. Allergy 2011;66:870-877.

10. Guhsl EE, Hofstetter G, Hemmer W, Ebner C, Vieths $\mathrm{S}$, Vogel L, et al. Vig r 6 , the cytokinin-specific binding protein from mung bean (Vigna radiata) sprouts, crossreacts with Bet $v$ 1-related allergens and binds IgE from birch pollen allergic patients' sera. Mol Nutr Food Res 2014;58:625-634.

11. Geroldinger-Simic $M$, Zelniker T, Aberer W, Ebner C, Egger C, Greiderer A, et al. Birch pollen-related food allergy: clinical aspects and the role of allergenspecific IgE and IgG4 antibodies. J Allergy Clin Immunol 2011;127:616-622.

12. Hauser M, Asam C, Himly M, Palazzo P, Voltolini $\mathrm{S}$, Montanari $\mathrm{C}$, et al. Bet $\vee$ 1-like pollen allergens of multiple Fagales species can sensitize atopic individuals. Clin Exp Allergy 2011;41:1804-1814.

13. Breiteneder $\mathrm{H}$, Ebner $\mathrm{C}$. Molecular and biochemical classification of plant-derived food allergens. J Allergy Clin Immunol 2000;106:27-36.

14. Vieths S, Scheurer S, Ballmer-Weber B. Current understanding of cross-reactivity of food allergens and pollen. Ann N Y Acad Sci 2002;964:47-68.

15. Kleine-Tebbe J, Vogel L, Crowell DN, Haustein UF, Vieths S. Severe oral allergy syndrome and anaphylactic reactions caused by a Bet $\vee 1$ - related PR-10 protein in soybean, SAM22. J Allergy Clin Immunol 2002;110:797-804.

16. Moneo I, Gómez M, Sánchez-Monge R, Alday E, de las Heras M, Esteban I, et al. Lack of crossreaction with Bet $v 1$ in patients sensitized to Dau c 1, a carrot allergen. Ann Allergy Asthma Immunol 1999;83:71-75.

17. Ballmer-Weber BK, Wuthrich B, Wangorsch A, Fotisch K, Altmann F, Vieths S. Carrot allergy: double-blinded, placebo-controlled food challenge and identification of allergens. J Allergy Clin Immunol 2001;108:301-307.

18. Moreno-Ancillo A, Gil-Adrados AC, DomìnguezNoche C, Cosmes PM, Pineda F. Occupational asthma due to carrot in a cook. Allergol Immunopathol (Madr) 2005;33:288-290.

19. Flinterman $A E$, Akkerdaas $\mathrm{JH}$, den Hartog Jager $\mathrm{CF}$, Rigby NM, Fernandez-Rivas M, Hoekstra MO, et al. Lipid transfer protein-linked hazelnut allergy in children from a non-Mediterranean birch-endemic area. J Allergy Clin Immunol 2008;121:423-428.

20. Bousquet PJ, Chinn S, Janson C, Kogevinas M, Burney $P$, Jarvis D. Geographical variation in the prevalence of positive skin tests to environmental aeroallergens in the European Community Respiratory Health Survey I. Allergy 2007;62:301-309.

21. Schmitz R, Ellert U, Kalcklosch M, Dahm S, Thamm M. Patterns of sensitization to inhalant and food allergens - findings from the German Health Interview and Examination Survey for Children and Adolescents. Int Arch Allergy Immunol 2013;162:263-270.

22. Haftenberger M, Laußmann D, Ellert U, Kalcklösch $M$, Langen $U$, Schlaud $M$, et al. Prävalenz von Sensibilisierungen gegen Inhalations- und Nahrungsmittelallergene. Ergebnisse der Studie zur Gesundheit Erwachsener in Deutschland (DEGS1). BundesgesundheitsblattGesundheitsforschung Gesundheitsschutz 2013;56:687-697.

23. Villalta $D$, Asero R. Is the detection of IgE to multiple Bet $\vee$ 1-homologous food allergens by means of allergen microarray clinically useful? J Allergy Clin Immunol 2010;125:1158-1161.

24. Cochrane SA, Salt LJ, Wantling E, Rogers A, Coutts $\mathrm{J}$, Ballmer-Weber BK, et al. Development of a standardized low-dose double-blind placebocontrolled challenge vehicle for the EuroPrevall project. Allergy 2012;67:107-113.

25. Bauermeister K, Ballmer-Weber BK, Bublin M, Fritsche P, Hanschmann KM, Hoffmann-Sommergruber $\mathrm{K}$, et al. Assessment of component-resolved in vitro diagnosis of celeriac allergy. J Allergy Clin Immunol 2009;124:1273-1281.

26. Bolhaar ST, Tiemessen MM, Zuidmeer L, van Leeuwen A, Hoffmann-Sommergruber K, Bruijnzeel-Koomen CA, et al. Efficacy of birch-pollen immunotherapy on crossreactive food allergy confirmed by skin tests and doubleblind food challenges. Clin Exp Allergy 2004;34:761769.

27. Hansen KS, Khinchi MS, Skov PS, Bindslev-Jensen C, Poulsen LK, Malling HJ. Food allergy to apple and specific immunotherapy with birch pollen. Mol Nutr Food Res 2004;48:441-448.

28. Mauro M, Russello M, Incorvaia C, Gazzola G, Frati $F$, Moingeon $P$, et al. Birch-apple syndrome treated with birch pollen immunotherapy. Int Arch Allergy Immunol 2011;156:416-422.

29. Asero R. Is there a role for birch pollen immunotherapy on oncomitant food allergy? Curr Treat Options Allergy 2015;2:83-89.

30. Purohit $A$, Niederberger $V$, Kronqvist $M$, Horak F, Grönneberg R, Suck R, et al. Clinical effects of immunotherapy with genetically modified recombinant birch pollen Bet $v 1$ derivatives. Clin Exp Allergy 2008;38:1514-1525.

31. Cromwell O, Niederberger V, Horak F, Fiebig H. Clinical experience with recombinant molecules for allergy vaccination. Curr Top Microbiol Immunol 2011;352:2742. 


\section{PART C}

\section{CROSS-REACTIVE MOLECULES \& THEIR CLINICAL RELEVANCE}

\section{NON-SPECIFIC LIPID TRANSFER PROTEINS (nsLTPs)}

\author{
Elide Pastorello, Domingo Barber
}

\section{THE PROTEIN}

Pru 3 3, the major allergen of peach, was the first nsLTP to be fully identified and characterized as a relevant food allergen $(1,2)$. It is the most broadly recognized allergen of the family although there are patients sensitized to other nsLTPs that might not develop IgE antibody reactive with Pru $\mathrm{p} 3$. It is located in vascular tissue and in the outer cell layers of the plant organs. Importantly, it is concentrated in the pericarp of fruits, while the pulp contains levels approximately 220-fold lower than the peel. Peach fuzz contains large amounts of Pru p 3. It is a small basic protein of 91 amino acids, with a molecular weight of 9,178 kDa. Structurally, Pru p 3 contains a highly-conserved domain consisting of eight cysteine residues which is a distinctive sign of the Prolamin superfamily. They form a network of four disulphide bridges which makes the protein resistant to high temperature and $\mathrm{pH}$ changes. The main structural motif of Pru p 3 consists in a compact C-terminal alpha-helical domain and four helices that are connected by short loops. The four disulfide bridges are responsible for the nsLTPs compact folding which forms a tunnellike hydrophobic cavity that runs through the whole molecule and is able to accommodate ligands such
nsLTPs are the most prevalent plant -food allergens in Southern Europe.

The clinical reactions can be systemic and severe, especially when not associated to birch pollinosis.

Pru p3, the major allergen of peach, plays a precursor role in the sensitization to other nsLTPs

Relevant nsLTPs containing plant-foods belong not only to Rosaceae family but also to the nut group and to cereals such as wheat, maize and rice. 


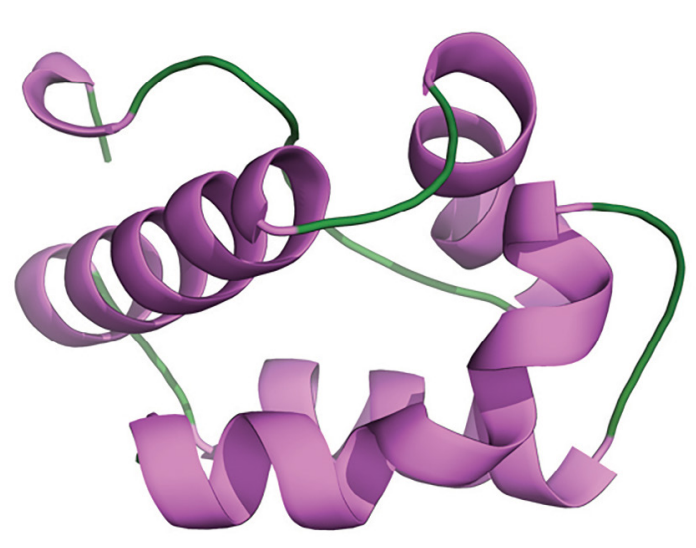

Figure 1

Crystal structure of peach Pru p 3 (PDB 2ALG), the prototypic member of the family of plant non-specific lipid transfer protein pan-allergens. Protein chains are colored. The structure was generated using PyMOL Molecular Graphics System v1.6.

as phospholipids, acyl-coenzyme A and fatty acids (Figure 1). Three IgE-binding epitopes on the LTP molecule have been identified: Pru p 311-20, Pru p 331-40, Pru p 371-80. These peptides are shared with other fruits including apple, apricot, plum, cherry, orange, strawberry, grape, with a sequence identity ranging from 62 to $81 \%$ (3). Pastorello et al. (4) have also identified two immunodominant T-cell reactive regions that have been called Pru p312-27 and Pru p 357-72. These peptides have the ability to induce the production of IL-4 from Pru $p$ 3-specific $T$ cells after allergen-specific stimulation which reflects a Th2-dominated response. The stable tertiary conformation of Pru $\mathrm{p} 3$ provides resistance to thermal degradation. In vitro IgE-antibody binding ability is maintained after $30 \mathrm{~min}$ at $121^{\circ} \mathrm{C}$ and after 160 min at $100^{\circ} \mathrm{C}(5,6)$. Pru $\mathrm{p} 3$ is also resistant to proteolytic digestion. After a proteolytic treatment with trypsin, $35 \%$ of the molecule remains intact and three high molecular weight (HMV) peptides are released in addition to the smaller peptide Pru $p$ 35772. These peptides still show IgE antibody and T-cell binding ability and thus have the potential to either sensitize or induce an allergic reaction (6).
Table 1

Basic protein characteristics of Pru p 3

\begin{tabular}{|ll|}
\hline \multicolumn{2}{|l|}{ Protein characteristics } \\
\hline Allergen source & Prunus persica, peach \\
\hline Protein family & Lipid Transfer Proteins \\
\hline $\begin{array}{l}\text { UniProtKB accession } \\
\text { No. }\end{array}$ & P81402 \\
\hline $\begin{array}{l}\text { Three dimensional } \\
\text { structure available }\end{array}$ & Yes \\
\hline Molecular structure & mainly alpha-helix \\
\hline Molecular weight & 9,178 kDa \\
\hline Length & 91 amino acids \\
\hline Ligand binding & mainly hydrophobic ligands \\
\hline Dimerization & no \\
\hline Glycosylation & no \\
\hline Disulfide bonds & 4 \\
\hline Isoelectric point & 9.25 \\
\hline Distribution & $\begin{array}{l}\text { seeds, fruits, leaves, roots, } \\
\text { pollens }\end{array}$ \\
\hline
\end{tabular}

\section{THE FAMILY}

Pru p 3 belongs to the family of non-specific Lipid Transfer Proteins (nsLTPs), which are the allergens most frequently involved in food induced allergic reactions in the adult population from the Mediterranean area (7). sLTPs belong to the superfamily of Prolamins that includes several families of allergens including the alpha-amylase inhibitors, $2 \mathrm{~S}$ albumins, and the non-specific lipid transfer proteins (nsLTPs). All the members of the Prolamin superfamily share the conserved amino acid pattern of eight cysteine residues. In contrast to the other members of the superfamily, nsLTPs are not restricted to seed tissues, but ubiquitously expressed throughout the plant. The concentration of nsLTPs is variable and depends on maturity, storage conditions, and cultivar of the fruit. nsLTPs have a role in the transport of hydrophobic molecules that comprise the cutin and suberin layers of plant tissues. Because of the suggestion that they could be involved in plant defense against bacterial and fungal infections, the nsLTPs have been classified as type 14 Pathogenesis-related Proteins. To date, the International Union of Immunological Societies 
Table 2

Representative Members of the nsLTP family and cross-reactivity between them

\begin{tabular}{|c|c|c|}
\hline $\begin{array}{l}\text { Botanical } \\
\text { family }\end{array}$ & Allergen source & Allergen \\
\hline \multicolumn{3}{|l|}{ Plant foods } \\
\hline \multirow[t]{3}{*}{ Rosaceae } & Peach (Prunus persica) & Pru p 3 \\
\hline & Apple (Malus domestica) & Mal d 3 \\
\hline & Cherry (Prunus avium) & Pru av 3 \\
\hline Vitaceae & Grape (Vitis vinifera) & Vit $\vee 1$ \\
\hline Rutaceae & Orange (Citrus sinensis) & Cit s 3 \\
\hline Solanaceae & $\begin{array}{l}\text { Tomato (Lycopersicon } \\
\text { esculentum) }\end{array}$ & Lyc e 3 \\
\hline Corylaceae & Hazelnut (Corylus avellana) & Cor a 8 \\
\hline Juglandaceae & Walnut (Juglans regia) & Jug r 3 \\
\hline Fabaceae & Peanut (Arachis hypogaea) & Ara h 9 \\
\hline Asteraceae & Lettuce (Lactuca sativa) & Lec s 1 \\
\hline \multirow[t]{2}{*}{ Poaceae } & Maize (Zea mays) & Zea m 14 \\
\hline & Wheat (Triticum aestivum) & Tri a 14 \\
\hline \multicolumn{3}{|c|}{ Occupational allergens } \\
\hline Euphorbiaceae & $\begin{array}{l}\text { Natural Rubber Latex } \\
\text { (Hevea brasiliensis) }\end{array}$ & Heb b 12 \\
\hline Asparagaceae & $\begin{array}{l}\text { Asparagus (Asparagus } \\
\text { officinalis) }\end{array}$ & Aspa o 1 \\
\hline \multicolumn{3}{|l|}{ Pollens } \\
\hline Urticaceae & $\begin{array}{l}\text { Parietaria (Parietaria } \\
\text { Judaica) }\end{array}$ & Par j 1 \\
\hline \multirow[t]{2}{*}{ Asteraceae } & $\begin{array}{l}\text { Ragweed (Ambrosia } \\
\text { artemisiifolia) }\end{array}$ & Amb a 6 \\
\hline & $\begin{array}{l}\text { Mugwort (Artemisia } \\
\text { vulgaris) }\end{array}$ & Art $\vee 3$ \\
\hline Oleaceae & Olive (Olea europoea) & Ole e 7 \\
\hline Platanaceae & Plane (Platanus acerifolia) & Pla a 3 \\
\hline
\end{tabular}

Allergen Nomenclature Sub-committee lists 41 allergenic nsLTPs that originate from fruits, pollen of trees and weeds, vegetables, nuts and seeds, and natural rubber latex. The most prominent members of the NsLTPs family are presented in Table 2. LTPs are the most important allergens of the Prunoideae subfamily which includes peach, apricot, plum and cherry (8).
A high degree of IgE anti-nsLPT cross-reactivity has been observed within the Rosaceae family and with citrus fruits, grape, tomato, vegetables (asparagus, lettuce, etc.), nuts (hazelnut, walnut, peanut, etc.), maize, onion, carrot, rice spelt (partial cross-reactivity) (3). The Arg39/Thr40 epitope is well conserved in Rosaceae nsLTPs and only partly conserved in cereal nsLTPs. In addition, relevant allergens from Parietaria, Artemisia, Platanus and Olea pollen are also members of the nsLTP family, but show rather low (Artemisia and Platanus) or absent (Parietaria and Olea) crossreactivity with Pru p 3 as a consequence of the lower sequence identity ( $<35 \%)$, and different lengths (5). In conclusion, conserved as well as divergent residues exist between the Rosaceae and other nsLTPs, but the sequence identity between i.e. Pru p 3 and Art v 3 (9) or Ara h 9 (10) is somehow limited.

\section{CLINICAL RELEVANCE}

Clinically relevant nsLTPs have been described both in foods and pollens. The current prevalence of nsLTPs, sensitization in Europe is unknown; however, in an epidemiological survey performed in Spain (11, 12) on pollen allergic patients, Pru p 3 sensitization affected $11 \%$ of the adults and $22 \%$ of the children tested. These data indicate that approximately $2 \%$ of adults and $4 \%$ of children manifest a positive slgE to Pru p 3, assuming a $20 \%$ prevalence of pollen allergy. Approximately $50 \%$ of the patients that were sensitized to Pru $\mathrm{p} 3$ were referred with a complaint of a possible food allergy. Considering the patients that are not pollen allergic and exclusively LTP-sensitized, the LTP-syndrome represents the most frequent type of food allergy in adults and adolescents in southern Mediterranean countries. In contrast, this nsLTP driven syndrome shows a low prevalence in Central and Northern Europe.

From a clinical point of view, the LTP-syndrome presents some peculiar aspects, which need to be appreciated when planning the appropriate management of nsLTP allergic patients.

\section{Clinical Reactivity}

LTP-induced allergic reactions can be mild but more often they are systemic and severe, as observed by several European investigators during the last decade. Different studies described the strong association between Pru p 3 positivity and the severity of systemic allergic symptoms (13-15). 


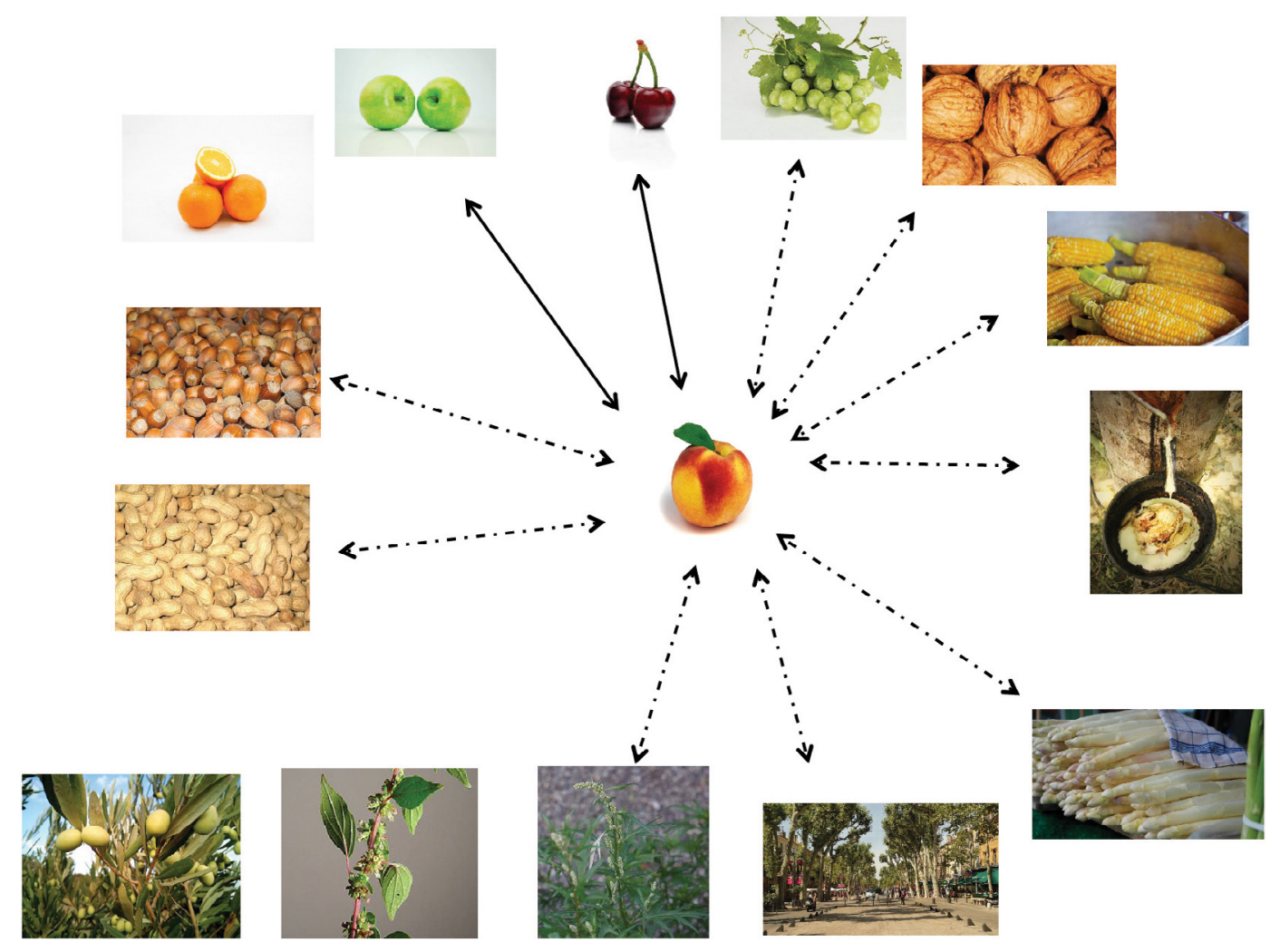

\section{Figure 2}

Cross-reactivity due to nsLTP molecules between different allergenic sources: continous lines indicate a high degree of cross-reactivity amon the Rosaceae family. Dashed lines indicate partial cross-reactivity.

A

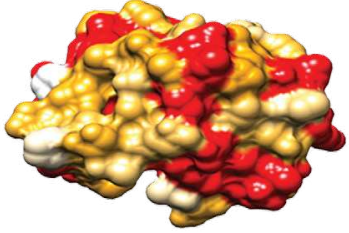

B

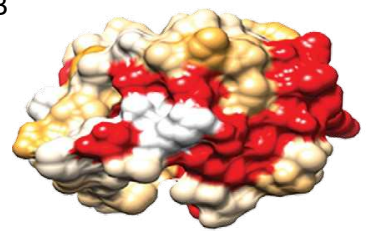

C

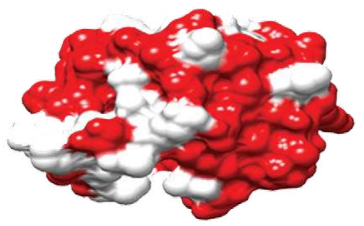

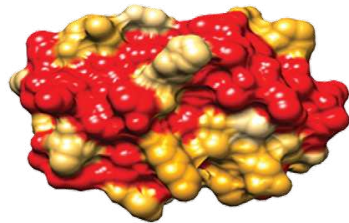
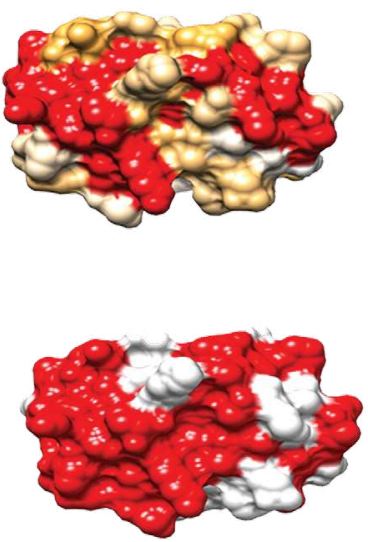

\section{Figure 3}

Homology between members of the nsLTP family. A) Surface residues on Pru p 3 conserved among 8 Rosaceae fruit nsLTP proteins. Red: Residues conserved in $90 \%$ or more proteins. Orange: Residues conserved in $40-90 \%$ of the proteins (gradient); White: Residues conserves in less than $40 \%$ of the proteins B) Surface residues conserved among Pru p 3 and 4 isoforms of Art $v$ 3. Red: Residues in Pru p 3 conserved in $90 \%$ or more of Art $\vee 3$ allergen isoforms. Orange: Residues conserved in 40-90\% of Art $\vee 3$ allergen isoforms (gradient); White: Residues conserved in less than $40 \%$ of Art $\vee 3$ allergen isoforms; C) Surface residues conerved among Pru p 3 and Ara h 9. Red: Residues in Pru p 3 conerved among Pru p 3 and Ara h 9. White: non-conserved residues. 
Interestingly, Pru p 3 (and other nsLTPs) can induce symptoms that range from an Oral Allergy Syndrome (OAS), with oral pruritus, edema, vesicles and erythema localized in the oro-pharyngeal cavity (16), to anaphylaxis, with gastrointestinal involvement, nausea, vomiting and diarrhea, generalized urticaria, as well as contact urticaria, and severe asthma. In the majority of the cases, systemic symptoms are preceded by OAS. Isolated systemic symptoms are very rare $(17,18)$.

\section{Geographical differences}

Another peculiar aspect of the LTP-syndrome is the clear-cut geographical difference in sensitization. The severe LTP-induced clinical manifestations seen in Mediterranean populations are usually in stark contrast to the mild clinical manifestations of the oral allergy syndrome that are associated to Birch pollen allergy which is caused by Bet $\mathrm{v} 1$ and Bet $\mathrm{v}$ 2 sensitization in Central and Northern Europe. As regards the peach model, the concept of the interdependence of the severity of the symptoms from Pru p 3 sensitization, especially in areas that are not exposed to Fagales, is now well accepted, as compared to mild Pru p 3 symptoms in subjects that are pollen-sensitized. These findings have also been confirmed by other investigators in the apple model (19).

In a European collaborative study on apple allergy, Mal d 3 sensitization was significantly more frequent in Spain, an almost birch-free area, as compared to birch-rich countries/regions such as The Netherlands, Austria and Northern Italy. These correlations suggest that the birch pollen exposure, typical of Central and Northern European populations, may confer a sort of "immune protection" to LTP sensitization.

\section{Pediatric patients}

Children sensitized to Pru p 3 present clinical symptoms earlier than those sensitized to the other pollen related allergens (20). In a clinical study carried out in Italian adults, 26 out of 48 subjects uniquely sensitized to Pru p 3 presented peach-related allergy symptoms between 2-15 years, earlier than subjects sensitized only to Pru 1 and/or Pru p 4. Furthermore, in the same patients, Pru p 3-sensitization correlated with the development of allergic reactions to a higher number of plant-foods than Pru p 1 and 4 sensitization alone.

\section{Role of Pru p 3}

Peach is the most frequent cause of nsLTP allergy, and Pru $\mathrm{p} 3$ sensitization seems to play a precursor role in the sensitization to other nsLTPs. The most frequently involved plant-foods are the fruits belonging to the Rosaceae family, which include apple, plum, apricot, cherry and pear. However, there are also botanically unrelated nsLTP-containing plant foods that appear to be strongly associated with peach, particularly in the nut group. In fact, walnut, hazelnut and peanut have been described as foods eliciting not only OAS but also severe systemic reactions in LTP-sensitized subjects. Also, cereals such as wheat, maize and rice can cause systemic reactions of various degrees of severity, as confirmed by DBPCFC in LTP-allergic patients. In particular, wheat has been recently described as the cause of exercise-related anaphylaxis in three young LTP-sensitized patients. nsLTP allergens have been identified also in green bean, fennel, orange, kiwi and lentil using sera of allergic patients who have been sensitized to peach nsLTP. A large number of the aforementioned studies have demonstrated that Pru p 3 sensitization dominates the immune response to nsLTPs in other foods containing structurally similar nsLTPs and that peach is almost always the food that initiates the nsLTP allergy syndrome. Importantly, however, there are cases in which Pru p 3 crossreactive slgE is present in individuals who experience no symptoms despite being positive for slgE to nsLTP in other foods. In these individuals, the IgE antinsLTP appears to have no clinical significance and should be considered as a risk factor for developing food allergies.

\section{nsLTPs and respiratory allergens}

As shown in table 2, nsLTPs have been identified as major and minor allergens in different tree and weed pollens. Interestingly, sensitization to inhaled pollen nsLTPs is associated with more severe clinical phenotypes. Pellitory pollen is the only pollen whose major allergen is an nsLTP. Accepted threshold pollen levels for sensitization are low and clinically, pellitory pollinosis is often linked to asthma. The presence of IgE antibody specific for Ole e 7, the nsLTP from Olive pollen, has been associated with a severe allergic olive pollen phenotype, with increased risk of asthma and concomitant-reactions during immunotherapy. In areas of heavy olive pollen exposure, this allergen can sensitize up to $50 \%$ of the pollen allergic population (11). Art $\vee 3$ and Pla a 3, are minor pollen 
allergens that display partial cross-reactivity with Pru p 3 (Figure 2). As a consequence, slgE to mugwort or Plane tree should always be evaluated for potential cross-reactivity with Pru $\mathrm{p} 3$ and other pollen nsLTPs. In Areas with high Plane tree (as Barcelona) or mugwort (as Gran Canaria) exposure, sensitization to these pollen nsLTPs has been associated with a more complex recognition pattern in nsLTP food allergic patients (21).

Importantly, nsLTP from fruits and vegetables have been reported to induce respiratory symptoms. Pru p 3 monosensitizes up to $10 \%$ of patients with respiratory allergy in areas with extensive orchard tree cultivation (12). It is present in high concentration in peach tree leaves and has been shown to induce respiratory allergy symptoms in sensitized patients. Asparagus nsLTP has been described as an occupational allergen that is able to induce respiratory symptoms (22).

\section{CLINICAL MANAGEMENT}

\section{Identification of clinically relevant allergens}

The clinical history should provide evidence for those plant-foods that are most frequently involved in the typical allergic reactions related to the nsLTP syndrome, including peach, apple and other Rosaceae fruits. Other foods that frequently are linked to nsLTP induced allergy symptoms include walnut, hazelnut, peanut, maize, wheat, rice, or other seeds. However consideration should be given to the fact that nsLTPs are present in all vegetable tissues and they all might theoretically induce vegetable-mediated allergic symptoms.

\section{Role of Cofactors}

A second critical point in the diagnosis associates with cofactors such as exercise, alcohol intake, antiacids and NSAIDs that can promote severe reactions. Thus, their potential involvement should always be considered in nsLTP-hypersensitive subjects. Furthermore, severe nsLTP-associated clinical reactions can also frequently occur in patients who are not pollen allergic.

\section{CRD Diagnosis}

Positive skin tests and/or extract-specific lgE that confirm plant-food sensitization should be further investigated by CRD. Skin prick tests with the commercial extracts, and a prick-to-prick with fresh vegetable foods should be performed according to European guidelines. A prick-to-prick test with fresh fruits and vegetables has proven to be more diagnostically sensitive in confirming a history of food allergy to plant-foods than a SPT using commercial food extracts (23). In order to test for LTP sensitization, LTPs containing allergenic commercial extracts of apple and peach are now commercially available. They can be useful for the identification of large numbers of LTP-sensitized patients. Pru p 3 containing reagents in particular have proven vital in the diagnosis of the nsLTP syndrome. However, Pru p 3 cannot be considered a general marker of nsLTP induced clinical allergy. As indicated above, Pru p 3 has very limited homology with pollen nsLTPs, such as Par j 1 or Ole e 7, and this means the potential for a complete lack of cross-reactivity. Other LTPs such as Tri a 14 that is connected to Baker's asthma, have proven to also be Pru p 3 independent sensitizers (24).

nsLTP-specific IgE antibody is an important marker for true food allergy with a high risk for severe systemic reactions. This is in contrast with the oral allergy syndrome-induced food allergy symptoms that are associated with sensitization to Bet $v 1$ and Bet $v 2$ homologues in birch allergic patients. As such, serological assays have been developed to detect anti-LTP specific IgE antibody (e.g., Thermofisher Scientific/Phadia ImmunoCAP) for use in the definitive diagnosis of plant-foods allergy.

In peach sensitized individuals, the simultaneous presence of both Pru p 3 and Pru p 1-specific IgE antibodies seems to lower the apparent risk of severe food allergic reactions, however similar data for other foods are still lacking. The availability of IgE antibody cut-off values that correlate with the severity of the symptoms could increase the efficacy of diagnosis. Recently (25) a proposed cut-off of $2.69 \mathrm{kUA} / \mathrm{l}$ has been reported for $r$ Pru $\mathrm{p} 3$ specific IgE antibody values that identifies peach-allergic patients at a higher risk for severe symptoms after peach ingestion. Further studies are needed to identify possible thresholds for IgE antibody levels to other relevant food nsLTP allergens.

At present, nine different nsLTPs are available on the multiplex ImmunoSorbent Allergen Chip (Thermofisher Scientific/ Phadia: ISAC). The clinical relevance of IgE antibody test results generated with this panel of allergenic specificities for predicting nsLTP clinical reactivity is still a subject of active 


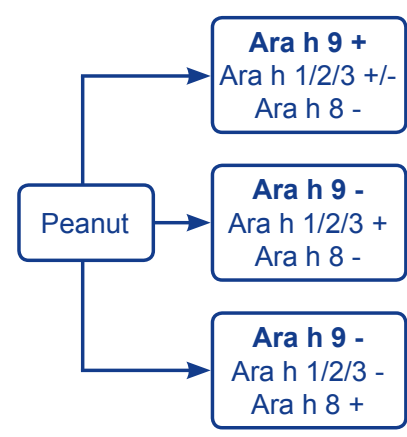

Secondary sensitization- cross -reactivity with birch pollen Local reactions (OAS)

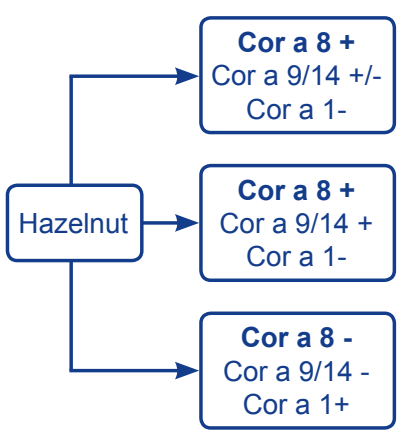

Primary sensitization Risk of severe reactions

Secondary sensitization- cross -reactivity with birch pollen Local reactions (OAS)

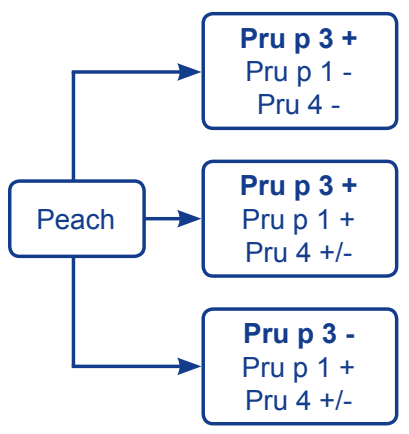

Primary sensitization

Risk of severe reactions

Co-sensitization

Protection agains severe systemic reactions

Secondary sensitization- cross -reactivity with birch pollen Local reactions (OAS)

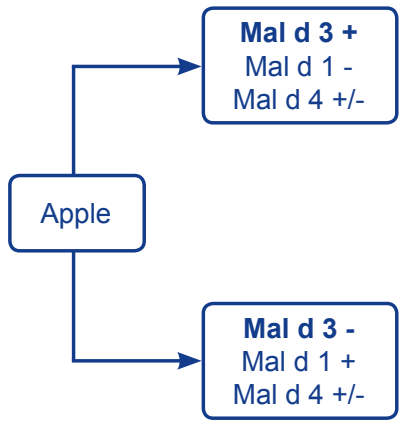

Primary sensitization Risk of severe reactions

Secondary sensitization- cross -reactivity with birch pollen Local reactions (OAS)

\section{Figure 4}

Diagnostic algorithms using component resolved diagnosis in the case of a positive serology or skin test to (a) peanut, (b) peach, (c) hazelnut, or (d) apple extracts (nsLTP allergens are displayed in bold).

research. In contrast to other pan-allergen allergen specificities, it may be necessary to incorporate a broader selection of nsLTP in the test panel to make a correct patient diagnosis. Pru p 3 seems to play a central role in nsLTP-mediated reactions. In vivo and in vitro diagnostic tools for Pru p 3 are commercially available and their inclusion in general patient screening is advisable, especially in geographic areas where the prevalence of nsLTPs sensitization is considerable.

\section{DBPCFCs for LTP-containing plant-foods}

The double-blind placebo-controlled food challenge (DBPCFC) is the gold standard for the diagnosis of food allergy. In routine clinical practice, DBPCFCs for LTP-containing plant-foods should be performed only for nutritionally relevant foods such as wheat, rice and maize. In some cases the challenge test should also be performed after exercise given that LTPs have been described as foods involved in food dependent exercise induced anaphylaxis (FDEIA) (26).

\section{Diagnosis Algorithms}

Figure 3 depicts diagnosis algorithms that can be used to evaluate a potential nsLTP mediated allergy.

Many patients show a progressive clinical recognition of nsLTPs. In some cases, they might be obliged to avoid almost any vegetable from the diet. How the complexity and intensity of the IgE antibody profile to the panel of nsLTPs might predict this evolution is subject of active research.

\section{Treatment}

The possibility of desensitization is being explored (27). In a recent congress communication (28), 90\% of treated patients with a sublingual peach extract vaccine tolerated a whole peach after one year of treatment. In contrast to the published study (27), about $50 \%$ of the patients were included, in spite of having had a previous anaphylactic reaction. This suggests that immunotherapy might be a feasible option for the treatment of severe nsLTP allergy. 
Currently there is only a peach-based sublingual vaccine commercially available in Spain.

\section{CLINICAL CASES}

\section{Case 1 (published (26))}

A 17 year-old male with a 12-years history of peachinduced systemic symptoms (urticaria, angioedema) and sporadic anaphylaxis of unknown origin. Specific IgE antibody levels were strongly positive to rPru $p$ 3 (34.7 kUA/L), and moderately positive to wheat (1.56 kUA/L), maize (4.92 kUA/L) and rice (1.46 $\mathrm{kUA} / \mathrm{L})$. slgE to omega 5 gliadin was negative. IgE immunoblotting was positive for reactivity to nsLTPs in three cereals. Wheat Open Exercise Food Challenge (OEFC) induced an anaphylactic reaction that was treated with Epinephrine. Rice and maize OEFCs were negative. A wheat-free diet allowed the patient to perform agonistic physical activity without any symptoms. He did not need to eliminate rice and maize. Thus, LTP sensitization detected to rice and maize was due to cross-reactivity with Pru $\mathrm{p} 3$. The message is that the challenge is mandatory in order to avoid unnecessary food elimination.

\section{Case 2 (unpublished real case)}

Female, 24 years old, with a recent episode of anaphylaxis (angioedema of lips and eyelids, abdominal pain and diarrhea), which occurred 15 minutes after the ingestion of hazelnut ice cream. She also reported oral allergy syndrome (OAS) and gastrointestinal symptoms (abdominal pain, nausea and vomiting) with most of the Rosaceae fruits that were particularly severe with peach. In addition, she reported a 5-years history of grass and pellitory pollen allergy. Prick-to-prick tests were positive for hazelnut, walnut, maize, peach, apple, plum and cherry. Specific IgE antibody responses were positive to hazelnut (4.61 kUA/I), peach (14.7 kUA/I) and all the Rosaceae fruits and nuts. The levels of IgE antibodies to selected nsLTP components were $10.9 \mathrm{kUA} / \mathrm{L}$ (rPru p 3) and $2.5 \mathrm{kUA} / \mathrm{L}$ (rCor a 8). IgE antibodies were negative to rPru p1, rCor a 1, rCor a 9, rCor a 14, milk, casein and eggs. In this patient, the rCor a 8 sensitization that caused an anaphylactic reaction following hazelnut exposure was shown to be secondary to Pru p 3 sensitization.

\section{Case 3 (published (26))}

Male, 15 year old boy with a history of atopic dermatitis, allergic asthma and OAS to Prunoideae fruits, and frequent episodes of severe anaphylaxis in the previous 2 years after eating any kind of cereal. A rice DBPCFC at rest was positive. The OEFCs with wheat and maize were positive and particularly severe with maize. The patient specific IgE antibody responses were positive to $\mathrm{rPru} p 3$ (6.94 kUA/L), wheat (5.70 kUA/L), rice (8.81 kUA/L) and maize $(9.86 \mathrm{kUA} / \mathrm{L})$ and they were negative for rw 5 gliadin. IgE immunoblotting was positive for nsLTPs present in all three cereals. In this patient, rPru p 3 sensitization was shown to be the cause of the observed sensitization to rice and maize nsLTPs that led to the rice induced anaphylaxis and the exercise-induced anaphylaxis (EIA) to maize. Both were much more severe than the allergic symptoms induced following wheat exposure. Importantly, the clinical effects of the cross-reactivity with Pru $p 3$ are virtually impossible to predict apriori.

\section{TOOLS}

Component resolved diagnosis by SPT is possible using peach extracts that have been highly enriched for Pru p 3 and have very low content of other allergens. As most of the patients with nsLTP mediated food allergic reactions will be positive to Pru p 3, this extract should be used in a general screening for respiratory as well as food-induced allergic disease.

Complex nsLTP syndrome patients will eventually react to multiple members of the nsLTP family. The ISAC-112 contains 9 different nsLTPs. In addition to the presence of Pru p 3, there are three nuts nsLTPs (Ara h 9, Cora a 8, Jug r 3), one cereal flour nsLTP (Tri a 14) and four pollen nsLTPs (Art v 3, Ole e 7, Pla a 3 and Par j2).

The slgE responses to the above mentioned nsLTP panel may reflect reactivity to a broader number of the nsLTP family recognition. IgE anti-nsLTP reactivity may be related to the severity of the LTP syndrome and the probability of future side reactions to new fruits and vegetables. However, to date, there is no validated approach or threshold limit values for slgE anti-nsLTP levels. The individual allergens are also available in the singleplex ImmunoCAP. 


\section{References}

1. Pastorello EA, Farioli L, Pravettoni V, Ortolani C, Ispano M, Monza M, et al. The major allergen of peach (Prunus persica) is a lipid transfer protein. J Allergy Clin Immunol 1999;103:520-526.

2. Sánchez-Monge $R$, Lombardero $M$, García-Sellés FJ, Barber D, Salcedo G. Lipid-transfer proteins are relevant allergens in fruit allergy. J Allergy Clin Immunol 1999;103:514-519.

3. Salcedo G, Sànchez-Monge R, Barber D, Dìaz-Perales A. Plant non-specific lipid transfer proteins: an interface between plant defence and human allergy. Biochim Biophys Acta 2007;1771:781-791.

4. Pastorello EA, Monza M, Pravettoni V, Longhi R, Bonara P, Scibilia J, et al. Characterization of the T-cell epitopes of the major peach allergen Pru p 3. Int Arch Allergy Immunol 2010;153:1-12.

5. Salcedo G, Sanchez-Monge R, Diaz-Perales A, GarciaCasado G, Barber D. Plant non-specific lipid transfer proteins as food and pollen allergens. Clin Exp Allergy 2004;34:1336-1341.

6. Cavatorta V, Sforza S, Aquino G, Galaverna G, Dossena A, Pastorello EA, et al. In vitro gastrointestinal digestion of the major peach allergen Pru p 3, a lipid transfer protein: molecular characterization of the products and assessment of their IgE binding abilities. Mol Nutr Food Res 2010;54:1452-1457.

7. Ortolani C, Ispano M, Pastorello E, Bigi A, Ansaloni R. The oral allergy syndrome. Ann allergy 1988;61:47-52.

8. Breiteneder $\mathrm{H}$, Ebner $\mathrm{C}$. Molecular and biochemical classification of plant-derived food allergens. J Allergy Clin Immunol 2000;106:27-36.

9. Gadermaier G, Harrer A, Girbl T, Palazzo P, Himly $M$, Vogel $L$, et al. Isoform identification and characterization of Art v 3, the lipid-transfer protein of mugwort pollen. Mol Immunol 2009;46:1919-1924.

10. Lauer I, Dueringer N, Pokoj S, Rehm S, Zoccatelli G, Reese $G$, et al. The non-specific lipid transfer protein, Ara $\mathrm{h} 9$, is an important allergen in peanut. Clin Exp Allergy 2009;39:1427-1437.

11. Barber D, de la Torre F, Feo F, Florido F, Guardia P, Moreno $C$, et al. Understanding patient sensitization profiles in complex pollen areas: a molecular epidemiological study. Allergy 2008;63:1550-1558.

12. Barber D, de la Torre F, Lombardero M, Antepara I, Colas C, Davila I, et al. Component-resolved diagnosis of pollen allergy based on skin testing with profilin, polcalcin and lipid transfer protein pan-allergens. Clin Exp Allergy 2009;39:1764-1773.

13. Fernandez-Rivas M, Bolhaar S, Gonzalez-Mancebo E, Asero $\mathrm{R}$, van Leeuwen A, Bohle B, et al. Apple allergy across Europe: how allergen sensitization profiles determine the clinical expression of allergies to plant foods. J Allergy Clin Immunol 2006;118:481-488.
14. Gamboa PM, Cáceres O, Antepara I, SánchezMonge R, Ahrazem O, Salcedo G, et al. Two different profiles of peach allergy in the north of Spain. Allergy 2007;62:408-414.

15. Asero R, Antonicelli L, Arena A, Bommarito L, Caruso B, Colombo $\mathrm{G}$, et al. Causes of food-induced anaphylaxis in Italian adults: a multi-centre study. Int Arch Allergy Immunol 2009;150:271-277.

16. Pastorello EA, Ortolani C, Farioli L, Pravettoni V, Ispano M, Borga A, et al. Allergenic cross-reactivity among peach, apricot, plum, and cherry in patients with oral allergy syndrome: an in vivo and in vitro study. J Allergy Clin Immunol 1994;94:699-707.

17. González-Mancebo E, Fernández-Rivas M. Outcome and safety of double-blind, placebo-controlled food challenges in 111 patients sensitized to lipid transfer proteins. J Allergy Clin Immunol 2008;121:1507-1508.

18. Asero R. Peach-induced contact urticaria is associated with lipid transfer protein sensitization. Int Arch Allergy Immunol 2011;154:345-348.

19. Gomez F, Aranda A, Campo P, Diaz-Perales A, BlancaLopez N, Perkins J, et al. High prevalence of lipid transfer protein sensitization in apple allergic patients with systemic symptoms. PLoS One 2014;9:e107304.

20. Pastorello EA, Farioli L, Stafylaraki C, Mascheri A, Scibilia J, Pravettoni V, et al. Anti-rPru p 3 IgE levels are inversely related to the age at onset of peachinduced severe symptoms reported by peach-allergic adults. Int Arch Allergy Immunol 2013;162:45-49.

21. Palacin A, Gomez-Casado C, Rivas LA, Aguirre J, Tordesillas L, Bartra J, et al. Graph based study of allergen cross-reactivity of plant lipid transfer proteins (LTPS) using microarray in a multicenter study. PLoS One 2012;7:e50799.

22. Diaz-Perales A, Tabar Al, Sànchez-Monge R, Garcia BE, Gomez B, Barber D, et al. Characterization of asparagus allergens: a relevant role of lipid transfer proteins. J Allergy Clin Immunol 2002;110:790-796.

23. Ortolani C, Ispano M, Pastorello EA, Ansaloni R, Magri GC. Comparison of results of skin prick tests (with fresh foods and commercial food extracts) and RAST in 100 patients with oral allergy syndrome. J Allergy Clin Immunol 1989;83:683-690.

24. Tordesillas L, Pacios LF, Palacin A, Quirce S, Armentia A, Barber D, et al. Molecular basis of allergen crossreactivity: non-specific lipid transfer proteins from wheat flour and peach fruit as models. Mol Immunol 2009;47:534-540.

25. Pastorello EA, Farioli L, Pravettoni V, Scibilia J, Mascheri A, Borgonovo L, et al. Pru p 3-sensitised Italian peachallergic patients are less likely to develop severe symptoms when also presenting IgE antibodies to Pru p 1 and Pru p 4. Int Arch Allergy immunol 2011;156:362372. 
26. Pastorello EA, Farioli L, Stafylaraki C, Scibilia J, Mirone $C$, Pravettoni $\mathrm{V}$, et al. Wheat-dependent exerciseinduced anaphylaxis caused by a lipid transfer protein and not by $\omega-5$ gliadin. Ann Allergy Asthma Immunol 2014;112:386-387.

27. Fernández-Rivas $M$, Garrido Fernández $S$, Nadal JA, Díaz de Durana MD, García BE, González-Mancebo $\mathrm{E}$, et al. Randomized double-blind, placebo-controlled trial of sublingual immunotherapy with a Pru $\mathrm{p} 3$ quantified peach extract. Allergy 2009;64:876-883.

28. Bagos G, Gómez F, Sala M, Campo P, Galindo L, Torres $M J$, et al. Evaluation of peach tolerance after one year of sublingual immunotherapy with LTP (Pru p 3) in allergic patients sensitised to food by LTPs. FAAM 2014, Dublin OP-14. 


\section{PART C}

\section{CROSS-REACTIVE}

MOLECULES \& THEIR CLINICAL RELEVANCE

\section{SERUM ALBUMINS}

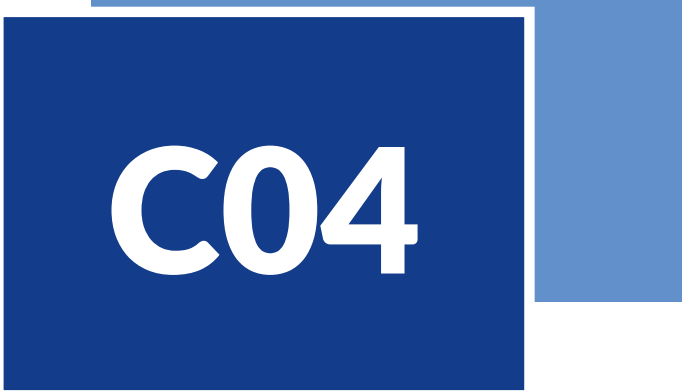

\section{Christiane Hilger}

\section{THE PROTEIN}

Bos d 6 - Bovine serum albumin (BSA), is a well characterized protein (Table 1) (1). It is synthesized in the liver and constitutes a major component of plasma. It is widely used in biochemical and immunological assays, as cell culture ingredient and in vaccines.

The protein architecture of Bos d 6 was resolved in 2012 (1) and revealed an a-helical structure, composed of three structurally similar domains arranged into a heart-shaped from Fig. 1. The molecule is stabilized by seventeen disulfide bonds. Bos $\mathrm{d} 6$ is denatured by heating above $50^{\circ} \mathrm{C}$. Helices are partially disrupted and heat-induced aggregation takes place at $60^{\circ} \mathrm{C}$.

Bos $\mathrm{d} 6$ is a respiratory and food allergen as it is present in bovine dander, in milk and meat. It is classified as minor allergen in animal dander, but it is an important meat and milk allergen in the case of uncooked food ingestion. In fact Bos $d 6$ is a thermolabile protein, and well done meat or boiled milk are safe for allergic patients. There is currently no assay available for quantification of Bos $d 6$ in the environment.

Minor respiratory allergen of animal dander

Highly conserved sequences with strong amino acid identity

Allergen implicated in pork-cat and birdegg syndrome

Meat and milk allergen

May elicit severe symptoms upon ingestion of not cooked or boiled food 


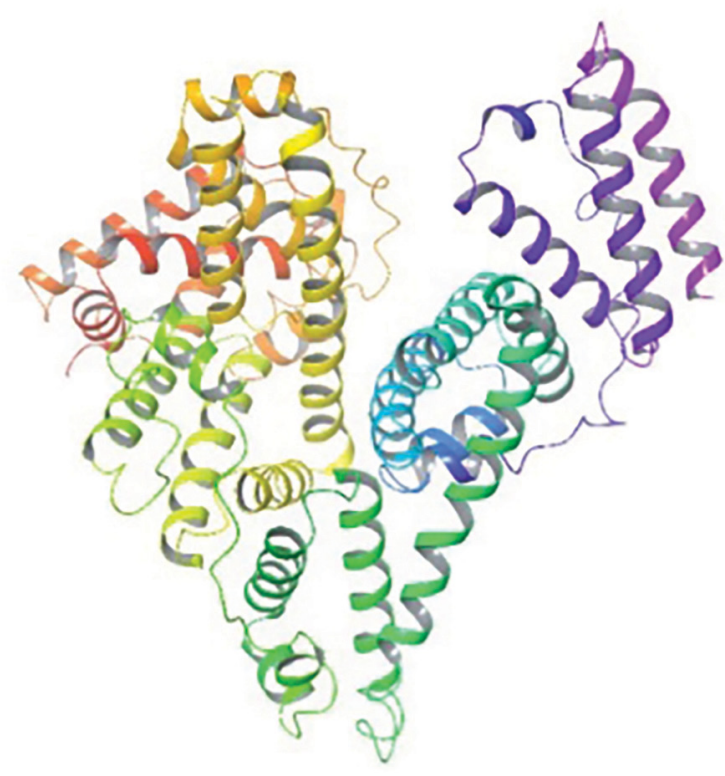

Figure 1

Ribbon model of the three-dimensional structure of Bos d 6 (3V03). N-terminal end in red, C-terminal end in purple.

\section{THE FAMILY}

Serum albumins are large globular proteins synthesized in the liver (2). They are abundant in plasma and contribute to the regulation of colloid osmotic pressure. Serum albumins transport a multitude of metabolites, nutrients, drugs and other molecules. They have an $\alpha$-helical structure with three domains stabilized by several disulfide bridges. Serum albumins change their conformation in order to bind diverse ligands. They are present in dander, saliva, milk and meat. They are thermolabile and are easily denatured in food by cooking. Chicken serum albumin, formerly $\alpha$-livetin, is an allergen of egg yolk. Seven allergens are officially recognized by the IUIS Allergen Nomenclature Subcommittee: Bos d 6 (bovine), Can f 3 (dog), Cav p 4 (guinea-pig), Equ c 3 (horse), Fel d 2 (cat), Sus s 1 (pig) and Gal d 5 (chicken) (Table 2). However, a number of serum albumins from different animals have been shown to bind IgE and to be cross-reactive: sheep, goat, rabbit, hamster and other mammals (3).

Serum albumins have a molecular weight of 65-69 $\mathrm{kDa}$. They are composed of 607-608 amino acids
Table 1

Basic protein characteristics of Bos d 6

\begin{tabular}{ll|}
\hline Protein characteristics \\
Allergen source & $\begin{array}{l}\text { Bos domesticus, domestic } \\
\text { cattle }\end{array}$ \\
\hline Protein family & Serum albumin \\
\hline $\begin{array}{l}\text { UniProtKB accession } \\
\text { No }\end{array}$ & P02769 \\
\hline $\begin{array}{l}\text { Three dimensional } \\
\text { structure available }\end{array}$ & Yes \\
\hline Molecular structure & mainly helical \\
\hline $\begin{array}{l}\text { Theoretical molecular } \\
\text { weight }\end{array}$ & 66.56 kDa \\
\hline Length & $\begin{array}{l}607 \text { amino acids; mature } \\
\text { protein: } 583 \text { amino acids }\end{array}$ \\
\hline Ligand binding & Yes \\
\hline Dimerization & No \\
\hline Glycosylation & No \\
\hline Disulfide bonds & 17 \\
\hline Isoelectric point & 5.6 \\
\hline Synthesis & liver, secreted \\
\hline Distribution & $\begin{array}{l}\text { Plasma, dander, saliva, milk, } \\
\text { meat, }\end{array}$ \\
\hline &
\end{tabular}

and during maturation, the signal peptide and a pro-peptide of 18 and 4 amino acids, respectively are cleaved off. Mammalian serum albumins have highly conserved amino acid sequences (2) and show sequence identities of $72-82 \%$ to human serum albumin (HSA) (Table 3). Avian serum albumins display lower identities (46-49\%) to HSA and to other mammalian serum albumins (42-48\%).

Table 3 displays two-by-two comparisons of amino acid identities between HSA and different serum albumins. The identity between HSA and mammalian serum albumins is very high. It was generally assumed that proteins with a sequence identity above $62 \%$ were rarely allergenic (4). Serum albumins constitute a remarkable exception to this rule. Other important animal allergen families like tropomyosins, $\beta$-parvalbumins and caseins lie below this threshold. IgE-cross-reactivity between mammalian serum albumins has been well documented (3). All pairs with a high sequence identity (>70\%) are potentially cross- 


\section{Textbox 1}

\section{Albumin Family Characteristics}

- Common tertiary $\alpha$-helical structure

- Highly conserved sequences with strong amino acid identity

- Large secreted molecules of 65 - 69 kDa

- Thermolabile

- Present in dander, secretions and meat

Table 2

Bos d 6-homologous allergens from animals

\begin{tabular}{|lll|}
\hline Animal family & Allergen source & Allergen \\
\hline Bovidae & $\begin{array}{l}\text { Domestic cattle (Bos } \\
\text { domesticus) }\end{array}$ & Bos d 6 \\
\hline Cavidae & Dog (Canis familiaris) & Can f 3 \\
\hline Equidae & $\begin{array}{l}\text { Guinea-pig (Cavia porcellus) } \\
\text { caballus) }\end{array}$ & Cav p 4 \\
\hline Felidae & Cat (Felis domesticus) & Fel d 2 \\
\hline Phasianidae & Chicken (Gallus domesticus) & Gal d 5 \\
\hline Suidae & Domestic pig (Sus scrofa) & Sus s 1 \\
\hline
\end{tabular}

reactive. It has been postulated that below $50 \%$, cross-reactivity is rare (5). Cross-reactivity between mammalian and the less conserved avian serum albumins seems actually to be rare, but has been documented in single case reports (6). Molecules displaying a low level of overall sequence identity may nevertheless share single epitopes composed of short stretches of sequence identity leading to patient-dependent IgE-cross-reactivity.

A comparison of the surfaces of some important allergenic mammalian serum albumins visualizes potential cross-reactive B cell epitopes (Fig. 2). Band T-cells recognizing HSA epitopes will be deleted during the immunological education process.

\section{CLINICAL RELEVANCE}

Serum albumins are respiratory allergens present in animal dander and fluids such as milk, serum, urine and saliva. They are considered minor allergens (7). Depending on the study population, about 14$50 \%$ of cat and dog-allergic patients present IgE to cat or dog albumins. Monosensitization to cat or dog serum albumin seems to be extremely rare in a primary sensibilisation. IgE to Fel d 2 would usually be detected along with IgE directed to a major allergen e.g. Fel $d$ 1. On the contrary, the presence of IgE to Can $f 3$ without any detectable IgE to other dog allergens (Can $f$ 1, Can $f$ 2) would be a marker of

\section{Table 3}

Amino acid identities (\%) between allergenic serum albumins and HSA (2)

\begin{tabular}{|c|c|c|c|c|c|c|c|}
\hline Bos d 6 & & & & & & & \\
\hline Can $\mathrm{f} 3$ & 76 & & & & & & \\
\hline Cavp 4 & 70 & 73 & & & & & \\
\hline Equ c 3 & 74 & 76 & 72 & & & & \\
\hline Fel d 2 & 78 & 87 & 76 & 78 & & & \\
\hline Gal d 5 & 43 & 46 & 43 & 43 & 45 & & \\
\hline HSA & 76 & 80 & 72 & 76 & 82 & 46 & \\
\hline Sus s 1 & 79 & 78 & 72 & 76 & 79 & 42 & 75 \\
\hline
\end{tabular}

Dark blue shaded areas indicate sequence identities $>80 \%$, light shaded areas display identities between 70 and $80 \%$. Figures in bold blue indicate documented lgE-cross-reactivity between albumins. HSA, human serum albumin. 

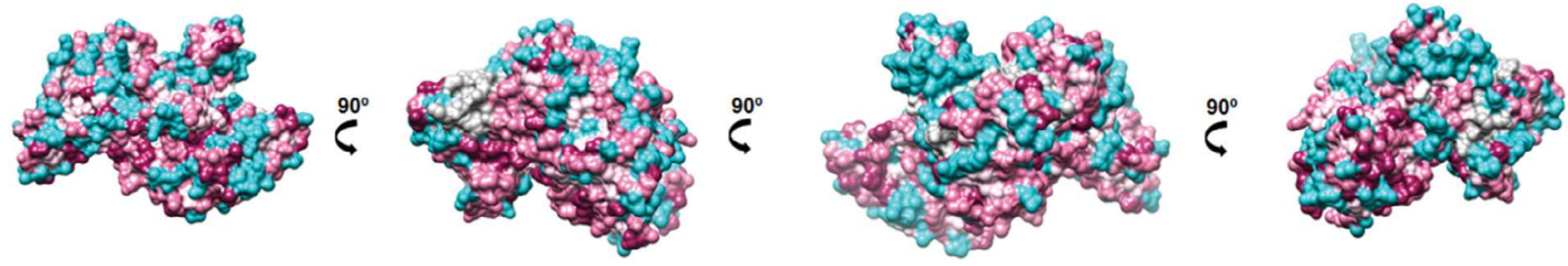

\section{Figure 2}

Surface representation of serum albumin comparisons. Comparison of the cross-reactive serum albumins from domestic cattle (Bos d 6), cat (Fel d 2), and pig (Sus s 1). Surface representation of Bos d 6 (3V03) colored by sequence conservation with Fel d 2 and Sus s 1, shown in 4 side views. Sequence conservation was calculated by Consurf (18). The highly variable sequence conservation was identified as deep blue, the average was in white and the conserved sequences are denoted in deep red (Figure provided by $\mathrm{K}$ Arumugam, Luxembourg Institute of Health).

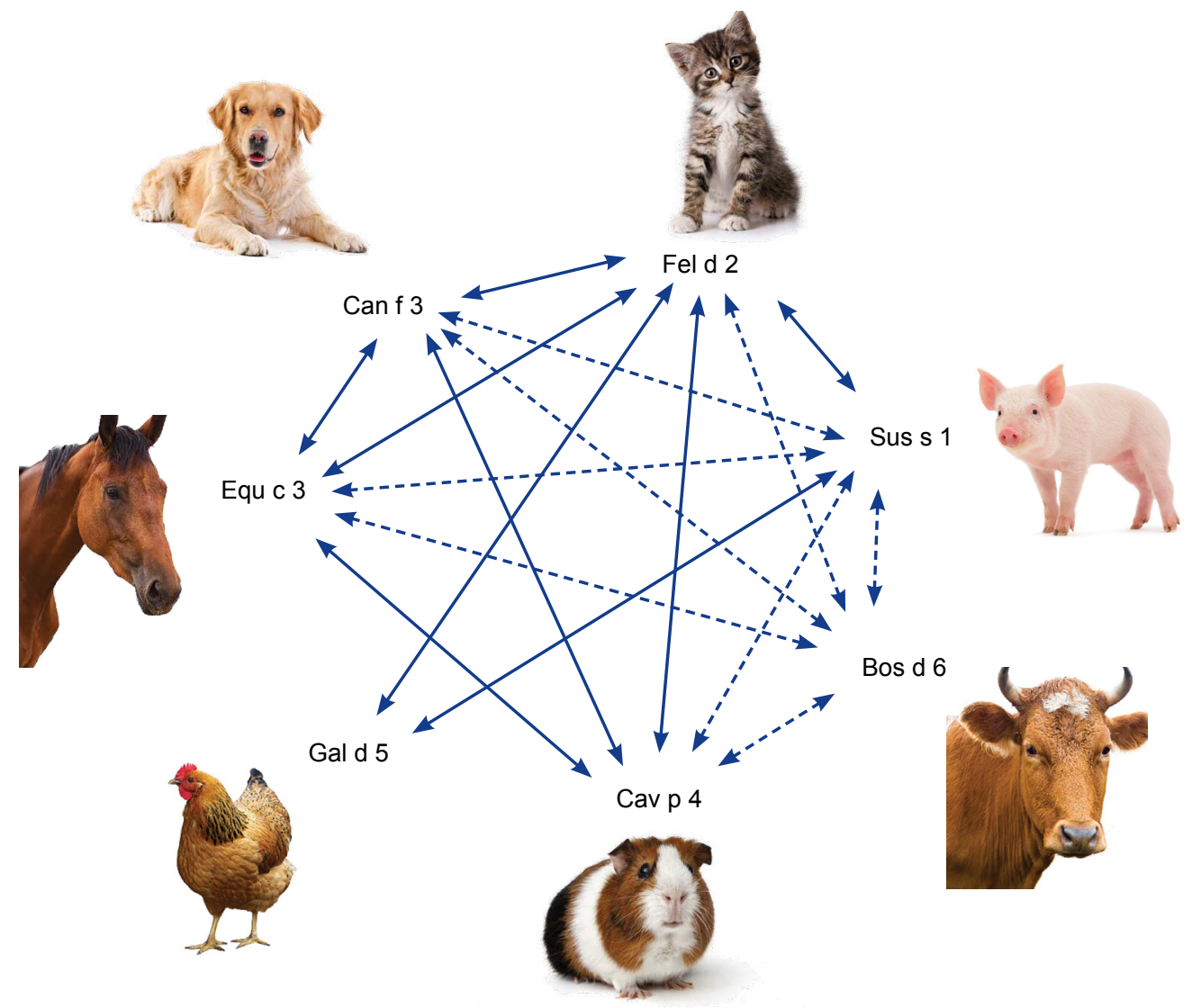

Figure 3

Cross-reactivities among allergenic serum albumins. All mammalian serum albumins are potentially IgE-cross-reactive. Clinical cross-reactivity between mammalian serum albumins of cat and pork and Gal d 5 are rare and have been documented only from mammal to bird. Solid lines represent documented IgE-cross-reactivity, dashed lines hypothetical cross-reactivity. 
cross-reactivity and the primary sensitization source should be investigated. The clinical relevance of IgE to serum albumins is difficult to explore as IgE to other animal allergens are always present in the same patient. It is generally assumed that they are of low relevance. However, two cases of occupational asthma triggered by inhalation of Bos $\mathrm{d} 6$ have been reported in a laboratory worker $(8,9)$. High levels of IgE-antibodies to Fel d 2 have been associated with atopic dermatitis in cat-allergic children (10).

In contrast to its role as respiratory allergen, serum albumin in food has been shown to elicit minor, moderate and severe clinical symptoms. Bos d 6 is a component of the milk whey fraction and constitutes about $1 \%$ of the total protein content of milk $(0.1-0.4 \mathrm{~g} /$ I). In a series of 60 children with immediate reactions to milk confirmed by DBPCFC, $61.3 \%$ had allergenspecific IgE-antibodies to Bos d 6 (11). Boiling milk for 10 min eliminated skin prick test responses in subjects reactive to BSA. Cross-reactivity has been described between different mammalian milks. Not only Bos $\mathrm{d}$ 6, but also the major bovine milk allergens caseins and $\beta$-lactoglobulin have homologues in milk from other species. Serum albumins are also an important allergen of meat. A high percentage of milk-allergic children (13-20\%) are also allergic to beef (12). In a series of 28 children diagnosed with beef allergy, 92.9\% were diagnosed allergic to milk by skin prick test and DBPCFC. In children with beef allergy, sensitization to Bos d 6 is a marker of cow's milk allergy (12). Because Bos $d 6$ is a thermolabile allergen, well-done meat is tolerated by most patients.

Albumins are also involved in cross-reactivity between animal dander and milk or meat. A patient allergic to horse developed a systemic reaction upon ingestion of mare's milk (13). The association between allergy to cat dander and pork meat, known as porkcat syndrome was already described more than a decade ago in Europe (14). Among 2 groups of catallergic patients, 14 and $23 \%$ had specific IgE to Fel d 2, 3-10\% had cross-reacting IgE to porcine albumin (15). Among those, one out of three experienced clinical symptoms upon ingestion of pork meat. Thus, statistically 1-3\% of cat-allergic patients could develop pork-cat syndrome. This syndrome has only recently been described in the US and should be differentiated from the delayed type of meat allergy based on IgE to alpha-gal (chapter B14) (16).

Chicken serum albumin (Gal d 5) is an inhalant and food allergen implicated in the bird-egg syndrome

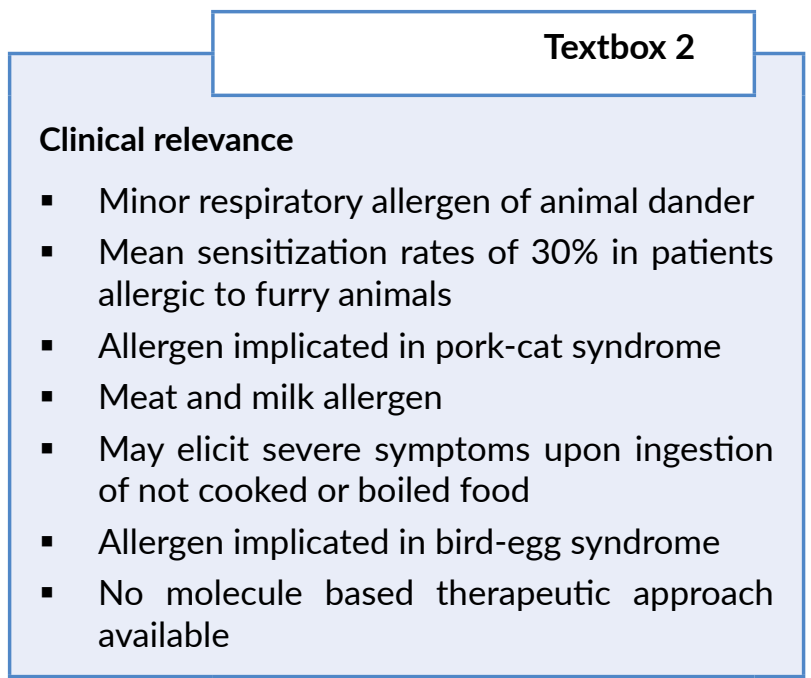

(17). It is present in egg yolk and it was also detected in domestic air samples. Bronchial challenges elicited asthmatic responses in 6 asthmatic patients. IgEreactivity was reduced to $88 \%$ after heating at $90^{\circ} \mathrm{C}$ for 30 min. Chicken serum albumin Gal d 5 does not share any sequence identity with ovalbumin Gal d 2, a storage protein and allergen of egg white.

The wide use of bovine serum albumin in cell culture media holds new risks. Several case reports have shown that Bos d 6, an ingredient of the culture medium of spermatozoids has provoked severe anaphylactic reactions upon artificial insemination (7). Some vaccines contain Bos d 6 but apparently no side effects have been described. Further studies are still needed.

As serum albumins are minor allergens, there is no current research on the development of hypoallergenic molecules. Current immunotherapies available contain animal dander extracts and albumin content may be variable.

\section{CLINICAL MANAGEMENT}

A careful record of the clinical history such as the presence of pets at home or regular pet contact is of great value. Skin prick test or allergen-specific IgE using animal dander will confirm animal sensitization. In order to define the primary sensitization source, specific IgE to major allergens such as Fel $d$ 1, Can $f$ 1, 2 or 5 should be determined. Unfortunately, the number of components is still limited and no allergens are available for small furry pets. IgE-antibodies 


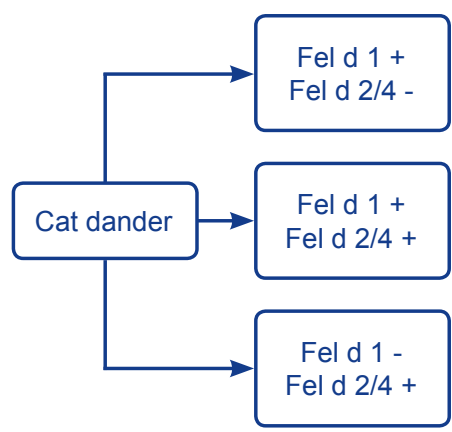

Primary sensitization to cat

Primary sensitization to cat, potentially cross-sensitization or cosensitization to other animal or food

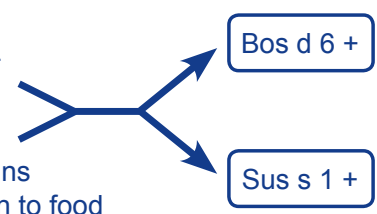

Marker of milk and beef allergy

Cross-sensitization to serum albumins and lipocalins, potential sensitization to food Check for primary source

Potential clinical symptoms upon pork ingestion; pork-cat syndrome

\section{Figure 4}

Added value of the use of single allergens in the case of a positive IgE test to cat dander.

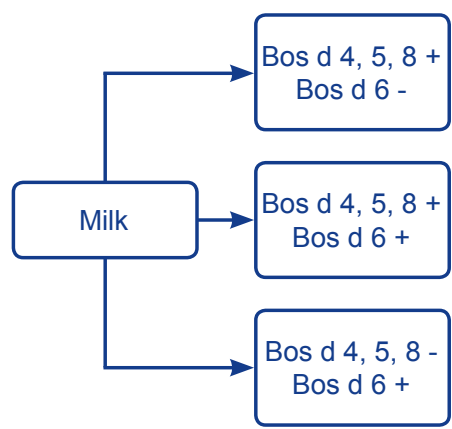

Primary sensitization to milk

Primary sensitization to milk, potentially cross-sensitization to meat or animal dander

Cross-sensitization to serum albumins, potential sensitization to food Check for primary source

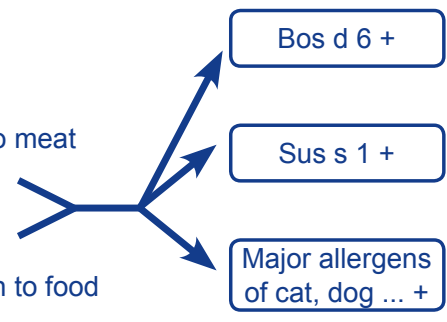

Marker of milk and beef allergy

Potential clinical symptoms upon pork ingestion; pork-cat syndrome

Primary sensitization to animal dander

Figure 5

Added value of the use of single allergens in the case of a positive IgE test to milk.

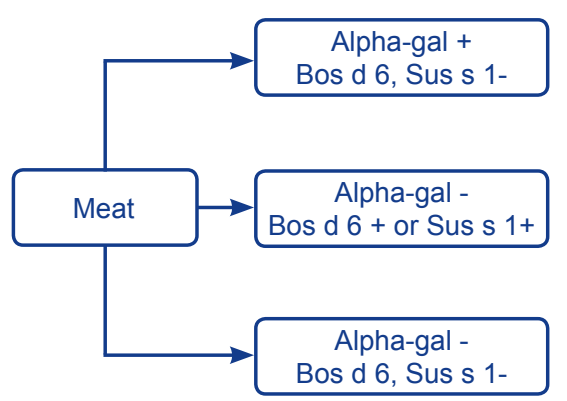

Delayed allergic reaction to meat

Immediate type reaction Sensitization to meat, potentially cross-sensitization to animal dander

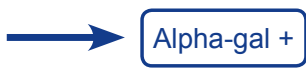

Sensitization to red meat possibly also to gelatin Sensitization to Cetuximab Sensitization to tick bites

Sensitization to other allergens or non-allergic reaction

Additional clinical testing

\section{Figure 6}

Added value of the use of single allergens in the case of a positive IgE test to meat. 
directed to serum albumins are a marker of crossreactivity, but do not mandatorily imply clinical crossreactivity. Patients with IgE to serum albumins should be advised to avoid mammalian pets as they may experience clinical symptoms upon contact with any pet. As mammalian serum albumins are highly crossreactive, the choice of serum albumins included in the determination of allergen-specific IgE should be guided by the clinical history. Specific IgE are mostly positive to Can $\mathrm{f} 3$ and Fel $\mathrm{d} 2$ whereas IgE-reactivity against Bos $d 6$ and Sus $s 1$ are less frequent. Gal d 5 should be considered as an independent allergen as homology to mammalian albumins is very low.

Patients with moderate to high levels of IgE to serum albumins are at risk to develop symptoms upon ingestion of unboiled milk and raw or medium cooked meat such as sausages, ham and steaks. IgE to Bos d 6 and Sus $s 1$ should be determined and patients should be carefully advised. As serum albumins are thermolabile, well-cooked meat and heated milk may be tolerated.

\section{CLINICAL CASES}

\section{Case 1 (published (15))}

Clinical history - A 17-year-old girl with a history of rhinitis and asthma when exposed to cat experienced anaphylactic reactions on two occasions when the only food consumed was pork (ham and sausage).

Test with extracts - The patient had positive skin prick tests to cat dander and pork. IgE were positive for cat dander (>100 kUA/L) and pork (22 kUA/L).

Test with molecules - Specific IgE to animal serum albumins were $165 \mathrm{kUA} / \mathrm{L}$ to Fel d 2, $37 \mathrm{kUA} / \mathrm{L}$ to Can f 3, $22 \mathrm{kUA} / \mathrm{L}$ to Sus s 1 and $2.5 \mathrm{kUA} / \mathrm{L}$ to Bos d 6. IgE to Sus s 1 could be totally inhibited by addition of Fel $d 2$, confirming primary sensitization to cat.

Conclusion - Results were consistent with pork-cat syndrome and the patient was advised to avoid eating pork.

\section{Case 2 (published (16))}

Clinical history - A 52-year-old man, experienced anaphylaxis 20 min after a meal of pork tenderloin, potatoes and green beans. As symptoms occurred quickly after the meal, the involvement of lgE directed to alpha-gal antibodies seemed unlikely.

Test with extracts - Skin prick tests were positive for pork, cat and dog dander. Allergen-specific IgE were positive for cat dander (3.74 kUA/L) and pork (0.65 kUA/L), negative to beef.

Test with molecules - The patient had specific IgE to Fel d 1 (1.58 kUA/L) and Fel d 2 (2.28 kUA/L). Specific IgE were negative for alpha-gal, and Bos d 6.

Conclusion - Results were consistent with pork-cat syndrome and the patient was advised to avoid eating pork.

Allergen nomenclature: Fel d 2, Bos d 6, Can $f$ 3, Sus $s$, Gal d 5: cat, bovine, dog, porcine and chicken serum albumins.

\section{References}

1. Majorek KA, Porebski PJ, Dayal A, Zimmerman MD, Jablonska K, Stewart AJ, et al. Structural and immunologic characterization of bovine, horse, and rabbit serum albumins. Mol Immunol 2012;52:174182.

2. Chruszcz M, Mikolajczak K, Mank N, Majorek KA, Porebski PJ, Minor W. Serum albumins-unusual allergens. Biochim Biophys Acta 2013;1830:53755381.

3. Spitzauer S, Pandjaitan B, Söregi G, Mühl S, Ebner $C$, Kraft D, et al. IgE cross-reactivities against albumins in patients allergic to animals. J Allergy Clin Immunol 1995;96:951-959.

4. Jenkins JA, Breiteneder H, Mills EN. Evolutionary distance from human homologs reflects allergenicity of animal food proteins. J Allergy Clin Immunol 2007;120:1399-1405.

5. Aalberse RC. Structural biology of allergens. J Allergy Clin Immunol 2000; 106: 228-238.

6. Hilger C, Swiontek K, Hentges F, Donnay C, de Blay F, Pauli G. Occupational inhalant allergy to pork followed by food allergy to pork and chicken: sensitization to hemoglobin and serum albumin. Int Arch Allergy Immunol 2010; 151:173-178.

7. Liccardi G, Asero R, D'Amato M, D'Amato G. Role of sensitization to mammalian serum albumin in allergic disease. Curr Allergy Asthma Rep 2011;11:421-426.

8. Choi GS, Kim JH, Lee HN, Sung JM, Lee JW, Park HS. Occupational asthma caused by inhalation of bovine serum albumin powder. Allergy Asthma Immunol Res 2009;1:45-47.

9. Voltolini $S$, Spigno $F$, Cioè A, Cagnati P, Bignardi D, Minale P. Bovine Serum Albumin: a double allergy risk. Eur Ann Allergy Clin Immunol 2013;45:144-147. 
10. Wisniewski JA, Agrawal R, Minnicozzi S, Xin W, Patrie J, Heymann PW, et al. Sensitization to food and inhalant allergens in relation to age and wheeze among children with atopic dermatitis. Clin Exp Allergy 2013;43:116070.

11. Restani P, Ballabio C, Di Lorenzo C, Tripodi S, Fiocchi A. Molecular aspects of milk allergens and their role in clinical events. Anal Bioanal Chem 2009;395:47-56.

12. Martelli A, De Chiara A, Corvo M, Restani P, Fiocchi A. Beef allergy in children with cow's milk allergy; cow's milk allergy in children with beef allergy. Ann Allergy Asthma Immunol 2002;89:38-43.

13. Fanta C, Ebner C. Allergy to mare's milk. Allergy 1998; 53:539-540.

14. Drouet M, Sabbah A, Le Sellin J, Bonneau JC, Gay G, Dubois-Gosnet C. [Fatal anaphylaxis after eating wild boar meat in a patient with pork-cat syndrome]. Allerg Immunol (Paris) 2001;33:163-165.
15. Hilger C, Kohnen M, Grigioni F, Lehners C, Hentges F. Allergic cross-reactions between cat and pig serum albumin. Study at the protein and DNA levels. Allergy 1997;52:179-187.

16. Posthumus J, James HR, Lane CJ, Matos LA, PlattsMills TA, Commins SP. Initial description of pork-cat syndrome in the United States. J Allergy Clin Immunol 2013;131:923-925.

17. Quirce $S$, Marañón F, Umpiérrez A, de las Heras M, Fernández-Caldas E, Sastre J. Chicken serum albumin ( $\mathrm{Gal} \mathrm{d} \mathrm{5}$ ) is a partially heat-labile inhalant and food allergen implicated in the bird-egg syndrome. Allergy 2001;56:754-762.

18. Landau M, Mayrose I, Rosenberg Y, Glaser F, Martz E, Pupko T, et al. ConSurf 2005: the projection of evolutionary conservation scores of residues on protein structures. Nucleic Acids Res 2005;33:W299302. 


\title{
PART C
}

CROSS-REACTIVE MOLECULES \& THEIR CLINICAL RELEVANCE

\section{TROPOMYOSINS}

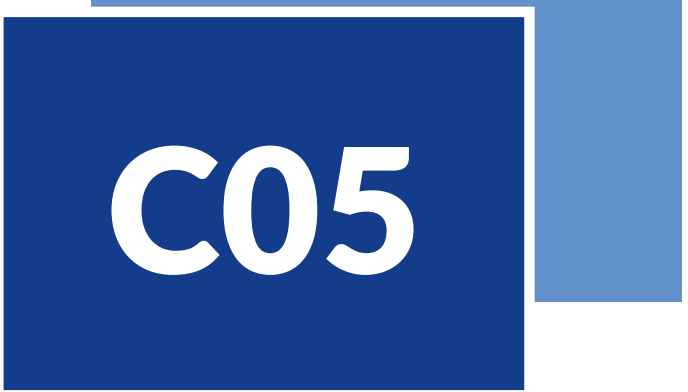

\author{
Luis Caraballo
}

\section{THE PROTEIN}

The shrimp (Penaeus aztecus) major allergen, Pen a 1 , is one of the most clinically relevant allergenic tropomyosins (1-3). Its basic characteristics are presented in Table 1. There is no three-dimensional structure available from any allergenic tropomyosin, but a predicted model of Ascaris lumbricoides tropomyosin (Asc | 3) is presented in Fig. 1. The secondary structure is a coiled-coil molecule formed by two parallel alpha-helices.

These proteins are heat-stable, which partially explains their high allergenicity. Together with actin and myosin play an important role in the contractile activity, as well as the regulation of cell morphology and motility. Shellfish allergenic tropomyosins were first described in shrimp (4-6) but they are important allergens in other sources. The relevance of sensitization to tropomyosins varies from low clinical impact to anaphylaxis.

Several lgE binding epitopes of shrimp tropomyosins have been reported. Ayuso et al. identified five major IgE binding sites on Pen a 1 that were cross-reactive epitopes among shrimp, lobster, house dust mite and cockroach $(7,8)$. The sites include eight IgE binding
Tropomyosins are highly allergenic crossreactive molecules from diverse sources (pan allergens) that can induce diverse symptoms including anaphylaxis.

Sensitization to tropomyosins can occur by ingestion (seafood), inhalation (mites, cockroaches) or parasite infection (ascariasis, anisakiasis).

The clinical impact of non-food allergenic tropomyosins may be greater than previously thought.

In vitro determination of IgE antibodies to tropomyosin could be more specific, with higher positive predictive value, than the whole extract in cases of shrimp allergy. 


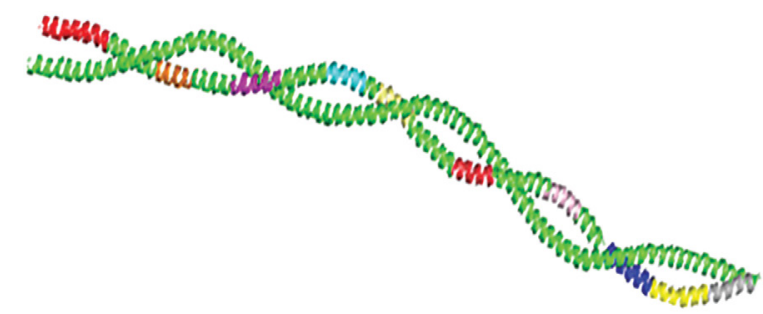

Figure 1

Molecular model of Ascaris lumbricoides tropomyosin generated by Swiss-Model (template Sus scrofa PDB 1c1g). Epitopes visualized with PyMOL.

epitopes: epitope 1 (residues 43 - 55) in region 1; epitope 2 (residues 87 - 101) in region 2; epitopes 3a (residues 137 - 141) and 3b (residues 144 151 ) in region 3; epitope 4 (residues 187 - 197) in region 4; and epitopes 5a (residues 249 - 259), 5b (residues 266 - 273) and 5c (residues 273 - 281) in region 5 . The sequence identity of these regions to homologous regions of other tropomyosins varies from 56\% (rabbit) to 98\% (lobster).

Further analyses of these epitopes and comparing them with other related sequences suggested that they can be classified into three types: epitope 5 a that is highly conserved among crustaceans, mollusks, insects and mites. The second type is composed of epitopes 2, 3 and 4 that represent all arthropods but not mollusks. The third type includes epitope $1,5 b$ and $5 c$, which are specific to crustacean (9). Epitope mapping of other tropomyosins such as Pen j 1, Pen $m 1$ and Pan b 1 from shrimp, Tur c 1 (snail), Cra g 1 (oyster) and Oct $v 1$ (octopus) have also been performed. The amino acid sequence of several tropomyosin epitopes is already known. In addition, $\mathrm{T}$ cell epitopes have been described. Ravkov $\mathrm{E}$ et al., using an in vitro MHC-peptide biding assay as well as ex vivo proliferation and cytokine release assays, identified and validated $17 \mathrm{~T}$ cell epitopes restricted to multiple $\mathrm{MHC}$ class II alleles. This finding is potentially useful for designing peptide-based immunotherapy of shrimp allergy. The challenge of further studies is to analyze which tropomyosins epitopes are species-specific markers.
Table 1

Basic protein characteristic s of Pen a 1

\begin{tabular}{ll|}
\hline \multicolumn{2}{l}{ Protein characteristics } \\
\hline Allergen source & Shrimp \\
\hline Protein family & Tropomyosin \\
\hline $\begin{array}{l}\text { UniProtKB accession } \\
\text { No }\end{array}$ & Q3Y8M6 \\
\hline $\begin{array}{l}\text { Three dimensional } \\
\text { structure available }\end{array}$ & No \\
\hline Molecular structure & Alpha-helical \\
\hline $\begin{array}{l}\text { Theoretical molecular } \\
\text { weight }\end{array}$ & 32.8 kDa \\
\hline Length & 284 amino acids \\
\hline Ligand binding & Yes \\
\hline Dimerization & Homodimer \\
\hline Glycosylation & No \\
\hline Disulfide bonds & No \\
\hline Isoelectric point & 4.72 \\
\hline Synthesis & Several isoforms \\
\hline Distribution & Muscle and non-muscle cells \\
\hline
\end{tabular}

\section{THE FAMILY}

Tropomyosin belongs to a family of proteins (Pfam PF00261) that includes a large number of cross reactive allergens, most of them from invertebrate sources, such as shrimp, lobster, crab, snail, abalone, whelk, clam, mussels, octopus, house dust mites, cockroaches and helminths (Table 2).

Around $40 \mathrm{lgE}$ binding tropomyosins has been described, some of them cloned and expressed as recombinant proteins and tested for allergenicity. However, only few (Pen a 1, Pen m 1, Ani s 3, Bla g 7 and Der $\mathrm{p}$ 10) have been included in commercial in vitro allergy testing. Tropomyosin amino acid sequence is highly conserved among shellfish and other invertebrates, where share over $70 \%$ identity; their comparison with vertebrate tropomyosins reveals 51 to $57 \%$ identity (1).

Leung $P$ et al. have shown that serum IgE to shrimp also binds tropomyosin of other shellfish such as greasy back shrimp, spiny lobster and Indo-Pacific swamp crab, abalone, whelk, green mussel, pen shell, 


\section{Table 2}

A list of clinically relevant tropomyosins

\begin{tabular}{|c|c|c|}
\hline Family & Allergen Source & Allergen \\
\hline \multirow[t]{5}{*}{ Penaeoidea } & Brown shrimp (Penaeus aztecus) & Pen a 1 \\
\hline & Northern Red Shrimp (Pandalus borealis) & Pan b 1 \\
\hline & Giant tiger prawn (Penaeus monodon) & Pen $\mathrm{m} 1$ \\
\hline & European Shrimp (Litopenaeus vannamei) & Lit v 1 \\
\hline & Common Shrimp (Crangon Crangon) & Cra c 1 \\
\hline Palinuridae & Spiny lobster (Panulirus stimpsoni) & Pan s 1 \\
\hline Cancridae & Common crab (Charybdis feriatus) & Cha f 1 \\
\hline Hellixidae & Brown garden snail (Helix aspersa) & Hel as 1 \\
\hline Mytilidae & Green mussel (Perna viridis) & Per v 1 \\
\hline Octopodidae & Common Octopus (Octopus vulgaris) & Oct v 1 \\
\hline Ommastephidae & Japanese flying squid (Todarodes pacificus) & Tod p 1 \\
\hline Osteidae & Pacific cupped oyster (Crassostrea gigas) & Cra g 1 \\
\hline Haliotidea & Abalone (Haliotis diversicolor) & Hal d 1 \\
\hline \multirow[t]{2}{*}{ Pyroglyphidae } & House dust mite (Dermatophagoides farinae) & Der $f 10$ \\
\hline & House dust mite (D. pteronyssinus) & Der $\mathrm{p} 10$ \\
\hline Echymiopodidae & Storage mite (Blomia tropicalis) & Blo t 10 \\
\hline Blattidae & American cockroach (Periplaneta americana) & Per a 7 \\
\hline Blattellidae & German cockroach (Blattella germanica) & Bla g 7 \\
\hline Anisakidae & Anisakis (Anisakis simplex) & Ani s 3 \\
\hline Ascaridae & Roundworm (Ascaris lumbricoides) & Asc 13 \\
\hline
\end{tabular}

\section{Table 3}

Amino acid identities (\%) between some tropomyosins

\begin{tabular}{|c|c|c|c|c|c|c|c|c|c|}
\hline Pen a 1 & & & & & & & & & \\
\hline Pen s 1 & $100 \%$ & & & & & & & & \\
\hline Cha f 1 & $100 \%$ & $91 \%$ & & & & & & & \\
\hline Hel as 1 & $71 \%$ & $63 \%$ & $63 \%$ & & & & & & \\
\hline Oct v 1 & $75 \%$ & $63 \%$ & $63 \%$ & $83 \%$ & & & & & \\
\hline Per v 1 & $55 \%$ & $55 \%$ & $55 \%$ & $70 \%$ & $70 \%$ & & & & \\
\hline Der p 10 & $71 \%$ & $80 \%$ & $82 \%$ & $64 \%$ & $63 \%$ & $55 \%$ & & & \\
\hline Bla g 7 & $76 \%$ & $82 \%$ & $84 \%$ & $63 \%$ & $63 \%$ & $56 \%$ & $80 \%$ & & \\
\hline \multirow[t]{2}{*}{ Asc I 3} & $62 \%$ & $72 \%$ & $74 \%$ & $64 \%$ & $64 \%$ & $57 \%$ & $73 \%$ & $70 \%$ & \\
\hline & Pen a 1 & Pen s 1 & Cha f 1 & Hel as 1 & Oct v 1 & Per v 1 & Der $\mathrm{p} 10$ & Bla g 7 & Asc I 3 \\
\hline
\end{tabular}

Figures in bold blue indicate tropomyosin pairs with $<70 \%$ amino acid sequence identity 


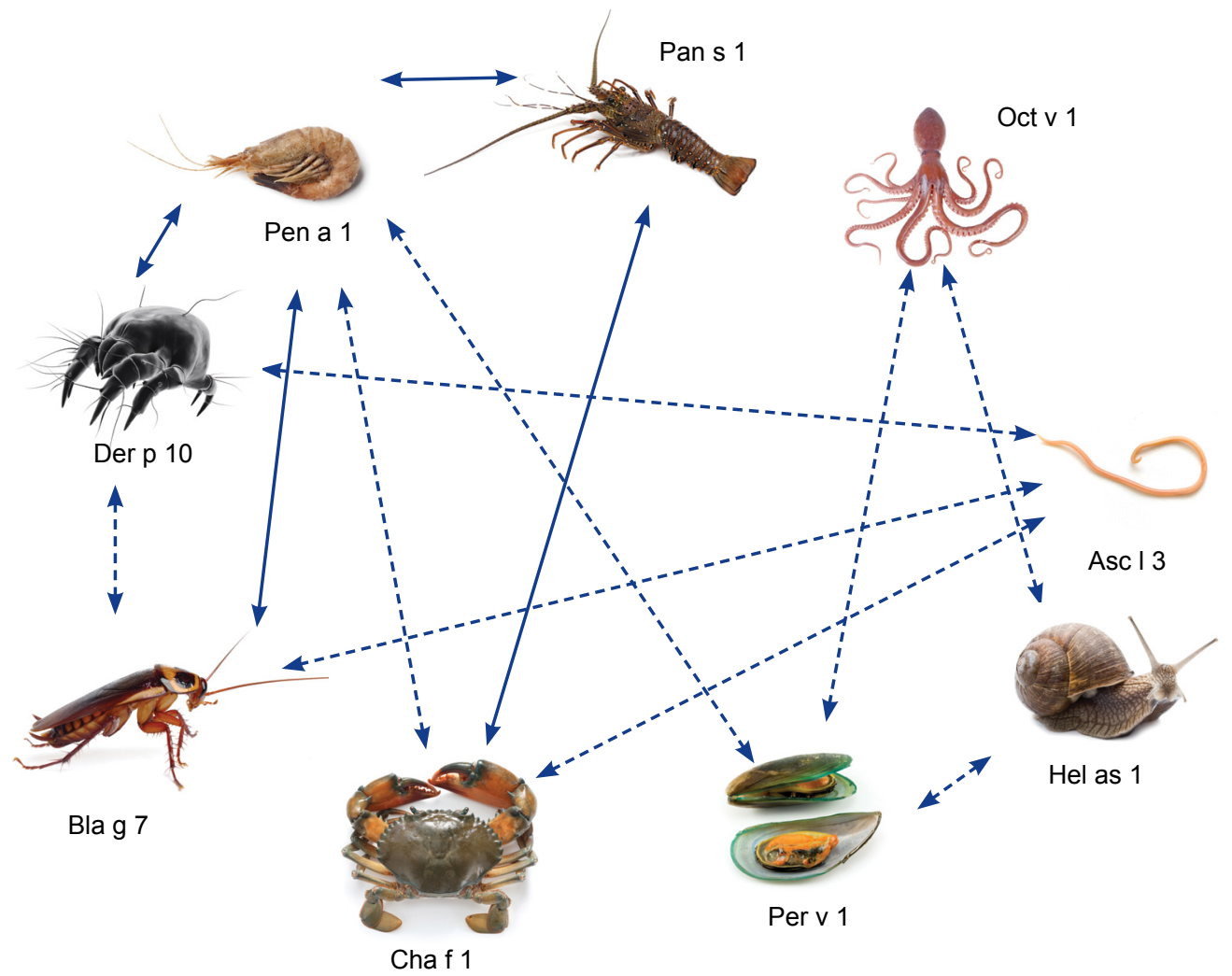

Figure 2

Cross-reactivity among allergenic tropomyosins from several sources. Lines represent documented IgEcorss-reactivity. Dotted lines represent potential IgE-cross-reactivity based on high sequence identities.

scallop, Pacific cupped oyster, cuttlefish, sword tip squid and octopus (10), which reflects the high cross reactivity of this family. Still, tropomyosins are not the only allergens of shellfish; other cross-reactive clinically relevant allergens have been reported (798).

For comparing the protein sequences of allergenic tropomyosins, Leung $M$ et al. (9) conducted a phylogenetic analysis and found that arthropods tropomyosins share $91.7 \%$ homology $(76.1$ $100 \%)$ and mollusks share $77.2 \%$ (65.1 - 99.3\%), which indicates that, at the whole sequence level, tropomyosins are not species-specific allergy markers. Homology between crustacean and mollusks tropomyosins is $56-68 \%$ and between vertebrate and invertebrate tropomyosins $53-57 \%$ (1). Table 3 displays two-by-two comparisons of amino acid identities between representative tropomyosins.

All pairs with a high sequence identity are potentially cross-reactive. Sequence alignments are good primary prediction tools for cross-reactivity, but most importantly, the use of IgE-inhibition studies with sera from clinically well characterized patients will allow defining clinically relevant cross-reactivity (Fig. 2). A comprehensive phylogenetic tree of allergenic tropomyosins from various taxonomic groups can be found in reference (9).

Mite (group 10) and cockroach (group 7) tropomyosins cross react with shrimp tropomyosins and have also been described as clinically relevant allergens. Besides, preliminary data show that $33 \%$ of sera with positive IgE against mosquito extract reacted to natural tropomyosin purified from and also showed cross reactivity with the shrimp allergens $n$ Pen $m 1$ and $r$ Lit $\vee 1$. The clinical impacts of these finding are currently under evaluation. The allergenicity and cross reactivity of Ascaris lumbricoides tropomyosin (Asc I 3) has been thoroughly analyzed (11-13); further studies have shown an important clinical impact by sensitizing the asthmatic populations in tropical underdeveloped countries, where helminthiases, together with perennial exposition to mite tropomyosins might increase asthma symptoms $(14,15)$. 
Cross-reactivity between house dust mite (HDM) allergens and some shellfish has been described and there are reports suggesting that it is clinically significant. Subcutaneous HDM immunotherapy in patients sensitized to shrimp or snail could increase allergy symptoms after ingestion of these foods. Sensitization to shrimp tropomyosin in Orthodox Jews has been demonstrated in HDM allergic patient (803). Although cross-reactive tropomyosins are good candidates for explaining these observations, other allergens may be involved. In addition, other authors have obtained opposite results after immunotherapy, suggesting that the adverse side effects are not universal and could be influenced by the type of immunotherapy and genetic factors determining the susceptibility to get sensitized by other allergens. Therefore, more research is needed to define this controversial effect of cross reactivity among arthropods on immunotherapy.

\section{CLINICAL RELEVANCE}

Tropomyosins from invertebrates are allergenic for genetically susceptible individuals and due to their extensive cross reactivity, are considered pan allergens. Recently the vertebrate tropomyosin, Ore $\mathrm{m} 4$, was described as a major allergen of tilapia (Oreochromis mossambicus) (17). Sensitization can occur by ingestion (seafood), inhalation (mites, cockroaches) or parasite infection (ascariasis, anisakiasis).

Most allergenic tropomyosins are major shellfish allergens. Symptoms may be induced by very low amounts of the offending food and sometimes by inhalation. They include immediate cutaneous reactions (urticaria, angioedema, rash) oral allergy syndrome (swelling in the lips and mouth), digestive symptoms (vomiting, abdominal cramping, and diarrhea), anaphylaxis and asthma. In Europe, sensitization to mite tropomyosin Der $\mathrm{p} 10$ is low and has been considered an effect of cross reactivity but also a marker for broad sensitization among HDM allergy patients. Component resolved diagnosis of D. pteronyssinus allergens Der $\mathrm{p} 1$, Der $\mathrm{p} 2$ and Der p 10 has been suggested for selecting patients for HDM immunotherapy. In this study, the frequency of IgE to Der $\mathrm{p} 10$ was low. However, the prevalence of sensitization to Der $f 10$ was found around $80 \%$ in Japan. In addition, sensitization to Der p 10 was found 55\% in Zimbabwe and 34\% in Colombia (14),

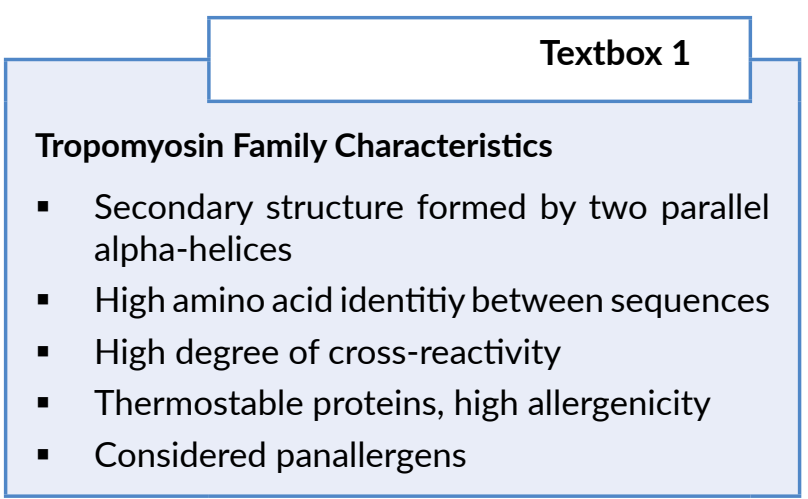

probably because of perennial exposure to shellfish and helminth infections. Therefore, the clinical impact of non-food allergenic tropomyosins may be greater than previously thought. In fact, it has been suggested that sensitization to tropomyosin from mite (14), cockroach, Ascaris $(14,15)$ and mosquito (18) could influence the prevalence and severity of asthma in places where co exposition to several sources of tropomyosin occurs. The influence of Ascaris tropomyosin sensitization on the outcome of immunotherapy for mite allergy has not been evaluated.

The frequency of IgE sensitization to tropomyosins in shellfish allergic patients ranges from 50 to $100 \%$. In addition, Pen a 1 binds up to $75 \%$ of all shrimp specific IgE antibodies (2), which is supported by histamine release experiments (1). Seafood allergy is one of the most common allergies and includes reaction to crustaceans, mollusks and fish. The prevalence of shellfish (crustacean and mollusks) allergy is $0.6 \%$ in the world population. In some regions of high consumption such as Singapore, the prevalence of seafood allergy in school children is around $5 \%$.

\section{CLINICAL MANAGEMENT}

Clinical history of adverse reaction suggesting allergy after intake of shellfish is crucial for starting diagnosis procedures. Whole extracts are very useful for diagnosing shellfish allergy by ST, although Prick-prick procedure is also useful. Tropomyosin sensitization is very important when evaluating shellfish allergy but other allergens also play a role (9). It has been suggested that in vitro determination of IgE antibodies to tropomyosin is more specific and has a higher positive predictive value than the whole extract in 


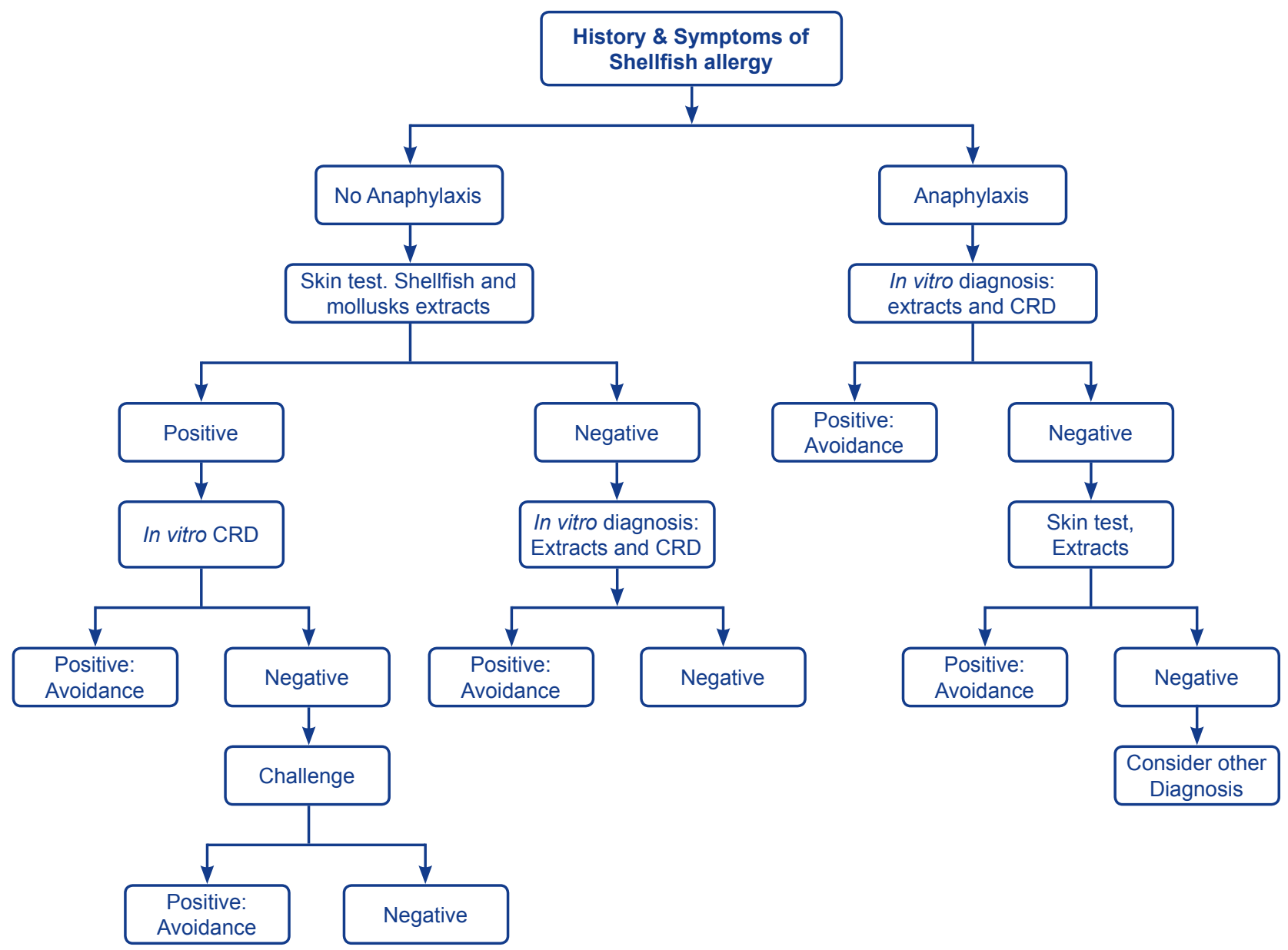

Figure 3

Diagnostic algorithm for shellfish allergy. In vitro tests for IgE to molecular allergens (CRD) include Pen a 1, Pen $\mathrm{m} 2$ and Pen $\mathrm{m} 4$.

cases of shrimp allergy. In addition, Thalayasingam et al. found that the presence of specific IgE to shrimp has diagnostic test sensitivity of $82 \%$ and specificity of $22.2 \%$ (19). This low specificity, mainly due to the high rate of false positives that in turn are a consequence of the high cross-reactivity between shrimp and other Arthropods allergens, explains why allergy to shellfish should be often diagnosed by challenge. Only two shellfish tropomyosins, Pen a 1 and Pen $\mathrm{m} 1$ (both from shrimp), are commercially available for in vitro testing. A possible diagnostic steps (Fig. 4) could be starting with ST with the whole extract, and detecting IgE antibodies to the extract, tropomyosin and other shellfish allergens, such as Pen $\mathrm{m} 2$ (Arginine kinase) and Pen m 4 (Sarcoplasmic calcium binding protein).
Since most shellfish tropomyosins share sequences and epitopes (cross-sensitization) and there is a great diversity of seafood (co-sensitization), it is currently difficult to define the primary allergenic source using component resolved diagnosis. Then the added value using single allergens for distinguishing the sensitizing source is still limited because there are no species-specific markers of sensitization. However, a panel of tropomyosin plus other five shrimp (C. crangon) allergens could be useful for comparing sensitization patterns from patients with different symptoms or severity of symptoms and identify clinically useful biomarkers. More recently Pascal $M$ et al. evaluated, in patients from USA, Brazil and Spain, the efficiency of several allergens to predict shrimp allergy. They found that tropomyosin and 
sarcoplasmic-calcium-binding-protein sensitization is associated with clinical reactivity; in addition, the tropomyosin epitope p51-55 seems to be of good value as a diagnostic test to confirm allergy. The authors present a very interesting flow diagram for shrimp allergy diagnosis (20).

Still, the gold standard for food allergy diagnosis is the double-blind placebo-controlled food challenge. An important aspect of management is the detection of tropomyosin in food samples to prevent accidental ingestion and anaphylactic reactions. Several approaches and techniques have been proposed.

Management of shellfish allergy is based on strictly avoidance based on clinical reactions. In general, if a patient is allergic to shrimp, avoidance of all crustaceans is recommended, although it is possible that allergy be limited to particular crustacean member. Avoidance of mollusks is advised if allergy to it is demonstrated. However, patients with high IgE reactivity to tropomyosin might be advised to avoid all shellfish. No immunotherapy is currently available for seafood allergy but experimental approaches to obtain appropriate compounds for specific immunotherapy have been developed. Hypoallergenic Pen a 1, hypoallergenic peptides from Met e 1, periodate treatment of crab tropomyosin and simulated gastric digestion of the whole shrimp extract are under analysis. Animal models for sensitization will help to obtain better reagents for diagnosis and treatment.

\section{CLINICAL CASES}

\section{Case 1 (published (21))}

Clinical History: A 30-year-old man with a 10-year history of mild persistent asthma and allergy to house dust mites and pollen presented generalized urticaria, facial erythema, and pharyngeal pruritus after eating shellfish on 3 separate occasions during a 2-year period. He associated the most recent episode with lobster. Since then, he has tolerated some crustaceans, mollusks, and fish, although he has avoided eating shrimp and lobster. No other food or drug allergies were reported, and he has not received immunotherapy for house dust mites.

Test with extracts: The patient had positive results for $D$ pteronyssinus $(21.4 \mathrm{kU} / \mathrm{L})$ and $D$ farinae extracts (12.6 kU/L). Weak SPT positivity with shrimp. IgE to lobster extracts $(2.9 \mathrm{kUA} / \mathrm{I})$ and PPT to lobster were positive $(6 \mathrm{~mm})$.

Food challenge: the patient tolerated up to $8 \mathrm{~g}$ of cooked shrimp during the challenge (and normal servings have been tolerated several times since). The study performed with lobster gave positive results by CAP (2.9 kUA/I) and PPT (6 $\mathrm{mm})$. However, the patient refused the oral food challenge with lobster.

Test with molecules: The patient had positive results to Der p 1 (4.7 kUA/L), Der p 2 (60.9 kUA/L), Der f 1 (0.4 kUA/L) and Der f 2 (47.2 kUA/L). Purified tropomyosins from shrimp (Pen a 1, $0.04 \mathrm{kU} / \mathrm{L}$ ) and $D$ pteronyssinus (Der p 10, 0 kU/L) were negative.

Conclusion: Selective allergy to lobster in a patient with primary sensitization to house dust mite.

\section{References}

1. Reese G, Schicktanz S, Lauer I, Randow S, Lüttkopf D, Vogel L et al. Structural, immunological and functional properties of natural recombinant Pen a 1, the major allergen of Brown Shrimp, Penaeus aztecus. Clin Exp Allergy 2006;36:517-524.

2. Daul CB, Slattery M, Reese G, Lehrer SB. Identification of the major brown shrimp (Penaeus aztecus) allergen as the muscle protein tropomyosin. Int Arch Allergy Immunol 1994;105:49-55.

3. Reese G, Jeoung BJ, Daoul CB, Lehrer SB. Characterization of recombinant shrimp allergen Pen a 1 (tropomyosin). Int Arch Allergy Immunol 1997;113: 240-242.

4. Hoffman DR, Day ED Jr, Miller JS. The major heat stable allergen of shrimp. Ann Allergy 1981;47:17-22.

5. Naqpal S, Rajappa L, Metcalfe DD, Rao PV. Isolation and characterization of heat-stable allergens from shrimp (Penaeus indicus). J Allergy Clin Immunol 1989;83:2636.

6. Leung PS, Chu KH, Chow WK, Ansari A, Bandea Cl, Kwan HS et al. Cloning, expression, and primary structure of Metapenaeus ensis tropomyosin, the major heat-stable shrimp allergen. J Allergy Clin Immunol 1994;94:882-890.

7. Ayuso R, Lehrer SB, Reese G. Identification of continuous, allergenic regions of the major shrimp allergen Pen a 1 (tropomyosin). Int Arch Allergy Immunol 2002;127:27-37.

8. Ayuso R, Reese G, Leong-Kee S, Plante M, Lehrer SB. Molecular basis of arthropod cross-reactivity: IgEbinding cross-reactive epitopes of shrimp, house dust mite and cockroach tropomyosins. Int Arch Allergy Immunol 2002;129:38-48. 
9. Leung NY, Wai CY, Shu S, Wang J, Kenny TP, Chu KH et al. Current immunological and molecular biological perspectives on seafood allergy: a comprehensive review. Clin Rev Allergy Immunol 2014;46:180-197.

10. Leung PS, Chow WK, Duffey S, Kwan HS, Gershwin $\mathrm{ME}, \mathrm{Chu} \mathrm{KH}$. IgE reactivity against a cross-reactive allergen in crustacea and mollusca: evidence for tropomyosin as the common allergen. J Allergy Clin Immunol 1996;98:954-961.

11. Santos AB, Rocha GM, Oliver C, Ferriani VP, Lima RC, Palma MS et al. Cross-reactive IgE antibody responses to tropomyosins from Ascaris lumbricoides and cockroach. J Allergy Clin Immunol 2008;121:10401046.

12. Acevedo N, Sánchez J, Erler A, Mercado D, Briza $P$, Kennedy $M$ et al. IgE cross-reactivity between Ascaris and domestic mite allergens: the role of tropomyosin and the nematode polyprotein ABA1. Allergy 2009;64:1635-1643.

13. Acevedo N, Erler A, Briza P, Puccio F, Ferreira F, Caraballo L. Allergenicity of Ascaris lumbricoides tropomyosin and IgE sensitization among asthmatic patients in a tropical environment. Int Arch Allergy Immunol 2011;154:195-206.

14. Ahumada V, García E, Dennis R, Rojas MX, Rondón MA, Pérez A et al. IgE responses to Ascaris and mite tropomyosins are risk factors for asthma. Clin Exp Allergy 2015;45:1189-1200.
15. Buendía E, Zakzuk J, Mercado D, Alvarez A, Caraballo L. The IgE response to Ascaris molecular components is associated with clinical indicators of asthma severity. World Allergy Organo J 2015;8:8.

16. Fernandes J, Reshef A, Patton L, Ayuso R, Reese G, Lehrer SB. Immunoglobulin $\mathrm{E}$ antibody reactivity to the major shrimp allergen, tropomyosin, in unexposed Orthodox Jews. Clin Exp Allergy 2003;3:956-961.

17. Liu R, Holck AL, Yang E, Liu C, Xue W. Tropomyosin from tilapia (Oreochromis mossambicus) as an allergen. Clin Exp Allergy 2013;43:365-377.

18. Cantillo JF, Fernandez-Caldas E, Puerta L. Immunological aspects of the immune response induced by mosquito allergens. Int Arch Allergy Immunol 2014;165:271-282.

19. Thalayasingam M, Gerez IF, Yap GC, Llanora GV, Chia IP, Chua L et al. Clinical and immunochemical profiles of food challenge proven or anaphylactic shrimp allergy in tropical Singapore. Clin Exp Allergy 2015;45:687-697.

20. Pascal M, Grishina G, Yang AC, Sánchez-García S, Lin J, Towle D et al. Molecular Diagnosis of Shrimp Allergy: Efficiency of Several Allergens to Predict Clinical Reactivity. J Allergy Clin Immunol Pract 2015;3:521529.

21. Iparraguirre $A$, Rodrìguez- Pérez $R$, Juste $S$, Ledesma $A$, Moneo I, Caballero ML. Selective allergy to lobster in a case of primary sensitization to house dust mites. $J$ Investig Allergol Clin Immunol 2009;19:409-413. 


\title{
PART C
}

CROSS-REACTIVE MOLECULES \& THEIR CLINICAL RELEVANCE

\section{POLCALCINS}

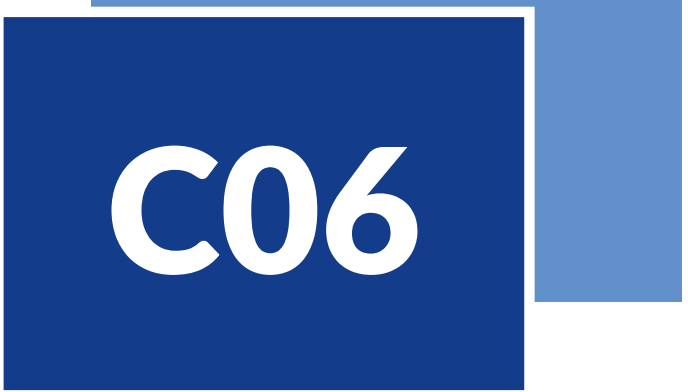

\author{
Joaquín Sastre
}

\section{THE PROTEIN}

The first cloned polcalcin was from birch pollen (Bet v 4) (1). But the most representative pollen polcalcin (1) is $\mathrm{Phl} p 7$ from Phleum pratense (Timothy grass). $\mathrm{Phl}$ $p 7$ belongs to a subfamily of two EF-hand calcium binding pollen allergens that are preferentially expressed in mature pollen of higher plants including monocotyledonic and dicotyledonic species.

$\mathrm{Phl}$ p 7 was detected only in pollen but not in root and leaf extracts. It seems that is completely eluted out of the pollen grains after a few minutes of hydration. It contains 78 amino acids with a molecular weight of 8,677 Da. Summaries of biochemical characteristics of $\mathrm{Phl} \mathrm{p} 7$ are showed in Table 1.

The physiological role of $\mathrm{Phl} p 7$ is likely related to the importance of regulating the calcium levels for pollen germination and pollen tube growth, as other calcium binding proteins contained in pollen.

rPhl p 7 exhibited a potent allergenic activity and is able to induced basophil histamine release and immediate type skin reactions. Phl p 7 has high stability (thermal and proteolysis) (1) and refolding capacity, a characteristic related to potent allergens.
Polcalcins are EF-hand calcium binding proteins only expressed in mature pollen

Extensive IgE cross-reactivity among pollen polcalcins

Specific IgE to polcalcins can be performed with any member of the family

Can be considered a marker of polysensitisation with unknown clinical relevance for respiratory symptoms 

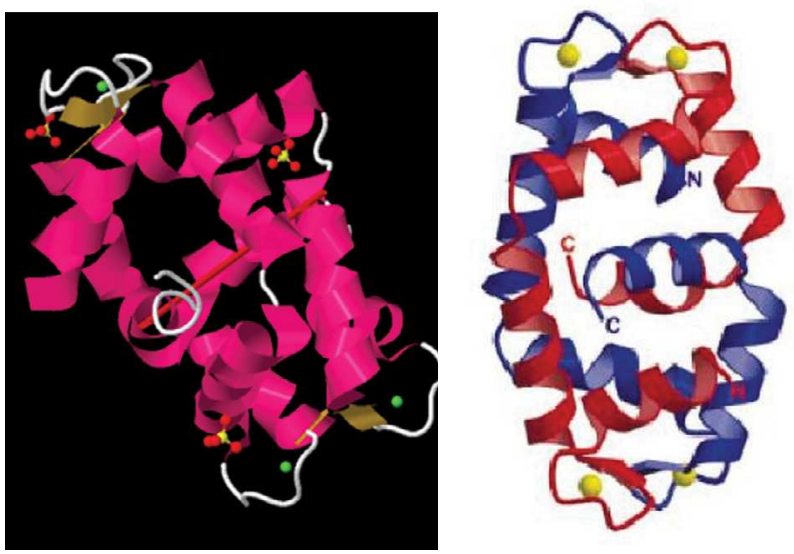

Figure 1

(A) Ribbon model of the three-dimensional structure of Phl p 7 (PDB 1K9U) solved by X-ray crystallography using DENZO and SCALEPACK software. (B) Stereo ribbon representation of the intertwined Phl p 7 dimer. Chain A is colored in blue, chain B is shown in red and the calcium ions are highlighted in yellow.

It contains calcium-modulated conformational lgE epitopes, which become accessible only in the calcium-bound form (open conformation), suggesting that IgE recognition is only activated by the calciumbound conformation.

\section{THE FAMILY}

Polcalcins belong to two EF-hand calcium-binding proteins. To date, 40 members of this allergen family have been identified in grasses, trees, bushes, weeds, and other flowering plants (Table 2). The two EF-hand allergens share a high degree of sequence homology (average sequence identity of $77 \%$ ), which explain the extensive cross-reactivity of allergic patients' IgE Abs with the various members of the family (Fig. 2-5). Although they do not present a complete immunological equivalence the diagnosis of patients sensitized to polcalcins can be performed with any member of the family $(809,810)$. The IgE binding capacity to polcalcins depends on conformational epitopes (2-6) (Fig. 4). It has been demonstrated that the Phl p 7 and related two EF-hand allergens do not share epitopes with other EF-hand allergens (e.g., Bet v 3, parvalbumin).
Table 1

Basic protein characteristics of Phl p 7

\begin{tabular}{|c|c|}
\hline \multicolumn{2}{|l|}{ Protein characteristics } \\
\hline Allergen source & $\begin{array}{l}\text { Phleum pratense (Timothy } \\
\text { grass) }\end{array}$ \\
\hline Protein family & Polcalcin \\
\hline $\begin{array}{l}\text { UniProtKB accession } \\
\text { No }\end{array}$ & O82040 \\
\hline $\begin{array}{l}\text { Three dimensional } \\
\text { structure available }\end{array}$ & yes \\
\hline Molecular structure & alpha-helical fold \\
\hline $\begin{array}{l}\text { Theoretical molecular } \\
\text { weight }\end{array}$ & 8,677 Da \\
\hline $\begin{array}{l}\text { Molecular weight } \\
\text { measured by MS }\end{array}$ & $14,22 \mathrm{kDa}$ \\
\hline Length & $\begin{array}{l}78 \text { amino acids, mature } \\
\text { protein }\end{array}$ \\
\hline Ligand binding & $\begin{array}{l}\text { EF-hand calcium-binding } \\
\text { allergen }\end{array}$ \\
\hline Dimerization & Dimer \\
\hline Glycosylation & no \\
\hline Disulfide bonds & no \\
\hline Isoelectric point & 4.39 \\
\hline Synthesis & Pollen grains \\
\hline Function & $\begin{array}{l}\text { Pollen germination and pollen } \\
\text { tube growth }\end{array}$ \\
\hline
\end{tabular}

Textbox 1

Polcalcin Family Characteristics

- Common tertiary structure with alpha-helical fold

- Calcium binding activity

- High sequence identity ( average 77\%)

- Small molecules of 8 kDa

- Conformational IgE epitopes (calciummodulated)

- Extensive IgE cross-reactivity among pollen polcalcins 


\section{Table 2}

Polcalcins described from different pollen

\begin{tabular}{|c|c|c|}
\hline $\begin{array}{l}\text { Botanical } \\
\text { family }\end{array}$ & Allergen source & Allergen \\
\hline \multirow[t]{19}{*}{ Grasses } & Crested Wheatgrass, Agropyron cristatum & Agr c 7 \\
\hline & Anthoxanthum odoratum & Ant o 7 \\
\hline & Cultivated Oat, Avena sativa & Ave s 7 \\
\hline & Tall Windmill Grass, Chloris elata & Chl el 7 \\
\hline & Bermuda grass, Cynodon dactylon & Cyn d 7 \\
\hline & Cocksfoot Grass, Dactylis glomerata & Dac g 7 \\
\hline & Salt grass, Distichlis spicata & Dis s 7 \\
\hline & Festuca elatior & Fes e 7 \\
\hline & Barley, Hordeum sativum & Hor v 7 \\
\hline & Cogon Grass, Imperata cylindrica & $\operatorname{Imp}$ c 7 \\
\hline & Rye grass, Lolium perenne & Lop p 7 \\
\hline & Rice, Oryza sativa & Orzs 7 \\
\hline & Timothy Grass, Phleum pratense & Phl p 7 \\
\hline & Kentucky Blue grass, Poa pratensis & Poa p 7 \\
\hline & Wheat, Triticum aestivum & Tri a 7 \\
\hline & Sorghum, Sorghum bicolor \& Sorghum halepense & Sor b $7 \&$ Sor h 7 \\
\hline & Wild Cane, Saccharum speciossisimum & Sac sp 7 \\
\hline & Smut Grass, Sporobolus indicus & Spo in 7 \\
\hline & Corn, Zea mays & Zea m 7 \\
\hline \multirow[t]{5}{*}{ Fagales } & Alder, Alnus glutinosa & Aln g 4 \\
\hline & Birch, Betula verrucosa & Bet $\vee 3$ Bet $\vee 4$ \\
\hline & Hornbeam, Carpinus betulus & Car b 4 \\
\hline & Beech, Fagus sylvatica & Fag s 4 \\
\hline & White Oak, Quercus alba & Que a 4 \\
\hline \multirow[t]{2}{*}{ Asteraceae } & Short Ragweed, Ambrosia artemisiifolia & Amb a 9, Amb a 10 \\
\hline & English Mugwort, Artemisia vulgaris & Art $\vee 5$ \\
\hline Brassicaceae & Rapeseed, Brassica napus & Bra n 4Bra n 7 \\
\hline Amaranthaceae & Goosefoot, Chenopodium album & Che a 3 \\
\hline \multirow[t]{3}{*}{ Cupressaceae } & Japanese cedar, Cryptomeria japonica & Cry j 4 \\
\hline & Arizona Cypress, Cupressus arizonica & Cup a 4 \\
\hline & Cedar, Juniperus oxycedrus & Jun o 4 \\
\hline \multirow[t]{3}{*}{ Oleaceae } & Ash, Fraxinus excelsior & Fra e 3 \\
\hline & Olive, Olea europeae & Ole e 3 Ole e 8 \\
\hline & Common Lilac, Syringa vulgaris & Syr v 3 \\
\hline Solanaceae & Tobacco, Nicotiana tabacum & Nic $\mathrm{t} 3$ \\
\hline Urticaceae & Parietaria, Pellitory, Parietaria judaica & Par j 4 \\
\hline Fabaceae & Locust Tree, Robinia pseudoacacia & Rob p 4 \\
\hline
\end{tabular}



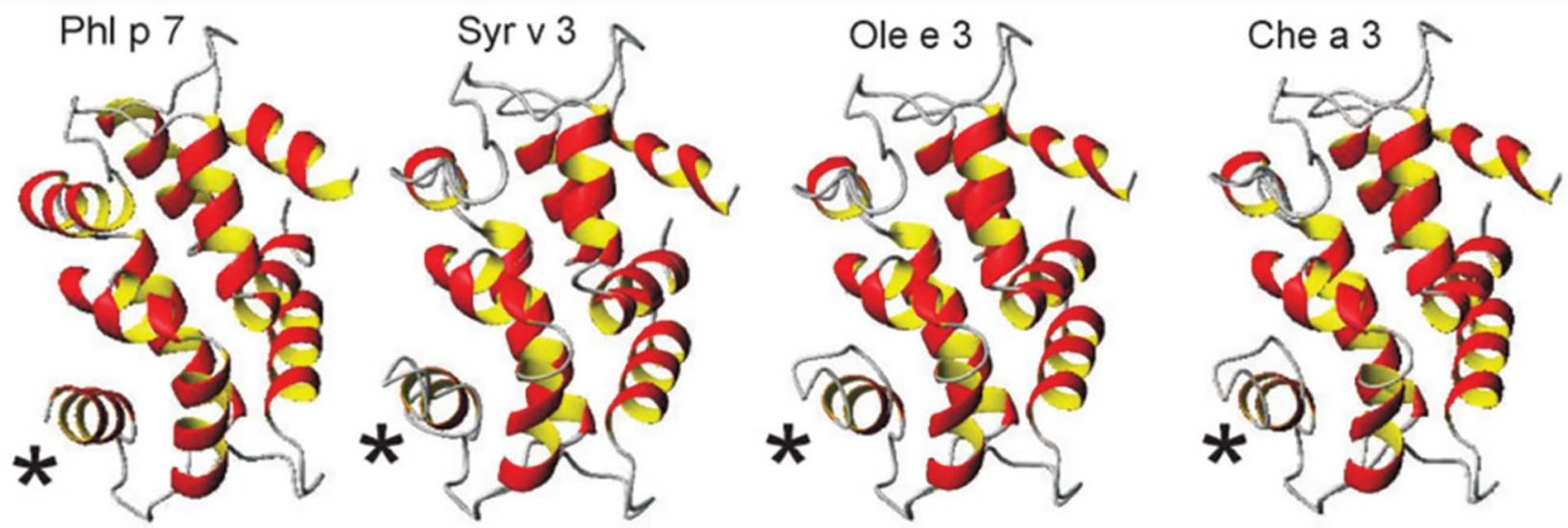

Figure 2

Backbone ribbon-type representation of the three-dimensional structure of Che a 3 (PDB 2OPO) and of structural models of Ole e 3 and Syr $\vee 3$ determined by using the services of the Swiss-Model Protein Modelling Server (811).

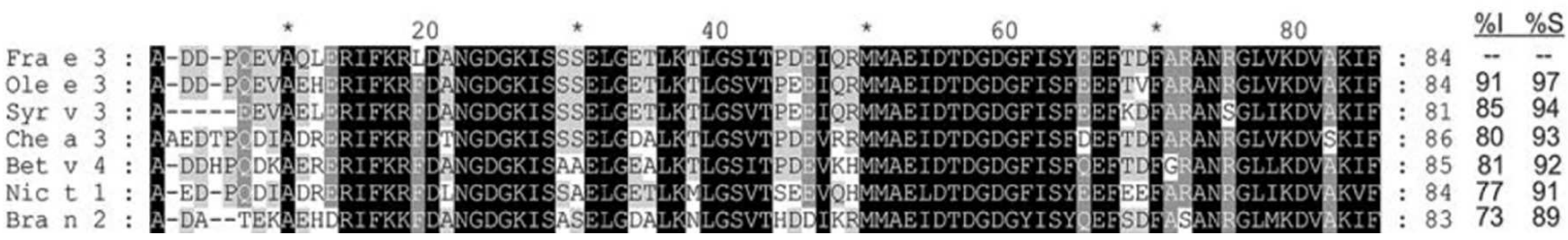

Figure 3

Comparison of the amino acid sequences of different polcalcins (7).

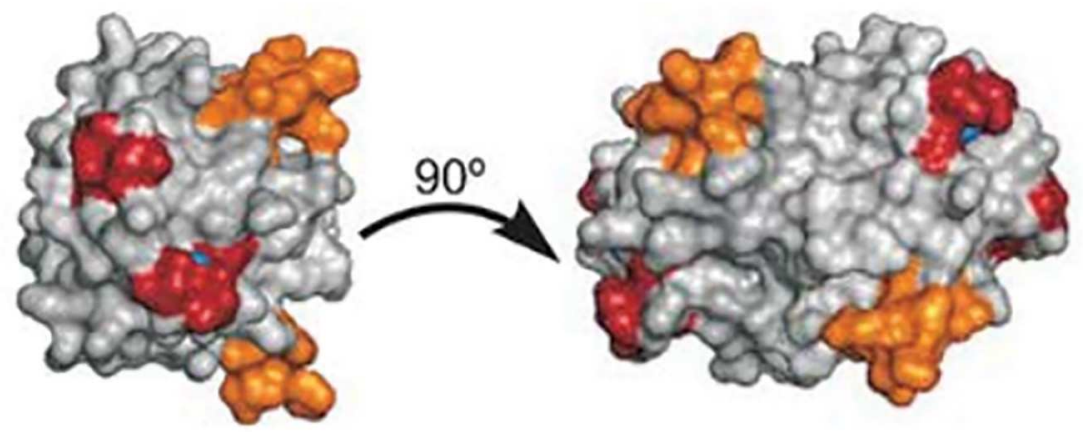

Figure 4

3D-modeling of Fra e 3 (dimer) (bottom). In orange, highly variable region; red, calcium-binding site; and blue, calcium ion. 


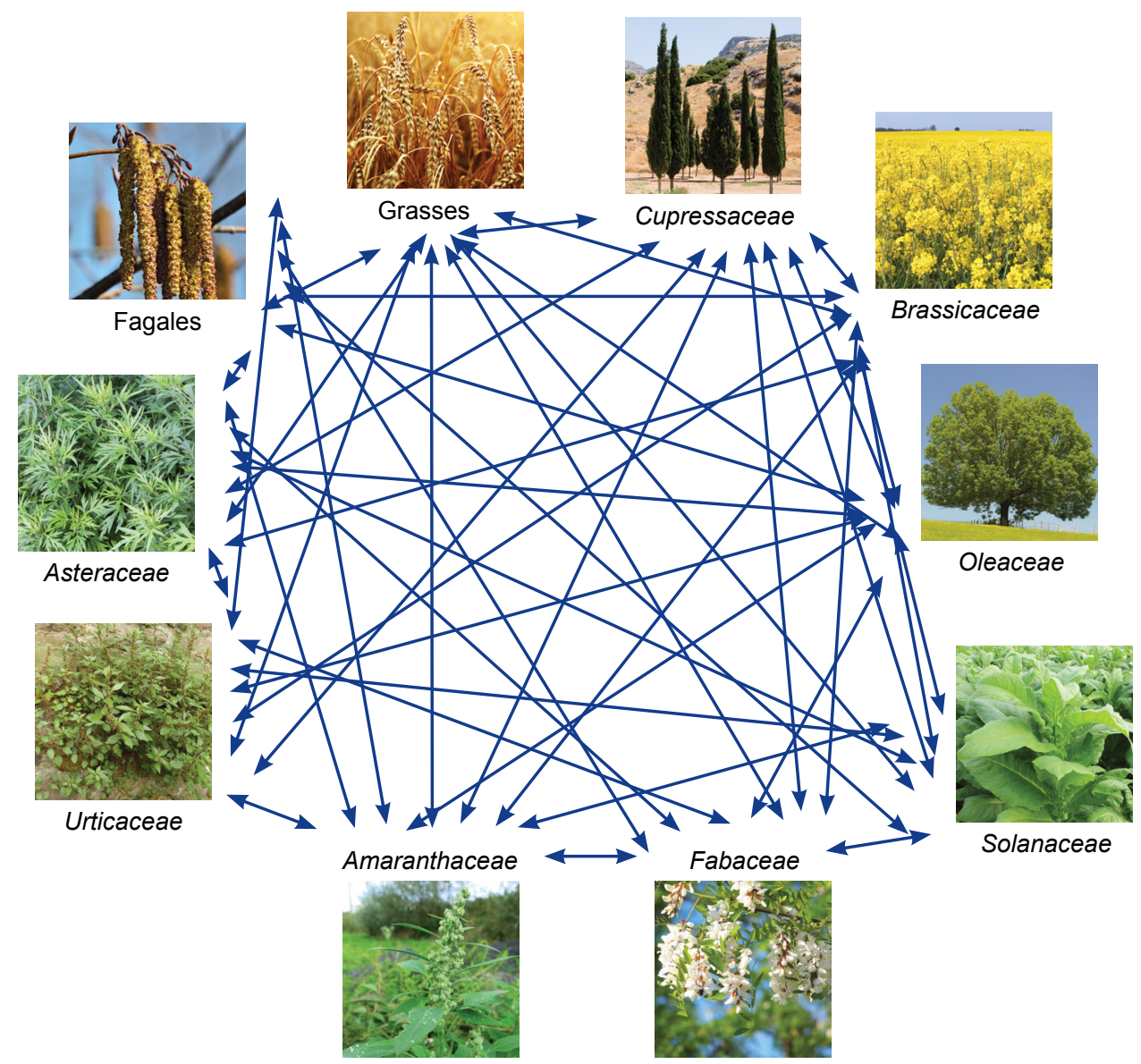

Figure 5

Cross-reactivity among polcalcins of different allergenic sources.

\section{CLINICAL RELEVANCE}

Themembers of this protein familyhavebeen identified as potent allergens. However, it is considered a minor allergen in all populations studied, since the reported allergenic prevalence of this family of proteins varies between 5 and $46 \%$. Che a 3 , a polcalcin from Chenopodium album, common in semi-desert areas, is an atypical polcalcin because it showed reactivity up to $46 \%$ of sera from individuals with chenopod allergy (8). The high prevalence of this panallergen in these patients might have a relationship with their characteristic polysensitization. Moverare et al. (9) compared different European populations regarding the reactivity of Bet $\mathrm{v}$, prevalence values between 5 and $11 \%$ for patients from North and Central Europe, and $27 \%$ for Italian patients. All these data point to the existence of a certain correlation between the poly-sensitization degree and geographical area and

\section{Textbox 2}

\section{Clinical relevance}

- Polcalcins are minor allergens in patients sensitised to grass, tree or weed pollen

- Are markers for cross-reactivity between pollen, but not present in plant foods.

- Unknown clinical relevance for respiratory symptoms

- Isolated polcalcins have extensive crossreactivity

- Diagnosis of patients sensitized to polcalcins can be performed with any member of the family

- Can be considered a marker of polysensitisation 


\section{Table 3}

Prevalence of polcalcin sensitisation in patients with pollen allergy

\begin{tabular}{|lcc|}
\hline $\begin{array}{l}\text { Main Sensitisation } \\
\text { of the Population } \\
\text { Studied }\end{array}$ & $\begin{array}{c}\text { Prevalence of sensitisation } \\
\text { to Polcalcins }\end{array}$ & Reference \\
\hline Birch & Bet v $4=5 \%$ & $(1)$ \\
\hline Chenopodium/Salsola & $\begin{array}{c}\text { Che a 3= 46\%; Che a 3= } \\
41 \%\end{array}$ & $(8,13)$ \\
\hline Olive & Ole e 3=20-30\% & $(11,14)$ \\
\hline Grass & Phl p 7 $=2-10 \%$ & $(11,15,16)$ \\
\hline Alnus & Aln g $4=18 \%$ & $(17)$ \\
\hline Robinia pseudoacacia & Che a 3=33\% & $(12)$ \\
\hline Ash & Fra a 3 $=16 \%$ & $(7)$ \\
\hline Cupresus/Cypress & Cup a $4=10 \%$ & $(18)$ \\
\hline Polysensised to pollen & Polcalcin= 31\% & $(19)$ \\
\hline
\end{tabular}
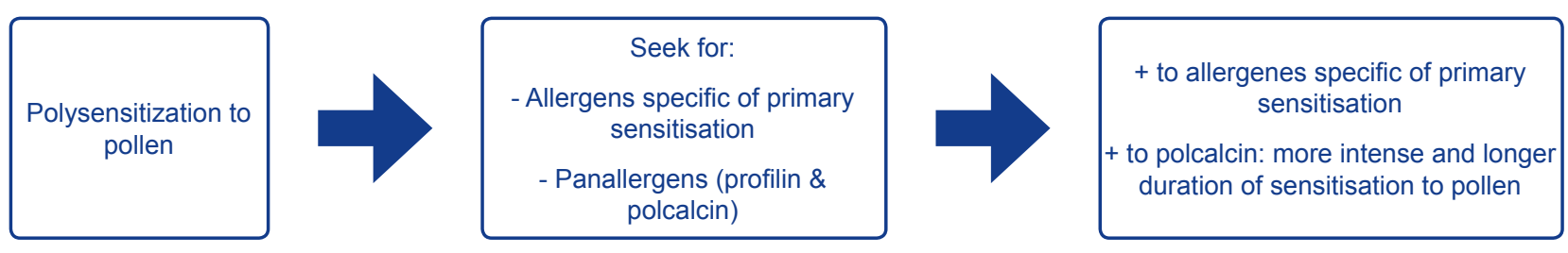

\section{Figure 5}

\section{Diagnostic algorithm for patients polysensitized to pollen}

prevalence of sensitisation minor allergens. In Table 87 are described the percentage of sensitisation to different polcalcins in patients with pollen allergy. Barber et al. (10) described that patients who were simultaneously sensitized to polcalcins and profilins, there was a duplication both in the number of sensitizations to major allergens and in the years of disease evolution. Similar findings were obtained by Orovitg et al. (11). Presence of IgE positivity to polcalcin in patients with pollen allergy is not a contraindication for AIT prescription. Otherwise, minimal quantity of polcalcin is present in commercial preparations for immunotherapy, thus making the treatment ineffective regarding this selected allergen.

\section{CLINICAL MANAGEMENT}

Polcalcins are only expressed in pollen, thus there is no connection with food allergy. It is considered as a panallergen and therefore could be a confounding factor in the diagnosis of polysensitized pollenallergic patients and may lead to a diagnosis of "allergy mirages" (12) (Fig. 5). Can also be considered a marker of a longer duration of sensitisation and of a more severe respiratory disease. Polcalcins are not commercially available for SPT, however, in some research articles an extract derived from palm pollen, which was prepared by ALK (Madrid, Spain) was used. For specific IgE determinations there are currently only two polcalcins available; Phl p 7 and Bet $v 4$. 


\section{Case 1}

Clinical History: Male, 26 years, with a 10-year history of rhinitis and asthma during spring. No complaint of adverse reaction to food.

Test with extracts: Skin prick test showed positive reaction to grass, olive, cypress, plantago. Due to extent polysensitisation to pollen a molecular diagnosis was performed before to give an indication for immunotherapy.

Test with molecules: Specific lgE was positive $\mathrm{Phl} p$ 1, Phl p 5, Phl p 12, Phl p 7 and negative to Ole e 1, Cup a 1, Pla I 1.

Conclusion: Results indicate a primary sensitisation only to grass pollen and panallergens (profilin and polcalcin), which confirm a longer duration of the respiratory symptoms and the severity of the disease. Immunotherapy with a grass pollen extract was prescribed.

\section{References}

1. Engel $E$, Richter $K$, Obermeyer $G$, Briza $P$, Kungl AJ, Simon B et al. Immunological and biological properties of Bet $v 4$, a novel birch pollen allergen with two EF-hand calcium-binding domains. J Biol Chem 1997;272:28630-28637.

2. Niederberger $\mathrm{V}$, Hayek B, Vrtala, Laffer S, Twardosz A, Vangelista L et al. Calcium-dependent immunoglobulin $\mathrm{E}$ recognition of the apo- and calcium-bound form of a cross-reactive two EF-hand timothy grass pollen allergen, Phl p 7. FASEB J 1999;13:843-856.

3. Henzl MT, Davis ME, Tan A. Polcalcin divalent ion-binding behavior and thermal stability: comparison of Bet v 4, Bra n 1, and Bra n 2 to Phl p 7. Biochemistry 2010;49:2256-2268.

4. Ledesma A, Barderas R, Westritschnig K, Quiralte J, Pascual CY, Valenta R et al. A comparative analysis of the cross-reactivity in the polcalcin family including Syr $v 3$, a new member from lilac pollen. Allergy 2006;61:477484.

5. Verdino P, Barderas R, Villalba M, Westritschnig K, Valenta $\mathrm{R}$, Rodriguez $\mathrm{R}$ et al. Three-dimensional structure of the cross-reactive pollen allergen Che a 3: visualizing crossreactivity on the molecular surfaces of weed, grass, and tree pollen allergens. J Immunol 2008;180:2313-2321.

6. Ohkuri T, Nagatomo S, Oda K, So T, Imoto T, Ueda T. A protein's conformational stability is an immunologically dominant factor: evidence that free-energy barriers for protein unfolding limit the immunogenicity of foreign proteins. J Immunol 2010;185:4199-4205.

7. Mas S, Garrido-Arandia M, Batanero, Westritschnig $\mathrm{K}$, Valenta R, Rodriguez $\mathrm{R}$ et al. Characterization of profilin and polcalcin panallergens from ash pollen. J Investig Allergol Clin Immunol 2014;24:257-266.

8. Barderas R, Villalba M, Pascual CY, Batanero E, Rodrìguez R. Profilin (Che a 2) and polcalcin (Che a 3) are relevant allergens of Chenopodium album pollen: isolation, amino acid sequences, and immunologic properties. J Allergy Clin Immunol 2004;113:1192-1198.

9. Movérare $\mathrm{R}$, Larsson $\mathrm{H}$, Carlsson $\mathrm{R}$, Holmquist I. Mugwort-sensitized individuals from North Europe, South Europe and North America show different IgE reactivity patterns. Int Arch Allergy Immunol 2011;154:164-172.

10. Barber D, de la Torre F, Lombardero M, Antépara I, Colas C, Dávila I, Tabar AI, Vidal C, Villalba M, Salcedo $\mathrm{G}$, Rodríguez R. Component-resolved diagnosis of pollen allergy based on skin testing with profilin, polcalcin and lipid transfer protein pan-allergens. Clin Exp Allergy 2009;39:1764-1773.

11. Orovitg A, Guardia P, Barber D, de la Torre F, Rodríguez $\mathrm{R}$, Villalba $\mathrm{M}$ et al. Enhanced diagnosis of pollen allergy using specific immunoglobulin $\mathrm{E}$ determination to detect major allergens and panallergens. J Investig Allergol Clin Immunol 2011;21:253-259.

12. Compés E, Hernández E, Quirce $S$, Palomares $O$, Rodríguez R, Cuesta J et al. Hypersensitivity to black locust (Robinia pseudoacacia) pollen: "allergy mirages". Ann Allergy Asthma Immunol 2006;96:586592.

13. Nouri $H$, Varasteh A, Afsharzadeh D, Tehrani M, Nasiraii LR, Sankian M. Diagnosis of Chenopodium album allergy with a cocktail of recombinant allergens as a tool for componentresolved diagnosis. Allergy 2011;66:530531.

14. Martínez A, Asturias JA, Monteseirín J, Moreno V, GarcíaCubillana A, Hernández $M$ et al. The allergenic relevance of profilin (Ole e 2) from Olea europea pollen. Allergy 2002;57(Suppl 71):17-23.

15. Darsow U, Brockow K, Pfab F, Jakob T, Petersson CJ, Borres MP et al. Allergens. Heterogeneity of molecular sensitization profiles in grass pollen allergy - implications for immunotherapy? Clin Exp Allergy 2014;44:778-786.

16. Sastre J, Rodríguez F, Campo P, Laffond E, Marín A, Alonso MD. Adverse reactions to immunotherapy are associated with different patterns of sensitization to grass allergens. Allergy 2015;70:598-600.

17. Hayek B, Vangelista L, Pastore A, Sperr WR, Valent $P$, Vrtala $S$ et al. Molecular and immunologic characterization of a highly cross-reactive two EFhand calcium-binding alder pollen allergen, Aln g 4: structural basis for calcium-modulated IgE recognition. J Immunol 1998;161:7031-7039. 
18. Pico de Coaña Y, Parody N, Fuertes MÁ, Carnés $\mathrm{J}$, Roncarolo D, Ariano R et al. Molecular cloning and characterization of Cup a 4, a new allergen from Cupressus arizonica. Biochem Biophys Res Commun 2010;401:451-457.
19. Asero R. Hypersensitivity to pollen panallergens (profilin and polcalcin) detected in vitro and in vivo: a comparative analysis. I Investig Allergol Clin Immunol 2011;21:323-324. 


\section{PART C}

CROSS-REACTIVE MOLECULES \& THEIR CLINICAL RELEVANCE

\section{LIPOCALINS}

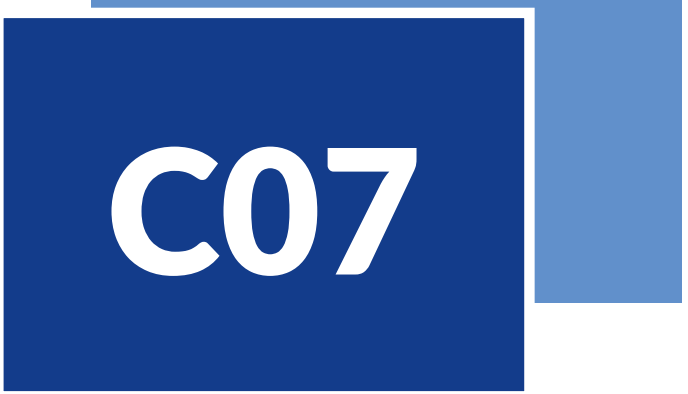

\section{Christiane Hilger}

\section{THE PROTEIN}

Equ $\mathrm{c}$ 1, the major allergen of horse, was one of the first lipocalins to be isolated, cloned and characterized (Table 1) (1). The determination of its three-dimensional structure classified it as lipocalin (Fig. 1) (2). Equ c 1 was found to have surfactant properties; it lowers the surface tension of liquids and could play a role in sweat evaporation. Equ c 1 purified from horse sweat contains oleamide, an endogenous bioactive substance, as well as other small organic molecules. The physiological role of Equ c 1 is still under investigation.

Horse allergens are readily detectable in stables (3). However they disperse poorly in the air as allergens are not detectable beyond $50 \mathrm{~m}$ from the stable. They stick to clothes and are readily transported home or to public places. They are detectable in classrooms when many children have regular contact with horses.

The characterization of B and T cell epitopes is still under investigation and no strategies have been developed so far for immunotherapy.

Common tertiary structure with low sequence identity among family members

Cross-reactive sub-group with high sequence identity

Airborne, easily spreading into indoor environment

Risk factor for respiratory symptoms and asthma

Impact of individual molecules on severity of symptoms is still unknown 


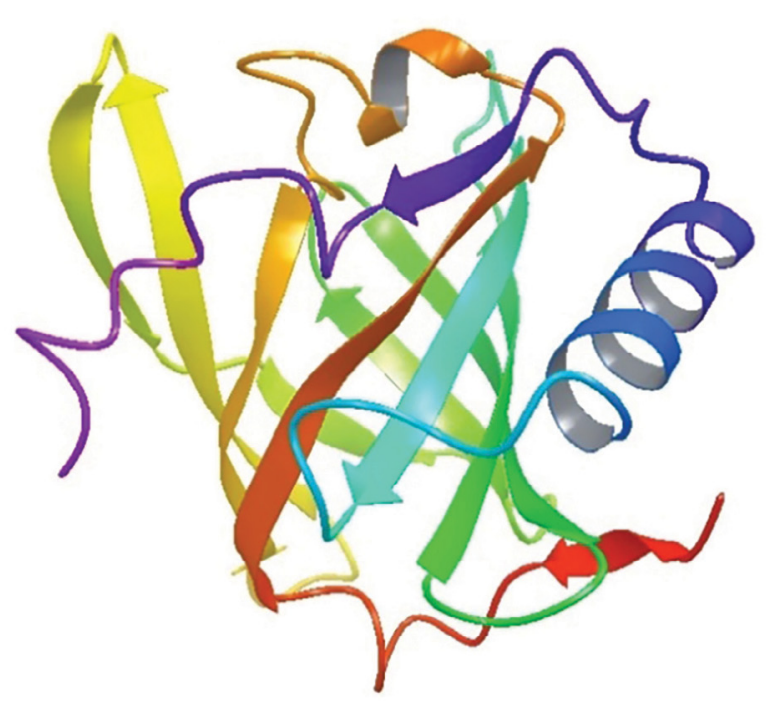

Figure 1

Ribbon model of the three-dimensional structure of Equ c 1 (PDB 1EW3). N-terminal end in red, C-terminal end in purple.

Lipocalin Family Characteristics
- Common tertiary structure with central
- Darrel
- Divergent sequences with low identity
- Sub-group with high sequence identity
- Airborne, easily spreading into indoor
environment

\section{THE FAMILY}

The majority of the mammalian allergens are lipocalins (4) (Table 2). Lipocalins are proteins that are ubiquitous; they are present also in arthropods, plants and bacteria, and have very diverse functions. They are characterized by a common tertiary structure composed of a central $\beta$-barrel formed of eight anti-parallel $\beta$-strands. The internal binding pocket carries small hydrophobic molecules such as retinol, steroids, pheromones and odorants. Mammalian allergens isolated so far are mostly odorant and pheromone binding lipocalins. Only few natural ligands have been characterized. Lipocalin

\section{Table 1}

Basic protein characteristics of Equ c 1

\begin{tabular}{|ll|}
\hline \multicolumn{2}{|l|}{ Protein characteristics } \\
\hline Allergen source & Equus caballus, horse \\
\hline Protein family & lipocalin \\
\hline $\begin{array}{l}\text { UniProtKB accession } \\
\text { No }\end{array}$ & Q95182 \\
\hline $\begin{array}{l}\text { Three dimensional } \\
\text { structure available }\end{array}$ & yes \\
\hline Molecular structure & mainly beta-sheet \\
\hline $\begin{array}{l}\text { Theoretical molecular } \\
\text { weight }\end{array}$ & 20.097 kDa \\
\hline $\begin{array}{l}\text { Molecular weight } \\
\text { measured by mass } \\
\text { spectrometry }\end{array}$ & 22.0 kDa \\
\hline Length & $\begin{array}{l}187 \text { amino acids, mature } \\
\text { protein } 172\end{array}$ \\
\hline Ligand binding & yes \\
\hline Dimerization & homodimer \\
\hline Glycosylation & yes \\
\hline Disulfide bonds & 1 \\
\hline Isoelectric point & 4.51 \\
\hline Synthesis & $\begin{array}{l}\text { Sublingual gland, low levels in } \\
\text { submaxillary gland and liver, } \\
\text { secreted }\end{array}$ \\
\hline Distribution & fur, saliva, urine \\
\hline
\end{tabular}

allergens are present in dander, saliva and urine. They stick to particles and become easily airborne and transported to public places such as schools or day-care centres (3-5).

Lipocalins are small, secreted molecules of 150-250 amino acids. Despite their highly conserved structure, they display little sequence identitiy, usually between 20 and $30 \%$. Therefore lipocalins were considered as species-specific allergy markers. The further isolation of newallergen molecules showed that some lipocalins have much higher sequence identities, which can be as high as $67 \%$. In inhibition studies they were able to cross-react at low doses (6-8). Representatives of this cross-reactive group are Equ c 1, Fel d 4 and Can f 6. However, even between molecules of low general sequence identity such as Fel d 4 and Can f 2 (25\% identity), single epitopes may have short stretches 
Table 2

Bos d 5-homologous allergens from animals

\begin{tabular}{|c|c|c|}
\hline $\begin{array}{l}\text { Animal } \\
\text { family }\end{array}$ & Allergen source & Allergen \\
\hline Bovidae & $\begin{array}{l}\text { Domestic cattle } \\
\text { (Bos domesticus) }\end{array}$ & $\begin{array}{l}\text { Bos d } 2 \\
\text { Bos d } 5\end{array}$ \\
\hline Canidae & Dog (Canis familiaris) & $\begin{array}{l}\text { Can f } 1 \\
\text { Can f } 2 \\
\text { Can f } 4 \\
\text { Can f } 6\end{array}$ \\
\hline Cavidae & Guinea-pig (Cavia porcellus) & $\begin{array}{l}\text { Cav p } 1 \\
\text { Cav p } 2 \\
\text { Cav p } 3 \\
\text { Cav p } 6\end{array}$ \\
\hline Cricetidae & $\begin{array}{l}\text { Golden hamster (Mesocricetus } \\
\text { auratus) }\end{array}$ & Mes a 1 \\
\hline Equidae & Domestic horse (Equus caballus) & $\begin{array}{l}\text { Equ c } 1 \\
\text { Equ c } 2\end{array}$ \\
\hline Felidae & Cat (Felis domesticus) & $\begin{array}{l}\text { Fel d } 4 \\
\text { Fel d } 7\end{array}$ \\
\hline Leporidae & Rabbit (Oryctolagus cuniculus) & $\begin{array}{l}\text { Ory c } 1 \\
\text { Ory c } 4\end{array}$ \\
\hline \multirow{2}{*}{ Muridae } & Mouse (Mus musculus) & Mus $\mathrm{m} 1$ \\
\hline & Rat (Rattus norvegicus) & Rat n 1 \\
\hline
\end{tabular}

of sequence identity and lead to patient-dependent IgE-cross-reactivity (9). Can $f 4$ has been reported to show some cross-reactivity with a putative bovine allergen with both molecules sharing only $37 \%$ of sequence identity (10).

Table 3 displays two-by-two comparisons of amino acid identities between a subgroup of lipocalins. All pairs with a high sequence identity are potentially cross-reactive. The challenge of further studies is to analyze which lipocalins are adequate speciesspecific markers and which are markers of crossreactivity. Sequence alignments are good primary prediction tools for cross-reactivity, but most importantly the use of IgE-inhibition studies with sera from clinically well-characterized patients will allow defining clinically relevant cross-reactivity.

A comparison of the surfaces of some members of the cross-reactive lipocalin subgroup visualizes potential cross-reactive B cell epitopes (Fig. 2A). In contrast, an analogous surface representation of a non-cross-reactive group of lipocalins shows a very limited epitope cross-reactivity (Fig. 2B).

The objective of further studies will be the identification of marker molecules for each animal species in order to clearly identify the sensitizing allergen source. Fig. 3 shows documented as well as putative cross-reactive molecules.

\section{Table 3}

Amino acid identities (\%) between lipocalins with high sequence identity

\begin{tabular}{|c|c|c|c|c|c|c|c|c|c|}
\hline Equ c 1 & & & & & & & & & \\
\hline Fel d 4 & 67 & & & & & & & & \\
\hline Fel d 7 & 26 & 20 & & & & & & & \\
\hline Can $\mathrm{f} 1$ & 28 & 26 & 62 & & & & & & \\
\hline Can $\mathrm{f} 6$ & 57 & 67 & 25 & 26 & & & & & \\
\hline Cav p 6 & 48 & 53 & 24 & 24 & 53 & & & & \\
\hline Mus $\mathrm{m} 1$ & 46 & 49 & 22 & 21 & 47 & 45 & & & \\
\hline Ory c 4 & 52 & 63 & 25 & 24 & 58 & 49 & 51 & & \\
\hline Rat $\mathrm{n} 1$ & 47 & 55 & 24 & 23 & 52 & 50 & 64 & 54 & \\
\hline & Equ c 1 & Fel d 4 & Fel d 7 & Can $\mathrm{f} 1$ & Can $\mathrm{f} 6$ & Cav p 6 & Mus m 1 & Ory c 4 & Rat $\mathrm{n} 1$ \\
\hline
\end{tabular}

Blue shaded areas indicate lipocalin pairs with $>50 \%$ amino acid sequence identity, figures in bold blue indicate documented lgE-cross-reactivity between lipocalins 

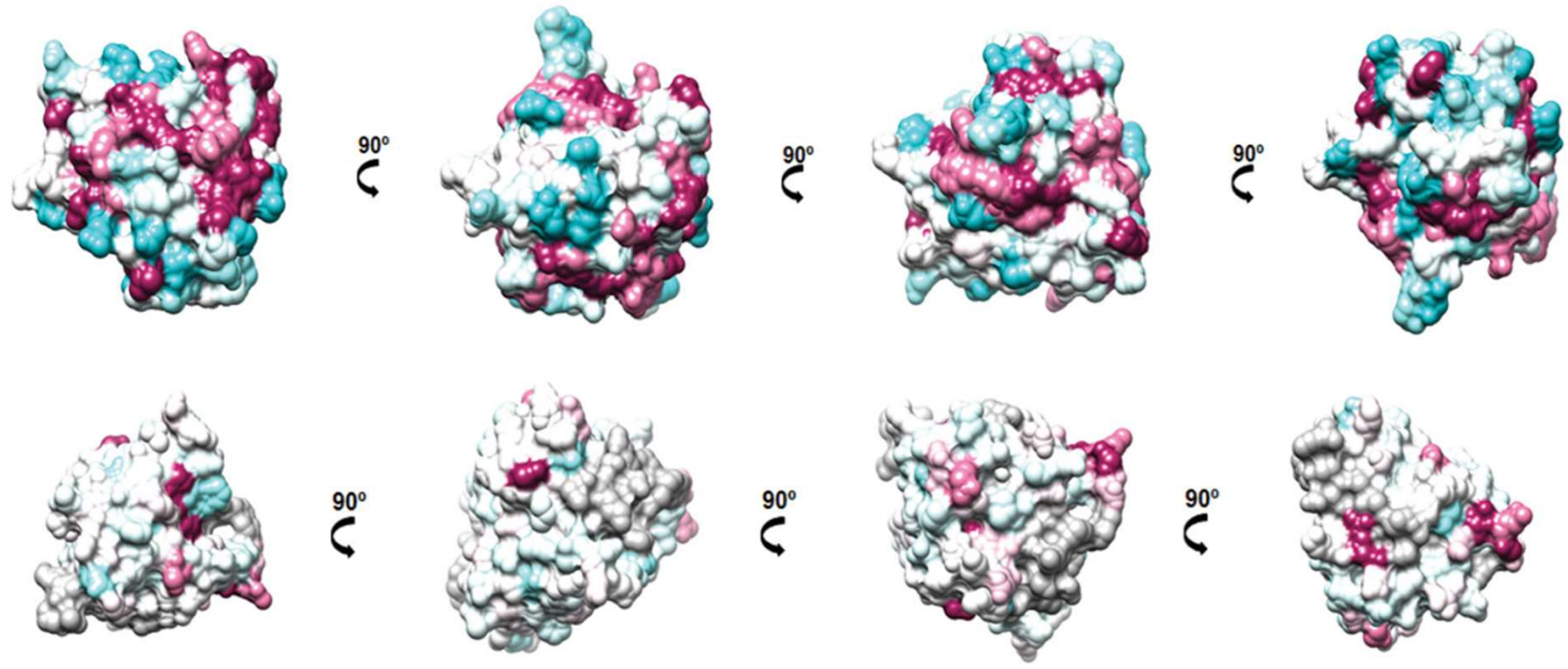

Figure 2

Surface representation of lipocalin comparisons A). Comparison of the cross-reactive lipocalin group from horse (Equ c 1), cat (Fel d 4), and dog (Can f 6). Surface representation of Equ c 1 (1EW3) colored by sequence conservation with Fel d 4, Can f 6. (figure kindly provided by Frontiers in Immunology (11) ) B) Comparison of non-cross-reactive lipocalins from dog (Can $f$ 1, Can $f$ 2, Can $f$ 4). Surface representation of Can $f 2$ (3L4R) colored by sequence conservation with Can $f 1$ and Can $f 4$. Sequence conservation was calculated by Consurf (16). The highly variable sequence conservation was identified as deep blue, the average was in white and the conserved sequences are denoted in deep red (Figure provided by $\mathrm{K}$ Arumugam, Luxembourg Institute of Health).

\section{CLINICAL RELEVANCE}

All mammalian lipocalin allergens are respiratory allergens, with the exception of the $\beta$-lactoglobulins (e.g.Bos d 5), which are present in milk (4). They are major allergens of different furry pets and are shed into the environment by animal dander and secretions. They stick to clothes and human hair and are passively transferred to public places (3). Allergens quantified in airborne dust in schools have been shown to attain levels that are able to sensitize children or to even stimulate asthma exacerbations. Up to $50 \%$ of the households in industrialized countries have a pet. Twenty-four percent of European households have a cat, $25 \%$ have a dog and about 6-8\% own a small mammal. Particularly families with children more frequently own a pet.

Allergy to furry animals is considered a risk factor for development of asthma (12). The role of single allergen molecules as markers of severe or mild disease has not yet been investigated in detail. A multi-sensitization to several components (lipocalin,

\section{Textbox 2}

\section{Clinical relevance}

- Up to $50 \%$ of households have a pet

- Risk factor for respiratory symptoms and asthma

- Impact of individual molecules on severity of symptoms is still unknown

- No molecule based therapeutic approach available

kallikrein, secretoglobin) seems to be associated with increased bronchial inflammation in severe asthmatics. In cat-, dog- or horse-allergic children, sensitization to Can $f 2$ and Equ c 1 was more common in severe than in controlled asthma (13).

For the moment, the best treatment seems to be allergen avoidance. However this is not always feasible as it is often difficult to get accepted by 


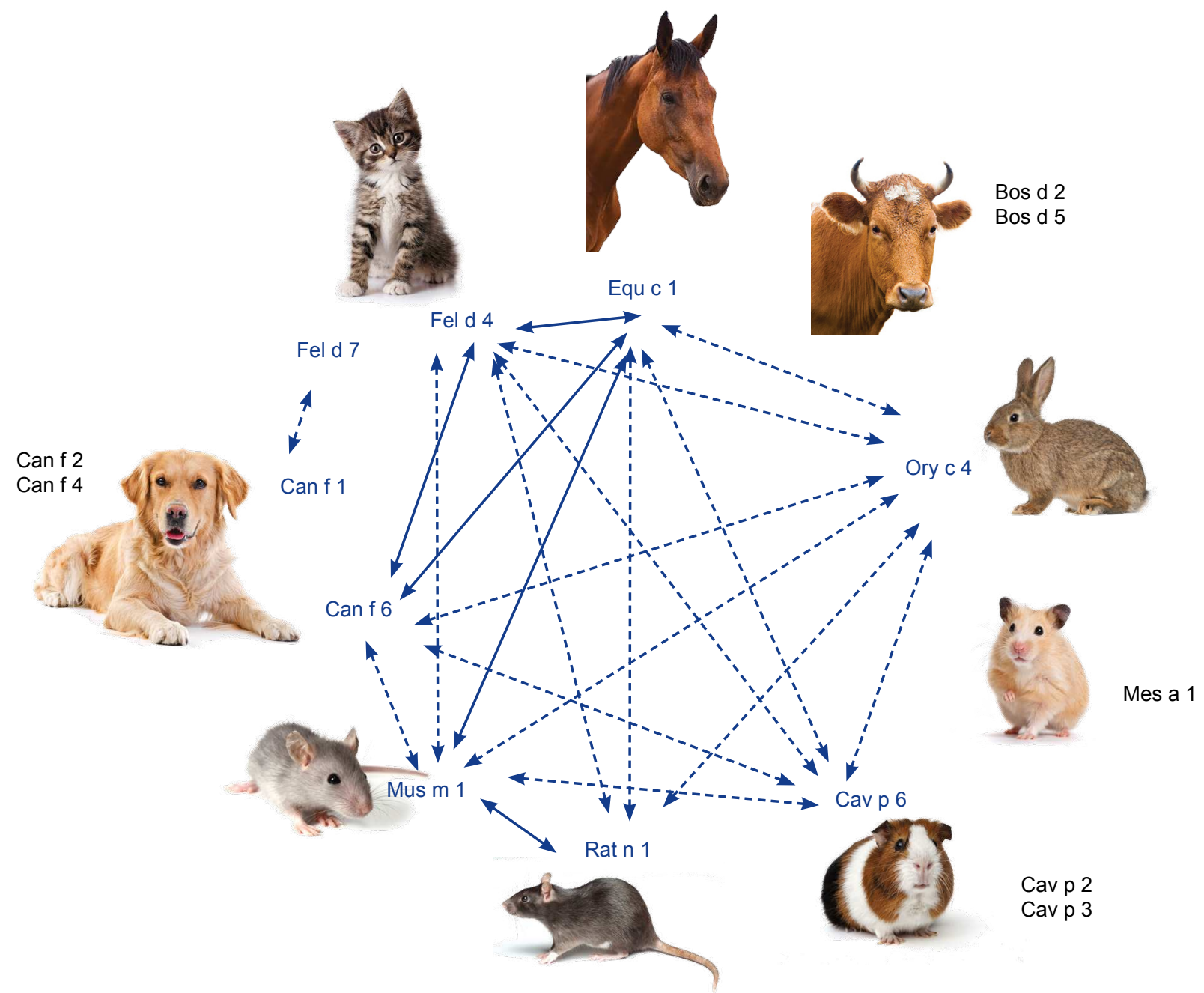

Figure 3

Cross-reactivities among allergenic lipocalins based on sequence identity. Lines represent documented IgE-cross-reactivity, dotted lines represent potential lgE-cross-reactivity based on high sequence identities.

the patients. In addition, as pets are kept in many households, severely allergic patients might get socially excluded if trying to avoid allergens. The only immunotherapies currently available are made of animal dander extracts. Results of subcutaneous immunotherapy (SCIT) have shown a benefit in catallergic patients with asthma and rhinoconjunctivitis. Results for dog SCIT are less convincing. A number of experimental therapies using peptides or recombinant designer molecules are being developed for Fel $\mathrm{d} 1$ (14). Before being able to develop analogous lipocalin vaccine reagents, much more research has to be done to determine the relevant molecules and their epitopes.

\section{CLINICAL MANAGEMENT}

A careful record of the clinical history such as the presence of pets at home or regular pet contact is of great value. Skin prick test or specific lgE using animal dander will confirm animal sensitization. As animal dander contains cross-reactive molecules such as serum albumins, some of the cross-reactive lipocalins and potentially other cross-reactive molecules, it is important to define the primary allergenic source, especially if a specific immunotherapy is intended. Co-sensitization has to be distinguished from crosssensitization. It is important to acknowledge that IgEcross-reactivity may not always imply clinical crossreactivity. 


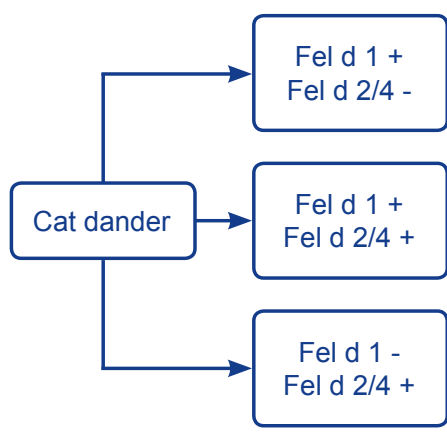

Figure 4
Primary sensitization to cat

Primary sensitization to cat, potentially cross-sensitization or cosensitization to other animal

Cross-sensitization to cat Check for primary source

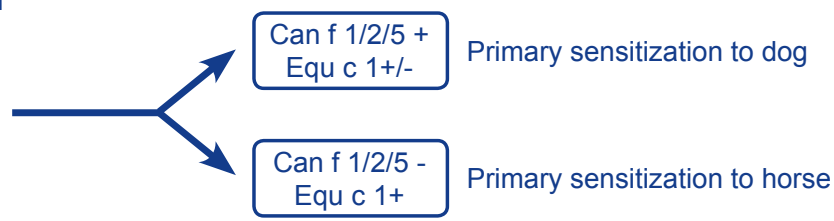

Added value of the use of single allergens in the case of a positive IgE test to cat dander.

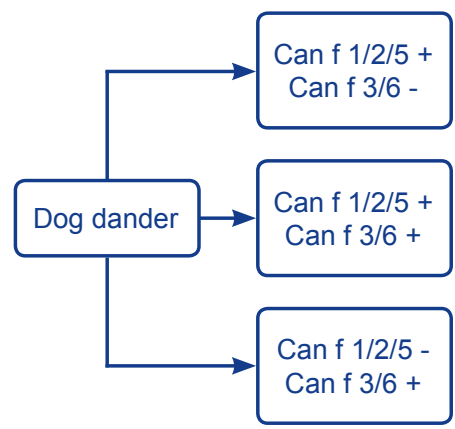

Primary sensitization to dog

Primary sensitization to dog, potentially cross-sensitization or cosensitization to other animal

Cross-sensitization to dog Check for primary source

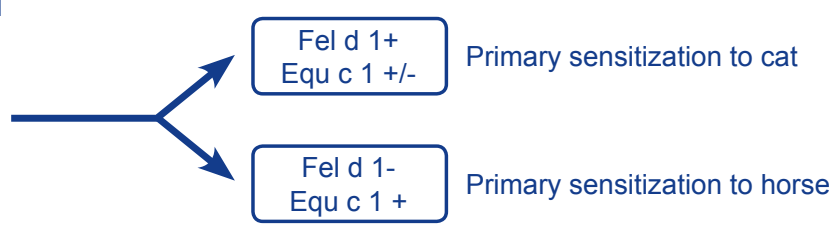

Figure 5

Added value of the use of single allergens in the case of a positive IgE test to dog dander.

At the current state of the art, Fel $d 1$, Can $f 1$, Can $\mathrm{f} 2$ and Can $\mathrm{f} 5$ are commercially available speciesspecific markers of sensitization. Equ c 1 may crossreact with Fel $d 4$ and Can $f$, Mus $m 1$ may crossreact with Rat $n 1$. If the clinical history does not allow a clear identification of the sensitizing animal, the following decision tree (Fig. 4-6) will help to orient the use of components. Unfortunately the number of commercially available components is still limited. The coverage is rather good for cat and dog, but only two cross-reactive molecules, Equ c 1 and Equ c 3, are available for horse and no molecules are available for component-resolved diagnosis of small furry pets.

\section{CLINICAL CASES}

\section{Case 1 (published (15))}

Clinical history: A 24-year-old man presented at the clinic with a 14-year history of rhinitis and asthma when exposed to horses and a 2 years history of rhinitis when exposed to dogs.

Test with extracts: Specific IgE to horse dander were elevated (92 kUA/L), they were moderate to dog (7.2 kUA/L).

Test with molecules: Specific IgE were detected to Equ c 1 (18 kUA/L). All commercially available dog allergens (Can $f 1,2,3,5$ ) were negative. However IgE to Can $f 6$ were clearly positive (3.7 kUA/L). Inhibition assays showed that IgE-recognition of Can $f \quad 6$ could be totally inhibited by low doses of Equ c 1.

Conclusion: In this particular case, clinical symptoms to dog were due to cross-reactivity of Can $\mathrm{f} 6$ with Equ c 1.

\section{Case 2 (published (7))}

Clinical history: A 30-year-old women had asthma upon exposure to her cat. 


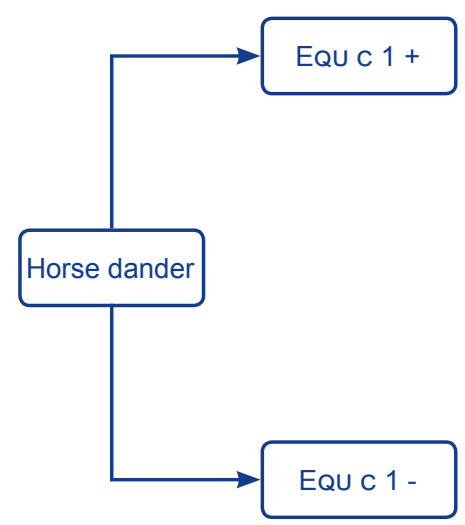

Could be primary sensitization to horse or cross-reactivity to other animals

$\lg$ E to different horse allergen, but primary source or

Cross-reactive allergen in dander Check for primary sensitization source
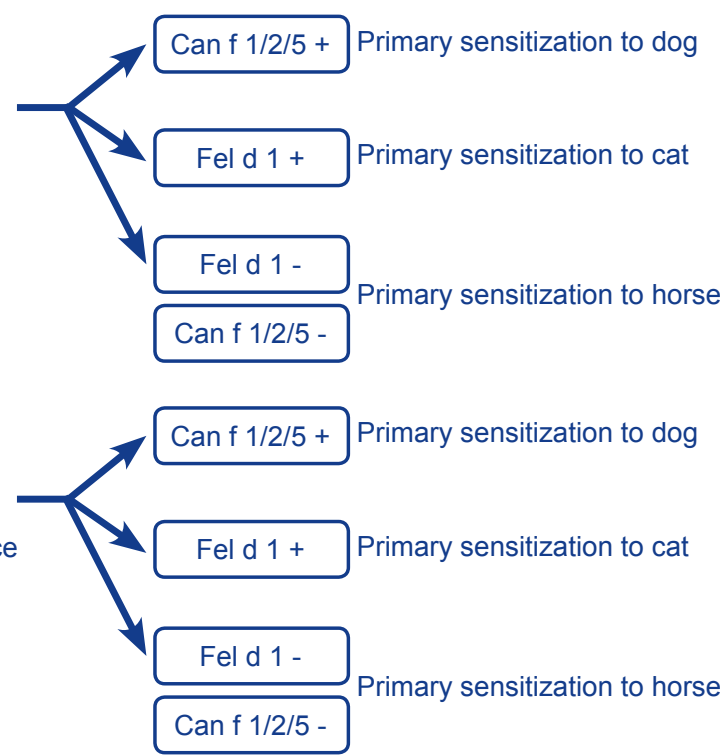

Added value of the use of single allergens in the case of a positive IgE test to horse dander.

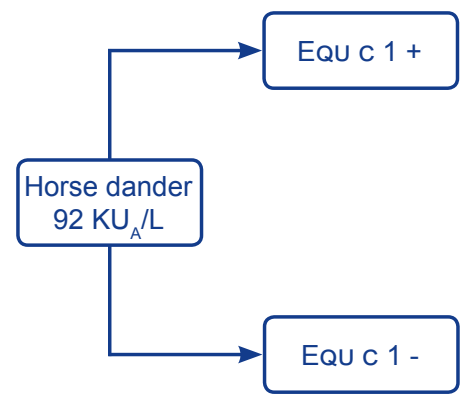

Could be primary sensitization to horse or cross-recativity to other animals

Figure 7

Diagnostic procedure for case 1.

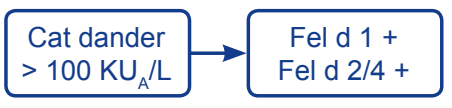

Figure 8
Primary sensitization to cat, potentially cross-sensitization to other animal. Check for cosensitization to dog.

Diagnostic procedure for case 2.

Test with extracts: Specific IgE were positive for cat (>100 kUA/L) and dog dander (9 kUA/L).

Test with molecules: The patient had specific IgE against Fel d 1 (51 kUA/L) and Fel d 4 (51 kUA/L), but Fel d 2, Can f 1, Can f 2 and Can $\mathrm{f} 3$ were negative. Specific lgE to the cross-reactive Can $f \quad 6$ were 18 kUA/L. These could be completely inhibited by Fel

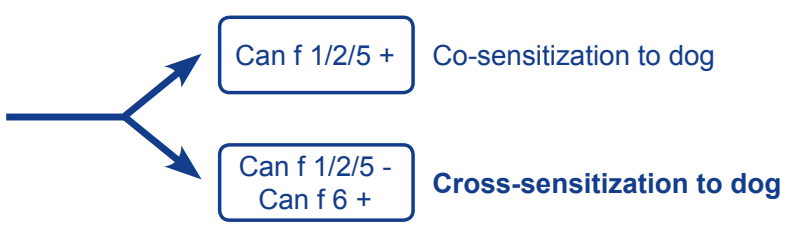

d 4, suggesting cat as the primary allergen source.

Conclusion: Specific IgE were positive to cat and dog, but the presence of specific lgE to the marker allergen Fel $d 1$ as well as the absence of specific IgE to Can $f 1$ or Can $f 2$ confirmed that cat was the primary allergen source and that Can $\mathrm{f} 6$ was a IgE-cross-reacting allergen in dog. 


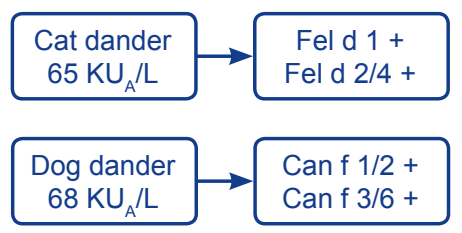

Primary sensitization to cat

potentially cross-sensitization to other animal.

Primary sensitization to dog

potentially cross-sensitization to other animal.

\section{Figure 9}

Diagnostic procedure to case 3.

\section{Case 3 (published (830))}

Clinical history: A 53-year-old man presents with respiratory symptoms upon exposure to cat and dog.

Test with extracts: Specific IgE were positive for cat ( $65 \mathrm{kUA} / \mathrm{L})$ and dog dander (68 kUA/L)

Test with molecules: The patient had specific IgE against Fel d 1 (35.8 kUA/L), Fel d 2 (0.7 kUA/L), Fel d 4 (45 kUA/L), Can f 1 (26 kUA/L), Can f 2 (13.5 $\mathrm{kUA} / \mathrm{L})$, Can $\mathrm{f} 3$ (0.2 kUA/L) and Can $\mathrm{f} 6$ (33 kUA/L).

Conclusion: The presence of IgE to the specific markers Fel $\mathrm{d} 1$, Can $\mathrm{f} 1$ and Can $\mathrm{f} 2$ argues for co-sensitization of cat and dog. Inhibition and cross-inhibition studies with Can $\mathrm{f} 6$ and Fel $\mathrm{d} 4$ showed weak inhibition, confirming the hypothesis of co-sensitization.

Allergen nomenclature: Fel d 1, cat uteroglobin; Can f 1, Can f 2, Can f 6, Equ c 1, Fel d 4, dog, horse and cat lipocalins; Can $f 3$, Fel $d$ 2: dog and cat serum albumins; Can $f$, dog kallikrein.

\section{References}

1. Gregoire C, Rosinski-Chupin I, Rabillon J, Alzari PM, David B, Dandeu JP. cDNA cloning and sequencing reveal the major horse allergen Equ c1 to be a glycoprotein member of the lipocalin superfamily. J Biol Chem 1996;271:32951-32959.

2. Lascombe MB, Gregoire C, Poncet $P$ et al. Crystal structure of the allergen Equ $\mathrm{c}$ 1. A dimeric lipocalin with restricted IgE-reactive epitopes. J Biol Chem 2000;275:21572-21577.

3. Zahradnik E., Raulf M. Animal Allergens and Their Presence in the Environment. Front Immunol. 2014;5: 76.

4. Hilger C, Kuehn A, Hentges F. Animal lipocalin allergens. Curr Allergy Asthma Rep 2012;12:438-447.

5. Salo PM, Sever ML, Zeldin DC. Indoor allergens in school and day care environments. J Allergy Clin Immunol 2009;124:185-192.

6. Saarelainen $S$, Rytkönen-Nissinen $M$, Rouvinen J, Taivainen A, Auriola S, Kauppinen A et al. Animalderived lipocalin allergens exhibit immunoglobulin $\mathrm{E}$ cross-reactivity. Clin Exp Allergy 2008;38:374-381.
7. Hilger C, Swiontek K, Arumugam K, Lehners C, Hentges F. Identification of a new major dog allergen highly cross-reactive with Fel d 4 in a population of cat- and dog-sensitized patients. J Allergy Clin Immunol 2012;129:1149-1151.

8. Nilsson OB, Binnmyr J, Zoltowska A, Saarne T, van Hage $M$, Grönlund $H$. Characterization of the dog lipocalin allergen Can $f$ 6: the role in cross-reactivity with cat and horse. Allergy 2012;67:751-757.

9. Madhurantakam $\mathrm{C}$, Nilsson $\mathrm{OB}$, Uchtenhagen $\mathrm{H}$, Konradsen J, Saarne T, Högbom E,et al. Crystal structure of the dog lipocalin allergen Can $f$ 2: implications for cross-reactivity to the cat allergen Fel d 4. J Mol Biol 2010;401:68-83.

10. Mattsson L, Lundgren T, Olsson P, Sundberg M, Lindholm J. Molecular and immunological characterization of Can $\mathrm{f}$ 4: a dog dander allergen cross-reactive with a 23 kDa odorant-binding protein in cow dander. Clin Exp Allergy 2010;40:1276-1287.

11. Hentges F, Leonard C, Arumugam K, Hilger C Immune responses to inhalant mammalian allergens. Front Immunol 2014;5:234.

12. Konradsen JR, Fujisawa T, van Hage M, Hedlin G, Hilger C, Kleine-Tebbe J, Matsui EC, Roberts G, Rönmark E, Platts-Mills T. Allergy to furry animals: New insights, diagnostic approaches, and challenges. J Allergy Clin Immunol 2014;pii: S0091-6749(14)01199-3.

13. Konradsen JR, Nordlund B, Onell A, Borres MP, Grönlund H, Hedlin G. Severe childhood asthma and allergy to furry animals: refined assessment using molecular-based allergy diagnostics. Pediatr Allergy Immunol 2014;25:187-192.

14. van Hage M, Pauli G. New vaccines for Mammalian allergy using molecular approaches. Front Immunol 2014;5:81.

15. Jakob T, Hilger $C$, Hentges F. Clinical relevance of sensitization to cross-reactive lipocalin Can f 6. Allergy 2013;68:690-691.

16. Landau M, Mayrose I, Rosenberg Y, Glaser F, Martz E, Pupko T, Ben-Tal N. ConSurf 2005: the projection of evolutionary conservation scores of residues on protein structures. Nucleic Acids Res 2005;33(Web Server issue):W299-302. 


\section{PART C}

CROSS-REACTIVE MOLECULES \& THEIR CLINICAL RELEVANCE

\section{PARVALBUMINS}

\author{
Annette Kuehn, Lars K. Poulsen, Martine \\ Morisset
}

\section{THE PROTEIN}

In the early seventies, Gad c 1 was the first parvalbumin identified as major fish allergen in Baltic cod. Subsequent cloning and biomolecular studies were performed with the parvalbumin Gad $\mathrm{m}$ 1, the homologue allergen from Atlantic cod (Table 1) (1). Currently, two isoallergens are listed in the allergen nomenclature database, Gad m 1.01 and Gad m 1.02. Each isoform has been characterized as two isoforms of high sequence homology. The protein structure of cod parvalbumin was first modeled on the basis of the x-ray structure from carp parvalbumin. Recently, the NMR-based protein structure was published revealing important characteristics on the folding and stability of parvalbumins (2). Gad $\mathrm{m} 1$ has a six $\alpha$-helical protein fold which is a common feature of parvalbumins (Fig. 1).

Gad $m 1$ was identified as the major allergen in cod muscle. However, food allergy to codfish might be also caused by other allergens, such as Gad $\mathrm{m} 2$ (cod enolase) and Gad $\mathrm{m} 3$ (cod aldolase) (3).

Cod parvalbumin is mainly found in fish muscle. It is a highly stable protein of low molecular weight (10-12 $\mathrm{kDa}$ ) binding $\mathrm{Ca}^{2+}$-ions (or $\mathrm{Mg}^{2+}$-ions) via two peptide
Parvalbumins are members of the $\mathrm{Ca}^{2+}$-binding EF-hand family.

Clinical cross-reactivity is explained by highly conserved parvalbumin lgE epitopes.

Monosensitized patients might have IgE to species-specific epitopes.

Parvalbumins are food and respiratory allergens.

Parvalbumins are fish panallergens.

Parvalbumins resist to food processing.

Perform skin testing with cod and salmon muscle

Be aware that a false negative result might be obtained with dark fish muscle!

Test specific lgE to cod and salmon extract Test specific lgE to cod and carp parvalbumin 


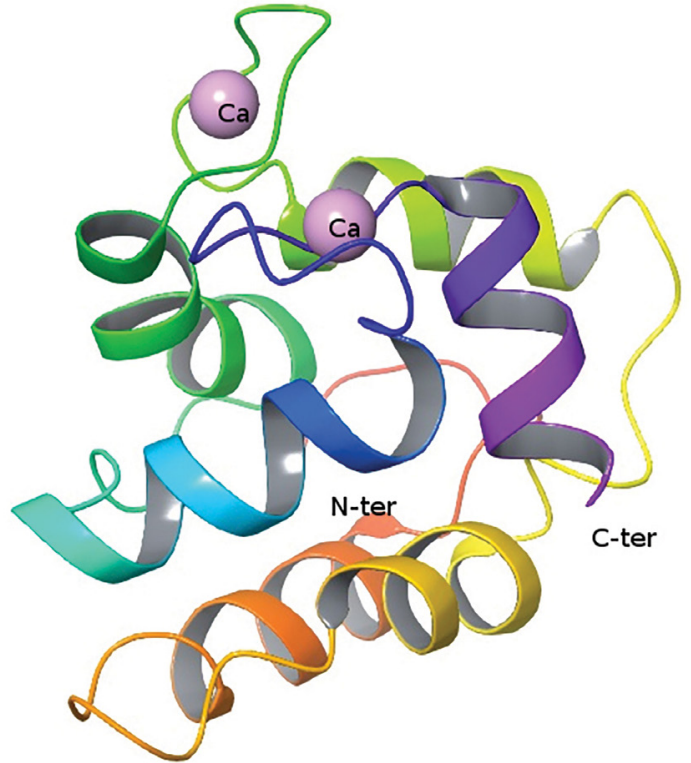

Figure 1

Three-dimensional structure of Gad m 1 (PDB 2MBX). Two $\mathrm{Ca}^{2+}$-molecules are bound to two functional EF-hand motifs.

loops called EF-hand motifs (4). It is involved in the regulation of the intracellular calcium concentration during muscle relaxation.

As for all bony fishes, cod has two types of muscles, the light and dark tissue differing by their physiological function. Cod belongs to the whitefishes having mainly light muscle tissue and only a small strip of dark tissue under the fish skin. Parvalbumins are commonly found in the light muscles but not in the dark muscles such as described for tuna (5). The allergen level in cod is up to $2 \mathrm{mg}$ parvalbumin per $\mathrm{g}$ of muscle tissue (6).

B cell epitopes have been analyzed for cod parvalbumin $(1,7)$. Several regions seem to be involved in the antigen-antibody interaction. A correlation was found between the severity of the allergic reaction and the number of epitopes. Allergic patients recognizing ten IgE-binding peptides including an important C-terminal epitope had more severe reactions than others. It was concluded that the number of linear epitopes should serve as a marker for the severity of the allergic reaction. A strategy for immunotherapy using hypoallergenic parvalbumins, including cod parvalbumin, is under development but it is not yet available for clinical practice (8). So far, successful oral immuntherapy for allergy to cod has been reported for patients treated with boiled fish (9).
Table 1

Basic protein characteristics of Gad m 1.

Protein characteristics

\begin{tabular}{|ll|}
\hline Allergen source & Gadus morhua, Atlantic cod \\
\hline Protein family & parvalbumin \\
\hline $\begin{array}{l}\text { UniProtKB accession } \\
\text { No }\end{array}$ & Q904LO \\
\hline $\begin{array}{l}\text { Three dimensional } \\
\text { structure available }\end{array}$ & yes \\
\hline Molecular structure & alpha-helical structure \\
\hline $\begin{array}{l}\text { Theoretical molecular } \\
\text { weight }\end{array}$ & $11.55 \mathrm{kDa}$ \\
\hline $\begin{array}{l}\text { Molecular weight } \\
\text { measured by mass } \\
\text { spectrometry }\end{array}$ & $11.36 \mathrm{kDa}$ \\
\hline Length & 109 amino acids \\
\hline Ligand binding & yes (Ca $\left.{ }^{2+}, \mathrm{Mg}^{2+}\right)$ \\
\hline Dimerization & dimer, oligomer \\
\hline Glycosylation & no \\
\hline Disulfide bonds & no \\
\hline Isoelectric point & 4.58 \\
\hline Synthesis & $\begin{array}{l}\text { muscle tissue, cytosolic } \\
\text { protein }\end{array}$ \\
\hline Distribution & muscle, swimbladder \\
\hline
\end{tabular}

\section{THE FAMILY}

Based on their protein characteristics, parvalbumins are attributed to two different phylogenetic origins, the $\alpha-$ and the $\beta$-lineage (10). Both subtypes can be found in different organs (central nervous system, endocrine tissue) but the highest expression rates have been determined in muscles (11). Muscles from mammals and birds express a-parvalbumins, which are considered as non-allergenic proteins.

Parvalbumins of the $\beta$-subtype have been characterized as panallergens in fish muscle (10). They belong to the EF-hand protein family comprising important allergens from both animal and plant origin (see also chapter 'Polcalcins'). These proteins share conserved domains consisting of $\mathrm{Ca}^{2+}$-binding peptide loops flanked on both sides by $a$-helices. These structures 
Table 2

Gad m 1-homologous allergens from fishes.

\begin{tabular}{|c|c|c|}
\hline Taxonomic order & Allergen source & Allergen \\
\hline \multirow{2}{*}{ Clupeiformes } & Herring (Clupea harengus) & Clu h $1^{*}$ \\
\hline & Pilchard (Sardinops sagax) & Sar sa $1^{*}$ \\
\hline \multirow{2}{*}{ Cypriniformes } & Carp (Cyprinus carpio) & Cyp с $1^{*}$ \\
\hline & Anchovy (Engraulis encrasicolus) & Eng e 1 \\
\hline \multirow{3}{*}{ Gadiformes } & Atlantic/Baltic cod (Gadus morhua/callarias) & Gad m 1/Gad c 1* \\
\hline & Hake (Merluccius merluccius) & Mer mr 1 \\
\hline & Pollock (Theragra chalcogramma) & The ch 1 \\
\hline \multirow{4}{*}{ Perciformes } & Barramundi (Lates calcarifer) & Lat c $1^{*}$ \\
\hline & Mackerel (Scomber scombrus) & Sco s 1 \\
\hline & Swordfish (Xiphias gladius) & Xip g $1^{*}$ \\
\hline & Tuna (Thunnus albacares) & Thu a $1^{*}$ \\
\hline \multirow{2}{*}{ Pleuronectiformes } & Megrim (Lepidorhombus whiffiagonis) & Lep w $1^{*}$ \\
\hline & Sole (Solea solea) & Sol so 1 \\
\hline \multirow{3}{*}{ Salmoniformes } & Char (Salvelinus fontinalis) & Sal f 1 \\
\hline & Rainbow trout (Oncorhynchus mykiss) & Onc m $1^{*}$ \\
\hline & Salmon (Salmo salar) & Sal s $1^{*}$ \\
\hline Scorpaeniformes & Redfish (Sebastes marinus) & Seb $\mathrm{m} 1^{*}$ \\
\hline
\end{tabular}

*www.allergen.org

are called EF-hand motif as both a-helices are arranged like the thumb and the forefinger of a hand. Fish parvalbumins have three EF-hand motifs (AB, CD, $E F$ ) whereas only the $C D$ - and EF-sites are functional and bind divalent ions $\left(\mathrm{Ca}^{2+}, \mathrm{Mg}^{2+}\right)$. Parvalbumin structures rearrange globally upon ion-binding and release. The ion-binding is essential for the stability of parvalbumins. $\mathrm{Ca}^{2+}$-depleted parvalbumins bind only weakly IgE-antibodies from fish-allergic patients $(11,12)$. Therefore, the functional calcium-binding motifs (CD, EF) have been suggested as important conformational B cell epitopes.

Allergens from the parvalbumin family have been identified and characterized in a number of fishes (1). Homologs from 12 species have been validated and included in the official allergen nomenclature database (www.allergen.org). Detailed data on molecular and allergenic properties are available for parvalbumins from fishes, which are commonly consumed in Europe such as cod, salmon, mackerel and tuna. The most important representatives of the parvalbumin family are summarized in Table 2.

Parvalbumins are small cytosolic molecules of 107-110 amino acids (1). Several but mostly two isoallergens can be found in the same fish muscle such as for salmon, cod and carp. These allergens were named $\beta 1$ - and $\beta 2$-parvalbumins sharing a sequence identity of $64 \%, 72 \%$ and $84 \%$, respectively. Not all isoforms are necessarily included in the official allergen nomenclature database. Table 3 illustrates pairwise comparisons of amino acid identities between parvalbumins from different fishes, which are commonly consumed in Europe. Identities highlighted in blue have been shown to be IgE cross-reactive molecules in in-vitro studies. This comparison shows that sequence homologies vary over a broad range. However, IgE cross-reactivity has not been only reported for highly similar (98 $\%$ identity) but even more distantly related fish parvalbumins (63\% identity). This complies with 
Table 3

Amino acid identities (\%) between parvalbumins from cod, carp, salmon, trout, herring, mackerel, tuna and whiff

\begin{tabular}{|c|c|c|c|c|c|c|c|c|}
\hline \multicolumn{3}{|l|}{ Gad $\mathrm{m} 1^{*}$} & & & & & & \\
\hline Сур с $1^{*}$ & 80 & & & & & & & \\
\hline Sal s $1^{*}$ & 64 & 64 & & & & & & \\
\hline Onc $\mathrm{m} 1^{*}$ & 65 & 65 & 98 & & & & & \\
\hline Clu h $1^{*}$ & 75 & 72 & 72 & 73 & & & & \\
\hline Sco s 1 & 72 & 76 & 64 & 65 & 75 & & & \\
\hline Thu a 1 & 77 & 88 & 67 & 68 & 74 & 70 & & \\
\hline \multirow[t]{2}{*}{ Lep w 1} & 63 & 68 & 63 & 65 & 63 & 69 & 69 & \\
\hline & Gad $m 1^{*}$ & Cyp с $1^{*}$ & Sal s $1^{*}$ & Onc $\mathrm{m} 1^{*}$ & Clu h $1^{*}$ & Sco s 1 & Thu a 1 & Lep w 1 \\
\hline
\end{tabular}

blue, documented IgE cross-reactivity; bold, > $80 \%$ amino acid sequence identity; * $\beta 1$-isoform used for comparison

A.

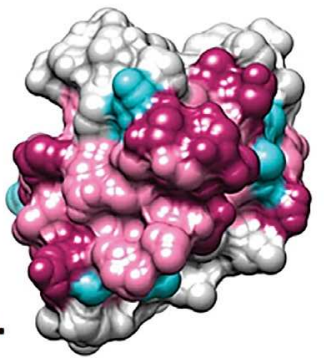

B.

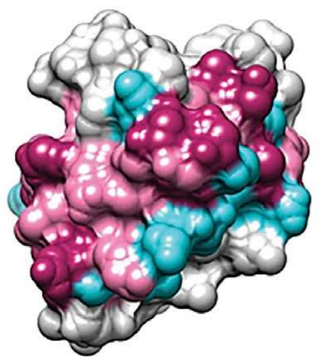

C.

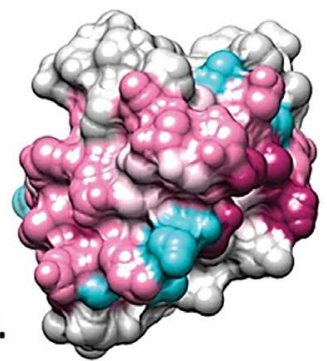

Figure 2

Surface comparisons of three different cross-reactive parvalbumins (based on PDB 2MBX, sequence conservation determined using ConSurf tool). A. cod Gad m 1, B. salmon Sal s 1, C. tuna Thu a 1; colors according to protein identity between depicted parvalbumin and the two others: blue, variable; white, average; red, conserved.

the fact that the global protein structures are highly conserved and argues for common conformational B-cell epitopes (11).

Fish parvalbumins are highly cross-reactive molecules, anti-parvalbumin IgE-antibodies recognize often homologs from different fishes supporting the fact that fish-allergic patients often react to multiple fishes (see chapter 'Fish allergy') (12). The molecular basis for this high IgE cross-reactivity is the remarkable structural homology, especially in the ion-binding regions. The surface comparison of selected cross-reactive fish allergens visualizes potential conformational B cell epitopes common to most parvalbumins (Fig. 2). However, a number of patients react only to specific or single fishes. Tolerance of single species might be explained by very low allergen contents such as for tuna (6). Another reason for this clinical mono-/oligo-sensitivity are species-specific IgE-binding epitopes present on parvalbumins $(13,14)$. Studies on monosensitivity to salmon/trout confirmed the presence of a salmonid- 
A.

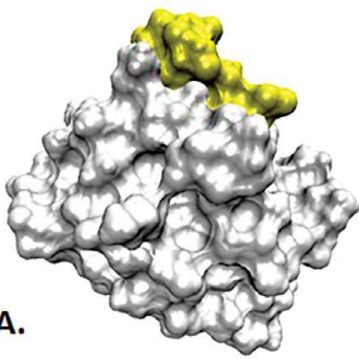

B.

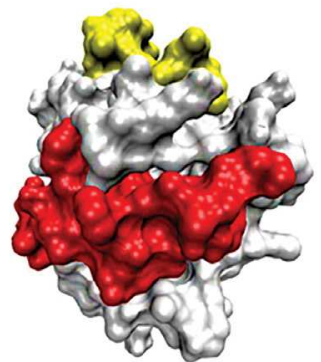

C.

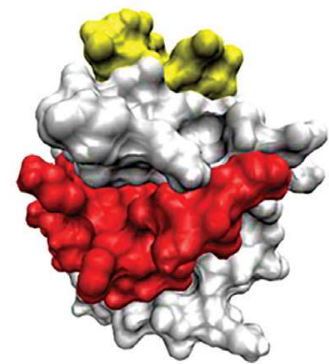

Figure 3

Surface comparisons of non cross-reactive parvalbumins in salmonid-monosensitized patients (based on PDB 2MBX, sequence conservation determined using ConSurf tool). A. cod Gad m 1, B. salmon Sal s 1 , C. trout Onc m 1. Yellow, Ca ${ }^{2+}$-binding sites; red, species-specific epitope.

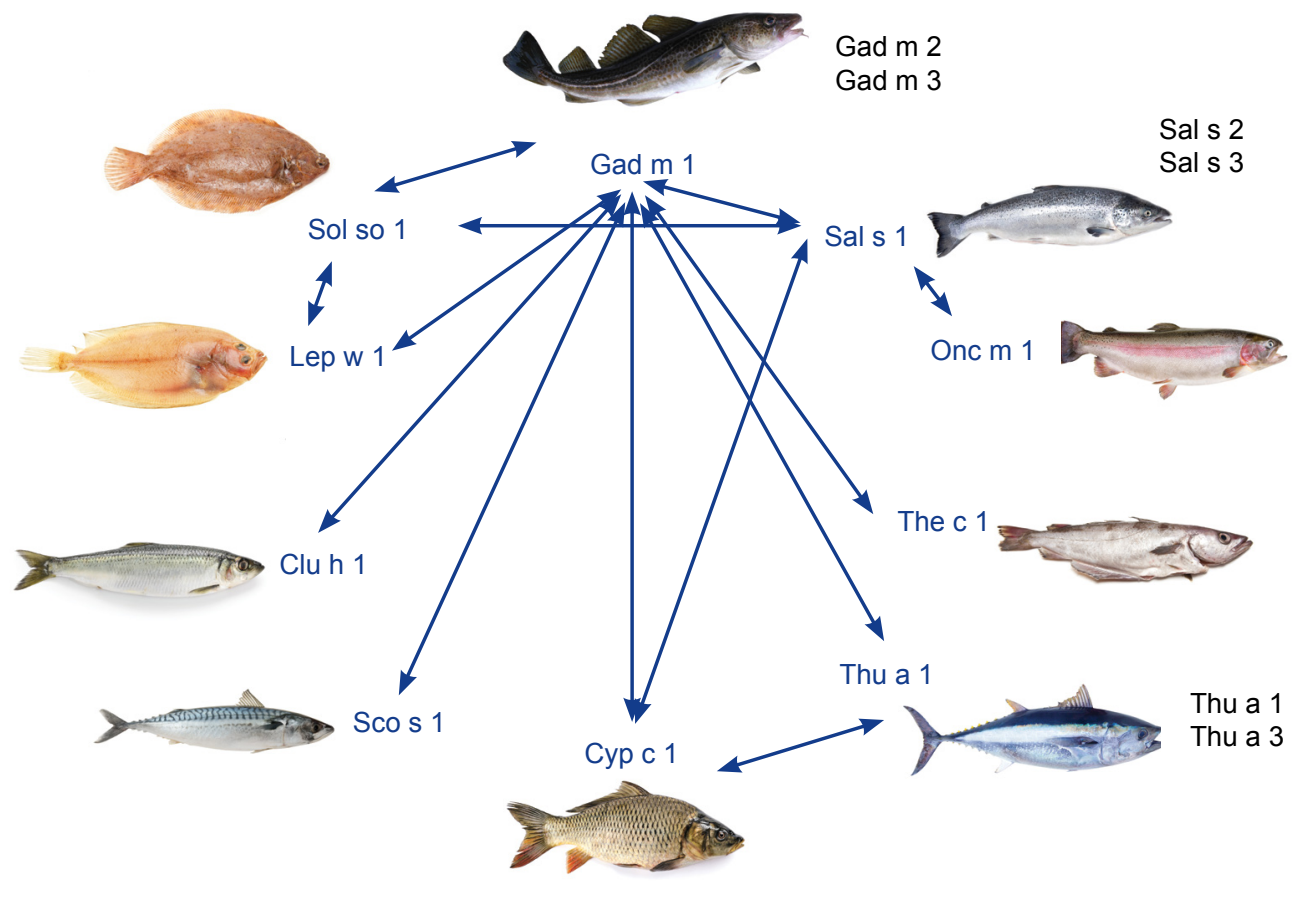

Figure 4

Cross-reactivities among allergenic fish parvalbumins. All parvalbumins have a high potential for cross-reactivity based on high sequence homology. Lines represent documented IgE-cross-reactivity. Clockwise: cod, salmon, trout, pollack, tuna, carp, mackerel, herring, whiff, sole.

specific parvalbumin epitope, which is unique for these fish allergens (Fig. 3).

Fig. 4 represents documented and putative crossreactivities among known fish parvalbumins. Recently, new fish allergens have been characterized (see chapter 'Fish allergy'). Fish beta-enolases and aldolases were identified as food allergens in cod
(Gad m 2, Gad m 3), salmon (Sal s 2, Sal s 3) and tuna (Thu a 2, Thu a 3) (Fig. 4) (3). Future studies will have to address the characterization of selective marker molecules, parvalbumins and the new fish allergens, for IgE-based diagnosis to discriminate between patients with clinical cross-reactivity and fish speciesspecific sensitization. 


\section{CLINICAL RELEVANCE}

Beta-parvalbumins are present in the fish muscle and skin but they become airborne upon handling and processing of fish (1). As such, they are both food and respiratory allergens. Fish allergy is both important in the domestic and occupational environment. Incidental episodes might occur upon inhalation of volatile allergens during fish preparation in the domestic environment. These allergenic molecules can even be found in unexpected environments such as mattress dust. Respiratory problems of the upper and lower airway tract have been reported in the occupational context among workers processing fish (15). A clear correlation has been shown for the development of work-related asthma and fish allergy as explained by high atmospheric fish allergen concentrations in the workplace.

Studies estimated that less than $1 \%$ of the general population suffers from an allergy to fish (see chapter 'Fish allergy') (11). Children often maintain their sensitization to fish during adolescence. Fish allergy is more frequent in countries with large coastal regions characterized by frequent fish consumption and settling of fish-processing industries. Concerning the prevalence of specific IgE to parvalbumins in fishallergic patients, it was stated for a long time that more than $90 \%$ are sensitized to this panallergen. According to results of recent studies, this prevalence seems to be considerably lower but it still requires further investigations for a better understanding (3).

The main route of sensitization to fish parvalbumins is by oral ingestion, thus by uptake through the gastrointestinal tract. Common clinical manifestations include mild (oral allergy syndrome, erythema) to moderate (urticaria, vomiting) or severe (angioedema, asthma, anaphylaxis) symptoms $(1,11)$. Sensitized patients with antacid medication are at higher risk to develop severe reactions than others.

A key feature of potent food allergens is their stability to thermal treatment. Fish parvalbumins are extremely heat-stable and therefore, they are still detectable in products processed by cooking, frying or in pickeled food (6). This emphasizes their undiminished allergenicity upon various preparation methods. Fish parvalbumins become glycosylated by heating in the presence of glucose through the Maillard reaction (16). However, the resulting effects on their allergenic potency, be it cumulative or diminishing, still require further investigations.
Currently, there is no causal therapy available to treat allergic sensitization to fish parvalbumins. The therapeutic desensibilization with increasing doses of fish is disadvised as the risk for anaphylactic reactions is important. A strict avoidance diet is recommended to fish-sensitized patients. According to established EU-labeling regulations, fish has to be indicated specifically with an allergy hazards note on the product of each commercial food product (guideline 2007/68/EG). Caution is advised with loose items and products from fish origin (fish gelatin), which might be contaminated with fish allergens. Another perspective for the future treatment of fish allergy is the development of hypoallergenic parvalbumins (8). Low IgE-bindings mutants have been first identified for carp parvalbumin and later on, further developed for homologue allergens from commonly consumed fish allergens. This therapeutic strategy is currently under investigation by an EU-funded project targeting the immunemodulatory efficiency of these hypoallergenic mutants.

\section{CLINICAL MANAGEMENT}

The mainstay for the diagnosis of fish allergy are the record of the patient's medical history, the analysis of the skin reactivity using fish extracts or authentic food, the quantification of serum IgE-antibodies and in some cases, oral food challenges (see Section B12 'Fish allergy'). IgE-quantification assays are available for approx. 30 extracts from different fish species as well as two parvalbumins, Gad c 1 from cod and Cyp c 1 from carp. Newly identified fish allergens such as enolases (cod Gad m 2, salmon Sal s 2, tuna Thu a 2), aldolases (cod Gad m 3, salmon Sal s 3, tuna Thu a 3) or fish gelatin are still missing for IgE-testing.

Highly sensitized patients often react to various fishes. First, they should be tested for skin reactivity to cod and salmon native muscle followed by analysis of specific lgE-binding to cod and salmon extract. Second, a polysensitization to fish can be confirmed by detecting specific IgE to the cross-reactive parvalbumins from cod (Gad m 1) and carp (Cyp c 1). Future IgE-testing for other allergens will entail a more specific diagnosis of these patients (Fig. 5).

However, a number of fish-allergic individuals react to specific fishes only (3). The challenge of future studies will be the identification of marker allergens for IgE-tests to discriminate between these poly- and 


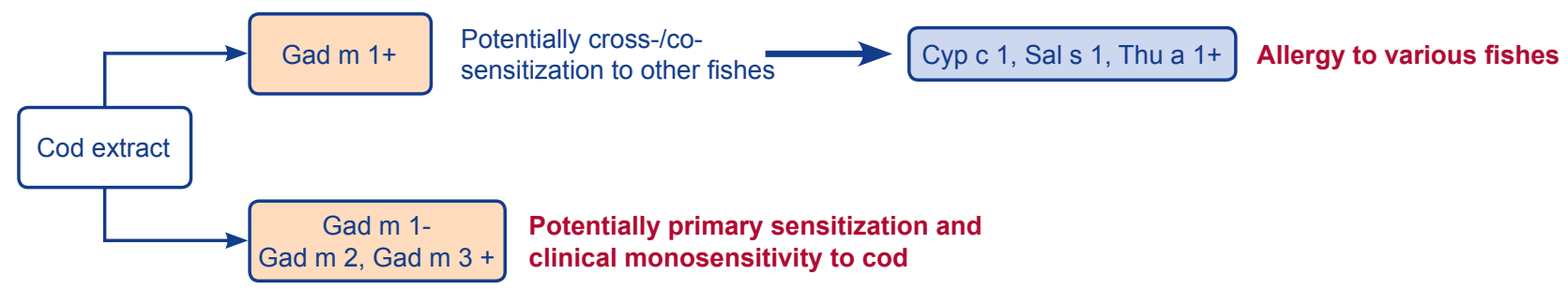

Figure 5

Added value of the use of single allergens in the case of a positive IgE test to cod extract.

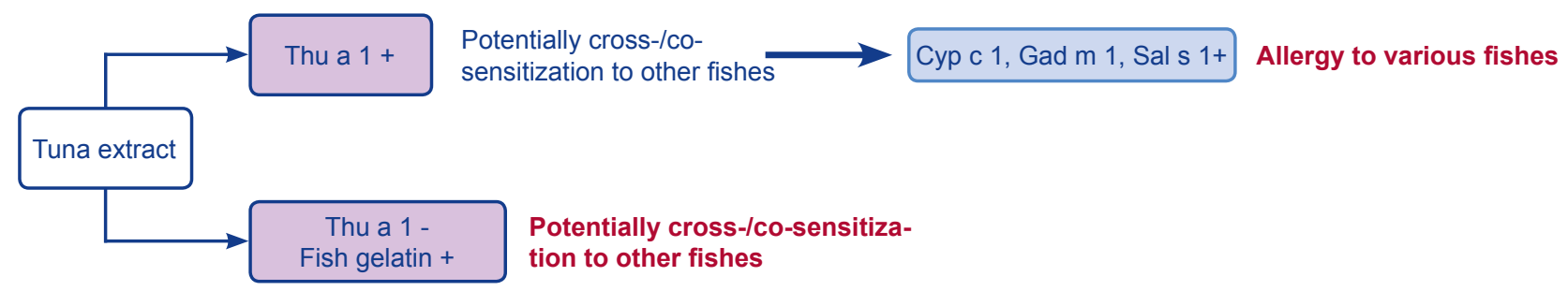

Figure 6

Added value of the use of single allergens in the case of a positive IgE test to tuna extract.

oligo-/mono-sensitized patients. As for now, the discriminative significance of anti-parvalbumin IgEantibodies seems to be low because they are often cross-reacting with various homologs which does not necessariliy imply a clinical reactivity. An exception has been reported for a subgroup of patients with monosensitivity to salmonid fishes. They might be diagnosed efficiently by determination of specific lgE to salmon and trout parvalbumin (see 'Clinical case 2') $(13,14)$. However, the positive predictive value (PPV) for single parvalbumins in the diagnosis of allergy to single fishes still needs to be defined.

Overall, allergens different from parvalbumins might be elicitors for fish allergy. Cod enolases and aldolases have been identified as responsible allergens in three clinical cases of monosensitivity to cod (see chapter 'Fish allergy') (17). Even other allergens such as fish gelatin might play a role in this context (Fig. 6) (18). Anyway, diagnostic conclusions from in vitro IgE-results, especially negative results, should be confirmed by further oral provocation tests in case of a strong suspicion of a fish allergy.

\section{CLINICAL CASES}

In the clinical cases presented in this paragraph, following parvalbumins been included in IgE-based diagnostic procedures during research studies: salmon Sal s 1, carp Cyp c 1, cod Gad m 1, tuna Thu a 1, trout Onc m 1, pangasius Pan h 1, catfish Sil g 1.

\section{Case 1 (published (3))}

Clinical History: A male child, 12 years, with a clinical history of fish allergy since early childhood presenting with angioedema and respiratory problems upon ingestion of different fishes as well as with acute urticaria when touching fish.

Test with extracts: Skin tests performed with commercial extracts were positive for cod, salmon and tuna. IgE to cod, salmon and tuna extract were positive (16 kUA/L, $32 \mathrm{kUA} / \mathrm{L}$ and $65 \mathrm{kUA} / \mathrm{L}$, respectively).

Food challenge: The parents of the child refused a food challenge. 


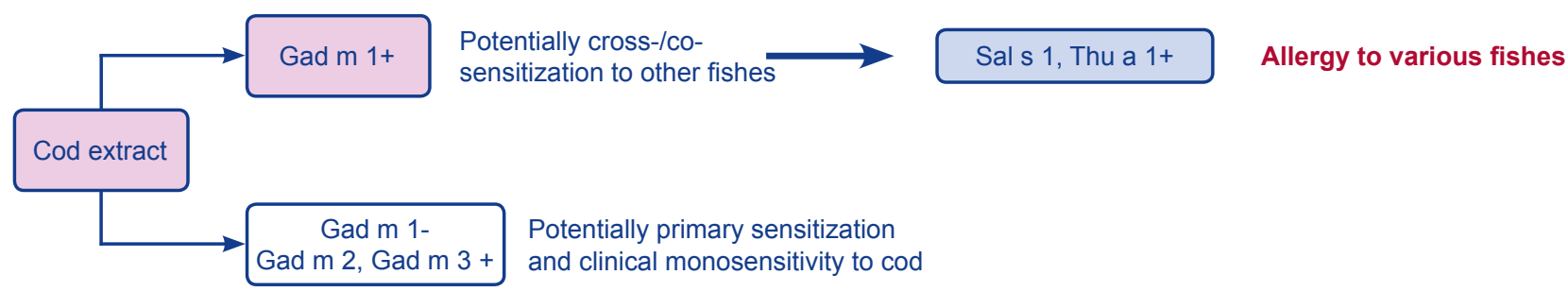

\section{Figure 7}

Diagnostic procedure case 1.
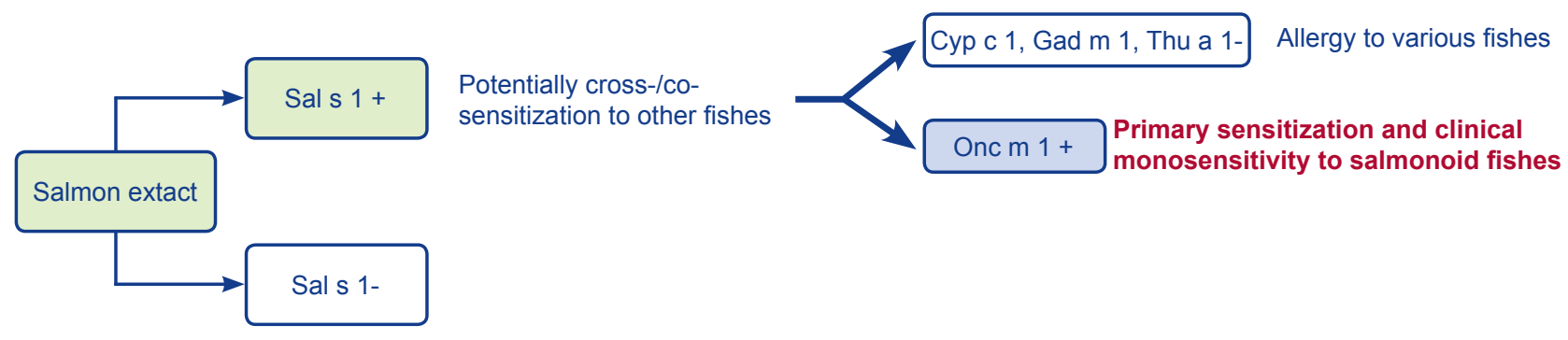

Figure 8

Diagnostic procedure case 2 .

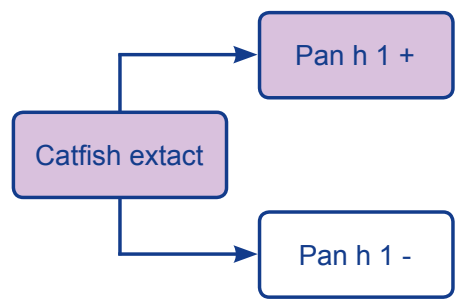

Potentially cross-/cosensitization to other fishes

$\lg E$ to other allergens
Сур с 1

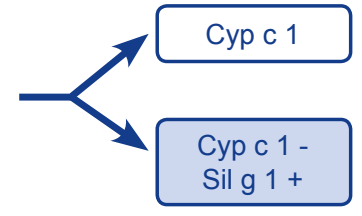

Allergy to various fishes

Specific sensitivity to fishes from the order Siluriformes

\section{Figure 9}

Diagnostic procedure case 3.

Test with molecules: Cod, salmon and tuna parvalbumins were positive in IgE ELISA (20 $\mathrm{kUA} / \mathrm{L}, 18 \mathrm{kUA} / \mathrm{L}$ and $30 \mathrm{kUA} / \mathrm{L}$, respectively).

Conclusion: The polysensitization to unlimited fishes was confirmed in this case by revealing cross-reactive IgE-antibodies to homologue parvalbumins from different fishes (Fig. 7).

\section{Case 2 (published (13))}

Clinical History: A female patient, 21 years, with a clinical history of fish allergy since childhood presenting with swellling of the tongue and oral mucosa, facial edema and vomiting minutes after ingestion of salmon or trout. 
Test with extracts: Skin tests performed with commercial extracts were positive for salmon and trout but negative for other fishes. Only lgE to salmon extract was slightly positive $(0.4 \mathrm{kUA} / \mathrm{L})$.

Food challenge: As the patient experienced repeatedly symptoms with salmonid fishes, she refused to be tested by oral provocation.

Test with molecules: As determined by IgE ELISA, salmon and trout parvalbumins were positive at 0.2-0.4 kUA/L. No inhibition assays were performed with other fish parvalbumins as IgEbinding was negative for cod, carp, mackerel, redfish and herring homologs.

Conclusion: In this case, clinical species-specific sensitivity to salmonid fishes was confirmed by specific IgE to salmon and trout parvalbumin (Fig. 8).

\section{Case 3 (published (19))}

Clinical History: Female patient, 36 years, presenting with anaphylaxis (oral itch, urticaria, angioedema, dizziness, hypotension) after eating a pangasius.

Test with extracts: All skin tests were negative. Specific IgE was not available for pangasius extract but it was positive for wels catfish extract $(0.5$ $\mathrm{kUA} / \mathrm{L})$, a closely related species.

Food challenge: The patient refused to be tested by oral provocation.

Test with molecules: IgE binding was found for parvalbumins from pangasius, wels catfish and angler which could not be abolished in IgE immunoblot cross-inhibition assays.

Conclusion: The species-specific sensitization to closely related fishes from the order Siluriformes was confirmed by component-based IgE-testing using parvalbumins from this fish family (Fig. 9).

\section{TOOLS}

Fish allergens validated by the World Health Organization and International Union of Immunological Societies (WHO/IUIS) Allergen Nomenclature Sub-committee can be checked at www.allergen.org. Informations about allergen families such as the EF-hand family can be obtained from the homepage of the AllFam database (www. meduniwien.ac.at/allergens/allfam/). Details about the new food labeling regulation are available at http://ec.europa.eu/food/food/labellingnutrition/ foodlabelling/index _ en.htm. Progress of the EUproject FAST on hypoallergenic carp parvalbumin Cyp c 1 can be found at the project homepage (www. allergome.org:8080/fast/index.jsp).

Fish allergens can be detected in authentic food samples using a DNA-based kit from R-Biopharm (http://www.r-biopharm.com/products/food-feedanalysis/allergens/fish/item/surefood-allergen-fish).

Two books might be useful for further reading about clinical features of fish allergy and biomolecular properties of food, including fish, proteins: 'Food allergy' by James JM, Burks W, Eigenmann PA, Elsevier 2012) and 'Chemical and biological properties of food allergens' by Jedrychowski L, Wichers HJ, CRC Press 2010.

\section{References}

1. Sharp MF, Lopata AL. Fish allergy: in review. Clin Rev Allergy Immunol 2014;46:258-271.

2. Moraes $\mathrm{AH}$, Ackerbauer $\mathrm{D}$, Kostadinova $\mathrm{M}$, Bublin $M$, de Oliveira GA, Ferreira F et al. Solution and highpressure NMR studies of the structure, dynamics, and stability of the cross-reactive allergenic cod parvalbumin Gad m 1. Proteins 2014;82:3032-3042.

3. Kuehn A, Hilger C, Lehners-Weber C, Codreanu-Morel $F$, Morisset M, Metz-Favre $C$ et al. Identification of enolases and aldolases as important fish allergens in cod, salmon and tuna: component resolved diagnosis using parvalbumin and the new allergens. Clin Exp Allergy 2013;43:811-822.

4. Schwaller B. The continuing disappearance of "pure" Ca2+ buffers. Cell Mol Life Sci 2009;66:275-300.

5. Kobayashi A, Tanaka H, Hamada Y, Ishizaki S, Nagashima Y, Shiomi K. Comparison of allergenicity and allergens between fish white and dark muscles. Allergy 2006;61:357-363.

6. Kuehn A, Scheuermann T, Hilger C, Hentges F. Important variations in parvalbumin content in common fish species: a factor possibly contributing to variable allergenicity. Int Arch Allergy Immunol 2010;153:359366.

7. Perez-Gordo M, Pastor-Vargas C, Lin J, Bardina L, Cases B, Ibáñez MD et al. Epitope mapping of the major allergen from Atlantic cod in Spanish population reveals different IgE-binding patterns. Mol Nutr Food Res 2013;57:1283-1290.

8. Zuidmeer-Jongejan L, Huber $\mathrm{H}$, Swoboda I, Rigby $\mathrm{N}$, Versteeg SA, Jensen BM et al. Development of a Hypoallergenic Recombinant Parvalbumin for First-inMan Subcutaneous Immunotherapy of Fish Allergy. Int 
Arch Allergy Immunol 2015;166:41-51.

9. Patriarca G, Nucera E, Pollastrini E, Roncallo C, De Pasquale T, Lombardo $C$ et al. Oral specific desensitization in food-allergic children. Dig Dis Sci 2007;52:1662-1672.

10. Radauer C, Bublin M, Wagner S, Mari A, Breiteneder $\mathrm{H}$. Allergens are distributed into few protein families and possess a restricted number of biochemical functions. J Allergy Clin Immunol 2008;121:847-852.

11. Kuehn A, Swoboda I, Arumugam K, Hilger C, Hentges F. Fish allergens at a glance: variable allergenicity of parvalbumins, the major fish allergens. Front Immunol 2014;5:179.

12. Bugajska-Schretter A, Grote M, Vangelista L, Valent $\mathrm{P}$, Sperr WR, Rumpold $\mathrm{H}$ et al. Purification, biochemical,and immunological characterisation of a major food allergen: different immunoglobulin $E$ recognition of the apo- and calcium-bound forms of carp parvalbumin. Gut 2000;46:661-669.

13. Kuehn A, Hutt-Kempf E, Hilger C, Hentges F. Clinical monosensitivity to salmonid fish linked to specific IgE-epitopes on salmon and trout betaparvalbumins. Allergy 2011;66:299-301.
14. Perez-Gordo M, Lin J, Bardina L, Pastor-Vargas C, Cases $\mathrm{B}$, Vivanco $\mathrm{F}$ et al. Epitope mapping of Atlantic salmon major allergen by peptide microarray immunoassay. Int Arch Allergy Immunol 2012;157:31-40.

15. Lopata AL, Jeebhay MF. Airborne seafood allergens as a cause of occupational allergy and asthma. Curr Allergy Asthma Rep 2013;13:288-297.

16. de Jongh HH, Taylor SL, Koppelman SJ. Controlling the aggregation propensity and thereby digestibility of allergens by Maillardation as illustrated for cod fish parvalbumin. J Biosci Bioeng 2011;111:204-211.

17. Kuehn A, Fischer J, Hilger C, Sparla C, Biedermann T, Hentges F. Correlation of clinical monosensitivity to cod with specific IgE to enolase and aldolase. Ann Allergy Asthma Immunol 2014;113:670-671.

18. Kuehn A, Hilger C, Hentges F. Anaphylaxis provoked by ingestion of marshmallows containing fish gelatin. J Allergy Clin Immunol 2009;123:708-709.

19. Raith M, Klug C, Sesztak-Greinecker G, Balic N, Focke $M$, Linhart $B$ et al. Unusual sensitization to parvalbumins from certain fish species. Ann Allergy Asthma Immunol 2014;113:571-572. 


\section{PART D}

APPENDICES 



\title{
APPENDICES
}

\section{IMPORTANT ALLERGENIC MOLECULES AND THEIR CHARACTERISTICS}

\author{
Stephanie Hofmaier, Karin Hoffmann- \\ Sommergruber, Heimo Breiteneder
}

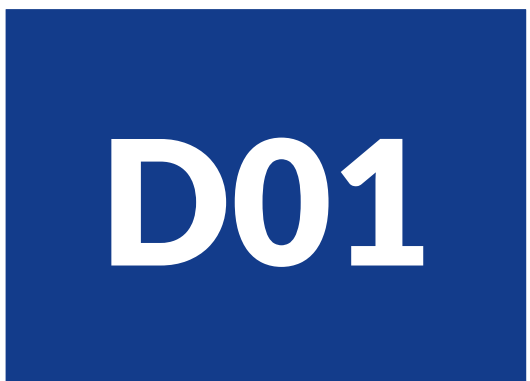

\begin{tabular}{|c|c|c|c|c|}
\hline $\mathrm{Nr}$ & Allergen & Source & Description & Chapter \\
\hline 1 & Act d 1 & $\begin{array}{l}\text { Green kiwifruit } \\
\text { (Actinidia deliciosa) }\end{array}$ & $\begin{array}{l}\text { This cysteine protease, actinidin is a major allergen from } \\
\text { kiwifruit and points towards kiwifruit monosensitization }\end{array}$ & B15 \\
\hline 2 & Act d 8 & $\begin{array}{l}\text { Green kiwifruit } \\
\text { (Actinidia deliciosa) }\end{array}$ & $\begin{array}{l}\text { This allergen is a PR-10 molecule (Bet } v 1 \text { homologue) from } \\
\text { kiwifruit, thus cross-sensitization may occur. }\end{array}$ & $\begin{array}{l}\mathrm{B} 15 \\
\mathrm{C} 02\end{array}$ \\
\hline 3 & Act d 9 & $\begin{array}{l}\text { Green kiwifruit } \\
\text { (Actinidia deliciosa) }\end{array}$ & $\begin{array}{l}\text { This allergen is a Profilin from kiwifruit. Sensitization to Act } \\
\text { d } 9 \text { (and to Act d 8) is a specific condition of patients with } \\
\text { pollen-kiwifruit allergies. }\end{array}$ & $\begin{array}{l}\text { B15 } \\
\text { C01 }\end{array}$ \\
\hline 4 & Amb a 1 & $\begin{array}{l}\text { Ragweed } \\
\text { (Ambrosia } \\
\text { artemisiifolia) }\end{array}$ & $\begin{array}{l}\text { This allergen is a marker of ragweed sensitization. Its } \\
\text { biological function is that of a pectase lyase and it shows } \\
\text { cross-reactivity with Art } v 6 \text { from Mugwort. }\end{array}$ & B03 \\
\hline 5 & Amb a 6 & $\begin{array}{l}\text { Ragweed (Ambrosia } \\
\text { artemisiifolia) }\end{array}$ & $\begin{array}{l}\text { Amb a 6, a non-specific Lipid Transfer Protein (nsLTP), is a } \\
\text { specific marker for sensitization to ragweed. }\end{array}$ & $\begin{array}{l}\mathrm{B} 03 \\
\mathrm{CO} 3\end{array}$ \\
\hline 6 & Api $\mathrm{g} 1$ & $\begin{array}{c}\text { Celery } \\
\text { (Apium graveolens) }\end{array}$ & $\begin{array}{l}\text { This allergen is a PR-10 molecule (Bet } v 1 \text { homologue) from } \\
\text { celery, thus cross-sensitization (up to } 75 \% \text { ) may occur in areas } \\
\text { where birch pollen is abundant. }\end{array}$ & B15 \\
\hline 7 & Api $m 1$ & $\begin{array}{l}\text { European, western } \\
\text { or common honey- } \\
\text { bee (Apis mellifera) }\end{array}$ & $\begin{array}{l}\text { This major bee venom allergen is recognized by up to } 97 \% \text { of } \\
\text { the patients who show a reaction to a sting of the common } \\
\text { honeybee. }\end{array}$ & B20 \\
\hline 8 & Api $m 2$ & $\begin{array}{l}\text { European, western } \\
\text { or common honey- } \\
\text { bee (Apis mellifera) }\end{array}$ & $\begin{array}{l}\text { IgE antibodies to this hyaluronidase may cross-react with Ves } \\
\text { v } 2 \text { in vespid venom, due to an identical CCD structure. }\end{array}$ & B20 \\
\hline
\end{tabular}




\begin{tabular}{|c|c|c|c|c|}
\hline $\mathrm{Nr}$ & Allergen & Source & Description & Chapter \\
\hline 9 & Api m 10 & $\begin{array}{l}\text { European, western } \\
\text { or common honey- } \\
\text { bee (Apis mellifera) }\end{array}$ & $\begin{array}{l}\text { This allergenic molecule is a specific marker protein for } \\
\text { honeybee venom allergy. }\end{array}$ & B20 \\
\hline 10 & Ara h 1 & $\begin{array}{c}\text { Peanut } \\
\text { (Arachis hypogaea) }\end{array}$ & $\begin{array}{l}\text { Ara } \mathrm{h} 1 \text { is a major peanut allergen with sensitization rates } \\
\text { between } 63 \% \text { and } 80 \% \text {. This heat stable molecule is a Cupin } \\
\text { (Vicillin-type, } 75 \text { globulin) }\end{array}$ & B18 \\
\hline 11 & Ara h 2 & $\begin{array}{c}\text { Peanut } \\
\text { (Arachis hypogaea) }\end{array}$ & $\begin{array}{l}\text { The heat stable peanut allergen Ara } \mathrm{h} 2 \text { is a Conglutin }(2 \mathrm{~S} \\
\text { albumin). An association between sensitization to Ara } \mathrm{h} \\
2 \text { and Ara } \mathrm{h} 6 \text { and systemic reactions to peanuts has been } \\
\text { demonstrated. }\end{array}$ & B18 \\
\hline 12 & Ara h 3 & $\begin{array}{c}\text { Peanut } \\
\text { (Arachis hypogaea) }\end{array}$ & $\begin{array}{l}\text { The } 11 \mathrm{~S} \text { (Cupin) is a heat stable and relevant food allergen } \\
\text { from peanut. Frequent cross-reactivity between Ara h } 1 \text { and } \\
\text { Ara } \mathrm{h} 3 \text { is observed. }\end{array}$ & B18 \\
\hline 13 & Ara h 6 & $\begin{array}{c}\text { Peanut } \\
\text { (Arachis hypogaea) }\end{array}$ & $\begin{array}{l}\text { Just as Ara h } 2 \text {, also Ara } \mathrm{h} 6 \text { is a heat stable Conglutin ( } 2 \mathrm{~S} \\
\text { albumin). In the US and Northern Europe } 76-96 \% \text { of the } \\
\text { patients with clinically relevant peanut allergy possess specific } \\
\text { IgE to Ara } \mathrm{h} 2 \text { and Ara } \mathrm{h} 6 \text {. }\end{array}$ & B18 \\
\hline 14 & Ara h 8 & $\begin{array}{c}\text { Peanut } \\
\text { (Arachis hypogaea) }\end{array}$ & $\begin{array}{l}\text { This peanut allergen is a PR.10-like molecule (Bet } v 1 \\
\text { homologue), which is not stable to heat. IgE sensitization to } \\
\text { Ara } \mathrm{h} 8 \text {, Ara } \mathrm{h} 5 \text { and glycoproteins (CCD) are usually caused by } \\
\text { cross-reactions to pollen allergens. }\end{array}$ & $\begin{array}{l}\mathrm{B} 18 \\
\mathrm{C} 02\end{array}$ \\
\hline 15 & Art $v 1$ & $\begin{array}{l}\text { Mugwort } \\
\text { (Artemisia vulgaris) }\end{array}$ & $\begin{array}{l}\text { Art } v 1 \text { is a marker of sensitization to mugwort. It is a defensin- } \\
\text { like protein and shows a partial cross-reactivity with Amb a } 4 \\
\text { from ragweed. }\end{array}$ & B03 \\
\hline 16 & Art v 6 & $\begin{array}{l}\text { Mugwort } \\
\text { (Artemisia vulgaris) }\end{array}$ & $\begin{array}{l}\text { This molecule is a Pectase lyase, which shows cross-reactivity } \\
\text { with Amb a } 1 \text { from Ragweed. }\end{array}$ & B03 \\
\hline 17 & Asp f 1 & $\begin{array}{l}\text { Fungus } \\
\text { (Aspergillus } \\
\text { fumigatus) }\end{array}$ & $\begin{array}{l}\text { This Ribotoxin is the major secreted allergen causing ABPA. } \\
\text { The spectrum of allergens produced by the fungus spans } \\
\text { a wide variety of different molecular structures covering } \\
\text { enzymes, secreted, intracellular, and structural proteins. }\end{array}$ & B07 \\
\hline 18 & Asp f 2 & $\begin{array}{l}\text { Fungus } \\
\text { (Aspergillus } \\
\text { fumigatus) }\end{array}$ & $\begin{array}{l}\text { Asp } f 2 \text { is an ABPA-related intracellular allergen of unknown } \\
\text { function. Even if further confirmatory studies are needed, it } \\
\text { seems that Asp } f 2 \text { is exclusively recognized by patients with } \\
\text { ABPA both, in asthma and CF. }\end{array}$ & B07 \\
\hline 19 & Asp f 6 & $\begin{array}{l}\text { Fungus } \\
\text { (Aspergillus fumigatus) }\end{array}$ & $\begin{array}{l}\text { This phylogenetically highly conserved intracellular allergen } \\
\text { shows a high degree of cross-reactivity with other fungal proteins. }\end{array}$ & $\begin{array}{l}\text { B07 } \\
\text { B08 }\end{array}$ \\
\hline 20 & Bet v 1 & $\begin{array}{l}\text { Birch pollen } \\
\text { (Betula verrucosa) }\end{array}$ & $\begin{array}{l}93 \% \text { of the birch allergic individuals produce specific lgE- } \\
\text { antibodies to this major birch pollen allergen. Homologues } \\
\text { of Bet } v 1 \text { are present in pollens from related early flowering } \\
\text { trees and have also been identified in a wide range of plant } \\
\text { foods. Through cross-reactivity, patients may not only suffer } \\
\text { from respiratory, but also from oropharyngeal symptoms, } \\
\text { coined oral allergy syndrome (OAS). }\end{array}$ & $\begin{array}{l}\mathrm{B} 01 \\
\mathrm{C} 02\end{array}$ \\
\hline 21 & Bet v 2 & $\begin{array}{c}\text { Birch } \\
\text { (Betula verrucosa) }\end{array}$ & $\begin{array}{l}\text { This allergenic molecule belongs to the profilin-like } \\
\text { superfamily. Profilins represent a major cause of cross- } \\
\text { reactivity among most plant sources due to their highly } \\
\text { conserved structure and ubiquitous distribution. }\end{array}$ & $\begin{array}{l}\text { B01 } \\
\text { C01 }\end{array}$ \\
\hline
\end{tabular}




\begin{tabular}{|c|c|c|c|c|}
\hline $\mathrm{Nr}$ & Allergen & Source & Description & Chapter \\
\hline 22 & Bla g 1 & $\begin{array}{l}\text { German } \\
\text { Cockroach } \\
\text { (Blattella } \\
\text { germanica) }\end{array}$ & $\begin{array}{l}\text { The presence of Bla g } 1 \text { in fecal particles makes this molecule, } \\
\text { together with Bla g } 2 \text {, a good marker of cockroach allergen } \\
\text { exposure. Cross-reactivity has been described between Bla g } \\
1 \text { and homologous proteins (such as Per a } 1 \text {, Per } f 1 \text { and Bla } \\
\text { o 1) from other cockroach species, as well as allergens from } \\
\text { other insects. }\end{array}$ & B05 \\
\hline 23 & Bla g 5 & $\begin{array}{l}\text { German } \\
\text { Cockroach } \\
\text { (Blattella } \\
\text { germanica) }\end{array}$ & $\begin{array}{l}\text { Bla g } 5 \text { is a sigma class glutathione S-transferase (GST), a } \\
\text { major cockroach allergen, which elicits high levels in IgE } \\
\text { responses among cockroach-sensitized individuals. Cross- } \\
\text { reactivity with GSTs of several sources has been described. }\end{array}$ & B05 \\
\hline 24 & Bla g 7 & $\begin{array}{l}\text { German } \\
\text { Cockroach } \\
\text { (Blattella } \\
\text { germanica) }\end{array}$ & $\begin{array}{l}\text { Bla g } 7 \text { belongs to the invertebrate tropomyosins, which are } \\
\text { important pan-allergens among dust mites, chironomids, } \\
\text { silverfish, crustaceans, nematodes and mollusks. IgE binding } \\
\text { frequency to cockroach tropomyosins are very different } \\
\text { in some populations, which may reflect differences in the } \\
\text { environmental conditions. }\end{array}$ & $\begin{array}{l}\text { B05 } \\
\text { C05 }\end{array}$ \\
\hline 25 & Blo t 1 & $\begin{array}{c}\text { Mite } \\
\text { (Blomia tropicalis) }\end{array}$ & $\begin{array}{l}\text { This molecule is a mite group } 1 \text { allergen (Cysteine protease). } \\
\text { Blo t } 1 \text { is a major marker of sensitization to the storage mite } \\
\text { Blomia tropicalis. }\end{array}$ & B04 \\
\hline 26 & Blo t 2 & $\begin{array}{c}\text { Mite } \\
\text { (Blomia tropicalis) }\end{array}$ & $\begin{array}{l}\text { This molecule is a mite group } 2 \text { allergen (NPC2 protein family). } \\
\text { Blo t } 2 \text { is a major marker of sensitization to the storage mite } \\
\text { Blomia tropicalis. }\end{array}$ & B04 \\
\hline 27 & Blo t 10 & $\begin{array}{c}\text { Mite } \\
\text { (Blomia tropicalis) }\end{array}$ & $\begin{array}{l}\text { Blo t } 10 \text { is a Tropomyosin from the storage mite Blomia } \\
\text { tropicalis. Especially its cross-reactivity with other } \\
\text { tropomyosins can be clinically relevant. }\end{array}$ & $\begin{array}{l}\text { B04 } \\
\text { C05 }\end{array}$ \\
\hline 28 & Bos d 4 & $\begin{array}{l}\text { Cow's milk (whey) } \\
\text { (Bos domesticus) }\end{array}$ & This alpha-lactalbumin is a major allergen from whey. & B10 \\
\hline 29 & Bos d 5 & $\begin{array}{l}\text { Cow's milk (whey) } \\
\text { (Bos domesticus) }\end{array}$ & $\begin{array}{l}\text { This Beta-lactoglobulin belongs to the group of lipocalins. } \\
\text { Therefore it may show cross-reactivity to a variety of } \\
\text { respiratory allergens. It is the only cow's milk protein that is } \\
\text { not present in human breast milk. }\end{array}$ & $\begin{array}{l}\mathrm{B} 10 \\
\mathrm{CO}\end{array}$ \\
\hline 30 & Bos d 6 & $\begin{array}{l}\text { Cow's milk (whey) } \\
\text { (Bos domesticus) }\end{array}$ & $\begin{array}{l}\text { Bos d } 6 \text { is a respiratory and food allergen as it is present in } \\
\text { bovine dander, in milk and meat. Also known as Bovine Serum } \\
\text { Albumin (BSA) it is classified as minor allergen in animal } \\
\text { dander, but is an important meat and milk allergen in the case } \\
\text { of uncooked food ingestion. }\end{array}$ & $\begin{array}{l}\text { B10/ } \\
\text { B14 } \\
\text { C04 }\end{array}$ \\
\hline 31 & Bos d 8 & $\begin{array}{l}\text { Cow's milk (curd) } \\
\text { (Bos domesticus) }\end{array}$ & $\begin{array}{l}\text { IgE to Casein (Bos d 8) can be found in } 53 \% \text { of the patients } \\
\text { reactive to cow's milk. High levels of specific IgE antibodies } \\
\text { directed against casein are predictive of clinical reactivity to } \\
\text { baked milk as it is more resistant to extensive heating than } \\
\text { other allergenic proteins. Due to the heterogeneity to the } \\
\text { casein fraction it has been further split into Bos d } 9 \text { - Bos } d 12 \text {. }\end{array}$ & B10 \\
\hline 32 & Bos d 9 & $\begin{array}{l}\text { Cow's milk (curd) } \\
\text { (Bos domesticus) }\end{array}$ & $\begin{array}{l}\text { This allergen represents the alphaS1- casein fraction from } \\
\text { whey and is regarded as a major food allergen. }\end{array}$ & B10 \\
\hline 33 & Bos d 10 & $\begin{array}{l}\text { Cow's milk (curd) } \\
\text { (Bos domesticus) }\end{array}$ & $\begin{array}{l}\text { This allergen represents the alphaS2-casein fraction from } \\
\text { whey and is regarded as a major food allergen. }\end{array}$ & B10 \\
\hline
\end{tabular}




\begin{tabular}{|c|c|c|c|c|}
\hline $\mathrm{Nr}$ & Allergen & Source & Description & Chapter \\
\hline 34 & Bos d 11 & $\begin{array}{l}\text { Cow's milk (curd) } \\
\text { (Bos domesticus) }\end{array}$ & $\begin{array}{l}\text { This allergen represents the beta-casein fraction from whey } \\
\text { and is regarded as a major food allergen. }\end{array}$ & B10 \\
\hline 35 & Bos d 12 & $\begin{array}{l}\text { Cow's milk (curd) } \\
\text { (Bos domesticus) }\end{array}$ & $\begin{array}{l}\text { This allergen represents the kappa-casein fraction from whey } \\
\text { and is regarded as a major food allergen. }\end{array}$ & B10 \\
\hline 36 & Can $\mathrm{f} 1$ & $\begin{array}{c}\text { Dog } \\
\text { (Canis familiaris) }\end{array}$ & $\begin{array}{l}\text { This major dog allergen (sensitization rate: } 50-76 \% \text { ) is a } \\
\text { species-specific marker of sensitization. Moreover, the } \\
\text { sensitization during childhood has been shown to be a } \\
\text { predictive marker of dog allergy in adolescence. Can } f 1 \text { is } \\
\text { a Lipocalin, synthesized in the salivary glands and dispersed } \\
\text { into the environment by saliva and dander. It has a moderate } \\
\text { risk of cross-reactivity with Fel d } 7 \text {. }\end{array}$ & $\begin{array}{l}\text { B06 } \\
\text { C07 }\end{array}$ \\
\hline 37 & Can $\mathrm{f} 3$ & $\begin{array}{l}\text { Dog } \\
\text { (Canis familiaris) }\end{array}$ & $\begin{array}{l}\text { This thermolabile protein is a serum albumin that has a high } \\
\text { risk of cross-reactivity with other serum albumins. }\end{array}$ & $\begin{array}{l}\mathrm{B} 06 \\
\mathrm{C} 04\end{array}$ \\
\hline 38 & Can $\mathrm{f} 6$ & $\begin{array}{l}\text { Dog } \\
\text { (Canis familiaris) }\end{array}$ & $\begin{array}{l}\text { This major dog allergen is a Lipocalin, synthesized in salivary } \\
\text { glands and dispersed into the environment by saliva and } \\
\text { dander. It has a moderate risk of cross-reactivity with Fel d } \\
4 \text { and Equ c } 1 \text {. }\end{array}$ & $\begin{array}{l}\text { B06 } \\
\text { C07 }\end{array}$ \\
\hline 39 & Che a 1 & $\begin{array}{l}\text { Goosefoot } \\
\text { (Chenopodium } \\
\text { album) }\end{array}$ & $\begin{array}{l}\text { This Ole e 1-like protein is a marker of sensitization to } \\
\text { goosefoot. It shows cross-reactivity with Sal k } 5 \text { of the } \\
\text { Russian Thistle. }\end{array}$ & B03 \\
\hline 40 & Cor a 1 & $\begin{array}{l}\text { Hazel (pollen and } \\
\quad \text { nuts) } \\
\text { (Corylus avellana) }\end{array}$ & $\begin{array}{l}\text { This Bet } v \text { 1-related food allergen belongs to the PR-10-like } \\
\text { proteins and is the major allergen in hazelnut allergy. One } \\
\text { isoform is mainly found in hazel pollen (Cor a 1.01), the other } \\
\text { mainly in the nut (Cor a 1.04). }\end{array}$ & $\begin{array}{l}\mathrm{B} 01 / \\
\mathrm{B} 19 \\
\mathrm{C} 02\end{array}$ \\
\hline 41 & Cor a 8 & $\begin{array}{l}\text { Hazelnut } \\
\text { (Corylus avellana) }\end{array}$ & $\begin{array}{l}\text { Cor a } 8 \text { is a non-specific Lipid Transfer Protein (nsLTP). Patients } \\
\text { with a sensitization to nsLTP mainly derived from peach (Pru p 3) } \\
\text { may develop cross-sensitization to other nsLTPs, such as Cor a } 8 .\end{array}$ & $\begin{array}{l}\mathrm{B} 19 \\
\mathrm{C} 03\end{array}$ \\
\hline 42 & Cor a 9 & $\begin{array}{c}\text { Hazelnut } \\
\text { (Corylus avellana) }\end{array}$ & $\begin{array}{l}\text { Cor a } 9 \text { is an abundant seed storage protein (11S globulin). } \\
\text { lgE antibodies against this allergen are linked with objective } \\
\text { symptoms for hazelnut allergy and a positive DBPCF for due } \\
\text { to hazelnut }\end{array}$ & B19 \\
\hline 43 & Cor a 14 & $\begin{array}{l}\text { Hazelnut } \\
\text { (Corylus avellana) }\end{array}$ & $\begin{array}{l}\text { Cor a } 14 \text { is a } 2 \mathrm{~S} \text {-albumin, which belongs to the seed storage } \\
\text { proteins. Severe allergic reactions to this allergenic molecule } \\
\text { have been reported in children and adults. Nevertheless it } \\
\text { doesn't qualify as major allergen, as most of the hazelnut } \\
\text { allergies are related to birch pollen (See Cor a 1) }\end{array}$ & B19 \\
\hline 44 & $\begin{array}{l}\text { Cross-reac- } \\
\text { tive carbo- } \\
\text { hydrate de- } \\
\text { terminants } \\
\text { (CCDs) }\end{array}$ & $\begin{array}{l}\text { Variable } \\
\text { (Plant derived) }\end{array}$ & $\begin{array}{l}\text { Cross-reactive carbohydrate determinants do not behave as } \\
\text { allergens in vivo and are therefore clinically insignificant, but } \\
\text { the presence of IgE to CCDs could lead to a misleading in vitro } \\
\text { reactivity also in the case of extract-based testing or when } \\
\text { using CCD-containing natural purified glycoproteins }\end{array}$ & B01 \\
\hline 45 & Cup a 1 & $\begin{array}{l}\text { Cypress } \\
\text { (Cupressus } \\
\text { arizonica) }\end{array}$ & $\begin{array}{l}\text { Cup a } 1 \text { is a specific marker allergen for a sensitization to } \\
\text { pollen of trees of the Cupressaceae family. The high sequence } \\
\text { identity, and therefore high degree of cross-reactivity among } \\
\text { Cupressaceae family members, suggests the use of Cup a } \\
1 \text { as a representative marker of the entire family for both } \\
\text { diagnostic testing and therapeutic approaches }\end{array}$ & B01 \\
\hline
\end{tabular}




\begin{tabular}{|c|c|c|c|c|}
\hline $\mathrm{Nr}$ & Allergen & Source & Description & Chapter \\
\hline 46 & Cyn d 1 & $\begin{array}{l}\text { Bermuda grass } \\
\text { (Cynodon dactylon) }\end{array}$ & $\begin{array}{l}\text { This major beta expansin group } 1 \text { allergen is a marker of } \\
\text { sensitization to the subtropical Bermuda grass. }\end{array}$ & B02 \\
\hline 47 & Сур с 1 & $\begin{array}{l}\text { Common Carp } \\
\text { (Cyprinus carpio) }\end{array}$ & $\begin{array}{l}\text { This major allergen in fish muscle is a parvalbumin. } \\
\text { Parvalbumins are small muscle proteins of remarkable stability } \\
\text { towards physicochemical effects by food processing. During } \\
\text { fish preparation, they can even become airborne. Most fish- } \\
\text { allergic patients have specific lgE to these allergens. }\end{array}$ & $\begin{array}{l}\text { B12 } \\
\text { C08 }\end{array}$ \\
\hline 48 & Der $\mathrm{p} 1$ & $\begin{array}{l}\text { House Dust Mite } \\
\text { (Dermatophagoides } \\
\text { pteronyssinus) }\end{array}$ & $\begin{array}{l}\text { Der p } 1 \text { is a major mite allergen (Prevalence among patients: } \\
70-100 \%) \text {. As an active cysteine protease, it has been } \\
\text { identified in fecal particles and is strongly associated with } \\
\text { asthma. Its important role for the symptoms of rhinitis and } \\
\text { asthma has been evidenced. }\end{array}$ & B04 \\
\hline 49 & Der $p 2$ & $\begin{array}{l}\text { House dust mite } \\
\text { (Dermatophagoides } \\
\text { pteronyssinus) }\end{array}$ & $\begin{array}{l}\text { Der p } 2 \text { is a major mite allergen (Prevalence among patients: } \\
80-100 \%) \text {. It has been identified in fecal particles and is } \\
\text { strongly associated with asthma. This molecule has activity } \\
\text { comparable to MD2. }\end{array}$ & B04 \\
\hline 50 & Der p 10 & $\begin{array}{l}\text { House dust mite } \\
\text { (Dermatophagoides } \\
\text { pteronyssinus) }\end{array}$ & $\begin{array}{l}\text { Der } \mathrm{p} 10 \text { is a Tropomyosin from house dust mite, present in } \\
\text { muscle and non-muscle cells. The tropomyosin amino acid } \\
\text { sequence is highly conserved among shellfish and other } \\
\text { invertebrates, which explains the high level of cross-reactivity. } \\
\text { Symptoms, which may depend on ingestion or inhalation, range } \\
\text { from milder reactions to anaphylaxis. HDM allergic patients in } \\
\text { Europe do not show high prevalences of lgE sensitization to Der } \\
\mathrm{p} 10 \text {. The observed sensitizations can be considered an effect } \\
\text { of cross-reactivity, but also a marker for broad sensitization. }\end{array}$ & $\begin{array}{l}\text { B04 } \\
\text { C05 }\end{array}$ \\
\hline 51 & Der $p 23$ & $\begin{array}{l}\text { House dust mite } \\
\text { (Dermatophagoides } \\
\text { pteronyssinus) }\end{array}$ & $\begin{array}{l}\text { Der p } 23 \text { is a house dust mite allergen identified in the fecal } \\
\text { particles and in the peritrophic lining of the gut. This recently } \\
\text { described molecule is a Peritrophin like protein that appears } \\
\text { to be associated with asthma. }\end{array}$ & B04 \\
\hline 52 & Equ c 1 & $\begin{array}{c}\text { Horse } \\
\text { (Equus Caballus) }\end{array}$ & $\begin{array}{l}\text { This major horse allergen is a species-specific marker of } \\
\text { sensitization. It is a Lipocalin, synthesized in salivary glands } \\
\text { and dispersed into the environment by saliva and dander. It } \\
\text { has a moderate risk of cross-reactivity with Fel d } 4 \text { and Can } \mathrm{f} \\
6 \text { and is known to have surfactant properties. }\end{array}$ & $\begin{array}{l}\text { B06 } \\
\text { C07 }\end{array}$ \\
\hline 53 & Equ c 3 & $\begin{array}{c}\text { Horse } \\
\text { (Equus Caballus) }\end{array}$ & $\begin{array}{l}\text { Equ c } 3 \text { is a serum albumin, a thermolabile protein synthesized } \\
\text { in the liver. This allergen is present in horse dander, secretions } \\
\text { and meat. It shows a high risk of cross-reactivity with other } \\
\text { serum albumins. }\end{array}$ & $\begin{array}{l}\text { B06 } \\
\text { C04 }\end{array}$ \\
\hline 54 & Fel d 1 & $\begin{array}{c}\text { Cat } \\
\text { (Felis domesticus) }\end{array}$ & $\begin{array}{l}\text { Fel } d 1 \text {, the major cat allergen, is a species-specific marker } \\
\text { of sensitization. Moreover, the sensitization during childhood } \\
\text { has been shown to be a predictive marker of cat allergy in } \\
\text { adolescence. This molecule, whose synthesis is related to } \\
\text { sexual hormones, is an uteroglobin expressed in skin and } \\
\text { salivary glands. }\end{array}$ & B06 \\
\hline 55 & Fel d 4 & $\begin{array}{c}\text { Cat } \\
\text { (Felis Domesticus) }\end{array}$ & $\begin{array}{l}\text { This major cat allergen is a Lipocalin, synthesized in salivary } \\
\text { glands and dispersed into the environment by saliva and } \\
\text { dander. It has a moderate risk of cross-reactivity with Can } \mathrm{f} 6 \\
\text { and Equ c } 1 \text {. }\end{array}$ & $\begin{array}{l}\mathrm{B} 06 \\
\mathrm{C} 07\end{array}$ \\
\hline
\end{tabular}




\begin{tabular}{|c|c|c|c|c|}
\hline $\mathrm{Nr}$ & Allergen & Source & Description & Chapter \\
\hline 56 & Gad c 1 & $\begin{array}{l}\text { Codfish } \\
\text { (Gadus callarias) }\end{array}$ & $\begin{array}{l}\text { The dominating major allergen in fish muscle is parvalbumin } \\
\text { of which Gad c } 1 \text { was the first to be identified. Parvalbumins } \\
\text { are small muscle proteins of remarkable stability towards } \\
\text { physicochemical effects by food processing. During fish } \\
\text { preparation, they can even become airborne. Parvalbumin } \\
\text { levels vary considerably in different fish tissues and species. } \\
\text { Most fish-allergic patients have specific IgE to these allergens. }\end{array}$ & $\begin{array}{l}\mathrm{B} 12 \\
\mathrm{C} 08\end{array}$ \\
\hline 57 & Gal d 1 & $\begin{array}{c}\text { Hen's egg } \\
\text { (Gallus domesticus) }\end{array}$ & $\begin{array}{l}\text { Ovomucoid is a heat-stable and highly allergenic egg white } \\
\text { protein. } 1 \text { IgE responses to Gal } d 1 \text { indicate a risk for reaction } \\
\text { to all forms of egg High levels of specific IgE may indicate } \\
\text { sustained egg allergy. }\end{array}$ & B11 \\
\hline 58 & Gal d 2 & $\begin{array}{c}\text { Hen's egg } \\
\text { (Gallus domesticus) }\end{array}$ & $\begin{array}{l}\text { Ovalbumin is the most abundant egg white protein. As it is } \\
\text { heat-labile, IgE responses to Gal d } 2 \text { indicate a risk for clinical } \\
\text { reaction to raw or slightly heated egg as well as certain vaccines. }\end{array}$ & B11 \\
\hline 59 & Gal d 3 & $\begin{array}{c}\text { Hen's egg } \\
\text { (Gallus domesticus) }\end{array}$ & $\begin{array}{l}\text { Ovotransferrin or conalbumin is a heat-labile egg white } \\
\text { protein with iron-binding capacity and antimicrobial activity. } \\
\text { IgE antibodies against Gal d } 3 \text { increase the risk for a clinical } \\
\text { reaction to raw or slightly heated egg. }\end{array}$ & B11 \\
\hline 60 & Gad m 1 & $\begin{array}{l}\text { Atlantic Codfish } \\
\text { (Gadus morhua) }\end{array}$ & $\begin{array}{l}\text { This molecule is a parvalbumin, the dominating major allergen } \\
\text { in fish muscle. Parvalbumins are small muscle proteins of } \\
\text { remarkable stability towards physicochemical effects by food } \\
\text { processing. Parvalbumin levels vary considerably in different } \\
\text { fish tissues and species. Most fish-allergic patients have } \\
\text { specific lgE to these allergens. }\end{array}$ & $\begin{array}{l}\mathrm{B} 12 \\
\mathrm{C} 08\end{array}$ \\
\hline 61 & Gly m 1 & $\begin{array}{c}\text { Soy } \\
\text { (Glycine max) }\end{array}$ & $\begin{array}{l}\text { Gly } m 1 \text { is a major respiratory allergen from soybean shells, to } \\
\text { which subjects are exposed through the inhalation of soybean } \\
\text { dust. Sensitization rates are still lacking. }\end{array}$ & B17 \\
\hline 62 & Gly m 4 & $\begin{array}{c}\text { Soy } \\
\text { (Glycine max) }\end{array}$ & $\begin{array}{l}\text { Gly } \mathrm{m} 4 \text {, an allergen from soy, is a PR-10-like protein (Bet } \\
\text { v } 1 \text { homologue) that has low thermal and digestive stability. } \\
\text { Soy allergy due to Bet v } 1 \text {-cross-reactions is considered the } \\
\text { most prevalent soy allergy in Northern and Middle Europe, } \\
\text { presumably also in the Northern parts of Asia, as well as } \\
\text { in North America (Canada, Northern states of the US), } \\
\text { depending on the degree of birch pollen exposure. }\end{array}$ & $\begin{array}{l}\mathrm{B} 17 \\
\mathrm{CO} 2\end{array}$ \\
\hline 63 & Gly m 5 & $\begin{array}{c}\text { Soy } \\
\text { (Glycine max) }\end{array}$ & $\begin{array}{l}\text { Sensitization to Gly } \mathrm{m} 5 \text {, a vicilin ( } 7 \mathrm{~S} \text { globulin) from soy points } \\
\text { towards an increased risk for atopic dermatitis and (severe) } \\
\text { food allergy to soy and related plant foods in children. } \\
\text { Prevalence rates are still unknown. }\end{array}$ & B17 \\
\hline 64 & Gly m 6 & $\begin{array}{c}\text { Soy } \\
\text { (Glycine max) }\end{array}$ & $\begin{array}{l}\text { Sensitization to Gly } \mathrm{m} 6 \text {, a legumin ( } 11 \mathrm{~S} \text { globulin) from soy } \\
\text { points towards an increased risk for atopic dermatitis and } \\
\text { (severe) food allergy to soy and related plant foods in children. } \\
\text { Prevalence rates are still unknown. }\end{array}$ & B17 \\
\hline 65 & Hev b 1 & $\begin{array}{c}\text { Rubber tree } \\
\text { (Hevea brasiliensis) }\end{array}$ & $\begin{array}{l}\text { Hev b } 1 \text { is a rubber elongation factor (REF) that is hard } \\
\text { to aerosolize because of its insolubility. Therefore, the } \\
\text { sensitization to this molecule seems to require contact with } \\
\text { blood or mucosal surfaces. Sensitization to Hev b } 1 \text { is less } \\
\text { common in health care workers, but it represents a major } \\
\text { allergen in spina bifida patients. }\end{array}$ & B22 \\
\hline
\end{tabular}




\begin{tabular}{|c|c|c|c|c|}
\hline $\mathrm{Nr}$ & Allergen & Source & Description & Chapter \\
\hline 66 & Hev b 3 & $\begin{array}{c}\text { Rubber tree } \\
\text { (Hevea brasiliensis) }\end{array}$ & $\begin{array}{l}\text { Hev b } 3 \text { is a small rubber particle protein that is hard } \\
\text { to aerosolize because of its insolubility. Therefore, the } \\
\text { sensitization to this molecule seems to require contact with } \\
\text { blood or mucosal surfaces. Sensitization to Hev b } 3 \text { is less } \\
\text { common in health care workers, but it represents a major } \\
\text { allergen in spina bifida patients. }\end{array}$ & B22 \\
\hline 67 & Hev b 5 & $\begin{array}{c}\text { Rubber tree } \\
\text { (Hevea brasiliensis) }\end{array}$ & $\begin{array}{l}\text { Hev b } 5 \text { is an acidic and heat- stable protein from rubber tree. } \\
\text { Currently rHev b 5, together with rHevb 6.01, is a major allergen } \\
\text { among health care workers sensitized to latex. Apart from this, } \\
\text { it is represents a pajor allergen for spina bifida patients. }\end{array}$ & B22 \\
\hline 68 & $\begin{array}{c}\text { Hev b } \\
6.01\end{array}$ & $\begin{array}{c}\text { Rubber tree } \\
\text { (Hevea brasiliensis) }\end{array}$ & $\begin{array}{l}\text { This rubber tree allergen is a Prohevein, that with } \\
\text { posttranslational cleavage proceeds in two further proteins: } \\
\text { Hev b } 6.02 \text { (hevein) and Hev b } 6.03 \text {. Currently } \mathrm{rHev} b 6.01 \text {, } \\
\text { together with rHev b } 5 \text {, is a major allergen among health care } \\
\text { workers sensitized to latex. Apart from this, it also represents } \\
\text { a major allergen for spina bifida patients. }\end{array}$ & B22 \\
\hline 69 & Hev b 8 & $\begin{array}{c}\text { Rubber tree } \\
\text { (Hevea brasiliensis) }\end{array}$ & $\begin{array}{l}\text { This rubber tree allergen is a profilin (Actin -binding protein), } \\
\text { that has been discussed as responsible for the latex -fruit } \\
\text { cross-reactivity. }\end{array}$ & $\begin{array}{l}\text { B22 } \\
\text { C01 }\end{array}$ \\
\hline 70 & Jug r 1 & $\begin{array}{c}\text { Walnut } \\
\text { (Juglans regia) }\end{array}$ & $\begin{array}{l}\text { Jug } r \text { is a } 2 S \text { albumin and sensitization to } 2 S \text { albumins from } \\
\text { tree nuts including walnut can be related with more severe } \\
\text { fodo allergic reactions. }\end{array}$ & B19 \\
\hline 71 & Jug r 3 & $\begin{array}{c}\text { Walnut } \\
\text { (Juglans regia) }\end{array}$ & $\begin{array}{l}\text { Jug r } 3 \text { is a non-specific lipid transfer protein (nsLTP). Patients } \\
\text { with a sensitization to nsLTP mainly derived from peach (Pru } \\
\text { p 3) may develop cross-sensitization to Walnut. }\end{array}$ & $\begin{array}{l}\mathrm{B} 19 \\
\mathrm{CO} 3\end{array}$ \\
\hline 72 & Mal d 1 & $\begin{array}{c}\text { Apple } \\
\text { (Malus domestica) }\end{array}$ & $\begin{array}{l}\text { This allergen is a PR.10-like molecule (Bet } \vee 1 \text { homologue) } \\
\text { from apple, thus cross-reactivity between pollen and food may } \\
\text { occur. }\end{array}$ & $\begin{array}{l}\mathrm{B} 15 \\
\mathrm{CO} 2\end{array}$ \\
\hline 73 & Mal d 3 & $\begin{array}{c}\text { Apple } \\
\text { (Malus domestica) }\end{array}$ & $\begin{array}{l}\text { Mal d } 3 \text { is a non-specific Lipid Transfer Protein, a small } \\
\text { stable protein not affected by low pH environment and } \\
\text { heat treatment. Patient with a sensitization to nsLTP mainly } \\
\text { derived from peach (Pru p 3) may develop cross-sensitization } \\
\text { to other fruit nsLTPs. The clinical manifestations vary from } \\
\text { local oropharyngeal symptoms up to anaphylaxis. The clinical } \\
\text { pattern is influenced by cofactors. }\end{array}$ & $\begin{array}{l}\mathrm{B} 15 \\
\mathrm{CO3}\end{array}$ \\
\hline 74 & Mala s 6 & $\begin{array}{l}\text { Fungus } \\
\text { (Malassezia } \\
\text { sympodialis) }\end{array}$ & $\begin{array}{l}\text { This allergen of Malassezia sympodialis is a Cyclophilin, } \\
\text { recognized by the } 92 \% \text { of the sensitized patients affected by } \\
\text { atopic dermatitis. }\end{array}$ & B08 \\
\hline 75 & Mala s 11 & $\begin{array}{l}\text { Fungus } \\
\text { (Malassezia } \\
\text { sympodialis) }\end{array}$ & $\begin{array}{l}\text { This allergen of Malassezia sympodialis is a Manganese } \\
\text { Superoxide Dismutase. In patients affected by atopic } \\
\text { dermatitis, the sensitization to this allergen correlates to } \\
\text { disease severity. It is highly cross-reacting with Asp f } 6 \text {. }\end{array}$ & B08 \\
\hline 76 & Mus $\mathrm{m} 1$ & $\begin{array}{l}\text { Mouse urine } \\
\text { (Mus musculus) }\end{array}$ & $\begin{array}{l}\text { This major mouse allergen is a prealbumin and lipocalin- } \\
\text { odorant-binding protein belonging to the rodent family of } \\
\text { major urinary proteins (MUP). MUPs are produced in the } \\
\text { liver and other exocrine glands under hormonal control } \\
\text { and secreted in urine. They seem to play a complex role in } \\
\text { chemosensory signaling among rodents. }\end{array}$ & $\begin{array}{l}\text { B23 } \\
\text { C07 }\end{array}$ \\
\hline
\end{tabular}




\begin{tabular}{|c|c|c|c|c|}
\hline $\mathrm{Nr}$ & Allergen & Source & Description & Chapter \\
\hline 77 & Ole e 1 & $\begin{array}{l}\text { Olive tree, } \\
\text { Plane tree } \\
\text { (Olea europaea) }\end{array}$ & $\begin{array}{l}\text { Ole e } 1 \text { is the most common sensitizing molecule in olive } \\
\text { pollen. It is utilized in both diagnostic and therapeutic } \\
\text { extracts for standardization purposes and can determine } \\
\text { immunological changes after olive pollen AIT }\end{array}$ & B01 \\
\hline 78 & Par j 1 & $\begin{array}{c}\text { Pellitory } \\
\text { (Parietaria judaica) }\end{array}$ & $\begin{array}{l}\text { Parietaria pollen is the only pollen whose major allergen, Par } \\
\mathrm{j} 1 \text {, is a non-specific lipid transfer (nsLTP) protein. Accepted } \\
\text { threshold pollen levels for sensitization are low and clinically, } \\
\text { Parietaria pollinosis is often linked to asthma. }\end{array}$ & $\begin{array}{l}\mathrm{B} 03 \\
\mathrm{CO} 3\end{array}$ \\
\hline 79 & Par j 2 & $\begin{array}{c}\text { Pellitory } \\
\text { (Parietaria judaica) }\end{array}$ & $\begin{array}{l}\text { Par j 2, a non-specific lipid Transfer Protein, is a highly specific } \\
\text { marker for sensitization to pellitory. }\end{array}$ & B03 \\
\hline 80 & Pen a 1 & $\begin{array}{l}\text { Brown Shrimp } \\
\text { (Penaeus aztecus) }\end{array}$ & $\begin{array}{l}\text { The shrimp major allergen, Pen a 1, is one of the most clinically } \\
\text { relevant allergenic tropomyosins. Five major IgE binding sites on } \\
\text { Pen a } 1 \text { have been identified that were cross-reactive epitopes } \\
\text { among shrimp, lobster, house dust mite and cockroach. Its } \\
\text { heat-stability partially explains its high allergenicity. }\end{array}$ & $\begin{array}{l}\text { B13 } \\
\text { C05 }\end{array}$ \\
\hline 81 & Per a 1 & $\begin{array}{l}\text { American Cockroach } \\
\text { (Periplaneta ameri- } \\
\text { cana) }\end{array}$ & $\begin{array}{l}\text { Per a } 1 \text { is a midgut microvilli protein homologue from the } \\
\text { American Cockroach It shows cross-reactivity with the } \\
\text { homologous protein Bla o } 1 \text { from the German Cockroach. }\end{array}$ & B05 \\
\hline 82 & Per a 2 & $\begin{array}{l}\text { American } \\
\text { Cockroach } \\
\text { (Periplaneta } \\
\text { americana) }\end{array}$ & $\begin{array}{l}\text { Per a } 2 \text { is an Aspartic protease-like from American Cockroach. } \\
\text { Sensitization to Per a } 2 \text { has been recognized more frequently } \\
\text { in patients with persistent asthma than in patients with } \\
\text { rhinitis only, suggesting that this allergen could be a marker } \\
\text { for more severe airway disease. }\end{array}$ & B05 \\
\hline 83 & Per a 7 & $\begin{array}{l}\text { American } \\
\text { Cockroach } \\
\text { (Periplaneta } \\
\text { americana) }\end{array}$ & $\begin{array}{l}\text { Per a } 7 \text { is a Tropomyosin from the American Cockroach. IgE } \\
\text { binding frequency to cockroach and mite tropomyosins are } \\
\text { very different according to different populations. Higher } \\
\text { values in tropical Countries and lower in the US and Europe } \\
\text { may reflect differences in environmental conditions. }\end{array}$ & $\begin{array}{l}\text { B05 } \\
\text { C05 }\end{array}$ \\
\hline 84 & Phl p 1 & $\begin{array}{l}\text { Timothy grass } \\
\text { (Phleum pratense) }\end{array}$ & $\begin{array}{l}\text { This major timothy grass pollen allergen is a marker of } \\
\text { genuine, species-specific, sensitization. It shares epitopes } \\
\text { with group } 1 \text { allergens from other grasses and shows IgE } \\
\text { cross-reactivity to most other group } 1 \text { allergens from grasses, } \\
\text { corns and monocots. Sensitization to Phl p } 1 \text { usually precedes } \\
\text { other grass pollen sensitizations and its specific lgE response } \\
\text { is the most prevalent in grass pollen allergic patients. }\end{array}$ & B02 \\
\hline 85 & Phl p 4 & $\begin{array}{l}\text { Timothy grass } \\
\text { (Phleum pratense) }\end{array}$ & $\begin{array}{l}\text { Phl p } 4 \text { is a tryptase-resistant glycoprotein. It can be classified } \\
\text { as a major allergen. It shows IgE cross-reactivity with other } \\
\text { group } 4 \text { grass pollen allergens. Moreover, cross-reactivity } \\
\text { to the major ragweed allergen Amb a } 1 \text { and to Oilseed Rape } \\
\text { pollen has been demonstrated. Natural Phl p } 4 \text { contains CCD, } \\
\text { which may lead to IgE cross-reactivity with a wide range of } \\
\text { plants and plant products. }\end{array}$ & B02 \\
\hline 86 & Phl p 5 & $\begin{array}{l}\text { Timothy grass } \\
\text { (Phleum pratense) }\end{array}$ & $\begin{array}{l}\text { Phl p } 5 \text { is another major pollen allergen of temperate grasses } \\
\text { with a lower sensitization prevalence, but often with high IgE- } \\
\text { levels. Phl p } 5 \text { is a cytoplasmatic ribonuclease, important in } \\
\text { the enzymatic degradation of RNA. It shows broad IgE cross- } \\
\text { reactivity with other group } 5 \text { allergens from the Pooideae } \\
\text { subfamily of temperate grasses. }\end{array}$ & B02 \\
\hline
\end{tabular}




\begin{tabular}{|c|c|c|c|c|}
\hline $\mathrm{Nr}$ & Allergen & Source & Description & Chapter \\
\hline 87 & Phl p 7 & $\begin{array}{l}\text { Timothy grass } \\
\text { (Phleum pratense) }\end{array}$ & $\begin{array}{l}\text { Phl } \mathrm{p} 7 \text { and } \mathrm{Phl} p 12 \text { are minor allergens, representing } \\
\text { pan-allergens from the plant world. Phl p } 7 \text {, polcalcin, is a } \\
\text { calcium binding protein present in many different pollen, } \\
\text { hence representing a broad cross-reacting allergen. Phl p } \\
\text { 7-sensitization can be used as a marker for a more general } \\
\text { pollen sensitization. }\end{array}$ & $\begin{array}{l}\mathrm{B} 02 \\
\mathrm{C} 06\end{array}$ \\
\hline 88 & Phl p 12 & $\begin{array}{l}\text { Timothy grass } \\
\text { Profilin } \\
\text { (Phleum pratense) }\end{array}$ & $\begin{array}{l}\text { Phl p } 12 \text { is a member of the profilin family, an actin-binding } \\
\text { protein that is present throughout the whole plant world. As } \\
\text { profilins are ubiquitous in plant cells, profilin sensitization } \\
\text { gives rise to a long range of cross-reacting plants and plant } \\
\text { products as birch, soybean, corn, latex and plant foods. }\end{array}$ & $\begin{array}{l}\mathrm{B} 02 \\
\mathrm{C} 01\end{array}$ \\
\hline 89 & Pla a 1 & $\begin{array}{l}\text { Plane tree } \\
\text { (Platanus } \\
\text { acerifolia) }\end{array}$ & $\begin{array}{l}\text { Pla a } 1 \text { and Pla a } 2 \text { may serve as a marker of primary } \\
\text { sensitization to plane tree pollen, therefore it is useful for } \\
\text { AIT selection, whilst the nsLTP Pla a } 3 \text { has been linked with } \\
\text { sensitization to plant-food LTPs }\end{array}$ & B01 \\
\hline 90 & Pla I 1 & $\begin{array}{l}\text { English plantain } \\
\text { (Plantago } \\
\text { lanceolata) }\end{array}$ & $\begin{array}{l}\text { Pla I 1, an Ole e 1-like protein, is a highly specific marker } \\
\text { allergen for English plantain because of the limited cross- } \\
\text { reactivity with the other protein family members. }\end{array}$ & B03 \\
\hline 91 & Pru p 3 & $\begin{array}{c}\text { Peach } \\
\text { (Prunus persica) }\end{array}$ & $\begin{array}{l}\text { Pru p } 3 \text { is the major allergen from peach. This molecule is a } \\
\text { non-specific lipid transfer protein, a small protein stable at } \\
\text { pH extremes, heat treatment and proteolytic digestion. It is } \\
\text { concentrated in the pericarp of fruits, while the pulp contains } \\
\text { levels approximately } 220 \text {-fold lower than the peel. It shows } \\
\text { from } 62 \text { to } 81 \% \text { of identity sequence with analogue protein } \\
\text { from apple, apricot, plum, cherry, orange, strawberry, grape. } \\
\text { Peach is the most frequent cause of nsLTP allergy, and } \\
\text { Pru p 3-sensitization seems to play a precursor role in the } \\
\text { sensitization to other nsLTPs. }\end{array}$ & $\begin{array}{l}\text { B15 } \\
\text { C03 }\end{array}$ \\
\hline 92 & Pru p 4 & $\begin{array}{c}\text { Peach } \\
\text { (Prunus persica) }\end{array}$ & $\begin{array}{l}\text { Pru p } 4 \text { is a Profilin from peach. Profilins are small proteins with } \\
\text { ubiquitous expression throughout the plant kingdom. They } \\
\text { are functional in various important cell-signalling pathways } \\
\text { and bind actin. These small proteins are of intermediate to } \\
\text { low stability when subjected to heat treatment. Sensitization } \\
\text { to profilin is frequently observed in patients, however it often } \\
\text { lacks clinical relevance. Allergens from the profilin family have } \\
\text { been identified also in apple, pear, cherry and strawberry. }\end{array}$ & $\begin{array}{l}\text { B15 } \\
\text { C01 }\end{array}$ \\
\hline 93 & Rat n 1 & $\begin{array}{l}\text { Rat urine } \\
\text { (Rattus norvegicus) }\end{array}$ & $\begin{array}{l}\text { Analogous to mouse allergens, the major rat (Rattus } \\
\text { norvegicus) allergen Rat } n 1 \text { is a prealbumin or alpha- } 2 \mathrm{u}- \\
\text { globulin that belongs to the lipocalin group and to the family } \\
\text { of MUPs (major urinary proteins). The amino acid identity } \\
\text { between mouse and rat MUPs is approximately } 65 \% \text {. Urine } \\
\text { collected from male rats contains much larger quantities of } \\
\text { Rat } n 1 \text { than urine from female rats. }\end{array}$ & $\begin{array}{l}\mathrm{B} 23 \\
\mathrm{C} 07\end{array}$ \\
\hline 94 & Sal k 1 & $\begin{array}{l}\text { Russian thistle } \\
\text { (Salsola kali) }\end{array}$ & $\begin{array}{l}\text { Sal k } 1 \text {, a Pectin Methylesterase, is a marker of sensitization } \\
\text { to Salsola. This allergen contains N-glycans, thus result might } \\
\text { be false positive if the patient is CCD positive. }\end{array}$ & B03 \\
\hline
\end{tabular}




\begin{tabular}{|c|c|c|c|c|}
\hline $\mathrm{Nr}$ & Allergen & Source & Description & Chapter \\
\hline 95 & Sal s 1 & $\begin{array}{l}\text { Atlantic Salmon } \\
\text { (Salmo salar) }\end{array}$ & $\begin{array}{l}\text { The dominating major allergen in fish muscle is parvalbumin. } \\
\text { Parvalbumins are small muscle proteins of remarkable } \\
\text { stability towards physicochemical effects by food processing. } \\
\text { During fish preparation, they can even become airborne. } \\
\text { Parvalbumin levels vary considerably in different fish tissues } \\
\text { and species. Most fish-allergic patients have specific IgE to } \\
\text { these allergens. }\end{array}$ & $\begin{array}{l}\mathrm{B} 12 \\
\mathrm{C} 08\end{array}$ \\
\hline 96 & Sus $\mathrm{s} 1$ & $\begin{array}{l}\text { Pig (Sus scrofa } \\
\text { domestica) }\end{array}$ & $\begin{array}{l}\text { Sus } s 1 \text { is the pork albumin. Its cross-reactivity with cat } \\
\text { albumin characterizes the cat pork syndrome. }\end{array}$ & B14 \\
\hline 97 & Tri a 14 & $\begin{array}{l}\text { Wheat } \\
\text { (Triticum aestivum) }\end{array}$ & $\begin{array}{l}\text { This molecule is a non-specific lipid transfer protein that has } \\
\text { high heat stability and probably does not cross-react with } \\
\text { grass pollen. It may be important both in wheat-dependent, } \\
\text { exercise-induced anaphylaxis (WDEIA) and in some cases of } \\
\text { food allergy. }\end{array}$ & $\begin{array}{l}\mathrm{B} 16 \\
\mathrm{C} 03\end{array}$ \\
\hline 98 & Tri a 19 & $\begin{array}{l}\text { Wheat } \\
\text { (Triticum aestivum) }\end{array}$ & $\begin{array}{l}\text { Tri a } 19 \text { is a wheat seed storage protein. This molecule is } \\
\text { an } \omega-5 \text {-gliadine not well represented in wheat extracts } \\
\text { because of its poor aqueous solubility. } 50 \%-70 \% \text { of wheat } \\
\text { allergic patients are sensitized to this allergen. Tri a } 19 \text { is } \\
\text { often responsible for wheat-dependent, exercise-induced } \\
\text { anaphylaxis (WDEIA). }\end{array}$ & B16 \\
\hline 99 & Ves v 1 & $\begin{array}{l}\text { Yellow Jacket } \\
\text { (Wasp) } \\
\text { (Vespula vulgaris) }\end{array}$ & $\begin{array}{l}\text { This Phospholipase A1 is the major allergen and therefore } \\
\text { marker protein in vespid venom allergy. }\end{array}$ & B21 \\
\hline 100 & Ves v 5 & $\begin{array}{l}\text { Yellow Jacket } \\
\quad \text { (Wasp) } \\
\text { (Vespula vulgaris) }\end{array}$ & $\begin{array}{l}\text { Ves } \vee 5 \text { is a marker protein for vespid venom allergy. It is a } \\
\text { protein of unknown function but high abundance in the } \\
\text { venom. With sensitization rates of } 84.5 \text { to } 100 \% \text { it is a major } \\
\text { allergen. }\end{array}$ & B21 \\
\hline
\end{tabular}




\section{APPENDICES}

GLOSSARY OF

IMMUNOLOGICAL

ASSAYS IN

MOLECULAR

ALLERGOLOGY

affinity in immunology, a measure of the attraction or force of association between a single antigenic site and a single antibody to that site ( $\mathrm{Ka})$.

allergen extract a mixture of molecules (some allergens and others not allergens), typically composed of proteins, glycoproteins, lipoproteins, or protein-conjugated chemicals/drugs that have been solubilized from a defined (usually biological) source and that a portion of which can elicit an immunoglobulin $\mathrm{E}$ antibody response in exposed persons;

Note: Allergen extracts have historically been referred to as "allergens" by diagnostic reagent manufacturers. This common practice should be replaced by the use of "allergen extract" to distinguish it from an allergen molecule.

allergen molecule individual native or recombinant allergen that has unique molecular and structural properties, including a defined molecular weight, isoelectric point, carbohydrate composition, nucleotide and/or amino acid sequence, and

Taken with permission from [59]

Note: this list is not exhaustive and has not yet been agreed on by all authors. A complete glossary will be provided in the future editions of this manuscript reactivity to a monospecific or monoclonal antibody. In addition, the allergenic property of the "major allergens" of a given allergen specificity needs to be verified by its ability to bind to immunoglobulin $E$ antibody and/or induce a positive skin test or histamine release from basophils from individuals who are clinically allergic to that allergen specificity.

allergen source (raw) material the starting raw material from which allergenic extracts are obtained;

Note: This material may or may not have been physically processed to remove extraneous, nonbiological materials, and it is typified by materials such as raw pollen, animal hair, mold cultures, drugs, venoms, foodstuffs, or recombinant expressed proteins.

allergenic epitope a submolecular structure or surface on the allergenic molecule that is primarily responsible for IgE antibody binding.

allergosorbent a solid phase material to which allergen extracts (mixtures of allergens) or individual native or recombinant allergen molecules are attached by covalent coupling methods or adsorption. 
antigen in immunology, any substance that can stimulate the production of antibodies by an organism and combine specifically with them.

analytical sensitivity quotient of the change in an indication and the corresponding change in the value of a quantity being measured (ISO 15193 ); Note 1: The term "analytical sensitivity" has been historically used to describe the lowest amount of a given substance in a biological specimen that is detectable in an assay system;

analytical specificity/selectivity ability of a measurement procedure to determine solely the quantity it purports to measure (ISO 1519323);

Note 1: Analytical specificity (selectivity) refers in general to the ability of an assay to measure one particular substance, rather than others, in a sample. Applied to immunoglobulin $E$ (IgE) assays with single allergens, "one particular substance" would indicate the repertoire of allergen-specific IgE to "one particular allergen molecule";

Note 2: In the previous editions of I/LA20, analytical specificity of IgE-detecting assays was solely linked to the capability to selectively measure IgE, instead of other immunoglobulin classes or subclasses (eg, immunoglobulin $G$, immunoglobulin $M)$. This definition refers to the "antibody-related analytical specificity (selectivity)" of IgE-detecting assays and is still an important definition for modern immunoassays;

Note 3: Allergen molecules for quantifying allergen-specific IgE will limit the detected IgE repertoire. Only antibodies binding to the selected molecule (eg, major cat allergen Fel d 1) will be detected instead of all allergen source-specific (eg, cat-specific) antibodies. Thus, the use of allergen molecules increases analytical specificity compared to the broad allergen-specific lgE repertoire directed toward a complex mixture of proteins in an extract (eg, from cat dander). This new definition refers to the "allergen-related analytical specificity (selectivity)" of IgE-detecting assays;

Note 4: The term "allergen-related analytical specificity (selectivity)" is particularly useful to understand and justify the concept of species-specific vs cross-reactive IgE antibodies toward defined allergen molecules. In case of particular physicochemical properties of certain allergen molecules (eg, high $\mathrm{pH}$ and digestion stability, high abundance in an allergen source), IgE detection with these molecules might be helpful to identify risk-associated allergen-specific IgE responses.

component resolved diagnosis [see: Molecularbased allergy diagnosis] Due to the misleading definitions of component, "Component resolved diagnosis" has been relabeled as "Molecular-based allergy (MA) diagnostics."

cross-reactivity in immunology, the reaction of an antibody with an antigen other than that which elicited its formation, as a result of shared, similar, or identical antigenic determinants;

Note1: Within the context of this document, crossreactivity has two meanings. First, it refers to a human immunoglobulin $E$ (IgE) antibody that binds to an allergenic epitope that is structurally similar to, but not identical with, the molecule that elicited its formation. Crossreactivity results from shared, similar, or identical allergenic determinants (allergen-related cross-reactivity). There are many illustrations of cross-reactive allergen moleculesfor example, among the Hymenoptera (vespid) venoms. Clinical cross-reactivity refers to the degree (percentage) of clinical reactions after exposure to taxonomically related allergen sources (eg, allergic reactions to goat's milk in case of clinically proven IgE-mediated allergy to cow's milk proteins).

Note 2: Second, cross-reactivity can refer to the degree to which the monoclonal or polyclonal antihuman IgE detection reagents bind to other human immunoglobulin isotypes (immunoglobulin $G$, immunoglobulin $A$, immunoglobulin $M$, immunoglobulin D)) (antibody-related cross-reactivity).

epitope/determinant the minimum molecular structure of the antigenic site that will react with a monoclonal antibody; any site on an antigen molecule at which an antibody can bind; the chemical structure of the site determining the specific combining antibody;

Note: In the context of immunoglobulin E (IgE) assays, allergenic epitopes are regions on allergens that bind directly to the IgE antibody binding site. They can be detected by monoclonal antibodies to 1) determine the level of allergens of a particular specificity in an environmental specimen (eg, Der $p 1$ and Der $f 1$ in house dust); and 2) demonstrate identity and qualify extracts during the manufacturing of allergen-containing reagents.

free immunoglobulin $E$ (IgE) human IgE that circulates in blood in an unbound state, free of therapeutically administered humanized immunoglobulin $\mathrm{G}$ anti-lgE, soluble IgE receptors, or other binding factors;

Note 1: Research assays for free lgE that use the alpha chain of the high affinity FcER1 (fragment crystallizableepsilon receptor 1) as the IgE detection reagent have been studied but they are not commercially available;

Note 2: See immunoglobulin $E$.

immunoglobulin $E$ (IgE) human $\operatorname{lgE}$ is an immunoglobulin of the approximate molecular weight of 190 000, which exists normally in monomeric form and constitutes approximately $0.0005 \%$ of the total serum immunoglobulins;

Note: It binds with high affinity to FceR1 (fragment crystallisable-epsilon receptor 1) mainly expressed on mast 
cells and basophils and low affinity FcER2 receptors on a number of other cells. IgE mediates the production and release of vasoactive mediators following the binding of allergen.

immunoglobulin a glycoprotein composed of two heavy and two light chains that functions as an antibody;

Note 1: Human immunoglobulins have been subdivided into different classes or isotypes (immunoglobulin $M$, immunoglobulin G (IgG)1, IgG2, immunoglobulin G3, immunoglobulin G4, immunoglobulin A (IgA)1, $\lg A 2$, immunoglobulin $D$, immunoglobulin $E$ [IgE]), each of which possesses a unique set of antigenic markers, physiochemical properties, and each of which produce a different pattern of effector functions (receptor binding, complement activation, opsonization). All antibodies are immunoglobulins, but it is not certain that all immunoglobulins possess antibody function;

isoallergens proteins (allergens) that are essentially identical except for minor differences in their primary amino acid composition or substituted side chains

Limit of detection (LoD)/minimum detectable dose lowest amount of analyte in a sample that can be detected with (stated) probability and defined accuracy (see also definition of analytical sensitivity)

Limits of Blank, Limits of Quantitation (LoQ) lowest amount of a measurand in a material that can be quantitatively determined with stated accuracy (as total error or as independent requirements for bias and precision), under stated experimental conditions.

major allergen generally regarded as an allergenic molecule to which $>50 \%$ of clinically allergic patients with an allergy to its source react.

minor allergen generally regarded as an allergenic molecule to which $<50 \%$ of clinically allergic patients with an allergy to its source react.

molecule-based allergy diagnosis (previously referred to as "Component resolved diagnosis") diagnosis of human allergy risks by evaluation of immunoglobulin $\mathrm{E}$ antibody-based sensitization profiles to individual allergen molecules (eg, Amb a 1 , first identified major allergen in common ragweed pollen);

Note 1: Molecule-based allergy diagnosis allows distinction of sensitization to primary (genuine) sensitizing allergens (eg, Bet $v 1$, major allergen from birch pollen) from secondary or cross-reactivity sensitization produced by panallergens (eg,
Bet $v 2$, profilin from birch pollen; Bet $v 4$, polcalcin from birch pollen);

Note 2: Due to the misleading definitions of "component," "component resolved diagnosis" has been relabeled as molecule-based allergy diagnosis.

multiplex assay a test delivering more than one test result based on a single addition of a test specimen; Note: Multiplex assays can be discriminated from singleplex assays where one addition of a specimen results in immunoglobulin $E$ (IgE) antibody measurement to one allergen specificity. A multi-allergen screen is not a multiplex assay despite the fact that it has been shown by the manufacturer to detect IgE antibody to each of the individual allergens in a mixture with a single serum addition. This is because the multiple allergens are in one allergen-containing reagent and the multi-allergen screen generates a single result, which defines it as a singleplex assay.

native allergenic molecules allergenic proteins, glycoproteins, and lipoproteins that have been purified by chemical, chromatographic, electrophoretic and/or immunoaffinity techniques from allergen extracts of natural biological materials and that can elicit an IgE antibody response.

omalizumab for asthma and allergy patients who receive subcutaneous injections of omalizumab, which is a humanized immunoglobulin G1-kappa antihuman immunoglobulin $E$ (IgE) fragment crystallizable $(\mathrm{Fc})$ therapy, their IgE will exist in two states: free IgE (unbound with omalizumab) and complexed omalizumab-bound IgE, which is blocked by the anti-lgE from binding to the alpha chain of the high affinity FceR1 (Fc-epsilon receptor 1 );

Note: Caution should be exercised when analyzing blood from allergy patients receiving omalizumab, since the therapeutically administered anti-IgE can alter the accuracy of some total and allergen-specific lgE assays.

qualitative assay an assay system that produces an indication of the presence or absence of an analyte but does not provide a precise estimate of the concentration of that analyte; a positive test result implies only that the assay signal exceeds the analytical threshold or positive cutoff point that has been set to obtain an arbitrary combination of diagnostic sensitivity and specificity;

Note: A positive assay signal should relate to a certain presence of immunoglobulin $E$ antibody specific to the allergen tested in the subject's blood. 
quantitative assay an assay system that produces an accurate and reproducible estimate of the concentration ofananalyte, suchasimmunoglobulin $\mathrm{E}$ (IgE) antibody, in the test specimen; its analysis can use homologous or heterologous interpolation from a calibration curve, which is referenced to a readily available standard reference preparation;

radioallergosorbent test (RAST) the acronym "RAST" should now be considered a historic term, because radioisotopes are rarely used today in clinical assays for immunoglobulin E (IgE) antibody; Note: In 1967, the RAST was developed as the first immunoassay system available for the measurement of human IgE antibodies to defined allergen specificities in human serum. In its original form, the RAST used a paper disc solid phase to which allergen was covalently attached (allergosorbent) to bind allergen-specific antibodies of all isotypes (primarily lgE, immunoglobulin $\mathrm{G}$, immunoglobulin A) from serum. Following a buffer wash to remove unbound serum proteins, bound IgE was detected with 1125-labeled polyclonal antihuman IgE. Results were reported in logrelated classes or in arbitrary units by interpolation from a heterologous IgE antibirch pollen reference curve. Many minor modifications of this original assay format have been commercialized and they are discussed in the text. When referring to serological lgE antibody assays, the term RAST should no longer be used as a generic descriptor to refer to an IgE antibody assay unless it describes a radioisotopic allergosorbent assay for human IgE antibody.

recombinant allergen molecules an allergenic molecule is produced in a heterologous cell system using a recombinant gene construct encoding the allergenic protein;

Note: A description of the process of cloning, sequencing, transfecting expression vectors, and expressing allergenic peptides and proteins is beyond the scope of I/LA20. Some immunoglobulin $E$ antibody assays currently use recombinant allergens to supplement natural allergen extracts and replace labile, low abundance, or absent allergens (eg, rHev b 5 in the Hevea brasiliensis latexcontaining reagent).

semi-quantitative assay a semiquantitative assay system provides an additional option over the qualitative assay in terms of defining the magnitude of the response. The variations in the positive signal detected by the assay are commonly presented in terms of a series of increasing grades or classes (eg, I to VI, low to high); in arbitrarily defined units per milliliter determined relative to a supplierspecific heterologous dose-response curve or an end-point dilution at which the assay signal becomes negative (eg, titer); or in comparison to a qualitative grading scheme (eg, color chart);
Note: Allergen-specific immunoglobulin $E$ assays that use normalized counts generated in two-point calibrated, modified, or alternative scoring systems are considered semiquantitative assays unless demonstrated otherwise. Classes, grades, or modified scoring units are considered historical reporting schemes that are out of date and should thus not be used. 


\section{APPENDICES}

\section{ABBREVIATIONS AND ACRONYMS}

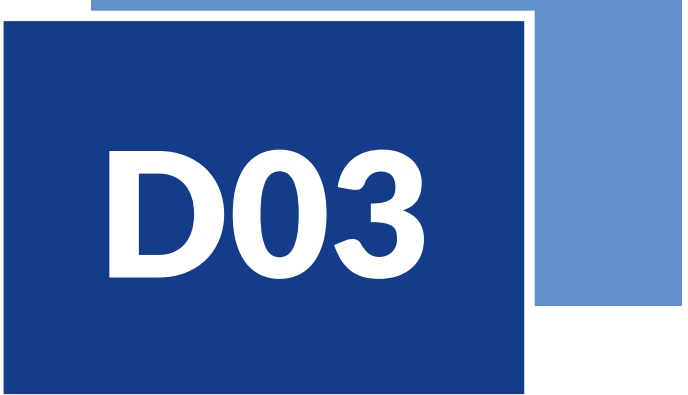

$\begin{array}{llll}\text { AAI } & \text { Adrenalin Autoinjector } & \text { BP } & \text { Birch Pollen } \\ \text { AAI } & \text { Alpha-Amylase Inhibitors } & \text { BSA } & \text { Bovine Serum Albumin } \\ \text { ABPA } & \text { Allergic Bronchopulmonary } & \text { Bt } & \text { Blomia tropicalis } \\ & \text { Aspergillosis } & \text { CCD(s) } & \begin{array}{l}\text { Cross-reactive Carbohydrate } \\ \text { Determinant(s) }\end{array} \\ \text { ACEI } & \begin{array}{l}\text { Angiotensin Converting Enzyme } \\ \text { Inhibitors }\end{array} & \text { CF } & \text { Cystic Fibrosis } \\ \text { AD } & \text { Atopic Dermatitis } & \text { CLSI } & \text { Clinical Laboratory Standards Institute } \\ \text { AEC } & \text { Absolute Eosinophil Count } & \text { CM } & \text { Cow's milk } \\ \text { aGAL } & \text { Alpha-GAL } & \text { CMA } & \text { Cow's Milk Allergy } \\ \text { AIT } & \text { Allergen Specific Immunotherapy } & \text { CRD } & \text { Component Resolved Diagnostics } \\ \text { AK } & \text { Arginine kinase } & \text { CRP } & \text { Carbohydrate-Rich Protein } \\ \text { AllFam } & \text { Allergen protein Families } & \text { CSBP } & \text { Cytokinin-Specific Binding Proteins } \\ \text { AR } & \text { Allergic Rhinitis } & \text { CT } & \text { Computed Tomography } \\ \text { ASA } & \text { Acetylsalicylic Acid } & \text { CXCL } & \text { Chemokine (C-X-C motif) Ligand 8 } \\ \text { BAMSE } & \text { Swedish abbreviation for Children, } & \text { DBPCFC } & \text { Double-Blind Placebo Controlled Oral } \\ & \text { Allergy, Milieu, Stockholm, } & \text { Food Challenge }\end{array}$


DPP IV Dipeptidylpeptidases IV EAACI European Academy of Allergy and
Clinical Immunology

EASI Eczema Area and Severity Index

EC Environmental Control

ECRHS European Community Respiratory Health Survey

EF-hand Conserved domain consisting of a 12 residue calcium-binding loop flanked on both sides by $a$-helices of 12 residues in length

EIA Exercise-Induced Anaphylaxis

ELISA Enzyme-Linked Immunosorbent Assay

EMD Emergency Department

EoE Eosinophilic Esophagitis

EPIT Epicutaneous Immunotherapy

ER Emergency Room

FA Food Allergy

FAST Food Allergy Specific ImmunoTherapy Here:

Title of an EU-project that aims at the development of safe and effective treatment of food allergies

FceRI High affinity receptor for the Fc part of IgE

FDEIA Food-Dependent Exercise-Induced Anaphylaxis

FPIES Food Protein-induced Enterocolitis Syndrome

fuc Fucose

GA²LEN Global Allergy and Asthma European Network

GCP Good Clinical Practices

GlcNAc N-acetylglucosamine

GMP Good Manufacturing Practices

GP Grass Pollen

GST Glutathione-S-Transferase

HB Honeybee

HBV Honeybee Venom

HCW Health-Care Workers

HDM House Dust Mite
HEPA High-Efficiency Particulate Arrestance

HMW High Molecular Weight

HRP horseradish peroxidase

HSA Human Serum Albumin

HWP Hydrolyzed Wheat Protein

Ig Immunoglobulin

IL (4....) Interleukin (4...)

ISAC Immune Solid phase Allergen Chip

ISU ISAC Standardized Units

IT Immunotherapy

IU International Unit

IUIS International Union of Immunological Societies

LAA Laboratory animal allergy

LAR Local Allergic Rhinitis

LLR Local reaction?

LMW Low Molecular Weight

LoB Limit of Blank

LoD Limit of Detection

LoQ Limit of Quantitation

LS lactose synthase

LTP Lipid Transfer Protein

MA Molecular based Allergy

man Mannose

MD2 Myeloid Differentiation Factor 2

MeDALL Mechanisms of the Development of ALLergy

MHC Major Histocompatibility Complex

MLC Myosin light chain

MLC Myosin Light Chain

MLP Major Latex Proteins

MnSOD Manganese-dependent Superoxide Dismutase

MSP Male-specific Submaxillary Gland Protein

MUP Major Urinary Proteins

MUXF3 Carbohydrate epitope which is found in many plant proteins (see "Glossary" for more information) 


\begin{tabular}{|c|c|c|c|}
\hline MW & Molecular Weight & SCIT & $\begin{array}{l}\text { Subcutaneous Injection } \\
\text { immunotherapy }\end{array}$ \\
\hline N.A. & Not Applicable & SDAP & $\begin{array}{l}\text { Structural Database of Allergenic } \\
\text { Proteins }\end{array}$ \\
\hline NCBI & $\begin{array}{l}\text { National Center for Biotechnology } \\
\text { Information }\end{array}$ & SIT & Specific Immunotherapy \\
\hline ND & Not Determined & slgE & Specific Immunoglobulin E \\
\hline NMR & Nuclear Magnetic Resonance & SLIT & Sublingual Immunotherapy \\
\hline NPC2 & Niemann-Pick disease, type C2 & SOB & Shortness Of Breath \\
\hline NPV & Negative Predictive Value & SOTI & Specific Oral Tolerance Induction \\
\hline NRL & Natural Rubber Latex & SPT & Skin Prick Tests \\
\hline NSAID & Nonsteroidal Anti-Inflammatory Drugs & SR & Systemic Reaction \\
\hline NSB & Non-Specific Binding & TH1 & T Helper 1 Cell \\
\hline nsLTP & Non-specific Lipid Transfer Protein & TH2 & T Helper 2 Cell \\
\hline OAS & Oral Allergy Syndrome & TIM & Triose phosphate isomerase \\
\hline OEFC & Open Exercise Food Challenge & $\operatorname{tlg} E$ & total Immunoglobulin E \\
\hline OFC & Oral Food Challenge & TLR & Toll-Like Receptor \\
\hline OIT & Oral Immunotherapy & TM & Tropomyosin \\
\hline PAMPS & $\begin{array}{l}\text { Pathogen Associated Molecular } \\
\text { Patterns }\end{array}$ & $\begin{array}{l}\text { TnC } \\
\text { TNF }\end{array}$ & $\begin{array}{l}\text { Troponin C } \\
\text { Tumor Necrosis Factor }\end{array}$ \\
\hline PAR & Protease-Activated Receptor & TVP & Textured Vegetable Protein \\
\hline PBMC & Peripheral Blood Mononuclear Cell & UCSF & University of California, San Francisco \\
\hline PDB & Protein Databank & UV & Ultraviolet \\
\hline Pfam & Protein family & VIT & Venom Specific Immunotherapy \\
\hline POCT & Point-Of-Care Testing & VV & Vespid Venom \\
\hline $\begin{array}{l}\text { PPT } \\
\text { PPV }\end{array}$ & $\begin{array}{l}\text { Prick by Prick Test } \\
\text { Positive Predictive Value }\end{array}$ & WDEIA & $\begin{array}{l}\text { Wheat-Dependent, Exercise-Induced } \\
\text { Anaphylaxis }\end{array}$ \\
\hline $\begin{array}{l}\text { PR-10 }(2 \\
3,5,14)\end{array}$ & $\begin{array}{l}\text { Pathogenesis Related Protein Group } \\
10(2,3,5,14)\end{array}$ & $\begin{array}{l}\text { WHO } \\
\text { YJ }\end{array}$ & $\begin{array}{l}\text { World Health Organization } \\
\text { Yellow Jacket }\end{array}$ \\
\hline PSA & Porcine Serum Albumin & YJV & Yellow Jacket Venom \\
\hline PW & Paper Wasp & & \\
\hline RAST & Radioallergosorbent Test & & \\
\hline REF & Rubber Elongation Factor & & \\
\hline ROC & Receiver Operating Characteristics & & \\
\hline RRP & Ripening-Related Proteins & & \\
\hline SAFE & $\begin{array}{l}\text { European Study on Plant Food } \\
\text { Allergies (Full title: 'Plant food } \\
\text { allergies: field to table strategies for } \\
\text { reducing their incidence in Europe') }\end{array}$ & & \\
\hline SCBP & Sarcoplasmic calcium binding protein & & \\
\hline
\end{tabular}





\section{APPENDICES}

\section{ACKNOWLEDGEMENTS}
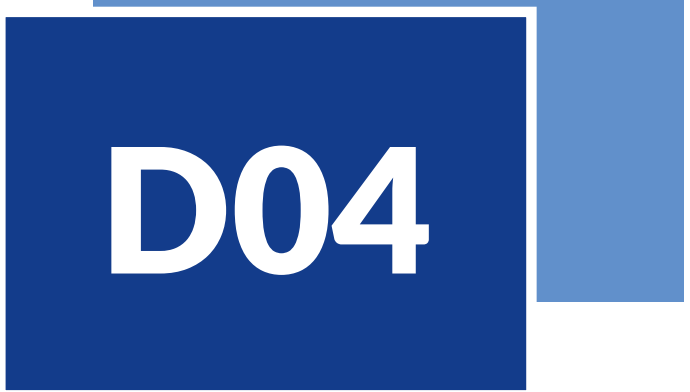

We are profoundly indebted with all the colleagues and coworkers who have contributed to the work of the EAACI Task Force promoting this document. In particular, we wish to thank the team of the Working Group on Molecular Allergology and Immunomodu-lation, Department of Pediatric Pneumology and Immunology, Charitè Medical University, Berlin, Germany, composed by Carla Mastrorilli (MD), Serena Perna (St Sc), Olympia Tsilochristou (MD), Alina Schwarz (Cand. med.) and Eissa Hakimeh, for their contribution in the organization of the Workshop on Molecular Alleregology held in Berlin on 13-15 April 2015. We also wish to thank Alessandro D'Onofrio for his contribution to the preparation of the manuscript.

We wish to acknowledge the support by the Austrian Science Fund grant SFB F4608. We would like to thank Araceli Diaz-Perales, from ETSIA UPM, Madrid, for providing the figure on melon profilin in Section C01, as well as Figure 1 in Section C03. For Figure 2 in Chapter CO3 we would like to thank. Elide Pastorella and DomingBarber would like to thank Claus Lundegaard, Jens Holm and Thomas Stranzl, ALK-Abello A/S, Denmark. 



\section{EAACI Headquarters}

Hagenholzstrasse 111

3rd Floor

8050 Zurich

Switzerland

Tel: +41442055533

Fax: +41442055539

info@eaaci.org

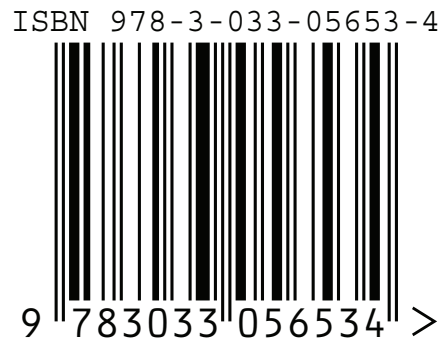

